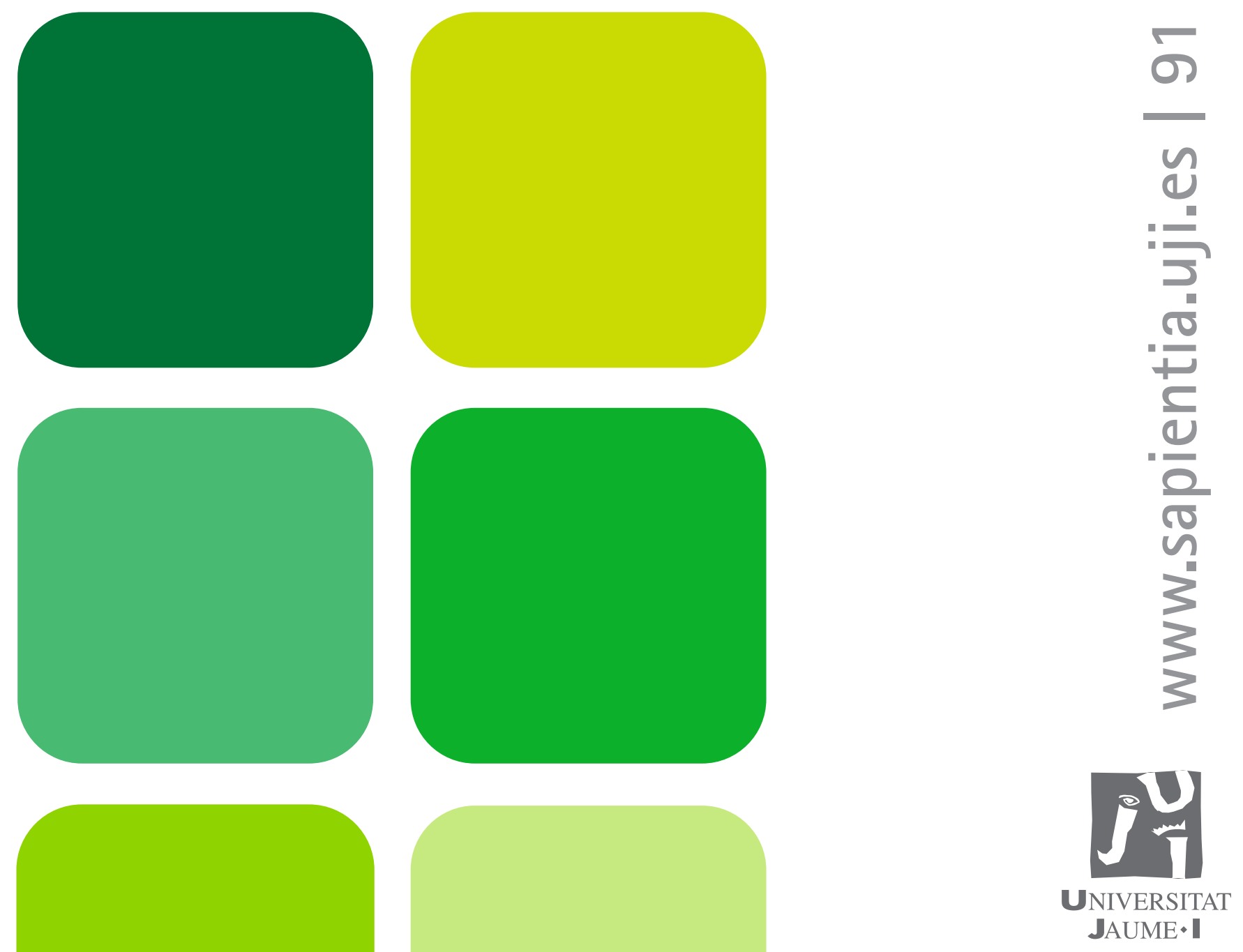

Reactores químicos y bioquímicos

A. Barba Juan

C. Clausell Terol 


\title{
Reactores químicos y bioquímicos
}

\author{
A. Barba Juan \\ C. Clausell Terol
}

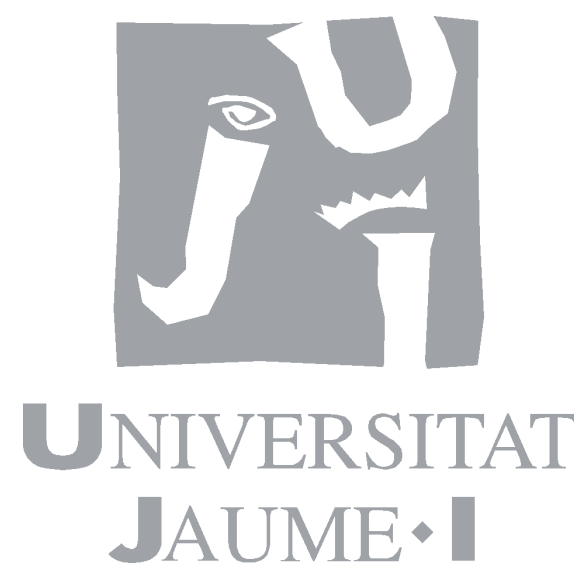

Departament d'Enginyeria Química

- Codi d'assignatura E01030 
Edita: Publicacions de la Universitat Jaume I. Servei de Comunicació i Publicacions Campus del Riu Sec. Edifici Rectorat i Serveis Centrals. 12071 Castelló de la Plana http://www.tenda.uji.es e-mail: publicacions@uji.es

Col·lecció Sapientia 91

www.sapientia.uji.es

Primera edició, 2014

ISBN: 978-84-695-9787-3

11 Publicacions de la Universitat Jaume I és una editorial membre de l'UNE, cosa que en garanteix la difusió de les obres en els àmbits nacional i inter-

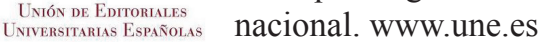

\section{(c) (i) (2)}

Reconeixement-CompartirIgual

\section{BY-SA}

Aquest text està subjecte a una llicència Reconeixement-CompartirIgual de Creative Commons, que permet copiar, distribuir i comunicar públicament l'obra sempre que s'especifique l'autor i el nom de la publicació fins i tot amb objectius comercials i també permet crear obres derivades, sempre que siguen distribuïdes amb aquesta mateixa llicència.

http://creativecommons.org/licenses/by-sa/3.0/legalcode 


\section{ÍNDICE}

Prólogo

Capítulo 1. Balances de energía en reactores químicos de flujo ideal . . . . .

1.1. Reactores químicos de flujo ideal $\ldots \ldots \ldots \ldots \ldots \ldots \ldots$

1.2. Balance general de energía en sistemas con reacción química ......

1.3. Balance general de energía en reactores de tanque agitado ........

1.3.1. Reactor continuo de tanque agitado en régimen no estacionario (RCTA) y reactor semicontinuo (RSCTA) . . . . . . . . . .

1.3.2. Reactor continuo de tanque agitado en régimen estacionario

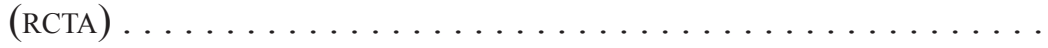

1.3.3. Reactor discontinuo de tanque agitado (RDTA) . . . . . . . .

1.4. Balance general de energía en reactores continuos tubulares de flujo de pistón (RFP)

Anexo I. Variables de composición y cálculo de la composición ..........

Anexo II. Balances de la materia . . . . . . . . . . . . . . . .

Anexo III. Balances de la energía

Anexo IV. Algunas consideraciones sobre el calor específico . ............

Problemas

Capítulo 2. Reactor continuo de tanque agitado (RCTA) con intercambio de calor y adiabático

2.1. Ecuaciones de diseño

2.2. Intercambio de calor

2.2.1. Dispositivos de intercambio de calor de temperatura constante

2.2.2. Dispositivos de intercambio de calor de temperatura variable

2.2.3. Sin dispositivos de intercambio de calor

2.2.4. Generalización de la ecuación de diseño (2)

2.3. Resolución de las ecuaciones de diseño

2.3.1. Método de Levenspiel

2.3.2. Método de Aris .

2.4. Estabilidad del RCTA

2.5. Sistemas en los que se desarrollan varias reacciones químicas .....

2.6. Temperatura de reacción óptima $\ldots \ldots \ldots \ldots \ldots \ldots \ldots \ldots$

Problemas 
Capítulo 3. Reactor discontinuo de tanque agitado (RDTA) intermedio y adiabático

3.1. Ecuaciones de diseño

3.2. Intercambio de calor

3.3. Resolución de las ecuaciones de diseño para un RDTA adiabático . ...

3.4. Resolución de las ecuaciones de diseño para un RDTA intermedio . . 3.4.1. RDTA intermedio a densidad de flujo de calor $q$ constante ..... 3.4.2. RDTA intermedio a $\mathrm{U} \mathrm{y}_{\mathrm{f}}$ constantes $\ldots \ldots \ldots \ldots \ldots \ldots \ldots$

3.5. Ciclo óptimo de temperatura de reacción $\ldots \ldots \ldots \ldots \ldots \ldots \ldots$ 3.5.1. Sistemas con una reacción química

3.5.2. Sistemas con varias reacciones químicas

3.6. RSCTA no isotermo

Problemas

Capítulo 4. Reactor continuo tubular de flujo de pistón (RFP) intermedio y adiabático

4.1. Ecuaciones de diseño

4.2. Intercambio de calor

4.3. Resolución de las ecuaciones de diseño para un RFP adiabático . . . . .

4.4. Resolución de las ecuaciones de diseño para un RFP intermedio . .... 4.4.1. RFP intermedio a densidad de flujo de calor $q$ constante . . . . . 4.4.2. RFP intermedio a U constante .

4.5. Perfil óptimo de temperatura de reacción

4.5.1. Sistemas con una reacción química . . . . . . . . . . .

4.5.2. Sistemas con varias reacciones químicas .............

Anexo I

Problemas

Capítulo 5. Asociación de reactores continuos

5.1. Asociación de RCTA . . . . . . . . . . . . . . . . . . . .

5.1.1. Cálculo del volumen de una serie de RCTA del mismo tamaño por el método de Aris . . . . . . . . . . . . . . . . . . .

5.1.2. Cálculo del volumen de una serie de RCTA de diferente tamaño por el método de Jones

5.1.3. Cálculo del volumen óptimo de cada RCTA de una serie . . . . .

5.1.4. Sistemas con varias reacciones químicas ...............

5.2. Asociación de RFP . . . . . . . . . . . . . . . . .

Anexo I. Balance de materia en asociaciones de RCTA . . . . . . . . . .

Anexo II. Balance de energía en asociaciones de RCTA . . . . . . . . . .

Anexo III. Balance de materia en asociaciones RFP . . . . . . . . . . .

Anexo IV. Balance de energía en asociaciones RFP . . . . . . . . . . .

Problemas

Capítulo 6. Consideraciones sobre el funcionamiento de reactores continuos adiabáticos

6.1. Curvas de velocidad de reacción constante ...............

6.2. Sistemas de un RCTA 
6.3. Secuencia de RCTA

6.4. Sistemas de un RFP

6.5. Secuencia de RFP

Problemas

Capítulo 7. Reactores bioquímicos. Fermentadores . .

7.1. Procesos celulares

7.2. Crecimiento celular

7.3. Estequiometría: coeficientes de rendimiento estequiométrico

7.4. Cinética microbiana

7.4.1. Ecuación de velocidad para el crecimiento celular

7.4.2 Ecuación de velocidad para el mantenimiento celular .......

7.4.3. Ecuación de velocidad para la muerte celular . . . . . . . . . .

7.4.4. Ecuación de velocidad para la formación de producto .......

7.4.5. Ecuación de velocidad para la desaparición de sustrato .......

7.5. Reactores bioquímicos: características y tipos de fermentadores . . . .

7.5.1. Fermentadores de Tanque Agitado (FTA) . . . . . . . . . .

7.5.2. Fermentadores Continuos Tubulares de Flujo de Pistón (FFP)

7.6. Balance de materia de fermentadores de tanque agitado (FTA) . . . . .

7.6.1. Fermentador discontinuo de tanque agitado (FDTA) . ........

7.6.2. Fermentador continuo de tanque agitado (FCTA), o quimiostato, en régimen estacionario y estéril

7.6.3. Fermentador continuo de tanque agitado (FCTA), o quimiostato, en régimen estacionario y estéril, con envenenamiento por producto

7.6.4. Fermentador continuo de tanque agitado (FCTA), o quimiostato, en régimen estacionario y con recirculación (no estéril)

7.7. Balance de materia de fermentadores continuos tubulares de flujo de pistón (FFP) con flóculos en suspensión

7.7.1. Fermentador continuo tubular de flujo de pistón (FFP) con flóculos en suspensión, en régimen estacionario

7.7.2. Fermentador continuo tubular de flujo de pistón (FFP) con flóculos en suspensión, en régimen estacionario, con envenenamiento por producto . . . . . . . . . . .

Anexo I. Cinética enzimática . . . . . . . . . . . . . . . . . Problemas

Bibliografía básica

Bibliografía complementaria 


\section{Prólogo}

Este libro, titulado Reactores químicos y bioquímicos, está concebido para los estudiantes de la Universitat Jaume I que cursan el tercer año del Grado en Ingeniería Química. En el segundo semestre de ese año deben cursar la asignatura obligatoria EQ1030-Reactores Químicos y Bioquímicos de 6 European Credit System Transfer (ECTS).

La asignatura EQ1030-Reactores Químicos y Bioquímicos está encuadrada en la materia ingeniería de la reacción química, y se imparte en el $2 .^{\circ}$ semestre del 3 . $^{\text {er }}$ curso del Grado en Ingeniería Química. En el 1. ${ }^{\text {er }}$ semestre del mismo curso se ha impartido la asignatura EQ1021-Ingeniería de la Reacción Química, que junto con la anterior asignatura componen la materia citada.

La Ingeniería Química estudia aquellos procesos en los que al menos en una de las operaciones unitarias que los constituyen se transforma la materia mediante una o varias reacciones químicas. Esta transformación se desarrolla en el interior de un dispositivo conocido como reactor químico. Por lo tanto, es fundamental que el alumno conozca los diferentes tipos de reactores químicos así como las leyes que los gobiernan: balance de materia, balance de energía, velocidad de reacción química y velocidad de transferencia de calor.

El objetivo es dotar al alumno de las herramientas necesarias para diseñar un reactor químico y para determinar las condiciones óptimas de su funcionamiento.

Para cursar con éxito la asignatura EQ1030-Reactores Químicos y Bioquímicos se recomienda haber superado las asignaturas EQ1013-Termodinámica Aplicada, EQ1015-Fundamentos de Ingeniería Química, EQ1019-Mecánica de Fluidos, EQ1020-Operaciones Básicas de Transmisión de Calor y, muy especialmente, EQ1021-Ingeniería de la Reacción Química (la otra asignatura que configura la materia).

En la asignatura EQ1021-Ingeniería de la Reacción Química el alumno debe haber adquirido unas competencias y unos conocimientos que son fundamentales para abordar el estudio de la asignatura EQ1030-Reactores Químicos y Bioquími$\cos$, ya que ambas asignaturas constituyen la primera y segunda parte de un cuerpo de conocimiento conocido en la literatura universal como ingeniería de la reacción química o ingeniería de reactores químicos.

En consecuencia, al iniciarse el estudio de la asignatura EQ1021-Reactores Químicos y Bioquímicos se recomienda que el alumno conozca los siguientes contenidos: modelo estequiométrico de un sistema reactante; cálculos de composición en los sistemas reactantes; fenomenología de las reacciones químicas e influencia de la composición y la temperatura sobre la velocidad de reacción; reactores ideales en condiciones isotermas: reactor de mezcla completa en operación continua, discontinua y semicontinua, reactor de flujo de pistón; sistemas de una sola reacción y 
de reacciones múltiples; y asociación de reactores continuos en condiciones isotermas.

Además el alumno debe saber: analizar, modelizar y calcular sistemas con reacción química en condiciones isotermas; determinar leyes de velocidad de reacción a partir de datos experimentales; aplicar los balances de materia a sistemas con reacción química isotermos; y resolver problemas de reactores químicos ideales, en condiciones isotermas.

El libro Reactores químicos y bioquímicos es el resultado de la experiencia de los autores que, durante los últimos 20 años, han venido impartiendo cursos de ingeniería de la reacción química y de reactores químicos. Pretende ser una herramienta de estudio del alumno que recoja los principales aspectos de los reactores químicos ideales no isotermos. Junto con los aspectos teóricos el alumno encontrará en cada capítulo una amplia colección de problemas que le ayudará a analizar, modelizar y calcular sistemas con reacción química no isotermos.

\author{
A. Barba Juan, C. Clausell Terol \\ Castellón, 2014
}




\section{CAPÍTULO 1}

\section{Balances de energía \\ en reactores químicos de flujo ideal}

\subsection{Reactores químicos de flujo ideal}

Existe una gran variedad de reactores químicos, pero todos ellos se pueden clasificar atendiendo a unos pocos criterios. De esta manera se puede sistematizar el conocimiento.

Los criterios básicos de clasificación son:

a) Según el modo de operación los reactores se clasifican en discontinuos (o intermitentes), semicontinuos y continuos, atendiendo a si las fases de operación (carga del reactor, reacción química y descarga del reactor, así como limpieza del mismo si se requiere) se realizan de forma simultánea o secuencial.

Los reactores intermitentes o discontinuos funcionan en régimen no estacionario, ya que la composición y las propiedades físicas de la mezcla de reacción varían con el tiempo de reacción. Se suelen utilizar para llevar a cabo reacciones a pequeña escala, para investigar nuevos procesos químicos, para la fabricación de pequeñas cantidades de producto y para la fabricación de productos químicos de alto valor añadido. Estos reactores presentan como características positivas su gran flexibilidad de operación y la relativamente baja inversión inicial requerida.

Los reactores continuos funcionan en régimen estacionario (tras un periodo de puesta en marcha inicial con comportamiento no estacionario), ya que la composición y las propiedades físicas de la mezcla de reacción en ningún caso varían con el tiempo. Se utilizan para llevar a cabo reacciones a gran escala y resulta relativamente sencillo establecer sistemas de control y automatización que permiten mantener la constancia de las condiciones de operación, sin embargo se requieren elevadas inversiones iniciales.

b) Según el modelo de flujo y contacto de la mezcla de reacción los reactores se clasifican en reactores de mezcla completa o perfecta, también denominados de tanque agitado, y reactores continuos tubulares de flujo de pistón. 
Combinando los criterios de modo de operación y modelo de flujo y contacto de la mezcla se obtienen cuatro tipos de Reactores Químicos de Flujo Ideal:

- Reactor Discontinuo de Tanque Agitado (RDTA) o Batch Reactor (figura 1)

- Reactor Semicontinuo de Tanque Agitado (RSCTA) o Semibatch Reactor (figura 2)

- Reactor Continuo de Tanque Agitado (RCTA) o Continuous-Stirred Tank Reactor (CSTR) or Backmix Reactor (figura 3)

- Reactor Continuo Tubular de Flujo de Pistón (RFP) o Plug-Flow Tubular Reactor (PFR) (figura 4)

En la asignatura previa EQ1021-Ingeniería de la Reacción Química que, junto con esta asignatura EQ1030-Reactores Químicos y Bioquímicos, constituye la materia Ingeniería de la Reacción Química, ya se ha explicado el funcionamiento isotermo de estos cuatro tipos de reactores. A continuación se muestran resumidamente las características fundamentales de funcionamiento de los Reactores Químicos de Flujo Ideal:

a) RDTA

- Agitación perfecta: $\forall, \mathrm{t}, \mathrm{c}_{\mathrm{j}}$ y $\mathrm{T}$ son uniformes en todo el volumen

- Régimen no estacionario: $c_{j}=f(t)$

- Intercambio de calor:

- Adiabático: $\mathrm{T}=\mathrm{f}(\mathrm{t})$

- No adiabático:

- Isotermo: $\mathrm{T} \neq \mathrm{f}(\mathrm{t})=$ constante

- No isotermo: $\mathrm{T}=\mathrm{f}(\mathrm{t})$

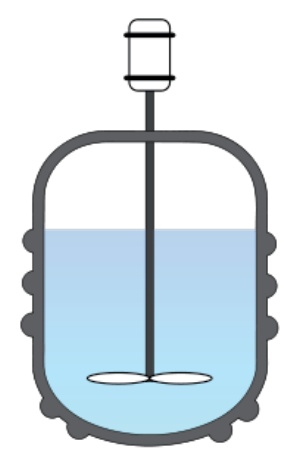

Figura 1. Reactor Discontinuo de Tanque Agitado (RDTA) o Batch Reactor

b) RSCTA

- Agitación perfecta: $\forall \mathrm{t}, \mathrm{c}_{\mathrm{j}} \mathrm{y}$ T son uniformes en todo el volumen

- A la entrada y a la salida $Q_{V}, T$ y $c_{j}$ no son necesariamente constantes

- Régimen no estacionario: $\mathrm{c}_{\mathrm{j}}=\mathrm{f}(\mathrm{t})$

- Intercambio de calor:

- Adiabático: $\mathrm{T}=\mathrm{f}(\mathrm{t})=$ constante

- No adiabático:

- Isotermo: $\mathrm{T} \neq \mathrm{f}(\mathrm{t})$

- No isotermo: $\mathrm{T}=\mathrm{f}(\mathrm{t})$ 


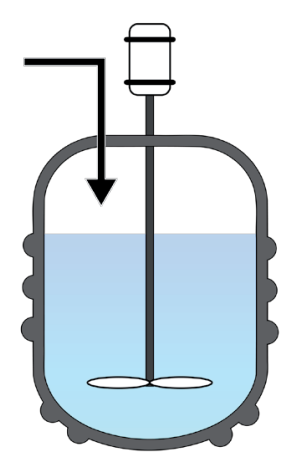

Figura 2. Reactor Semicontinuo de Tanque Agitado (RSCTA) o Semibatch Reactor

c) RCTA (funcionando en régimen estacionario, ya que en régimen no estacionario su comportamiento es el de un RSCTA)

- Agitación perfecta: $\forall \mathrm{t}, \mathrm{c}_{\mathrm{j}} \mathrm{y} \mathrm{T}$ son uniformes en todo el volumen

- Régimen estacionario: $c_{j} \neq \mathrm{f}(\mathrm{t}), \mathrm{T} \neq \mathrm{f}(\mathrm{t})$

- A la entrada y a la salida $Q_{V}, T$ y c son constantes

- Intercambio de calor:

- Adiabático

- No adiabático

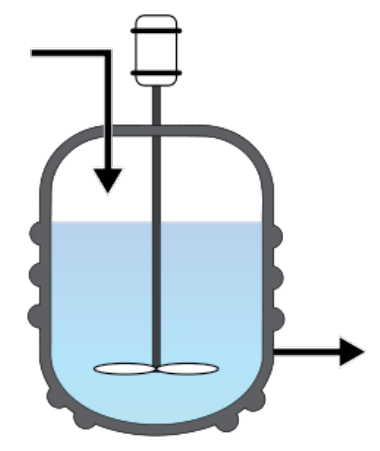

Figura 3. Reactor Continuo de Tanque Agitado (RCTA) o Continuous-Stirred Tank Reactor (CSTR) or Backmix Reactor

d) RFP

- No hay mezcla longitudinal

- Régimen estacionario: $\mathrm{c}_{\mathrm{j}} \neq \mathrm{f}(\mathrm{t}), \mathrm{c}_{\mathrm{j}}=\mathrm{f}(\ell)$

- A la entrada y a la salida $Q_{V}, T$ y c son constantes

- Intercambio de calor:

- Adiabático: $\mathrm{T}=\mathrm{f}(\ell)$ )

- No adiabático:

- Isotermo: $\mathrm{T} \neq \mathrm{f}(\ell)$

- No isotermo: $\mathrm{T}=\mathrm{f}(\mathrm{l})$ 


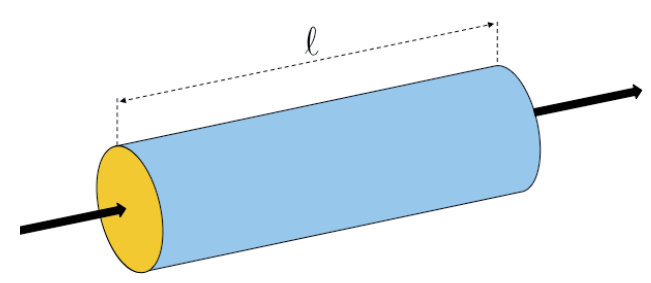

Figura 4. Reactor Continuo Tubular de Flujo de Pistón (RFP) o Plug-Flow Tubular Reactor (PFR)

Para diseñar o determinar las condiciones de funcionamiento de cualquiera de estos reactores es necesario conoce:

- la estequiometría

- las condiciones de equilibrio químico

- la ecuación de conservación de materia (balance de materia)

- la ecuación de velocidad de reacción

- la ecuación de conservación de energía (balance de energía)

- la ecuación de velocidad de transmisión de calor

En el Anexo I se recogen las variables más habituales para expresar la composición de un sistema con reacción química así como la forma de calcular la composición del sistema a medida que avanza la reacción. Los balances de materia se han descrito en profundidad en la asignatura EQ1021-Ingeniería de la Reacción Química, mostrándose en el Anexo II un resumen de los mismos.

Otros conceptos introducidos en la asignatura EQ1021-Ingeniería de la Reacción Química que conviene tener presente en la presente asignatura son, para los reactores continuos en régimen estacionario, el tiempo espacial y el tiempo de residencia.

Se define tiempo espacial el cociente entre el volumen de reacción y el caudal volumétrico medido a las condiciones de la corriente de entrada al reactor:

$$
\tau=\frac{V}{Q_{v_{0}}}(s)
$$

Dado que el caudal volumétrico está referido exclusivamente a las condiciones de la corriente de entrada, esta definición es independiente de que el fluido circulante sea de densidad constante (líquido y gas a $\mathrm{P}, \mathrm{T}$ constantes e $\Delta v=0$ ) o de densidad variable (gas a $\mathrm{P} y / \mathrm{o} \mathrm{T}$ variable constantes y/o $\Delta v \neq 0$ ). Asimismo, la definición es idéntica para cualquier reactor continuo: el reactor continuo de tanque agitado o el reactor de flujo de pistón.

El significado físico de este parámetro es el tiempo de reloj que una porción de la mezcla reactante pasaría en el reactor (tiempo que necesitaría para atravesar el volumen de reacción, desde la corriente de entrada hasta la corriente de salida). Obsérvese que el tiempo verbal utilizado es el condicional, puesto que el tiempo 
real de paso a través del volumen de reacción puede ser distinto si las condiciones de operación se modifican en dicho volumen.

Se define tiempo de residencia el cociente entre el volumen de reacción y el caudal volumétrico a lo largo del reactor. Dado que este caudal puede variar entre las condiciones de la corriente de entrada, del volumen de reacción y de la corriente de salida, se calcula de forma diferente para cada tipo de reactor continuo.

i) Caso del RCTA

Por la propia configuración del RCTA la corriente de salida representa una muestra exacta de las condiciones del volumen de reacción. En consecuencia, el tiempo de residencia se calcula como sigue:

$$
\theta=\frac{\mathrm{V}}{\mathrm{Q}_{\mathrm{v}}}(\mathrm{s})
$$

para fluidos de densidad constante (líquido y gas a $\mathrm{P}, \mathrm{T}$ constantes e $\Delta v=0$ ) coincide con el tiempo espacial:

$$
\theta=\frac{\mathrm{V}}{\mathrm{Q}_{\mathrm{v}}}=\frac{\mathrm{V}}{\mathrm{Q}_{\mathrm{v} 0}}=\tau \quad \text { (s) }
$$

para fluidos de densidad variable (gas a P y/o T variable y/o $\Delta v \neq 0$ ):

$$
\theta=\frac{v}{Q_{v}}=\frac{v}{Q_{v} \cdot \frac{P}{P} \cdot \frac{T}{T_{0}} \cdot\left(1+\varepsilon_{k} \cdot x\right)}=\frac{\tau}{\frac{P_{0}}{P} \cdot \frac{T}{T_{0}} \cdot\left(1+\varepsilon_{k} \cdot x\right)} \text { (s) }
$$

ii) Caso del RFP

Por la propia configuración del RFP la mezcla de reacción puede ir cambiando de condiciones a medida que avanza por el interior del reactor y hasta que alcanza la corriente de salida. En consecuencia, el tiempo de residencia se calcula como:

$d \theta=\frac{d V}{Q_{v}(\ell)}$ y considerando el balance de la materia $d \theta=F_{k 0} \cdot \frac{d X}{\left(-v_{k}\right) \cdot r(X) \cdot Q_{v}(X)}(s)$

para fluidos de densidad constante (líquido y gas a $\mathrm{P}, \mathrm{T}$ constantes e $\Delta v=0$ ) coincide con el tiempo espacial:

$$
\theta=\int_{0}^{v} \frac{d V}{Q_{v}(\ell)}=\int_{0}^{v} \frac{d V}{Q_{v_{0}}}=\frac{1}{Q_{v_{0}}} \cdot \int_{0}^{v} d V=\frac{V}{Q_{v_{0}}}=\tau(s)
$$


para fluidos de densidad variable (gas a $\mathrm{P} y / \mathrm{o} T$ variable y/o $\Delta v \neq 0$ ):

$$
\theta=F_{k 0} \cdot \int_{0}^{X} \frac{d X}{\left(-v_{k}\right) \cdot r(X) \cdot Q_{v}(X)}=\frac{F_{k 0}}{Q_{v 0}} \cdot \int_{0}^{X} \frac{d X}{\left(-v_{k}\right) \cdot r(X) \cdot \frac{P_{0}}{P} \cdot \frac{T}{T_{0}} \cdot\left(1+\varepsilon_{k} \cdot X\right)}(s)
$$

El significado físico del tiempo de residencia es único para todos los tipos de reactores continuos: es el tiempo de reloj que una porción de la mezcla reactante pasa en el reactor (tiempo que necesita para atravesar el volumen de reacción, desde la corriente de entrada hasta la corriente de salida). Obsérvese que el tiempo verbal utilizado es el presente, puesto que se trata del tiempo real de paso a través del volumen de reacción.

\subsection{Balance general de energía en sistemas con reacción química}

El balance general de energía para un sistema con reacción química se puede expresar de forma cualitativa mediante la siguiente igualdad:

$$
\begin{gathered}
\text { Salida }- \text { Entrada }+ \text { Acumulación }=\text { Generación }+ \text { Transmisión } \\
\qquad \mathrm{S}-\mathrm{E}+\mathrm{A}=\mathrm{G}+\mathrm{T}
\end{gathered}
$$

En primer lugar se harán algunas consideraciones sobre dos de estos términos, G y T:

i) Al tratarse de reactores químicos (en los que tienen lugar reacciones químicas) no existe generación de energía, por lo que $\mathrm{G}=0$. Sólo en el caso de reactores nucleares se podría producir la transformación de materia en energía.

ii) El término de transmisión indica la transferencia de energía, en forma de calor y trabajo, entre el sistema con reacción y los alrededores. Por lo tanto se puede expresar en la forma:

$$
\mathrm{T}=\text { calor }+ \text { trabajo }=\mathrm{Q}^{*}+\mathrm{W}^{*}(\mathrm{~kJ} / \mathrm{s})
$$

siendo la simplificación más habitual que el trabajo efectuado es despreciable frente al calor transferido:

$$
\mathrm{Q}^{*}>>>\mathrm{W}^{*}
$$

donde el trabajo y el calor son positivos si se transfieren de los alrededores al sistema y negativos en caso contrario. Este término de transmisión es independiente de la cantidad de materia presente en el sistema químico. 
Seguidamente, se harán unas consideraciones sobre los términos de salida, entrada y acumulación. Estos términos indican que la energía va asociada a la materia que entra, sale y se acumula en el reactor. En consecuencia conviene recordar las siguientes definiciones de las posibles energías asociadas a la materia:

- $\mathrm{e}_{\mathrm{p}}$ : energía potencial $(\mathrm{kJ} / \mathrm{kmol}$ totales $)$

- $\mathrm{e}_{\mathrm{k}}$ : energía cinética $(\mathrm{kJ} / \mathrm{kmol}$ totales $)$

- u: energía interna $(\mathrm{kJ} / \mathrm{kmol}$ totales $)$

- $\mathrm{P} / \rho_{\mathrm{m}}$ : energía de flujo, que considera los efectos de compresión y expansión del flujo que se pueden producir, respectivamente, a la entrada y salida del reactor $(\mathrm{kJ} / \mathrm{kmol}$ totales $)$

Por tanto la energía asociada a la materia es la suma de:

$$
\left(e_{p}+e_{k}+u\right)\left(\frac{k J}{k m o l \text { totales }}\right)
$$

que en el caso de que haya efecto de expansión y/o compresión hay que escribir como:

$$
\left(e_{p}+e_{k}+u+\frac{p}{\rho_{m}}\right)\left(\frac{k J}{\text { kmol totales }}\right)
$$

siendo esta última ecuación solo operativa en las puntos de entrada y salida del reactor. La simplificación y transformación más habitual es:

$$
e_{p}+e_{k} \ll<u+\frac{p}{\rho_{m}}
$$

y a partir de la termodinámica clásica:

$$
\begin{aligned}
& \mathrm{H}=\mathrm{U}+\mathrm{P} \cdot \mathrm{V} \quad(\mathrm{kJ}) \\
& \frac{\mathrm{H}}{\mathrm{N}_{\mathrm{t}}}=\frac{\mathrm{U}}{\mathrm{N}_{\mathrm{t}}}+\mathrm{P} \cdot \frac{\mathrm{V}}{\mathrm{N}_{\mathrm{t}}} \quad(\mathrm{kJ} / \text { kmoltotales }) \\
& \mathrm{h}=\mathrm{U}+\frac{\mathrm{P}}{\rho_{\mathrm{m}}}
\end{aligned}
$$

Por lo tanto, los términos del balance que se están comentando, al tener en consideración la energía asociada a la materia del sistema, se obtienen necesariamente de considerar la cantidad de materia indicada por el balance de materia (kmol totales/s) y multiplicarla por la energía asociada a esa materia $(\mathrm{kJ} / \mathrm{kmol}$ totales), obteniéndose de esa manera los términos del balance de energía $(\mathrm{kJ} / \mathrm{s})$.

A continuación se definirá el balance general de energía en sistemas con reacción química, que se puede construir mediante las siguientes igualdades: 


$$
\begin{aligned}
S-E & =\int_{A}\left(e_{p}+e_{k}+u+\frac{P}{\rho_{m}}\right) \cdot \rho_{m} \cdot(\vec{v} \cdot \vec{n}) \cdot d A\left(\frac{\mathrm{kJ}}{\mathrm{kmol} \text { totales }} \cdot \frac{\mathrm{kmol} \text { totales }}{\mathrm{s}}=\frac{\mathrm{kJ}}{\mathrm{s}}\right) \\
A & =\frac{\mathrm{d}}{\mathrm{dt}} \int_{V}\left(e_{p}+e_{k}+\mathrm{u}\right) \cdot \rho_{\mathrm{m}} \cdot d V\left(\frac{1}{\mathrm{~s}} \cdot \frac{\mathrm{kJ}}{\mathrm{kmol} \text { totales }} \cdot \frac{\mathrm{kmol} \text { totales }}{\mathrm{m}^{3}} \cdot \mathrm{m}^{3}=\frac{\mathrm{kJ}}{\mathrm{s}}\right)
\end{aligned}
$$

Teniendo en cuenta las consideraciones realizadas sobre los términos G y T:

$$
\int_{A}\left(e_{p}+e_{k}+u+\frac{P}{\rho_{m}}\right) \cdot \rho_{m} \cdot(\vec{v} \cdot \vec{n}) \cdot d A+\frac{d}{d t} \int_{V}\left(e_{p}+e_{k}+u\right) \cdot \rho_{m} \cdot d V=Q^{*} \quad(k J / s)
$$

así como sobre términos en que la energía va asociada a la materia (E, A y S), el balance general de energía queda como:

$$
\int_{A} h \cdot \rho_{m} \cdot(\vec{v} \cdot \vec{n}) \cdot d A+\frac{d}{d t} \int_{V}\left(h-\frac{P}{\rho_{m}}\right) \cdot \rho_{m} \cdot d V=Q^{*} \quad(k J / s)
$$

$$
\int_{A} d\left(F_{t} \cdot h\right)+\frac{d}{d t} \int_{V}\left(h \cdot \rho_{m}-P\right) \cdot d V=Q^{*} \quad(k J / s)
$$

Antes de establecer el balance de energía de cada reactor ideal conviene recordar algunos conceptos de la termodinámica química y definir algunos parámetros:

- Entalpía de una especie química $\mathrm{A}_{\mathrm{j}}$.

- En la corriente de entrada o en el tiempo inicial:

$$
h_{j 0}=\left(\Delta H_{f, j}^{0}\right)_{298}+\int_{298}^{T_{0}} C_{p j} \cdot d T \quad\left(k J / k m o l d e A_{j}\right)
$$

y en la corriente de salida, a lo largo del reactor o durante el tiempo de reacción:

$$
h_{j}=\left(\Delta H_{f, j}^{0}\right)_{298}+\int_{298}^{T} C_{p j} \cdot d T \quad\left(k J / k m o l ~ d e ~ A_{j}\right)
$$

aunque el calor específico es una función de la temperatura, si se toman valores medios del mismo, independientes de la temperatura, se simplifican notablemente las expresiones matemáticas:

$$
\begin{aligned}
& h_{j 0}=\left(\Delta H_{f, j}^{0}\right)_{298}+\int_{298}^{T_{0}} \bar{C}_{p j} \cdot d T=\left(\Delta H_{f, j}^{0}\right)_{298}+\bar{C}_{p j} \cdot\left(T_{0}-298\right) \quad\left(k J / k m o l ~ d e ~ A_{j}\right) \\
& h_{j}=\left(\Delta H_{f, j}^{0}\right)_{298}+\int_{298}^{T} \bar{C}_{p j} \cdot d T=\left(\Delta H_{f, j}^{0}\right)_{298}+\bar{C}_{p j} \cdot(T-298) \quad\left(k J / k m o l ~ d e ~ A_{j}\right)
\end{aligned}
$$

y su correspondiente diferencial:

$$
\mathrm{dh}_{\mathrm{j}}=\overline{\mathrm{C}}_{\mathrm{pj}} \cdot \mathrm{dT} \quad\left(\mathrm{kJ} / \mathrm{kmol} \mathrm{de} \mathrm{A}_{\mathrm{j}}\right)
$$


- Entalpía de una reacción química $i$, por mol de componente clave o de componente de referencia, a la temperatura de reacción:

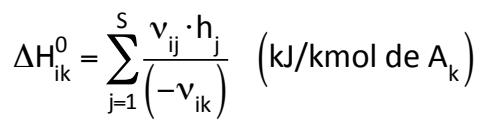

y su correspondiente diferencial:

$$
\mathrm{d}\left(\Delta H_{\mathrm{ik}}^{0}\right)=\sum_{\mathrm{j}=1}^{\mathrm{S}} \frac{v_{\mathrm{ij}} \cdot \mathrm{dh}}{\left(-v_{\mathrm{ik}}\right)} \quad\left(\mathrm{kJ} / \mathrm{kmol} \text { de } \mathrm{A}_{\mathrm{k}}\right)
$$

- Parámetro $\mathrm{J}_{\mathrm{i}}$

A partir de la relación molar en la corriente de entrada o en el tiempo inicial:

$$
\theta_{\mathrm{j}}=\mathrm{n}_{\mathrm{j} 0}=\frac{\mathrm{N}_{\mathrm{j} 0}}{N_{\mathrm{k} 0}}=\frac{F_{\mathrm{j} 0}}{F_{\mathrm{k} 0}}\left(\mathrm{kmol} \mathrm{A} / \mathrm{kmol} \mathrm{A} \mathrm{k}_{\mathrm{j}}\right)
$$

se puede definir el parámetro $\mathrm{J}_{\mathrm{i}}$ de cada reacción química:

$$
J_{i}=\frac{-\Delta H_{i k}^{0}}{\sum_{j=1}^{S} \theta_{j} \cdot \bar{C}_{p j}}(K)
$$

Seguidamente se obtendrán los balances de energía para los tres tipos de reactores químicos de flujo ideal.

\subsection{Balance general de energía en reactores de tanque agitado}

En los reactores continuos de tanque agitado ideales se aplican las siguientes hipótesis: $i$ ) el flujo de circulación de los fluidos es normal a la superficie de entrada y de salida, ii) la mezcla en el interior del reactor es perfecta y iii) las propiedades de la corriente de salida coinciden con las propiedades de cualquier punto del volumen de reacción, por lo que la ecuación (1) queda como:

$$
\int_{F_{t} h_{0}}^{F_{t} \cdot h} d\left(F_{t} \cdot h\right)+\frac{d}{d t} \int_{V}\left(h \cdot \rho_{m}-P\right) \cdot d V=Q^{*}(k J / s)
$$

y al ser todas las variables independientes de la posición espacial:

$$
F_{t} \cdot h-F_{t 0} \cdot h_{0}+\frac{d}{d t}\left[\left(h \cdot \rho_{m}-P\right) \cdot v\right]=Q^{*} \quad(k J / s)
$$

en esta ecuación conviene simplificar en la medida de lo posible el término de acumulación:

$$
\frac{d}{d t}\left[\left(h \cdot \rho_{m}-P\right) \cdot V\right]=\frac{d\left(h \cdot \rho_{m} \cdot V\right)}{d t}-\frac{d(P \cdot V)}{d t}=\frac{d\left(h \cdot N_{t}\right)}{d t}-P \cdot \frac{d V}{d t}-V \cdot \frac{d P}{d t} \quad(k J / s)
$$


y considerando que en muchos sistemas, especialmente en el caso de líquidos y régimen estacionario, no hay variación del volumen ni de la presión con el tiempo o, en todo caso, estas variaciones son despreciables frente al término entálpico:

$$
\frac{d}{d t}\left[\left(h \cdot \rho_{m}-P\right) \cdot v\right] \approx \frac{d\left(h \cdot N_{t}\right)}{d t}=\frac{d}{d t}\left(\sum_{j=1}^{s} h_{j} \cdot N_{j}\right) \quad(k J / s)
$$

En definitiva, el balance de energía para un reactor de tanque agitado se puede escribir como:

$$
\sum_{j=1}^{S} F_{j} \cdot h_{j}-\sum_{j=1}^{S} F_{j 0} \cdot h_{j 0}+\frac{d}{d t}\left(\sum_{j=1}^{S} N_{j} \cdot h_{j}\right)=Q^{*} \quad(k J / s)
$$

\subsubsection{Reactor continuo de tanque agitado en régimen no estacionario (RCTA) y reactor semicontinuo (RSCTA)}

Al no estar en régimen estacionario no se puede prescindir de ninguno de los términos de la ecuación (2) que constituyen el balance general de energía de los reactores de tanque agitado. Dado que en sistemas semicontinuos resulta generalmente difícil definir el grado de conversión, suele ser más conveniente transformar la ecuación (2) haciendo uso del balance de materia y no de las relaciones estequiométricas. Así, para el caso de sistemas con una única reacción química, a partir del correspondiente balance de materia:

$$
\begin{aligned}
& F_{j}-F_{j 0}+\frac{d\left(c_{j} \cdot V\right)}{d t}=\Re_{j} \cdot V \\
& F_{j}-F_{j 0}+\frac{d N_{j}}{d t}=v_{j} \cdot r \cdot V \\
& F_{j}=F_{j 0}-\frac{d N_{j}}{d t}+v_{j} \cdot r \cdot V \quad\left(\mathrm{kmol} \mathrm{A}_{j} / s\right)
\end{aligned}
$$

se obtiene:

$$
\begin{gathered}
\sum_{j=1}^{S}\left(F_{j 0}-\frac{d N_{j}}{d t}+v_{j} \cdot r \cdot V\right) \cdot h_{j}-\sum_{j=1}^{S} F_{j 0} \cdot h_{j 0}+\frac{d}{d t}\left(\sum_{j=1}^{S} N_{j} \cdot h_{j}\right)=Q^{*} \\
\sum_{j=1}^{S} F_{j 0} \cdot h_{j}-\sum_{j=1}^{S} \frac{d N_{j} \cdot h_{j}}{d t}+r \cdot V \cdot \sum_{j=1}^{S} v_{j} \cdot h_{j}-\sum_{j=1}^{S} F_{j 0} \cdot h_{j 0}+\sum_{j=1}^{S} \frac{d N_{j} \cdot h_{j}}{d t}+\sum_{j=1}^{S} \frac{N_{j} \cdot d h_{j}}{d t}=Q^{*} \\
\sum_{j=1}^{S} F_{j 0} \cdot\left(h_{j}-h_{j 0}\right)+r \cdot V \cdot \sum_{j=1}^{S} v_{j} \cdot h_{j}+\sum_{j=1}^{S} \frac{N_{j} \cdot d h_{j}}{d t}=Q^{*} \quad(k J / s)
\end{gathered}
$$

Finalmente, considerando la definición de la entalpía de una especie química, así como su forma diferencial, y la entalpía de la reacción se llega a: 


$$
\sum_{j=1}^{S} N_{j} \cdot \bar{C}_{p j} \cdot \frac{d T}{d t}=Q^{*}+\left(T_{0}-T\right) \cdot \sum_{j=1}^{S} F_{j 0} \cdot \bar{C}_{p j}+v_{k} \cdot \Delta H_{k}^{0} \cdot r \cdot V \quad(k J / s) \quad \text { ECUACIÓN (2.1) }
$$

Ejercicio 1. Siguiendo el mismo procedimiento el alumno puede obtener el balance de energía correspondiente a un sistema con varias reacciones químicas:

$$
\sum_{j=1}^{S} N_{j} \cdot \bar{C}_{p j} \cdot \frac{d T}{d t}=Q^{*}+\left(T_{0}-T\right) \cdot \sum_{j=1}^{S} F_{j 0} \cdot \bar{C}_{p j}+\left(\sum_{i=1}^{R} v_{i k} \cdot \Delta H_{i k}^{0} \cdot r_{i}\right) \cdot V \quad(k J / S)
$$

\subsubsection{Reactor continuo de tanque agitado en régimen estacionario (RCTA)}

$\mathrm{Al}$ estar funcionando en régimen estacionario el sistema permanece invariable con el tiempo no apareciendo el término de acumulación en el balance. La ecuación (2) queda como:

$$
\sum_{j=1}^{S} F_{j} \cdot h_{j}-\sum_{j=1}^{S} F_{j 0} \cdot h_{j 0}=Q^{*} \quad(k J / s)
$$

Esta ecuación se puede desarrollar introduciendo una variable de composición (normalmente el grado de conversión X) en lugar del caudal molar y la temperatura $(\mathrm{T})$ en lugar de la entalpía.

Para introducir el grado de conversión se utiliza la correspondiente relación estequiométrica que, para el caso de que tenga lugar una única reacción química, es:

$$
F_{j}=F_{j 0}-F_{k 0} \cdot \frac{v_{j}}{v_{k}} \cdot X
$$

resultando:

$$
\begin{aligned}
& \sum_{j=1}^{S} F_{j 0} \cdot h_{j}+\sum_{j=1}^{S} F_{k 0} \cdot \frac{v_{j}}{\left(-v_{k}\right)} \cdot X \cdot h_{j}-\sum_{j=1}^{S} F_{j 0} \cdot h_{j 0}=Q^{*} \\
& \sum_{j=1}^{S} F_{j 0} \cdot\left(h_{j}-h_{j 0}\right)+F_{k 0} \cdot X \cdot \sum_{j=1}^{S} \frac{v_{j} \cdot h_{j}}{\left(-v_{k}\right)}=Q^{*} \quad(k J / s)
\end{aligned}
$$

Considerando la definición de la entalpía de una especie química y la entalpía de la reacción se llega a:

$$
\left(T-T_{0}\right) \cdot \sum_{j=1}^{S} F_{j 0} \cdot \bar{C}_{p j}+F_{k 0} \cdot X \cdot \Delta H_{k}^{0}=Q^{*} \quad(k J / s)
$$

Finalmente, considerando la definición del parámetro J de la reacción se obtiene: 


$$
\left(T-T_{0}\right)-X \cdot J=\frac{Q^{*}}{F_{k 0} \cdot \sum_{j=1}^{S} \theta_{j} \cdot \bar{C}_{p j}}(K)
$$

Si se define el parámetro $Q=\frac{Q^{*}}{F_{k 0} \cdot \sum_{j=1}^{S} \theta_{j} \cdot \bar{C}_{p j}}$, el balance se puede reescribir como:

$$
T-T_{0}-J \cdot X=Q \quad(K)
$$

ECUACIÓN (2.2)

Si en lugar de $\mathrm{X}$ se sustituye el correspondiente balance de materia, se obtiene otra expresión del balance de energía para un RCTA en el que tiene lugar una reacción química:

$$
T-T_{0}-J \cdot\left[\frac{\left(-v_{k}\right) \cdot r \cdot \tau}{c_{k 0}}\right]=Q \quad(K)
$$

Ejercicio 2. Siguiendo el mismo procedimiento el alumno puede obtener los siguientes balances de energía:

i) Para cualquier reactor $n$ de una serie de RCTA en los que tiene lugar una única reacción química:

$$
\left(T_{n}-\bar{T}_{n}\right)-J \cdot\left(X_{n}-\bar{X}_{n}\right)=Q_{n} \text { O }\left(T_{n}-\bar{T}_{n}\right)-J \cdot\left[\frac{\left(-v_{k}\right) \cdot r_{n} \cdot \tau_{n}}{c_{k 0}}\right]=Q_{n}
$$

ii) Para un RCTA en el que tienen lugar varias reacciones químicas simultáneas:

solo para reacciones químicas paralelas $\mathrm{T}-\mathrm{T}_{0} \cdot \sum_{\mathrm{i}=1}^{\mathrm{R}} \mathrm{J}_{\mathrm{i}} \cdot \mathrm{X}_{\mathrm{i}}=\mathrm{Q}$

para cualquier sistema de reacciones químicas $T-T_{0}-\frac{\tau}{c_{k 0}} \cdot \sum_{i=1}^{R}\left(-v_{i k}\right) \cdot r_{i} \cdot J_{i}=Q$

iii) Para cualquier reactor $n$ de una serie de RCTA en los que tienen lugar varias reacciones químicas simultáneas:

solo para reacciones químicas paralelas $\left(T_{n}-\bar{T}_{n}\right)-\sum_{i=1}^{R} J_{i} \cdot\left(X_{i n}-\bar{X}_{i n}\right)=Q_{n}$

para cualquier sistema de reacciones químicas $\left(T_{n}-\bar{T}_{n}\right)-\frac{\tau_{n}}{c_{k 0}} \cdot \sum_{i=1}^{R}\left(-v_{i k}\right) \cdot r_{i n} \cdot J_{i}=Q_{n}$ 


\subsubsection{Reactor discontinuo de tanque agitado (RDTA)}

Al no estar funcionando en régimen estacionario el sistema varía con el tiempo, pero dado que no hay corrientes de entrada ni de salida, la ecuación (2) queda como:

$$
\begin{aligned}
\frac{d}{d t}\left(\sum_{j=1}^{s} N_{j} \cdot h_{j}\right) & =Q^{*} \quad(k J / s) \\
\sum_{j=1}^{S} N_{j} \cdot \frac{d h_{j}}{d t}+\sum_{j=1}^{S} \frac{d N_{j}}{d t} \cdot h_{j} & =Q^{*} \quad(k J / s)
\end{aligned}
$$

Esta ecuación se puede desarrollar introduciendo una variable de composición (normalmente el grado de conversión X) en lugar del número de moles y la temperatura $(\mathrm{T})$ en lugar de la entalpía.

Para introducir el grado de conversión se utiliza la correspondiente relación estequiométrica que, para el caso de que tenga lugar una única reacción química, es:

$$
\begin{array}{r}
N_{j}=N_{j 0}-N_{k 0} \cdot \frac{v_{j}}{v_{k}} \cdot X \\
d N_{j}=N_{k 0} \cdot \frac{v_{j}}{\left(-v_{k}\right)} \cdot d X
\end{array}
$$

resultando:

$$
\sum_{j=1}^{S} N_{j 0} \cdot \frac{d h_{j}}{d t}+N_{k 0} \cdot X \cdot \frac{\sum_{j=1}^{S} \frac{v_{j} \cdot d h_{j}}{\left(-v_{k}\right)}}{d t}+N_{k 0} \cdot \frac{d X}{d t} \cdot \sum_{j=1}^{S} \frac{v_{j} \cdot h_{j}}{\left(-v_{k}\right)}=Q^{*} \quad(k J / s)
$$

Considerando la definición de la entalpía de una especie química, la entalpía de la reacción y sus correspondientes diferenciales, así como suponiendo que la entalpía de la reacción no cambia con el tiempo de reacción (aunque cambien las condiciones de operación del reactor) se llega a:

$$
\frac{d T}{d t} \cdot \sum_{j=1}^{S} N_{j 0} \cdot \bar{C}_{p j}+N_{k 0} \cdot \Delta H_{k}^{0} \cdot \frac{d X}{d t}=Q^{*} \quad(k J / s)
$$

Finalmente, considerando la definición del parámetro J de la reacción se obtiene:

$$
\frac{d T}{d t}-J \cdot \frac{d X}{d t}=\frac{Q^{*}}{N_{k 0} \cdot \sum_{j=1}^{s} \theta_{j} \cdot \bar{C}_{p j}}(K / s)
$$

Si se define el parámetro $Q^{\prime}=\frac{Q^{*}}{N_{k 0} \cdot \sum_{j=1}^{S} \theta_{j} \cdot \bar{C}_{p j}}$, el balance se puede reescribir como: 


$$
\frac{d T}{d t}-J \cdot \frac{d X}{d t}=Q^{\prime} \quad(K / s)
$$

Si en lugar de dX se sustituye el correspondiente balance de materia, se obtiene otra expresión del balance de energía para un RCTA en el que tiene lugar una reacción química:

$$
\frac{\mathrm{dT}}{\mathrm{dt}}+\frac{\mathrm{v}_{\mathrm{k}} \cdot \mathrm{r}}{\mathrm{c}_{\mathrm{k} 0}} \cdot \mathrm{J}=\mathrm{Q}^{\prime} \quad(\mathrm{K} / \mathrm{s})
$$

Ejercicio 3. Siguiendo el mismo procedimiento el alumno puede obtener los siguientes balances de energía para sistemas con varias reacciones químicas:

solo para reacciones químicas paralelas $\frac{\mathrm{dT}}{\mathrm{dt}}-\sum_{\mathrm{i}=1}^{\mathrm{R}} \mathrm{J}_{\mathrm{i}} \cdot \frac{\mathrm{dX}}{\mathrm{dt}}=\mathrm{Q}^{\prime}$

para cualquier sistema de reacciones químicas $\frac{\mathrm{dT}}{\mathrm{dt}}+\frac{1}{\mathrm{c}_{\mathrm{k} 0}} \cdot \sum_{\mathrm{i}=1}^{\mathrm{R}} \mathrm{v}_{\mathrm{ik}} \cdot \mathrm{r}_{\mathrm{i}} \cdot \mathrm{J}_{\mathrm{i}}=\mathrm{Q}^{\prime}$

\subsection{Balance general de energía en reactores continuos tubulares de flujo de pistón (RFP)}

En el caso de los reactores continuos tubulares de flujo de pistón el flujo de circulación de los fluidos es normal a la superficie de entrada y de salida, pero las propiedades de la mezcla de reacción cambian a medida que avanza a lo largo de la longitud del reactor. Este comportamiento específico obliga a aplicar el balance de materia general, ecuación (1), de forma diferencial a un elemento de volumen $\mathrm{dV}$. En consecuencia, si solo se considera el funcionamiento en régimen estacionario,

$$
d\left(F_{t} \cdot h\right)=d Q^{*}(k J / s)
$$

que se transforma, para el caso en consideración, en:

$$
\sum_{j=1}^{s} F_{j} \cdot d h_{j}+\sum_{j=1}^{s} h_{j} \cdot d F_{j}=d Q^{*} \quad(k J / s)
$$

ECUACIÓN (3)

Al igual que se ha hecho en los reactores de tanque agitado, esta ecuación se puede desarrollar introduciendo una variable de composición (normalmente el grado de conversión X) en lugar del caudal molar y la temperatura (T) en lugar de la entalpía. 
Para introducir el grado de conversión se utiliza la correspondiente relación estequiométrica que, para el caso de que tenga lugar una única reacción química, es:

$$
\begin{aligned}
& F_{j}=F_{j 0}-F_{k 0} \cdot \frac{v_{j}}{v_{k}} \cdot X \\
& d F_{j}=F_{k 0} \cdot \frac{v_{j}}{\left(-v_{k}\right)} \cdot d X
\end{aligned}
$$

resultando:

$$
\begin{aligned}
& \sum_{j=1}^{S}\left(F_{j 0}-F_{k 0} \cdot \frac{v_{j}}{v_{k}} \cdot X\right) \cdot d h_{j}+\sum_{j=1}^{S} h_{j} F_{k 0} \cdot \frac{v_{j}}{\left(-v_{k}\right)} \cdot d X=d Q^{*} \\
& \sum_{j=1}^{S} F_{j 0} \cdot d h_{j}+F_{k 0} \cdot X \cdot \frac{\sum_{j=1}^{S} v_{j} \cdot d h_{j}}{\left(-v_{k}\right)}+F_{k 0} \cdot d X \cdot \frac{\sum_{j=1}^{S} v_{j} \cdot h_{j}}{\left(-v_{k}\right)}=d Q^{*}(k J / s)
\end{aligned}
$$

Considerando la definición de la entalpía de una especie química, la entalpía de la reacción y sus correspondientes diferenciales, así como suponiendo que la entalpía de la reacción no cambia con la longitud del reactor (aunque cambien las condiciones de operación del reactor) se llega a:

$$
\left(\sum_{j=1}^{s} F_{j 0} \cdot \bar{C}_{p j}\right) \cdot d T+F_{k 0} \cdot \Delta H_{k}^{0} \cdot d X=d Q^{*} \quad(k J / s)
$$

Finalmente, considerando la definición del parámetro J de la reacción se obtiene:

$$
d T-J \cdot d X=\frac{d Q^{*}}{F_{k 0} \cdot \sum_{j=1}^{S} \theta_{j} \cdot \bar{C}_{p j}}(K)
$$

Si se define el parámetro $d Q=\frac{d^{*}}{s}$, el balance se puede reescribir como:

$$
\begin{aligned}
& F_{k 0} \cdot \sum_{j=1}^{S} \theta_{j} \cdot \bar{C}_{p j} \\
& d T-J \cdot d X=d Q \quad(K)
\end{aligned}
$$

o bien, a lo largo de la longitud del reactor:

$$
\frac{\mathrm{dT}}{\mathrm{d} \ell}-\mathrm{J} \cdot \frac{\mathrm{dX}}{\mathrm{d} \ell}=\frac{\mathrm{dQ}}{\mathrm{d} \ell}(\mathrm{K} / \mathrm{m})
$$

Si en lugar de X se sustituye el correspondiente balance de materia, se obtiene otra expresión del balance de energía para un RCTA en el que tiene lugar una reacción química:

$$
\frac{\mathrm{dT}}{\mathrm{d} \ell}+\frac{v_{\mathrm{k}} \cdot \mathrm{r}}{\mathrm{G}_{\mathrm{k} 0}} \cdot \mathrm{J}=\frac{\mathrm{dQ}}{\mathrm{d} \ell} \quad(\mathrm{K} / \mathrm{m})
$$


Ejercicio 4. Siguiendo el mismo procedimiento el alumno puede obtener los siguientes balances de energía para sistemas con varias reacciones químicas:

solo para reacciones químicas paralelas $\frac{\mathrm{dT}}{\mathrm{d} \ell}-\sum_{\mathrm{i}=1}^{\mathrm{R}} \mathrm{J}_{\mathrm{i}} \cdot \frac{\mathrm{dX}}{\mathrm{d} \ell}=\frac{\mathrm{dQ}}{\mathrm{d} \ell}$ para cualquier sistema de reacciones químicas $\frac{\mathrm{dT}}{\mathrm{d} \ell}+\frac{1}{\mathrm{G}_{\mathrm{k} 0}} \cdot \sum_{\mathrm{i}=1}^{\mathrm{R}} v_{\mathrm{ik}} \cdot \mathrm{r}_{\mathrm{i}} \cdot \mathrm{J}_{\mathrm{i}}=\frac{\mathrm{dQ}}{\mathrm{d} \ell}$

En el Anexo III se recogen los balances de energía más habituales.

En el Anexo IV se detallan algunas consideraciones sobre las diferentes formas de expresar el calor específico de una mezcla de reacción. 
ANEXO I

VARIABLES DE COMPOSICIÓN Y CÁLCULO

DE LA COMPOSICIÓN

\section{Variables de composición}

- Extensivas: el número de moles, $\mathrm{N}_{\mathrm{j}}$, y el caudal molar $\mathrm{F}_{\mathrm{j}}$.

- Intensivas:

\begin{tabular}{|c|c|c|}
\hline Variable de composición & Reactores discontinuos & Reactores continuos \\
\hline Concentración $\left(\mathrm{kmol} \mathrm{A}_{\mathrm{j}} / \mathrm{m}^{3}\right)$ & $\mathrm{c}_{\mathrm{j}}=\frac{\mathrm{N}_{\mathrm{j}}}{\mathrm{V}}$ & $\mathrm{c}_{\mathrm{j}}=\frac{\mathrm{F}_{\mathrm{j}}}{\mathrm{Q}_{\mathrm{V}}}$ \\
\hline $\begin{array}{c}\text { Fracción molar } \\
(\mathrm{kmol} \mathrm{A} / \mathrm{kmol} \mathrm{totales})\end{array}$ & $\mathrm{x}_{\mathrm{j}}=\frac{\mathrm{N}_{\mathrm{j}}}{\mathrm{N}_{\mathrm{t}}}$ & $\mathrm{x}_{\mathrm{j}}=\frac{\mathrm{F}_{\mathrm{j}}}{\mathrm{F}_{\mathrm{t}}}$ \\
\hline Presión parcial (Pa) & $\mathrm{p}_{\mathrm{j}}=\mathrm{N}_{\mathrm{j}} \cdot \frac{\mathrm{R} \cdot \mathrm{T}}{\mathrm{V}}$ & $\mathrm{p}_{\mathrm{j}}=\mathrm{F}_{\mathrm{j}} \cdot \frac{\mathrm{R} \cdot \mathrm{T}}{\mathrm{Q}_{\mathrm{V}}}$ \\
\hline $\begin{array}{c}\text { Relación molar } \\
\left(\mathrm{kmol} \mathrm{A} / \mathrm{kmol} \mathrm{A} \mathrm{A}_{\mathrm{k}}\right)\end{array}$ & $\mathrm{n}_{\mathrm{j}}=\frac{\mathrm{N}_{\mathrm{j}}}{\mathrm{N}_{\mathrm{k} 0}}$ & $\mathrm{n}_{\mathrm{j}}=\frac{\mathrm{F}_{\mathrm{j}}}{\mathrm{F}_{\mathrm{k} 0}}$ \\
\hline
\end{tabular}

Cambios de composición en sistemas con una única reacción química

- A partir de variables de composición extensivas

$$
\begin{gathered}
\mathrm{N}_{\mathrm{j}}=\mathrm{N}_{\mathrm{j} 0}-\mathrm{N}_{\mathrm{k} 0} \cdot \frac{v_{\mathrm{j}}}{v_{\mathrm{k}}} \cdot \mathrm{X} \\
\mathrm{F}_{\mathrm{j}}=\mathrm{F}_{\mathrm{j} 0}-\mathrm{F}_{\mathrm{k} 0} \cdot \frac{v_{\mathrm{j}}}{v_{\mathrm{k}}} \cdot \mathrm{X}
\end{gathered}
$$

- A partir de variables de composición intensivas

$\S$ concentración:

- en reactores discontinuos: $c_{j}=\frac{N_{j}}{V}=\frac{N_{j 0}-N_{k 0} \cdot \frac{v_{j}}{v_{k}} \cdot X}{V}$

i) en el caso de sistemas líquidos el volumen se considera constante $\mathrm{V}=\mathrm{V}_{0}$

$$
c_{j}=\frac{N_{j}}{V_{0}}=\frac{N_{j 0}-N_{k 0} \cdot \frac{v_{j}}{v_{k}} \cdot X}{V_{0}}=c_{j 0}-c_{k 0} \cdot \frac{v_{j}}{v_{k}} \cdot X
$$


ii) en el caso de los gases el volumen puede variar según las condiciones:

$$
c_{j}=\frac{N_{j}}{V}=\frac{N_{j 0}-N_{k 0} \cdot \frac{v_{j}}{v_{k}} \cdot X}{V_{0} \cdot \frac{p_{0}}{p} \cdot \frac{T}{T_{0}} \cdot\left(1+\varepsilon_{k} \cdot X\right)}=\frac{c_{j 0}-c_{k 0} \cdot \frac{v_{j}}{v_{k}} \cdot X}{\frac{p_{0}}{p} \cdot \frac{T}{T_{0}} \cdot\left(1+\varepsilon_{k} \cdot X\right)}
$$

- en reactores continuos: $c_{j}=\frac{F_{j}}{Q_{V}}=\frac{F_{j 0}-F_{k 0} \cdot \frac{v_{j}}{v_{k}} \cdot X}{Q_{V}}$

i) en el caso de sistemas líquidos el caudal se considera constante $\mathrm{Q}_{\mathrm{V}}=\mathrm{Q}_{\mathrm{V} 0}$

$$
c_{j}=\frac{F_{j}}{Q_{V 0}}=\frac{F_{j 0}-F_{k 0} \cdot \frac{v_{j}}{v_{k}} \cdot X}{Q_{v 0}}=c_{j 0}-c_{k 0} \cdot \frac{v_{j}}{v_{k}} \cdot X
$$

ii) en el caso de los gases el caudal puede variar según las condiciones:

$$
c_{j}=\frac{F_{j}}{Q_{V}}=\frac{F_{j 0}-F_{k 0} \cdot \frac{v_{j}}{v_{k}} \cdot X}{Q_{V 0} \cdot \frac{p_{0}}{p} \cdot \frac{T}{T_{0}} \cdot\left(1+\varepsilon_{k} \cdot X\right)}=\frac{c_{j 0}-c_{k 0} \cdot \frac{v_{j}}{v_{k}} \cdot X}{\frac{p_{0}}{p} \cdot \frac{T}{T_{0}} \cdot\left(1+\varepsilon_{k} \cdot X\right)}
$$

siendo en ambos casos: $\varepsilon_{\mathrm{k}}=\mathrm{x}_{\mathrm{k} 0} \cdot \frac{\sum_{\mathrm{j}=1}^{\mathrm{S}} v_{\mathrm{j}}}{\left(-v_{\mathrm{k}}\right)}$

$\S$ fracción molar:

- en reactores discontinuos:

$$
x_{j}=\frac{N_{j}}{N_{t}}=\frac{N_{j 0}-N_{k 0} \cdot \frac{v_{j}}{v_{k}} \cdot X}{N_{t 0} \cdot\left(1+\varepsilon_{k} \cdot X\right)}=\frac{x_{j 0}-x_{k 0} \cdot \frac{v_{j}}{v_{k}} \cdot X}{\left(1+\varepsilon_{k} \cdot X\right)}
$$

- en reactores continuos:

$$
\begin{gathered}
\mathrm{x}_{\mathrm{j}}=\frac{\mathrm{F}_{\mathrm{j}}}{\mathrm{F}_{\mathrm{t}}}=\frac{\mathrm{F}_{\mathrm{j} 0}-\mathrm{F}_{\mathrm{k} 0} \cdot \frac{v_{\mathrm{j}}}{v_{\mathrm{k}}} \cdot \mathrm{X}}{\mathrm{F}_{\mathrm{t} 0} \cdot\left(1+\varepsilon_{\mathrm{k}} \cdot \mathrm{X}\right)}=\frac{\mathrm{x}_{\mathrm{j} 0}-\mathrm{x}_{\mathrm{k} 0} \cdot \frac{v_{\mathrm{j}}}{v_{\mathrm{k}}} \cdot \mathrm{X}}{\left(1+\varepsilon_{\mathrm{k}} \cdot \mathrm{X}\right)} \\
\text { siendo en ambos casos } \varepsilon_{\mathrm{k}}=\mathrm{x}_{\mathrm{k} 0} \cdot \frac{\sum_{\mathrm{j}=1}^{\mathrm{S}} v_{\mathrm{j}}}{\left(-v_{\mathrm{k}}\right)}
\end{gathered}
$$


$\S$ relación molar

- en reactores discontinuos:

$$
n_{j}=\frac{N_{j}}{N_{k} 0}=\frac{N_{j 0}-N_{k 0} \cdot \frac{v_{j}}{v_{k}} \cdot X}{N_{k 0}}=n_{j 0}-\frac{v_{j}}{v_{k}} \cdot X=\theta_{j}-\frac{v_{j}}{v_{k}} \cdot X
$$

- en reactores continuos:

$$
n_{j}=\frac{F_{j}}{F_{k} 0}=\frac{F_{j 0}-F_{k 0} \cdot \frac{v_{j}}{v_{k}} \cdot X}{F_{k 0}}=n_{j 0}-\frac{v_{j}}{v_{k}} \cdot X=\theta_{j}-\frac{v_{j}}{v_{k}} \cdot X
$$

Cambios de composición en sistemas con varias reacciones químicas paralelas

A partir de variables de composición extensivas

$$
\begin{gathered}
N_{j}=N_{j 0}-N_{k 0} \cdot \sum_{j=1}^{S} \frac{v_{i j}}{v_{i k}} \cdot X_{i} \\
F_{j}=F_{j 0}-F_{k 0} \cdot \sum_{j=1}^{S} \frac{v_{i j}}{v_{i k}} \cdot X_{i}
\end{gathered}
$$




\section{ANEXO II}

\section{BALANCES DE LA MATERIA}

\section{Reactor Discontinuo de Tanque Agitado, RDTA (Batch Reactor)}

Balance general de materia

$$
\frac{\mathrm{d}\left(\mathrm{c}_{\mathrm{j}} \cdot \mathrm{V}\right)}{\mathrm{dt}}=\mathfrak{R}_{\mathrm{j}} \cdot \mathrm{V}
$$

\begin{tabular}{|c|c|c|c|}
\hline \multirow{2}{*}{$\begin{array}{l}\text { Variable } \\
\text { de compo- } \\
\text { sición }\end{array}$} & \multicolumn{3}{|c|}{ Sistemas con una reacción química } \\
\hline & Ecuación general & $\begin{array}{c}\text { Ecuación a V } \\
\text { constante }\end{array}$ & Ecuación a V variable \\
\hline$c_{j}$ & $\frac{d\left(c_{j} \cdot V\right)}{d t}=v_{j} \cdot r \cdot V$ & $\mathrm{dt}=\frac{\mathrm{dc} \mathrm{j}_{\mathrm{j}}}{\mathrm{v}_{\mathrm{j}} \cdot \mathrm{r}}$ & $d t=\frac{d c_{j}}{v_{j} \cdot r}+c_{j} \cdot \frac{d \ln V}{v_{j} \cdot r}$ \\
\hline$X$ & $\mathrm{dt}=\frac{\mathrm{N}_{\mathrm{k} 0}}{\mathrm{~V}} \cdot \frac{\mathrm{dX}}{\left(-\mathrm{v}_{\mathrm{k}}\right) \cdot \mathrm{r}}$ & $\mathrm{dt}=\frac{\mathrm{N}_{\mathrm{k} 0}}{\mathrm{~V}_{0}} \cdot \frac{\mathrm{dX}}{\left(-\mathrm{v}_{\mathrm{k}}\right) \cdot \mathrm{r}}$ & $\mathrm{dt}=\frac{\mathrm{N}_{\mathrm{k} 0}}{\mathrm{~V}_{0} \cdot \frac{\mathrm{P}_{0}}{\mathrm{P}} \cdot \frac{\mathrm{T}}{\mathrm{T}_{0}} \cdot\left(1+\varepsilon_{\mathrm{k}} \cdot \mathrm{X}\right)} \cdot \frac{\mathrm{dX}}{\left(-\mathrm{v}_{\mathrm{k}}\right) \cdot \mathrm{r}}$ \\
\hline $\mathrm{n}_{\mathrm{j}}$ & $\mathrm{dt}=\frac{\mathrm{N}_{\mathrm{k} 0}}{\mathrm{~V}} \cdot \frac{\mathrm{dn}_{\mathrm{j}}}{\mathrm{v}_{\mathrm{j}} \cdot \mathrm{r}}$ & $\mathrm{dt}=\frac{\mathrm{N}_{\mathrm{k} 0}}{\mathrm{~V}_{0}} \cdot \frac{\mathrm{dn}_{\mathrm{j}}}{\mathrm{v}_{\mathrm{j}} \cdot \mathrm{r}}$ & $\mathrm{dt}=\frac{\mathrm{N}_{\mathrm{k} 0}}{\mathrm{~V}} \cdot \frac{\mathrm{dn}_{\mathrm{j}}}{\mathrm{v}_{\mathrm{j}} \cdot \mathrm{r}}$ \\
\hline $\begin{array}{l}\text { Variable } \\
\text { de compo- } \\
\text { sición más } \\
\text { adecuada }\end{array}$ & & Sistemas con varias 1 & eacciones químicas \\
\hline $\mathrm{X}_{\mathrm{i}}$ & $\frac{\mathrm{dX}}{\mathrm{dt}}=\frac{\mathrm{V}}{\mathrm{N}_{\mathrm{k}}}$ & $-\left(-v_{i k}\right) \cdot r_{i}$ & $\mathrm{i}=1,2 \ldots \ldots . \mathrm{R}$, paralelas \\
\hline $\mathrm{n}_{\mathrm{j}}$ & $\frac{\mathrm{dn}_{\mathrm{j}}}{\mathrm{dt}}=\frac{\mathrm{V}}{\mathrm{N}_{\mathrm{k} 0}}$ & $\sum_{i=1}^{R} v_{i j} \cdot r_{i}$ & $\mathrm{j}=1,2 \ldots \ldots . \mathrm{S}$, cualesquiera \\
\hline
\end{tabular}


Reactor Semicontinuo de Tanque Agitado, RSCTA (Semibatch Reactor)

Balance general de materia

$$
\mathrm{F}_{\mathrm{j}}-\mathrm{F}_{\mathrm{j} 0}+\frac{\mathrm{d}\left(\mathrm{c}_{\mathrm{j}} \cdot \mathrm{V}\right)}{\mathrm{dt}}=\Re_{\mathrm{j}} \cdot \mathrm{V}
$$

\begin{tabular}{|c|c|c|}
\hline $\begin{array}{c}\text { Condiciones } \\
\text { habituales a la } \\
\text { entrada del reactor }\end{array}$ & $\begin{array}{c}\text { Condiciones } \\
\text { habituales a la } \\
\text { salida del reactor }\end{array}$ & $\begin{array}{c}\text { Ecuación particular para un determinado RSCTA } \\
\text { y la puesta en marcha de un RCTA }\end{array}$ \\
\hline $\mathrm{F}_{\mathrm{j} 0}=$ cte; $\mathrm{Q}_{\mathrm{v} 0}=$ cte & $\mathrm{F}_{\mathrm{j}}=\mathrm{Q}_{\mathrm{V}}=0$ & $\frac{\mathrm{dc}_{\mathrm{j}}}{\mathrm{dt}}+\frac{\mathrm{Q}_{\mathrm{v} 0}}{\mathrm{~V}_{0}+\mathrm{Q}_{\mathrm{v} 0} \cdot \mathrm{t}} \cdot\left(\mathrm{c}_{\mathrm{j}}-\mathrm{c}_{\mathrm{j} 0}\right)=\mathrm{v}_{\mathrm{j}} \cdot \mathrm{r}$ \\
\hline $\mathrm{F}_{\mathrm{j} 0}=$ cte; $\mathrm{Q}_{\mathrm{v} 0}=$ cte & $\mathrm{Q}_{\mathrm{v} 0}>\mathrm{Q}_{\mathrm{v}}$ & $\frac{\mathrm{dc}_{\mathrm{j}}}{\mathrm{dt}}+\frac{\mathrm{Q}_{\mathrm{v} 0}}{\mathrm{~V}_{0}+\left(\mathrm{Q}_{\mathrm{v} 0}-\mathrm{Q}_{\mathrm{v}}\right) \cdot \mathrm{t}} \cdot\left(\mathrm{c}_{\mathrm{j}}-\mathrm{c}_{\mathrm{j} 0}\right)=\mathrm{v}_{\mathrm{j}} \cdot \mathrm{r}$ \\
\hline $\mathrm{F}_{\mathrm{j} \mathrm{0}}=$ cte; $\mathrm{Q}_{\mathrm{V} 0}=\mathrm{cte}$ & $\mathrm{Q}_{\mathrm{v} 0}=\mathrm{Q}_{\mathrm{v}}$ & $\mathrm{c}_{\mathrm{j}}-\mathrm{c}_{\mathrm{j} 0}+\tau \cdot \frac{\mathrm{dc}}{\mathrm{dt}}=\mathrm{v}_{\mathrm{j}} \cdot \mathrm{r} \cdot \tau$ \\
\hline
\end{tabular}

Reactor Continuo de Tanque Agitado, RCTA (Continuous-Stirred Tank Reactor, CSTR, or Backmixed Reactor)

Balance general de materia

$$
\mathrm{F}_{\mathrm{j}}-\mathrm{F}_{\mathrm{j} 0}=\Re_{\mathrm{j}} \cdot \mathrm{V}
$$

\begin{tabular}{|c|c|}
\hline & istemas con una reacción química \\
\hline \multirow{2}{*}{$\begin{array}{l}\text { Un RCTA } \\
\left(\mathrm{X}_{0}=0\right)\end{array}$} & $c_{k 0} \cdot X+v_{k} \cdot r \cdot \tau=0$ \\
\hline & $c_{j 0}-c_{j}+v_{j} \cdot r \cdot \tau=0$ \\
\hline \multirow{2}{*}{$\begin{array}{l}\text { Varios RCTA } \\
\left(\overline{\mathrm{X}}_{\mathrm{n}} \neq 0\right)\end{array}$} & $c_{k 0} \cdot\left(X_{n}-\bar{X}_{n}\right)+v_{k} \cdot r_{n} \cdot \tau_{n}=0$ \\
\hline & $\bar{c}_{j n}-c_{j n}+v_{j} \cdot r_{n} \cdot \tau_{n}=0$ \\
\hline \multicolumn{2}{|r|}{ Sistemas con varias reacciones químicas } \\
\hline \multirow{2}{*}{$\begin{array}{l}\text { Un RCTA } \\
\left(\mathrm{X}_{\mathrm{i} 0}=0\right)\end{array}$} & $\mathrm{c}_{\mathrm{k} 0} \cdot \mathrm{X}_{\mathrm{i}}+\tau \cdot v_{\mathrm{ik}} \cdot \mathrm{r}_{\mathrm{i}}=0$, paralelas \\
\hline & $\mathrm{c}_{\mathrm{j} 0}-\mathrm{c}_{\mathrm{j}}+\tau \cdot \sum_{\mathrm{i}=1}^{\mathrm{R}} \mathrm{v}_{\mathrm{ij}} \cdot \mathrm{r}_{\mathrm{i}}=0$, cualesquiera \\
\hline \multirow{2}{*}{$\begin{array}{l}\text { Varios RCTA } \\
\left(\overline{\mathrm{X}}_{\text {in }} \neq 0\right)\end{array}$} & $\mathrm{c}_{\mathrm{k} 0} \cdot\left(\mathrm{X}_{\mathrm{in}}-\overline{\mathrm{X}}_{\mathrm{in}}\right)+\mathrm{v}_{\mathrm{ik}} \cdot \mathrm{r}_{\mathrm{in}} \cdot \tau_{\mathrm{n}}=0$, paralelas \\
\hline & $\bar{c}_{\mathrm{jn}}-\mathrm{c}_{\mathrm{jn}}+\tau_{\mathrm{n}} \cdot \sum_{\mathrm{i}=1}^{\mathrm{R}} \mathrm{v}_{\mathrm{ij}} \cdot \mathrm{r}_{\mathrm{in}}=0$, cualesquiera \\
\hline
\end{tabular}


Reactor Continuo Tubular de Flujo de Pistón, RFP (Plug-Flow Tubular Reactor, PFR)

Balance general de materia

$$
\Re_{\mathrm{j}}=\frac{\mathrm{dF}_{\mathrm{j}}}{\mathrm{dV}}
$$

\begin{tabular}{|c|}
\hline Sistemas con una reacción química \\
\hline $\mathrm{dV}=\mathrm{Q}_{\mathrm{v} 0} \cdot \frac{\mathrm{dc_{ \textrm {j } }}}{v_{\mathrm{j}} \cdot \mathrm{r}}$, densidad constante \\
\hline $\mathrm{dV}=\mathrm{F}_{\mathrm{k} 0} \cdot \frac{\mathrm{dX}}{\left(-v_{\mathrm{k}}\right) \cdot \mathrm{r}}$, densidad variable \\
\hline$\frac{\mathrm{dX}}{\mathrm{dV}}=\frac{\left(-v_{\mathrm{i} k}\right) \cdot \mathrm{r}_{\mathrm{i}}}{\mathrm{F}_{\mathrm{k} 0}} \quad \mathrm{Sistemas}$ con varias reacciones químicas \\
\hline$\frac{\mathrm{dn}}{\mathrm{dV}}=\frac{\sum_{\mathrm{i}=1}^{\mathrm{R}} \mathrm{v}_{\mathrm{ij}} \cdot \mathrm{r}_{\mathrm{i}}}{\mathrm{F}_{\mathrm{k} 0}} \quad \mathrm{j}=1,2 \ldots \ldots . . \mathrm{R}$, paralelas \\
\end{tabular}


Balances de materia más habituales para sistemas con una Reacción Química que tiene lugar en un único Reactor Químico de Flujo Ideal

Balance general de materia

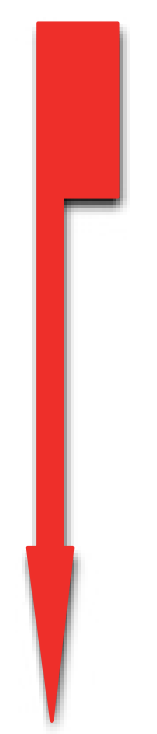

$$
\begin{gathered}
\int_{A} c_{j} \cdot(\vec{v} \cdot \vec{n}) \cdot d A+\frac{d}{d t} \int_{V} c_{j} \cdot d V=\int_{V} \Re R_{j} \cdot d V\left(\frac{\mathrm{kmol} \mathrm{A}_{j}}{s}\right) \\
\int_{A} d F_{j}+\frac{d}{d t} \int_{V} c_{j} \cdot d V=\int_{V} \Re_{j} \cdot d V\left(\frac{\mathrm{kmol} \mathrm{A}}{s}\right)
\end{gathered}
$$

Reactor Continuo Tubular de Flujo de Pistón, RFP (Plug-Flow Tubular Reactor, PFR)

$$
\begin{gathered}
d F_{j}=\Re_{j} \cdot d V\left(\frac{k m o l}{s}\right) \\
d V=F_{k 0} \cdot \frac{d X}{\left(-v_{k}\right) \cdot r}\left(m^{3}\right)
\end{gathered}
$$

Reactores de Tanque Agitado

$F_{j}-F_{j 0}+\frac{d}{d t} \int_{V} c_{j} \cdot d V=\int_{V} \Re_{j} \cdot d V\left(\frac{k m o l}{A_{j}}\right)$

Reactor Continuo de Tanque Agitado, RCTA

(Continuous-Stirred Tank Reactor, CSTR,

or Backmixed Reactor)

$\mathrm{F}_{\mathrm{j}}-\mathrm{F}_{\mathrm{j} 0}=\Re_{\mathrm{j}} \cdot \mathrm{V} \quad(\mathrm{kmol} \mathrm{A} / \mathrm{s})$

$c_{k 0} \cdot X+v_{k} \cdot r \cdot \tau=0 \quad\left(k m o l A_{k} / m^{3}\right)$

Reactor Discontinuo de Tanque Agitado, RDTA

(Batch Reactor)

$$
\begin{aligned}
& \frac{d}{d r}\left(c_{j} \cdot V\right)=\Re_{j} \cdot V \quad\left(k m o l ~ A_{j} / s\right) \\
& d t=\frac{N_{k 0}}{V} \cdot \frac{d X}{\left(-v_{k}\right) \cdot r} \quad(s)
\end{aligned}
$$

Reactor Semicontinuo de Tanque Agitado, RSCTA (Semibatch Reactor)

$$
\begin{aligned}
& F_{j}-F_{j 0}+\frac{d}{d t} \int_{V} c_{j} \cdot d V=\int_{V} \Re_{j} \cdot d V\left(\frac{k m o l A_{j}}{s}\right) \\
& c_{j}-c_{j 0}+\tau \cdot \frac{d c_{j}}{d t}=v_{j} \cdot r \cdot \tau\left(\frac{k m o l A_{j}}{m^{3}}\right)
\end{aligned}
$$




\section{ANEXO III}

\section{BALANCES DE LA ENERGÍA}

Reactor Discontinuo de Tanque Agitado, RDTA (Batch Reactor)

Balance general de energía

$$
\frac{\mathrm{d}}{\mathrm{dt}}\left(\sum_{\mathrm{j}=1}^{\mathrm{S}} \mathrm{N}_{\mathrm{j}} \cdot \mathrm{h}_{\mathrm{j}}\right)=\mathrm{Q}^{*}
$$

\begin{tabular}{|c|}
\hline$\frac{\mathrm{dT}}{\mathrm{dt}}-\mathrm{J} \cdot \frac{\mathrm{dX}}{\mathrm{dt}}=\mathrm{Q}^{\prime}$ \\
\hline$\frac{\mathrm{dT}}{\mathrm{dt}}+\frac{\mathrm{v}_{\mathrm{k}} \cdot \mathrm{r}}{\mathrm{c}_{\mathrm{k} 0}} \cdot \mathrm{J}=\mathrm{Q}^{\prime}$ \\
\hline Sistemas con varias reacciones químicas \\
\hline$\frac{\mathrm{dT}}{\mathrm{dt}}-\sum_{\mathrm{i}=1}^{\mathrm{R}} \mathrm{J}_{\mathrm{i}} \cdot \frac{\mathrm{dX}}{\mathrm{dt}}=\mathrm{Q}^{\prime}$, paralelas \\
\hline$\frac{\mathrm{dT}}{\mathrm{dt}}+\frac{1}{\mathrm{c}_{\mathrm{k} 0}} \cdot \sum_{\mathrm{i}=1}^{\mathrm{R}} \mathrm{v}_{\mathrm{ik}} \cdot \mathrm{r}_{\mathrm{i}} \cdot \mathrm{J}_{\mathrm{i}}=\mathrm{Q}^{\prime}$, cualesquiera \\
\hline
\end{tabular}

Reactor Semicontinuo de Tanque Agitado, RSCTA (Semibatch Reactor) Ecuación para un RSCTA y la puesta en marcha de un RCTA

Balance general de energía

$$
\begin{gathered}
\sum_{j=1}^{S} \mathrm{~F}_{j} \cdot \mathrm{h}_{\mathrm{j}}-\sum_{\mathrm{j}=1}^{\mathrm{S}} \mathrm{F}_{\mathrm{j} 0} \cdot \mathrm{h}_{\mathrm{j} 0}+\frac{\mathrm{d}}{\mathrm{dt}}\left(\sum_{\mathrm{j}=1}^{\mathrm{S}} \mathrm{N}_{\mathrm{j}} \cdot \mathrm{h}_{\mathrm{j}}\right)=\mathrm{Q}^{*} \\
\sum_{\mathrm{j}=1}^{\mathrm{S}} \mathrm{N}_{\mathrm{j}} \cdot \overline{\mathrm{C}}_{\mathrm{pj}} \cdot \frac{\mathrm{dT}}{\mathrm{dt}}=\mathrm{Q}^{*}+\sum_{\mathrm{j}=1}^{\mathrm{S}} \mathrm{F}_{\mathrm{j} 0} \cdot \overline{\mathrm{C}}_{\mathrm{pj}} \cdot\left(\mathrm{T}_{0}-\mathrm{T}\right)+\mathrm{v}_{\mathrm{k}} \cdot \Delta \mathrm{H}_{\mathrm{k}}^{0} \cdot \mathrm{r} \cdot \mathrm{V} \\
\text { Sistemas con varias reacciones químicas }_{\mathrm{j}=1}^{\mathrm{S}} \mathrm{N}_{\mathrm{j}} \cdot \overline{\mathrm{C}}_{\mathrm{pj}} \cdot \frac{\mathrm{dT}}{\mathrm{dt}}=\mathrm{Q}^{*}+\left(\mathrm{T}_{0}-\mathrm{T}\right) \cdot \sum_{\mathrm{j}=1}^{\mathrm{S}} \mathrm{F}_{\mathrm{j} 0} \cdot \overline{\mathrm{C}}_{\mathrm{pj}}+\left(\sum_{\mathrm{i}=1}^{\mathrm{R}} v_{\mathrm{ik}} \cdot \Delta \mathrm{H}_{\mathrm{ik}}^{0} \cdot \mathrm{r}_{\mathrm{i}}\right) \cdot \mathrm{V}
\end{gathered}
$$


Reactor Continuo de Tanque Agitado, RCTA (Continuous-Stirred Tank Reactor, CSTR, or Backmixed Reactor)

Balance general de energía

$$
\sum_{j=1}^{S} F_{j} \cdot h_{j}-\sum_{j=1}^{S} F_{j 0} \cdot h_{j 0}=Q^{*}
$$

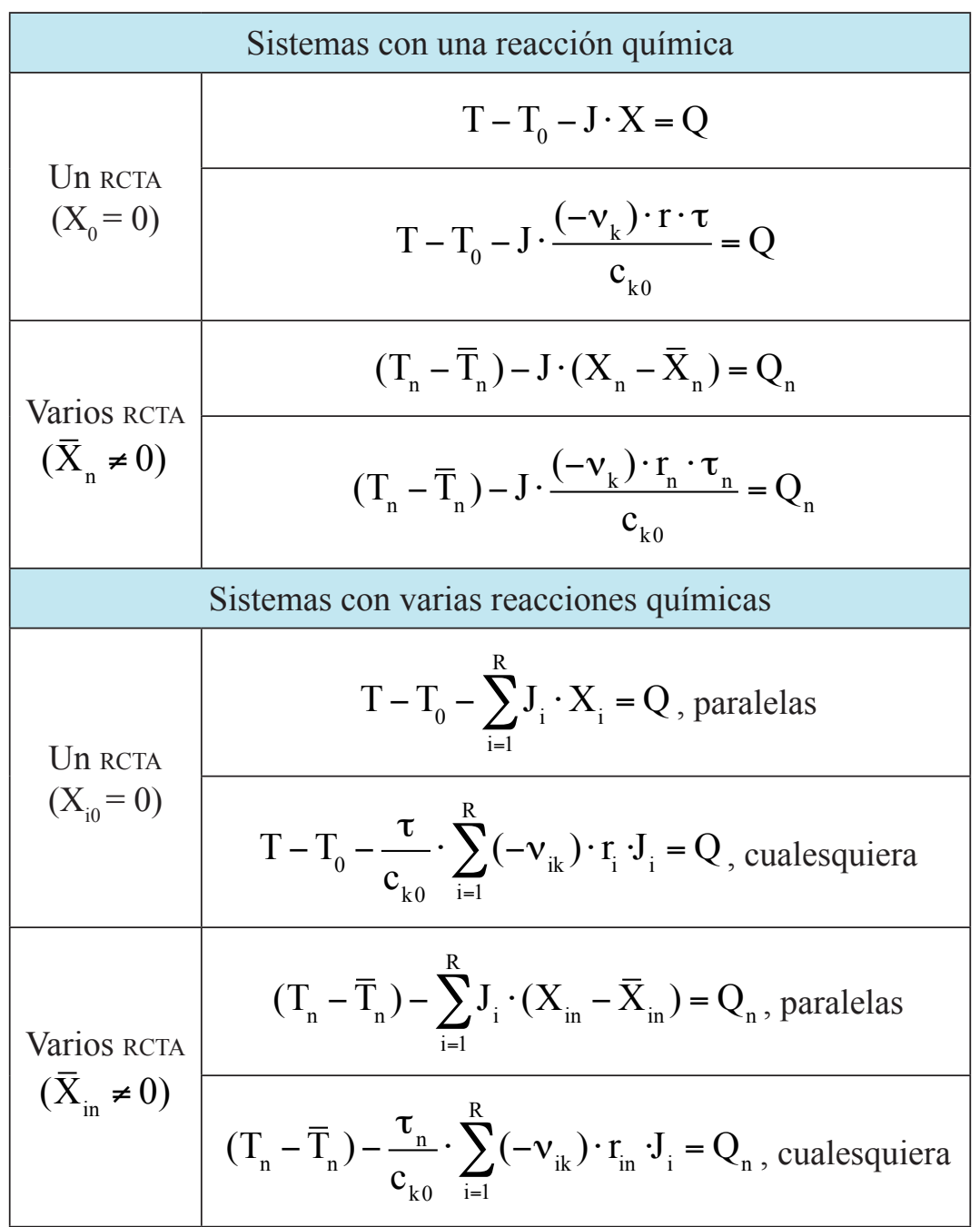


Reactor Continuo Tubular de Flujo de Pistón, RFP (Plug-Flow Tubular Reactor, PFR)

Balance general de energía

$$
\sum_{j=1}^{S} F_{j} \cdot d h_{j}+\sum_{j=1}^{S} h_{j} \cdot d F_{j}=d Q^{*}
$$

\begin{tabular}{|c|}
\hline Sistemas con una reacción química \\
\hline$\frac{\mathrm{dT}}{\mathrm{d} \ell}-\mathrm{J} \cdot \frac{\mathrm{dX}}{\mathrm{d} \ell}=\frac{\mathrm{dQ}}{\mathrm{d} \ell}$ \\
$\frac{\mathrm{dT}}{\mathrm{d} \ell}+\frac{v_{\mathrm{k}} \cdot \mathrm{r}}{\mathrm{G}_{\mathrm{k} 0}} \cdot \mathrm{J}=\frac{\mathrm{dQ}}{\mathrm{d} \ell}$ \\
\hline$\frac{\mathrm{dT}}{\mathrm{d} \ell}-\sum_{\mathrm{i}=1}^{\mathrm{R}} \mathrm{J}_{\mathrm{i}} \cdot \frac{\mathrm{dX}}{\mathrm{d} \ell}=\frac{\mathrm{dQ}}{\mathrm{d} \ell}$, paralelas \\
\hline$\frac{\mathrm{dT}}{\mathrm{d} \ell}+\frac{1}{\mathrm{G}_{\mathrm{k} 0}} \cdot \sum_{\mathrm{i}=1}^{\mathrm{R}} v_{\mathrm{ik}} \cdot \mathrm{r}_{\mathrm{i}} \cdot \mathrm{J}_{\mathrm{i}}=\frac{\mathrm{dQ}}{\mathrm{d} \ell}$, cualesquiera \\
\hline
\end{tabular}


Balances de energía más habituales para sistemas con una Reacción Química que tiene lugar en un único Reactor Químico de Flujo Ideal

\section{Balance general de energía}

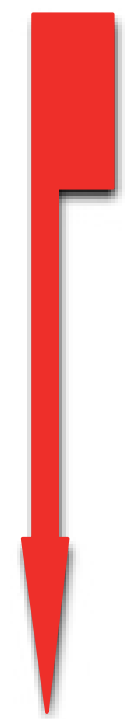

$$
\begin{gathered}
\int_{A}\left(e_{p}+e_{k}+u+\frac{P}{\rho_{m}}\right) \cdot \rho_{m} \cdot(\vec{v} \cdot \vec{n}) \cdot d A+\frac{d}{d t} \int_{V}\left(e_{p}+e_{k}+u\right) \cdot \rho_{m} \cdot d V=Q^{*} \quad(k J / s) \\
\int_{A} h \cdot \rho_{m} \cdot(\vec{v} \cdot \vec{n}) \cdot d A+\frac{d}{d t} \int_{V}\left(h-\frac{P}{\rho_{m}}\right) \cdot \rho_{m} \cdot d V=Q^{*} \quad(k J / s) \\
\int_{A} d\left(F_{t} \cdot h\right)+\frac{d}{d t} \int_{V}\left(h \cdot \rho_{m}-P\right) \cdot d V=Q^{*}(k J / s) \text { ECUACIÓN (1) }
\end{gathered}
$$

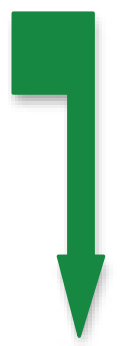

Reactor Continuo Tubular de Flujo de Pistón, RFP (Plug-Flow Tubular Reactor, PFR)

$$
\begin{aligned}
& \text { ECUACIÓN (3) } \sum_{\mathrm{j}=1}^{\mathrm{S}} \mathrm{F}_{\mathrm{j}} \cdot d \mathrm{~d}_{\mathrm{j}}+\sum_{\mathrm{j}=1}^{\mathrm{S}} \mathrm{h}_{\mathrm{j}} \cdot \mathrm{dF_{ \textrm {j } }}=\mathrm{dQ}^{*} \quad(\mathrm{~kJ} / \mathrm{s}) \\
& \text { ECUACIÓN (3.1) } \frac{\mathrm{dT}}{\mathrm{d} \ell}-\mathrm{J} \cdot \frac{\mathrm{dX}}{\mathrm{d} \ell}=\frac{\mathrm{dQ}}{\mathrm{d} \ell} \quad(\mathrm{K} / \mathrm{m})
\end{aligned}
$$

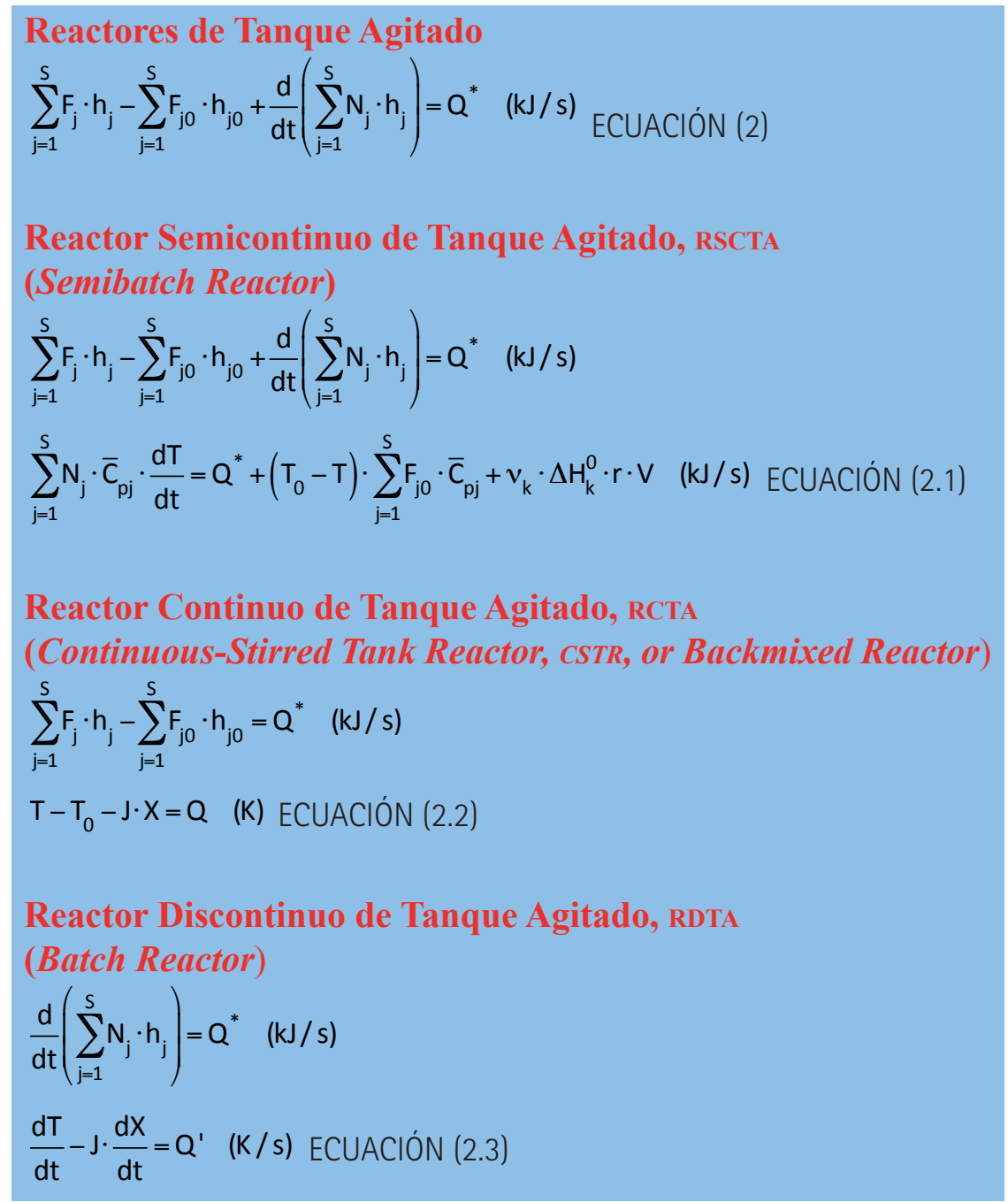




\section{ANEXO IV}

\section{ALGUNAS CONSIDERACIONES SOBRE EL CALOR ESPECÍFICO}

A continuación se muestran las correlaciones entre el calor específico medio $\left(\mathrm{C}_{\mathrm{pm}}\right)$ de una mezcla y los calores específicos de las especies químicas $\left(\mathrm{C}_{\mathrm{pj}}\right)$ :

$$
\begin{aligned}
& \sum_{\mathrm{j}=1}^{\mathrm{S}} \theta_{\mathrm{j}} \cdot \overline{\mathrm{C}}_{\mathrm{pj}}=\frac{\mathrm{N}_{10}}{\mathrm{~N}_{\mathrm{k} 0}} \cdot \overline{\mathrm{C}}_{\mathrm{p} 1}+\frac{\mathrm{N}_{20}}{\mathrm{~N}_{\mathrm{k} 0}} \cdot \overline{\mathrm{C}}_{\mathrm{p} 2}+\ldots \ldots+\frac{\mathrm{N}_{\mathrm{k} 0}}{\mathrm{~N}_{\mathrm{k} 0}} \cdot \overline{\mathrm{C}}_{\mathrm{pk}} \cdots \cdots \cdot \frac{\mathrm{N}_{\mathrm{S} 0}}{\mathrm{~N}_{\mathrm{k} 0}} \cdot \overline{\mathrm{C}}_{\mathrm{pS}}=
\end{aligned}
$$

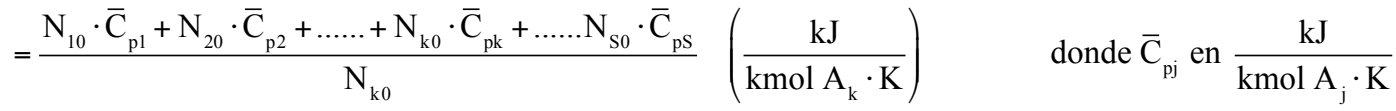

$$
\begin{aligned}
& \overline{\mathrm{C}}_{\mathrm{pm}}=\frac{\mathrm{N}_{10} \cdot \overline{\mathrm{C}}_{\mathrm{p} 1}+\mathrm{N}_{20} \cdot \overline{\mathrm{C}}_{\mathrm{p} 2}+\ldots \ldots+\mathrm{N}_{\mathrm{k} 0} \cdot \overline{\mathrm{C}}_{\mathrm{pk}}+\ldots \ldots . \mathrm{N}_{\mathrm{s} 0} \cdot \overline{\mathrm{C}}_{\mathrm{pS}}}{\mathrm{N}_{10}+\mathrm{N}_{20}+\ldots \ldots+\mathrm{N}_{\mathrm{k} 0}+\ldots \ldots . \mathrm{N}_{\mathrm{s} 0}}= \\
& =\frac{\mathrm{N}_{10} \cdot \overline{\mathrm{C}}_{\mathrm{p} 1}+\mathrm{N}_{20} \cdot \overline{\mathrm{C}}_{\mathrm{p} 2}+\ldots \ldots+\mathrm{N}_{\mathrm{k} 0} \cdot \overline{\mathrm{C}}_{\mathrm{pk}}+\ldots \ldots . \mathrm{N}_{\mathrm{S} 0} \cdot \overline{\mathrm{C}}_{\mathrm{pS}}}{\mathrm{N}_{\mathrm{t} 0}}= \\
& =\mathrm{x}_{10} \cdot \overline{\mathrm{C}}_{\mathrm{p} 1}+\mathrm{x}_{20} \cdot \overline{\mathrm{C}}_{\mathrm{p} 2}+\ldots \ldots+\mathrm{x}_{\mathrm{k} 0} \cdot \overline{\mathrm{C}}_{\mathrm{pk}}+\ldots \ldots . \mathrm{x}_{\mathrm{s} 0} \cdot \overline{\mathrm{C}}_{\mathrm{ps}}\left(\frac{\mathrm{kJ}}{\mathrm{kmol} \text { totales } \cdot \mathrm{K}}\right) \quad \text { donde } \overline{\mathrm{C}}_{\mathrm{pj}} \text { en } \frac{\mathrm{kJ}}{\mathrm{kmol} \mathrm{A} \cdot \mathrm{K}}
\end{aligned}
$$

Comparando ambas definiciones:

$$
\begin{gathered}
\mathrm{N}_{\mathrm{t} 0} \cdot \overline{\mathrm{C}}_{\mathrm{pm}}=\mathrm{N}_{\mathrm{k} 0} \cdot \sum_{\mathrm{j}=1}^{\mathrm{S}} \theta_{\mathrm{j}} \cdot \overline{\mathrm{C}}_{\mathrm{pj}} \\
\mathrm{obien} \\
\overline{\mathrm{C}}_{\mathrm{pm}}=\mathrm{x}_{\mathrm{k} 0} \cdot \sum_{\mathrm{j}=1}^{\mathrm{S}} \theta_{\mathrm{j}} \cdot \overline{\mathrm{C}}_{\mathrm{pj}}
\end{gathered}
$$

En sistemas continuos se cumplen las mismas igualdades sin más que sustituir $\mathrm{N}_{\mathrm{j} 0}$ por $\mathrm{F}_{\mathrm{j} 0}$

Suele ser bastante habitual encontrar en la bibliografía que el valor medio del calor específico de una mezcla esté referido al volumen de la mezcla $\left(\mathrm{m}^{3}\right)$ a la masa de la mezcla $(\mathrm{kg})$ en lugar de la número de kmoles totales. En este caso, antes de aplicar las igualdades anteriores hay que referir el valor medio del calor específico de la mezcla al número de kmoles, según se muestra a continuación:

i) si el valor medio del calor específico de una mezcla está referido al volumen de la mezcla $\left(\mathrm{m}^{3}\right)$

$$
\overline{\mathrm{C}}_{\mathrm{pm}}\left(\frac{\mathrm{kJ}}{\mathrm{kmol} \text { totales } \cdot \mathrm{K}}\right)=\overline{\mathrm{C}}_{\mathrm{pm}}\left(\frac{\mathrm{kJ}}{\mathrm{m}^{3} \cdot \mathrm{K}}\right) \cdot \frac{1}{\mathrm{c}_{\mathrm{t} 0}}\left(\frac{\mathrm{m}^{3}}{\mathrm{kmol} \text { totales }}\right)
$$

ii) si el valor medio del calor específico de una mezcla está referido a la masa de la mezcla $(\mathrm{kg})$

$$
\overline{\mathrm{C}}_{\mathrm{pm}}\left(\frac{\mathrm{kJ}}{\mathrm{kmol} \text { totales } \cdot \mathrm{K}}\right)=\overline{\mathrm{C}}_{\mathrm{pm}}\left(\frac{\mathrm{kJ}}{\mathrm{kg} \cdot \mathrm{K}}\right) \cdot \rho\left(\frac{\mathrm{kg}}{\mathrm{m}^{3}}\right) \cdot \frac{1}{\mathrm{c}_{\mathrm{t} 0}}\left(\frac{\mathrm{m}^{3}}{\mathrm{kmol} \text { totales }}\right)
$$




\section{PROBLEMAS}

Problema 01. Un RDTA adiabático se carga con una solución acuosa que contiene $500 \mathrm{~kg}$ de anhídrido acético, a $15.5^{\circ} \mathrm{C}(288.5 \mathrm{~K})$, cuya concentración es de 0.49 $\mathrm{kmol}$ de anhídrido acético $/ \mathrm{m}^{3}$. La reacción que se ha de desarrollar en dicho reactor se puede representar por el esquema:

$$
\left(\mathrm{CH}_{3} \mathrm{CO}\right)_{2} \mathrm{O}(\ell)+\mathrm{H}_{2} \mathrm{O}(\ell) \rightarrow 2 \mathrm{CH}_{3} \mathrm{COOH}(\ell)
$$

y su cinética es de primer orden respecto al anhídrido acético. Por cada kmol de anhídrido acético que se hidrolice se desprenden $209370 \mathrm{~kJ}$ (puede admitirse que $\Delta \mathrm{H}^{\circ}$ prácticamente no varía con la temperatura en el intervalo de operación).

Calcular el tiempo necesario para alcanzar un grado de conversión del 80 \%.

\section{Datos y notas}

La densidad de la disolución inicial es de $1050 \mathrm{~kg} / \mathrm{m}^{3}$ y su calor específico es $3767 \mathrm{~J} / \mathrm{kg} \mathrm{K}$. Ambas propiedades físicas puede admitirse que permanecen constantes durante toda la reacción.

La variación de la constante de velocidad con la temperatura viene dada en la tabla siguiente:

\begin{tabular}{|c|c|c|c|c|c|c|}
\hline $\mathrm{T}(\mathrm{K})$ & 277.6 & 283.1 & 288.7 & 294.3 & 299.8 & 305.4 \\
\hline $\mathrm{k}\left(\mathrm{min}^{-1}\right)$ & 0.035 & 0.057 & 0.084 & 0.123 & 0.174 & 0.245 \\
\hline
\end{tabular}

Problema 02. En un reactor de mezcla completa, perfectamente aislado térmicamente, tiene lugar una reacción química de primer orden, irreversible, exotérmica y en fase líquida, de la forma:

$$
\mathrm{A}_{1} \rightarrow \mathrm{A}_{2}
$$

Con la finalidad de que la temperatura de la mezcla reactante permanezca constante durante todo el período de reacción, se añade a la mezcla, continuamente, un líquido inerte (perfectamente miscible con la mezcla reactante) que actúa como refrigerante. El caudal con que se alimenta el líquido frío se va modificando de acuerdo con las necesidades de refrigeración del sistema. En la figura adjunta se esquematiza la instalación. 


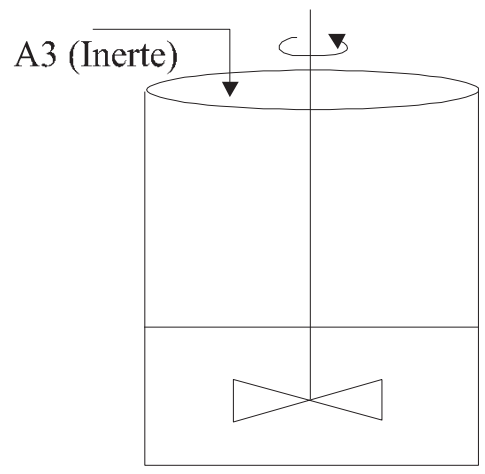

Calcular:

a) El caudal volumétrico de líquido inerte refrigerante $\left(\mathrm{Q}_{\mathrm{V} 0}\right)$ al cabo de dos horas de iniciarse la reacción.

b) El volumen de la mezcla reactante que hay en el reactor cuando han transcurrido dos horas desde el comienzo del ciclo de reacción.

\section{Datos y notas}

Temperatura de reacción: $310 \mathrm{~K}$

Constante de velocidad de reacción: $1.2 \cdot 10^{-4} \mathrm{~s}^{-1}$ ( a $310 \mathrm{~K}$ )

Temperatura de entrada del líquido inerte: $300 \mathrm{~K}$

Calor específico de todos los compuestos: $2.1 \mathrm{~kJ} / \mathrm{kg} \mathrm{K}$

Densidad de todos los componentes: $800 \mathrm{~kg} / \mathrm{m}^{3}$

Calor de reacción a $310 \mathrm{~K}:-58000 \mathrm{~kJ} / \mathrm{kmol}$ de $\mathrm{A}_{1}$

Inicialmente el reactor solo contiene $\mathrm{A}_{1}$ con una concentración de $8 \mathrm{kmol}$ de $\mathrm{A}_{1} / \mathrm{m}^{3}$

El reactante $A_{1}$ ocupa inicialmente (para $\left.t=0\right)$ un volumen de reactor de $1.5 \mathrm{~m}^{3}$

Problema 03. En un RCTA se desarrolla una reacción química elemental irreversible en fase líquida, según el esquema:

$$
\mathrm{A}_{1}+\mathrm{A}_{2} \rightarrow 2 \mathrm{~A}_{3}
$$

Dado que la reacción es endotérmica, el reactor va provisto de una camisa de vapor de calefacción (figura adjunta). A partir de los datos que se detallan a continuación, calcular la temperatura de la mezcla reactante (temperatura de reacción) si el citado reactor opera en régimen estacionario, alcanzando un grado de conversión del $95 \%$. 


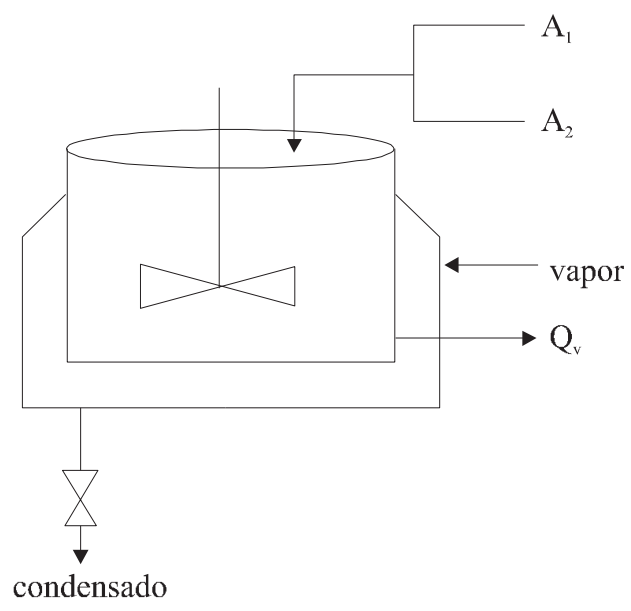

\section{Datos y notas}

Volumen del reactor: $0.50 \mathrm{~m}^{3}$.

Superficie efectiva de transmisión de calor: $0.90 \mathrm{~m}^{2}$.

Temperatura de condensación del vapor de calefacción: $458 \mathrm{~K}$.

Coeficiente global de transmisión de calor desde el vapor calefactor a la mezcla reactante: $852 \mathrm{~J} / \mathrm{m}^{2} \cdot \mathrm{s} \cdot \mathrm{K}$.

Potencia del agitador: $0,25 \mathrm{Hp}$.

Calor de reacción: $46500 \mathrm{~kJ} / \mathrm{kmol}$ de $\mathrm{A}_{1}$ (supóngase independiente de la temperatura).

\begin{tabular}{|c|c|c|c|}
\cline { 2 - 4 } \multicolumn{1}{c|}{} & $\mathrm{A}_{1}$ & $\mathrm{~A}_{2}$ & $\mathrm{~A}_{3}$ \\
\hline Caudal de alimentación $(\mathrm{mol} / \mathrm{h})$ & 100 & 100 & 0 \\
\hline $\begin{array}{c}\text { Temperatura de alimento }(\mathrm{K}) \\
\text { Calor específico }(\mathrm{kJ} / \mathrm{mol} . \mathrm{K})(\text { Independiente de } \\
\text { la T) }\end{array}$ & 300 & 300 & --- \\
\hline Peso molecular & 184 & 200 \\
\hline Densidad $\left(\mathrm{kg} / \mathrm{m}^{3}\right)$ & 128 & 94 & --- \\
\hline
\end{tabular}

El volumen de reacción permanece constante lógicamente durante toda la operación (régimen estacionario) y es igual al volumen del reactor.

Problema 04. La descomposición de la fosfamina tiene lugar a temperaturas elevadas con arreglo al esquema siguiente:

$$
4 \mathrm{PH}_{3}(\mathrm{~g}) \rightarrow \mathrm{P}_{4}(\mathrm{~g})+6 \mathrm{H}_{2}(\mathrm{~g})
$$

La reacción es endotérmica e irreversible, ajustándose bien a una cinética de primer orden, según la ecuación $-\mathfrak{R}_{\mathrm{PH}_{3}}=\mathrm{k} \cdot \mathrm{c}_{\mathrm{PH}_{3}}$ 
Si se parte de fosfamina pura, a $945 \mathrm{~K}$, y la reacción se desarrolla adiabáticamente, mediante un balance energético, puede determinarse la variación de la temperatura con el grado de conversión. Dicha variación se detalla en la tabla siguiente junto con los valores de la constante de velocidad referida a la desaparición de fosfamina correspondientes a las diferentes temperaturas:

\begin{tabular}{|c|c|c|c|c|c|}
\hline $\mathrm{X}$ & 0.00 & 0.10 & 0.20 & 0.25 & 0.30 \\
\hline $\mathrm{T}(\mathrm{K})$ & 945 & 885 & 826 & 797 & 768 \\
\hline $\mathrm{k}\left(\mathrm{s}^{-1}\right)$ & 37.2 & 1.42 & $3.65 \cdot 10^{-2}$ & $3 \cdot 10^{-3}$ & $5.83 \cdot 10^{-4}$ \\
\hline
\end{tabular}

¿Qué tamaño de reactor tubular se requerirá para alcanzar una conversión del 30\%, si el reactor opera a la presión atmosférica, y el alimento se introduce (en fase gaseosa) con un caudal másico de $22.7 \mathrm{~kg}$ de fosfamina pura $/ \mathrm{h}$, a una temperatura de $945 \mathrm{~K}$ ?

Problema 05. Las reacciones de craqueo de hidrocarburos se suelen llevar a cabo en reactores tipo RFP. Dado que se trata de reacciones endotérmicas, se utilizan diferentes técnicas para suministrar al sistema el flujo de calor necesario. La temperatura máxima a la entrada está determinada por problemas con los materiales de construcción o con la aparición de reacciones no deseadas como la coquización. Si se ha fijado la temperatura a la entrada del RFP $\left(\mathrm{T}_{0}\right)$ y la temperatura a lo largo del reactor puede disminuir, a pesar de la posible aportación energética, este efecto, junto con la disminución de la concentración de los reactivos por la reacción química, contribuirá a la disminución de la velocidad de ésta. Una forma de reducir este efecto consiste en utilizar una elevada proporción de inerte en la corriente alimento.

Considérese una reacción de craqueo de la forma:

$$
\mathrm{A}_{1} \rightarrow \text { productos }
$$

cuya cinética de primer orden responde a la ecuación:

$$
\mathrm{r}=10^{14} \cdot \exp \left(\frac{-24000}{\mathrm{~T}}\right) \cdot \mathrm{c}_{1} \quad\left(\mathrm{~g} / \mathrm{s} \cdot \mathrm{m}^{3}\right)
$$

donde $\mathrm{T}$ va en $\mathrm{K}$ y $\mathrm{c}_{1}$ en $\mathrm{g} / \mathrm{m}^{3}$.

Esta reacción se desarrolla en un RFP adiabático con un tiempo espacial de $0.3 \mathrm{~s}$. Si la concentración en la corriente de entrada es de $132 \mathrm{~g} / \mathrm{m}^{3}$ de $\mathrm{A}_{1}$ y $270 \mathrm{~g} / \mathrm{m}^{3}$ de inerte, calcular la concentración de $\mathrm{A}_{1}$ y la temperatura de la corriente de salida. Repetir el cálculo anterior para una corriente de entrada cuyas concentraciones son $270 \mathrm{~g} / \mathrm{m}^{3}$ de $A_{1}$ y $132 \mathrm{~g} / \mathrm{m}^{3}$ de inerte e interpretar los resultados obtenidos. 


\section{Datos y notas}

Calor específico de $\mathrm{A}_{1}$ y de sus productos: $0.4 \mathrm{cal} / \mathrm{g} \cdot \mathrm{K}$

Calor específico del inerte: $0.5 \mathrm{cal} / \mathrm{g} \cdot \mathrm{K}$

La densidad del sistema se puede considerar constante en primera aproximación. Para el valor de esta densidad se tomará la de entrada, que se calculará como la suma de las densidades (composiciones) de los dos componentes.

$\Delta \mathrm{H}^{0}{ }_{1}=203 \mathrm{cal} / \mathrm{g}$

$\mathrm{T}_{0}=798 \mathrm{~K}$

Problema 06. En un RDTA se desarrolla la reacción elemental, irreversible, en fase líquida:

$$
\mathrm{A}_{1}+\mathrm{A}_{2} \rightarrow \mathrm{A}_{3}+\mathrm{A}_{4}
$$

El alimento consiste en una mezcla equimolecular de $\mathrm{A}_{1}$ y $\mathrm{A}_{2}$ puros, a $100{ }^{\circ} \mathrm{C}$.

a) Deducir una expresión que relacione el grado de conversión con el tiempo de reacción, cuando el RDTA opera de forma isoterma a la temperatura del alimento.

b) Deducir una expresión que relacione la temperatura con el grado de conversión, cuando el RDTA opera de forma adiabática.

c) Determinar la variación del grado de conversión con el tiempo de reacción cuando el RDTA opera de forma adiabática. ¿Se podrán utilizar dispositivos de control que tengan accesorios situados dentro del reactor que se deterioren a temperaturas superiores a $250^{\circ} \mathrm{C}$ ?

d) Si el reactor funciona de forma isoterma, ¿a qué temperatura ha de operar para conseguir una conversión de 0.95 en tres horas? ¿Existiría en este caso algún problema con los accesorios de los elementos de control mencionados en el apartado anterior?

Datos y notas

$\Delta \mathrm{H}^{0}{ }_{1}=-10^{5} \mathrm{~kJ} / \mathrm{kmol}$ de $\mathrm{A}_{1}$

$\mathrm{k}=1.5 \cdot 10^{11} \cdot \exp (-14000 / \mathrm{T}) \quad\left(\mathrm{m}^{3} / \mathrm{kmol} \cdot \mathrm{s}\right)$

Calor específico del alimento: $3.6 \mathrm{~kJ} / \mathrm{K} \cdot \mathrm{kg}$ (constante para todo el periodo de reacción)

Densidad del alimento: $1100 \mathrm{~kg} / \mathrm{m}^{3}$ (constante para todo el periodo de reacción)

$\mathrm{M}_{1}=100 \mathrm{~kg} / \mathrm{kmol}$

$\mathrm{M}_{2}=80 \mathrm{~kg} / \mathrm{kmol}$

Problema 07. Se desea diseñar una planta piloto para la producción de $\mathrm{A}_{3}$, según la siguiente reacción irreversible en fase gas:

$$
\mathrm{A}_{1}+\mathrm{A}_{2} \rightarrow \mathrm{A}_{3}+\mathrm{A}_{4}
$$


El alimento al reactor consiste en una mezcla de 4 moles de $\mathrm{A}_{2}$ por cada mol de $\mathrm{A}_{1}$, a $200{ }^{\circ} \mathrm{C}$. El caudal molar total del alimento es $0.17 \cdot 10^{-3} \mathrm{kmol} / \mathrm{h}$. El reactor está constituido por un tubo de 5 pulgadas de diámetro interno y perfectamente aislado del exterior para que su comportamiento se pueda considerar adiabático. Teniendo en cuenta que el producto $\mathrm{A}_{3}$ se descompone a una temperatura superior a los $546 \mathrm{~K}$, calcular la máxima posible longitud del reactor para evitar la descomposición de $\mathrm{A}_{3}$.

\section{Datos y notas}

$\Delta \mathrm{H}_{\mathrm{k}}^{\circ}=-26800 \mathrm{kcal} / \mathrm{kmol}$

$\mathrm{r}=206 \cdot 10^{6} \cdot \exp \left(\frac{-14233}{\mathrm{~T}}\right) \cdot \mathrm{p}_{1} \cdot \mathrm{p}_{2}$, en la que si las presiones parciales

se expresan en atm la velocidad viene en $\mathrm{kmol} / \mathrm{m}^{3} \cdot \mathrm{h}$

Los calores específicos son $8.6,25.3,28$ y $7.2 \mathrm{kcal} / \mathrm{kmol} \cdot \mathrm{K}$ para $\mathrm{A}_{1}, \mathrm{~A}_{2}$, $\mathrm{A}_{3} \mathrm{y} \mathrm{A}_{4}$ respectivamente.

La presión total es constante e igual a 2 atm.

Problema 08. La hidrólisis de soluciones acuosas diluidas de anhídrido acético es una reacción irreversible y de segundo orden total (primer orden parcial con respecto a cada uno de los reactivos). El esquema de reacción es:

$$
\left(\mathrm{CH}_{3} \mathrm{CO}\right)_{2} \mathrm{O}(\ell)+\mathrm{H}_{2} \mathrm{O}(\ell) \rightarrow 2 \mathrm{CH}_{3} \mathrm{COOH}(\ell)
$$

Esta reacción se va a llevar a cabo en un RDTA que se va a cargar inicialmente con 200 litros de disolución acuosa de anhídrido acético, con una concentración de $0.216 \mathrm{kmol} / \mathrm{m}^{3}$, y a $15^{\circ} \mathrm{C}$.

a) Si el RDTA es isotermo (utilizando el sistema refrigerante adecuado), calcular el tiempo de reacción necesario para alcanzar una conversión del 70 \%.

b) Si el RDTA está perfectamente aislado y su comportamiento puede considerarse adiabático, calcular el tiempo de reacción necesario para alcanzar una conversión del $70 \%$.

Datos y notas

\begin{tabular}{|c|c|c|c|c|}
\hline $\mathrm{T}(\mathrm{K})$ & 283 & 288 & 298 & 313 \\
\hline $\mathrm{r}\left(\mathrm{kmol} / \mathrm{min} \cdot \mathrm{m}^{3}\right)$ & $0.0567 \times \mathrm{c}$ & $0.0806 \times \mathrm{c}$ & $0.1580 \times \mathrm{c}$ & $0.3800 \times \mathrm{c}$ \\
\hline
\end{tabular}

siendo c la concentración de anhídrido acético, en $\mathrm{kmol} / \mathrm{m}^{3}$.

Para la mezcla de reacción $\mathrm{C}_{\mathrm{p}}=3.77 \mathrm{~kJ} / \mathrm{kg} \cdot \mathrm{K}$

Para la mezcla de reacción $\rho=1050 \mathrm{~kg} / \mathrm{m}^{3}$

$\Delta \mathrm{H}_{\mathrm{k}}^{\circ}=-209300 \mathrm{~kJ} / \mathrm{kmol}$ 


\section{Reactor continuo de tanque agitado (RCTA) con intercambio de calor y adiabático}

\subsection{Ecuaciones de diseño}

En este capítulo se tratarán fundamentalmente los sistemas constituidos por un único Reactor Continuo de Tanque Agitado (RCTA) funcionando en régimen estacionario en el que tiene lugar una única reacción química en fase líquida (en la práctica industrial esto es lo habitual puesto que los RDTA se utilizan en sistemas de densidad constante, normalmente fases líquidas) cuya velocidad de reacción es normal (no autocatalítica). En consecuencia, tal y como se recogía en Anexo II del capítulo 1, el balance de materia se puede expresar mediante cualquiera de las dos ecuaciones siguientes:

$$
\begin{gathered}
c_{j 0}-c_{j}+v_{j} \cdot r \cdot \tau=0 \quad(j=1,2 \ldots S) \\
c_{k 0} \cdot X+v_{k} \cdot r \cdot \tau=0
\end{gathered}
$$

Para poder aplicar estas ecuaciones es necesario conocer la ecuación de la velocidad de reacción química, en función de la temperatura y de la concentración o el grado de conversión:

$$
r=r[X, T] \quad 0 \quad r=r\left[C_{j}, T\right]
$$

quedando:

$$
\begin{gathered}
c_{j 0}-c_{j}+v_{j} \cdot r\left(c_{j}, T\right) \cdot \tau=0 \quad(j=1,2 \ldots S) \\
c_{k 0} \cdot X+v_{k} \cdot r(X, T) \cdot \tau=0
\end{gathered}
$$

Estas dos ecuaciones indican que el volumen de reacción o el tiempo espacial siempre es inversamente proporcional a la velocidad de reacción, por lo que la representación de la inversa de $r$ frente a $c_{j}$ o X, permite calcular el volumen de reacción de forma gráfica según se representa en la figura 1: 

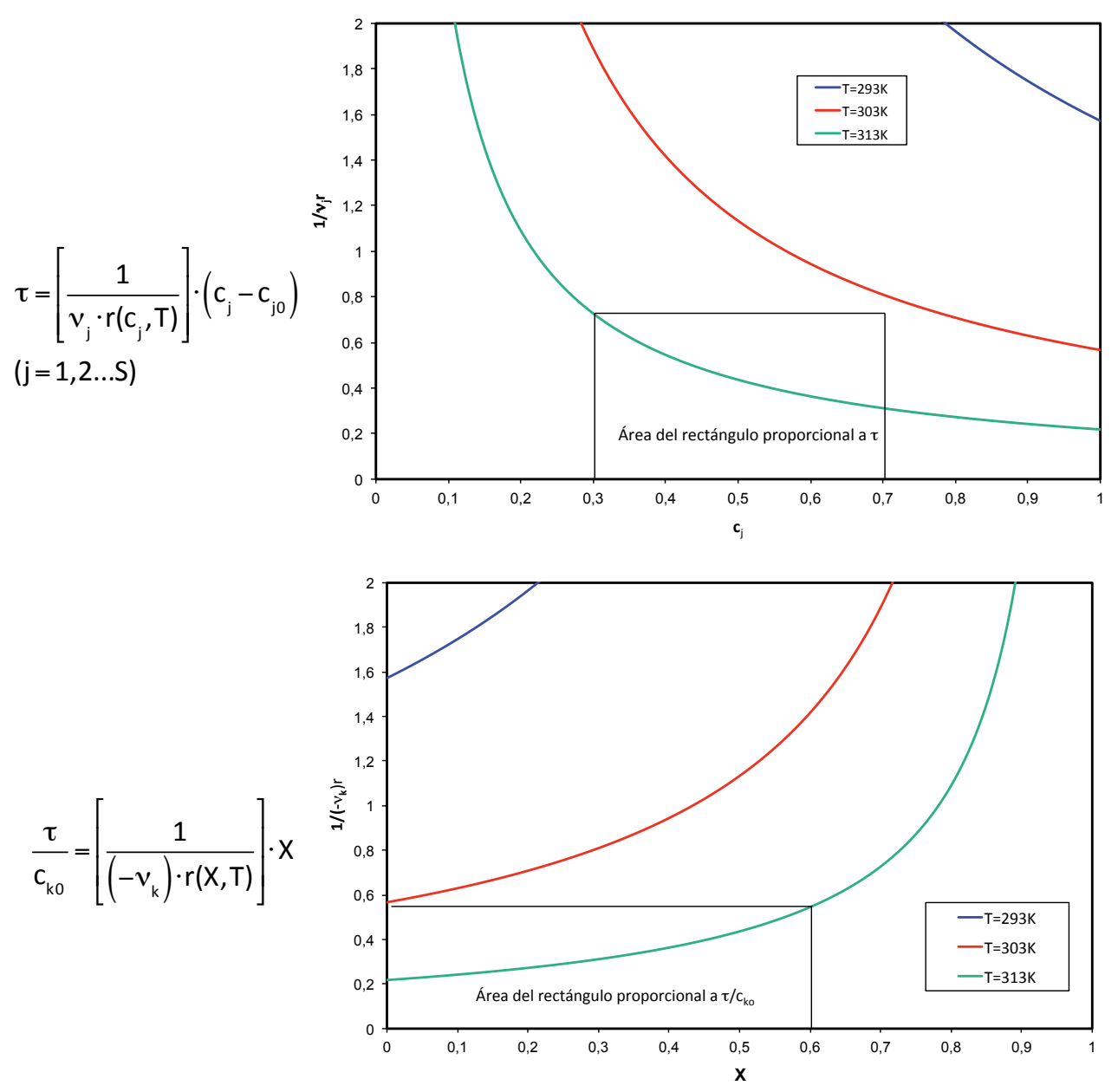

Figura 1. Cálculo del volumen de reacción o del tiempo espacial a partir de la función inversa de la velocidad de reacción

Análogamente, en el capítulo 1 también se han mostrado los balances de energía que, para el sistema constituido por un RCTA en el que se desarrolla una única reacción química, se puede expresar mediante una de las dos ecuaciones siguientes:

$$
\begin{gathered}
T-T_{0}-J \cdot X=Q \\
T-T_{0}+J \cdot \frac{v_{k} \cdot r \cdot \tau}{c_{k 0}}=Q
\end{gathered}
$$

Para poder aplicar estas ecuaciones es necesario conocer la ecuación de la velocidad de transmisión de calor, cuya forma depende del sistema de intercambio de calor utilizado.

En resumen, las dos ecuaciones más habituales para el diseño de un RCTA con una reacción química son:

$$
\begin{gathered}
c_{k 0} \cdot X+v_{k} \cdot r(X, T) \cdot \tau=0 \\
T-T_{0}-J \cdot X=Q
\end{gathered}
$$$$
\text { ECUACIÓN DE DISEÑO (1) }
$$ 


\subsection{Intercambio de calor}

En la ecuación de diseño (2), que es la forma habitual del balance de energía, se debe sustituir la velocidad de transmisión de calor por la correspondiente función del gradiente de temperaturas (entre el fluido intercambiador y la mezcla de reacción), del coeficiente global de transmisión de calor y del área de transmisión de calor:

$$
d Q^{*}=U \cdot\left(T_{f}-T\right) \cdot d A
$$

Puede apreciarse que, si el fluido intercambiador actúa como calefactor de la mezcla reactante $\left(\mathrm{T}_{\mathrm{f}}>>>\mathrm{T}\right), \mathrm{dQ}^{*}$ es positivo $\mathrm{y}$, si actúa como refrigerante $\left(\mathrm{T}_{\mathrm{f}}<<<\mathrm{T}\right)$, $\mathrm{dQ}^{*}$ es negativo. Al sustituir esta ecuación en la ecuación de diseño (2) se añade una incógnita más $\left(\mathrm{T}_{\mathrm{f}}\right)$, que sería conveniente eliminar. Esto se puede hacer aplicando un balance de energía al sistema de intercambio de calor utilizado.

(Para este apartado es conveniente revisar los conceptos vistos en la asignatura EQ1020-Operaciones Básicas de Transmisión de Calor.)

\subsubsection{Dispositivos de intercambio de calor de temperatura constante}

Se trata de un intercambiador de doble pared o camisa:

a) camisa de calefacción o de condensación: el fluido intercambiador es vapor que condensa;

b) camisa de enfriamiento o de vaporización: el fluido intercambiador es un líquido que hierve.

La temperatura del fluido intercambiador se mantiene constante, e igual a $\mathrm{T}_{\mathrm{f}}$, porque se produce un cambio de fase (condensación o vaporización) poniéndose en juego exclusivamente el calor latente de dicho cambio de fase. Asimismo, por tratarse de un RCTA en régimen estacionario la temperatura de la mezcla reactante y de la corriente de salida, T, también se mantiene constante. Estas condiciones de funcionamiento se esquematizan en la figura 2.

Bajo estas condiciones el balance de energía aplicado al intercambiador es:

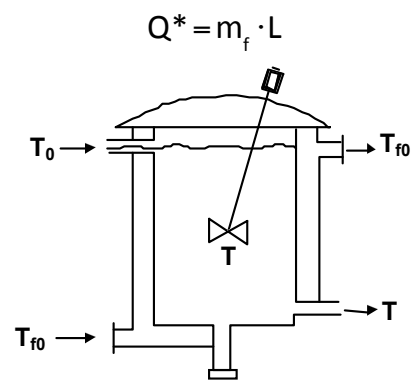

Figura 2. Condiciones de funcionamiento de un intercambiador de camisa

y si se conoce $\mathrm{m}_{\mathrm{f}}$ se puede calcular $\mathrm{Q}^{*}$. 
La ecuación de velocidad es integrable directamente:

$$
\int d Q^{*}=\int U \cdot\left(T_{f 0}-T\right) \cdot d A=U \cdot\left(T_{f 0}-T\right) \cdot \int d A
$$

y:

$$
Q^{*}=U \cdot\left(T_{f 0}-T\right) \cdot A
$$

En consecuencia, en la ecuación de diseño (2) se puede sustituir Q* quedando:

$$
\begin{gathered}
T-T_{0}-J \cdot X=Q=\frac{Q^{*}}{F_{k 0} \cdot \sum_{j=1}^{S} \theta_{j} \cdot C_{p j}}=\frac{U \cdot A}{F_{k 0} \cdot \sum_{j=1}^{S} \theta_{j} \cdot C_{p j}} \cdot\left(T_{f 0}-T\right) \\
T-T_{0}-J \cdot X=\kappa \cdot\left(T_{f 0}-T\right)
\end{gathered}
$$

siendo $\kappa$ :

$$
\kappa=\frac{U \cdot A}{F_{k 0} \cdot \sum_{j=1}^{s} \theta_{j} \cdot C_{p j}}
$$

\subsubsection{Dispositivos de intercambio de calor de temperatura variable}

Se pueden clasificar en dos grupos:

a) un intercambiador, sea serpentín o no, sumergido en la mezcla reactante, por el que circula un líquido que no cambia de fase (figura 3).

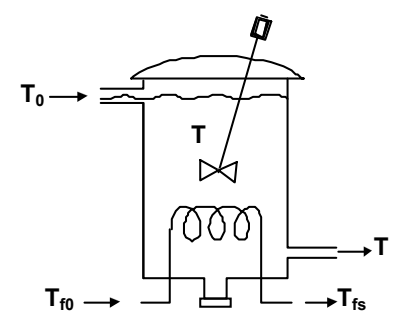

Figura 3. Intercambiador de calor sumergido en la mezcla reactante

b) la mezcla reactante se bombea a través de un intercambiador de calor externo (figura 4).

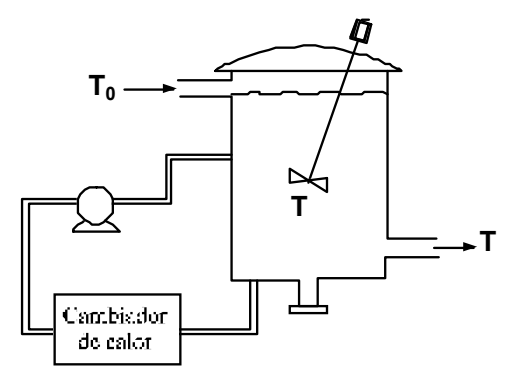

Figura 4. Intercambiador de calor externo 
En cualquiera de estas dos posibilidades la temperatura del fluido intercambiador variará desde $\mathrm{T}_{\mathrm{f} 0}$ hasta $\mathrm{T}_{\mathrm{fs}}$. Mientras que, por tratarse de un RCTA, la temperatura de la mezcla reactante y de la corriente de salida, $T$, se mantiene constante.

Bajo estas condiciones el balance de energía aplicado al fluido intercambiador es:

$$
Q^{*}=m_{f} \cdot C_{f} \cdot\left(T_{f 0}-T_{f s}\right)
$$

donde se cumple el criterio de signos elegido, pues si el fluido intercambiador actúa como refrigerante, $\mathrm{T}_{\mathrm{fs}}>\mathrm{T}_{\mathrm{fo}}$ y por tanto, $\mathrm{Q}^{*}<0$, y si opera como fluido calefactor, $\mathrm{T}_{\mathrm{fs}}<\mathrm{T}_{\mathrm{f} 0}$ y por tanto, $\mathrm{Q}^{*}>0$. Si se conoce $\mathrm{m}_{\mathrm{f}}, \mathrm{T}_{\mathrm{f0}} \mathrm{y} \mathrm{T}_{\mathrm{fs}}$ se puede calcular $\mathrm{Q}^{*}$. La integración de la ecuación de velocidad:

$$
\int d Q^{*}=\int U \cdot\left(T_{f}-T\right) \cdot d A
$$

conduce a:

$$
Q^{*}=U \cdot A \cdot(\Delta T)_{m l}=U \cdot A \cdot \frac{\left(T_{f 0}-T\right)-\left(T_{f s}-T\right)}{\ln \frac{\left(T_{f 0}-T\right)}{\left(T_{f s}-T\right)}}
$$

Si se conoce $\mathrm{T}_{\mathrm{f} 0}$ y $\mathrm{T}_{\mathrm{fs}}$ se puede calcular $\mathrm{Q}^{*}$.

Si no se conoce $T_{f s}$ se debe poner en función de las temperaturas $T_{f 0} y t$, ya que $\mathrm{T}_{\mathrm{f} 0}$ debe ser conocida mientras que $\mathrm{T}$ es una incógnita pero que ya estaba presente tanto en el balance de materia como en el de energía. Para ello se deben combinar la ecuación de velocidad de transmisión de calor y el balance de energía del fluido intercambiador:

$$
m_{f} \cdot C_{f} \cdot\left(T_{f 0}-T_{f s}\right)=U \cdot A \cdot \frac{\left(T_{f 0}-T\right)-\left(T_{f s}-T\right)}{\ln \frac{\left(T_{f 0}-T\right)}{\left(T_{f s}-T\right)}}=U \cdot A \cdot \frac{\left(T_{f 0}-T_{f s}\right)}{\ln \frac{\left(T_{f 0}-T\right)}{\left(T_{f s}-T\right)}}
$$

de donde:

$$
T_{f s}=T+\left(T_{f 0}-T\right) \cdot\left\{1-\exp \left[\frac{-U \cdot A}{m_{f} \cdot C_{f}}\right]\right\}
$$

sustituyendo en el balance de energía del intercambiador:

$$
Q^{*}=m_{f} \cdot C_{f} \cdot\left(T_{f 0}-T_{f s}\right)=m_{f} \cdot C_{f} \cdot\left\{1-\exp \left[\frac{-U \cdot A}{m_{f} \cdot C_{f}}\right]\right\} \cdot\left(T_{f 0}-T\right)
$$

Así, en la ecuación de diseño (2) se puede sustituir Q* quedando:

$$
\begin{gathered}
T-T_{0}-J \cdot X=Q=\frac{Q^{*}}{F_{k 0} \cdot \sum_{j=1}^{S} \theta_{j} \cdot C_{p j}}=\frac{m_{f} \cdot C_{f} \cdot\left(T_{f 0}-T_{f s}\right)}{F_{k 0} \cdot \sum_{j=1}^{S} \theta_{j} \cdot C_{p j}}=\frac{m_{f} \cdot C_{f}}{F_{k 0} \cdot \sum_{j=1}^{S} \theta_{j} \cdot C_{p j}} \cdot\left[1-\exp \left(\frac{-U \cdot A}{m_{f} \cdot C_{f}}\right)\right] \cdot\left(T_{f 0}-T\right) \\
T-T_{0}-J \cdot X=\kappa \cdot\left(T_{f 0}-T\right)
\end{gathered}
$$


siendo $\kappa$ :

$$
\kappa=\frac{m_{f} \cdot C_{f}}{F_{k 0} \cdot \sum_{j=1}^{s} \theta_{j} \cdot C_{p j}} \cdot\left[1-\exp \left(\frac{-U \cdot A}{m_{f} \cdot C_{f}}\right)\right]
$$

\subsubsection{Sin dispositivos de intercambio de calor}

Cuando el RCTA no intercambia calor y se admite que está perfectamente aislado, se denomina adiabático. En este caso $\mathrm{Q}^{*}=0 \mathrm{y}$, por tanto, $\mathrm{Q}=0$. Aplicando esta condición a la ecuación de diseño (2) se obtiene:

$$
T-T_{0}-J \cdot X=0
$$

que indica, como se muestra en la figura 5, que X es una función lineal de T:

$$
T=T_{0}+J \cdot X \quad O \quad X=\frac{-T_{0}}{J}+\frac{1}{J} \cdot T
$$

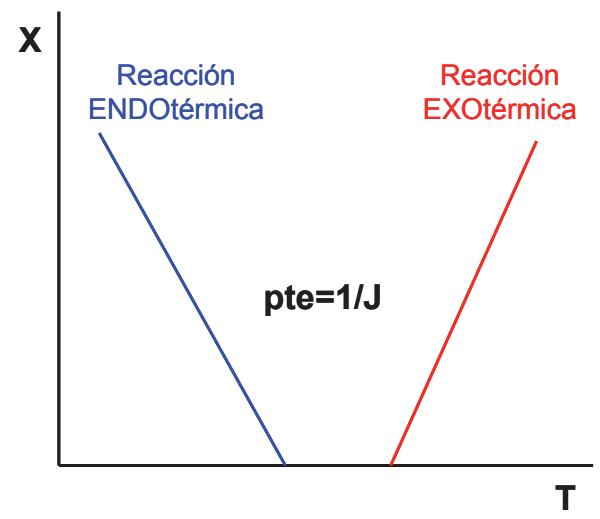

Figura 5. Recta adiabática $\mathrm{X}$ versus $\mathrm{T}$

La ordenada en el origen y la pendiente son positivas o negativas dependiendo del valor de J; es decir, según la reacción sea exotérmica o endotérmica.

\subsubsection{Generalización de la ecuación de diseño (2)}

De acuerdo con los tres tipos de intercambio de calor indicados, la ecuación de diseño (2) se puede generalizar a la ecuación de diseño (3):

$$
T-T_{0}-J \cdot X=\kappa \cdot\left(T_{f 0}-T\right)
$$

donde: 
- $\kappa=\frac{U \cdot A}{\sum_{j}^{s} \theta_{j} \cdot C}$ para un sistema de intercambio de calor con $T_{f}$ constante $\mathrm{F}_{\mathrm{k} 0} \cdot \sum_{\mathrm{j}=1}^{\mathrm{s}} \theta_{\mathrm{j}} \cdot \mathrm{C}_{\mathrm{pj}}$

- $\quad \kappa=\frac{m_{f} \cdot C_{f}}{T_{f} F_{k 0} \cdot \sum_{j=1}^{s} \theta_{j} \cdot C_{p j}} \cdot\left[1-\exp \left(\frac{-U \cdot A}{m_{f} \cdot C_{f}}\right)\right]$, para un sistema de intercambio de calor con variable

- $\kappa=0$, para un RCTA aislado sin sistema de intercambio de calor (adiabático)

\subsection{Resolución de las ecuaciones de diseño}

El diseño de un RCTA en el que se desarrolla una única reacción química o el cálculo de las condiciones de funcionamiento supone resolver simultáneamente las ecuaciones de diseño (1) y (3):

$$
\begin{aligned}
& c_{k 0} \cdot X+\nu_{k} \cdot r(X, T) \cdot \tau=0 \\
& T-T_{0}-J \cdot X=\kappa \cdot\left(T_{f 0}-T\right)
\end{aligned}
$$

Se trata, pues, de resolver un sistema de dos ecuaciones que solo puede tener dos incógnitas. Esta resolución suele ser un problema matemático sencillo excepto en el caso de que las dos incógnitas sean X y T, como consecuencia de la relación exponencial que existe entre la temperatura y la velocidad de reacción química (ley de Arrhenius). Existen dos métodos que permiten abordar este problema:

El Método de Levenspiel, propuesto por el profesor Octave Levenspiel y que se encuentra recogido en sus numerosos libros publicados sobre la materia, como

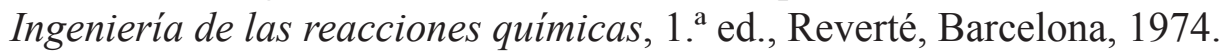

El Método de Aris, propuesto por el profesor Rutherford Aris y que se encuentra recogido en sus numerosos libros publicados sobre la materia, como Análisis de reactores, 1. ${ }^{a}$ ed., Alhambra, Madrid, 1973.

A continuación se detallará el procedimiento a seguir para aplicar cada uno de los métodos indicados. 


\subsubsection{Método de Levenspiel}

Este método se basa en el siguiente procedimiento:

i) A partir de la ecuación de diseño (1) $c_{k 0} \cdot X+v_{k} \cdot r(X, T) \cdot \tau=0$ obtener una expresión de la forma $X=f(T)$

$$
X=f(T)
$$

ECUACIÓN (4)

cuya representación en la forma $\mathrm{X}$ versus $\mathrm{T}$ es, con carácter general para cualquier tipo de reacción química y cualquier expresión de la velocidad de reacción química, una curva sigmoidal tal y como se muestra cualitativamente en la figura 6.

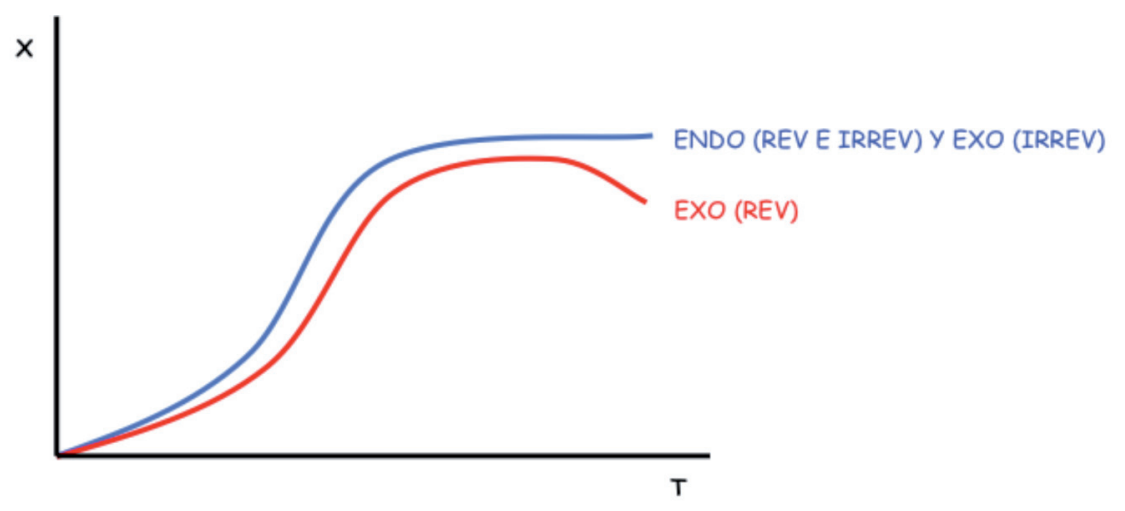

Figura 6. Representación del balance de materia en la forma $\mathrm{X}$ versus T según el método de Levenspiel

Ejercicio 1. El alumno puede comprobar esta forma de la curva para el caso de una reacción irreversible de primer orden de la forma $A_{1} \rightarrow A_{2}$, procediendo de la siguiente forma:

$$
\begin{array}{r}
r=k \cdot c_{10} \cdot(1-X)=A \cdot \exp \left[-\frac{E}{R \cdot T}\right] \cdot c_{10} \cdot(1-X) \\
c_{10} \cdot X+(-1) \cdot r \cdot \tau=c_{10} \cdot X-A \cdot \exp \left[-\frac{E}{R \cdot T}\right] \cdot c_{10} \cdot(1-X) \cdot \tau=0
\end{array}
$$

y despejando $\mathrm{X}$ :

$$
X=\frac{\tau \cdot A \cdot \exp \left[-\frac{E}{R \cdot T}\right]}{1+\tau \cdot A \cdot \exp \left[-\frac{E}{R \cdot T}\right]}=\frac{\tau \cdot k}{1+\tau \cdot k}
$$

Para poder hacer la representación gráfica el alumno debe buscar en la bibliografía los datos para una reacción química concreta. 
ii) A partir de la ecuación de diseño (3) $T-T_{0}-J \cdot X=\kappa \cdot\left(T_{f 0}-T\right)$ obtener la expresión

$$
X=-\frac{\left(T_{0}+\kappa \cdot T_{f 0}\right)}{J}+\frac{(1+\kappa)}{J} \cdot T
$$

ECUACIÓN (5)

cuya representación en la forma $\mathrm{X}$ versus T es, siempre, una línea recta cuya pendiente depende del carácter exotérmico o endotérmico de la reacción química. En efecto, teniendo en cuenta que $\kappa \geq 0$, para reacciones exotérmicas $\mathrm{J}>0$ y para reacciones endotérmicas $\mathrm{J}<0$. Esta recta corta al eje de abscisas cuando $X=0$ en el siguiente punto:

$$
T^{\cdot}=\frac{T_{0}+\kappa \cdot T_{f 0}}{1+\kappa}
$$

que, en el caso de que el RCTA sea adiabático $(\kappa=0)$, se convierte en $\mathrm{T}^{*}=\mathrm{T}_{0}$

iii) La representación conjunta de las funciones matemáticas descritas por las ecuaciones (4) y (5) permite la resolución simultánea de ambas ecuaciones mediante la determinación de los puntos de corte. Estos puntos de corte o soluciones de las ecuaciones de diseño se denominan estados estacionarios.

\section{Resolución para reacciones endotérmicas (reversibles e irreversibles)}

En la figura 7 se muestra la representación de las ecuaciones (4) y (5) para el caso en el que la reacción química que se desarrolla en el RCTA sea endotérmica $(\mathrm{J}<0)$, tanto si es reversible como si es irreversible. Puede observarse que, como consecuencia de la pendiente negativa de la ecuación (5), solo existe una solución matemática o estado estacionario. En consecuencia, las ecuaciones (4) y (5) también se podrían resolver analíticamente utilizando un método numérico.

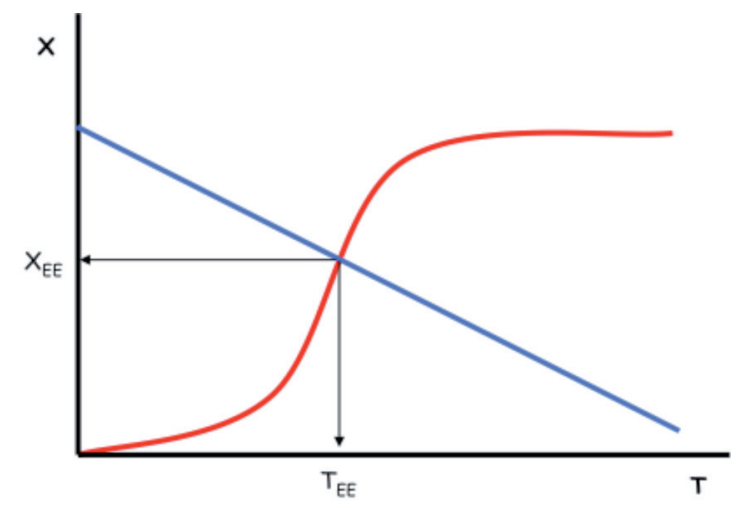

Figura 7. Representación de las ecuaciones (4) y (5) para una reacción endotérmica reversible o irreversible 
Resolución para reacciones exotérmicas (reversibles e irreversibles)

En la figura 8 se muestra la representación de las ecuaciones (4) y (5) para el caso en el que la reacción química que se desarrolla en el RCTA sea exotérmica $(\mathrm{J}>0)$, tanto si es reversible como si es irreversible. Puede observarse que, como consecuencia de la pendiente positiva de la ecuación (5), pueden existir varias soluciones matemáticas o estados estacionarios. En esta figura se muestra el caso de que exista una única solución con un valor de $\mathrm{X}$ elevado, siempre que la temperatura correspondiente $\left(\mathrm{T}_{\mathrm{EEI}}\right)$ sea menor que la máxima permisible para el sistema $\left(\mathrm{T}^{*}\right)$.

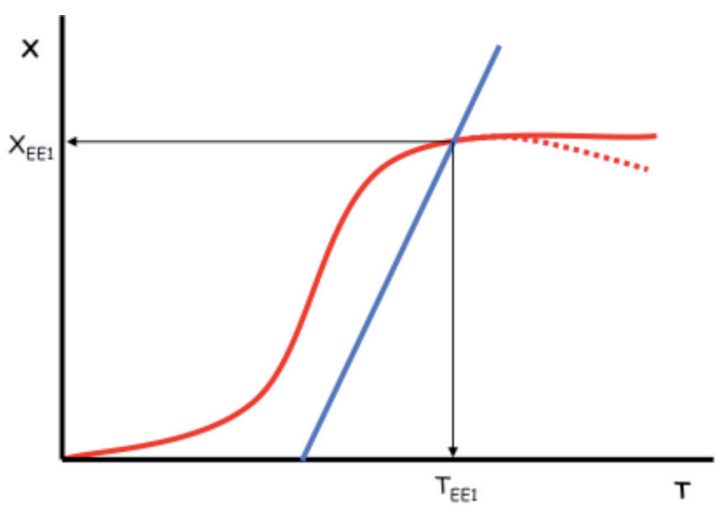

Figura 8. Representación de las ecuaciones (4) y (5) para una reacción exotérmica reversible o irreversible

La posición relativa de las ecuaciones (4) y (5) puede ser tal que conduzca a la existencia de dos posibles estados estacionarios: un punto de corte y un punto de tangencia, como se muestra en la figura 9. En este caso el punto de tangencia es un estado estacionario inestable, ya que cualquier pequeña variación del sistema que hiciera que la ecuación (5) se desplazara hacia la derecha (cambio de la recta continua a la discontinua) provocaría que el sistema tuviera un único estacionario al que le correspondería una temperatura relativamente elevada. Este proceso se conoce como encendido del reactor, y es un proceso indeseado que hay que tratar de evitar. Obviamente, a priori, el segundo estado estacionario es el mejor puesto que conduce a un valor de $\mathrm{X}$ elevado, siempre que la temperatura correspondiente $\left(\mathrm{T}_{\mathrm{EE} 2}\right)$ sea menor que la máxima permisible para el sistema $\left(\mathrm{T}^{*}\right)$.

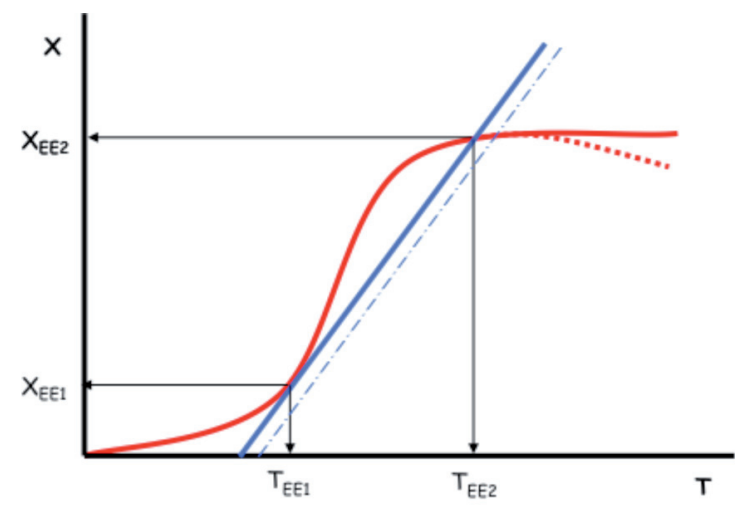

Figura 9. Posición relativa de las ecuaciones (4) y (5) con dos estados estacionarios 
La posición relativa de las ecuaciones (4) y (5) puede ser tal que conduzca a la existencia de dos posibles estados estacionarios: un punto de corte y un punto de tangencia, como se muestra en la figura 10. En este caso el punto de tangencia es un estado estacionario inestable, ya que cualquier pequeña variación del sistema que hiciera que la ecuación (5) se desplazara hacia la izquierda (cambio de la recta continua a la discontinua) provocaría que el sistema tuviera un único estacionario al que le correspondería un grado de conversión muy pequeño. Este proceso se conoce como apagado del reactor, y es un proceso indeseado que hay que tratar de evitar.

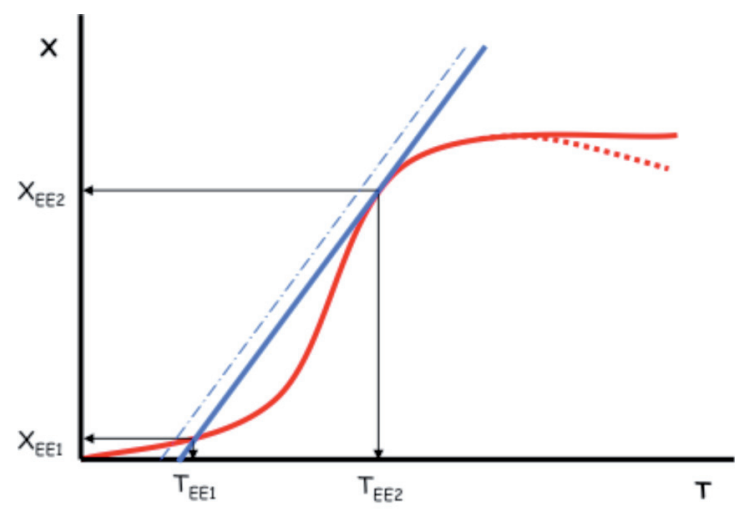

Figura 10. Posición relativa de las ecuaciones (4) y (5) con dos estados estacionarios

Finalmente, la posición relativa de las ecuaciones (4) y (5) también puede ser tal que conduzca a la existencia de hasta tres posibles estados estacionarios, como se muestra en la figura 11. Como se verá más adelante (en el apartado dedicado al método de Aris), el primer y tercer estado estacionario son estables mientras que el segundo estado estacionario es inestable.

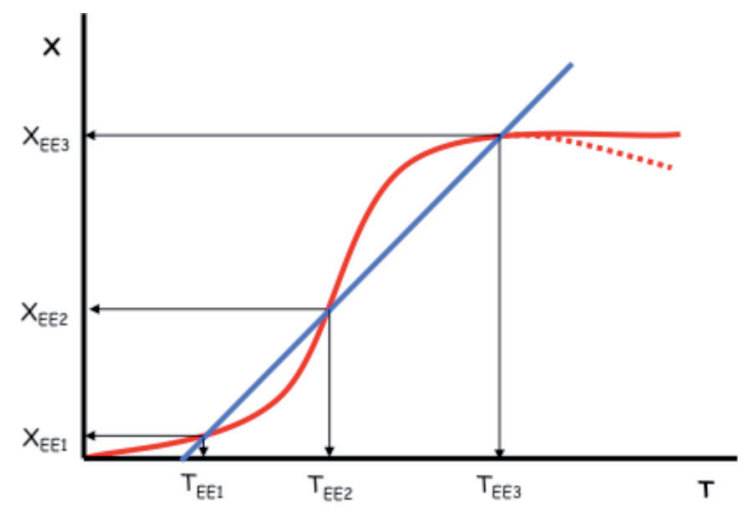

Figura 11. Posición relativa de las ecuaciones (4) y (5) con tres estados estacionarios

¿Cómo actuar para modificar las condiciones de estado estacionario?

A la vista de la multiplicidad de los estados estacionarios en el caso de las reacciones exotérmicas, lo más recomendable es utilizar las ecuaciones (4) y (5) para asegurarse que el sistema tiene una única solución. Es decir, existe un único estado 
estacionario estable que conduce a un grado de conversión elevado y a una temperatura inferior a la máxima permisible por el sistema. Para ello, lo más sencillo es actuar sobre el sistema intentando aumentar la pendiente $(1+\kappa) / \mathrm{J}$ y disminuir su ordenada en el origen $-\left(\mathrm{T}_{0}+\kappa \cdot \mathrm{T}_{\mathrm{f0}}\right) / \mathrm{J}$. Esto se puede conseguir aumentando $\kappa \mathrm{y}$ aumentando $\mathrm{T}_{0} \mathrm{y} / \mathrm{o} \mathrm{T}_{\mathrm{f} 0}$. De esta forma resulta fácil conseguir una única solución como muestra la línea continua de la figura 12.

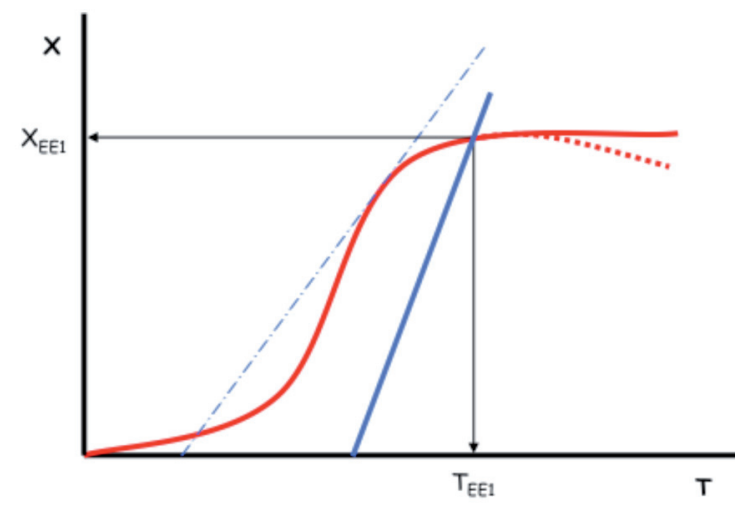

Figura 12. Posición relativa de las ecuaciones (4) y (5) con una única solución

\subsubsection{Método de Aris}

El Método de Aris se basa en resolver las ecuaciones de diseño (1) y (3) utilizando el procedimiento de sustitución:

$$
\begin{aligned}
& c_{k 0} \cdot X+v_{k} \cdot r(X, T) \cdot \tau=0 \\
& T-T_{0}-J \cdot X=\kappa \cdot\left(T_{f 0}-T\right)
\end{aligned}
$$

1) Despejar $X$ en función de $T$ de la ecuación de diseño (1): $X=X(T)$

2) Sustituir $X$ por $X(T)$ en la ecuación de diseño (3)

De esta forma se obtiene una ecuación con una única incógnita, la T, de la forma (cuyas unidades son grados K):

$$
\mathrm{T}-\mathrm{T}_{0}-\mathrm{J} \cdot \mathrm{X}(\mathrm{T})=\kappa \cdot\left(\mathrm{T}_{\mathrm{f} 0}-\mathrm{T}\right)
$$

que se puede reordenar como:

$$
J \cdot X(T)=-\left(T_{0}+\kappa \cdot T_{f 0}\right)+(1+\kappa) \cdot T
$$

Aunque la ecuación (6) solo tiene una incógnita puede que no sea sencilla de resolver analíticamente por las siguientes consideraciones:

1) La función $X=X(T)$ es complicada: contiene la velocidad de reacción en 
la que la variable $\mathrm{T}$ forma parte, al menos, de una exponencial. Incluso la expresión de la velocidad de reacción y, por tanto, de la ecuación de diseño (1) puede ser tan complicada que no sea posible despejar $\mathrm{X}$ y obtener $\mathrm{X}=\mathrm{X}(\mathrm{T})$, como se acaba de apuntar.

2) Puede tener una o varias soluciones, todas ellas con sentido físico. Por ello es necesario establecer una metodología que permita analizar todas las posibles soluciones.

En consecuencia, resulta mucho más operativo resolver la ecuación (6) utilizando un método gráfico. Para ello se definen dos nuevas funciones:

$$
Q_{G}=J \cdot X(T)
$$

ECUACIÓN (7)

$$
Q_{e}=-\left(T_{0}+\kappa \cdot T_{f 0}\right)+(1+\kappa) \cdot T
$$

ECUACIÓN (8)

y se representan ambas funciones en la forma de $Q_{G}$ y $Q_{e}$ versus $T$; los puntos de corte (es decir, los puntos en que se cumple que $Q_{G} y_{e}$ ) serán los que satisfagan la ecuación (6) y se denominan estados estacionarios (EE).

Obsérvese que la función $\mathrm{Q}_{\mathrm{G}}$ contiene la entalpía (en $\mathrm{J}$ ) y la velocidad de reacción (en $\mathrm{X}(\mathrm{T})$ ), por lo que representa el «calor generado» durante el proceso de reacción química. Análogamente, la función $\mathrm{Q}_{\mathrm{e}}$ contiene los parámetros característicos del sistema de intercambio de calor (en $\kappa$ ) y todas las temperaturas $\left(\mathrm{T}, \mathrm{T}_{0} \mathrm{y} \mathrm{T}_{\mathrm{fo}}\right)$, representando el «calor eliminado». Los términos «calor generado» y «calor eliminado» aparecen entrecomillados por las siguientes razones:

a) las unidades de las ecuaciones (7) y (8) son grados K.

b) el «calor generado» puede ser positivo o negativo, en tanto en cuanto que la reacción puede ser exotérmica o endotérmica.

c) el «calor eliminado» puede ser positivo, negativo o nulo, ya que el sistema de intercambio de calor puede calentar o refrigerar al RCTA o, simplemente, aislar perfectamente al RCTA (caso del reactor adiabático).

\section{Análisis de la función $Q_{e}$}

El análisis matemático de esta función es:

1) al representar $Q_{e}$, en ordenadas, frente a $T$, en abscisas, se obtiene una línea recta cuya ordenada en el origen es $-\left(T_{0}+\kappa \cdot T_{f 0}\right)$ y la pendiente vale $(1+\kappa)$.

2) $\kappa$ puede valer 0 , para un RCTA adiabático, o ser mayor que 0 (para un RCTA con intercambio de calor); por lo tanto la pendiente tiene que estar entre 1 e $\infty$.

3) el punto de corte de la recta con el eje de ordenadas carece de interés porque se producirá a la temperatura de $0 \mathrm{~K}$. 
4) el punto de corte de la recta con el eje de abscisas es $\left(T^{\bullet}, 0\right)$. El valor de $T^{\bullet}$ se puede calcular:

$$
\begin{gathered}
Q_{e}=-\left(T_{0}+\kappa \cdot T_{f 0}\right)+(1+\kappa) \cdot T \\
0=-\left(T_{0}+\kappa \cdot T_{f 0}\right)+(1+\kappa) \cdot T^{\cdot} \\
T^{\cdot}=\frac{T_{0}+\kappa \cdot T_{f 0}}{1+\kappa}
\end{gathered}
$$

que para un RCTA adiabático $(\kappa=0)$ se simplifica a $\mathrm{T}^{\bullet}=\mathrm{T}_{0}$. Obsérvese que $\mathrm{T}^{\bullet}$ solo representa la corriente de entrada del alimento al RCTA $\left(\mathrm{T}_{0}\right)$ en el caso de que este sea adiabático.

La figura 13 muestra la representación de la función $\mathrm{Q}_{\mathrm{e}}$.

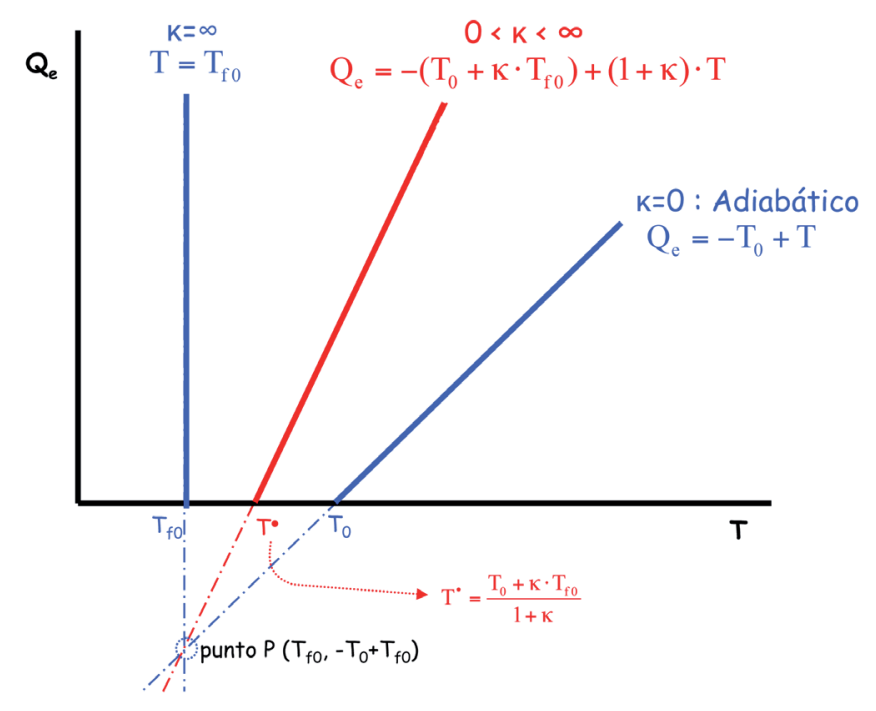

Figura 13. Representación de la función $\mathrm{Q}_{\mathrm{e}}$ del método de Aris

INFLUENCIA DE $\mathrm{T}_{0} \mathrm{Y} / \mathrm{O} \mathrm{T}_{\mathrm{f} 0}$ SOBRE LA ORDENADA EN EL ORIGEN DE LA RECTA $\mathrm{Q}_{\mathrm{e}}$

$\mathrm{Al}$ aumentar tanto $\mathrm{T}_{0}$ como $\mathrm{T}_{\mathrm{f} 0}$ la ordenada en el origen disminuye (se hace más negativa) mientras que la pendiente se mantiene invariable. El efecto gráfico es que la recta se desplaza en paralelo hacia valores crecientes del eje de abscisas (figura 14). 


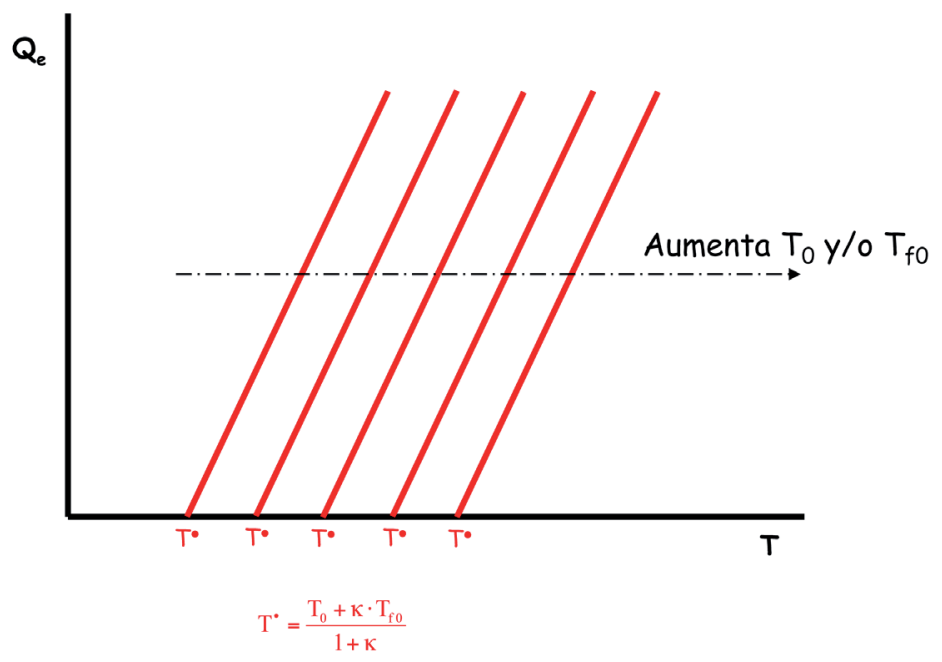

Figura 14. Efecto del aumento de $\mathrm{T}_{0} \mathrm{y} / \mathrm{o} \mathrm{T}_{\mathrm{f} 0}$ sobre la función $\mathrm{Q}_{\mathrm{e}}$

INFLUENCIA DE $\kappa$ SOBRE LA ORDENADA EN EL ORIGEN Y LA PENDIENTE DE LA RECTA $Q_{e}$

Al aumentar el valor de $\kappa$ se producen dos efectos simultáneos: disminuye la ordenada en el origen y aumenta la pendiente. El efecto gráfico neto es que la recta gira de derecha a izquierda pivotando sobre un punto $\mathrm{P}$, como se muestra en la figura 15. Las coordenadas de este punto $P\left(T_{P}, Q_{e P}\right)$ se pueden calcular como el punto de intersección de dos rectas cualesquiera con diferentes valores de $\kappa$ :

$$
\begin{aligned}
& Q_{e P}=-\left(T_{0}+\kappa_{1} \cdot T_{f 0}\right)+\left(1+\kappa_{1}\right) \cdot T_{P} \\
& Q_{e P}=-\left(T_{0}+\kappa_{2} \cdot T_{f 0}\right)+\left(1+\kappa_{2}\right) \cdot T_{P}
\end{aligned}
$$

de donde:

$$
\begin{aligned}
& T_{p}=T_{f 0} \\
& Q_{e P}=T_{f 0}-T_{0}
\end{aligned}
$$

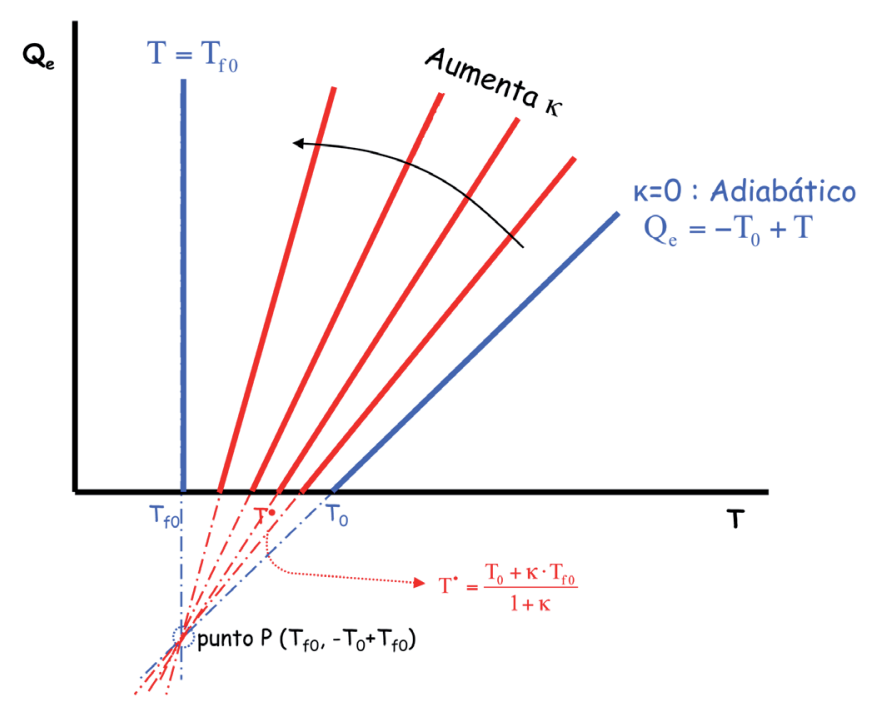

Figura 15. Efecto del aumento de $k$ sobre la función $\mathrm{Q}_{\mathrm{e}}$ 
Análisis de la función $Q_{G}$

Conviene recordar que $\mathrm{X}=\mathrm{X}(\mathrm{T})$ es una función complicada que resulta de despejar X de la ecuación de diseño (1).

Para realizar el análisis matemático de la función $\mathrm{Q}_{\mathrm{G}}$ habría que:

a) Definir previamente $\mathrm{r}(\mathrm{X}, \mathrm{T})$, recordando que, de forma general, $r$ es:

$$
r=A \cdot \exp \left[-\frac{E}{R \cdot T}\right] \cdot \prod_{j=1}^{S} c_{j}^{\beta_{j}}-A^{\prime} \cdot \exp \left[-\frac{E^{\prime}}{R \cdot T}\right] \cdot \prod_{j=1}^{S} c_{j}^{\gamma_{j}}
$$

b) Tener en cuenta que, dado que $\mathrm{X}$ se encuentra comprendido necesariamente en el intervalo $[0,1]$, el signo de la función depende de $\mathrm{J}$ :

$$
J=-\frac{\Delta H_{k}^{0}}{\sum_{j=1}^{s} \theta_{j} \cdot \bar{C}_{p, j}}
$$

y, por lo tanto,

i) Reacciones exotérmicas: $\Delta \mathrm{H}^{0}{ }_{\mathrm{k}}<0, \mathrm{~J}>0$ y $\mathrm{Q}_{\mathrm{G}}>0$ (la función $\mathrm{Q}_{\mathrm{G}}$ aparece en el cuadrante positivo)

ii) Reacciones endotérmicas: $\Delta \mathrm{H}^{0}{ }_{\mathrm{k}}>0, \mathrm{~J}<0$ y $\mathrm{Q}_{\mathrm{G}}<0$ (la función $\mathrm{Q}_{\mathrm{G}}$ aparece en el cuadrante negativo)

Se puede demostrar que la función $\mathrm{Q}_{\mathrm{G}}$ tiene alguna de las formas sigmoidales que se muestran en la figura 16 y que para:

- las reacciones endotérmicas (tanto reversibles como irreversibles) y las exotérmicas irreversibles presenta una asíntota en $\mathrm{Q}_{\mathrm{G}}=\mathrm{J}$

- las reacciones exotérmicas reversibles presenta un máximo.

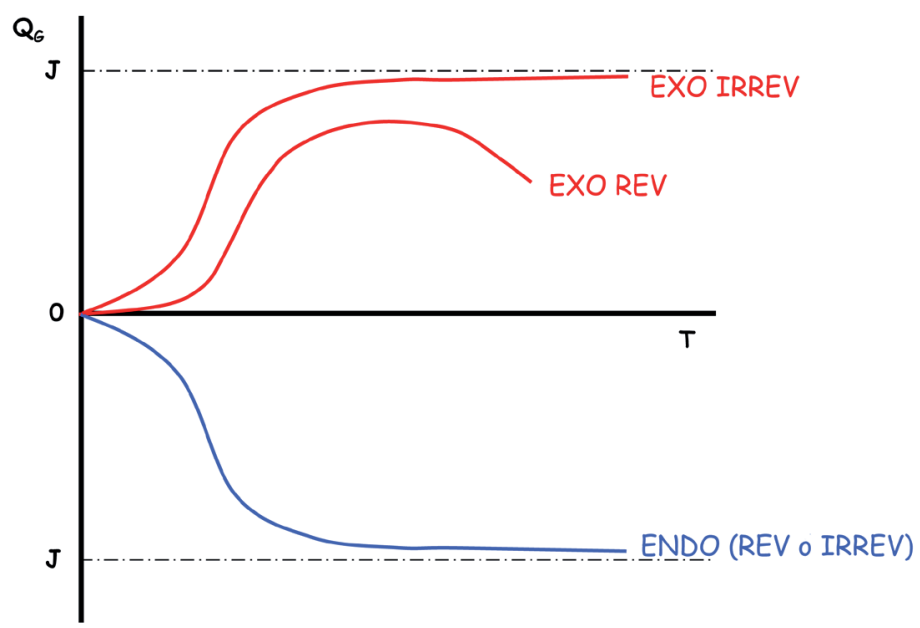

Figura 16. Representación de la función $\mathrm{Q}_{\mathrm{G}}$ del método de Aris 
Ejercicio 2. El alumno puede comprobar esta forma de la curva para el caso de una reacción irreversible de primer orden de la forma $A_{1} \rightarrow A_{2}$, procediendo de la siguiente forma:

$$
r=k \cdot c_{10} \cdot(1-X)=A \cdot \exp \left[-\frac{E}{R \cdot T}\right] \cdot c_{10} \cdot(1-X)
$$

teniendo en cuenta el balance de materia:

$$
C_{10} \cdot X+(-1) \cdot r \cdot \tau=c_{10} \cdot X-A \cdot \exp \left[-\frac{E}{R \cdot T}\right] \cdot c_{10} \cdot(1-X) \cdot \tau=0
$$

y despejando $\mathrm{X}$ :

$$
X=\frac{\tau \cdot A \cdot \exp \left[-\frac{E}{R \cdot T}\right]}{1+\tau \cdot A \cdot \exp \left[-\frac{E}{R \cdot T}\right]}=\frac{\tau \cdot k}{1+\tau \cdot k}
$$

se puede construir la función:

$$
Q_{G}=J \cdot X(T)=J \cdot \frac{\tau \cdot A \cdot \exp \left[-\frac{E}{R \cdot T}\right]}{1+\tau \cdot A \cdot \exp \left[-\frac{E}{R \cdot T}\right]}=J \cdot \frac{\tau \cdot k}{1+\tau \cdot k}
$$

Demuéstrese para la función $\mathrm{Q}_{\mathrm{G}}$ que:

i) la primera derivada es $\frac{d Q_{G}}{d T}=J \cdot \frac{E}{R \cdot T^{2}} \cdot \frac{\tau \cdot k}{(1+\tau \cdot k)^{2}}$, pudiendo ser mayor o menor que cero (según la reacción sea exotérmica o endotérmica, respectivamente), lo que indica que la curva es monotónicamente creciente o decreciente

ii) igualando la segunda derivada a cero se puede calcular el punto de inflexión, situado a la temperatura $T_{i}: T i=\frac{E}{R} \cdot\left[\left(\frac{1}{2}\right)-\left(\frac{\tau \cdot k}{1+\tau \cdot k}\right)\right]$

iii) la función tienda a infinito: $\lim _{\mathrm{T} \rightarrow \infty} \mathrm{Q}_{\mathrm{G}}=\mathrm{J}$

INFLUENCIA DE $\mathrm{Q}_{\mathrm{V} 0}$ SOBRE LA FUNCIÓN $\mathrm{Q}_{\mathrm{G}}$

Al aumentar el caudal volumétrico de entrada al reactor la pendiente de la curva $\mathrm{Q}_{\mathrm{G}}$ disminuye. El efecto neto es que la curva sigmoidal se suaviza aunque sigue tendiendo asintóticamente al mismo valor de J (figura 17). 


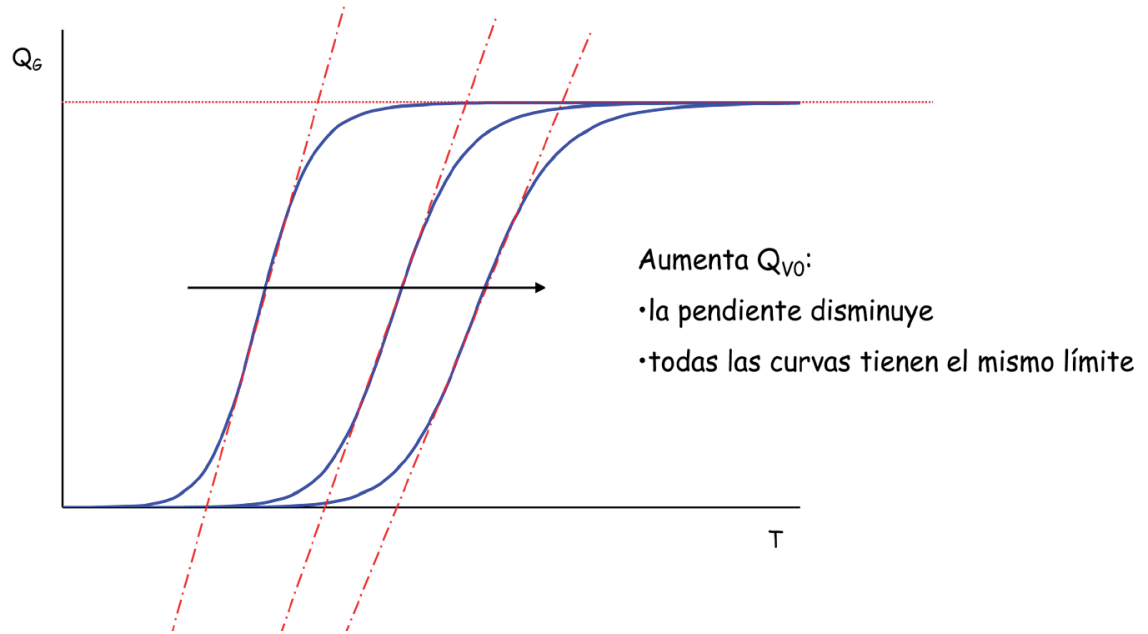

Figura 17. Efecto del aumento de $\mathrm{Q}_{\mathrm{V} 0}$ sobre la función $\mathrm{Q}_{\mathrm{G}}$

INFLUENCIA DE $\theta_{\mathrm{j}}$ SOBRE LA FUNCIÓN $\mathrm{Q}_{\mathrm{G}}$

$\mathrm{Al}$ aumentar el valor de $\theta_{\mathrm{j}}$ la pendiente de la curva $\mathrm{Q}_{\mathrm{G}}$ disminuye. El efecto neto es que la curva sigmoidal se suaviza y, además, tiende asintóticamente a un valor de $\mathrm{J}$ menor, de acuerdo con la propia definición de J (figura 18).

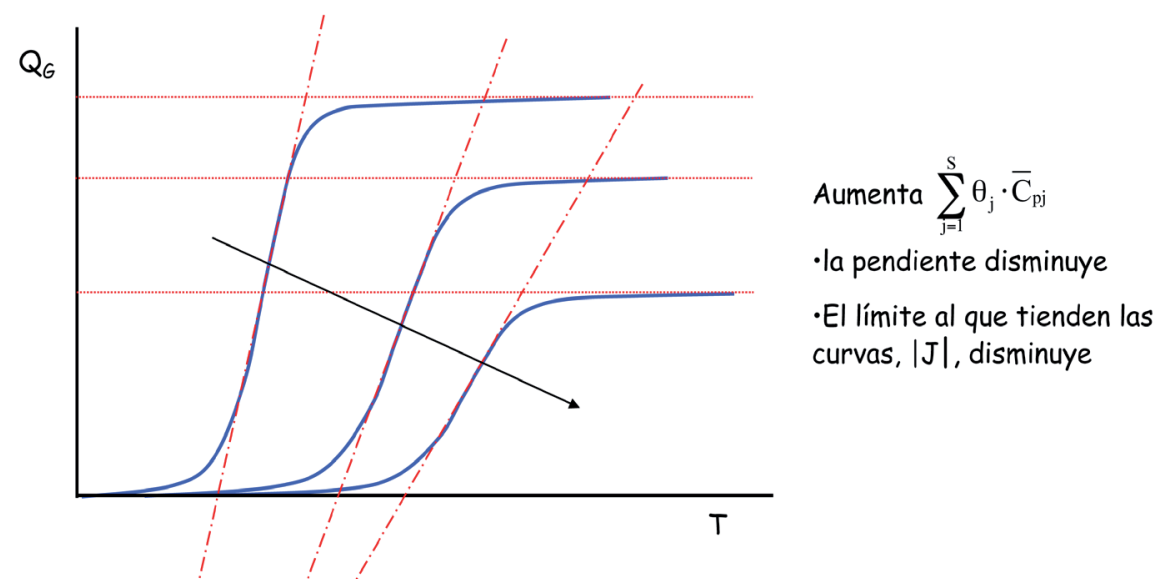

Figura 18. Efecto del aumento de $\theta_{j}$ sobre la función $\mathrm{Q}_{\mathbf{G}}$

\section{Cálculo de los estados estacionarios}

Como se ha indicado, la ecuación (6) se resuelve definiendo previamente las funciones $\mathrm{Q}_{\mathrm{G}}$, ecuación (7), y $\mathrm{Q}_{\mathrm{e}}$, ecuación (8). Cuando se representan ambas funciones en la misma gráfica en la forma de $\mathrm{Q}_{\mathrm{G}}$ y $\mathrm{Q}_{\mathrm{e}}$ versus $\mathrm{T}$ el punto o los puntos de corte son los que satisfacen la ecuación (6) y cada uno se denomina estado estacionario. El número de estados estacionarios posibles depende del tipo de reacción química que se desarrolle en el RCTA:

- Reacción endotérmica (reversible o irreversible): 1 estado estacionario (figura 19). 


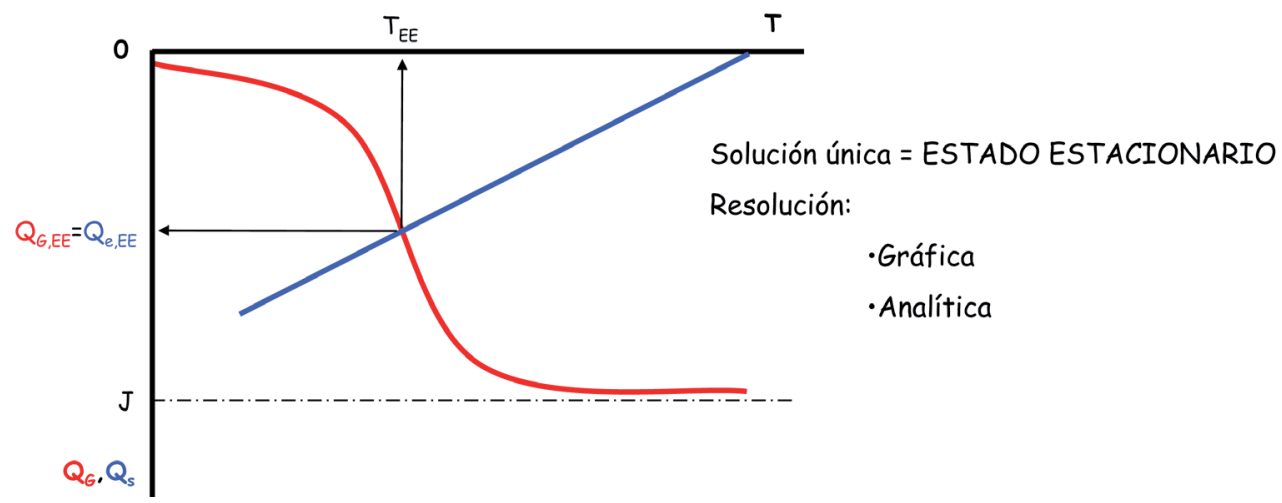

Figura 19. Representación de las ecuaciones (7) y (8) para una reacción endotérmica reversible o irreversible

- Reacción exotérmica (reversible o irreversible): 1, 2 o 3 estados estacionarios. Algunas consideraciones sobre el número de estados estacionarios:

1. La mejor situación posible es tener un único estado estacionario, siempre que corresponda a una temperatura inferior a la máxima permisible y a una conversión suficientemente elevada (figura 20).

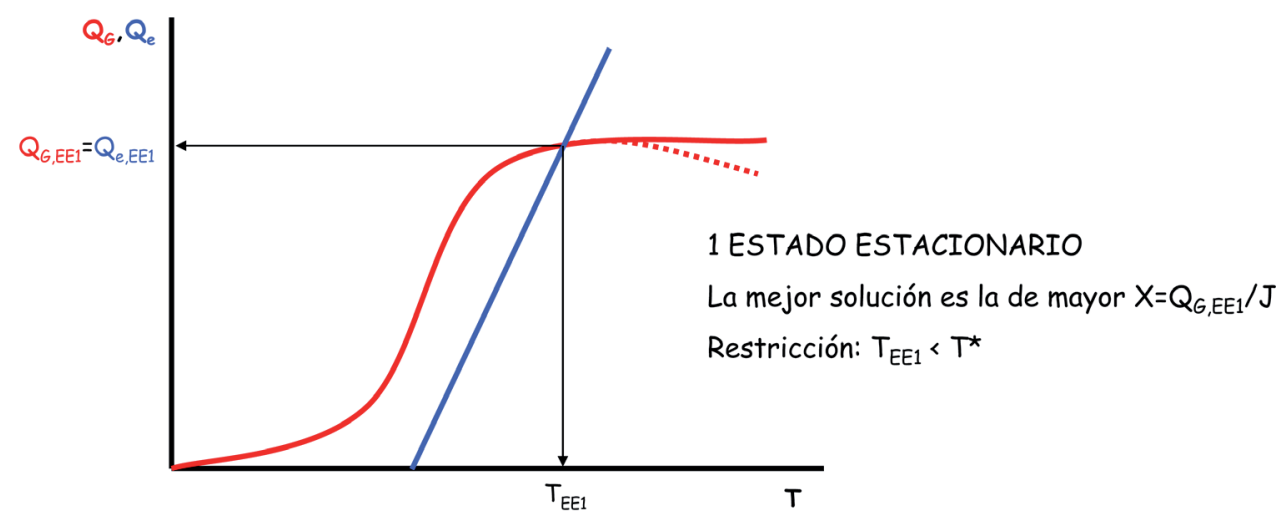

Figura 20. Posición relativa de las ecuaciones (7) y (8) con un único estado estacionario

2. La peor situación posible es tener dos estados estacionarios ya que se puede producir un encendido o un apagado de la reacción (figuras 21 y 22).

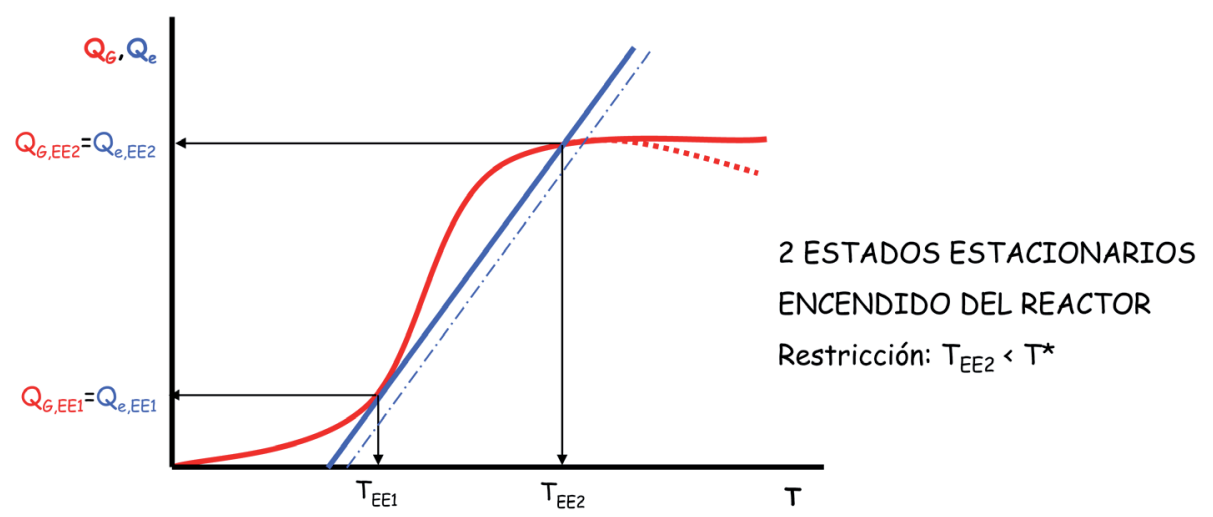

Figura 21. Posición relativa de las ecuaciones (7) y (8) con dos estados estacionarios 


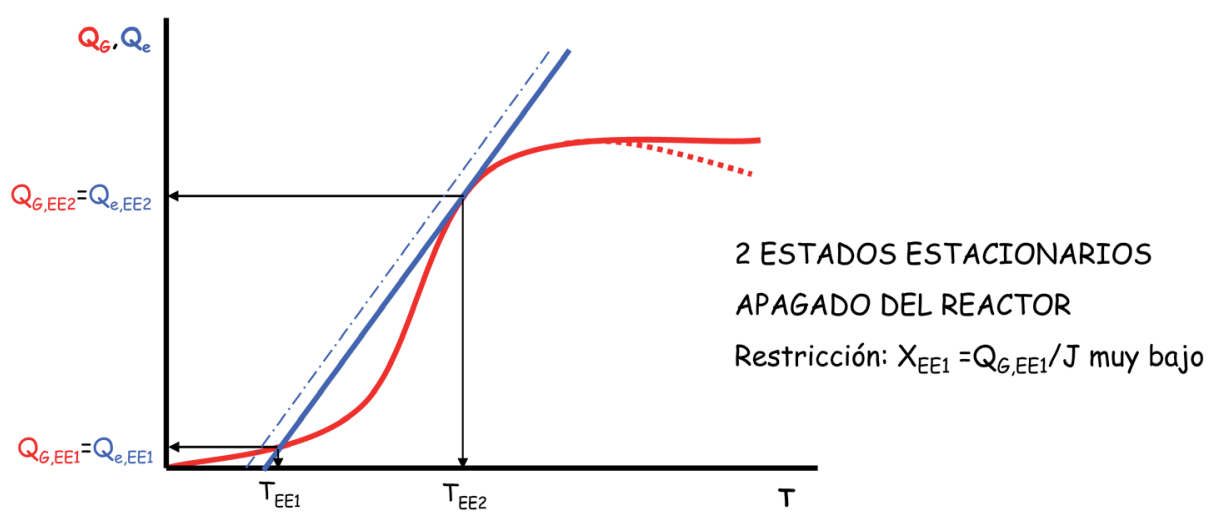

Figura 22. Posición relativa de las ecuaciones (7) y (8) con dos estados estacionarios

3. Una situación intermedia es la de tener tres estados estacionarios como se muestra en la figura 23 (como se verá más adelante dos de los estados estacionarios serán estables y uno inestable, y los sistemas químicos solo pueden alcanzar los estados estacionarios estables).

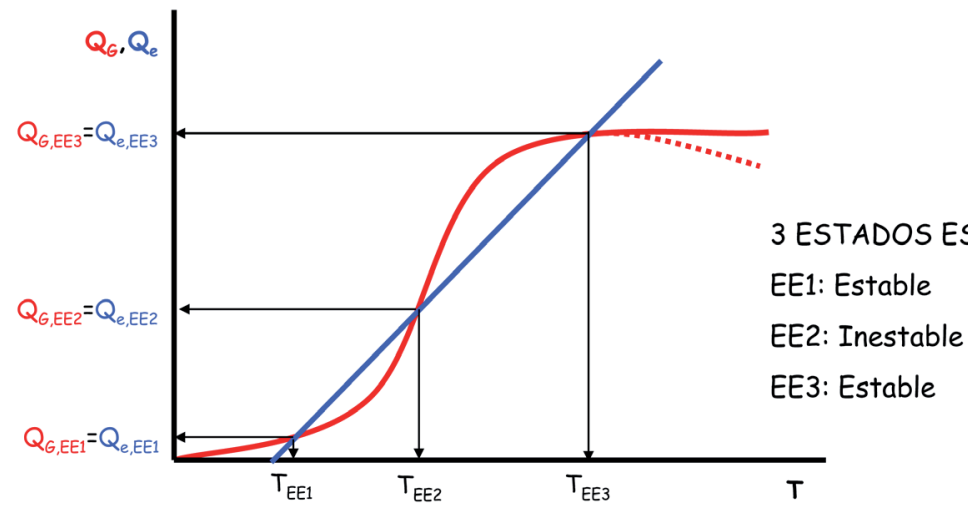

Figura 23. Posición relativa de las ecuaciones (7) y (8) con tres estados estacionarios

Modificando convenientemente las funciones $\mathrm{Q}_{\mathrm{G}}$ y $\mathrm{Q}_{\mathrm{e}}$ puede obtenerse un único estado estacionario. A continuación se mostrará como las variables $\mathrm{T}_{0} \mathrm{y}_{\mathrm{v} 0}$ afectan a dichas funciones $\mathrm{y}$, en consecuencia, a los estados estacionarios que se obtienen.

\section{INFLUENCIA DE $\mathrm{T}_{0}$ SOBRE LOS ESTADOS ESTACIONARIOS}

Supóngase un reactor en el que la temperatura del alimento $\mathrm{T}_{0}$ (el efecto sería el mismo actuando sobre la temperatura del fluido intercambiador $\mathrm{T}_{\mathrm{f0}}$ ) pueda variarse dentro de un pequeño intervalo mientras las demás variables de operación permanecen constantes.

Para cinco temperaturas diferentes del alimento $\left(\mathrm{T}_{01}<\mathrm{T}_{02}<\mathrm{T}_{03}<\mathrm{T}_{04}<\mathrm{T}_{05}\right)$ la función $\mathrm{Q}_{\mathrm{G}}$ permanece invariable mientras que la función $\mathrm{Q}_{\mathrm{e}}$ se desplaza paralelamente hacia la derecha. Se podrían tener hasta las cinco posibles diferentes situaciones que se recogen en la tabla 1, para cinco posiciones diferentes de la función $\mathrm{Q}_{\mathrm{e}}$. 
Tabla 1. Posibles estados estacionarios para cinco temperaturas diferentes del alimento

\begin{tabular}{|c|c|c|}
\hline Nomenclatura de la recta $\mathrm{Q}_{\mathrm{e}}$ & Número de EE & Nomenclatura de los EE \\
\hline $\mathrm{T}_{01}$ & 1 & EE1 $\mathrm{i}$ \\
\hline $\mathrm{T}_{02}$ & 2 & EE2 $\mathrm{i}-\mathrm{EE} 2 \mathrm{~s}$ \\
\hline $\mathrm{T}_{03}$ & 3 & EE3i $-\mathrm{EE} 3 \mathrm{c}-\mathrm{EE} 3 \mathrm{~s}$ \\
\hline $\mathrm{T}_{04}$ & 2 & EE4i $-\mathrm{EE} 4 \mathrm{~s}$ \\
\hline $\mathrm{T}_{05}$ & 1 & EE5s \\
\hline
\end{tabular}

Al representar las cinco rectas $\mathrm{Q}_{\mathrm{e}}$ (una para cada temperatura de alimentación al reactor diferente) junto con la curva $\mathrm{Q}_{\mathrm{G}}$ se observan los estados estacionarios posibles (1,2 o hasta 3$)$ según la posición relativa de las funciones matemáticas, tal y como se muestra en la figura 24 (obsérvese que la temperatura de entrada del alimento sirve como nomenclatura de cada recta $\mathrm{Q}_{\mathrm{e}}$ pero en ningún caso representa el punto de corte entre dichas rectas y el eje de abscisas; solo en el caso de que el reactor fuera adiabático dicho punto de corte coincidiría con la temperatura de entrada del alimento).

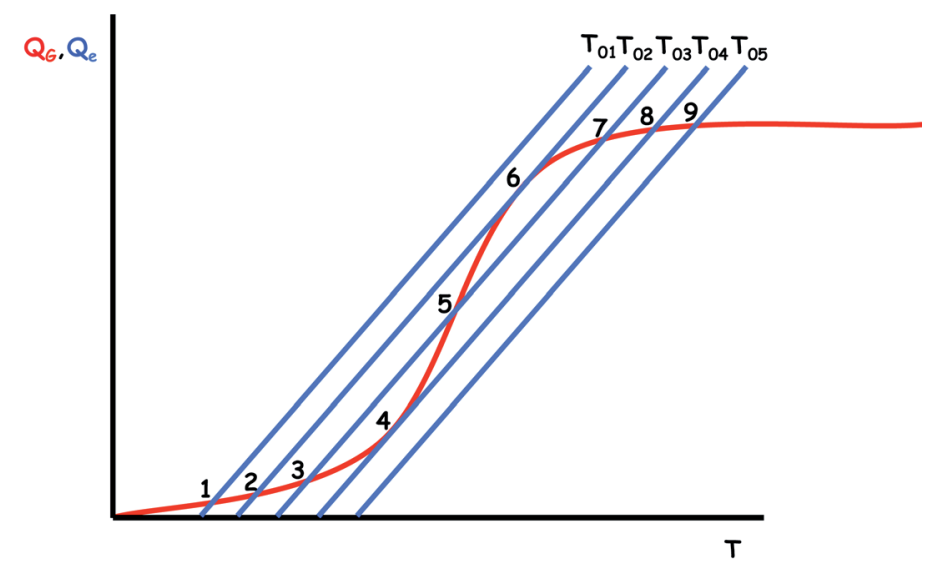

Figura 24. Posibles estados estacionarios para cinco temperaturas diferentes del alimento

Sin embargo, si existe o se provoca deliberadamente una perturbación, y el valor de $\mathrm{T}_{0}$ se aumenta con suficiente lentitud desde $\mathrm{T}_{01}$ (para que en cada una de las cinco funciones $\mathrm{Q}_{\mathrm{e}}$ escogidas se alcance el régimen estacionario) la secuencia de estados estacionarios que se seguirá se muestra en la tabla 2.

Tabla 2. Posibles estados estacionarios al incrementar la temperatura del alimento

\begin{tabular}{|c|c|}
\hline Temperatura de entrada & EE \\
\hline $\mathrm{T}_{01}$ & $1 \mathrm{i}$ \\
\hline $\mathrm{T}_{02}$ & $2 \mathrm{i}$ \\
\hline $\mathrm{T}_{03}$ & $3 \mathrm{i}$ \\
\hline $\mathrm{T}_{04}$ & $4 \mathrm{i}$ \\
\hline$>\mathrm{T}_{04}$ & A la derecha del 4s (Encendido) \\
\hline
\end{tabular}


Análogamente, si existe o se provoca deliberadamente una perturbación, y el valor de $\mathrm{T}_{0}$ se disminuye con suficiente lentitud desde $\mathrm{T}_{05}$ (para que en cada una de las cinco funciones $\mathrm{Q}_{\mathrm{e}}$ escogidas se alcance el régimen estacionario) la secuencia de estados estacionarios que se seguirá se muestra en la tabla 3.

Tabla 3. Posibles estados estacionarios al disminuir la temperatura del alimento

\begin{tabular}{|c|c|}
\hline Temperatura de entrada & EE \\
\hline $\mathrm{T}_{05}$ & $5 \mathrm{~s}$ \\
\hline $\mathrm{T}_{04}$ & $4 \mathrm{~s}$ \\
\hline $\mathrm{T}_{03}$ & $3 \mathrm{~s}$ \\
\hline $\mathrm{T}_{02}$ & $2 \mathrm{~s}$ \\
\hline$<\mathrm{T}_{02}$ & A la izquierda del 2i (Apagado) \\
\hline
\end{tabular}

Como puede observarse nunca se alcanza el estado estacionario $3 \mathrm{c}$, por lo que se dice que es inestable.

Al representar las temperatura del reactor correspondiente a cada estado estacionario $\left(\mathrm{T}_{\mathrm{EE}}\right)$ frente a la temperatura de entrada del alimento al reactor se obtiene una curva sigmoidal como la que se muestra en la figura 25 . De nuevo se observa que al aumentar $\mathrm{T}_{0}$ se produce el encendido del reactor pasando del estado estacionario $4 \mathrm{i}$ al $4 \mathrm{~s}$, mientras que al disminuir $\mathrm{T}_{0}$ se produce el apagado del reactor pasando del estado estacionario $2 \mathrm{~s}$ al $2 \mathrm{i}$.

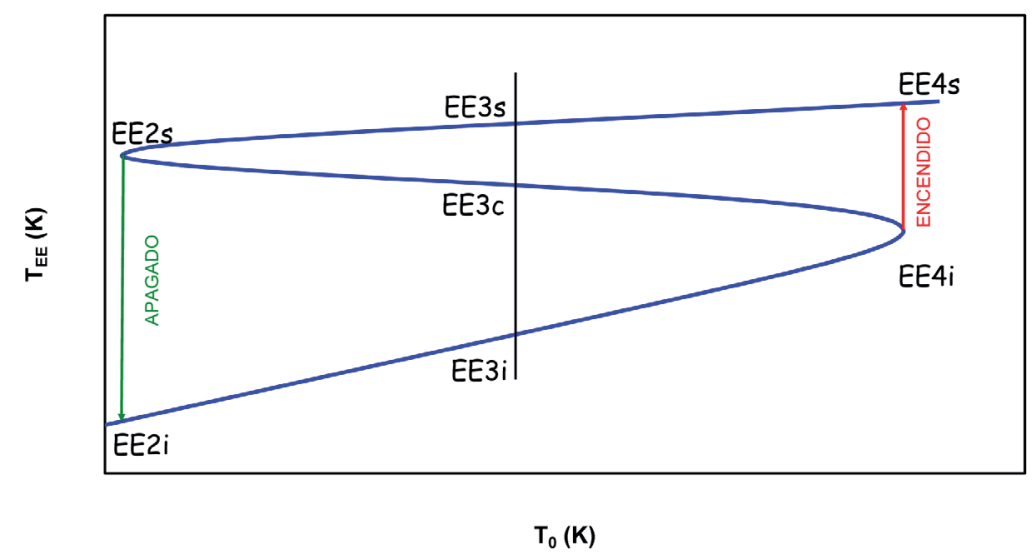

Figura 25. Relación entre la temperatura del estado estacionario y la temperatura del alimento 
Ejercicio 3. El alumno puede observar esta forma de la curva para el caso de una reacción reversible de primer orden de la forma $A_{1}(\ell) \leftrightarrow A_{2}(\ell)$, que se lleva a cabo en un RCTA de $0,667 \mathrm{~m}^{3}$ y con las siguientes condiciones de la corriente de entrada: $\mathrm{c}_{10}=1.73 \mathrm{kmol} / \mathrm{m}^{3} ; \mathrm{F}_{10}=0.0126 \mathrm{kmol} / \mathrm{min}$.

Los datos termodinámicos y cinéticos de la reacción química son:

$$
\begin{aligned}
& \Delta \mathrm{H}_{\mathrm{k}}^{\mathrm{o}}=-83620 \mathrm{~kJ} / \mathrm{kmol} ; \theta_{1} \cdot \overline{\mathrm{C}}_{\mathrm{p} 1}=41.81 \mathrm{~kJ} / \mathrm{kmol} \cdot \mathrm{K} ; \\
& \mathrm{k}=3 \cdot 10^{13} \cdot \exp \left[-\frac{15000}{\mathrm{~T}}\right] \mathrm{min}^{-1} \mathrm{k}^{\prime}=5.44 \cdot 10^{23} \cdot \exp \left[-\frac{25050.5}{\mathrm{~T}}\right] \mathrm{min}^{-1}
\end{aligned}
$$

El RCTA intercambia calor con el medio ambiente, que se encuentra a $T_{f 0}=10{ }^{\circ} \mathrm{C}$ siendo $\mathrm{U} \cdot \mathrm{A}=2.09 \mathrm{~kJ} / \mathrm{min} \cdot \mathrm{K}$.

Demuéstrese que la relación que se obtiene entre la temperatura de la corriente de alimentación $\left(\mathrm{T}_{0}\right)$ y la temperatura de los estados estacionarios del reactor $(\mathrm{T})$ es:

$$
\mathrm{T}_{0}=4.967 \cdot \mathrm{T}-1122.67-\frac{183160 \cdot 3 \cdot 10^{13} \cdot \exp \left(-\frac{15000}{\mathrm{~T}}\right)}{1+91.58 \cdot\left[3 \cdot 10^{13} \cdot \exp \left(-\frac{15000}{\mathrm{~T}}\right)+5.44 \cdot 10^{23} \cdot \exp \left(-\frac{25050.5}{\mathrm{~T}}\right)\right]}
$$

y hágase la correspondiente representación teniendo en cuenta que la temperatura del reactor puede oscilar entre 250 y $500 \mathrm{~K}$.

INFLUENCIA DE $\mathrm{Q}_{\mathrm{V} 0}$ SOBRE LOS ESTADOS ESTACIONARIOS

Supóngase un reactor en el que el caudal volumétrico del alimento $\mathrm{Q}_{\mathrm{v} 0}$ pueda variarse dentro de un pequeño intervalo mientras las demás variables de operación permanecen constantes.

Para cinco caudales volumétricos diferentes del alimento $\left(\mathrm{Q}_{\mathrm{V} 01}>\mathrm{Q}_{\mathrm{V} 02}>\mathrm{Q}_{\mathrm{V} 03}>\right.$ $\mathrm{Q}_{\mathrm{V} 04}>\mathrm{Q}_{\mathrm{V} 05}$ ) tanto la función $\mathrm{Q}_{\mathrm{G}}$ como la función $\mathrm{Q}_{\mathrm{e}}$ varían. Se podrían tener hasta las cinco posibles diferentes situaciones para cinco posiciones diferentes de ambas funciones que se recogen en la tabla 4 y se muestran en la figura 26.

Tabla 4. Posibles estados estacionarios para cinco caudales diferentes del alimento

\begin{tabular}{|c|c|c|}
\hline Nomenclatura de las funciones $\mathrm{Q}_{\mathrm{G}} \mathrm{y} \mathrm{Q}_{\mathrm{e}}$ & Número de EE & Nomenclatura de los EE \\
\hline $\mathrm{Q}_{\mathrm{v} 01}$ & 1 & EE1i \\
\hline $\mathrm{Q}_{\mathrm{V} 02}$ & 2 & EE2i $-\mathrm{EE} 2 \mathrm{~s}$ \\
\hline $\mathrm{Q}_{\mathrm{v} 03}$ & 3 & EE3i $-\mathrm{EE} 3 \mathrm{c}-\mathrm{EE} 3 \mathrm{~s}$ \\
\hline $\mathrm{Q}_{\mathrm{v} 04}$ & 2 & EE4i $-\mathrm{EE} 4 \mathrm{~s}$ \\
\hline $\mathrm{Q}_{\mathrm{v} 05}$ & 1 & EE5s \\
\hline
\end{tabular}




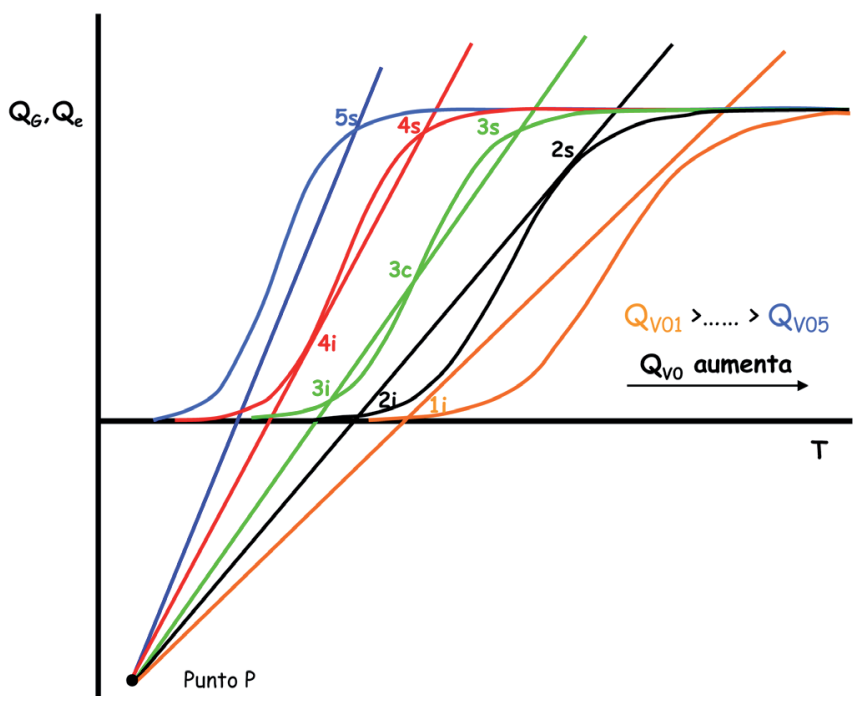

Figura 26. Posibles estados estacionarios para cinco caudales diferentes del alimento

Sin embargo, si existe o se provoca deliberadamente una perturbación, y el valor de $Q_{\mathrm{V} 0}$ se aumenta con suficiente lentitud desde $\mathrm{Q}_{\mathrm{V} 05}$ (para que en cada una de las cinco situaciones escogidas se alcance el régimen estacionario) la secuencia de estados estacionarios que se seguirá se muestra en la tabla 5.

Tabla 5. Posibles estados estacionarios al incrementar el caudal del alimento

\begin{tabular}{|c|c|}
\hline Caudal volumétrico de entrada & EE \\
\hline $\mathrm{Q}_{\mathrm{V} 05}$ & $5 \mathrm{~s}$ \\
\hline $\mathrm{Q}_{\mathrm{V} 04}$ & $4 \mathrm{~s}$ \\
\hline $\mathrm{Q}_{\mathrm{V} 03}$ & $3 \mathrm{~s}$ \\
\hline $\mathrm{Q}_{\mathrm{V} 02}$ & $2 \mathrm{~s}$ \\
\hline$>\mathrm{Q}_{\mathrm{V} 02}$ & A la izquierda del 2i (Apagado) \\
\hline
\end{tabular}

Análogamente, si existe o se provoca deliberadamente una perturbación, y el valor de $\mathrm{Q}_{\mathrm{V} 0}$ se disminuye con suficiente lentitud desde $\mathrm{Q}_{\mathrm{V} 01}$ (para que en cada una de las cinco situaciones escogidas se alcance el régimen estacionario) la secuencia de estados estacionarios que se seguirá se muestra en la tabla 6 .

Tabla 6. Posibles estados estacionarios al disminuir el caudal del alimento

\begin{tabular}{|c|c|}
\hline Caudal volumétrico de entrada & $\mathrm{EE}$ \\
\hline $\mathrm{Q}_{\mathrm{V} 01}$ & $1 \mathrm{i}$ \\
\hline $\mathrm{Q}_{\mathrm{V} 02}$ & $2 \mathrm{i}$ \\
\hline $\mathrm{Q}_{\mathrm{V} 03}$ & $3 \mathrm{i}$ \\
\hline $\mathrm{Q}_{\mathrm{V} 04}$ & $4 \mathrm{i}$ \\
\hline$<\mathrm{Q}_{\mathrm{V} 04}$ & A la derecha del 4s (Encendido) \\
\hline
\end{tabular}


Como puede observarse nuevamente nunca se alcanza el estado estacionario $3 \mathrm{c}$, por lo que se dice que es inestable.

Al representar las temperatura del reactor correspondiente a cada estado estacionario $\left(\mathrm{T}_{\mathrm{EE}}\right)$ frente a la temperatura de entrada del alimento al reactor se obtiene una curva sigmoidal como la que se muestra en la figura 27. De nuevo se observa que al disminuir $\mathrm{Q}_{\mathrm{v} 0}$ se produce el encendido del reactor pasando del estado estacionario $4 \mathrm{i}$ al $4 \mathrm{~s}$, mientras que al aumentar $\mathrm{Q}_{\mathrm{V} 0}$ se produce el apagado del reactor pasando del estado estacionario $2 \mathrm{~s}$ al $2 \mathrm{i}$.

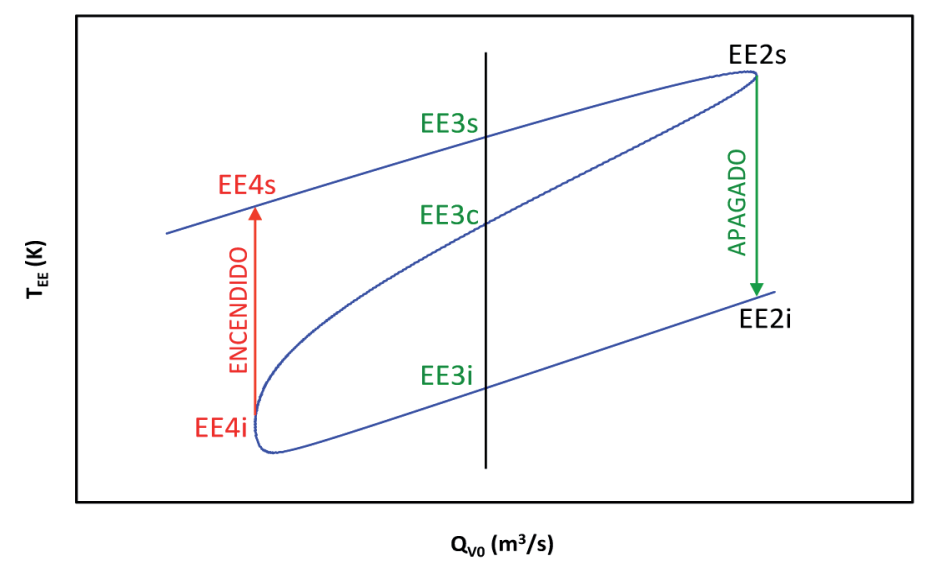

Figura 27. Relación entre la temperatura del estado estacionario y el caudal del alimento

Ejercicio 4. El alumno puede observar esta forma de la curva para el caso de una reacción irreversible de primer orden de la forma $A_{1}(\ell) \rightarrow$ productos $(\ell)$, que se lleva a cabo en un RCTA de $1 \mathrm{~m}^{3}$ y con las siguientes condiciones de la corriente de entrada: $\mathrm{c}_{10}=1 \mathrm{kmol} / \mathrm{m}^{3} ; \mathrm{T}_{0}=320 \mathrm{~K}$

Los datos termodinámicos y cinéticos de la reacción química son:

$$
\begin{aligned}
& \Delta \mathrm{H}_{\mathrm{k}}^{\mathrm{o}}=-52300 \mathrm{~kJ} / \mathrm{kmol} ; \theta_{1} \cdot \overline{\mathrm{C}}_{\mathrm{p} 1}=514.6 \mathrm{~kJ} / \mathrm{kmol} \cdot \mathrm{K} ; \\
& \mathrm{k}=7.1 \cdot 10^{12} \cdot \exp \left[-\frac{13200}{\mathrm{~T}}\right] \mathrm{min}^{-1}
\end{aligned}
$$

El RCTA es adiabático y no intercambia calor.

Demuéstrese que la relación que se obtiene entre el caudal volumétrico de la corriente de alimentación $\left(\mathrm{Q}_{\mathrm{V} 0}\right)$ y la temperatura de los estados estacionarios del reactor $(\mathrm{T})$ es:

$$
\mathrm{Q}_{\mathrm{V} 0}=\frac{101.63 \cdot 7.1 \cdot 10^{12} \cdot \exp \left[-\frac{13200}{\mathrm{~T}}\right]}{(\mathrm{T}-320)}-7.1 \cdot 10^{12} \cdot \exp \left[-\frac{13200}{\mathrm{~T}}\right]
$$

donde $\mathrm{T}$ va en $\mathrm{K}$ y $\mathrm{Q}_{\mathrm{VO}}$ en $\mathrm{m}^{3} / \mathrm{min}$. Hágase la correspondiente representación teniendo en cuenta que el caudal volumétrico puede oscilar entre los valores 0.1 $1 / \mathrm{min}$ y $100 \mathrm{l} / \mathrm{min}$. 


\subsection{Estabilidad del RCTA}

En la fase de diseño del RCTA es muy importante tener presente las consideraciones anteriores de la influencia de $\mathrm{T}_{0}, \mathrm{~T}_{\mathrm{f} 0} \mathrm{y} \mathrm{Q}_{\mathrm{V} 0}$ sobre el estado estacionario que se alcanza. En efecto, pequeñas perturbaciones de estas variables pueden llevar al RCTA a un estado estacionario en el que se produzca el encendido o el apagado de la reacción.

En el caso de que matemáticamente sean posibles tres estados estacionarios, se puede demostrar que físicamente solo dos son estables, mientras que el otro es inestable. En la figura 28 se representa la curva $Q_{G}$ y la recta $Q_{e}$ para el caso de una reacción exotérmica con tres estados estacionarios A, B y C.

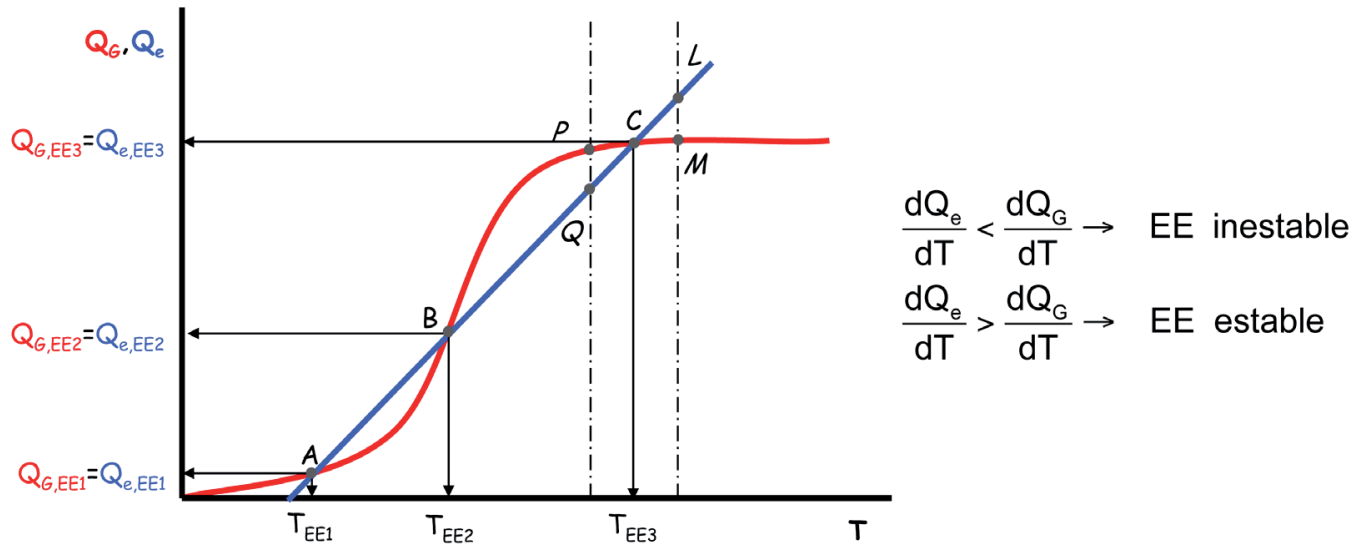

Figura 28. Funciones $\mathrm{Q}_{\mathrm{G}}$ y $\mathrm{Q}_{\mathrm{e}}$ para el caso de una reacción exotérmica con tres estados estacionarios A, B y C

Se puede realizar el siguiente análisis:

- Punto $C$. Un ligero aumento de la temperatura en el reactor $\left(\mathrm{T}_{\mathrm{C}}\right)$ conduciría a la zona donde la recta de eliminación de energía $\left(\mathrm{Q}_{\mathrm{e}}\right)$ está por encima de la curva de generación de energía $\left(\mathrm{Q}_{\mathrm{G}}\right)$, como puede apreciarse en los puntos L y M. Por tanto, para temperaturas ligeramente superiores a las del estado estacionario indicado tiene lugar una eliminación neta de energía (ya que el valor de la función de eliminación de energía es siempre mayor que el valor de la función de generación de energía) que tendería a reducir la temperatura en el reactor, retornando el sistema de forma espontánea al estado estacionario $\mathrm{C}$ al que le corresponde la temperatura $\mathrm{T}_{\mathrm{C}}$. Por el contrario, un ligero descenso de la temperatura en el reactor conduciría a una zona donde la generación de energía (correspondiente al punto $\mathrm{P}$ ) sería superior a la eliminación de la misma (punto Q), de modo que se produciría un aumento de la temperatura de la mezcla reactante hasta $T_{C}$. Es decir, el reactor tiene una propensión intrínseca a permanecer en el estado estacionario $\mathrm{C}$, debido al hecho de que la pendiente de la recta de eliminación de energía es mayor que la de la curva de generación en las regiones inmediatas al punto C. 
- Punto A. Puede hacerse un razonamiento análogo al realizado para el punto $\mathrm{C}$.

- Punto B. En cambio, en los alrededores del estado estacionario representado por $\mathrm{B}$, la pendiente de la curva de generación de energía es mayor que la de eliminación y, por tanto, el comportamiento del sistema es completamente diferente. Un ligero aumento de temperatura en el reactor por encima de $\mathrm{T}_{\mathrm{B}}$ produce una generación neta de energía que tendería a aumentar más la temperatura del reactor hasta alcanzar $T_{C}$. Por el contrario, un descenso en la temperatura $T_{B}$ traería consigo una eliminación neta de energía que supondría un descenso de la temperatura del reactor hasta $\mathrm{T}_{\mathrm{A}}$. Por tanto, puede afirmarse que el estado intermedio representado por el punto $\mathrm{B}$ es inestable.

En resumen puede generalizarse que un estado estacionario será inestable, cuando se cumpla en el punto de intersección entre la recta $Q_{e}$ y la curva $Q_{G}$ la desigualdad:

$$
\frac{d Q_{e}}{d T}<\frac{d Q_{G}}{d T}
$$

es decir, si la pendiente de la recta de eliminación de energía es menor que la pendiente de la curva de generación de energía en ese punto. Esta condición es suficiente para garantizar la inestabilidad. En cambio, si ocurre lo contrario el estado estacionario suele ser estable:

$$
\frac{d Q_{e}}{d T}>\frac{d Q_{G}}{d T}
$$

(En realidad, esta condición es necesaria para asegurar la estabilidad del estado estacionario pero no suficiente; para tener la completa seguridad de la estabilidad del estado estacionario habría que aplicar las ecuaciones del balance de materia y del balance de energía en un RCTA funcionando en régimen no estacionario, régimen en que está operando desde el momento en que se ha producido alguna perturbación, y deducir una segunda condición de estabilidad. Sin embargo esta discusión queda fuera de un curso introductorio).

\subsection{Sistemas en los que se desarrollan varias reacciones químicas}

En este apartado se tratarán, solo a modo de ejemplo, los sistemas constituidos por un único Reactor Continuo de Tanque Agitado (RCTA) funcionando en régimen estacionario en el que tienen lugar varias reacciones químicas simultáneamente (consecutivas, paralelas o mezcladas). En consecuencia, tal y como se recogía en Anexo II del capítulo 1, el balance de materia se puede expresar mediante cualquiera de las dos ecuaciones siguientes: 
a) para reacciones químicas paralelas: $\mathrm{c}_{\mathrm{k} 0} \cdot \mathrm{X}_{\mathrm{i}}+\tau \cdot \mathrm{v}_{\mathrm{ik}} \cdot \mathrm{r}_{\mathrm{i}}=0 \quad \mathrm{i}=1,2, \ldots \mathrm{R}$

b) para cualquier sistema de reacciones químicas:

$$
c_{j 0}-c_{j}+\tau \cdot \sum_{i=1}^{R} v_{i j} \cdot r_{i}=0 \quad j=1,2, \ldots S
$$

Para poder aplicar estas ecuaciones es necesario conocer las ecuaciones de la velocidad de cada reacción química, en función de la temperatura y de la concentración o el grado de conversión:

$$
r_{i}=r_{i}\left[X_{i}, T\right] \quad \circ \quad r=r\left[c_{j}, T\right]
$$

De esta forma se podrán escribir R o S ecuaciones de diseño:

$$
\begin{array}{ll}
c_{k 0} \cdot X_{i}+\tau \cdot v_{i k} \cdot r_{i}\left(X_{i}, T\right)=0 & i=1,2, \ldots R \\
c_{j 0}-c_{j}+\tau \cdot \sum_{i=1}^{R} v_{i j} \cdot r_{i}\left(c_{j}, T\right)=0 & j=1,2, \ldots S
\end{array}
$$

Análogamente, en el capítulo 1 también se han mostrado los balances de energía que, para el sistema constituido por un RCTA en el que se desarrollan varias reacciones químicas, se puede expresar mediante una de las dos ecuaciones siguientes:

a) para reacciones químicas paralelas: $\mathrm{T}-\mathrm{T}_{0}-\sum_{\mathrm{i}=1}^{\mathrm{R}} \mathrm{J}_{\mathrm{i}} \cdot \mathrm{X}_{\mathrm{i}}=\mathrm{Q}$

b) para cualquier sistema de reacciones químicas:

$$
\mathrm{T}-\mathrm{T}_{0}-\frac{\tau}{\mathrm{c}_{\mathrm{k} 0}} \cdot \sum_{\mathrm{i}=1}^{\mathrm{R}}\left(-\mathrm{v}_{\mathrm{ik}}\right) \cdot \mathrm{r}_{\mathrm{i}} \cdot \mathrm{J}_{\mathrm{i}}=\mathrm{Q}
$$

Para poder aplicar cualquiera de estas dos ecuaciones es necesario conocer la ecuación de la velocidad de transmisión de calor, cuya forma depende del sistema de intercambio de calor utilizado, como se ha visto en el apartado 2.2 de este capítulo, pero que siempre se puede expresar como:

$$
\mathrm{Q}=\kappa \cdot\left(\mathrm{T}_{\mathrm{f0}}-\mathrm{T}\right)
$$

quedando las dos posibles ecuaciones del balance de energía como:

a) para reacciones químicas paralelas: $\mathrm{T}-\mathrm{T}_{0}-\sum_{\mathrm{i}=1}^{\mathrm{R}} \mathrm{J}_{\mathrm{i}} \cdot \mathrm{X}_{\mathrm{i}}=\mathrm{Q}$

b) para cualquier sistema de reacciones químicas:

$$
\mathrm{T}-\mathrm{T}_{0}-\frac{\tau}{\mathrm{c}_{\mathrm{k} 0}} \cdot \sum_{\mathrm{i}=1}^{\mathrm{R}}\left(-\mathrm{v}_{\mathrm{ik}}\right) \cdot \mathrm{r}_{\mathrm{i}} \cdot \mathrm{J}_{\mathrm{i}}=\mathrm{Q}
$$


El diseño de un RCTA en el que se desarrollan varias reacciones químicas o el cálculo de las condiciones de funcionamiento supone resolver simultáneamente un conjunto de sistemas de ecuaciones de diseño:

a) para reacciones químicas paralelas, hay que resolver $\mathrm{R}+1$ ecuaciones:

$$
\begin{gathered}
\mathrm{c}_{\mathrm{k} 0} \cdot \mathrm{X}_{\mathrm{i}}+\tau \cdot \mathrm{v}_{\mathrm{ik}} \cdot \mathrm{r}_{\mathrm{i}}\left(\mathrm{X}_{\mathrm{i}}, \mathrm{T}\right)=0 \quad \mathrm{i}=1,2, \ldots \mathrm{R} \\
\mathrm{T}-\mathrm{T}_{0}-\sum_{\mathrm{i}=1}^{\mathrm{R}} \mathrm{J}_{\mathrm{i}} \cdot \mathrm{X}_{\mathrm{i}}=\kappa \cdot\left(\mathrm{T}_{\mathrm{f} 0}-\mathrm{T}\right)
\end{gathered}
$$

en donde solo puede haber $\mathrm{R}+1$ incógnitas.

b) para cualquier sistema de reacciones químicas, hay que resolver $\mathrm{S}+1$ ecuaciones:

$$
\begin{gathered}
c_{j 0}-c_{j}+\tau \cdot \sum_{i=1}^{R} v_{i j} \cdot r_{i}\left(c_{j}, T\right)=0 \quad j=1,2, \ldots S \\
T-T_{0}-\frac{\tau}{c_{k 0}} \cdot \sum_{i=1}^{R}\left(-v_{i k}\right) \cdot r_{i} \cdot J_{i}=\kappa \cdot\left(T_{f 0}-T\right)
\end{gathered}
$$

en donde solo puede haber $\mathrm{S}+1$ incógnitas.

La resolución matemática de cualquiera de estos sistemas de ecuaciones no es trivial y su complejidad varía con el tipo de reacción química (reversible o irreversible) y con el orden de reacción. En algunos pocos casos la resolución puede ser relativamente sencilla e, incluso, se puede aplicar alguno de los métodos utilizados para el diseño de un reactor en el que tiene lugar una única reacción. En efecto, véase, a modo de ejemplo, el siguiente caso.

Diseño de un RCTA en el que transcurren dos reacciones simultáneas consecutivas, irreversibles y de primer orden

Supóngase un sistema de reacción constituido por dos reacciones químicas simultáneas, consecutivas, irreversibles y de primer orden con las concentraciones en la corriente de alimento al RCTA que se indican:

$$
\mathrm{A}_{1} \stackrel{\mathrm{i}=1}{\longrightarrow} \mathrm{A}_{2} \stackrel{\mathrm{i}=2}{\longrightarrow} \mathrm{A}_{3} \quad \mathrm{r}_{1}=\mathrm{k}_{1} \cdot \mathrm{c}_{1} \quad \mathrm{r}_{2}=\mathrm{k}_{2} \cdot \mathrm{c}_{2} \quad \mathrm{c}_{10}, \mathrm{c}_{20}=0, \mathrm{c}_{30}=0
$$

Dado que hay tres especies químicas, al aplicar el balance de materia se obtienen las tres ecuaciones siguientes: 


$$
\begin{aligned}
& c_{1}=c_{10} \cdot \frac{1}{1+\tau \cdot k_{1}} \\
& c_{2}=c_{10} \cdot \frac{\tau \cdot k_{1}}{1+\tau \cdot k_{1}} \cdot \frac{1}{1+\tau \cdot k_{2}} \\
& c_{3}=c_{10} \cdot \frac{\tau \cdot k_{1}}{1+\tau \cdot k_{1}} \cdot \frac{\tau \cdot k_{2}}{1+\tau \cdot k_{2}}
\end{aligned}
$$

Análogamente, aplicando el balance de energía se obtiene:

$$
T-T_{0}-\frac{\tau}{C_{10}} \cdot\left(J_{1} \cdot k_{1} \cdot c_{1}+J_{2} \cdot k_{2} \cdot C_{2}\right)=\kappa \cdot\left(T_{f 0}-T\right)
$$

Por lo tanto si las incógnitas son $\mathrm{c}_{1}, \mathrm{c}_{2}, \mathrm{c}_{3} \mathrm{y} \mathrm{T}$ se dispone de un sistema de cuatro ecuaciones con cuatro incógnitas que se puede resolver por cualquier método matemático. Ahora bien, en este caso concreto también se puede resolver aplicando el método de Aris descrito para sistemas con una única reacción química. En efecto, si en el balance de energía se sustituyen las concentraciones resulta:

$$
\frac{\tau}{c_{10}} \cdot\left(J_{1} \cdot k_{1} \cdot c_{10} \cdot \frac{1}{1+\tau \cdot k_{1}}+J_{2} \cdot k_{2} \cdot c_{10} \cdot \frac{\tau \cdot k_{1}}{1+\tau \cdot k_{1}} \cdot \frac{1}{1+\tau \cdot k_{2}}\right)=T-T_{0}-K \cdot\left(T_{f 0}-T\right)
$$

Es decir, una ecuación cuya única incógnita es la temperatura y que, reordenándola, se puede escribir como:

$$
\frac{\tau \cdot k_{1}}{1+\tau \cdot k_{1}} \cdot\left(J_{1}+J_{2} \cdot \frac{\tau \cdot k_{2}}{1+\tau \cdot k_{2}}\right)=-\left(T_{0}+\kappa \cdot T_{f 0}\right)+(1+\kappa) \cdot T
$$

Dado que esta ecuación puede resultar difícil de resolver analíticamente, es conveniente hacerlo mediante un método gráfico sin más que definir la función:

$$
Q_{e}=-\left(T_{0}+\kappa \cdot T_{f 0}\right)+(1+\kappa) \cdot T
$$

que es una línea recta, al igual que en el caso de una única reacción química, puesto que no interviene la velocidad de reacción y la función:

$$
Q_{G}=\frac{\tau \cdot k_{1}}{1+\tau \cdot k_{1}} \cdot\left(J_{1}+J_{2} \cdot \frac{\tau \cdot k_{2}}{1+\tau \cdot k_{2}}\right)
$$

que es una curva complicada cuya forma depende de la entalpía de cada reacción $\left(\mathrm{J}_{1} \mathrm{y} \mathrm{J}_{2}\right)$, de las energías de activación $\left(\mathrm{E}_{1} \mathrm{y} \mathrm{E}_{2}\right)$ y de los factores preexponenciales $\left(A_{1}\right.$ y $\left.A_{2}\right)$. Representando ambas funciones en la forma de $Q_{G}$ y $Q_{e}$ versus $T$ los puntos de corte (es decir, los puntos en que se cumple que $\mathrm{Q}_{\mathrm{G}}$ y $\mathrm{Q}_{\mathrm{e}}$ ) serán los que satisfagan la ecuación en $\mathrm{T}$ anterior y se denominan estados estacionarios (EE). 
Las posibilidades que se pueden dar para el ejemplo en cuestión se muestran cualitativamente en las figuras 29 a 34, indicando el número de posibles estados estacionarios.

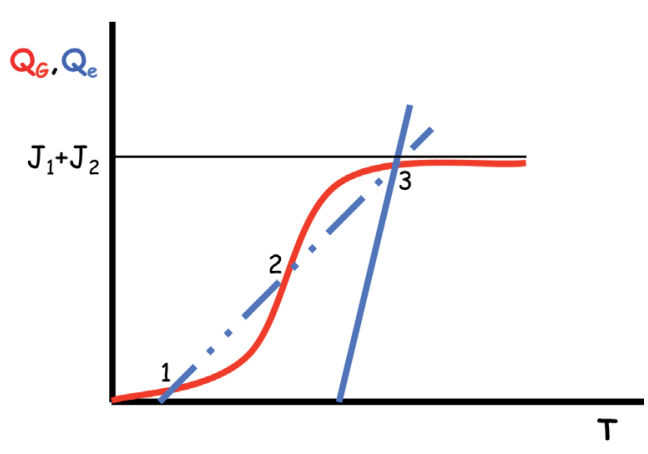

Figura 29. Las dos reacciones son exotérmicas con $\mathrm{E}_{1} \approx \mathrm{E}_{2} \circ \mathrm{E}_{1}>\mathrm{E}_{2}$

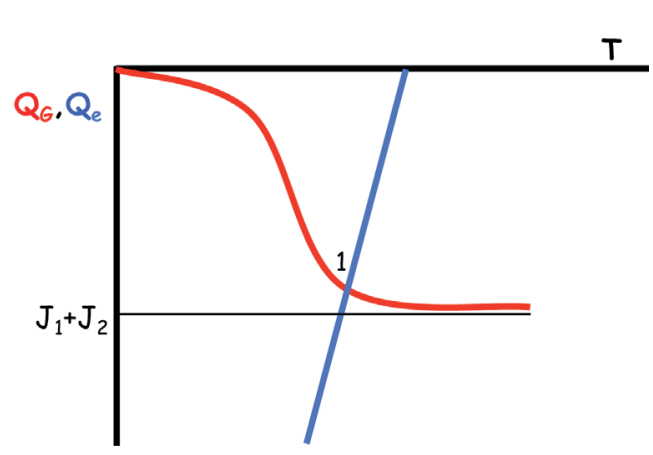

Figura 31. Las dos reacciones son endotérmicas con $\mathrm{E}_{1} \approx \mathrm{E}_{2}$ o $\mathrm{E}_{1}>\mathrm{E}_{2}$

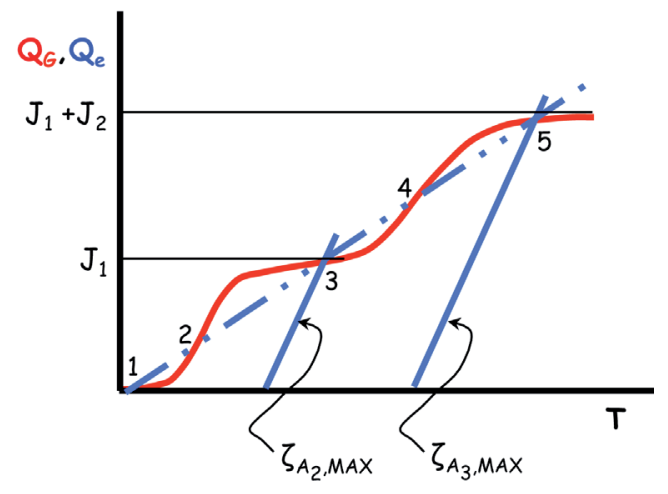

Figura 30. Las dos reacciones son exotérmicas con $\mathrm{E}_{1}<\mathrm{E}_{2}$

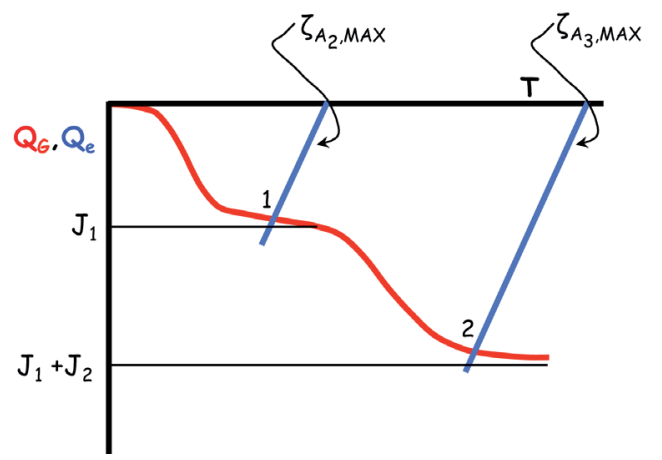

Figura 32. Las dos reacciones son endotérmicas con $\mathrm{E}_{1}<\mathrm{E}_{2}$

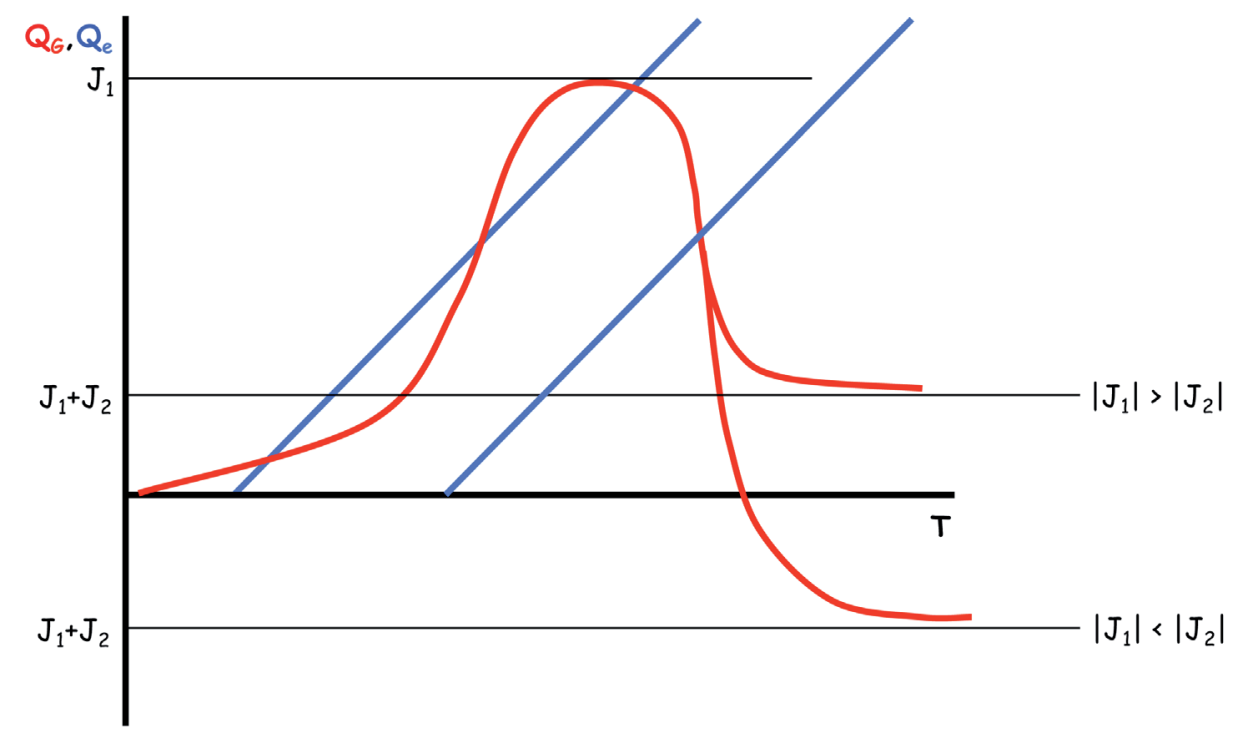

Figura 33. La reacción $i=1$ es exotérmica mientras que $i=2$ es endotérmica 


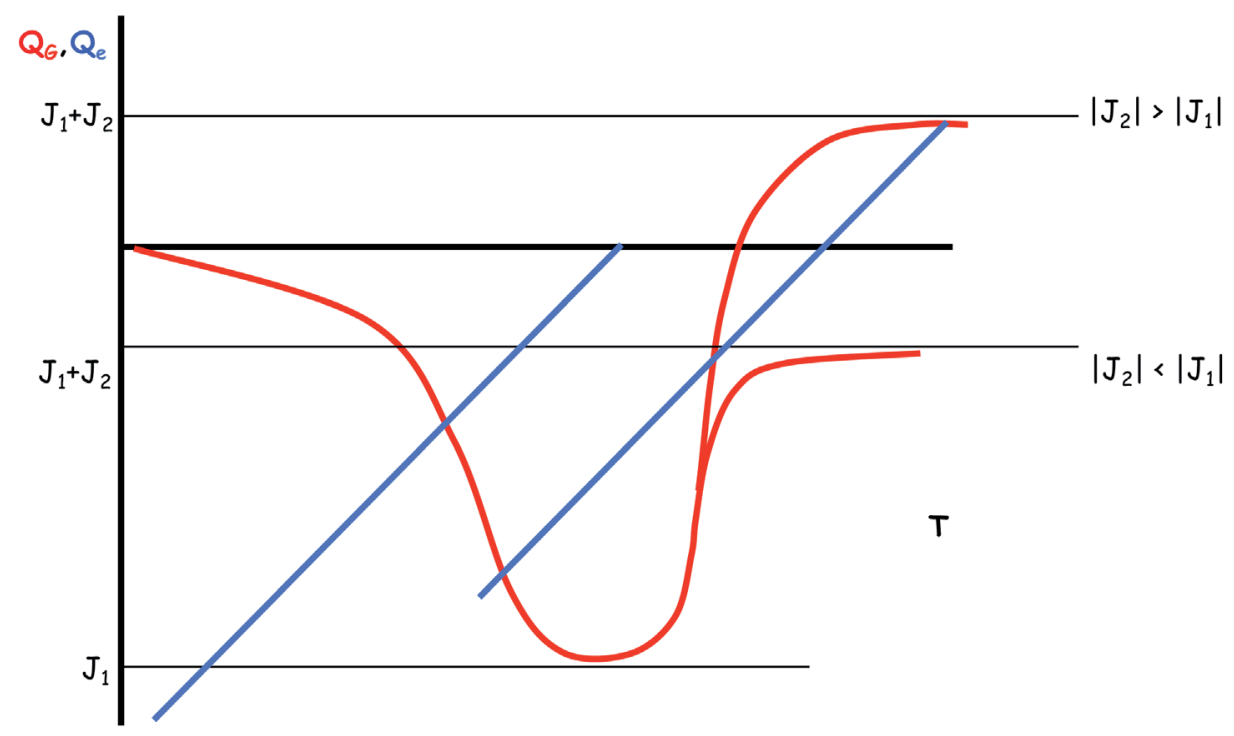

Figura 34. La reacción $i=1$ es endotérmica mientras que la $i=2$ es exotérmica

\subsection{Temperatura de reacción óptima}

Tal y como se ha mostrado en los apartados anteriores y en la asignatura EQ1021 Ingeniería de la Reacción Química, la temperatura de reacción junto con el grado de conversión determinan el valor de la velocidad de reacción. Así pues, el par de valores de X y T que definen cada estado estacionario marca la velocidad a la que se produce la reacción química en el RCTA. La temperatura de reacción óptima, para un grado de conversión determinado, es la que hace que la velocidad de reacción sea máxima puesto que, en consecuencia, el volumen de reacción será mínimo, de acuerdo con la ecuación de diseño (1).

La velocidad de reacción máxima posible, en sistemas con una reacción química, se puede estimar fácilmente para cada tipo de reacción siempre que se conozcan las curvas de velocidad de reacción constante estudiadas en la asignatura EQ1021 Ingeniería de la Reacción Química. Si el sistema fuera de varias reacciones químicas se requiere el uso de una serie de RCTA, lo que se estudiará en el capítulo 5.

\section{Reacciones endotérmicas, reversibles e irreversibles, y reacciones exotérmicas} irreversibles

Para estos tres tipos de reacciones las curvas de velocidad de reacción constante son de la forma que se muestra en las figuras 35 y 36. 


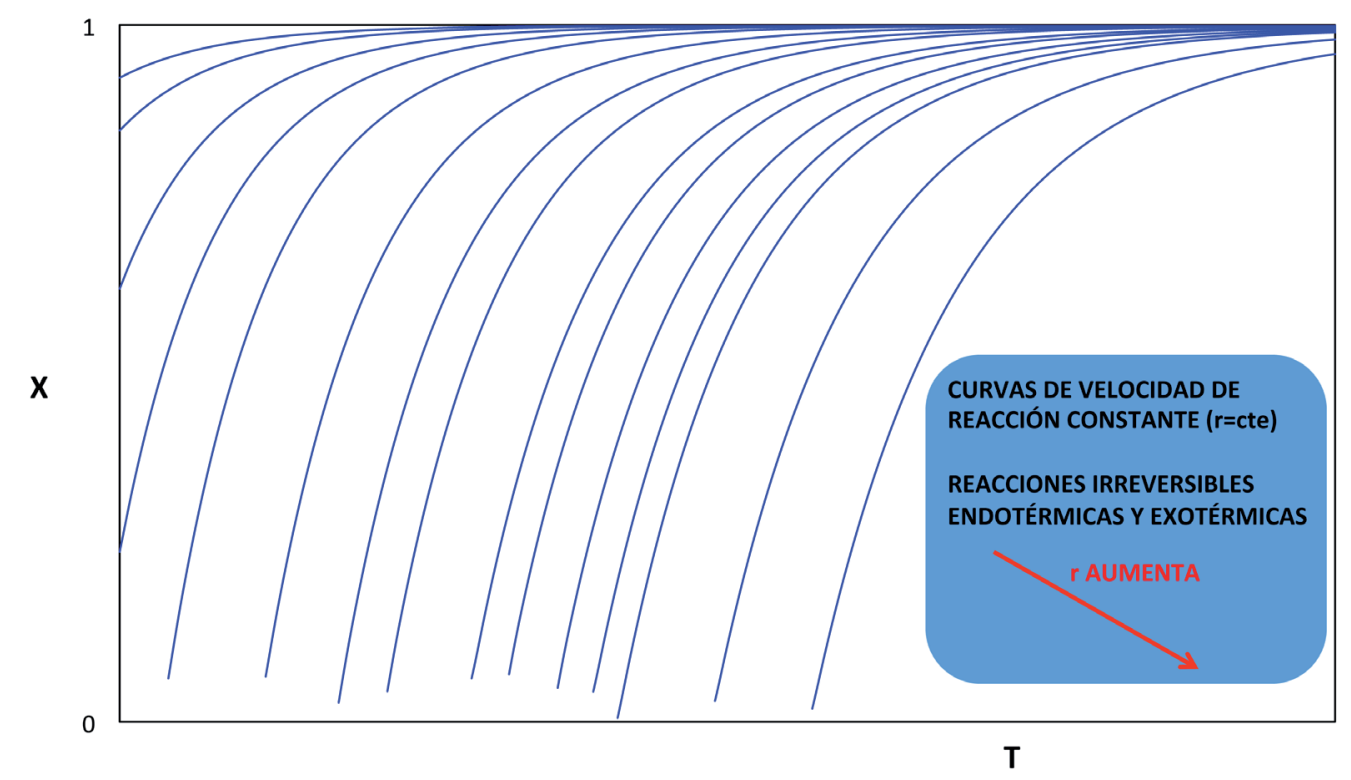

Figura 35. Curvas de velocidad de reacción constante para reacciones irreversibles endotérmicas y exotérmicas

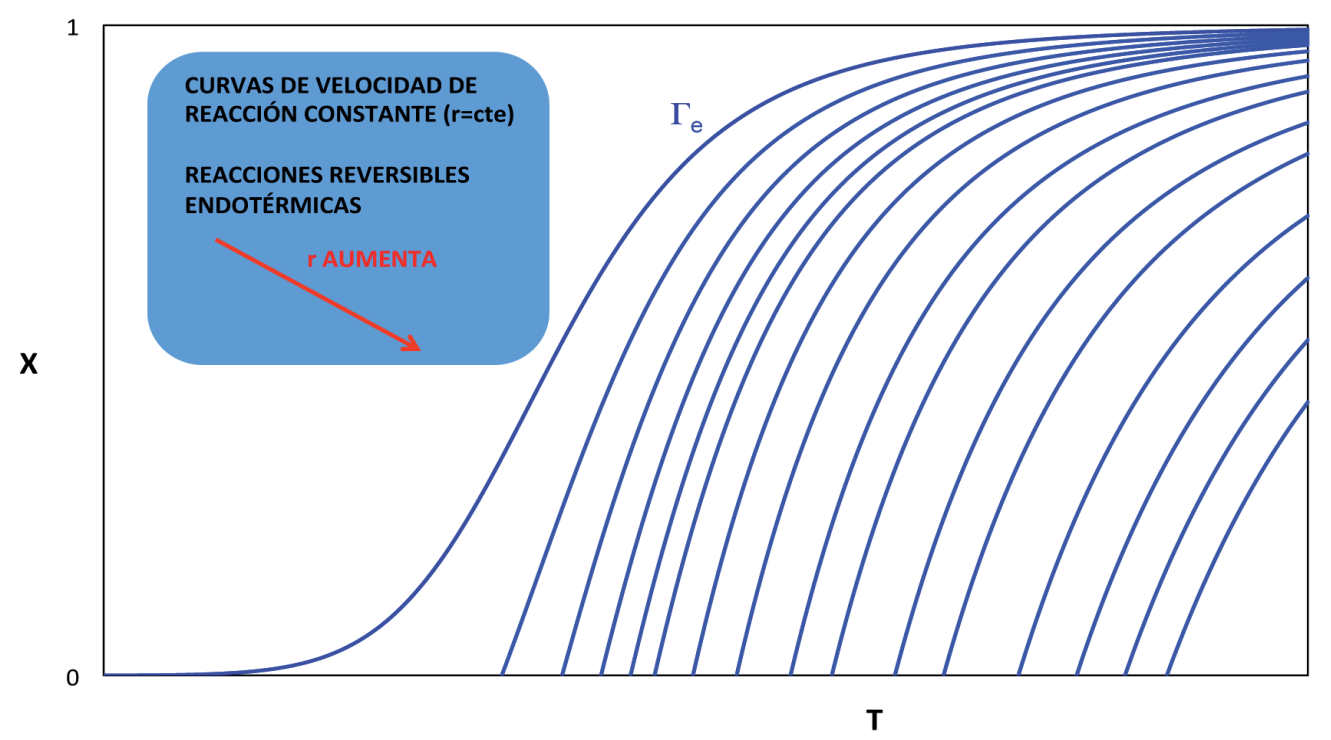

Figura 36. Curvas de velocidad de reacción constante para reacciones reversibles endotérmicas

A partir del análisis de estas curvas se deduce fácilmente (figura 37) que la mejor forma de funcionamiento de un RCTA, en el que se desarrolle algún tipo de estas tres reacciones químicas, es que el estado estacionario se sitúe en la máxima temperatura permisible (o ligeramente inferior) por el sistema $\left(\mathrm{T}^{*}\right)$, ya que de esta forma la velocidad de reacción es máxima para el correspondiente grado de conversión del estado estacionario.

En las reacciones endotérmicas reversibles debe recordarse que el máximo grado de conversión que se puede alcanzar a cada temperatura fijada viene determinado por la curva de equilibrio. 


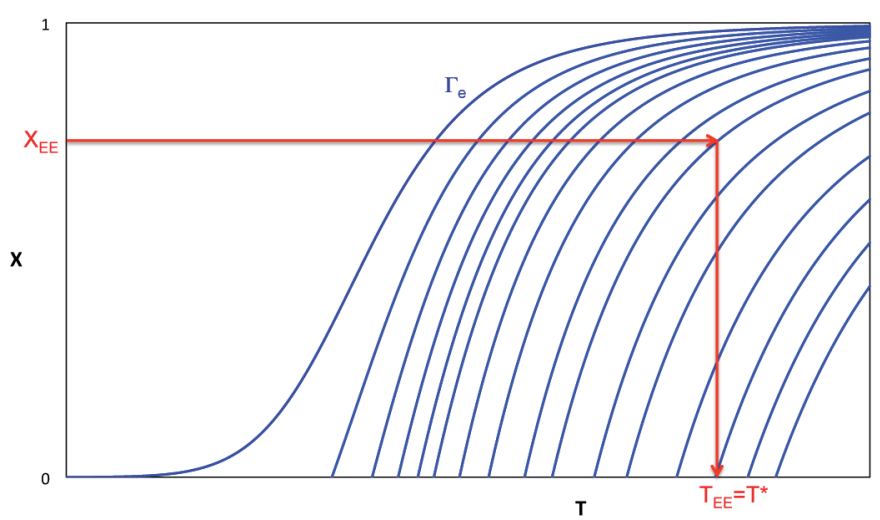

Figura 37. Determinación de la temperatura óptima en reacciones endotérmicas o exotérmicas irreversibles

\section{Reacciones exotérmicas reversibles}

Para este tipo de reacción las curvas de velocidad de reacción constante son de la forma que se muestra en la figura 38.

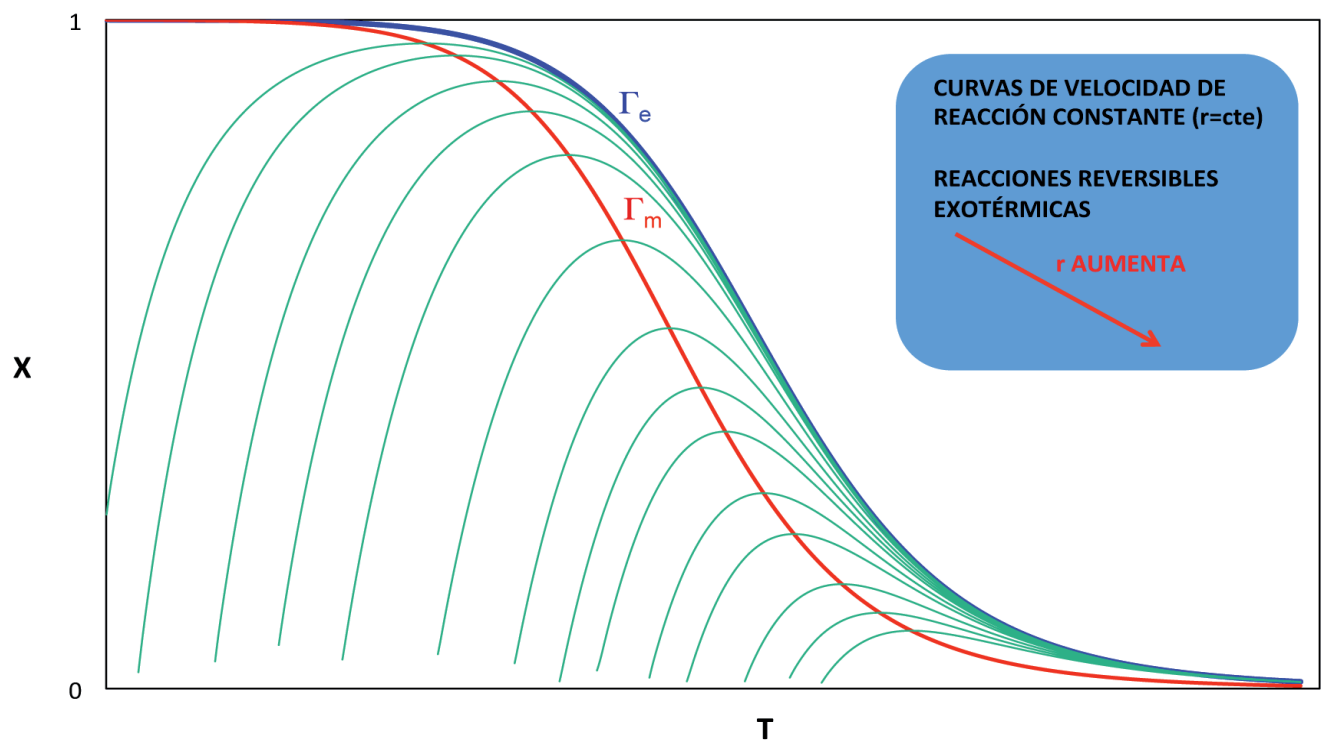

Figura 38. Curvas de velocidad de reacción constante para reacciones reversibles exotérmicas

Del análisis de las curvas se deduce que la pareja de valores $\mathrm{X}, \mathrm{T}$ del estado estacionario debe situarse sobre la curva $\Gamma_{\mathrm{m}}$ de máxima velocidad de reacción (figura 39).

En las reacciones exotérmicas reversibles debe recordarse que el máximo grado de conversión que se puede alcanzar a cada temperatura fijada viene determinado por la curva de equilibrio. 


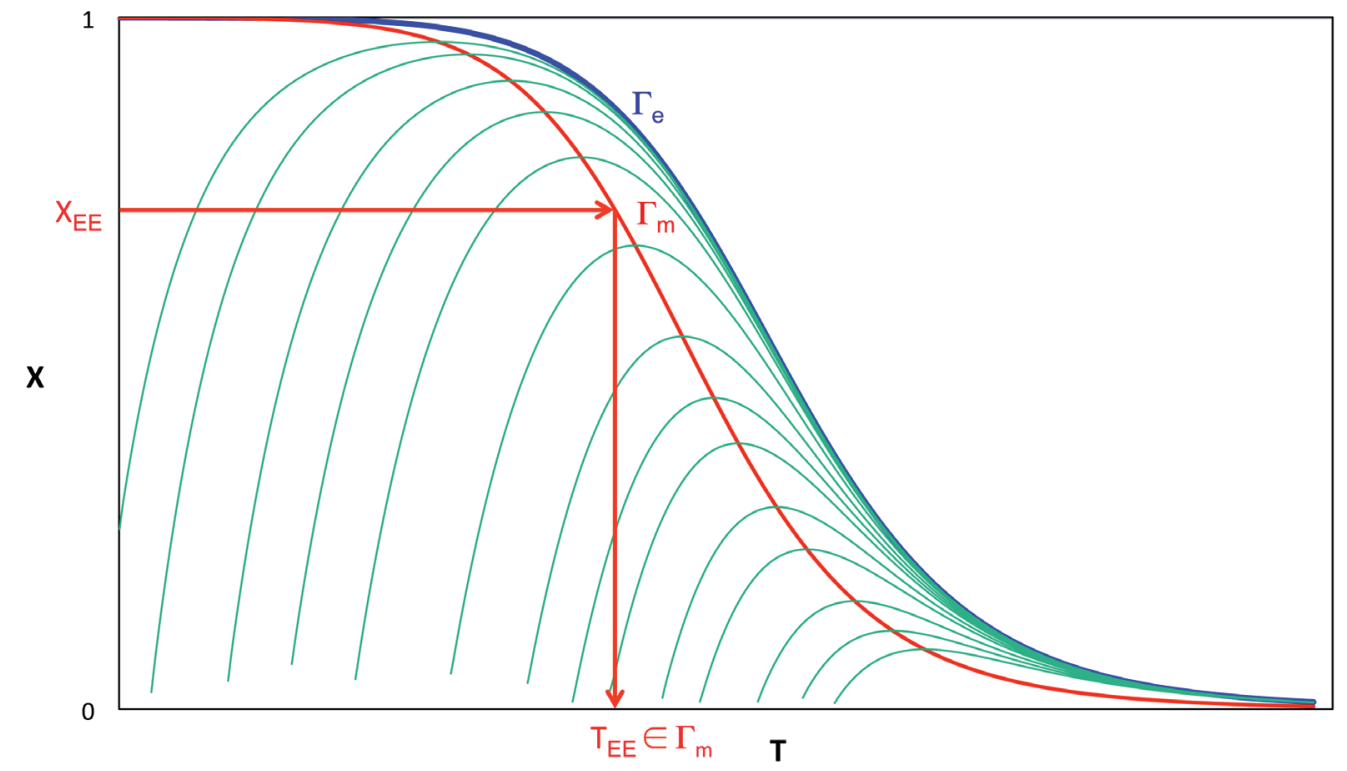

Figura 39. Determinación de la temperatura óptima en reacciones exotérmicas reversibles 


\section{PROBLEMAS}

Problema 01. En un proceso continuo se hidroliza una solución acuosa de un éster de ácido orgánico monobásico por reacción acuosa de $\mathrm{NaOH}$ en un RCTA que contiene $6 \mathrm{~m}^{3}$ de líquido. En el interior del reactor hay un serpentín sumergido que mantiene la temperatura de reacción a $25^{\circ} \mathrm{C}$. A partir de los datos que se dan a continuación estimar el área de transmisión de calor necesaria si el agua de enfriamiento entra en el serpentín a $15^{\circ} \mathrm{C}$ y lo abandona a $20^{\circ} \mathrm{C}$. Despréciense las pérdidas de calor desde el reactor a los alrededores.

\section{Datos y notas}

Solución de éster: caudal: $0.025 \mathrm{~m}^{3} / \mathrm{s}$; temperatura: $25^{\circ} \mathrm{C}$; concentración: $1 \mathrm{kmol} / \mathrm{m}^{3}$

Solución de álcali: caudal: $0.010 \mathrm{~m}^{3} / \mathrm{s}$; temperatura: $20{ }^{\circ} \mathrm{C}$; concentración: $5 \mathrm{kmol} / \mathrm{m}^{3}$

Constante de velocidad de reacción a $25{ }^{\circ} \mathrm{C}: 0.11 \mathrm{~m}^{3} / \mathrm{kmol} \cdot \mathrm{s}$

Calor de reacción: $-1.46 \cdot 10^{7} \mathrm{~J} / \mathrm{kmol}$

Coeficiente global de transmisión de calor: $2280 \mathrm{~W} / \mathrm{m}^{2} \cdot \mathrm{K}$

Problema 02. En un RCTA se desarrolla la reacción endotérmica irreversible en fase líquida $A_{1}+A_{2} \rightarrow A_{3}$. El reactor se calienta mediante una camisa de vapor a la presión adecuada. El alimento consiste en una mezcla de $\mathrm{A}_{1} \mathrm{y} \mathrm{A}_{2}$, en la que la concentración de ambos componentes es la misma e igual a $2 \mathrm{kmol} / \mathrm{m}^{3}$, que se introduce en el reactor a la temperatura de $300 \mathrm{~K}$, con un caudal volumétrico de $30 \cdot 10^{-3} \mathrm{~m}^{3} / \mathrm{min}$. El volumen total de reacción es de $1.2 \mathrm{~m}^{3}$ y se desea obtener un grado de conversión de 0.6 de $\mathrm{A}_{1}$

a) Encontrar la temperatura de estado estacionario a que debe funcionar el reactor para alcanzar el grado de conversión deseado.

b) Calcular la temperatura a la que debe condensar el vapor de calefacción.

c) Determinar los kg de vapor de calefacción que condensarán por hora.

\section{Datos y notas}

La velocidad de reacción se puede expresar como $\mathrm{r}=\mathrm{k} \cdot \mathrm{c}_{1} \cdot \mathrm{c}_{2}$ en la que $\mathrm{k}$ viene expresada en $\mathrm{m}^{3} / \mathrm{kmol} \cdot \mathrm{h}$, valiendo 1.035 para una temperatura de $300 \mathrm{~K}$, varía con la temperatura según la ley de Arrhenius siendo su energía de activación de $10000 \mathrm{~kJ} / \mathrm{kmol}$.

El calor de reacción a $300 \mathrm{~K}$ vale $41860 \mathrm{~kJ} / \mathrm{kmol}$ de $\mathrm{A}_{1}$ y los calores específicos molares medios, para el intervalo de temperaturas del problema, puede considerarse que tienen los valores siguientes:

$$
\overline{\mathrm{C}}_{\mathrm{p} 1}=\overline{\mathrm{C}}_{\mathrm{p} 2}=83.7 \mathrm{~kJ} / \mathrm{kmol} \cdot \mathrm{K} \quad \overline{\mathrm{C}}_{\mathrm{p} 3}=167.4 \mathrm{~kJ} / \mathrm{kmol} \cdot \mathrm{K}
$$


El área de la superficie de transmisión de calor es de $6 \mathrm{~m}^{2}$ y el coeficiente global de transmisión de calor desde el fluido calefactor a la mezcla reactante vale $15070 \mathrm{~kJ} / \mathrm{h} \cdot \mathrm{m}^{2} \cdot \mathrm{K}$. El calor latente de vaporización del agua (en $\mathrm{kcal} / \mathrm{kg}$ ) en función de la temperatura (en grados Celsius) se puede expresar como $\mathrm{H}_{\mathrm{v}}=606.5-0.695 \cdot \mathrm{T}$

Problema 03. En un RCTA con un volumen útil de $10^{-3} \mathrm{~m}^{3}$ se desarrolla la reacción irreversible en fase líquida y de segundo orden $A_{1}+A_{2} \rightarrow 2 A_{3}$. El alimento se introduce con un caudal de $6 \cdot 10^{-3} \mathrm{~m}^{3} / \mathrm{h}$ y está constituido por una mezcla equimolecular de $\mathrm{A}_{1} \mathrm{y} \mathrm{A}_{2}$, en la que dichas especies están presentes con una concentración de $6.67 \mathrm{kmol} / \mathrm{m}^{3}$. Si la temperatura del alimento es de $42.4{ }^{\circ} \mathrm{C}$ y el reactor está rodeado por una camisa de refrigeración que se mantiene a una temperatura prácticamente uniforme e igual a $87^{\circ} \mathrm{C}$ :

a) Encontrar los posibles estados estacionarios indicando su estabilidad o inestabilidad. Resolver utilizando los métodos de Levenspiel y Aris, y comparar los resultados obtenidos.

b) Estimar la temperatura de encendido de la reacción en el supuesto que la temperatura de entrada del alimento se fuera aumentando lentamente.

\section{Datos y notas}

La constante de velocidad de reacción se puede estimar como:

$$
\mathrm{k}=5.5 \cdot 10^{7} \cdot \exp \left(\frac{-10000}{\mathrm{~T}}\right) \mathrm{m}^{3} / \mathrm{kmol} \cdot \mathrm{s}
$$

El calor de reacción vale $-80 \mathrm{~kJ} / \mathrm{mol}$ de $\mathrm{A}_{1} \mathrm{y}$ el calor específico medio de la mezcla alimento es $2.72 \cdot 10^{3} \mathrm{~kJ} / \mathrm{m}^{3} \cdot \mathrm{K}$

El área de la superficie de transmisión de calor es de $2.5 \cdot 10^{-2} \mathrm{~m}^{2} \mathrm{y}$ el coeficiente global de transmisión de calor vale $69.8 \mathrm{~W} / \mathrm{m}^{2} \cdot \mathrm{K}$

Problema 04. Una mezcla que contiene dos reactantes $\mathrm{A}_{1}$ y $\mathrm{A}_{2}$ en igual concentración $\left(6.67 \mathrm{kmol} / \mathrm{m}^{3}\right)$ se alimenta a un RCTA, adiabático, donde reacciona a $120{ }^{\circ} \mathrm{C}$, con el esquema $A_{1}+A_{2} \rightarrow A_{3}$. La reacción es irreversible, de segundo orden y su

constante de velocidad vale $\mathrm{k}=5.5 \cdot 10^{7} \cdot \exp \left(\frac{-10000}{\mathrm{~T}}\right) \mathrm{m}^{3} / \mathrm{kmol} \cdot \mathrm{s}$

¿Cuál debe ser el caudal volumétrico y la temperatura de entrada del alimento si el reactor tiene un volumen de un litro y se desea alcanzar un grado de conversión de 0.5? Discutir la estabilidad del estado estacionario.

\section{Datos y notas}

El calor de reacción vale $-80 \mathrm{~kJ} / \mathrm{mol}$ de $\mathrm{A}_{1} \mathrm{y}$ el calor específico medio de la mezcla alimento es $2.72 \cdot 10^{3} \mathrm{~kJ} / \mathrm{m}^{3} \cdot \mathrm{K}$. 
Problema 05. La reacción homogénea en fase líquida irreversible y de primer orden $\mathrm{A}_{1} \rightarrow$ productos se quiere desarrollar en un RCTA adiabático. La concentración de $\mathrm{A}_{1}$ en el alimento es de $3 \mathrm{kmol} / \mathrm{m}^{3}$ y se introduce a la temperatura de $298 \mathrm{~K}$ y con un caudal volumétrico de $6 \cdot 10^{-5} \mathrm{~m}^{3} / \mathrm{s}$ en un reactor de $18 \cdot 10^{-3} \mathrm{~m}^{3}$. La densidad de la mezcla reactante es $1000 \mathrm{~kg} / \mathrm{m}^{3}$.

La constante de velocidad vale $\mathrm{k}=4.48 \cdot 10^{6} \cdot \exp \left(\frac{-7500}{\mathrm{~T}}\right) \mathrm{s}^{-1}$

a) Determinar los valores de $\mathrm{X}$ y $\mathrm{T}$ de todos los estados estacionarios posibles y discútase su estabilidad.

b) Calcular la temperatura a la que sería conveniente introducir el alimento para obtener un único estado estacionario con un grado de conversión elevado.

\section{Datos y notas}

El calor de reacción vale $-209000 \mathrm{~kJ} / \mathrm{kmol}$ de $\mathrm{A}_{1}$ y el calor específico medio de la mezcla alimento es $4.16 \mathrm{~kJ} / \mathrm{kg} \cdot \mathrm{K}$

Problema 06. En un reactor cuyo comportamiento fluidodinámico puede asimilarse a un RCTA se desarrolla la reacción gaseosa reversible y de primer orden $\mathrm{A}_{1} \leftrightarrow \mathrm{A}_{2}$. Operando a $300 \mathrm{~K}$ el volumen de reacción necesario para obtener una conversión de 0.6 es de $0.1 \mathrm{~m}^{3}$.

a) Utilizando el mismo caudal volumétrico de alimento calcular el volumen del reactor necesario para obtener el mismo grado de conversión si la temperatura de operación es de $400 \mathrm{~K}$.

b) Calcular la temperatura de operación que hará mínimo el volumen del reactor, para el caudal volumétrico y grado de conversión anteriores.

\section{Datos y notas}

La constante de velocidad de la reacción directa vale:

$$
\mathrm{k}=10^{3} \cdot \exp \left(\frac{-2400}{\mathrm{~T}}\right) \mathrm{s}^{-1}
$$

A $300 \mathrm{~K}$ la constante de equilibrio vale 10 .

El calor de reacción a $300 \mathrm{~K}$ vale $-33490 \mathrm{~J} / \mathrm{mol}$ de $\mathrm{A}_{1}$ y el calor específico molar de las dos especies que participan en la reacción puede admitirse que tiene el mismo valor para cualquier temperatura.

Admítase que $\mathrm{T}_{0}=\mathrm{T}$ en todos los casos considerados. 
Problema 07. En un RCTA se está desarrollando en fase líquida la reacción irreversible de primer orden $A_{1} \rightarrow A_{2}$. El reactor, de $10 \mathrm{~m}^{3}$ de volumen, funciona en régimen estacionario y el alimento (exento de $\mathrm{A}_{2}$ ) se introduce con un caudal volumétrico de $10^{-2} \mathrm{~m}^{3} / \mathrm{s}$ y con una concentración de $5 \mathrm{kmol}$ de $\mathrm{A}_{1} / \mathrm{m}^{3}$, siendo la densidad de la solución de $850 \mathrm{~kg} / \mathrm{m}^{3}$.

a) Para evitar que tenga lugar una reacción secundaria de formación de un producto indeseable se ha de procurar que la temperatura de reacción no supere los $340 \mathrm{~K}$. Demostrar que si el alimento se introduce a $310 \mathrm{~K}$ y se monta un serpentín refrigerante, manteniendo la temperatura del fluido refrigerante al valor constante de $310 \mathrm{~K}$, se podrá alcanzar un grado de conversión de 0.8 sin que se produzca el encendido de la reacción. Calcular el valor que el producto $\mathrm{U} \cdot \mathrm{A}$ debe tener.

b) En el caso de que la temperatura del alimento se redujera a $300 \mathrm{~K}$, demostrar que, una vez alcanzado el estado estacionario, se continuaría obteniendo un grado de conversión de 0.8 si la temperatura del refrigerante se elevara hasta $331 \mathrm{~K}$ (manteniéndose prácticamente constante a lo largo de todo el serpentín). Comentar el papel que juega en este caso el serpentín de refrigeración. Supóngase que el valor del producto $\mathrm{U} \cdot \mathrm{A}$ es el obtenido en el apartado anterior.

Datos y notas

La constante de velocidad de la reacción vale $\mathrm{k}=10^{13} \cdot \exp \left(\frac{-12000}{\mathrm{~T}}\right) \mathrm{s}^{-1}$

El calor de reacción vale $-20000 \mathrm{~kJ} / \mathrm{kmol}$ de $\mathrm{A}_{1}$ y el calor específico medio de la solución vale $2.2 \mathrm{~kJ} / \mathrm{kg} \cdot \mathrm{K}$

Problema 08. La reacción entre el tiosulfato sódico y el peróxido de hidrógeno (agua oxigenada) se lleva a cabo en un RCTA adiabático. Aunque se trata de una reacción muy compleja, en las condiciones de operación elegidas se puede representar mediante el esquema $\mathrm{H}_{2} \mathrm{O}_{2}+0.5 \mathrm{Na}_{2} \mathrm{~S}_{2} \mathrm{O}_{3} \rightarrow$ productos :

a) Determinar, utilizando el método de Aris, los posibles estados estacionarios si el alimento entra al RCTA a $6{ }^{\circ} \mathrm{C}$.

b) Si la temperatura del alimento crece de forma continua y gradual hasta alcanzar los $9{ }^{\circ} \mathrm{C}$ ¿Qué sucede con los estados estacionarios? ¿Se sigue obteniendo un grado de conversión bajo, como en el caso del apartado anterior?

\section{Datos y notas}

La densidad y los calores específicos permanecen constantes durante la reacción.

$\mathrm{c}_{\text {peróxido }, 0}=1 \mathrm{kmol} / \mathrm{m}^{3} ; \mathrm{c}_{\text {tiosulfato }, 0}=1.5 \mathrm{kmol} / \mathrm{m}^{3}$ 
$\Delta \mathrm{H}^{0}=-293854 \mathrm{~kJ} / \mathrm{kmol}$ de peróxido; calor específico del alimento $=$ $=5141.4 \mathrm{~kJ} / \mathrm{K} \times \mathrm{kmol}$ de peróxido

$\mathrm{t}=15 \mathrm{~s}$

$$
r=6.81 \cdot 10^{11} \cdot c_{1} \cdot c_{2} \cdot \exp \left[-\frac{9260}{T}\right] \quad(\mathrm{mol} / \ell \cdot \mathrm{s})
$$

Problema 09. El propilenglicol se obtiene por hidrólisis del óxido de propileno (OP), en presencia de ácido sulfúrico, según la reacción:

$$
\mathrm{C}_{3} \mathrm{H}_{6} \mathrm{O}+\mathrm{H}_{2} \mathrm{O} \stackrel{\mathrm{H}_{3} \mathrm{SO}_{9}}{\longrightarrow} \mathrm{CH}_{2} \mathrm{OH}-\mathrm{CHOH}-\mathrm{CH}_{3}
$$

que se desarrolla a temperaturas próximas a la ambiental cuando se utiliza ácido sulfúrico como catalizador.

Un ingeniero químico está encargado de supervisar el funcionamiento de un RCTA adiabático en el que se produce a escala industrial propilenglicol por el método descrito. Desgraciadamente el reactor está comenzando a deteriorarse, puesto que el ácido sulfúrico ataca al acero con el que está construido, y hay que reemplazarlo por uno nuevo. Buscando en el almacén el ingeniero químico encuentra un RCTA de $1.14 \mathrm{~m}^{3}$ de volumen útil (con un revestimiento interior de vidrio que impedirá que el ácido sulfúrico ataque al acero con el que está construido el reactor) que no se usa y que se podría estudiar su utilización para producir el propilenglicol.

En la instalación actual se están alimentando $19.54 \mathrm{kmol} / \mathrm{h}$ de OP. El alimento consta de dos corrientes:

Corriente A: una mezcla equivolumétrica de OP $\left(1.32 \mathrm{~m}^{3} / \mathrm{h}\right)$ y de metanol $(1.32$ $\left.\mathrm{m}^{3} / \mathrm{h}\right)$.

Corriente B: una corriente de agua que contiene un $0.1 \%$ de ácido sulfúrico, siendo su caudal volumétrico $6.6 \mathrm{~m}^{3} / \mathrm{h}$.

El caudal molar de alimentación del metanol es $32.63 \mathrm{kmol} / \mathrm{h}$ y el del agua 364.5 $\mathrm{kmol} / \mathrm{h}$. La temperatura de las dos corrientes es $14.5^{\circ} \mathrm{C}$, antes de mezclarlas, pero hay un incremento instantáneo de $9.5^{\circ} \mathrm{C}$ en la corriente que resulta debido al elevado calor de mezcla. En consecuencia la temperatura de entrada del alimento al reactor es $24{ }^{\circ} \mathrm{C}$.

Furosawa estableció que en condiciones parecidas a las que opera el RCTA, la reacción es de primer orden respecto a la concentración de OP y de orden aparente cero respecto al agua que se utiliza en exceso. La variación de la constante cinética con la temperatura es de la forma:

$$
\mathrm{k}=16.96 \cdot 10^{12} \cdot \exp [-75362 /(\mathrm{R} \cdot \mathrm{T})]\left(\mathrm{h}^{-1}\right)
$$

viniendo la energía de activación expresada en $\mathrm{J} / \mathrm{mol}$. 
En la operación hay una importante restricción: el OP es una sustancia de bajo punto de ebullición (a $1 \mathrm{~atm}$. abs. hierve a $34.3^{\circ} \mathrm{C}$ ). Por lo tanto, con la mezcla que se está utilizando, el reactor no debe sobrepasar los $57^{\circ} \mathrm{C}$ para evitar las pérdidas de OP a través del sistema de ventilación del reactor.

a) ¿Puede utilizarse el RCTA del almacén para sustituir el que se está deteriorando, funcionando de forma adiabática? Si se puede emplear, ¿cuál será la conversión de $\mathrm{OP}$ en propilenglicol en estado estacionario?

b) En el mismo almacén se encuentra también un serpentín en desuso que tiene un área útil de transmisión de calor de $5 \mathrm{~m}^{2}$. Si el agua de refrigeración que se utiliza circula a través del serpentín con un caudal suficientemente grande para admitir que su temperatura permanece constante e igual a $29.5^{\circ} \mathrm{C}$, y para este caudal y las condiciones del reactor se sabe que el coeficiente global de transmisión de calor vale $1500 \mathrm{~kJ} / \mathrm{h} \cdot \mathrm{m}^{2} \cdot \mathrm{K}$, ¿podría instalarse este serpentín en el reactor del almacén para llevar a cabo la reacción, con las restricciones antes citadas, en el caso de que se incremente a $27^{\circ} \mathrm{C}$ la temperatura de entrada del alimento? ¿Qué conversión y temperatura de estado estacionario resultarían?

\section{Datos y notas}

Entalpía de reacción $=-85300 \mathrm{~kJ} / \mathrm{kmol}$ de OP

\begin{tabular}{|c|c|c|c|}
\hline Especie & $\rho\left(\mathrm{kg} / \mathrm{m}^{3}\right)$ & $\mathrm{C}_{\mathrm{p}}(\mathrm{J} / \mathrm{mol} \cdot \mathrm{K})$ & $\mathrm{M}(\mathrm{g} / \mathrm{mol})$ \\
\hline OP & 859 & 146.5 & 58.1 \\
\hline Agua & 994 & 75.35 & 18 \\
\hline Propilenglicol & 1036 & 192.6 & 76.1 \\
\hline Metanol & 791 & 81.63 & 32 \\
\hline
\end{tabular}

Problema 10. Una reacción exotérmica, de primer orden, en fase líquida, de la forma $A_{1} \rightarrow$ productos, tiene lugar en un RCTA de $4 \mathrm{~m}^{3}$. Los parámetros de la ecuación de Arrhenius para este sistema son $\mathrm{A}=2 \cdot 10^{13} \mathrm{~s}^{-1} \mathrm{y} \mathrm{E}=100000 \mathrm{~kJ} / \mathrm{kmol}$. La entalpía es $-50000 \mathrm{~kJ} / \mathrm{kmol}$ y el calor específico de la mezcla de reacción $4 \mathrm{~kJ} / \mathrm{kg} \cdot \mathrm{K}$. La densidad media de la mezcla de reacción es $1000 \mathrm{~kg} / \mathrm{m}^{3}$.

a) Si la concentración del alimento es $4 \mathrm{kmol} / \mathrm{m}^{3}$, el caudal $0.005 \mathrm{~m}^{3} / \mathrm{s}$, y el RCTA opera de forma adiabática, calcular los estados estacionarios cuando la temperatura del alimento es 290, 297 y $305 \mathrm{~K}$.

b) ¿Cuál es el mínimo valor de la temperatura del alimento, en condiciones adiabáticas, y cuales son los valores de X y T del estado estacionario que le corresponde?

c) Si la temperatura del alimento es $297 \mathrm{~K}$, se desea obtener un grado de conversión de 0.932 y que únicamente exista un estado estacionario, es necesario recurrir a una RCTA con intercambio de calor. Calcular el valor de U · A del sistema de intercambio de calor si se dispone de un fluido intercambiador que se encuentra a $360 \mathrm{~K}$ y puede admitirse que su temperatura permanece prácticamente constante durante la operación de intercambio. 
Problema 11. Una reacción endotérmica, de primer orden, en fase líquida, de la forma $A_{1} \rightarrow$ productos tiene lugar en un RCTA. La concentración del alimento es 1.25 $\mathrm{kmol} / \mathrm{m}^{3}$, el caudal de alimentación $0.02 \mathrm{~m}^{3} / \mathrm{s}$, y el volumen del reactor es $20 \mathrm{~m}^{3}$. La entalpía de la reacción es $50000 \mathrm{~kJ} / \mathrm{kmol}$ de A, el calor específico de la mezcla de reacción es $3 \mathrm{~kJ} / \mathrm{kg} \cdot \mathrm{K}$ y su densidad $900 \mathrm{~kg} / \mathrm{m}^{3}$.

a) Si la temperatura del alimento es de $300 \mathrm{~K}$, calcular la temperatura de un fluido intercambiador de calor que circula por el interior de un serpentín, para que la temperatura de reacción coincida con la temperatura del alimento. ¿Qué grado de conversión se obtendrá?

b) Si no existiera el serpentín y el RCTA fuera adiabático, ¿cuál debería ser la temperatura del alimento para obtener el mismo resultado?

\section{Datos y notas}

A $300 \mathrm{~K}$ la constante de velocidad es $\mathrm{k}=4 \cdot 10^{-3} \mathrm{~s}^{-1}$

Para el serpentín puede asumirse que $\mathrm{U} \cdot \mathrm{A}=10 \mathrm{~kJ} / \mathrm{s} \cdot \mathrm{K}$ y que la temperatura del fluido intercambiador permanece prácticamente constante durante el proceso de intercambio.

Problema 12. La reacción de primer orden $A_{1} \rightarrow A_{2}$ se desarrolla en un RCTA adiabático. Determinar los posibles estados estacionarios (como parejas temperatura - grado de conversión) para las siguientes condiciones de operación:

$$
\begin{aligned}
& \rho_{\text {media }}=1000 \mathrm{~kg} / \mathrm{m}^{3} ; \mathrm{c}_{10}=3 \mathrm{kmol} / \mathrm{m}^{3} ; \mathrm{T}_{0}=298 \mathrm{~K} ; \mathrm{Q}_{\mathrm{vo}}=6 \cdot 10^{-5} \mathrm{~m}^{3} / \mathrm{s} \\
& \mathrm{V}=0.018 \mathrm{~m}^{3} \\
& \mathrm{r}=\mathrm{k} \cdot \mathrm{c}_{1} ; \mathrm{k}=\mathrm{e}^{\left[15.32-\frac{7550}{\mathrm{~T}}\right]} \mathrm{s}^{-1} \\
& \Delta \mathrm{H}_{1}^{0}=-50000 \mathrm{kcal} / \mathrm{kmol} ; \mathrm{C}_{\mathrm{p}, \text { media }}=1 \mathrm{kcal} / \mathrm{kg} \cdot \mathrm{K}
\end{aligned}
$$

Problema 13. La reacción endotérmica, en fase líquida, de primer orden e irreversible $A_{1} \rightarrow A_{2}+A_{3}$ tiene lugar en un RCTA que opera en estado estacionario. Si la temperatura del alimento es $310 \mathrm{~K}$, calcular la temperatura a la que se debe mantener el fluido calefactor, que circula por un serpentín, para conseguir una conversión de 0.75 .

\section{Datos y notas}

$$
\begin{aligned}
& \rho_{\text {fase líquida }}=950 \mathrm{~kg} / \mathrm{m}^{3} ; \mathrm{c}_{10}=0.8 \mathrm{kmol} / \mathrm{m}^{3} ; \mathrm{Q}_{\mathrm{V} 0}=8.3 \cdot 10^{-3} \mathrm{~m}^{3} / \mathrm{s} \\
& \mathrm{V}=15 \mathrm{~m}^{3} \\
& \mathrm{~A}=3.5 \cdot 10^{13} \mathrm{~s}^{-1} ; \mathrm{E}=100000 \mathrm{~kJ} / \mathrm{kmol} \\
& \Delta \mathrm{H}_{\mathrm{k}}{ }^{0}=51000 \mathrm{~kJ} / \mathrm{kmol} ; \mathrm{C}_{\mathrm{p} \text {,fase líquida }}=3.5 \mathrm{~kJ} / \mathrm{kg} \cdot \mathrm{K} \\
& \mathrm{U} \cdot \mathrm{A}=10 \mathrm{~kJ} / \mathrm{s} \cdot \mathrm{K}
\end{aligned}
$$


Problema 14. Se desea polimerizar estireno en un RCTA adiabático. La reacción de polimerización es irreversible y de primer orden. En un experimento de laboratorio se ha encontrado que el incremento de temperatura cuando el alimento se convierte totalmente es de $400 \mathrm{~K}$.

a) Determinar la temperatura y el grado de conversión a la salida del reactor.

b) Si la temperatura máxima permisible es de $540 \mathrm{~K}$, ¿resulta adecuada la operación adiabática?

c) Si la respuesta al apartado anterior es negativa, ¿qué prestaciones debería tener el equipo de refrigeración y a qué condiciones de temperatura y grado de conversión trabajaría el reactor? Discutir la estabilidad de dichas condiciones.

\section{Datos y notas}

$$
\mathrm{T}_{0}=300 \mathrm{~K} ; \mathrm{t}=2 \mathrm{~h} ; \mathrm{k}=10^{10} \cdot \exp \left[-\frac{10000}{\mathrm{~T}}\right]\left(\mathrm{h}^{-1}\right)
$$

Problema 15. En un RCTA con intercambio de calor se llevan a cabo en fase líquida dos reacciones consecutivas, irreversibles y de primer orden, que responden a los esquemas de reacción:

$$
A_{1} \stackrel{i=1}{\longrightarrow} A_{2} \stackrel{i=2}{\longrightarrow} A_{3} .
$$

El tiempo espacial del RCTA es de 10 minutos. Se alimenta A puro a $273 \mathrm{~K}$.

a) Determinar el número de estados estacionarios posibles, calculando los valores de $\mathrm{c}_{\mathrm{j}} \mathrm{y} \mathrm{T}$ e indicando si son estables o inestables.

b) Proponer unas condiciones de trabajo que permitan aumentar la selectividad de $\mathrm{A}_{2}$. Justificarlo gráficamente.

c) Proponer unas condiciones de trabajo que permitan aumentar la selectividad de $\mathrm{A}_{3}$. Justificarlo gráficamente.

\section{Datos y notas}

$\Delta \mathrm{H}^{0}{ }_{1}=-41840 \mathrm{~kJ} / \mathrm{kmol} ; \Delta \mathrm{H}^{0}{ }_{2}=-50208 \mathrm{~kJ} / \mathrm{kmol} ; \mathrm{C}_{\mathrm{pAl}}=12.55 \mathrm{~kJ} / \mathrm{kmol} \cdot \mathrm{K}$

$\mathrm{k}_{1}=2.5 \cdot 10^{9} \cdot \exp [-8000 / \mathrm{T}] \mathrm{min}^{-1} ; \mathrm{k}_{2}=2 \cdot 10^{8} \cdot \exp [-11000 / \mathrm{T}] \mathrm{min}^{-1}$

$\mathrm{T}_{\mathrm{f} 0}=273 \mathrm{~K} ; \kappa=20$

Problema 16. En un RCTA adiabático se lleva a cabo una reacción irreversible, de primer orden, en fase líquida, de la forma: $\mathrm{A}_{1} \rightarrow$ Productos .

a) Determinar la temperatura del alimento $\left(\mathrm{T}_{0}\right)$ en que se produce el encendido cuando el caudal volumétrico de alimentación es 0.1 1/min, 10 l/min y $100 \mathrm{l} /$ min. Comentar el resultado. 
b) Si la temperatura inferior a la que se puede se puede introducir el alimento es $15^{\circ} \mathrm{C}$, calcular el caudal máximo del alimento para el que se puede conseguir el apagado del reactor por descenso de la temperatura de alimentación. Construir la curva de $\mathrm{Q}_{\mathrm{V} 0}=\mathrm{f}\left(\mathrm{T}_{\mathrm{EE}}\right)$ para la temperatura de alimento de $15^{\circ} \mathrm{C}$.

\section{Datos y notas}

Volumen de reacción $=1 \mathrm{~m}^{3}$

Entalpía de reacción $=-52300 \mathrm{~kJ} / \mathrm{kmol}$ de $\mathrm{A} ; \sum_{\mathrm{j}=1}^{\mathrm{j}=\mathrm{s}} \theta_{\mathrm{j}} \cdot \overline{\mathrm{C}}_{\mathrm{pj}}=514.6 \mathrm{~kJ} / \mathrm{kmol} \cdot \mathrm{K}$
$\mathrm{E} / \mathrm{R}=13200 \mathrm{~K} ; \mathrm{A}=7.1 \cdot 10^{12} \mathrm{~min}^{-1}$

Problema 17. La reacción en fase líquida, exotérmica, irreversible y de orden cero $\mathrm{A}_{1} \rightarrow \mathrm{A}_{2}$ tiene lugar a $85^{\circ} \mathrm{C}$ en un RCTA de $0.2 \mathrm{~m}^{3}$ provisto de una camisa de intercambio de calor. La temperatura del fluido refrigerante se mantiene constante a $0{ }^{\circ} \mathrm{C}$. El coeficiente de transmisión de calor es $120 \mathrm{~W} / \mathrm{m}^{2} \cdot \mathrm{K}$. Determinar:

a) El grado de conversión alcanzado

b) El área de transmisión de calor

c) Haciendo uso de las funciones $\mathrm{Q}_{\mathrm{G}} \mathrm{y} \mathrm{Q}_{\mathrm{e}}$, $\mathrm{y}$ de su representación, indicar qué sucedería si:

c1) si aumentara el área de transmisión de calor

c2) si disminuyera el área de transmisión de calor

\section{Datos y notas}

La densidad de la solución es $0.90 \mathrm{~kg} / \mathrm{dm}^{3}$

La temperatura del alimento es $40{ }^{\circ} \mathrm{C}$ y el caudal de alimentación $90 \mathrm{~kg} / \mathrm{min}$

El peso molecular de $\mathrm{A}_{1}$ es $90 \mathrm{~g} / \mathrm{mol}$

$\mathrm{k}=1.127 \mathrm{kmol} / \mathrm{m}^{3} \cdot \min$ a $40{ }^{\circ} \mathrm{C} ; \mathrm{k}=1.421 \mathrm{kmol} / \mathrm{m}^{3} \cdot \min$ a $50{ }^{\circ} \mathrm{C}$

El calor de reacción es $-250 \mathrm{~J} / \mathrm{g}$ y el calor específico de la solución es 2

$\mathrm{J} / \mathrm{g} \cdot \mathrm{K}$

Problema 18. En un RCTA se han de desarrollar las reacciones elementales $A_{1} \stackrel{k_{1}}{\longrightarrow} A_{2} \stackrel{k_{2}}{\longrightarrow} A_{3}$.

Calcular gráficamente la temperatura de estado estacionario del reactor, el volumen del reactor y el área de intercambio de calor del serpentín, para alcanzar una conversión del 0.5 del $\mathrm{A}_{1}$ alimentado y una relación $\mathrm{c}_{\mathrm{A} 2} / \mathrm{c}_{\mathrm{A} 3}=10$.

\section{Datos y notas}

$\mathrm{x}_{\mathrm{A} 0}=1$; Caudal volumétrico: $0.225 \mathrm{~m}^{3} / \mathrm{s}$; Caudal molar: $0.025 \mathrm{kmol} / \mathrm{s}$

Temperatura del alimento: $205^{\circ} \mathrm{C}$ 
$\mathrm{A}_{1}=5 \cdot 10^{8} \mathrm{~s}^{-1} ; \mathrm{A}_{2}=1 \cdot 10^{11} \mathrm{~s}^{-1} ; \mathrm{E}_{1}=72106 \mathrm{~kJ} / \mathrm{kmol} ; \mathrm{E}_{2}=97692 \mathrm{~kJ} / \mathrm{kmol}$

$\Delta \mathrm{H}_{01}=34890 \mathrm{~kJ} / \mathrm{kmol} ; \Delta \mathrm{H}_{02}=46520 \mathrm{~kJ} / \mathrm{kmol}$

Calor específico de todas las especies químicas: $105 \mathrm{~kJ} / \mathrm{kmol} \cdot \mathrm{K}$

El fluido calefactor es vapor de agua saturado a $177^{\circ} \mathrm{C}$

Coeficiente global de transmisión de calor: $142 \mathrm{~W} / \mathrm{K} \cdot \mathrm{m}^{2}$

Problema 19. En un RCTA se va a llevar a cabo la reacción en fase líquida $\mathrm{A}_{1}(\ell) \leftrightarrow \mathrm{A}_{2}(\ell)$. El alimento se introduce en el reactor a $375 \mathrm{~K}$, con un cauda mola de $0.0126 \mathrm{kmol} / \mathrm{min}$ y una concentración de $1.73 \mathrm{kmol}$ de $\mathrm{A}_{1} / \mathrm{m}^{3}$. El volumen de reacción es de $0.667 \mathrm{~m}^{3}$.

El reactor intercambia calor con los alrededores, cuya temperatura puede oscilar entre $10,20,30$ y $40^{\circ} \mathrm{C}$ de invierno a verano. Calcular la influencia de la temperatura exterior sobre el grado de conversión obtenido y la máxima disminución que puede experimentar ese grado de conversión al pasar del invierno al verano.

\section{Datos y notas}

$$
\begin{aligned}
& \Delta \mathrm{H}_{\mathrm{k}}^{\mathrm{o}}=-83620 \mathrm{~kJ} / \mathrm{kmol} ; \theta_{1} \cdot \overline{\mathrm{C}}_{\mathrm{p} 1}=41.81 \mathrm{~kJ} / \mathrm{kmol} \cdot \mathrm{K} \\
& \mathrm{r}=\mathrm{k} \cdot \mathrm{c}_{1}-\mathrm{k}^{\prime} \cdot \mathrm{c}_{2}=3 \cdot 10^{13} \cdot \exp \left[-\frac{15000}{\mathrm{~T}}\right] \cdot \mathrm{c}_{10} \cdot(1-\mathrm{X})-5.44 \cdot 10^{23} \cdot \exp \left[-\frac{25050.5}{\mathrm{~T}}\right] \cdot \mathrm{c}_{10} \cdot \mathrm{X} \quad \mathrm{kmol} / \mathrm{m}^{3} \cdot \min \\
& \mathrm{U} \cdot \mathrm{A}=2.09 \mathrm{~kJ} / \mathrm{min} \cdot \mathrm{K}
\end{aligned}
$$

Problema 20. En un RCTA de $1 \mathrm{~m}^{3}$ se va a realizar la reacción: $\mathrm{A}_{1}(\ell) \rightarrow$ productos $(\ell)$. El caudal volumétrico del alimento puede oscilar entre los valores $0.11 / \mathrm{min} \leq \mathrm{Q}_{\mathrm{V} 0}$ $\leq 100 \mathrm{l} / \mathrm{min}$. El RCTA intercambia calor con el aire que lo rodea, que se encuentra a $273 \mathrm{~K}$, siendo $\mathrm{U} \cdot \mathrm{A}=257.3 \mathrm{~J} / \mathrm{min} \cdot \mathrm{K}$.

a) calcular la evolución de $\mathrm{T}_{0}$, a la que se produce el encendido, con $\mathrm{Q}_{\mathrm{V} 0}$. Determinar la pareja de valores $(\mathrm{X}, \mathrm{T})$ correspondientes a los estados estacionarios estable e inestable.

b) si $_{0}=320 \mathrm{~K}$, obtener la variación de las temperaturas correspondientes a los estados estacionarios con $\mathrm{Q}_{\mathrm{V} 0}$

\section{Datos y notas}

$$
\begin{aligned}
& \mathrm{c}_{10}=1 \mathrm{kmol} / \mathrm{m}^{3} \\
& \Delta \mathrm{H}_{\mathrm{k}}^{\mathrm{o}}=-52300 \mathrm{~kJ} / \mathrm{kmol} ; \theta_{1} \cdot \overline{\mathrm{C}}_{\mathrm{p} 1}=514.6 \mathrm{~kJ} / \mathrm{kmol} \cdot \mathrm{K} \\
& \mathrm{r}=\mathrm{k} \cdot \mathrm{c}_{1}=7.1 \cdot 10^{12} \cdot \exp \left[-\frac{13200}{\mathrm{~T}}\right] \cdot \mathrm{c}_{10} \cdot(1-\mathrm{X}) \quad \mathrm{kmol} / \mathrm{m}^{3} \cdot \mathrm{min}
\end{aligned}
$$




\section{Reactor discontinuo de tanque agitado (RDTA) intermedio y adiabático}

\subsection{Ecuaciones de diseño}

En este capítulo se tratarán los sistemas constituidos por un reactor discontinuo de tanque agitado (RDTA) en el que fundamentalmente tiene lugar una única reacción química. En consecuencia, tal y como se recogía en Anexo II del capítulo 1, el balance de materia se puede expresar mediante el conjunto de expresiones recogidas en la tabla 1, que dependen de la variable de composición que se utilice.

Tabla 1. Balances de materia para un reactor discontinuo de tanque agitado

\begin{tabular}{|c|c|c|c|}
\hline \multirow{2}{*}{$\begin{array}{l}\text { Variable de } \\
\text { composición }\end{array}$} & \multicolumn{3}{|c|}{ Sistemas con una reacción química } \\
\hline & Ecuación general & Ecuación a V constante & Ecuación a V variable \\
\hline$c_{j}$ & $\frac{d\left(c_{j} \cdot V\right)}{d t}=v_{j} \cdot r \cdot V$ & $d t=\frac{d c_{j}}{v_{j} \cdot r}$ & $d t=\frac{d c_{j}}{v_{j} \cdot r}+c_{j} \cdot \frac{d \ln V}{v_{j} \cdot r}$ \\
\hline $\mathrm{X}$ & $\mathrm{dt}=\frac{\mathrm{N}_{\mathrm{k} 0}}{\mathrm{~V}} \cdot \frac{\mathrm{dX}}{\left(-\mathrm{v}_{\mathrm{k}}\right) \cdot \mathrm{r}}$ & $\mathrm{dt}=\frac{\mathrm{N}_{\mathrm{k} 0}}{\mathrm{~V}_{0}} \cdot \frac{\mathrm{dX}}{\left(-\mathrm{v}_{\mathrm{k}}\right) \cdot \mathrm{r}}$ & $\mathrm{dt}=\frac{\mathrm{N}_{\mathrm{k} 0}}{\mathrm{~V}_{0} \cdot \frac{\mathrm{P}_{0}}{\mathrm{P}} \cdot \frac{\mathrm{T}}{\mathrm{T}_{0}} \cdot\left(1+\varepsilon_{\mathrm{k}} \cdot \mathrm{X}\right)} \cdot \frac{\mathrm{dX}}{\left(-\mathrm{v}_{\mathrm{k}}\right) \cdot \mathrm{r}}$ \\
\hline $\mathrm{n}_{\mathrm{j}}$ & $\mathrm{dt}=\frac{\mathrm{N}_{\mathrm{k} 0}}{\mathrm{~V}} \cdot \frac{\mathrm{dn}_{\mathrm{j}}}{v_{\mathrm{j}} \cdot \mathrm{r}}$ & $\mathrm{dt}=\frac{\mathrm{N}_{\mathrm{k} 0}}{\mathrm{~V}_{0}} \cdot \frac{\mathrm{dn}_{\mathrm{j}}}{\mathrm{v}_{\mathrm{j}} \cdot \mathrm{r}}$ & $\mathrm{dt}=\frac{\mathrm{N}_{\mathrm{k} 0}}{\mathrm{~V}} \cdot \frac{\mathrm{dn}_{\mathrm{j}}}{\mathrm{v}_{\mathrm{j}} \cdot \mathrm{r}}$ \\
\hline
\end{tabular}

En este capítulo se estudiará en profundidad el caso de sistemas con una única reacción química y de volumen constante (en la práctica industrial esto es lo habitual puesto que los RDTA se utilizan en sistemas de densidad constante, normalmente fases líquidas), en los que la velocidad de reacción es normal (no autocatalítica) y cuya composición se exprese utilizando el grado de conversión o la concentración. En consecuencia, las expresiones de los balances de materia que se utilizarán son: 


$$
\begin{gathered}
\mathrm{dt}=\frac{\mathrm{N}_{\mathrm{k} 0}}{\mathrm{~V}_{0}} \cdot \frac{\mathrm{dX}}{\left(-\mathrm{v}_{\mathrm{k}}\right) \cdot \mathrm{r}}=\mathrm{c}_{\mathrm{k} 0} \cdot \frac{\mathrm{dX}}{\left(-\mathrm{v}_{\mathrm{k}}\right) \cdot \mathrm{r}} \\
\mathrm{dt}=\frac{\mathrm{dc} \mathrm{c}_{\mathrm{j}}}{\mathrm{v}_{\mathrm{j}} \cdot \mathrm{r}}
\end{gathered}
$$

Para poder aplicar estas ecuaciones es necesario conocer la ecuación de la velocidad de reacción química, en función de la temperatura y de la concentración o el grado de conversión:

$$
r=r[X, T] \text { o } r=r\left[C_{j}, T\right]
$$

quedando:

$$
\begin{gathered}
d t=\frac{N_{k 0}}{V_{0}} \cdot \frac{d X}{\left(-v_{k}\right) \cdot r(X, T)}=c_{k 0} \cdot \frac{d X}{\left(-v_{k}\right) \cdot r(X, T)} \\
d t=\frac{d c_{j}}{v_{j} \cdot r(X, T)}
\end{gathered}
$$

Análogamente, en el capítulo 1 también se han mostrado los balances de energía que, para el sistema constituido por un RDTA en el que se desarrolla una única reacción química, se puede expresar mediante una de las dos ecuaciones siguientes:

$$
\begin{aligned}
& \frac{\mathrm{dT}}{\mathrm{dt}}-\mathrm{J} \cdot \frac{\mathrm{dX}}{\mathrm{dt}}=\mathrm{Q}^{\prime} \\
& \frac{\mathrm{dT}}{\mathrm{dt}}+\frac{\mathrm{v}_{\mathrm{k}} \cdot \mathrm{r}}{\mathrm{c}_{\mathrm{k} 0}} \cdot \mathrm{J}=\mathrm{Q}^{\prime}
\end{aligned}
$$

Para poder aplicar estas ecuaciones es necesario conocer la ecuación de la velocidad de transmisión de calor, cuya forma depende del sistema de intercambio de calor utilizado.

En resumen, las dos ecuaciones más habituales para el diseño de un RDTA con una reacción química son:

$$
\begin{array}{cl}
\mathrm{dt}=\mathrm{c}_{\mathrm{k} 0} \cdot \frac{\mathrm{dX}}{\left(-v_{\mathrm{k}}\right) \cdot \mathrm{r}(\mathrm{X}, \mathrm{T})} & \text { ECUACIÓN DE DISEÑO (1) } \\
\frac{\mathrm{dT}}{\mathrm{dt}}-\mathrm{J} \cdot \frac{\mathrm{dX}}{\mathrm{dt}}=\mathrm{Q}^{\prime} & \text { ECUACIÓN DE DISEÑO (2) }
\end{array}
$$

Obviamente la resolución simultánea de las ecuaciones de diseño permite calcular, entre otras variables, el tiempo de reacción (t) necesario para alcanzar un determinado grado de conversión. Posteriormente se obtiene el volumen del reactor $(\mathrm{V})$, 
para una producción dada, según se explicó en la asignatura EQ1021-Ingeniería de la Reacción Química. A continuación se resume el procedimiento:

a) $\mathrm{Q}_{\mathrm{V}}$ se definió como el volumen de mezcla (reactante y posibles disolventes inertes presentes en el reactor) a tratar por unidad de tiempo. Hay que resaltar que aunque las unidades de este parámetro son $\mathrm{m}^{3} / \mathrm{s}$ no se trata de un caudal volumétrico puesto que el sistema es discontinuo

b) $\mathrm{t}_{\mathrm{C}}, \mathrm{t}_{0}, \mathrm{t}_{\mathrm{D}} \mathrm{y} \mathrm{t}$ se definieron, respectivamente, como los tiempos de carga, de paro o limpieza, de descarga y de reacción, de modo que el tiempo total del ciclo de reacción $\left(\mathrm{t}_{\mathrm{T}}\right)$ viene dado por la expresión:

$$
\mathrm{t}_{\mathrm{T}}=\mathrm{t}_{\mathrm{C}}+\mathrm{t}_{0}+\mathrm{t}_{\mathrm{D}}+\mathrm{t}=\mathrm{t}^{\prime}+\mathrm{t} \quad(\mathrm{s} / \text { ciclo })
$$

donde t' es el tiempo de inactividad en cada ciclo.

c) el cálculo del volumen de reactor discontinuo necesario para desarrollar la reacción con la extensión y producción previstas se efectúa por la expresión:

$$
\mathrm{V}=\mathrm{Q}_{\mathrm{V}} \cdot \mathrm{t}_{\mathrm{T}}
$$

Desde un punto de vista térmico los RDTA pueden tener un comportamiento isotermo, adiabático o intermedio, manteniéndose la temperatura constante o variable con el tiempo de reacción. En la figura 1 se muestra para cada caso la evolución de la temperatura con el tiempo de reacción, teniendo en cuenta que la reacción que tiene lugar puede ser exotérmica o endotérmica.
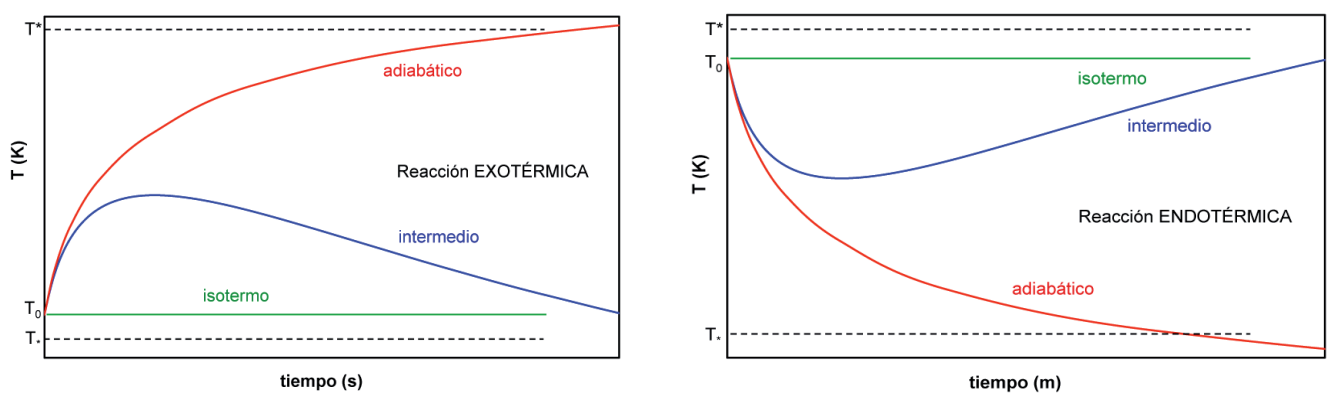

Figura 1. Evolución de la temperatura con el tiempo según el tipo de reacción y las características del reactor

\subsection{Intercambio de calor}

En la ecuación de diseño (2), que es la forma habitual del balance de energía, hay que recordar que:

$$
Q^{\prime}=\frac{Q^{*}}{N_{k 0} \cdot \sum_{j=1}^{S} \theta_{j} \cdot \bar{C}_{p j}}
$$

por lo que se debe sustituir la velocidad de transmisión de calor por la correspondiente expresión. 
Se considerarán dos tipos básicos de intercambio de calor:

a) Reactores en los que se mantiene constante la densidad de flujo de calor, $q$ en $\mathrm{J} / \mathrm{s} \cdot \mathrm{m}^{2}$ (el suministro de calor por unidad de tiempo y unidad de área de intercambio de calor), durante toda la operación:

$$
Q^{*}=\mathrm{q} \cdot \mathrm{A}
$$

b) Reactores en los que se mantiene constante el coeficiente global de transmisión de calor $U$, así como la temperatura del fluido intercambiador, durante todo el período de reacción.

$$
Q^{*}=U \cdot\left(T_{f}-T\right) \cdot A
$$

Toda reacción química va acompañada de un consumo o desprendimiento de energía, según sea endotérmica o exotérmica respectivamente. Esta circunstancia se acentúa en el caso de los reactores industriales debido a que, en ellos, se suelen tratar mezclas de concentraciones elevadas a fin de conseguir producciones altas con el menor volumen de reactor posible. De aquí que los reactores tipo tanque agitado o tipo autoclave vayan generalmente equipados con dispositivos que permitan el calentamiento o el enfriamiento de la mezcla reactante, con vistas a controlar la temperatura de la misma, durante la reacción (excepto los que operan en condiciones adiabáticas).

Para calentar la mezcla de reacción, cuando la reacción es endotérmica, se puede suministrar calor mediante dispositivos eléctricos (mantas calefactoras o resistencias sumergidas), vapor que condensa (camisas de vapor) o líquidos calientes que no cambian de fase (con intercambiadores externos o serpentines internos o externos). Los sistemas preferibles son las camisas de vapor o los serpentines externos ya que de este modo se puede utilizar, a efectos de la reacción, todo el volumen del reactor y se consigue mejor agitación.

Para enfriar la mezcla de reacción, cuando la reacción es exotérmica, se puede eliminar calor mediante líquidos que hierven (camisas externa), líquidos fríos que no cambian de fase. (con intercambiadores externos y serpentines internos o externos) o aire en radiadores externos (intercambiadores de calor con tubos provistos de aletas). Los sistema preferibles son las camisas externas o, sobre todo, los serpentines ya que se puede hacer circular el fluido refrigerante a mayor velocidad por su interior, obtener elevados coeficientes de transmisión de calor, lo que, a su vez, permite tener una superficie de transmisión de calor más pequeña que si se utiliza una camisa de refrigeración.

\subsection{Resolución de las ecuaciones de diseño para un RDTA adiabático}

El diseño de un RDTA en el que se desarrolla una única reacción química a volumen constante o el cálculo de las condiciones de funcionamiento supone resolver simultáneamente las ecuaciones de diseño (1) y (2): 


$$
\begin{array}{cl}
\mathrm{dt}=\mathrm{c}_{\mathrm{k} 0} \cdot \frac{\mathrm{dX}}{\left(-v_{\mathrm{k}}\right) \cdot \mathrm{r}(\mathrm{X}, \mathrm{T})} & \text { ECUACIÓN DE DISEÑO (1) } \\
\frac{\mathrm{dT}}{\mathrm{dt}}-\mathrm{J} \cdot \frac{\mathrm{dX}}{\mathrm{dt}}=\mathrm{Q}^{\prime} & \text { ECUACIÓN DE DISEÑO (2) }
\end{array}
$$

El procedimiento consiste en:

a) Resolver la ecuación de diseño (2), teniendo en cuenta que el sistema no intercambia calor por lo que se cumple que $Q^{*}=0=Q^{\prime}$. En consecuencia:

$$
\begin{gathered}
\frac{\mathrm{dT}}{\mathrm{dt}}-\mathrm{J} \cdot \frac{\mathrm{dX}}{\mathrm{dt}}=0 \\
\mathrm{dT}=\mathrm{J} \cdot \mathrm{dX}
\end{gathered}
$$

y esta ecuación de variables separadas se puede integrar fácilmente entre las condiciones iniciales (a tiempo cero) y las condiciones a cualquier tiempo de reacción:

$$
\begin{gathered}
\int_{\mathrm{T}_{0}}^{\mathrm{T}} \mathrm{dT}=\mathrm{J} \cdot \int_{0}^{\mathrm{X}} \mathrm{dX} \\
\mathrm{T}-\mathrm{T}_{0}=\mathrm{J} \cdot \mathrm{X}
\end{gathered}
$$

y reordenando variables, la integración de la ecuación de diseño (2) ha permitido obtener la recta adiabática mostrada en la figura 2:

$$
\begin{gathered}
\mathrm{T}=\mathrm{T}_{0}+\mathrm{J} \cdot \mathrm{X} \\
\mathrm{X}=-\frac{\mathrm{T}_{0}}{\mathrm{~J}}+\frac{1}{\mathrm{~J}} \cdot \mathrm{T}
\end{gathered}
$$

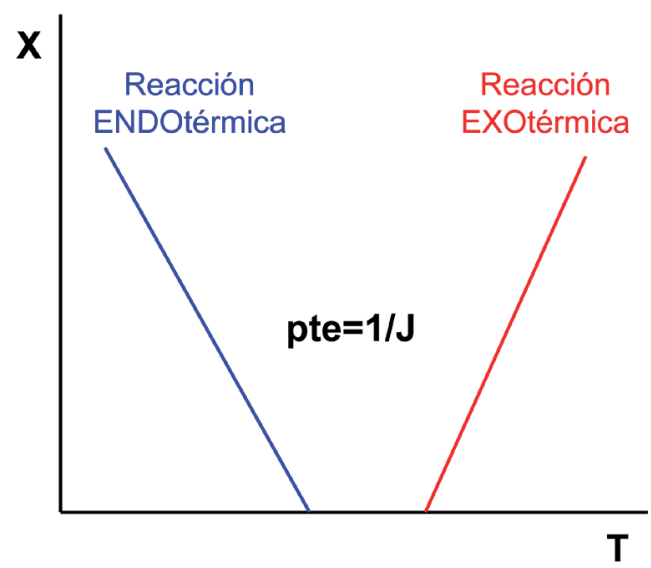

Figura 2. Recta adiabática X versus $\mathrm{T}$ 
b) Resolver la ecuación de diseño (1), teniendo en cuenta la relación entre X y T mostrada por la adiabática de conversión:

$$
\mathrm{dt}=\mathrm{c}_{\mathrm{k} 0} \cdot \frac{\mathrm{dX}}{\left(-v_{\mathrm{k}}\right) \cdot \mathrm{r}(\mathrm{X}, \mathrm{T})}=\mathrm{c}_{\mathrm{k} 0} \cdot \frac{\mathrm{dX}}{\left(-\mathrm{v}_{\mathrm{k}}\right) \cdot \mathrm{r}\left(\mathrm{X}, \mathrm{T}_{0}+\mathrm{J} \cdot \mathrm{X}\right)}
$$

de esta forma la ecuación de diseño (1) tiene variables separadas y se puede integrar, analítica o numéricamente, para calcular el tiempo de reacción:

$$
\int_{0}^{t} d t=t=c_{k 0} \cdot \int_{0}^{X} \frac{d X}{\left(-v_{k}\right) \cdot r\left(X, T_{0}+J \cdot X\right)}
$$

\subsection{Resolución de las ecuaciones de diseño para un RDTA intermedio}

Los RDTA intermedios son aquellos que no tienen ni comportamiento isotermo ni adiabático. Son consecuencia de que no están perfectamente aislados de los alrededores (por lo que no son adiabáticos), pero el calor que intercambian no es suficiente para que el reactor se mantenga isotermo. Es decir:

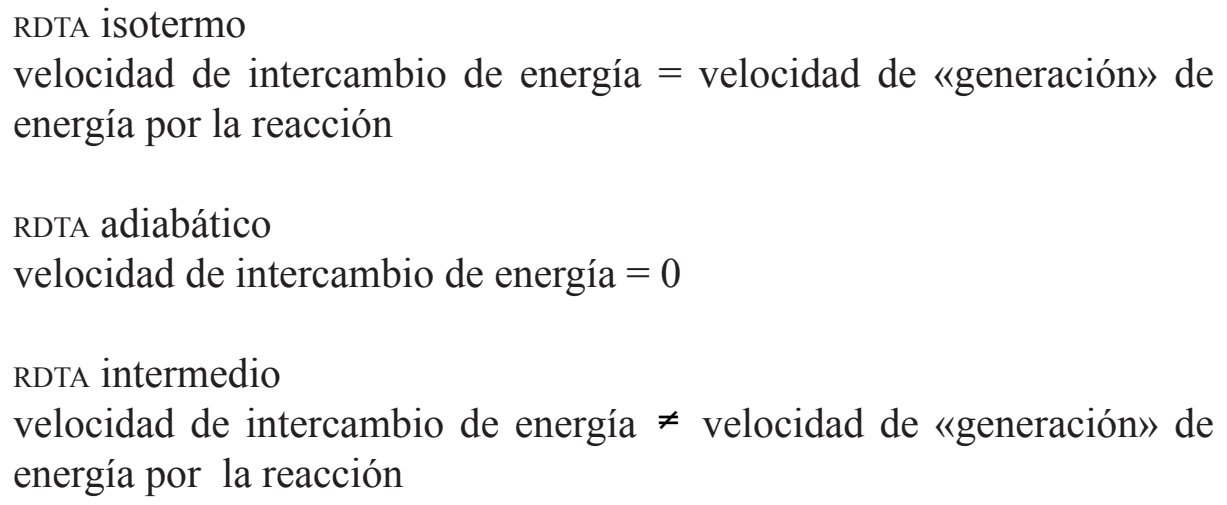

En consecuencia, el diseño de un RDTA en el que se desarrolla una única reacción química a volumen constante o el cálculo de las condiciones de funcionamiento supone resolver simultáneamente las ecuaciones de diseño (1) y (2):

$$
\begin{gathered}
d t=c_{k 0} \cdot \frac{d X}{\left(-v_{k}\right) \cdot r(X, T)} \\
\frac{d T}{d t}-J \cdot \frac{d X}{d t}=Q^{\prime}
\end{gathered}
$$

Se trata de dos ecuaciones diferenciales que hay que resolver simultáneamente, bien analíticamente o bien, el caso más habitual, numéricamente utilizando un método 
numérico como el de Runge-Kutta de $4 .^{\circ}$ orden. Para ello conviene expresar las ecuaciones como:

$$
\frac{d t}{d X}=\frac{c_{k 0}}{\left(-v_{k}\right) \cdot r(X, T)}
$$

$$
\frac{\mathrm{dT}}{\mathrm{dt}}=\mathrm{Q}^{\prime}+\mathrm{J} \cdot \frac{\mathrm{dX}}{\mathrm{dt}}
$$

Como puede observarse, este par de ecuaciones consta de una variable independiente (el tiempo $t$ ) y dos variables dependientes del tiempo (X y T). Antes de proceder a su integración simultánea es conveniente ponerlas en la forma en que las dos diferenciales estén con respecto a la misma variable: $\mathrm{dt} / \mathrm{dX}$ y dt/dT, $\mathrm{dX} / \mathrm{dt}$ y dT/dt o dt/dX y dT/dX. Cualquier pareja es buena para realizar la integración, pero la más conveniente y habitual es la que resulta de transformar la ecuación de diseño (2) de la siguiente forma:

$$
\begin{gathered}
\frac{d T}{d t}-J \cdot \frac{d X}{d t}=Q^{\prime} \\
Q^{\prime} \cdot d t=d T-J \cdot d X
\end{gathered}
$$

sustituir Q’ por su definición y dt por la ecuación de diseño (1):

$$
\frac{\mathrm{Q}^{*}}{\mathrm{~N}_{\mathrm{k} 0} \cdot \sum_{\mathrm{j}=1}^{\mathrm{S}} \theta_{\mathrm{j}} \cdot \overline{\mathrm{C}}_{\mathrm{pj}}} \cdot \mathrm{c}_{\mathrm{k} 0} \cdot \frac{\mathrm{dX}}{\left(-\mathrm{v}_{\mathrm{k}}\right) \cdot \mathrm{r}(\mathrm{X}, \mathrm{T})}=\mathrm{dT}-\mathrm{J} \cdot \mathrm{dX}
$$

y reordenando:

$$
\frac{\mathrm{dT}}{\mathrm{dX}}=\mathrm{J}+\frac{\mathrm{c}_{\mathrm{k} 0} \cdot \mathrm{Q}^{*}}{\mathrm{~N}_{\mathrm{k} 0} \cdot \sum_{\mathrm{j}=1}^{\mathrm{S}} \theta_{\mathrm{j}} \cdot \overline{\mathrm{C}}_{\mathrm{pj}} \cdot\left(-\mathrm{v}_{\mathrm{k}}\right) \cdot \mathrm{r}(\mathrm{X}, \mathrm{T})}
$$

Por lo tanto, el estudio de los RDTA intermedios requiere la integración simultánea de las ecuaciones de diseño (3) y (4):

$$
\begin{array}{cc}
\frac{\mathrm{dt}}{\mathrm{dX}}=\frac{\mathrm{c}_{\mathrm{k} 0}}{\left(-v_{\mathrm{k}}\right) \cdot \mathrm{r}(\mathrm{X}, \mathrm{T})} & \text { ECUACIÓN DE DISEÑO (3) } \\
\frac{\mathrm{dT}}{\mathrm{dX}}=\mathrm{J}+\frac{\mathrm{c}_{\mathrm{k} 0} \cdot \mathrm{Q}^{*}}{\mathrm{~N}_{\mathrm{k} 0} \cdot \sum_{\mathrm{j}=1}^{\mathrm{S}} \theta_{\mathrm{j}} \cdot \overline{\mathrm{C}}_{\mathrm{pj}} \cdot\left(-v_{\mathrm{k}}\right) \cdot \mathrm{r}(\mathrm{X}, \mathrm{T})} & \text { ECUACIÓN DE DISEÑO (4) }
\end{array}
$$

en las que el valor de Q* dependerá del sistema de intercambio de calor utilizado. 


\subsubsection{RDTA intermedio a densidad de flujo de calor $q$ constante}

Este tipo de intercambio de calor solo sirve para el caso de que se esté desarrollando una reacción endotérmica. La ecuación de velocidad de transmisión de calor es:

$$
Q^{*}=q \cdot A
$$

por lo que las ecuaciones de diseño (3) y (4) quedan como:

$$
\begin{gathered}
\frac{d t}{d X}=\frac{c_{k 0}}{\left(-v_{k}\right) \cdot r(X, T)} \\
\frac{d T}{d X}=J+\frac{c_{k 0} \cdot q \cdot A}{N_{k 0} \cdot \sum_{j=1}^{S} \theta_{j} \cdot \bar{C}_{p j} \cdot\left(-v_{k}\right) \cdot r(X, T)}
\end{gathered}
$$

Estas dos ecuaciones se pueden integrar simultáneamente conocidos los valores iniciales de las variables, habitualmente: $\mathrm{t}=0, \mathrm{X}=0 \mathrm{y} \mathrm{T}=\mathrm{T}_{0}$. La integración numérica permite la obtención de una tabla de valores $t, X, T$, cuyas representaciones gráficas son de la forma que se muestra en la figura 3.
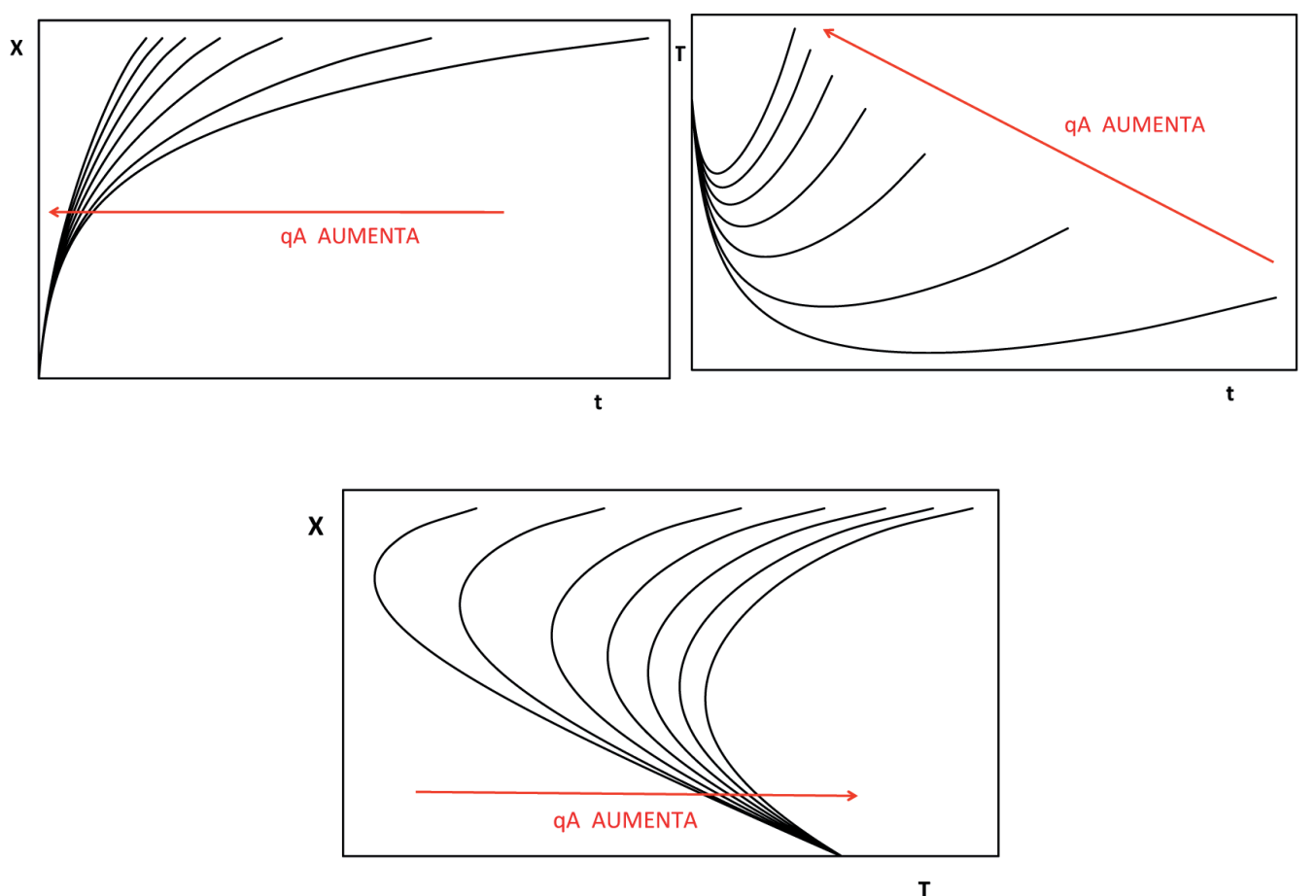

Figura 3. Evolución de las variables t, X y T en un RDTA intermedio a densidad de flujo de calor constante 


\subsection{2. $\mathrm{RDTA}$ intermedio a $\mathrm{U} \mathrm{y}_{\mathrm{f}}$ constantes}

Este tipo de intercambio de calor sirve tanto para el caso de que se esté desarrollando una reacción endotérmica como exotérmica. La ecuación de velocidad de transmisión de calor es:

$$
Q^{*}=U \cdot\left(T_{f}-T\right) \cdot A
$$

en la que al ser $\mathrm{U}_{\text {y }} \mathrm{T}_{\mathrm{f}}$ constantes además de $\mathrm{A}$, no introduce ninguna variable adicional puesto que $\mathrm{T}$ ya estaba presente en las ecuaciones de diseño; por lo tanto, las ecuaciones de diseño (3) y (4) quedan como:

$$
\begin{gathered}
\frac{d t}{d X}=\frac{c_{k 0}}{\left(-v_{k}\right) \cdot r(X, T)} \\
\frac{d T}{d X}=J+\frac{c_{k 0} \cdot U \cdot A \cdot\left(T_{f}-T\right)}{N_{k 0} \cdot \sum_{j=1}^{S} \theta_{j} \cdot \bar{C}_{p j} \cdot\left(-v_{k}\right) \cdot r(X, T)}
\end{gathered}
$$

Estas dos ecuaciones se pueden integrar simultáneamente conocidos los valores iniciales de las variables, habitualmente: $\mathrm{t}=0, \mathrm{X}=0 \mathrm{y} \mathrm{T}=\mathrm{T}_{0}$. La integración numérica permite la obtención de una tabla de valores $\mathrm{t}, \mathrm{X}, \mathrm{T}$, cuyas representaciones gráficas depende de que la reacción sea exotérmica o endotérmica. Para el caso de una reacción endotérmica son de la forma que se muestran en la figura 4.
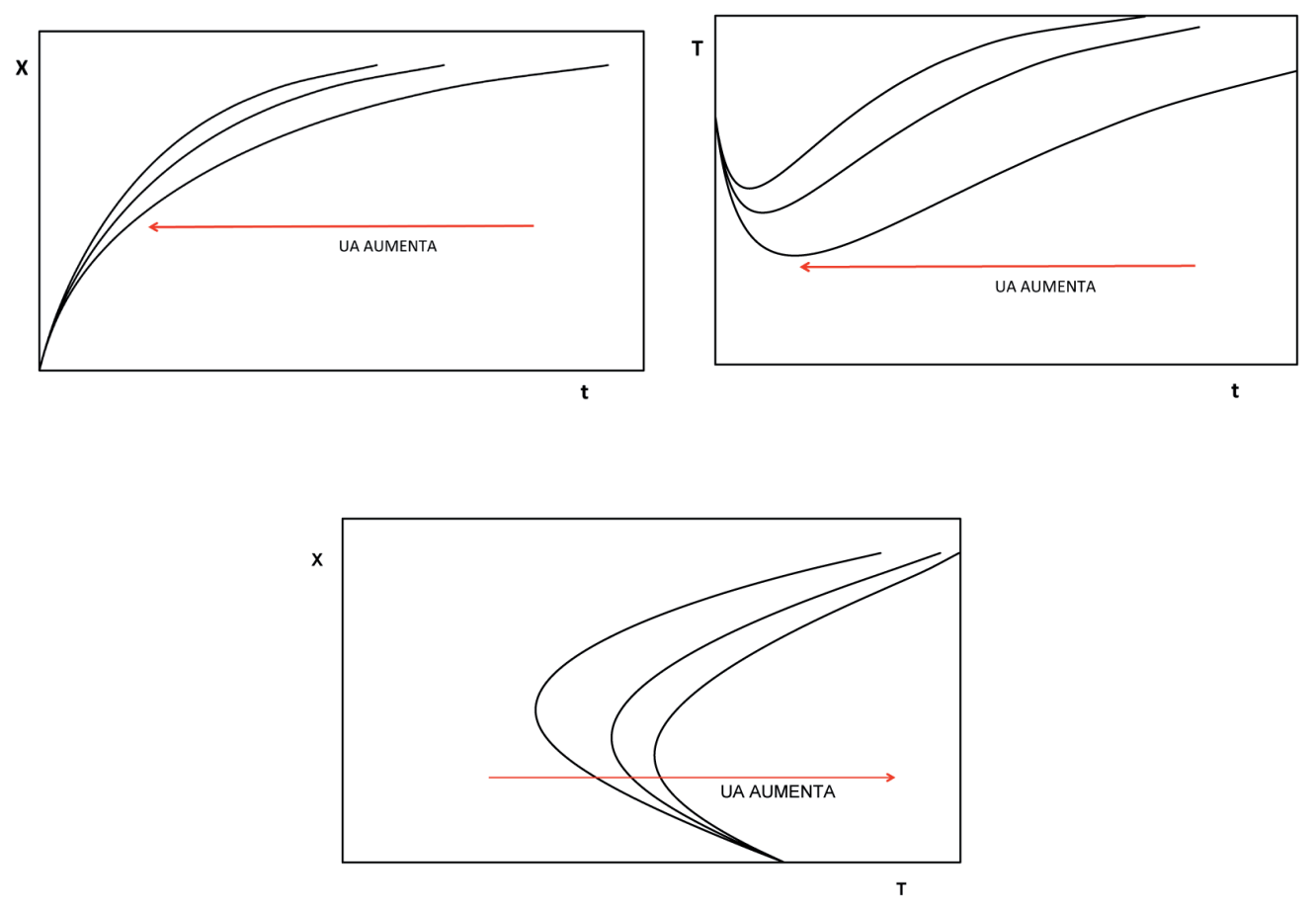

Figura 4. Evolución de las variables t, $\mathrm{X}$ y T en un RDTA intermedio a $\mathrm{U}_{\mathrm{y}} \mathrm{T}_{\mathrm{f}}$ constantes 


\subsection{Ciclo óptimo de temperatura de reacción}

Tal y como se ha mostrado en los apartados anteriores y en la asignatura EQ1021 Ingeniería de la Reacción Química, la temperatura de reacción puede permanecer constante o variar con el tiempo de reacción. El ciclo óptimo de temperatura a lo largo del periodo de reacción es el que maximiza el beneficio, concepto que se verá más adelante en este capítulo, lo que implica:

- En sistemas con una sola reacción química, el ciclo óptimo de temperaturas de reacción es aquel que requiere el tiempo de reacción más pequeño para alcanzar un grado de conversión final dado, lo cual supone que el volumen del reactor será el mínimo necesario para obtener una producción dada. Ello equivale a tener unas cargas económicas fijas (relacionadas con el volumen del reactor) mínimas y un coste de reacción mínimo por ser el tiempo de reacción el menor posible. Al cumplirse ambas consideraciones simultáneamente se consigue que el beneficio económico sea máximo.

- En sistemas con varias reacciones químicas simultáneas el perfil óptimo temperaturas es el que, además de requerir un volumen de reacción lo mas pequeño posible, determine la mayor selectividad del producto principal. Ambas circunstancias conducen a obtener un beneficio mas alto.

\subsubsection{Sistemas con una reacción química}

De acuerdo con la ecuación de diseño (1) el tiempo mínimo de reacción se alcanza cuando la velocidad de reacción es lo más elevada posible. Esto se puede estimar fácilmente para cada tipo de reacción siempre que se conozcan las curvas de velocidad de reacción constante estudiadas en la asignatura EQ1021-Ingeniería de la Reacción Química.

Reacciones endotérmicas, reversibles e irreversibles, y reacciones exotérmicas irreversibles

Para estos tres tipos de reacciones las curvas de velocidad de reacción constante son de la forma que se muestra en las figuras 5 y 6. 


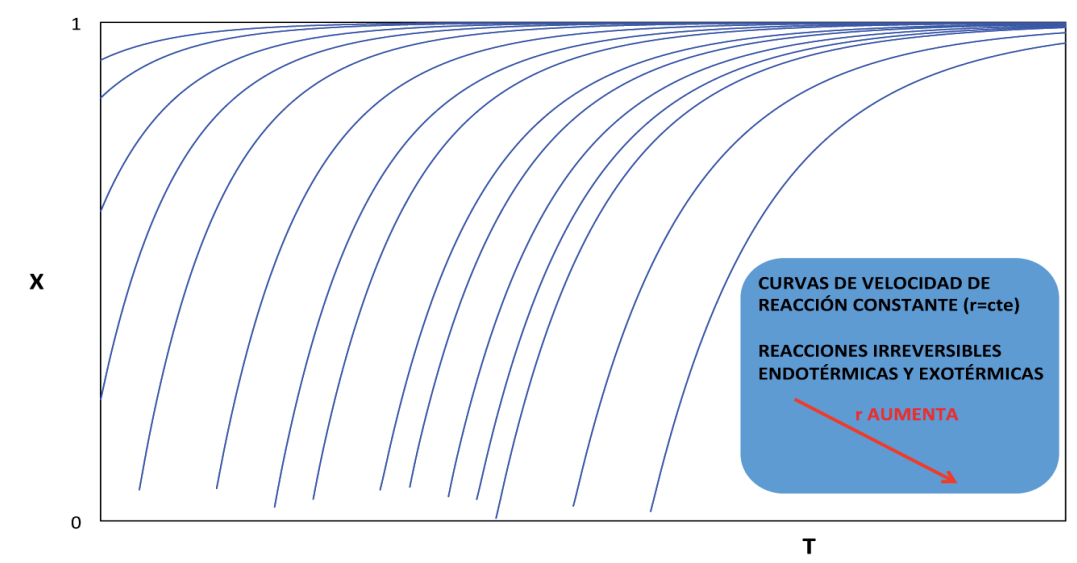

Figura 5. Curvas de velocidad reacción constante para reacciones irreversibles endotérmicas y exotérmicas

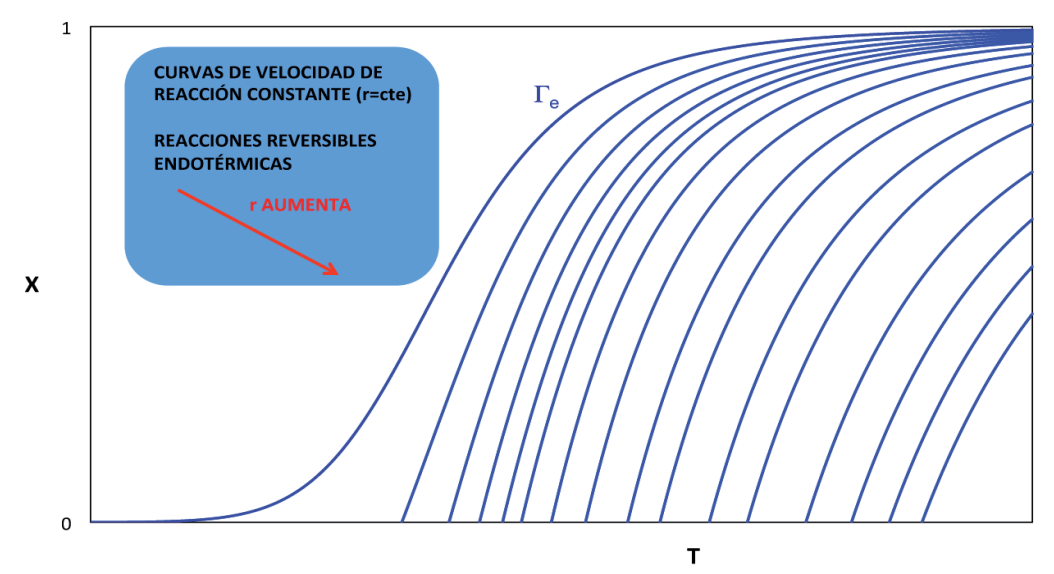

Figura 6. Curvas de velocidad reacción constante para reacciones reversibles endotérmicas

A partir del análisis de estas curvas se deduce fácilmente (figura 7) que la mejor forma de funcionamiento de un RDTA, en el que se desarrolle algún tipo de estas tres reacciones químicas, es la isoterma a la máxima temperatura permisible (o ligeramente inferior) por el sistema $\left(\mathrm{T}^{*}\right)$, ya que de esta forma la velocidad de reacción es máxima para cada grado de conversión y, en consecuencia, para cada tiempo de reacción.

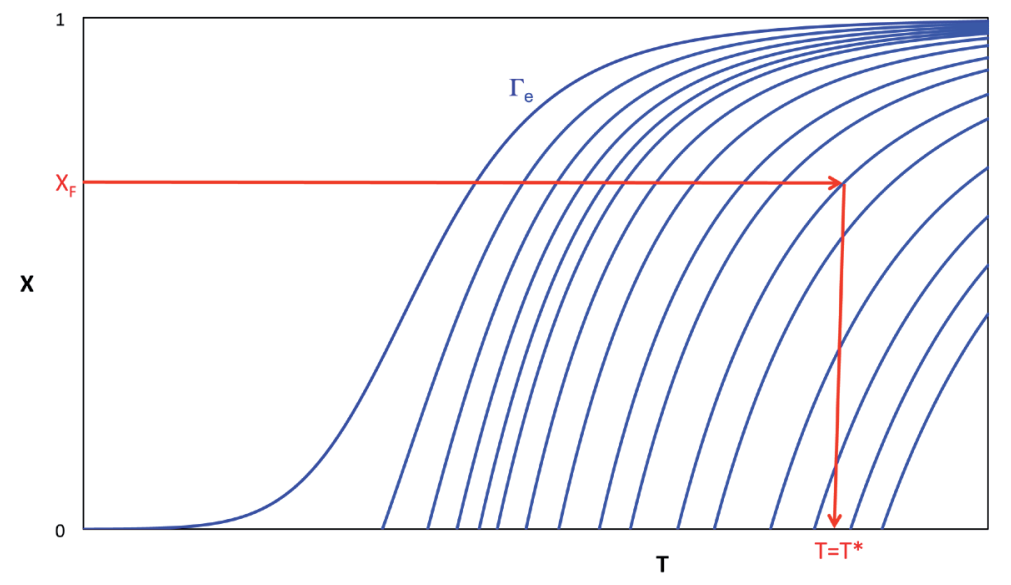

Figura 7. Determinación de la temperatura óptima en reacciones endotérmicas o exotérmicas irreversibles 
En las reacciones endotérmicas reversibles debe recordarse que el máximo grado de conversión que se puede alcanzar a cada temperatura fijada viene determinado por la curva de equilibrio.

\section{Reacciones exotérmicas reversibles}

Para este tipo de reacción las curvas de velocidad de reacción constante son de la forma que se muestra en la figura 8 .

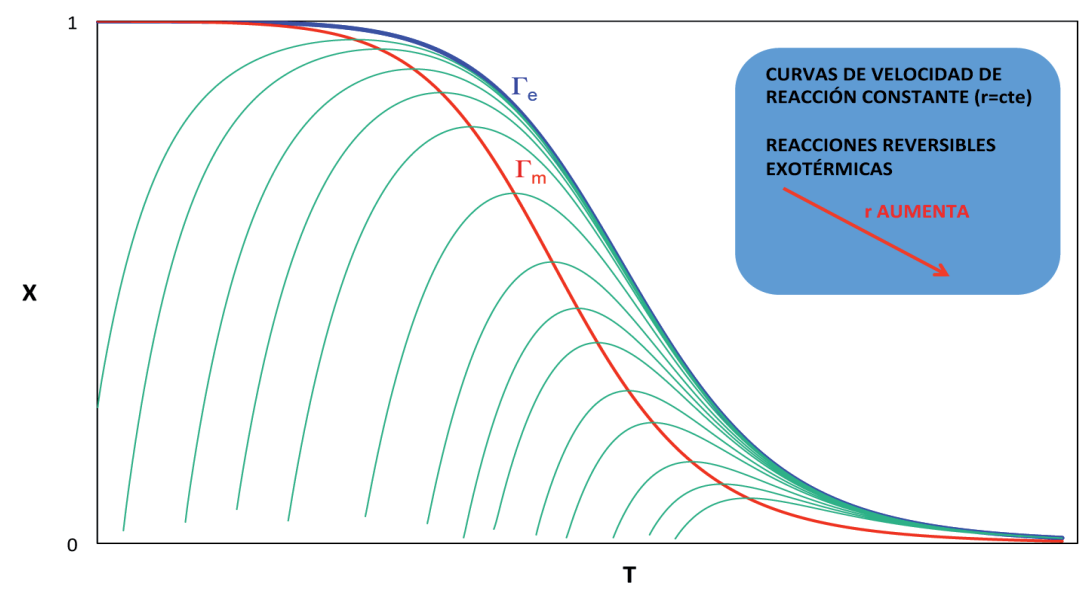

Figura 8. Curvas de velocidad de reacción constante para reacciones reversibles exotérmicas

Del análisis de las curvas se extraen dos conclusiones:

a) si el RDTA es isotermo para alcanzar un determinado grado de conversión final la temperatura de trabajo tiene que ser la que, para dicho grado de conversión, se sitúe sobre la curva $\Gamma_{\mathrm{m}}$ de máxima velocidad de reacción (figura 9).

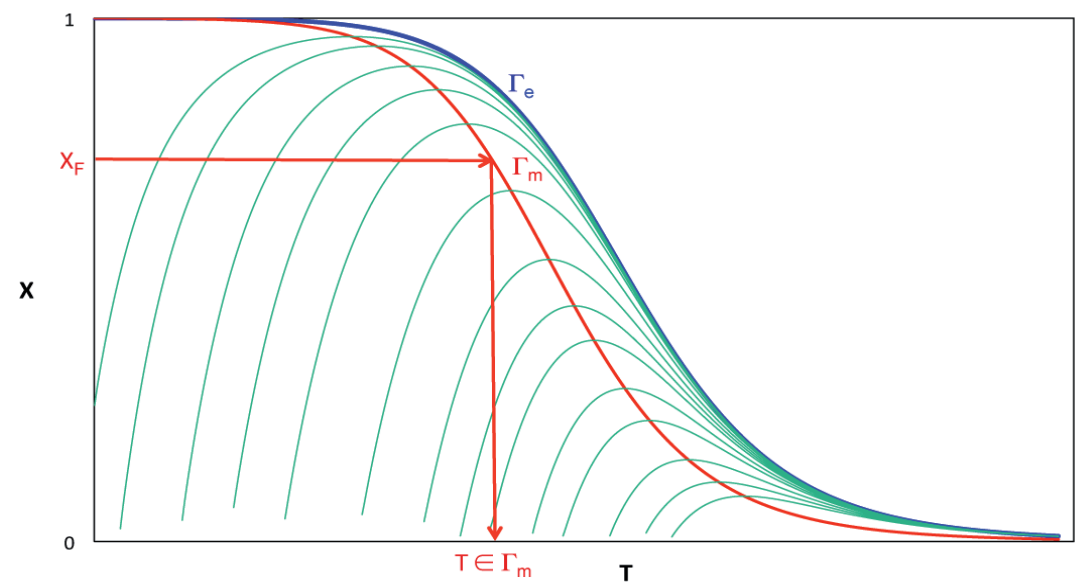

Figura 9. Determinación de la temperatura óptima en reacciones exotérmicas reversibles

b) si el RDTA es intermedio se debe cumplir que, a medida que avanza el tiempo de reacción, todas las parejas de valores $(\mathrm{X}, \mathrm{T})$ deben situarse sobre la curva 
$\Gamma_{\mathrm{m}}$ (figura 10). Es decir, es como si el RDTA se desplazara por la curva $\Gamma_{\mathrm{m}}$ desde el grado de conversión inicial (a tiempo cero) hasta el grado de conversión final (a tiempo final). De esta forma, en cualquier tiempo de reacción la reacción química se está desarrollando a la máxima velocidad de reacción posible.

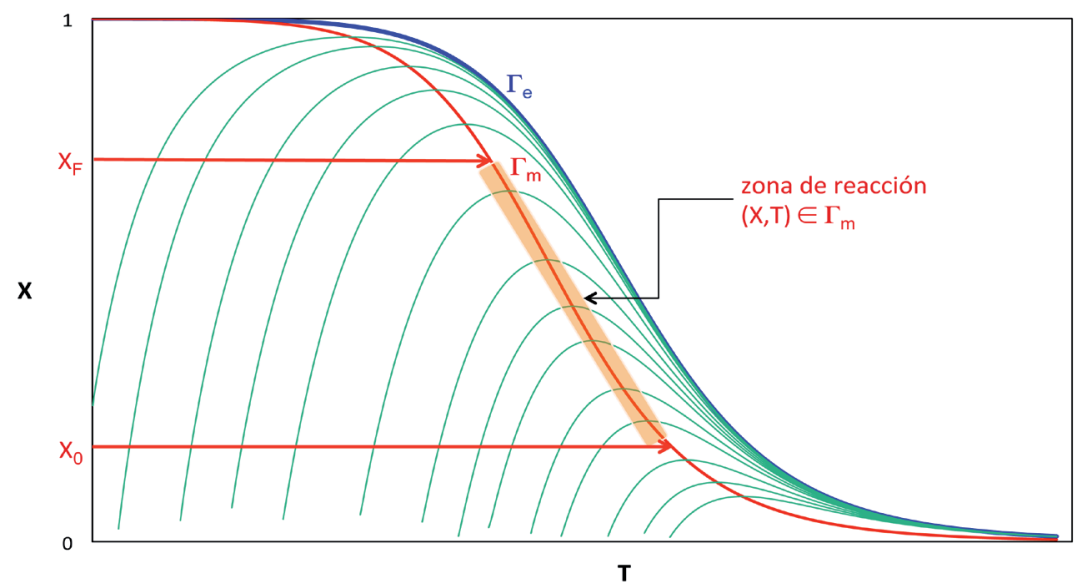

Figura 10. Evolución óptima de la temperatura en reacciones exotérmicas reversibles

En este caso resulta evidente que si se parte de un grado de conversión cero, como es habitual, se requeriría una temperatura inicial excesivamente alta. Por ello, lo más conveniente es que el RDTA opere inicialmente de forma isoterma, a la temperatura máxima permisible $\left(\mathrm{T}^{*}\right)$ hasta que alcance la curva de velocidades de reacción máximas $\Gamma_{\mathrm{m}}$. Análogamente, si se desea llegar a un grado de conversión muy elevado es posible que la temperatura llegue a ser excesivamente baja. En este caso lo más conveniente es que el reactor vuelva a operar desde la temperatura mínima permisible $\left(\mathrm{T}_{*}\right)$ de forma isoterma hasta alcanzar el grado de conversión final. El ciclo completo se muestra en la figura 11. En la tabla 2 se resumen los valores de $\mathrm{X}$ y $\mathrm{T}$ que el RDTA debe seguir a lo largo del tiempo de reacción.

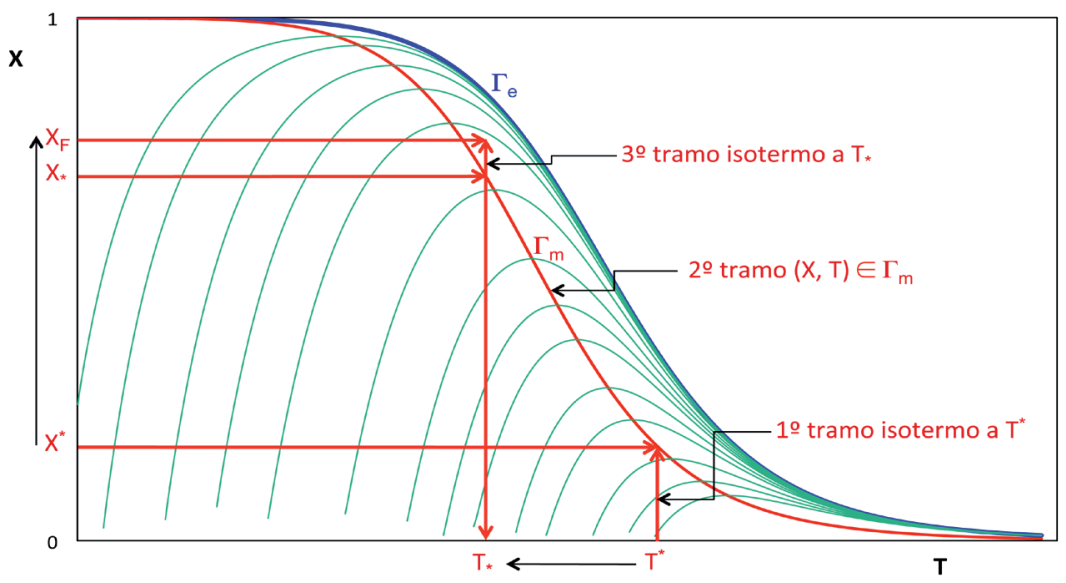

Figura 11. Ciclo óptimo de temperatura en reacciones exotérmicas reversibles 
Tabla 2. Ciclo óptimo de temperatura en reacciones exotérmicas reversibles

\begin{tabular}{|c|c|c|c|}
\hline RDTA & Intervalo de $\mathrm{X}$ & Intervalo de $\mathrm{T}$ & Velocidad de reacción \\
\hline Isotermo & $0 \leq \mathrm{X} \leq \mathrm{X}^{*}$ & $\mathrm{~T}=\mathrm{T}^{*}=$ constante & $\mathrm{r}=\mathrm{r}\left(\mathrm{X}, \mathrm{T}^{*}\right)$ \\
\hline Intermedio & $\mathrm{X}^{*} \leq \mathrm{X} \leq \mathrm{X}_{*}$ & $\mathrm{~T}^{*} \leq \mathrm{T} \leq \mathrm{T}_{*} /(\mathrm{X}, \mathrm{T}) \in \Gamma_{\mathrm{m}}$ & $\mathrm{r}=\mathrm{r}\left[\mathrm{X}, \mathrm{T}_{\mathrm{m}}(\mathrm{X})\right]$ \\
\hline Isotermo & $\mathrm{X}_{*} \leq \mathrm{X} \leq \mathrm{X}_{\mathrm{F}}$ & $\mathrm{T}=\mathrm{T}_{*}=$ constante & $\mathrm{r}=\mathrm{r}\left(\mathrm{X}, \mathrm{T}_{*}\right)$ \\
\hline
\end{tabular}

En las reacciones exotérmicas reversibles debe recordarse que el máximo grado de conversión que se puede alcanzar a cada temperatura fijada viene determinado por la curva de equilibrio.

Ejercicio 2. Una práctica habitual, en el caso de las reacciones exotérmicas reversibles, consiste en sustituir el primer tramo isotermo por un funcionamiento adiabático. Represéntese esta situación en una gráfica $X=f(T)$ que contenga las curvas de velocidad de reacción constante, las curvas $\Gamma_{\mathrm{m}}$ y $\Gamma_{\mathrm{e}}$, indicando claramente los parámetros $\mathrm{X}^{*}, \mathrm{X}_{*}, \mathrm{~T}^{*}$ y $\mathrm{T}_{*} \mathrm{y}$ la temperatura inicial $\mathrm{T}_{0}$ de la mezcla de reacción.

Un problema adicional que se plantea en este tipo de funcionamiento de un RDTA, en el que tiene lugar una reacción exotérmica reversible, es el diseño del sistema de intercambio de calor para conseguir que la variación de la temperatura y la conversión de la mezcla de reacción siga la trayectoria de perfil óptimo de temperaturas. Se puede realizar a partir de la ecuación de diseño (4):

$$
\frac{\mathrm{dT}}{\mathrm{dX}}=\mathrm{J}+\frac{\mathrm{c}_{\mathrm{k} 0} \cdot \mathrm{Q}^{*}}{\mathrm{~N}_{\mathrm{k} 0} \cdot \sum_{\mathrm{j}=1}^{\mathrm{S}} \theta_{\mathrm{j}} \cdot \overline{\mathrm{C}}_{\mathrm{pj}} \cdot\left(-\mathrm{v}_{\mathrm{k}}\right) \cdot \mathrm{r}(\mathrm{X}, \mathrm{T})}
$$

de donde:

$$
-Q^{*}=\left(J-\frac{d T}{d X}\right) \cdot \frac{N_{k 0} \cdot \sum_{j=1}^{S} \theta_{j} \cdot \bar{C}_{p j}}{c_{k 0}} \cdot\left(-v_{k}\right) \cdot r(X, T)
$$

Así, Q* se puede calcular en los diferentes tramos de reacción:

a) En los dos tramos isotermos $\mathrm{dT} / \mathrm{dX}=0 \mathrm{y}$ :

$$
-Q^{*}=J \cdot \frac{\mathrm{N}_{\mathrm{k} 0} \cdot \sum_{\mathrm{j}=1}^{\mathrm{S}} \theta_{j} \cdot \overline{\mathrm{C}}_{\mathrm{pj}}}{\mathrm{c}_{\mathrm{k} 0}} \cdot\left(-v_{\mathrm{k}}\right) \cdot \mathrm{r}(\mathrm{X}, \mathrm{T})
$$

siendo $\mathrm{T}$ un valor constante e igual a $\mathrm{T}^{*} \mathrm{y}_{*}$ en cada tramo. 
b) En el tramo intermedio:

$$
-Q^{*}=\left(J-\frac{d T}{d X}\right) \cdot \frac{N_{k 0} \cdot \sum_{j=1}^{S} \theta_{j} \cdot \bar{C}_{p j}}{c_{k 0}} \cdot\left(-v_{k}\right) \cdot r(X, T)
$$

siendo $\mathrm{T}$ la temperatura de velocidad de reacción máxima $\left(\mathrm{T}_{\mathrm{m}}\right)$ que, según lo visto en la asignatura EQ1021-Ingeniería de la Reacción Química, se puede correlacionar con $\mathrm{X}$ mediante la expresión:

$$
T_{m}(X)=\frac{-\Delta H_{k}^{0}}{R \cdot \ln \left[\frac{E^{\prime}}{E} \cdot \frac{A^{\prime}}{A} \cdot \prod_{j=1}^{S} c_{j}^{v_{j}}(X)\right]}
$$

\subsubsection{Sistemas con varias reacciones químicas}

En estos sistemas, el criterio que permite seleccionar el ciclo de temperatura más adecuado consiste en optimizar la selectividad del producto principal, favoreciendo el transcurso de las reacciones por las que este se forma y dificultando las reacciones por las que desaparece o aquéllas que conducen a otros productos no deseados, procurando que el reactor opere a la temperatura más alta que convenga, para reducir, a la vez (en lo posible) el volumen del reactor necesario.

A continuación se mostrarán de forma cualitativa algunos ejemplos.

a) Sistema de dos reacciones consecutivas irreversibles de primer orden

$$
\begin{gathered}
\mathrm{A}_{1} \stackrel{\mathrm{i}=1}{\longrightarrow} \mathrm{A}_{2} \stackrel{\mathrm{i}=2}{\longrightarrow} \mathrm{A}_{3} \\
\mathrm{r}_{1}=\mathrm{k}_{1}(\mathrm{~T}) \cdot \mathrm{c}_{1}(\mathrm{t}) \quad ; \quad \mathrm{r}_{2}=\mathrm{k}_{2}(\mathrm{~T}) \cdot \mathrm{c}_{2}(\mathrm{t})
\end{gathered}
$$

Hay que actuar sobre las velocidades de reacción dependiendo del producto que se desee obtener. $\mathrm{Y}$ en estas ecuaciones hay que recordar que intervienen dos parámetros: la temperatura, a través de la constante de velocidad, y la concentración de reactivo, que va variando con el tiempo de reacción. Ambos factores hay que tenerlos en consideración en un razonamiento cualitativo.

Supóngase, por ejemplo, que se desea obtener $\mathrm{A}_{2}$, por lo que hay que favorecer la reacción $\mathrm{i}=1$ e intentar minimizar la reacción $\mathrm{i}=2$, y que la relación de las energías de activación es $\mathrm{E}_{1}<<<\mathrm{E}_{2}$.

En primer lugar conviene hacer una representación cualitativa de la variación de las constantes de velocidad con la temperatura (figura 12). Es decir, en general conviene operar a temperaturas bajas donde $\mathrm{k}_{1}>\mathrm{k}_{2}$ 


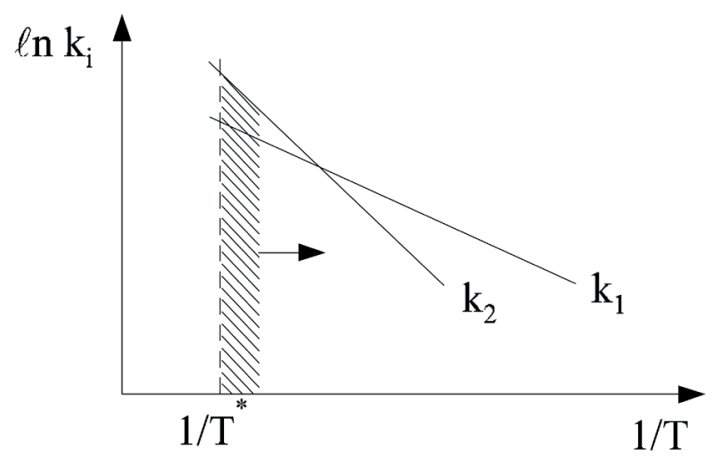

Figura 12. Variación de las constantes de velocidad con la temperatura

Ahora bien, a pesar de esta diferencia de las constantes de velocidad, es evidente que en los inicios de la reacción hay una elevada concentración de $\mathrm{A}_{1}$ pero muy pequeña de $\mathrm{A}_{2}$, por lo que se puede trabajar a temperaturas más altas (aunque sea $\mathrm{k}_{2}>\mathrm{k}_{1}$ ) porque probablemente se cumplirá que $\mathrm{r}_{1}>\mathrm{r}_{2}$. Obviamente, a medida que aumenta el tiempo de reacción también lo hace la concentración de $\mathrm{A}_{2} \mathrm{y}$, para que se siga cumpliendo que $r_{1}>r_{2}$, el RDTA ha de comenzar a operar a temperaturas cada vez menores donde $\mathrm{k}_{1}>\mathrm{k}_{2}$

En conclusión el RDTA comenzaría a operar a temperaturas altas y, a medida que avanzara el tiempo de reacción, debería ir disminuyendo la temperatura (figura 12).

\section{Ejercicio 3. Hágase el mismo estudio cualitativo para el sistema de tres reaccio-} nes paralelas y consecutivas, irreversibles de primer orden:

$$
\begin{gathered}
\mathrm{A}_{1} \stackrel{\mathrm{i}=1}{\longrightarrow} \mathrm{A}_{2} \\
\mathrm{~A}_{2} \stackrel{\mathrm{i}=2}{\longrightarrow} \mathrm{A}_{3} \\
\mathrm{~A}_{2} \stackrel{\mathrm{i}=3}{\longrightarrow} \mathrm{A}_{4} \\
\mathrm{r}_{1}=\mathrm{k}_{1}(\mathrm{~T}) \cdot \mathrm{c}_{1}(\mathrm{t}) \quad ; \quad \mathrm{r}_{2}=\mathrm{k}_{2}(\mathrm{~T}) \cdot \mathrm{c}_{2}(\mathrm{t}) \quad ; \quad \mathrm{r}_{3}=\mathrm{k}_{3}(\mathrm{~T}) \cdot \mathrm{c}_{2}(\mathrm{t})
\end{gathered}
$$

en que $\mathrm{E}_{2}>\mathrm{E}_{1}$ y $\mathrm{E}_{2}<\mathrm{E}_{3}$ y el producto principal que se desea obtener sea $\mathrm{A}_{3}$

Ejercicio 4. Hágase el mismo estudio cualitativo para el sistema de tres reacciones paralelas y consecutivas, irreversibles de primer orden:

$$
\begin{gathered}
\mathrm{A}_{1} \stackrel{\mathrm{i}=1}{\longrightarrow} \mathrm{A}_{2} \stackrel{\mathrm{i}=3}{\longrightarrow} \mathrm{A}_{3} \\
\mathrm{~A}_{1} \stackrel{\mathrm{i}=2}{\longrightarrow} \mathrm{A}_{4} \\
\mathrm{r}_{1}=\mathrm{k}_{1}(\mathrm{~T}) \cdot \mathrm{c}_{1}(\mathrm{t}) \quad ; \quad \mathrm{r}_{2}=\mathrm{k}_{2}(\mathrm{~T}) \cdot \mathrm{c}_{1}(\mathrm{t}) \quad ; \quad \mathrm{r}_{3}=\mathrm{k}_{3}(\mathrm{~T}) \cdot \mathrm{c}_{2}(\mathrm{t})
\end{gathered}
$$

en que $E_{1}>E_{2}$ y $E_{1}<E_{3}$ y el producto principal que se desea obtener sea $A_{2}$ 


\subsection{RSCTA no isotermo}

Algunos reactores semicontinuos (RSCTA) podrían definirse como un reactor continuo de tanque agitado (RCTA) que opera en régimen no estacionario. El comportamiento semicontinuo se presenta, pues, cuando un reactor de tanque agitado continuo (RCTA) se encuentra en la etapa de puesta en marcha, cuando sus condiciones de operación se cambian de un estado estacionario a otro, o cuando se encuentra en la fase de parada.

Además de la situación mencionada en el párrafo anterior, con frecuencia se utilizan reactores semicontinuos con una finalidad concreta. Así, por ejemplo:

i) En ocasiones, es ventajoso introducir inicialmente uno de los reactantes dentro del reactor e ir añadiendo el otro, poco a poco, de forma continua (figura 13). En efecto, cuando el calor de reacción es grande, la temperatura de reacción puede controlarse regulando la velocidad de adición de uno de los reactantes, de modo que la energía liberada (o consumida) pueda ser más fácilmente eliminada por el fluido refrigerante (o suministrada por el fluido calefactor) sin modificarse demasiado la temperatura del reactor. Esta es una forma de compensar la baja capacidad de transmisión de calor que tienen los reactores de tanque agitado. Este método de operación (semicontinuo) permite un elevado grado de control sobre la concentración de la mezcla reactante $y$, por tanto, de la velocidad de reacción, que no es posible realizar en los RDTA o en los reactores continuos. Si la velocidad de reacción es muy grande y puede considerarse prácticamente instantánea, puede regularse controlando el caudal de entrada de un reactivo, ya que, en estas circunstancias, la velocidad de reacción está controlada por la velocidad de adición, cumpliéndose $\Re_{\text {reactivo }}=\mathrm{F}_{\text {reactivo }, 0} / \mathrm{V}(\mathrm{t}) \quad\left(\mathrm{kmol}\right.$ de reactivo $\left./ \mathrm{s} \cdot \mathrm{m}^{3}\right)$.

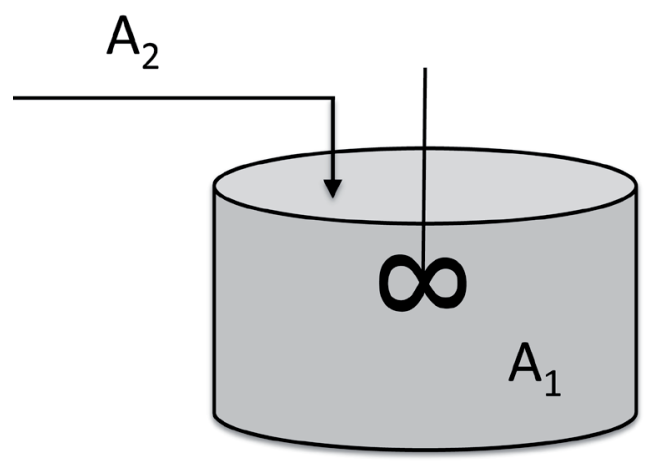

Figura 13. RSCTA con velocidad de reacción controlada por la velocidad de adición

ii) Otra forma de operar un RSCTA consiste en introducir todos los reactantes simultáneamente, en el instante inicial, en el reactor, y operar en condiciones de temperatura tales que se vaya eliminando uno de los productos continuamente (por ejemplo, la eliminación de vapor de agua formado en las reacciones de esterificación, cuando los reactivos y el producto principal tienen 
una temperatura de ebullición superior a la del agua) (figura 14). La ventaja radica en que aumenta la velocidad de reacción, al eliminar uno de los productos de una reacción reversible y se aumenta, a la vez, la concentración de los reactivos.

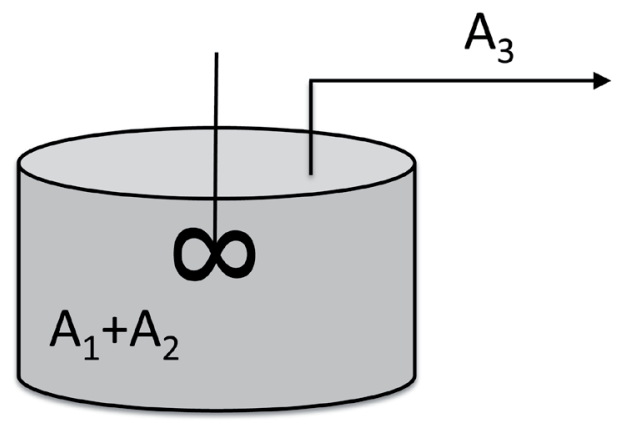

Figura 14. RSCTA con eliminación continua de producto

En este apartado se tratarán los sistemas constituidos por un Reactor SemiContinuo de Tanque Agitado (RSCTA) en el que fundamentalmente tiene lugar una única reacción química. En consecuencia, tal y como se recogía en Anexo II del capítulo 1, el balance de materia se puede expresar mediante:

$$
F_{j}-F_{j 0}+\frac{d\left(c_{j} \cdot V\right)}{d t}=\Re_{j} \cdot V \quad(j=1,2 \ldots . . S)
$$

ECUACIÓN DE DISEÑO (5)

y en la asignatura EQ1021-Ingeniería de la Reacción Química se han visto algunos casos particulares recogidos en la tabla 3 .

Tabla 3. Tipos de RSCTA según las condiciones de funcionamiento

\begin{tabular}{|c|c|c|c|}
\hline $\begin{array}{c}\text { Tipo de } \\
\text { RSCTA }\end{array}$ & $\begin{array}{c}\text { Condiciones } \\
\text { habituales a la } \\
\text { entrada del } \\
\text { reactor }\end{array}$ & $\begin{array}{c}\text { Condiciones } \\
\text { habituales a } \\
\text { la salida del } \\
\text { reactor }\end{array}$ & $\begin{array}{c}\text { Ecuación particular para un determinado } \\
\text { RSCTA y la puesta en marcha de un RCTA }\end{array}$ \\
\hline$A_{1}$ & $\mathrm{~F}_{\mathrm{j} 0}=$ cte; $\mathrm{Q}_{\mathrm{V} 0}=$ cte & $\mathrm{Q}_{\mathrm{V} 0}=\mathrm{Q}_{\mathrm{V}}$ & $\mathrm{c}_{\mathrm{j}}-\mathrm{c}_{\mathrm{j} 0}+\tau \cdot \frac{\mathrm{dc}}{\mathrm{dt}}=\mathrm{v}_{\mathrm{j}} \cdot \mathrm{r} \cdot \tau$ \\
\hline $\mathrm{A}_{2}$ & $\mathrm{~F}_{\mathrm{j} 0}=$ cte; $\mathrm{Q}_{\mathrm{V} 0}=\mathrm{cte}$ & $\mathrm{Q}_{\mathrm{V} 0}>\mathrm{Q}_{\mathrm{V}}$ & $\frac{\mathrm{dc} \mathrm{c}_{\mathrm{j}}}{\mathrm{dt}}+\frac{\mathrm{Q}_{\mathrm{v} 0}}{\mathrm{~V}_{0}+\left(\mathrm{Q}_{\mathrm{v} 0}-\mathrm{Q}_{\mathrm{v}}\right) \cdot \mathrm{t}} \cdot\left(\mathrm{c}_{\mathrm{j}}-\mathrm{c}_{\mathrm{j} 0}\right)=\mathrm{v}_{\mathrm{j}} \cdot \mathrm{r}$ \\
\hline $\mathrm{B}$ & $\mathrm{F}_{\mathrm{j} 0}=\mathrm{cte} ; \mathrm{Q}_{\mathrm{V} 0}=\mathrm{cte}$ & $\mathrm{F}_{\mathrm{j}}=\mathrm{Q}_{\mathrm{V}}=0$ & $\frac{\mathrm{dc}}{\mathrm{dt}}+\frac{\mathrm{Q}_{\mathrm{v} 0}}{\mathrm{~V}_{0}+\mathrm{Q}_{\mathrm{v} 0} \cdot \mathrm{t}} \cdot\left(\mathrm{c}_{\mathrm{j}}-\mathrm{c}_{\mathrm{j} 0}\right)=v_{\mathrm{j}} \cdot \mathrm{r}$ \\
\hline
\end{tabular}

Los tipos A y B corresponden, respectivamente, a los esquemas mostrados en las figuras $15 \mathrm{y} 16$. 


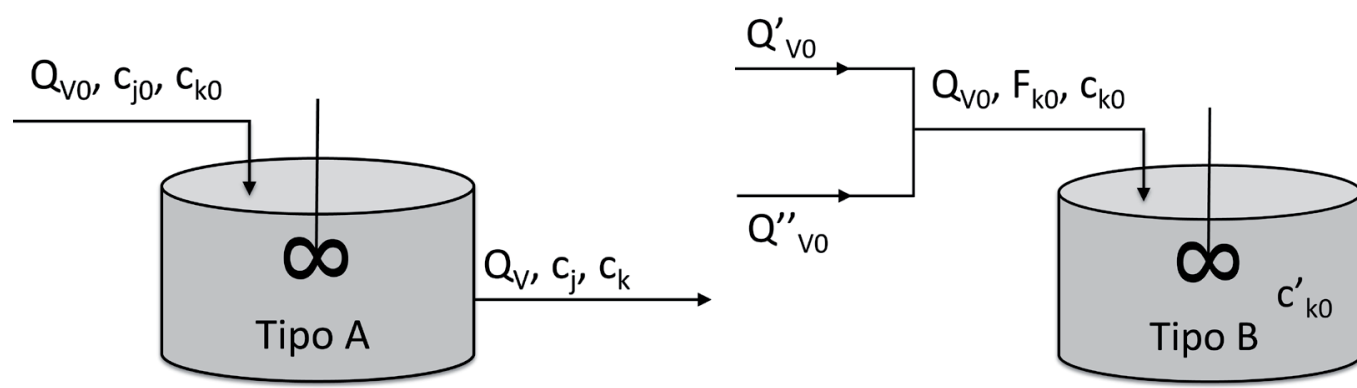

Figura 15. RSCTA tipo A

Figura 16. RSCTA tipo B

Análogamente, en el capítulo 1 también se ha mostrado que el balance de energía, para un sistema constituido por un RSCTA en el que se desarrolla una única reacción química, se puede expresar como:

$$
\sum_{\mathrm{j}=1}^{\mathrm{S}} \mathrm{N}_{\mathrm{j}} \cdot \overline{\mathrm{C}}_{\mathrm{pj}} \cdot \frac{\mathrm{dT}}{\mathrm{dt}}=\mathrm{Q}^{*}+\sum_{\mathrm{j}=1}^{\mathrm{S}} \mathrm{F}_{\mathrm{j} 0} \cdot \overline{\mathrm{C}}_{\mathrm{pj}} \cdot\left(\mathrm{T}_{0}-\mathrm{T}\right)+v_{\mathrm{k}} \cdot \Delta \mathrm{H}_{\mathrm{k}}^{0} \cdot \mathrm{r} \cdot \mathrm{V} \quad \text { ECUACIÓN DE DISEÑO (6) }
$$

En general, cada problema planteado un RSCTA requiere de una resolución particular. En el caso más general pasa por la resolución simultánea del conjunto de ecuaciones diferenciales constituido por: la ecuación de diseño (5) aplicada a cada especie química y por la ecuación de diseño (6). En algunos casos concretos el procedimiento matemático puede simplificarse, dependiendo de las condiciones de operación del reactor.

Ejercicio 5. El alumno puede demostrar que, para un RSCTA del tipo B isotermo (manteniendo la temperatura del reactor constante e igual a la temperatura de la corriente de entrada), en el que tiene lugar una reacción en fase líquida, irreversible y de primer orden de la forma $\mathrm{A}_{1} \rightarrow \mathrm{A}_{2}$, donde el volumen inicial es $\mathrm{V}_{0}$ exento de $\mathrm{A}_{1}$ y la corriente de $\mathrm{A}_{1}$ es pura, se cumple:

a) a partir del balance de materia $\frac{\mathrm{c}_{1}}{\mathrm{c}_{10}}=\frac{[1-\exp (-\mathrm{k} \cdot \mathrm{t})]}{\mathrm{k} \cdot\left(\mathrm{t}+\frac{\mathrm{V}_{0}}{\mathrm{Q}_{\mathrm{V}}}\right)} \mathrm{y} \frac{\mathrm{c}_{2}}{\mathrm{c}_{10}}=\frac{\mathrm{k} \cdot \mathrm{t}-[1-\exp (-\mathrm{k} \cdot \mathrm{t})]}{\mathrm{k} \cdot\left(\mathrm{t}+\frac{\mathrm{V}_{0}}{\mathrm{Q}_{\mathrm{V} 0}}\right)}$

b) a partir del balance de energía $\mathrm{Q}^{*}=\mathrm{r} \cdot \mathrm{V} \cdot \Delta \mathrm{H}_{1}^{0} \mathrm{O} \mathrm{Q}^{*}=\mathrm{c}_{10} \cdot \mathrm{Q}_{\mathrm{V} 0} \cdot \Delta \mathrm{H}_{1}^{0} \cdot[1-\exp (-\mathrm{k} \cdot \mathrm{t})]$ 


\section{PROBLEMAS}

Problema 01. Al estudiar la producción de aceites secantes por descomposición del aceite de ricino acetilado según el esquema de reacción:

aceite ricino acetilado $(\ell) \rightarrow \mathrm{CH}_{3} \mathrm{COOH}(\mathrm{g})+$ aceite secante $(\ell)$

Gruummit y Flemming pudieron correlacionar los resultados experimentales suponiendo que la ecuación de velocidad era de la forma $r=k \cdot c_{1}$, en el intervalo de temperaturas comprendido entre 568 y $613 \mathrm{~K}$, siendo la energía de activación de $186277 \mathrm{~kJ} / \mathrm{kmol}$ y el factor preexponencial $1.937 \cdot 10^{15} \mathrm{~min}^{-1}$.

Si se utiliza un RDTA adiabático para obtener una producción de aceite secante de $500 \mathrm{~kg} / \mathrm{h}$, cuya temperatura inicial es de $613 \mathrm{~K}$ y los tiempos de carga, descarga y limpieza suman 50 minutos, calcular:

a) El tiempo de reacción necesario para alcanzar un grado de conversión de 0.6 construyendo previamente la curva X-t.

b) El volumen de reacción para obtener la producción media fijada.

\section{Datos y notas}

La descomposición completa de $1 \mathrm{~kg}$ de aceite de ricino acetilado originaría $0.156 \mathrm{~kg}$ de ácido acético.

La densidad de la mezcla de reacción es de $900 \mathrm{~kg} / \mathrm{m}^{3}$.

$\Delta \mathrm{H}_{\mathrm{k}}^{\mathrm{o}}=62790 \mathrm{~kJ} / \mathrm{kmol}$

El calor específico medio molar es $966 \mathrm{~kJ} / \mathrm{kmol} \cdot \mathrm{K}$

Problema 02. Para fabricar aceites secantes se hidroliza aceite de ricino acetilado en un RDTA según el esquema de reacción

$$
\text { aceite ricino acetilado }(\ell) \rightarrow \mathrm{CH}_{3} \mathrm{COOH}(\mathrm{g})+\text { aceite secante }(\ell)
$$

Si en cada ciclo de operación se cargan en el reactor $277 \mathrm{~kg}$ de aceite de ricino acetilado y la temperatura inicial de $613 \mathrm{~K}$, calcular:

a) La potencia que habrá que suministrar a la mezcla reactante, mediante calentadores eléctricos sumergidos, para que se alcance un grado de conversión de 0.7 para un tiempo de reacción de 20 minutos.

b) La superficie de transmisión de calor si la máxima diferencia de temperatura permisible entre la superficie de los calentadores y la mezcla de reacción es de $150 \mathrm{~K}$ y el coeficiente global de transmisión de calor (referido al área externa de los calentadores) vale $340 \mathrm{~J} / \mathrm{s} \cdot \mathrm{m}^{2} \cdot \mathrm{K}$. 


\section{Datos y notas}

En el problema 01 puede encontrarse la información complementaria necesaria para resolver este problema.

El peso molecular del aceite de ricino acetilado es $385 \mathrm{~kg} / \mathrm{kmol}$.

Problema 03. Un RDTA de $2 \mathrm{~m}^{3}$ se carga con $50 \mathrm{kmol}$ en total a $450 \mathrm{~K}$ para desarrollar la reacción irreversible en fase líquida $\mathrm{A}_{1}+\mathrm{A}_{2} \rightarrow \mathrm{A}_{3}$, en presencia de un inerte $\mathrm{A}_{4}$. La composición de la mezcla inicial es $\mathrm{x}_{10}=0.4, \mathrm{x}_{20}=0.4, \mathrm{x}_{30}=0.1 \mathrm{y} \mathrm{x}_{40}=0.1$

El calor de reacción a $560 \mathrm{~K}$ es $\Delta \mathrm{H}_{\mathrm{k}}^{\mathrm{o}}=10050 \mathrm{~kJ} / \mathrm{kmol} \mathrm{A}$, y los calores específicos molares de las especies químicas presentes son $\mathrm{C}_{\mathrm{p} 1}=41.8 \mathrm{~kJ} / \mathrm{kmol} \cdot \mathrm{K}, \mathrm{C}_{\mathrm{p} 2}=$ $33.5 \mathrm{~kJ} / \mathrm{kmol} \cdot \mathrm{K}, \mathrm{C}_{\mathrm{p} 3}=50.2 \mathrm{~kJ} / \mathrm{kmol} \cdot \mathrm{K} \mathrm{y} \mathrm{C}_{\mathrm{p} 4}=25.1 \mathrm{~kJ} / \mathrm{kmol} \cdot \mathrm{K}$.

La cinética es de primer orden parcial respecto a cada uno de los reactivos (de segundo orden total). Experimentalmente se ha determinado los valores siguientes para la constante de velocidad:

\begin{tabular}{|c|c|c|}
\hline $\mathrm{T}(\mathrm{K})$ & 350 & 400 \\
\hline $\mathrm{k}\left(\mathrm{m}^{3} / \mathrm{kmol} \cdot \mathrm{h}\right)$ & 0.06 & 0.36 \\
\hline
\end{tabular}

Para calentar el sistema de reacción se utiliza vapor Dowtherm que puede condensar a 200 o $250{ }^{\circ} \mathrm{C}$.

a) Las curvas $\mathrm{T}=\mathrm{f}(\mathrm{t}), \mathrm{X}=\mathrm{f}(\mathrm{t})$ y $\mathrm{T}=\mathrm{f}(\mathrm{X})$ para valores de $\mathrm{U} \cdot \mathrm{A}$ comprendidos entre 0 (adiabático) y $7 \mathrm{~kJ} / \mathrm{s} \cdot \mathrm{K}$ y para valores de $\mathrm{X}$ comprendidos entre 0 y 0.9 .

b) A partir de las gráficas anteriores escoger el valor del producto $U$ $\cdot$ A que permite realizar la reacción con una desviación mínima de $20 \mathrm{~K}$ con respecto a la temperatura inicial y con un tiempo de reacción mínimo, para alcanzar un grado de conversión de 0.8 .

c) Proyectar el reactor sabiendo que el coeficiente individual de transmisión de calor del lado de la mezcla reactiva puede oscilar a voluntad (modificando la velocidad del agitador) entre 418.6 y $1046.5 \mathrm{~kJ} / \mathrm{h} \cdot \mathrm{m}^{2} \cdot \mathrm{K}$.

\section{Datos y notas}

El sistema puede considerarse de densidad constante.

La conductividad térmica de la pared del reactor es $\mathrm{k}=155 \mathrm{~kJ} / \mathrm{h} \cdot \mathrm{m} \cdot \mathrm{K}$ y su espesor es de $3 \mathrm{~mm}$.

El coeficiente individual de transmisión de calor del vapor Dowtherm que condensa vale:

\begin{tabular}{|c|c|c|}
\hline $\mathrm{T}(\mathrm{K})$ & 473 & 523 \\
\hline $\mathrm{h}_{\mathrm{v}}\left(\mathrm{kJ} / \mathrm{h} \cdot \mathrm{m}^{2} \cdot \mathrm{K}\right)$ & 31400 & 24500 \\
\hline
\end{tabular}


Problema 04. Se desea fabricar hexametilentetramina (нмт), en un reactor semicontinuo de tanque agitado, adicionando una cantidad acuosa de amoniaco (25\% en peso de $\mathrm{NH}_{3}$ ) con un caudal volumétrico de $0.454 \mathrm{~m} / \mathrm{h}$, a una carga inicial de 0.90 $\mathrm{m}^{3}$ (a $25^{\circ} \mathrm{C}$ ) de una solución acuosa de formaldehido (42\% en peso de $\mathrm{HCHO}$ ). La temperatura inicial de la solución de formaldehido se eleva a $50^{\circ} \mathrm{C}$ para comenzar la reacción. La temperatura de la disolución de $\mathrm{NH}_{3}$ que se introduce continuamente es de $25^{\circ} \mathrm{C}$.

Puede admitirse que el calor desprendido durante la reacción, que tiene lugar en fase líquida, es independiente de la temperatura y la concentración y su valor es $2230 \mathrm{~kJ} / \mathrm{kg}$ de HMT.

Si el reactor opera a $100^{\circ} \mathrm{C}$ la velocidad de reacción es muy rápida (prácticamente instantánea). No es aconsejable que el reactor opere a temperaturas superiores a los $100{ }^{\circ} \mathrm{C}$ para evitar la vaporización del agua con el consiguiente aumento de la presión (el reactor está herméticamente cerrado).

Se propone enfriar el reactor mediante serpentines por cuyo interior circula agua. El coeficiente global de trasmisión de calor entre la mezcla reactante y el agua de refrigeración vale $482.6 \mathrm{~J} / \mathrm{s} \cdot \mathrm{m}^{2} \cdot \mathrm{K}$. La velocidad de circulación del agua de refrigeración a través de los serpentines es tal que su temperatura apena varía, por lo que puede tomarse un valor medio de $25^{\circ} \mathrm{C}$.

Calcular el área de transmisión de calor y la duración de la primera etapa de reacción (régimen transitorio).

\section{Datos y notas}

La velocidad de reacción inversa es despreciable, por lo que el esquema de reacción es:

$$
4 \mathrm{NH}_{3}+6 \mathrm{HCHO} \rightarrow \mathrm{N}_{4}\left(\mathrm{CH}_{2}\right)_{6}+6 \mathrm{H}_{2} \mathrm{O}
$$

Densidad de la solución amoniacal $910 \mathrm{~kg} / \mathrm{m}^{3}$; densidad de la solución de formaldehido $1100 \mathrm{~kg} / \mathrm{m}^{3}$.

Calor específico de la mezcla reactante $75.3 \mathrm{~kJ} / \mathrm{kmol} \cdot \mathrm{K}$ (constante e independiente de la composición).

Calor específico de la disolución acuosa de amoniaco $75.3 \mathrm{~kJ} / \mathrm{kmol} \cdot \mathrm{K}$.

El reactor funciona del modo siguiente durante cada ciclo de operación:

a) En una primera etapa, y para que la mezcla de reacción alcance rápidamente la temperatura a que se desea tenga lugar la reacción $\left(100^{\circ} \mathrm{C}\right)$, se cortará el paso de agua de refrigeración.

b) Durante la segunda etapa la reacción se desarrollará a la temperatura constante de $100^{\circ} \mathrm{C}$. 
Problema 05. Se desea hidrolizar anhídrido acético en un reactor semicontinuo de tanque agitado isotermo en el que inicialmente se introducen $0.01 \mathrm{~m}^{3}$ de una solución acuosa que contiene $0.5 \cdot 10^{-4} \mathrm{kmol}$ de anhídrido acético por cada metro cúbico de la misma. En una operación típica se conecta el agitador, una vez se ha introducido la carga inicial, y se calienta el reactor hasta que la mezcla alcanza la temperatura de reacción de $313 \mathrm{~K}$. A continuación comienza a introducirse en el reactor una corriente de una disolución acuosa de anhídrido acético (cuya concentración es $3 \cdot 10^{-4} \mathrm{kmol}$ de anhídrido por metro cúbico) con un caudal volumétrico de $0.12 \mathrm{~m}^{3} / \mathrm{h}$, mientras que se drena una corriente de producto de igual caudal volumétrico.

Si la reacción es irreversible y de primer orden respecto al anhídrido acético, calcular la concentración de dicho componente y de ácido acético en la disolución que sale del reactor al cabo de 3 minutos de reacción y al cabo de un tiempo de reacción infinito.

\section{Datos y notas}

Admítase que la densidad de la mezcla de reacción permanece constante durante toda la operación.

A la temperatura de reacción la constante de velocidad vale $6.33 \cdot 10^{-3} \mathrm{~s}^{-1}$.

Supóngase que la reacción está prácticamente congelada hasta el momento en que comienza a introducirse la disolución de anhídrido acético en el reactor.

Problema 06. Para estudiar la cinética de una reacción química en fase líquida, a escala de laboratorio, es frecuente la práctica de sumergir el reactor en un baño de agua a temperatura uniforme, con objeto de que la temperatura de reacción varíe lo menos posible a lo largo del experimento. Se dispone de un montaje de este tipo consistente en un RDTA de 0.4 litros de volumen, sumergido en un baño de agua cuya temperatura se mantendrá constante e igual a $20^{\circ} \mathrm{C}$ durante el experimento. En dicho reactor se va a desarrollar la reacción irreversible en fase líquida de polimerización del estireno, en presencia del catalizador adecuado, según el esquema $\mathrm{A}_{1} \rightarrow \mathrm{A}_{2}$

La ecuación de velocidad es $\mathrm{r}=\mathrm{k} \cdot \mathrm{c}^{1 / 2} \cdot \mathrm{c}_{1}$, donde $\mathrm{c}_{1}$ es la concentración de estireno en la solución bencénica y c la concentración de catalizador (a las condiciones de operación puede admitirse que permanece constante e igual a $0.01 \mathrm{~mol} / 1$ para cualquier valor del tiempo de reacción). La variación de la constante de velocidad con la temperatura es $\mathrm{k}=2.321 \cdot 10^{10} \cdot \exp \left(\frac{-7356.07}{\mathrm{~T}}\right) \quad(\ell / \mathrm{mol})^{1 / 2} \cdot \mathrm{min}^{-1}$ 
Si la superficie de intercambio de calor entre el reactor y el baño de agua es de $0.0322 \mathrm{~m}^{2}$, la temperatura inicial de la mezcla de reacción es de $20^{\circ} \mathrm{C}$ y la concentración inicial de estireno es de $2 \mathrm{kmol} / \mathrm{m}^{3}$ :

a) Calcular la variación de la temperatura de la mezcla de reacción con el grado de conversión, hasta el valor de 0.6.

b) Calcular el tiempo necesario para alcanzar la conversión del 0.6.

\section{Datos y notas}

El coeficiente global medio de transmisión de calor vale $142 \mathrm{~J} / \mathrm{s} \cdot \mathrm{m}^{2} \cdot \mathrm{K}$. El calor de reacción medio en el intervalo de temperaturas considerado es $-69.5 \mathrm{~kJ} / \mathrm{mol}$.

Puede tomarse para la capacidad calorífica media de la mezcla de reacción, en el intervalo de temperaturas de operación, el valor de $183 \mathrm{~J} / \mathrm{mol}$ de $A_{1} \cdot K$.

Problema 07. Se quiere desarrollar la reacción irreversible en fase líquida $A_{1} \rightarrow A_{2}$ en un RDTA de $5 \mathrm{~m}^{3}$ de volumen. La velocidad de reacción varía con la temperatura en la forma $\mathrm{k}=4 \cdot 10^{6} \cdot \exp \left(\frac{-7900}{\mathrm{~T}}\right) \mathrm{s}^{-1}$

La temperatura inicial de la mezcla de reacción es de $20{ }^{\circ} \mathrm{C}$ y la máxima temperatura de reacción permisible es de $95^{\circ} \mathrm{C}$. El reactor va provisto de un intercambiado de calor de $3.3 \mathrm{~m}^{2}$ de área que puede operar con vapor de agua saturado $\left(\mathrm{T}_{\mathrm{f}}=120^{\circ} \mathrm{C}\right.$ $\left.\mathrm{y} \mathrm{U}=1360 \mathrm{~J} / \mathrm{s} \cdot \mathrm{m}^{2} \cdot \mathrm{K}\right)$ o con agua fría $\left(\mathrm{T}_{\mathrm{f}}=15^{\circ} \mathrm{C} \mathrm{y} \mathrm{U}=1180 \mathrm{~J} / \mathrm{s} \cdot \mathrm{m}^{2} \cdot \mathrm{K}\right)$.

El tiempo requerido para llenar y vaciar el reactor es de 10 y 15 minutos respectivamente, no requiriéndose el limpiado del mismo.

Si la mezcla inicial consiste en $\mathrm{A}_{1}$ puro, se desea obtener un grado de conversión igual o mayor a 0.9 y el reactor opera en cada ciclo con la siguiente secuencia de etapas:

$1^{\mathrm{o}}$ se precalienta la mezcla inicial hasta $55^{\circ} \mathrm{C}$ utilizando el dispositivo de calefacción

2..$^{\circ}$ a partir de ese momento el reactor funciona adiabáticamente

$3^{\circ}$ cuando la temperatura de la mezcla de reacción llega a $95^{\circ} \mathrm{C}$, o el grado de conversión a 0.9 , se enfría la mezcla de reacción hasta $45^{\circ} \mathrm{C}$ utilizando el dispositivo refrigerante

calcular:

a) El tiempo de reacción y el tiempo total del ciclo de reacción en el caso hipotético de que la reacción solo tenga lugar durante la etapa $2 .{ }^{a}$. 
b) Caso de que la reacción se iniciara en el momento de introducir la mezcla en el reactor $\left(\mathrm{a} 20^{\circ} \mathrm{C}\right)$ desarrollándose durante la etapa $1 .{ }^{\mathrm{a}}$ de precalentamiento, así como durante la etapa $2 .^{\mathrm{a}}$, prosiguiendo durante la etapa $3 .^{\mathrm{a}}$ de enfriamiento, deducir las ecuaciones diferenciales que, adecuadamente integradas entre los límites que se indicará, permitirían calcular la variación de T y X con el tiempo de reacción durante las etapas de precalentamiento y enfriamiento. Sustituir en dichas ecuaciones todas las magnitudes que se conozcan de modo que queden en función solo de $\mathrm{X}, \mathrm{T}$ y $\mathrm{t}$.

\section{Datos y notas}

Considérese $\Delta \mathrm{H}_{1}^{\mathrm{o}}=-1670 \mathrm{~kJ} / \mathrm{kg} \quad ; \quad \rho_{\mathrm{m}} \cdot \overline{\mathrm{C}}_{\mathrm{p}}=4.2 \cdot 10^{6} \quad \mathrm{~J} / \mathrm{m}^{3} \cdot \mathrm{K}$, constantes e independientes de la composición, siendo $\rho_{\mathrm{m}}$ la densidad molar de la mezcla de reacción.

$\mathrm{c}_{10}=1 \mathrm{kmol} / \mathrm{m}^{3} ; \mathrm{M}_{1}=100 \mathrm{~kg} / \mathrm{kmol}$

Problema 08. La reacción química reversible $\mathrm{A}_{1} \leftrightarrow \mathrm{A}_{2}$ tiene lugar en fase líquida y se ha comprobado que es de primer orden. Dicha reacción va a llevarse a cabo en un RDTA que solo puede funcionar de forma no adiabática (isoterma y/o intermedia). Por problemas de ebullición la temperatura máxima permisible es $77^{\circ} \mathrm{C}$. Calcular el tiempo mínimo de reacción necesario para que el $80 \%$ de los reactivos se conviertan en productos.

\section{Datos y notas}

La mezcla inicial es una disolución acuosa (con un $10 \%$ de $\mathrm{A}_{1}$ y un $90 \%$ de agua, porcentajes molares) en la que la concentración de $\mathrm{A}_{1}$ es $1 \mathrm{~mol} / \mathrm{l}$ $r=6.34 \cdot 10^{10} \cdot \exp \left[\frac{-9500}{T}\right] \cdot c_{1}-3.56 \cdot 10^{32} \cdot \exp \left[\frac{-26900}{T}\right] \cdot c_{2} \quad$ en $\mathrm{mol} /(\min \cdot 1)$ cuando

las concentraciones se expresan en $\mathrm{mol} / \mathrm{l}$.

$\Delta \mathrm{H}_{1}^{\mathrm{o}}=-144670 \mathrm{~J} / \mathrm{mol} ; \mathrm{C}_{\mathrm{p} 1}=655 \mathrm{~J} / \mathrm{mol} \cdot \mathrm{K} ; \mathrm{C}_{\mathrm{p} 2}=60 \mathrm{~J} / \mathrm{mol} \cdot \mathrm{K} ;$

$\mathrm{C}_{\text {pagua }}=75 \mathrm{~J} / \mathrm{mol} \cdot \mathrm{K}$

Problema 09. La reacción elemental, irreversible, en fase gas $A_{1}+2 A_{2} \rightarrow A_{3}$ tiene lugar en un RDTA a 2 atmósferas de presión constante. A $50{ }^{\circ} \mathrm{C}$ la constante de velocidad vale $110\left(\mathrm{~m}^{3}\right)^{2} /\left(\mathrm{kmol}^{2} \cdot \mathrm{min}\right)$. La mezcla inicial consiste en una mezcla equimolar de $\mathrm{A}_{1} \mathrm{y}_{2}$, en la que no hay ni $\mathrm{A}_{3}$ ni inertes. La energía de activación es $20000 \mathrm{kcal} / \mathrm{kmol}$.

Calcular:

a) El valor de las velocidades de reacción $\mathrm{r}, \Re_{\mathrm{A} 1}, \Re_{\mathrm{A} 2}$ y $\Re_{\mathrm{A} 3}$ cuando $\mathrm{t}=0$. 
b) El valor de $\mathrm{r}$ cuando ha reaccionado la cuarta parte de $\mathrm{A}_{1}$ inicialmente introducida en el RDTA.

c) El valor de $\mathrm{r}$ cuando $\mathrm{t}=0$ si la temperatura se aumentará a $60{ }^{\circ} \mathrm{C}$.

d) El tiempo necesario para que reaccione la cuarta parte de $\mathrm{A}_{1}$ inicialmente presente, si el RDTA es isotermo y se mantiene a $60{ }^{\circ} \mathrm{C}$.

\section{Datos y notas}

Recuérdese que en los apartados $a$ ) y $b$ ) la temperatura del reactor es $50^{\circ} \mathrm{C}$.

Problema 10. La reacción en fase líquida $A_{1}+A_{2} \rightarrow A_{3}$ se lleva a cabo en un RDTA de 50 litros. La reacción es irreversible y de primer orden con respecto a cada reactivo.

a) Determinar el tiempo de reacción necesario para alcanzar un grado de conversión del 0.9 si:

a.1) el RDTA es adiabático

a.2) el RDTA es isotermo a la temperatura $T_{0}$

b) Determinar las funciones del tiempo de reacción $\mathrm{Q}^{*} \mathrm{y} \mathrm{T}_{\mathrm{f}}$, en el supuesto de que se disponga de un serpentín en el interior del RDTA que permita mantener las condiciones isotermas del subapartado a.2.

c) Calcular y representar el perfil de X y T con el tiempo de reacción para las condiciones adiabáticas del subapartado a.1.

\section{Datos y notas}

$$
\begin{aligned}
& \rho_{\text {fase líquida }}=750 \mathrm{~kg} / \mathrm{m}^{3} ; \mathrm{c}_{10}=0.50 \mathrm{kmol} / \mathrm{m}^{3} ; \mathrm{c}_{20}=0.75 \mathrm{kmol} / \mathrm{m}^{3} \\
& \mathrm{~T}_{0}=400 \mathrm{~K} \\
& \Delta \mathrm{H}_{1}^{\mathrm{o}}=-145000 \mathrm{~kJ} / \mathrm{kmol} ; \mathrm{C}_{\mathrm{p} \text { fase líquida }}=3.8 \mathrm{~kJ} / \mathrm{kg} \cdot \mathrm{K} \\
& \mathrm{k}=1.4 \cdot 10^{7} \cdot \exp \left[\frac{-7700}{\mathrm{~T}}\right] \mathrm{m}^{3} / \mathrm{kmol} \cdot \mathrm{min} \\
& \mathrm{U} \cdot \mathrm{A}=0.1 \mathrm{~kJ} / \mathrm{s} \cdot \mathrm{K}(\text { solo para el apartado } b)
\end{aligned}
$$

Problema 11. La reacción irreversible, de segundo orden, en fase líquida, $\mathrm{A}_{1} \rightarrow$ productos se desarrolla en un RDTA provisto de un intercambiador de calor. La mezcla inicial está constituida por $\mathrm{A}_{1}$ puro, con una concentración de $16 \mathrm{~mol} / \mathrm{l}$, que se encuentra a $350 \mathrm{~K}$.

a) Calcular la evolución de la temperatura y el grado de conversión con el tiempo de reacción cuando el valor de $\mathrm{U} \cdot \mathrm{A} / \mathrm{V}$ es $5500 \mathrm{~J} / \mathrm{h} \cdot 1 \cdot \mathrm{K}$

b) ¿Qué sucedería si el RDTA fuera adiabático? 
Datos y notas

$$
\begin{aligned}
& \mathrm{c}_{10}=16 \mathrm{~mol} / \mathrm{l} \\
& \Delta \mathrm{H}_{1}^{\mathrm{o}}=-(11625+11.625 \cdot \mathrm{T}) \mathrm{J} / \mathrm{mol} ; \mathrm{C}_{\mathrm{p} 1}=1860 \mathrm{~J} / \ell \cdot \mathrm{K} \\
& \mathrm{k}=554726 \cdot \exp \left[\frac{-5000}{\mathrm{~T}}\right] \ell / \mathrm{mol} \cdot \mathrm{h} \\
& \mathrm{T}_{\mathrm{f} 0}=300 \mathrm{~K}
\end{aligned}
$$

Problema 12. Se quiere desarrollar, en fase líquida, la reacción endotérmica irreversible de tercer orden $3 \mathrm{~A}_{1} \rightarrow 2 \mathrm{~A}_{2}+\mathrm{A}_{3}$. Para ello se utilizará un RDTA adiabático. La mezcla de reacción está constituida inicialmente por $\mathrm{A}_{1}$ puro y se calentará dentro del reactor hasta $400{ }^{\circ} \mathrm{C}$. Durante este periodo de calentamiento se alcanza un grado de conversión de 0.1. A partir de ese momento, calcular el tiempo de reacción necesario para alcanzar un grado de conversión de 0.7 .

Datos y notas

$$
\begin{aligned}
& \mathrm{m}_{10}=950 \mathrm{~kg} ; \mathrm{N}_{10}=10.2 \mathrm{kmol} \\
& \mathrm{V}=1 \mathrm{~m}^{3} \\
& \Delta \mathrm{H}_{1}^{\mathrm{o}}=104650 \mathrm{~kJ} / \mathrm{kmol} ; \mathrm{C}_{\mathrm{pl}}=2.48 \mathrm{~kJ} / \mathrm{kg} \cdot \mathrm{K} \\
& \text { Ink }=\frac{-5000}{\mathrm{~T}}+5 \mathrm{ken}\left(\mathrm{m}^{3} / \mathrm{kmol}\right)^{2} \cdot \mathrm{h}^{-1}
\end{aligned}
$$




\section{Reactor continuo tubular de flujo de pistón (RFP) intermedio y adiabático}

\subsection{Ecuaciones de diseño}

En este capítulo se tratarán fundamentalmente los sistemas constituidos por un único reactor continuo tubular de flujo de pistón (RFP) funcionando en régimen estacionario en el que tiene lugar una única reacción química cuya velocidad de reacción es normal (no autocatalítica). En consecuencia, tal y como se recogía en Anexo II del capítulo 1, el balance de materia se puede expresar mediante cualquiera de las dos ecuaciones siguientes que dependen de la variable de composición que se utilice:

i) sistemas de densidad constante: $\mathrm{dV}=\mathrm{Q}_{\mathrm{v} 0} \cdot \frac{\mathrm{dc}_{\mathrm{j}}}{v_{\mathrm{j}} \cdot \mathrm{r}}$

ii) sistemas de densidad variable (o de densidad constante): $d V=F_{k 0} \cdot \frac{d X}{\left(-v_{k}\right) \cdot r}$

De las dos resulta más conveniente aplicar la segunda, puesto que es válida para cualquier sistema, independientemente de que su densidad permanezca constante o varíe; además como los reactores son de sección $(\mathrm{S})$ constante $\mathrm{dV}=\mathrm{S} \cdot \mathrm{dl}$ por lo que la ecuación más recomendable de balance de materia es:

$$
\mathrm{d} \ell=\frac{\mathrm{F}_{\mathrm{k} 0}}{\mathrm{~S}} \cdot \frac{\mathrm{dX}}{\left(-v_{\mathrm{k}}\right) \cdot \mathrm{r}}
$$

Para poder aplicar estas ecuaciones es necesario conocer la ecuación de la velocidad de reacción química, en función de la temperatura y de la concentración o el grado de conversión:

$$
r=r[X, T] \quad 0 \quad r=r\left[C_{j}, T\right]
$$

Análogamente, en el capítulo 1 también se han mostrado los balances de energía que, para el sistema constituido por un RFP en el que se desarrolla una única reacción química, se puede expresar mediante una de las dos ecuaciones siguientes: 


$$
\begin{aligned}
& \frac{\mathrm{dT}}{\mathrm{d} \ell}-\mathrm{J} \cdot \frac{\mathrm{dX}}{\mathrm{d} \ell}=\frac{\mathrm{dQ}}{\mathrm{d} \ell} \\
& \frac{\mathrm{dT}}{\mathrm{d} \ell}+\frac{v_{\mathrm{k}} \cdot \mathrm{r}}{\mathrm{G}_{\mathrm{k} 0}} \cdot \mathrm{J}=\frac{\mathrm{dQ}}{\mathrm{d} \ell}
\end{aligned}
$$

Para poder aplicar estas ecuaciones es necesario conocer la ecuación de la velocidad de transmisión de calor, cuya forma depende del sistema de intercambio de calor utilizado.

En resumen, las dos ecuaciones más habituales para el diseño de un RFP con una reacción química son:

$$
\begin{array}{ll}
\mathrm{d} \ell=\frac{\mathrm{F}_{\mathrm{k} 0}}{\mathrm{~S}} \cdot \frac{\mathrm{dX}}{\left(-v_{\mathrm{k}}\right) \cdot \mathrm{r}}=\mathrm{G}_{\mathrm{k} 0} \cdot \frac{\mathrm{dX}}{\left(-v_{\mathrm{k}}\right) \cdot \mathrm{r}} & \text { ECUACIÓN DE DISEÑO (1) } \\
\frac{\mathrm{dT}}{\mathrm{d} \ell}-\mathrm{J} \cdot \frac{\mathrm{dX}}{\mathrm{d} \ell}=\frac{\mathrm{dQ}}{\mathrm{d} \ell} & \text { ECUACIÓN DE DISEÑO (2) }
\end{array}
$$

Obviamente la resolución simultánea de las ecuaciones de diseño permite calcular, entre otras variables, la longitud del reactor (1) necesaria para alcanzar un determinado grado de conversión.

En el anexo I se muestran algunos de los parámetros geométricos de este tipo de reactores y algunas de las variables más utilizadas en su análisis.

Desde un punto de vista térmico, los RFP pueden tener un comportamiento isotermo, adiabático o intermedio, manteniéndose la temperatura constante o variable a lo largo de la longitud del reactor. En la figura 1 se muestra para cada caso la evolución de la temperatura con la longitud del reactor, teniendo en cuenta que la reacción que tiene lugar puede ser exotérmica o endotérmica
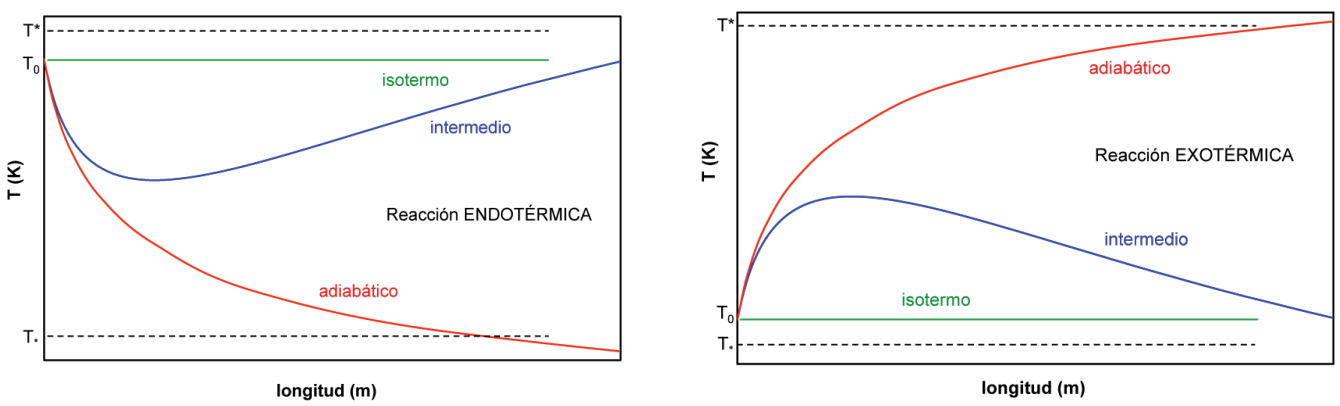

Figura 1. Evolución de la temperatura con la longitud según el tipo de reacción y las características del reactor 


\subsection{Intercambio de calor}

En la ecuación de diseño (2), que es la forma habitual del balance de energía, hay que recordar que:

$$
d Q=\frac{d Q^{*}}{F_{k 0} \cdot \sum_{j=1}^{S} \theta_{j} \cdot \bar{C}_{p j}}
$$

por lo que se debe sustituir la velocidad de transmisión de calor por la correspondiente expresión.

Se considerarán dos tipos básicos de intercambio de calor:

a) Reactores en los que se mantiene constante la densidad de flujo de calor, $q$ en $\mathrm{J} / \mathrm{s} \cdot \mathrm{m}^{2}$ (el suministro de calor por unidad de tiempo y unidad de área de intercambio de calor), durante toda la operación:

$$
d Q^{*}=q \cdot d A
$$

Obsérvese que en la ecuación de diseño (2) aparece dQ/d1, por lo que:

$$
\frac{d Q}{d \ell}=\frac{d Q^{*}}{\left(F_{k 0} \cdot \sum_{j=1}^{S} \theta_{j} \cdot \bar{C}_{p j}\right) \cdot d \ell}=\frac{q}{\left(F_{k 0} \cdot \sum_{j=1}^{S} \theta_{j} \cdot \bar{C}_{p j}\right)} \cdot \frac{d A}{d \ell}=\frac{q}{\left(F_{k 0} \cdot \sum_{j=1}^{S} \theta_{j} \cdot \bar{C}_{p j}\right)} \cdot \pi \cdot D=\frac{q}{\left(F_{k 0} \cdot \sum_{j=1}^{S} \theta_{j} \cdot \bar{C}_{p j}\right)} \cdot 2 \cdot \pi \cdot R
$$

b) Reactores en los que se mantiene constante el coeficiente global de transmisión de calor U, pudiendo la temperatura del fluido intercambiador permanecer también constante o variar con la longitud.

$$
d Q^{*}=U \cdot\left(T_{f}-T\right) \cdot d A
$$

Obsérvese que en la ecuación de diseño (2) aparece dQ/dl, por lo que:

$$
\frac{\mathrm{dQ}}{\mathrm{d} \ell}=\frac{\mathrm{dQ} *}{\left(\mathrm{~F}_{\mathrm{k} 0} \cdot \sum_{\mathrm{j}=1}^{\mathrm{S}} \theta_{\mathrm{j}} \cdot \overline{\mathrm{C}}_{\mathrm{pj}}\right) \cdot \mathrm{d} \ell}=\frac{\mathrm{U} \cdot\left(\mathrm{T}_{\mathrm{f}}-\mathrm{T}\right)}{\left(\mathrm{F}_{\mathrm{k} 0} \cdot \sum_{\mathrm{j}=1}^{\mathrm{S}} \theta_{\mathrm{j}} \cdot \overline{\mathrm{C}}_{\mathrm{pj}}\right)} \cdot \frac{\mathrm{dA}}{\mathrm{d} \ell}=\frac{\mathrm{U} \cdot\left(\mathrm{T}_{\mathrm{f}}-\mathrm{T}\right)}{\left(\mathrm{F}_{\mathrm{k} 0} \cdot \sum_{\mathrm{j}=1}^{\mathrm{S}} \theta_{\mathrm{j}} \cdot \overline{\mathrm{C}}_{\mathrm{pj}}\right)} \cdot \mathrm{A}_{\mathrm{S}}=\frac{\left(\mathrm{T}_{\mathrm{f}}-\mathrm{T}\right) \cdot \mathrm{St}}{\mathrm{R}_{\mathrm{h}}}
$$

Para una eficaz eliminación o suministro de calor, el diámetro de un reactor tubular cilíndrico debiera ser tan pequeño como fuera posible, a fin de reducir al máximo la distancia a lo largo de la cual se ha de transmitir el calor dentro del reactor, evitando así la formación de gradientes radiales de temperatura. Cuando el diámetro del reactor ha de ser grande por razones ajenas a las del fenómeno de transporte de calor, sobre todo en reactores catalíticos, la refrigeración se realiza mediante serpentines o haces de tubos. 
Hay diferentes formas para efectuar un control de temperatura a través de las paredes del reactor. El más simple, en teoría, aunque no en la práctica, consiste en mantener la temperatura uniforme en toda la pared del reactor. Para una reacción endotérmica, en la que hay que suministrar calor a la mezcla reactante, es frecuente situar el reactor tubular dentro de un horno si se desarrolla a temperatura muy elevada, o calentar con resistencias eléctricas arrolladas alrededor del tubo, si éste no es muy largo. Para temperaturas próximas a $100{ }^{\circ} \mathrm{C}$ podría utilizarse una camisa de vapor que condensa o vapor de un líquido térmico. Para una reacción exotérmica se suelen utilizar camisas de enfriamiento que pueden consistir en un líquido que hierve a temperatura elevada, o bien un líquido de alto punto de ebullición, que no experimente cambios de fase y que pueda circular a lo largo de las paredes del reactor en contracorriente o en paralelo respecto a la mezcla reactante. En este último caso, la temperatura del fluido refrigerante variará a lo largo del reactor, por lo que se complica algo el diseño, como se verá más adelante.

\subsection{Resolución de las ecuaciones de diseño para un RFP adiabático}

El diseño de un RFP en el que se desarrolla una única reacción química o el cálculo de las condiciones de funcionamiento supone resolver simultáneamente las ecuaciones de diseño (1) y (2):

$$
\begin{array}{cl}
\mathrm{d} \ell=\frac{\mathrm{F}_{\mathrm{k} 0}}{\mathrm{~S}} \cdot \frac{\mathrm{dX}}{\left(-v_{\mathrm{k}}\right) \cdot \mathrm{r}}=\mathrm{G}_{\mathrm{k} 0} \cdot \frac{\mathrm{dX}}{\left(-v_{\mathrm{k}}\right) \cdot \mathrm{r}} & \text { ECUACIÓN DE DISEÑO (1) } \\
\frac{\mathrm{dT}}{\mathrm{d} \ell}-\mathrm{J} \cdot \frac{\mathrm{dX}}{\mathrm{d} \ell}=\frac{\mathrm{dQ}}{\mathrm{d} \ell} & \text { ECUACIÓN DE DISEÑO (2) }
\end{array}
$$

El procedimiento consiste en:

a) Resolver la ecuación de diseño (2), teniendo en cuenta que el sistema no intercambia calor por lo que se cumple que $\mathrm{dQ}^{*} / \mathrm{d} \ell=0=\mathrm{dQ} / \mathrm{d} \ell$. En consecuencia:

$$
\begin{gathered}
\frac{\mathrm{dT}}{\mathrm{d} \ell}-\mathrm{J} \cdot \frac{\mathrm{dX}}{\mathrm{d} \ell}=0 \\
\mathrm{dT}=\mathrm{J} \cdot \mathrm{dX}
\end{gathered}
$$

y esta ecuación de variables separadas se puede integrar fácilmente entre las condiciones iniciales (a tiempo cero) y las condiciones a cualquier tiempo de reacción:

$$
\begin{gathered}
\int_{\mathrm{T}_{0}}^{\mathrm{T}} \mathrm{dT}=\mathrm{J} \cdot \int_{0}^{\mathrm{X}} \mathrm{dX} \\
\mathrm{T}-\mathrm{T}_{0}=\mathrm{J} \cdot \mathrm{X}
\end{gathered}
$$


y reordenando variables, la integración de la ecuación de diseño (2) ha permitido obtener la recta adiabática mostrada en la figura 2.

$$
\begin{gathered}
\mathrm{T}=\mathrm{T}_{0}+\mathrm{J} \cdot \mathrm{X} \\
\mathrm{X}=-\frac{\mathrm{T}_{0}}{\mathrm{~J}}+\frac{1}{\mathrm{~J}} \cdot \mathrm{T}
\end{gathered}
$$

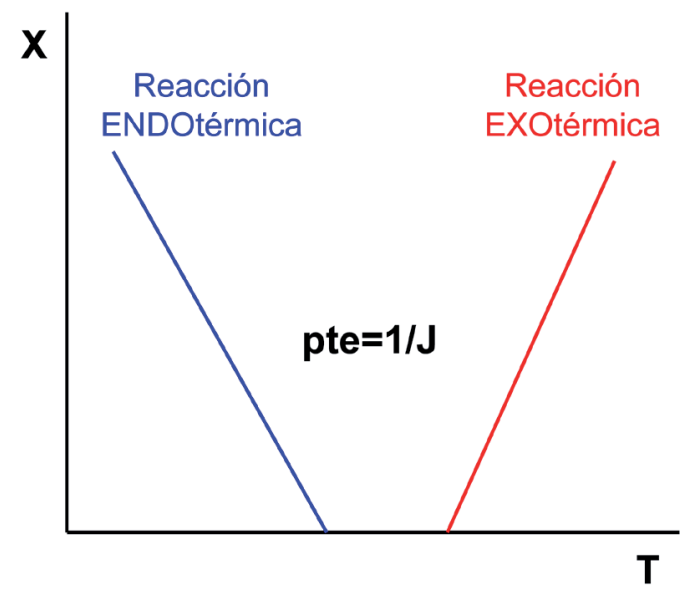

Figura 2. Recta adiabática X versus $\mathrm{T}$

b) Resolver la ecuación de diseño (1), teniendo en cuenta la relación entre X y T mostrada por la adiabática de conversión:

$$
\mathrm{d} \ell=\mathrm{G}_{\mathrm{k} 0} \cdot \frac{\mathrm{dX}}{\left(-\mathrm{v}_{\mathrm{k}}\right) \cdot \mathrm{r}(\mathrm{X}, \mathrm{T})}=\mathrm{c}_{\mathrm{k} 0} \cdot \frac{\mathrm{dX}}{\left(-\mathrm{v}_{\mathrm{k}}\right) \cdot \mathrm{r}\left(\mathrm{X}, \mathrm{T}_{0}+\mathrm{J} \cdot \mathrm{X}\right)}
$$

de esta forma la ecuación de diseño (1) tiene variables separadas y se puede integrar, analítica o numéricamente, para calcular el tiempo de reacción:

$$
\int_{0}^{\mathrm{L}} \mathrm{d} \ell=\mathrm{L}=\mathrm{G}_{\mathrm{k} 0} \cdot \int_{0}^{\mathrm{X}} \frac{\mathrm{dX}}{\left(-\mathrm{v}_{\mathrm{k}}\right) \cdot \mathrm{r}\left(\mathrm{X}, \mathrm{T}_{0}+\mathrm{J} \cdot \mathrm{X}\right)}
$$

\subsection{Resolución de las ecuaciones de diseño para un RFP intermedio}

Los RFP intermedios son aquellos que no tienen ni comportamiento isotermo ni adiabático. Son consecuencia de que no están perfectamente aislados de los alrededores (por lo que no son adiabáticos), pero el calor que intercambian no es suficiente para que el reactor se mantenga isotermo. Es decir:

RDTA isotermo

velocidad de intercambio de energía = velocidad de «generación» de energía por la reacción 
RDTA adiabático

velocidad de intercambio de energía $=0$

RDTA intermedio

velocidad de intercambio de energía $\neq$ velocidad de «generación» de energía por la reacción

En consecuencia, el diseño de un RFP en el que se desarrolla una única reacción química a volumen constante o el cálculo de las condiciones de funcionamiento supone resolver simultáneamente las ecuaciones de diseño (1) y (2):

$$
\begin{gathered}
\mathrm{d} \ell=\frac{\mathrm{F}_{\mathrm{k} 0}}{\mathrm{~S}} \cdot \frac{\mathrm{dX}}{\left(-v_{\mathrm{k}}\right) \cdot \mathrm{r}}=\mathrm{G}_{\mathrm{k} 0} \cdot \frac{\mathrm{dX}}{\left(-v_{\mathrm{k}}\right) \cdot \mathrm{r}} \\
\frac{\mathrm{dT}}{\mathrm{d} \ell}-\mathrm{J} \cdot \frac{\mathrm{dX}}{\mathrm{d} \ell}=\frac{\mathrm{dQ}}{\mathrm{d} \ell}
\end{gathered}
$$

Se trata de dos ecuaciones diferenciales que hay que resolver simultáneamente bien analíticamente o bien, el caso más habitual, numéricamente utilizando un método numérico como el de Runge-Kutta de $4 .^{\circ}$ orden. Para ello conviene expresar las ecuaciones como:

$$
\frac{\mathrm{d} \ell}{\mathrm{dX}}=\frac{\mathrm{G}_{\mathrm{k} 0}}{\left(-v_{\mathrm{k}}\right) \cdot \mathrm{r}}
$$

$$
\frac{\mathrm{dT}}{\mathrm{d} \ell}=\mathrm{J} \cdot \frac{\mathrm{dX}}{\mathrm{d} \ell}+\frac{\mathrm{dQ}}{\mathrm{d} \ell}
$$

Como puede observarse, este par de ecuaciones consta de una variable independiente (la longitud 1) y dos variables dependientes de la longitud (X y T). Antes de proceder a su integración simultánea es conveniente ponerlas en la forma en que las dos diferenciales estén con respecto a la misma variable: $\mathrm{dl} / \mathrm{dX}$ y dl/dT, $\mathrm{dX} / \mathrm{dl}$ y dT/dl o dl/dX y dT/dX. Cualquier pareja es buena para realizar la integración, pero la más conveniente y habitual es la que resulta de transformar la ecuación de diseño (2) de la siguiente forma:

$$
\begin{gathered}
\frac{\mathrm{dT}}{\mathrm{d} \ell}=\frac{\mathrm{dQ}}{\mathrm{d} \ell}+\mathrm{J} \cdot \frac{\mathrm{dX}}{\mathrm{d} \ell} \\
\frac{\mathrm{dT}}{\mathrm{dX}}=\mathrm{J}+\frac{\mathrm{dQ}}{\mathrm{dX}}
\end{gathered}
$$

sustituir en el término de la derecha dX por la ecuación de diseño (1):

$$
\frac{\mathrm{dT}}{\mathrm{dX}}=\mathrm{J}+\frac{\mathrm{G}_{\mathrm{k} 0}}{\left(-v_{\mathrm{k}}\right) \cdot \mathrm{r}(\mathrm{X}, \mathrm{T})} \cdot \frac{\mathrm{dQ}}{\mathrm{d} \ell}
$$


Por lo tanto, el estudio de los RFP intermedios requiere la integración simultánea de las ecuaciones de diseño (3) y (4):

$$
\begin{gathered}
\frac{d \ell}{d X}=\frac{G_{k 0}}{\left(-v_{k}\right) \cdot r} \\
\frac{d T}{d X}=J+\frac{G_{k 0}}{\left(-v_{k}\right) \cdot r(X, T)} \cdot \frac{d Q}{d \ell}
\end{gathered}
$$

ECUACIÓN DE DISEÑO (3)

ECUACIÓN DE DISEÑO (4)

en las que el valor de $\mathrm{dQ} / \mathrm{dl}$ dependerá del sistema de intercambio de calor utilizado.

\subsubsection{RFP intermedio a densidad de flujo de calor $q$ constante}

Este tipo de intercambio de calor solo sirve para el caso de que se esté desarrollando una reacción endotérmica. La ecuación de velocidad de transmisión de calor es:

$$
\frac{d Q}{d \ell}=\frac{d Q^{*}}{\left(F_{k 0} \cdot \sum_{j=1}^{S} \theta_{j} \cdot \bar{C}_{p j}\right) \cdot d \ell}=\frac{q}{\left(F_{k 0} \cdot \sum_{j=1}^{S} \theta_{j} \cdot \bar{C}_{p j}\right)} \cdot \frac{d A}{d \ell}=\frac{q}{\left(F_{k 0} \cdot \sum_{j=1}^{S} \theta_{j} \cdot \bar{C}_{p j}\right)} \cdot \pi \cdot D=\frac{q}{\left(F_{k 0} \cdot \sum_{j=1}^{S} \theta_{j} \cdot \bar{C}_{p j}\right)} \cdot 2 \cdot \pi \cdot R
$$

por lo que las ecuaciones de diseño (3) y (4) quedan como:

$$
\begin{gathered}
\frac{d \ell}{d X}=\frac{G_{k 0}}{\left(-v_{k}\right) \cdot r} \\
\frac{d T}{d X}=J+\frac{G_{k 0}}{\left(-v_{k}\right) \cdot r(X, T)} \cdot \frac{q}{\left(F_{k 0} \cdot \sum_{j=1}^{S} \theta_{j} \cdot \bar{C}_{p j}\right)} \cdot \pi \cdot D=J+\frac{1}{\left(-v_{k}\right) \cdot r(X, T)} \cdot \frac{q}{R_{h} \cdot \sum_{j=1}^{S} \theta_{j} \cdot \bar{C}_{p j}}
\end{gathered}
$$

Estas dos ecuaciones se pueden integrar simultáneamente conocidos los valores iniciales de las variables, habitualmente: $1=0, X=0 \mathrm{y} \mathrm{T}=\mathrm{T}_{0}$. La integración numérica permite la obtención de una tabla de valores $1, \mathrm{X}, \mathrm{T}$, cuyas representaciones gráficas son de la forma que se muestra en la figura 3.
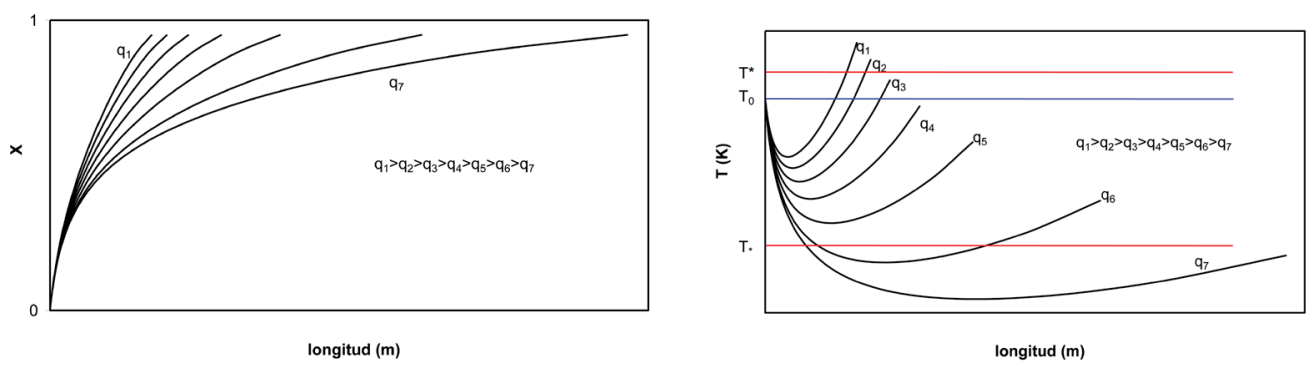


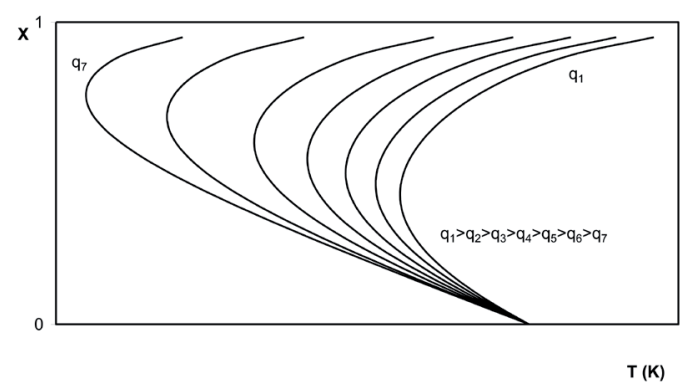

Figura 3. Evolución de las variables 1, X y T en un RFP a densidad de flujo de calor constante

Para calcular el valor de $q$ óptimo se debe fijar un valor final de $\mathrm{X}$ y resolver simultáneamente las ecuaciones de diseño (3) y (4), lo que permite determinar el valor de L para el X fijado, para diferentes valores de $q$. La representación de $q$ versus $\mathrm{L}$ (para el valor final de $\mathrm{X}$ deseado) da una curva como la que se muestra en la figura 4, fijándose el valor óptimo de $q$ en la zona de cambio brusco de pendiente.

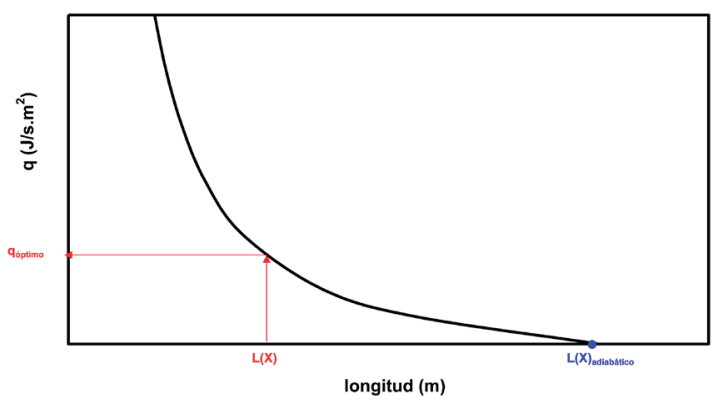

Figura 4. Cálculo del valor óptimo de $q$

\subsubsection{RFP intermedio a U constante}

Este tipo de intercambio de calor sirve tanto para el caso de que se esté desarrollando una reacción endotérmica como exotérmica. La ecuación de velocidad de transmisión de calor es:

$$
\frac{\mathrm{dQ}}{\mathrm{d} \ell}=\frac{\mathrm{dQ} *}{\left(\mathrm{~F}_{\mathrm{k} 0} \cdot \sum_{\mathrm{j}=1}^{\mathrm{S}} \theta_{\mathrm{j}} \cdot \overline{\mathrm{C}}_{\mathrm{pj}}\right) \cdot \mathrm{d} \ell}=\frac{\mathrm{U} \cdot\left(\mathrm{T}_{\mathrm{f}}-\mathrm{T}\right)}{\left(\mathrm{F}_{\mathrm{k} 0} \cdot \sum_{\mathrm{j}=1}^{\mathrm{S}} \theta_{\mathrm{j}} \cdot \overline{\mathrm{C}}_{\mathrm{pj}}\right)} \cdot \frac{\mathrm{dA}}{\mathrm{d} \ell}=\frac{\mathrm{U} \cdot\left(\mathrm{T}_{\mathrm{f}}-\mathrm{T}\right)}{\left(\mathrm{F}_{\mathrm{k} 0} \cdot \sum_{\mathrm{j}=1}^{\mathrm{S}} \theta_{\mathrm{j}} \cdot \overline{\mathrm{C}}_{\mathrm{pj}}\right)} \cdot \mathrm{A}_{\mathrm{S}}=\frac{\left(\mathrm{T}_{\mathrm{f}}-\mathrm{T}\right) \cdot \mathrm{St}}{\mathrm{R}_{\mathrm{h}}}
$$

en la que U (o el módulo de Stanton) es constante; por lo tanto, las ecuaciones de diseño (3) y (4) quedan como sigue:

$$
\begin{gathered}
\frac{d \ell}{d X}=\frac{G_{k 0}}{\left(-v_{k}\right) \cdot r} \\
\frac{d T}{d X}=J+\frac{G_{k 0}}{\left(-v_{k}\right) \cdot r(X, T)} \cdot \frac{\left(T_{f}-T\right) \cdot S t}{R_{h}}
\end{gathered}
$$


Estas dos ecuaciones se pueden integrar simultáneamente conocidos los valores iniciales de las variables, habitualmente: $1=0, X=0$ y $T=T_{0}$. La integración numérica permite la obtención de una tabla de valores $1, \mathrm{X}, \mathrm{T}$. Se distinguirán dos posibles casos según $T_{f}$ sea constante o variable:

\section{Caso 1. El valor de $T_{f}$ permanece constante}

En este caso las ecuaciones:

$$
\begin{gathered}
\frac{d \ell}{d X}=\frac{G_{k 0}}{\left(-v_{k}\right) \cdot r} \\
\frac{d T}{d X}=J+\frac{G_{k 0}}{\left(-v_{k}\right) \cdot r(X, T)} \cdot \frac{\left(T_{f}-T\right) \cdot S t}{R_{h}}
\end{gathered}
$$

solo tienen tres variables (la independiente 1, y las dos dependientes de la longitud X y T) por lo que se pueden integrar simultáneamente. La representación gráfica de la tabla de valores $1, X, T$ que se obtiene en la integración depende de que la reacción sea exotérmica o endotérmica y son de la forma que se muestra en la figura 5.

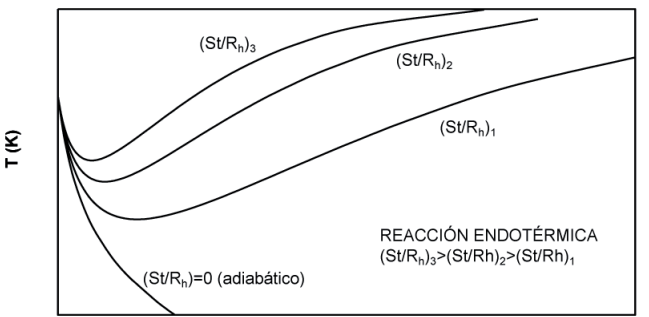

longitud ( $\mathrm{m})$

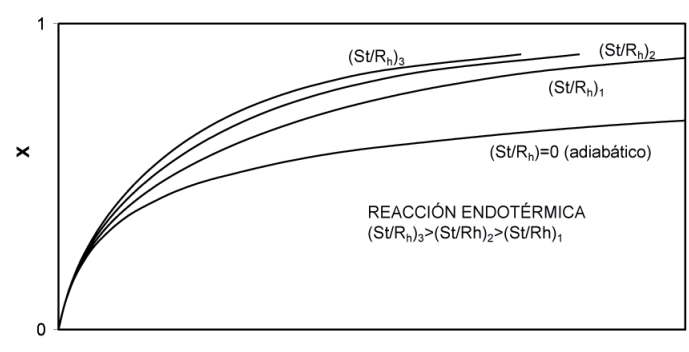

longitud ( $m$ )

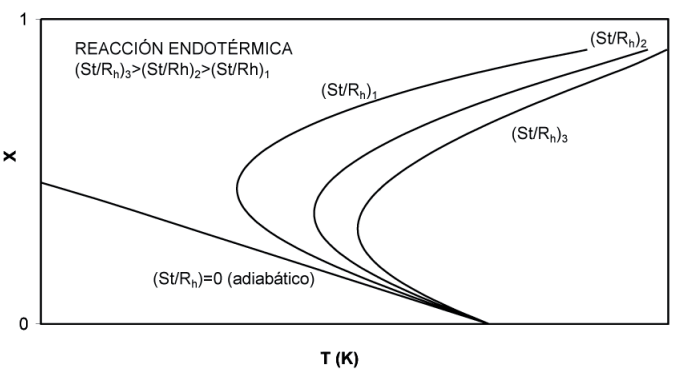

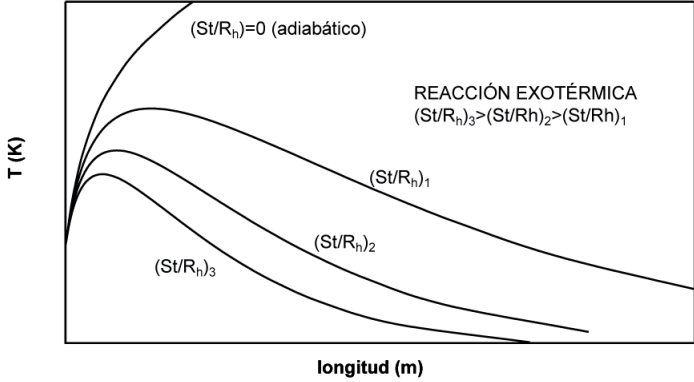
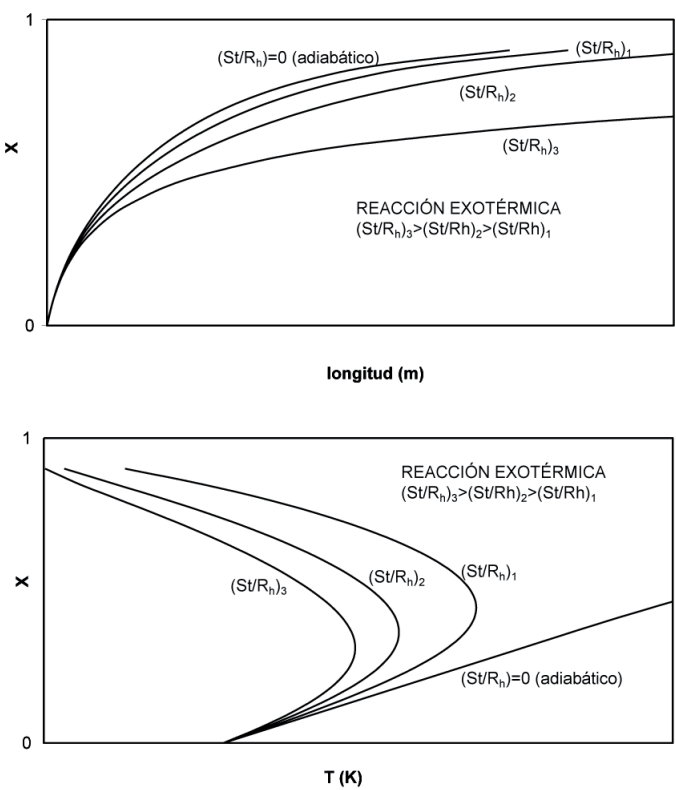

Figura 5. Evolución de las variables $1, \mathrm{X}$ y $\mathrm{T}$ en un RFP intermedio con $\mathrm{T}_{\mathrm{f}}$ constante 
Para calcular el valor de $\mathrm{St} / \mathrm{R}_{\mathrm{h}}$ óptimo se debe fijar un valor final de $\mathrm{X}$ y resolver simultáneamente las ecuaciones de diseño (3) y (4), lo que permite determinar el valor de $L$ para el $X$ fijado, para diferentes valores de $S t / R_{h}$. La representación de $S t / R_{h}$ versus $L$ (para el valor final de $X$ deseado) da una curva como la que se muestra en la figura 6, fijándose el valor óptimo de $S t / R_{h}$ en la zona de cambio brusco de pendiente.
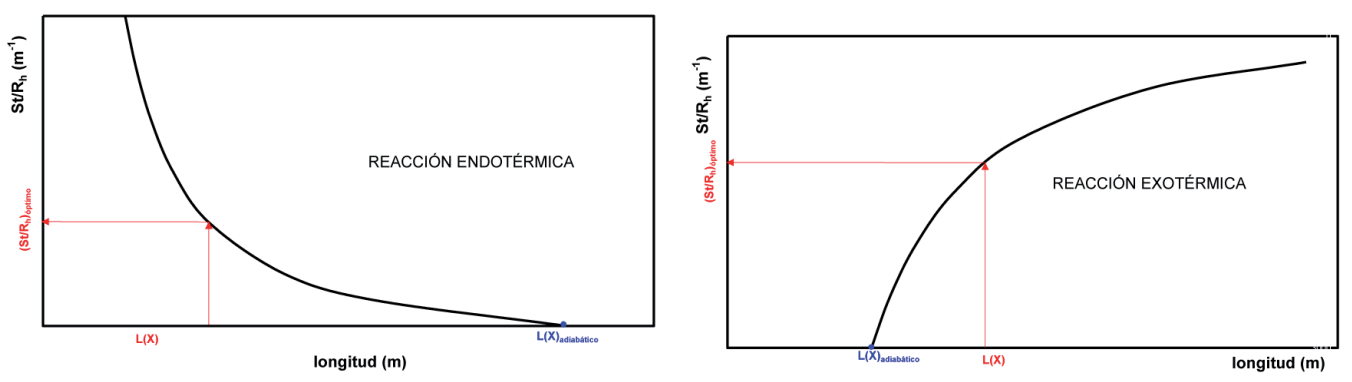

Figura 6. Cálculo del valor óptimo de $\mathrm{St} / \mathrm{R}_{\mathrm{h}}$

Como se ha indicado, la integración simultánea de las ecuaciones de diseño (3) y (4) depende de las condiciones iniciales que, habitualmente, son $1=0, X=0 \mathrm{y}$ $\mathrm{T}=\mathrm{T}_{0}$. Resulta evidente que, de estas tres condiciones iniciales, es la temperatura la que puede optar por diferentes valores, influyendo de forma determinante en los resultados de la integración.

INFLUENCIA DE $\mathrm{T}_{0}$ SOBRE LAS CURVAS X-T PARA UNA REACCIÓN EXOTÉRMICA

La ecuación de diseño (4) para este tipo de intercambio de calor se ha utilizado en la forma:

$$
\frac{d T}{d X}=J+\frac{G_{k 0}}{\left(-v_{k}\right) \cdot r(X, T)} \cdot \frac{\left(T_{f}-T\right) \cdot S t}{R_{h}}
$$

donde se cumple que por ser la reacción exotérmica:

i) $\mathrm{J}=\frac{-\Delta \mathrm{H}_{\mathrm{k}}^{0}}{\sum_{\mathrm{j}=1}^{\mathrm{S}} \theta_{\mathrm{j}} \cdot \overline{\mathrm{C}}_{\mathrm{pj}}}>0$

ii) el fluido del intercambiador de calor actúa como refrigerante $\mathrm{T}_{\mathrm{f}}<\mathrm{T}_{0} ; \mathrm{T}_{\mathrm{f}} \leq \mathrm{T}$

Por lo tanto la ecuación $\mathrm{dT} / \mathrm{dX}$ se puede reescribir:

$$
\frac{\mathrm{dT}}{\mathrm{dX}}=|\mathrm{J}|-\left|\frac{\left(\mathrm{T}_{\mathrm{f}}-\mathrm{T}\right) \cdot \mathrm{St}}{\mathrm{R}_{\mathrm{h}}} \cdot \frac{\mathrm{G}_{\mathrm{k} 0}}{\left(-v_{\mathrm{k}}\right) \cdot \mathrm{r}[\mathrm{X}, \mathrm{T}]}\right| \begin{gathered}
<0 \\
=0 \\
>0
\end{gathered}
$$


en la que $J$ es constante mientras que el segundo término de la parte derecha de la igualdad depende del valor de la temperatura porque afecta a la diferencia $\mathrm{T}_{\mathrm{f}}-\mathrm{T}\left[\mathrm{T}_{0}\right.$ en $1=0$ y $X=0$ y T en $1(X)]$ y a la velocidad de reacción $r$. Por lo tanto se pueden plantear dos preguntas:

a) ¿Cómo es el inicio de la curva T,X en función de dónde se sitúe $T_{0}$ ?

Se responde a la pregunta analizando $\mathrm{dT} / \mathrm{dX}$ en el punto de inicio $\mathrm{l}=0, \mathrm{X}=$ 0 y $\mathrm{T}=\mathrm{T}_{0}$, resultando:

$$
\left[\frac{\mathrm{dT}}{\mathrm{dX}}\right]_{\mathrm{X}=0}=|\mathrm{J}|-\left|\frac{\left(\mathrm{T}_{\mathrm{f}}-\mathrm{T}_{0}\right) \cdot \mathrm{St}}{\mathrm{R}_{\mathrm{h}}} \cdot \frac{\mathrm{G}_{\mathrm{k} 0}}{\left(-\mathrm{v}_{\mathrm{k}}\right) \cdot \mathrm{r}_{0}\left[0, \mathrm{~T}_{0}\right]}\right| \begin{aligned}
& <0, \text { es decir } \mathrm{T}_{0} \text { próximo a } \mathrm{T}_{\mathrm{f}} \\
& =0 \\
& >0 \text {, es decir } \mathrm{T}_{0} \text { próximo a } \mathrm{T}_{\mathrm{f}}
\end{aligned}
$$

que en la figura 7 corresponde a los puntos:

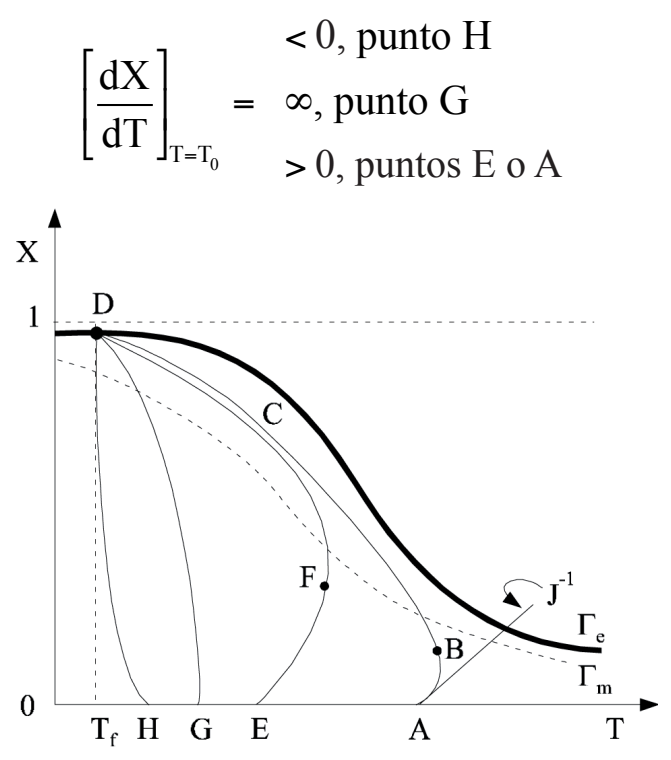

Figura 7. Influencia de $\mathrm{T}_{0}$ sobre la curva $\mathrm{X}$ versus $\mathrm{T}$

b) ¿Cómo afecta $\mathrm{T}_{0}$ a la forma de una curva $\mathrm{T}, \mathrm{X}$ ?

Se responde a la pregunta analizando $\mathrm{dT} / \mathrm{dX}$ en cualquier punto intermedio del reactor $1, \mathrm{X}$ y $\mathrm{T}$, resultando:

$$
\frac{\mathrm{dT}}{\mathrm{dX}}=|\mathrm{J}|-\left|\frac{\left(\mathrm{T}_{\mathrm{f}}-\mathrm{T}\right) \cdot \mathrm{St}}{\mathrm{R}_{\mathrm{h}}} \cdot \frac{\mathrm{G}_{\mathrm{k} 0}}{\left(-v_{\mathrm{k}}\right) \cdot \mathrm{r}[\mathrm{X}, \mathrm{T}]}\right| \begin{aligned}
& >0, \text { es decir X pequeños } \\
& =0 \\
& <0, \text { es decir X grandes }
\end{aligned}
$$

que en la figura 7 , donde se representa $\mathrm{X}$ frente a $\mathrm{T}$, corresponde a los puntos:

$$
\begin{aligned}
\frac{\mathrm{dX}}{\mathrm{dT}}= & >0, \text { punto } \mathrm{B} \text { o } \mathrm{F}, \text { punto caliente } \\
& <0, \text { punto } \mathrm{C}
\end{aligned}
$$


INFLUENCIA DE $\mathrm{T}_{0}$ SOBRE LAS CURVAS X-T PARA UNA REACCIÓN ENDOTÉRMICA

La ecuación de diseño (4) para este tipo de intercambio de calor se ha utilizado en la forma:

$$
\frac{d T}{d X}=J+\frac{G_{k 0}}{\left(-v_{k}\right) \cdot r(X, T)} \cdot \frac{\left(T_{f}-T\right) \cdot S t}{R_{h}}
$$

donde se cumple que por ser la reacción endotérmica:

i) $\mathrm{J}=\frac{-\Delta \mathrm{H}_{\mathrm{k}}^{0}}{\sum_{\mathrm{j}=1}^{\mathrm{S}} \theta_{\mathrm{j}} \cdot \overline{\mathrm{C}}_{\mathrm{pj}}}<0$

ii) el fluido del intercambiador de calor actúa como calefactor $\mathrm{T}_{\mathrm{f}}>\mathrm{T}_{0} ; \mathrm{T}_{\mathrm{f}} \geq \mathrm{T}$

Por lo tanto la ecuación $\mathrm{dT} / \mathrm{dX}$ se puede reescribir:

$$
\frac{\mathrm{dT}}{\mathrm{dX}}=\left|\frac{\left(\mathrm{T}_{\mathrm{f}}-\mathrm{T}\right) \cdot \mathrm{St}}{\mathrm{R}_{\mathrm{h}}} \cdot \frac{\mathrm{G}_{\mathrm{k} 0}}{\left(-v_{\mathrm{k}}\right) \cdot \mathrm{r}[\mathrm{X}, \mathrm{T}]}\right|-|\mathrm{J}| \begin{array}{r}
<0 \\
=0 \\
>0
\end{array}
$$

en la que $\mathrm{J}$ es constante mientras que el segundo término de la parte derecha de la igualdad depende del valor de la temperatura porque afecta a la diferencia $T_{f}-T$ $\left[\mathrm{T}_{0}\right.$ en $1=0$ y $\mathrm{X}=0$ y $\mathrm{T}$ en $\left.1(\mathrm{X})\right]$ y a la velocidad de reacción $\mathrm{r}$. Por lo tanto se pueden plantear dos preguntas:

a) ¿Cómo es el inicio de la curva T,X en función de dónde se sitúe $\mathrm{T}_{0}$ ?

Se responde a la pregunta analizando $\mathrm{dT} / \mathrm{dX}$ en el punto de inicio $1=0, \mathrm{X}=$ 0 y $\mathrm{T}=\mathrm{T}_{0}$, resultando:

$$
\left[\frac{\mathrm{dT}}{\mathrm{dX}}\right]_{\mathrm{X}=0}=\left|\frac{\left(\mathrm{T}_{\mathrm{f}}-\mathrm{T}_{0}\right) \cdot \mathrm{St}}{\mathrm{R}_{\mathrm{h}}} \cdot \frac{\mathrm{G}_{\mathrm{k} 0}}{\left(-v_{\mathrm{k}}\right) \cdot \mathrm{r}_{0}\left[0, \mathrm{~T}_{0}\right]}\right|-|\mathrm{J}| \begin{aligned}
& <0, \text { es decir } \mathrm{T}_{0} \text { próximo a } \mathrm{T}_{\mathrm{f}} \\
& \quad 0 \\
& >0, \text { es decir } \mathrm{T}_{0} \text { alejado de } \mathrm{T}_{\mathrm{f}}
\end{aligned}
$$

que en la figura 8 , donde se representa $\mathrm{X}$ frente a $\mathrm{T}$, corresponde a los puntos:

$$
\left[\frac{\mathrm{dX}}{\mathrm{dT}}\right]_{\mathrm{T}=\mathrm{T}_{0}}=\stackrel{<0, \text { punto } \mathrm{A}}{>0 \text { puntos } \mathrm{F} \text { o G }}
$$




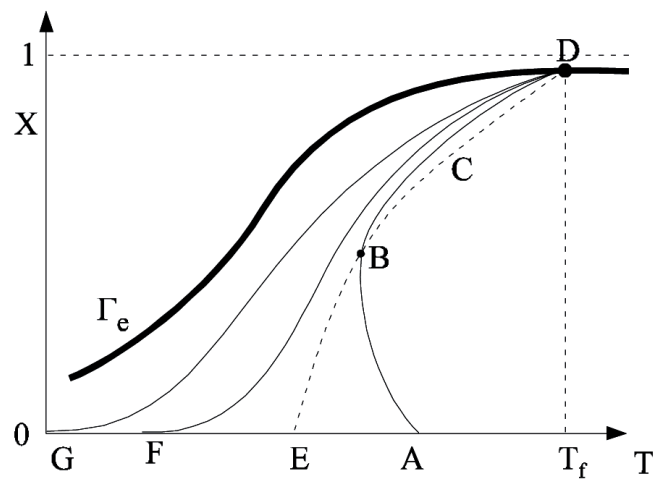

Figura 8. Influencia de $\mathrm{T}_{0}$ sobre la curva $\mathrm{X}$ versus $\mathrm{T}$

b) ¿Cómo afecta $T_{0}$ a la forma de una curva $T, X$ ?

Se responde a la pregunta analizando $\mathrm{dT} / \mathrm{dX}$ en cualquier punto intermedio del reactor $1, \mathrm{X}$ y $\mathrm{T}$, resultando:

$$
\frac{\mathrm{dT}}{\mathrm{dX}}=\left|\frac{\left(\mathrm{T}_{\mathrm{f}}-\mathrm{T}\right) \cdot \mathrm{St}}{\mathrm{R}_{\mathrm{h}}} \cdot \frac{\mathrm{G}_{\mathrm{k} 0}}{\left(-v_{\mathrm{k}}\right) \cdot \mathrm{r}[\mathrm{X}, \mathrm{T}]}\right|-|\mathrm{J}| \begin{aligned}
& <0, \text { es decir X pequeños } \\
& =0,
\end{aligned}
$$

que en la figura 8 , donde se representa $\mathrm{X}$ frente a $\mathrm{T}$, corresponde a los puntos:

$$
\begin{aligned}
& <0, \text { punto } \\
\frac{\mathrm{dX}}{\mathrm{dT}}= & \infty, \text { punto } \mathrm{B}, \text { punto frío } \\
& <0, \text { punto } \mathrm{D}
\end{aligned}
$$

Caso 2. El valor de $T_{f}$ varía a lo largo de la longitud del reactor

En este caso las ecuaciones:

$$
\begin{gathered}
\frac{d \ell}{d X}=\frac{G_{k 0}}{\left(-v_{k}\right) \cdot r} \\
\frac{d T}{d X}=J+\frac{G_{k 0}}{\left(-v_{k}\right) \cdot r(X, T)} \cdot \frac{\left(T_{f}-T\right) \cdot S t}{R_{h}}
\end{gathered}
$$

solo cuatro variables (la independiente 1 , las dos dependientes de la longitud X y T y $\mathrm{T}_{\mathrm{f}}$ ). En consecuencia, para poder integrarlas simultáneamente lo primero que se requiere es encontrar una función que relacione $\mathrm{T}_{\mathrm{f}}$ con $\mathrm{X}$.

En este capítulo se van a estudiar las cuatro posibles disposiciones de este tipo de RFP que se muestran en la figura 9. En todas ellas el fluido intercambiador circula 
a lo largo de la pared del reactor, de modo que $\mathrm{T}_{\mathrm{f}}$ es una función de 1, así como la conversión $(\mathrm{X})$ y la temperatura $(\mathrm{T})$ dentro del reactor:

Disposiciones $a$ ) y $b$ ): el fluido intercambiador es distinto de la mezcla de reacción y sirven tanto para reacciones exotérmicas como endotérmicas.

Disposiciones $c$ ) y $d$ ): el fluido intercambiador es el mismo que la mezcla de reacción y sirven solo para reacciones exotérmicas, especialmente las catalíticas. Se denominan reactores RFP autotérmicos

En las disposiciones $a$ ) y c) los fluidos circulan en paralelo, mientras que en la $b$ ) y $d$ ) lo hacen en contracorriente.

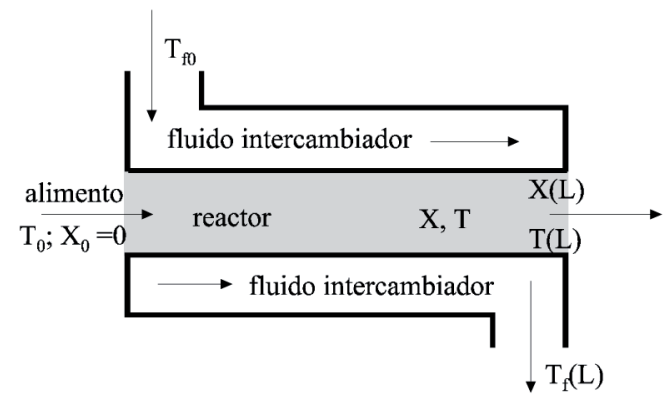

a) Paralelo. Fluido refrigerante o calefactor independiente.

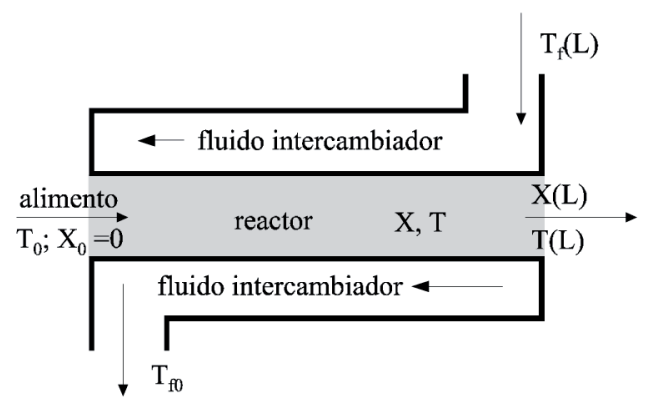

b) Contracorriente. Fluido refrigerante o calefactor independiente.

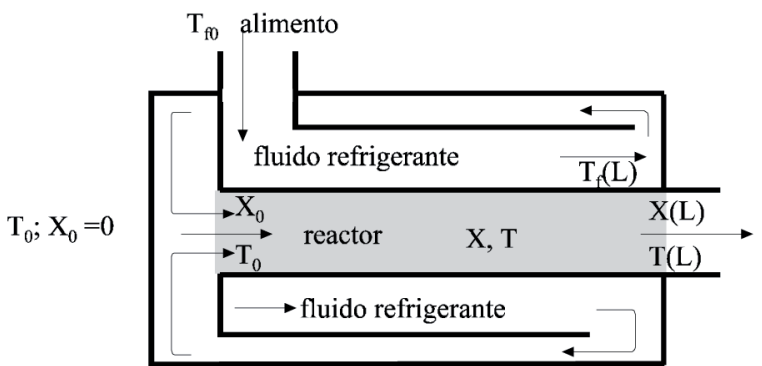

c) Paralelo. Alimento como fluido refrigerante.

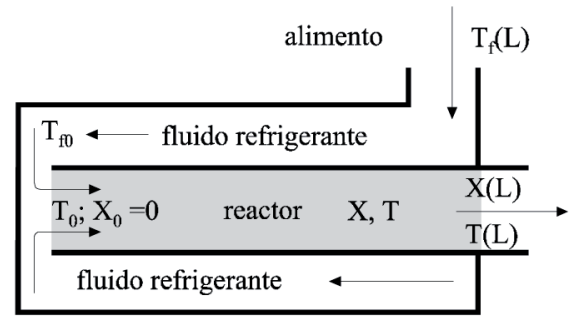

d) Contracorriente. Alimento como fluido refrigerante.

Figura 9. Disposiciones básicas de un RFP intermedio con $T_{f}$ variable

Como puede apreciarse en la figura 10, que pretende simplificar las cuatro disposiciones anteriores y que recoge las dos formas de circulación, en adelante se designará por plano 0 el de la izquierda de cualquier disposición mientras que el plano L será el de la derecha de cualquier disposición, independientemente de que trate de entradas o salidas de fluidos. Todas las variables en el plano 0 llevarán el mismo como subíndice y todas las variables en el plano L lo indicarán en la forma (L). Así, por ejemplo, en la circulación en paralelo $\mathrm{T}_{\mathrm{f}}(\mathrm{L})$ representa la temperatura de salida del fluido intercambiador, pero en circulación en contracorriente representa la temperatura de entrada del fluido intercambiador. 


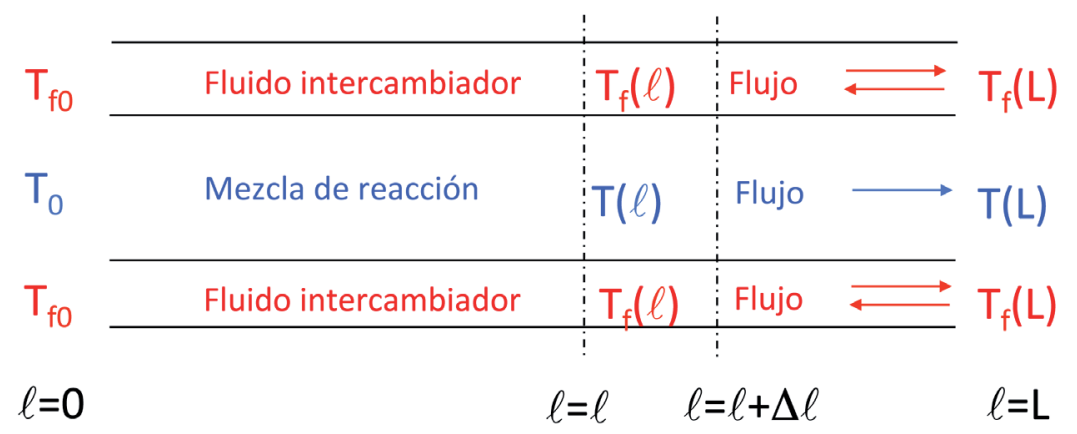

Figura 10. Puntos de referencia

Para correlacionar $\mathrm{T}_{\mathrm{f}}$ con $\mathrm{X}$ se requiere aplicar un balance de energía al sistema de intercambio de calor (conviene aquí recordar los conceptos aprendidos en la asignatura EQ1020-Operaciones Básicas de Transmisión de Calor), que junto con el de la mezcla de reacción permite obtener dicha relación, la cual depende de que la circulación sea en paralelo o en contracorriente. A continuación se muestra cómo obtener la citada relación en ambos casos.

\section{Circulación en PARAlelo}

El balance de energía aplicado al fluido intercambiador es $\mathrm{dQ}^{*}=-\mathrm{m}_{\mathrm{f}} \cdot \overline{\mathrm{C}}_{\mathrm{pf}} \cdot \mathrm{dT}_{\mathrm{f}} \mathrm{y}$ el de la mezcla de reacción, como ya se ha visto $\mathrm{dT}-\mathrm{J} \cdot \mathrm{dX}=\mathrm{dQ}=\frac{\mathrm{dQ}^{*}}{\mathrm{~F}_{\mathrm{k} 0} \cdot \sum_{\mathrm{j}=1}^{\mathrm{S}} \theta_{\mathrm{j}} \cdot \overline{\mathrm{C}}_{\mathrm{pj}}}$ Igualando ambas ecuaciones, puesto que $\mathrm{dQ}^{*}$ tiene que ser el mismo:

$$
\begin{aligned}
& \frac{-\mathrm{m}_{\mathrm{f}} \cdot \overline{\mathrm{C}}_{\mathrm{pf}} \cdot \mathrm{dT}_{\mathrm{f}}}{\mathrm{F}_{\mathrm{k} 0} \cdot \sum_{\mathrm{j}=1}^{\mathrm{j}=\mathrm{S}} \theta_{\mathrm{j}} \cdot \overline{\mathrm{C}}_{\mathrm{pj}}}=\mathrm{dT}-\mathrm{J} \cdot \mathrm{dX} \\
& \quad \text { si se define el parámetro } \gamma: \gamma=\frac{\mathrm{F}_{\mathrm{k} 0} \cdot \sum_{\mathrm{j}=1}^{\mathrm{j}=\mathrm{S}} \theta_{\mathrm{j}} \cdot \overline{\mathrm{C}}_{\mathrm{pj}}}{\mathrm{m}_{\mathrm{f}} \cdot \overline{\mathrm{C}}_{\mathrm{pf}}} \\
& -\mathrm{dT}_{\mathrm{f}}=\gamma \cdot \mathrm{dT}-\gamma \cdot \mathrm{J} \cdot \mathrm{dX}
\end{aligned}
$$

e integrando entre los planos 0 y L:

$$
-\int_{\mathrm{T}_{\mathrm{f} 0}}^{\mathrm{T}_{\mathrm{f}}} \mathrm{dT}_{\mathrm{f}}=\gamma \cdot \int_{\mathrm{T}_{0}}^{\mathrm{T}} \mathrm{dT}-\gamma \cdot \mathrm{J} \cdot \int_{0}^{\mathrm{X}} \mathrm{dX}
$$

se obtiene:

1. Para la disposición $a) \mathrm{T}_{\mathrm{f}}=\mathrm{T}_{\mathrm{f} 0}+\gamma \cdot\left(\mathrm{T}_{0}-\mathrm{T}+\mathrm{J} \cdot \mathrm{X}\right)$

2. Para la disposición $c$ ) (teniendo en cuenta que $\gamma=1$ por coincidir el fluido intercambiador con el de la mezcla de reacción) $\mathrm{T}_{\mathrm{f}}=\mathrm{T}_{\mathrm{f} 0}+\left(\mathrm{T}_{0}-\mathrm{T}+\mathrm{J} \cdot \mathrm{X}\right)$ 


\section{Circulación En CONTRACORRIEnTE}

El balance de energía aplicado al fluido intercambiador es $\mathrm{dQ}^{*}=\mathrm{m}_{\mathrm{f}} \cdot \overline{\mathrm{C}}_{\mathrm{pf}} \cdot \mathrm{dT}_{\mathrm{f}} \mathrm{y}$ el de la mezcla de reacción, como ya se ha visto $\mathrm{dT}-\mathrm{J} \cdot \mathrm{dX}=\mathrm{dQ}=\frac{\mathrm{dQ}^{*}}{\mathrm{~F}_{\mathrm{k} 0} \cdot \sum_{\mathrm{j}=1}^{\mathrm{S}} \theta_{\mathrm{j}} \cdot \overline{\mathrm{C}}_{\mathrm{pj}}}$

Igualando ambas ecuaciones, puesto que $\mathrm{dQ}^{*}$ tiene que ser el mismo:

$$
\frac{\mathrm{m}_{\mathrm{f}} \cdot \overline{\mathrm{C}}_{\mathrm{pf}} \cdot \mathrm{dT}_{\mathrm{f}}}{\mathrm{F}_{\mathrm{k} 0} \cdot \sum_{\mathrm{j}=1}^{\mathrm{j}=\mathrm{S}} \theta_{\mathrm{j}} \cdot \overline{\mathrm{C}}_{\mathrm{pj}}}=\mathrm{dT}-\mathrm{J} \cdot \mathrm{dX}
$$

si se define el parámetro $\gamma: \gamma=\frac{\mathrm{F}_{\mathrm{k} 0} \cdot \sum_{\mathrm{j}=1}^{\mathrm{j}=\mathrm{S}} \theta_{\mathrm{j}} \cdot \overline{\mathrm{C}}_{\mathrm{pj}}}{\mathrm{m}_{\mathrm{f}} \cdot \overline{\mathrm{C}}_{\mathrm{pf}}}$

$$
\mathrm{dT}_{\mathrm{f}}=\gamma \cdot \mathrm{dT}-\gamma \cdot \mathrm{J} \cdot \mathrm{dX}
$$

e integrando entre los planos 0 y L:

$$
\int_{\mathrm{T}_{\mathrm{f} 0}}^{\mathrm{T}_{\mathrm{f}}} \mathrm{dT}_{\mathrm{f}}=\gamma \cdot \int_{\mathrm{T}_{0}}^{\mathrm{T}} \mathrm{dT}-\gamma \cdot \mathrm{J} \cdot \int_{0}^{\mathrm{X}} \mathrm{dX}
$$

se obtiene:

1. Para la disposición $b) \mathrm{T}_{\mathrm{f}}=\mathrm{T}_{\mathrm{f} 0}-\gamma \cdot\left(\mathrm{T}_{0}-\mathrm{T}+\mathrm{J} \cdot \mathrm{X}\right)$

2. Para la disposición $d$ ) (teniendo en cuenta que $\gamma=1$ por coincidir el fluido intercambiador con el de la mezcla de reacción) $\mathrm{T}_{\mathrm{f}}=\mathrm{T}_{\mathrm{f} 0}-\left(\mathrm{T}_{0}-\mathrm{T}+\mathrm{J} \cdot \mathrm{X}\right)$

\section{CONCLUSIÓN}

En la tabla 1 se recogen las cuatro posibles expresiones de $T_{f}$ según el tipo de circulación para un RFP.

Tabla 1. Expresiones de $\mathrm{T}_{\mathrm{f}}$ según circulación y disposición en el RFP

\begin{tabular}{|c|c|c|c|}
\hline \multirow{2}{*}{ en paralelo } & Disposición & $\mathrm{T}_{\mathrm{f}}$ & Tipo de reactor \\
\cline { 2 - 4 } & $\mathrm{a}$ & $\mathrm{T}_{\mathrm{f}}=\mathrm{T}_{\mathrm{f} 0}+\gamma \cdot\left(\mathrm{T}_{0}-\mathrm{T}+\mathrm{J} \cdot \mathrm{X}\right)$ & RFP \\
\hline \multirow{2}{*}{ en contracorriente } & $\mathrm{c}$ & $\mathrm{T}_{\mathrm{f}}=\mathrm{T}_{\mathrm{f} 0}+\left(\mathrm{T}_{0}-\mathrm{T}+\mathrm{J} \cdot \mathrm{X}\right)$ & RFP autotérmico \\
\cline { 2 - 4 } & $\mathrm{b}$ & $\mathrm{T}_{\mathrm{f}}=\mathrm{T}_{\mathrm{f} 0}-\gamma \cdot\left(\mathrm{T}_{0}-\mathrm{T}+\mathrm{J} \cdot \mathrm{X}\right)$ & RFP \\
\cline { 2 - 4 } & $\mathrm{d}$ & $\mathrm{T}_{\mathrm{f}}=\mathrm{T}_{\mathrm{f} 0}-\left(\mathrm{T}_{0}-\mathrm{T}+\mathrm{J} \cdot \mathrm{X}\right)$ & RFP autotérmico \\
\hline
\end{tabular}


Caso 2. Disposición a)

En este caso las ecuaciones a integrar simultáneamente son:

$$
\begin{gathered}
\frac{d \ell}{d X}=\frac{G_{k 0}}{\left(-v_{k}\right) \cdot r} \\
\frac{d T}{d X}=J+\frac{G_{k 0}}{\left(-v_{k}\right) \cdot r(X, T)} \cdot \frac{\left(T_{f}-T\right) \cdot S t}{R_{h}}
\end{gathered}
$$

junto con:

$$
\mathrm{T}_{\mathrm{f}}=\mathrm{T}_{\mathrm{f} 0}+\gamma \cdot\left(\mathrm{T}_{0}-\mathrm{T}+\mathrm{J} \cdot \mathrm{X}\right)
$$

donde:

$\mathrm{T}_{\mathrm{f} 0}=$ temperatura de entrada del fluido intercambiador (conocida)

$\mathrm{T}_{f}(1)=$ temperatura en una posición intermedia del fluido intercambiador (desconocida)

$\mathrm{T}_{\mathrm{f}}(\mathrm{L})=$ temperatura a la salida del fluido intercambiador (desconocida)

$\mathrm{T}_{0}=$ temperatura de entrada de la mezcla de reacción (conocida)

$\mathrm{T}(\mathrm{l})=$ temperatura en una posición intermedia de la mezcla de reacción (desconocida)

$\mathrm{T}(\mathrm{L})=$ temperatura a la salida de la mezcla de reacción (desconocida)

El procedimiento de resolución consiste en sustituir $\mathrm{T}_{\mathrm{f}}$ por su función de $\mathrm{X}$ quedando de nuevo dos ecuaciones diferenciales:

$$
\begin{gathered}
\frac{\mathrm{d} \ell}{\mathrm{dX}}=\frac{\mathrm{G}_{\mathrm{k} 0}}{\left(-v_{\mathrm{k}}\right) \cdot \mathrm{r}} \\
\frac{\mathrm{dT}}{\mathrm{dX}}=\mathrm{J}+\frac{\mathrm{G}_{\mathrm{k} 0}}{\left(-v_{\mathrm{k}}\right) \cdot \mathrm{r}(\mathrm{X}, \mathrm{T})} \cdot \frac{\left[\mathrm{T}_{\mathrm{f} 0}+\gamma \cdot\left(\mathrm{T}_{0}-\mathrm{T}+\mathrm{J} \cdot \mathrm{X}\right)-\mathrm{T}\right] \cdot \mathrm{St}}{\mathrm{R}_{\mathrm{h}}}
\end{gathered}
$$

que se integran simultáneamente para valores conocidos de $\mathrm{T}_{\mathrm{f} 0} \mathrm{y}$ las condiciones iniciales habituales de $\mathrm{T}_{0}, 1=0$ y $\mathrm{X}=0$, obteniéndose una tabla de valores de $\mathrm{X}, \mathrm{T}$, 1. Si en esta tabla se desean conocer los valores de $\mathrm{T}_{\mathrm{f}}$ se pueden calcular mediante $\mathrm{T}_{\mathrm{f}}=\mathrm{T}_{\mathrm{f} 0}+\gamma \cdot\left(\mathrm{T}_{0}-\mathrm{T}+\mathrm{J} \cdot \mathrm{X}\right)$

\section{Caso 2. Disposición b)}

En este caso las ecuaciones a integrar simultáneamente son:

$$
\begin{gathered}
\frac{d \ell}{d X}=\frac{G_{k 0}}{\left(-v_{k}\right) \cdot r} \\
\frac{d T}{d X}=J+\frac{G_{k 0}}{\left(-v_{k}\right) \cdot r(X, T)} \cdot \frac{\left(T_{f}-T\right) \cdot S t}{R_{h}}
\end{gathered}
$$


junto con:

$$
\mathrm{T}_{\mathrm{f}}=\mathrm{T}_{\mathrm{f} 0}-\gamma \cdot\left(\mathrm{T}_{0}-\mathrm{T}+\mathrm{J} \cdot \mathrm{X}\right)
$$

donde:

$\mathrm{T}_{\mathrm{f} 0}=$ temperatura a la salida del fluido intercambiador (desconocida)

$T_{f}(1)=$ temperatura en una posición intermedia del fluido intercambiador (desconocida)

$\mathrm{T}_{\mathrm{f}}(\mathrm{L})=$ temperatura de entrada del fluido intercambiador (conocida)

$\mathrm{T}_{0}=$ temperatura de entrada de la mezcla de reacción (conocida)

$\mathrm{T}(\mathrm{l})=$ temperatura en una posición intermedia de la mezcla de reacción (desconocida)

$\mathrm{T}(\mathrm{L})=$ temperatura a la salida de la mezcla de reacción (desconocida)

El procedimiento de resolución consiste en sustituir $\mathrm{T}_{\mathrm{f}}$ por su función de $\mathrm{X}$ quedando de nuevo dos ecuaciones diferenciales:

$$
\begin{gathered}
\frac{d \ell}{d X}=\frac{G_{k 0}}{\left(-v_{k}\right) \cdot r} \\
\frac{d T}{d X}=J+\frac{G_{k 0}}{\left(-v_{k}\right) \cdot r(X, T)} \cdot \frac{\left[T_{f 0}-\gamma \cdot\left(T_{0}-T+J \cdot X\right)-T\right] \cdot S t}{R_{h}}
\end{gathered}
$$

que se integran las condiciones iniciales habituales de $\mathrm{T}_{0}, 1=0$ y $\mathrm{X}=0$, obteniéndose una tabla de valores de X, T, 1. Para ello, y dado que se desconoce el valor de $\mathrm{T}_{\mathrm{f} 0}$, hay que ir probando valores de $\mathrm{T}_{\mathrm{f} 0}$. En esta tabla se puede calcular $\mathrm{T}_{\mathrm{f}}$ mediante $\mathrm{T}_{\mathrm{f}}=\mathrm{T}_{\mathrm{f} 0}+\gamma \cdot\left(\mathrm{T}_{0}-\mathrm{T}+\mathrm{J} \cdot \mathrm{X}\right)$

El valor adecuado de $\mathrm{T}_{\mathrm{f} 0}$ es aquel que permite obtener unos valores $1=\mathrm{L}, \mathrm{X}=$ $\mathrm{X}(\mathrm{L}), \mathrm{T}=\mathrm{T}(\mathrm{L})$ y $\mathrm{T}_{\mathrm{f}}=\mathrm{T}_{\mathrm{f}}(\mathrm{L})$ en el que $\mathrm{T}_{\mathrm{f}}=\mathrm{T}_{\mathrm{f}}(\mathrm{L})$ coincide con el valor conocido.

\section{Caso 3. Disposición c)}

En este caso las ecuaciones a integrar simultáneamente son:

$$
\begin{gathered}
\frac{d \ell}{d X}=\frac{G_{k 0}}{\left(-v_{k}\right) \cdot r} \\
\frac{d T}{d X}=J+\frac{G_{k 0}}{\left(-v_{k}\right) \cdot r(X, T)} \cdot \frac{\left(T_{f}-T\right) \cdot S t}{R_{h}}
\end{gathered}
$$

junto con:

$$
\mathrm{T}_{\mathrm{f}}=\mathrm{T}_{\mathrm{f} 0}+\left(\mathrm{T}_{0}-\mathrm{T}+\mathrm{J} \cdot \mathrm{X}\right)
$$


donde:

$\mathrm{T}_{\mathrm{f} 0}=$ temperatura de entrada del fluido intercambiador (conocida)

$\mathrm{T}_{\mathrm{f}}(1)=$ temperatura en una posición intermedia del fluido intercambiador (desconocida)

$\mathrm{T}_{\mathrm{f}}(\mathrm{L})=$ temperatura a la salida del fluido intercambiador (desconocida)

$\mathrm{T}_{0}=$ temperatura de entrada de la mezcla de reacción (desconocida)

$\mathrm{T}(1)=$ temperatura en una posición intermedia de la mezcla de reacción (desconocida)

$\mathrm{T}(\mathrm{L})=$ temperatura a la salida de la mezcla de reacción (desconocida)

con la importante restricción de que $\mathrm{T}_{\mathrm{f}}(\mathrm{L})=\mathrm{T}_{0}$

El procedimiento de resolución consiste en sustituir $\mathrm{T}_{\mathrm{f}}$ por su función de $\mathrm{X}$ quedando de nuevo dos ecuaciones diferenciales:

$$
\begin{gathered}
\frac{d \ell}{d X}=\frac{G_{k 0}}{\left(-v_{k}\right) \cdot r} \\
\frac{d T}{d X}=J+\frac{G_{k 0}}{\left(-v_{k}\right) \cdot r(X, T)} \cdot \frac{\left[T_{f 0}+\left(T_{0}-T+J \cdot X\right)-T\right] \cdot S t}{R_{h}}
\end{gathered}
$$

que se integran simultáneamente para el valor conocido de $\mathrm{T}_{\mathrm{f} 0} \mathrm{y}$ las condiciones iniciales habituales de $1=0$ y $X=0$. Dado que no se conoce $T_{0}$, hay que dar valores arbitrarios, obteniéndose una tabla de valores de $\mathrm{X}, \mathrm{T}, \mathrm{l}$. Si en esta tabla se desean conocer los valores de $T_{f}$ se pueden calcular mediante $T_{f}=T_{f 0}+\left(T_{0}-T+J \cdot X\right)$.

El valor adecuado de $\mathrm{T}_{0}$ es aquel que permite obtener unos valores $1=\mathrm{L}, \mathrm{X}=$

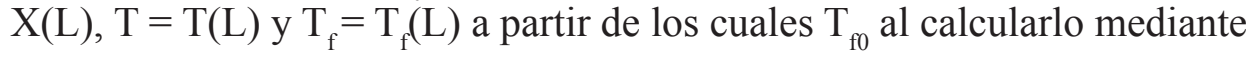

$$
\begin{gathered}
\mathrm{T}_{\mathrm{f}}=\mathrm{T}_{\mathrm{f} 0}+\left(\mathrm{T}_{0}-\mathrm{T}+\mathrm{J} \cdot \mathrm{X}\right) \\
\mathrm{T}_{\mathrm{f} 0}=\mathrm{T}_{\mathrm{f}}(\mathrm{L})-\mathrm{T}_{0}+\mathrm{T}(\mathrm{L})-\mathrm{J} \cdot \mathrm{X}(\mathrm{L})=\mathrm{T}(\mathrm{L})-\mathrm{J} \cdot \mathrm{X}(\mathrm{L})
\end{gathered}
$$

coincide con el valor conocido.

\section{Caso 4. Disposición d)}

En este caso las ecuaciones a integrar simultáneamente son:

$$
\begin{gathered}
\frac{d \ell}{d X}=\frac{G_{k 0}}{\left(-v_{k}\right) \cdot r} \\
\frac{d T}{d X}=J+\frac{G_{k 0}}{\left(-v_{k}\right) \cdot r(X, T)} \cdot \frac{\left(T_{f}-T\right) \cdot S t}{R_{h}}
\end{gathered}
$$

junto con:

$$
\mathrm{T}_{\mathrm{f}}=\mathrm{T}_{\mathrm{f} 0}-\left(\mathrm{T}_{0}-\mathrm{T}+\mathrm{J} \cdot \mathrm{X}\right)
$$


donde:

$\mathrm{T}_{\mathrm{f} 0}=$ temperatura a la salida del fluido intercambiador (desconocida)

$\mathrm{T}_{\mathrm{f}}(1)=$ temperatura en una posición intermedia del fluido intercambiador (desconocida)

$\mathrm{T}_{\mathrm{f}}(\mathrm{L})=$ temperatura de entrada del fluido intercambiador (conocida)

$\mathrm{T}_{0}=$ temperatura de entrada de la mezcla de reacción (desconocida)

$\mathrm{T}(1)=$ temperatura en una posición intermedia de la mezcla de reacción (desconocida)

$\mathrm{T}(\mathrm{L})=$ temperatura a la salida de la mezcla de reacción (desconocida)

con la importante restricción de que $\mathrm{T}_{\mathrm{f0}}=\mathrm{T}_{0}$.

El procedimiento de resolución consiste en sustituir $\mathrm{T}_{\mathrm{f}}$ por su función de $\mathrm{X}$ quedando de nuevo dos ecuaciones diferenciales:

$$
\begin{gathered}
\frac{d \ell}{d X}=\frac{G_{k 0}}{\left(-v_{k}\right) \cdot r} \\
\frac{d T}{d X}=J+\frac{G_{k 0}}{\left(-v_{k}\right) \cdot r(X, T)} \cdot \frac{(-J \cdot X) \cdot S t}{R_{h}}
\end{gathered}
$$

que se integran simultáneamente para las condiciones iniciales habituales de $1=0$ $\mathrm{y} \mathrm{X}=0$. Dado que no se conoce $\mathrm{T}_{0}$, hay que dar valores arbitrarios, obteniéndose una tabla de valores de $\mathrm{X}, \mathrm{T}, 1$. Si en esta tabla se desean conocer los valores de $\mathrm{T}_{\mathrm{f}}$ se pueden calcular mediante $\mathrm{T}_{\mathrm{f}}=\mathrm{T}_{\mathrm{f} 0}-\left(\mathrm{T}_{0}-\mathrm{T}+\mathrm{J} \cdot \mathrm{X}\right)=\mathrm{T}-\mathrm{J} \cdot \mathrm{X}$

El valor adecuado de $T_{0}$ es aquel que permite obtener unos valores $1=\mathrm{L}, \mathrm{X}=$ $\mathrm{X}(\mathrm{L}), \mathrm{T}=\mathrm{T}(\mathrm{L})$ y $\mathrm{T}_{\mathrm{f}}=\mathrm{T}_{\mathrm{f}}(\mathrm{L})$ tales que $\mathrm{T}_{\mathrm{f}}(\mathrm{L})$ coincide con el expermiental:

$$
\begin{gathered}
\mathrm{T}_{\mathrm{f}}=\mathrm{T}_{\mathrm{f} 0}-\left(\mathrm{T}_{0}-\mathrm{T}+\mathrm{J} \cdot \mathrm{X}\right)=\mathrm{T}-\mathrm{J} \cdot \mathrm{X} \\
\mathrm{T}_{\mathrm{f}}(\mathrm{L})=\mathrm{T}(\mathrm{L})-\mathrm{J} \cdot \mathrm{X}(\mathrm{L})
\end{gathered}
$$

\subsection{Perfil óptimo de temperaturas de reacción}

Tal y como se ha mostrado en los apartados anteriores y en la asignatura EQ1021 Ingeniería de la Reacción Química, la temperatura de reacción puede permanecer constante o variar a lo largo de la longitud del reactor. El perfil óptimo de temperatura a lo largo del RFP es el que minimiza el volumen de reacción.

\subsubsection{Sistemas con una reacción química}

De acuerdo con la ecuación de diseño (1) el volumen (y la longitud) mínimo de reacción se alcanza cuando la velocidad de reacción es lo más elevada posible. Esto se puede estimar fácilmente para cada tipo de reacción siempre que se conozcan las curvas de velocidad de reacción constante estudiadas en la asignatura EQ1021-Ingeniería de la Reacción Química. 
Reacciones endotérmicas, reversibles e irreversibles, y reacciones exotérmicas irreversibles

Para estos tres tipos de reacciones las curvas de velocidad de reacción constante son de la forma que se muestra en las figuras 11 y 12 .

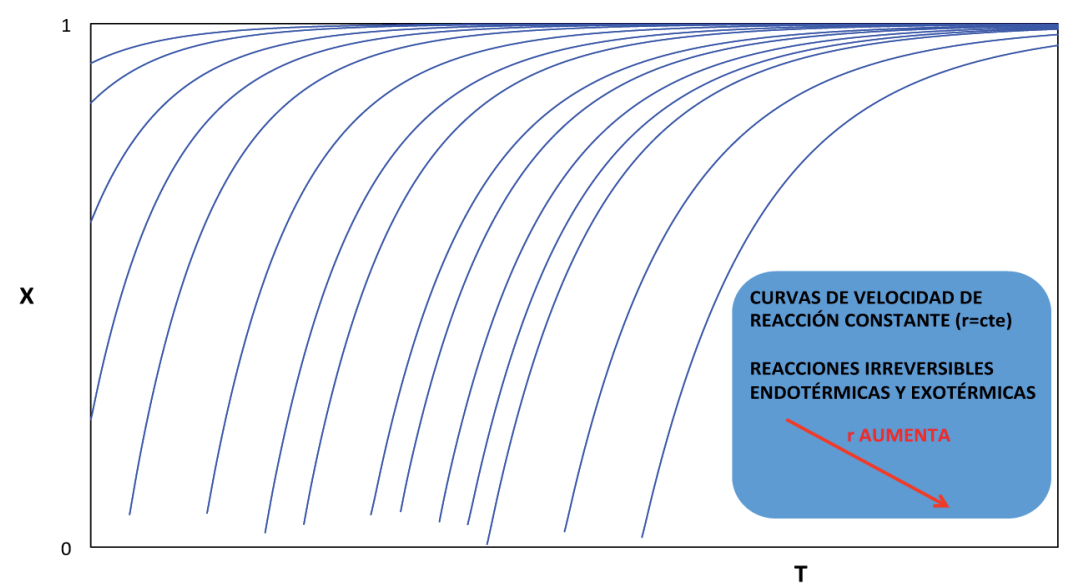

Figura 11. Curvas de velocidad de reacción constante para reacciones irreversibles endotérmicas y exotérmicas

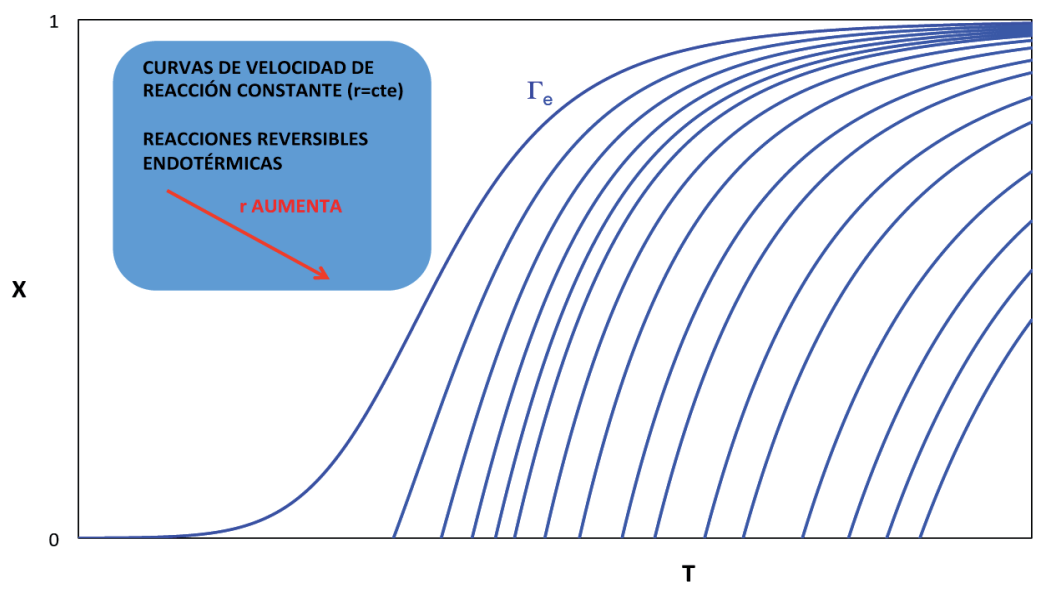

Figura 12. Curvas de velocidad de reacción constante para reacciones reversibles endotérmicas

A partir del análisis de estas curvas se deduce fácilmente (figura 13) que la mejor forma de funcionamiento de un RFP, en el que se desarrolle algún tipo de estas tres reacciones químicas, es la isoterma a la máxima temperatura permisible (o ligeramente inferior) por el sistema $\left(\mathrm{T}^{*}\right)$, ya que de esta forma la velocidad de reacción es máxima para cada grado de conversión. 


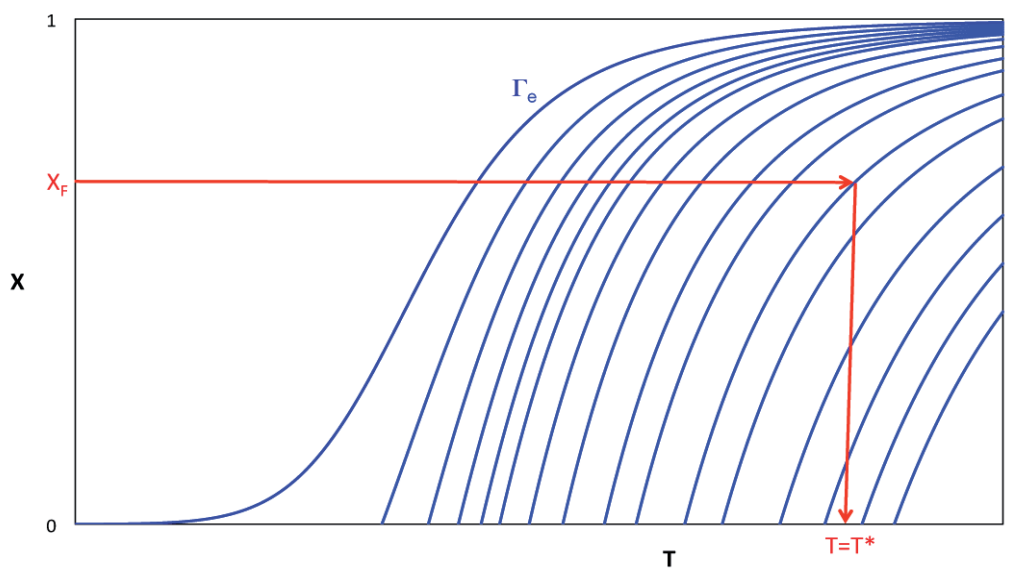

Figura 13. Determinación de la temperatura óptima en reacciones endotérmicas o exotérmicas irreversibles

En las reacciones endotérmicas reversibles debe recordarse que el máximo grado de conversión que se puede alcanzar a cada temperatura fijada viene determinado por la curva de equilibrio.

\section{Reacciones exotérmicas reversibles}

Para este tipo de reacción las curvas de velocidad de reacción constante son de la forma que se muestran en la figura 14.

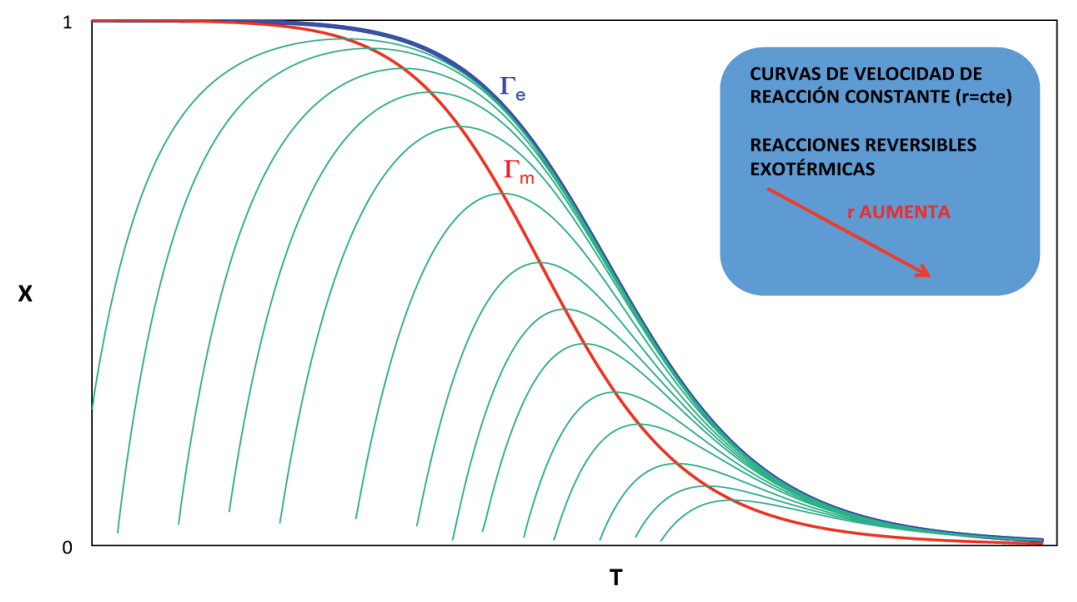

Figura 14. Curvas de velocidad de reacción constante para reacciones reversibles exotérmicas

Del análisis de las curvas se extraen dos conclusiones:

a) si el RFP es isotermo, para alcanzar un determinado grado de conversión final la temperatura de trabajo tiene que ser la que, para dicho grado de conversión, se sitúe sobre la curva $\Gamma_{\mathrm{m}}$ de máxima velocidad de reacción (figura 15). 


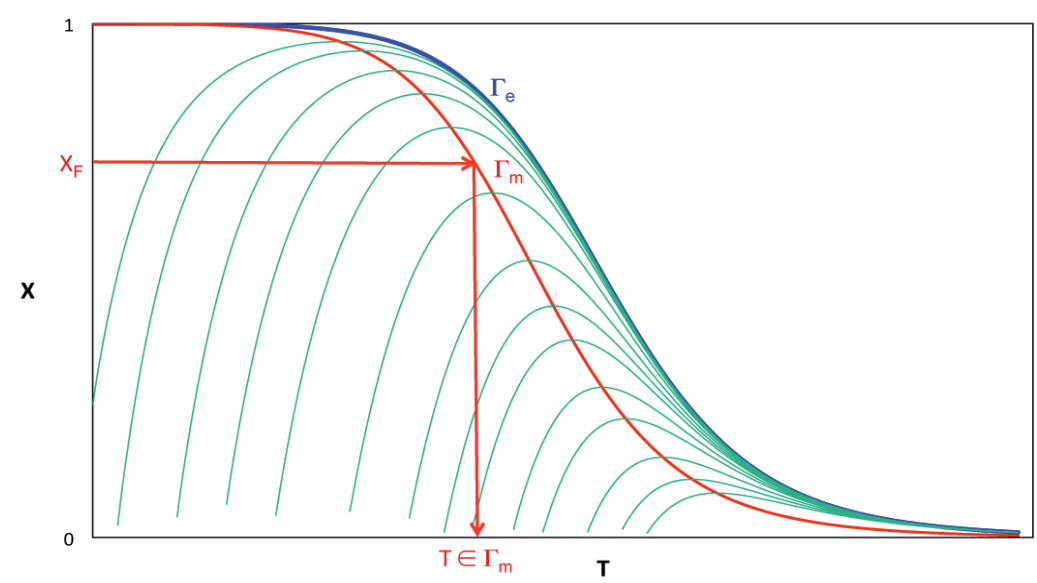

Figura 15. Determinación de la temperatura óptima en reacciones exotérmicas reversibles

b) Si el RFP es intermedio se debe cumplir que, a medida que avanza el tiempo de reacción, todas las parejas de valores $(X, T)$ deben situarse sobre la curva $\Gamma_{\mathrm{m}}$ (figura 16). Es decir, es como si al avanzar a lo largo de la longitud del RFP se avanzara sobre la curva $\Gamma_{\mathrm{m}}$ desde el grado de conversión inicial hasta el grado de conversión final. De esta forma, en cualquier punto del RFP la la reacción química se está desarrollando a la máxima velocidad de reacción posible.

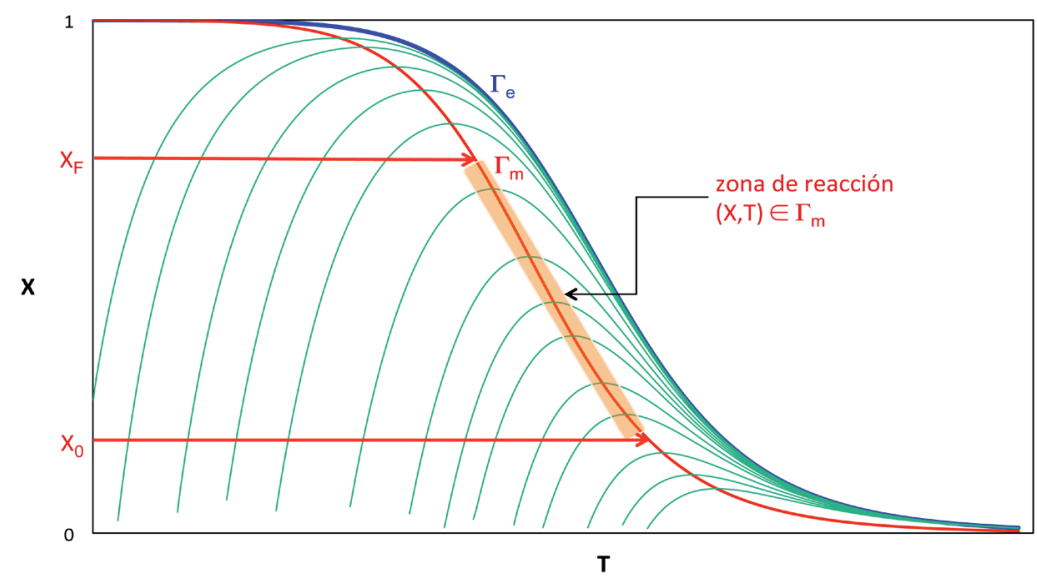

Figura 16. Evolución óptima de la temperatura en reacciones exotérmicas reversibles

En este caso resulta evidente que si se parte de un grado de conversión cero, como es habitual, se requeriría una temperatura inicial excesivamente alta. Por ello, lo más conveniente es que el RFP opere inicialmente de forma isoterma, a la temperatura máxima permisible $\left(\mathrm{T}^{*}\right)$ hasta que alcance la curva de velocidades de reacción máximas $\Gamma_{\mathrm{m}}$. Análogamente, si se desea llegar a un grado de conversión muy elevado es posible que la temperatura llegue a ser excesivamente baja. En este caso lo más conveniente es que el reactor vuelva a operar desde la temperatura mínima permisible $\left(\mathrm{T}_{*}\right)$ de forma isoterma hasta alcanzar el grado de conversión final. El camino se muestra en la figura 17 . En la tabla 2 se resumen los valores de X y T que el RFP debe seguir a lo largo de su longitud. 


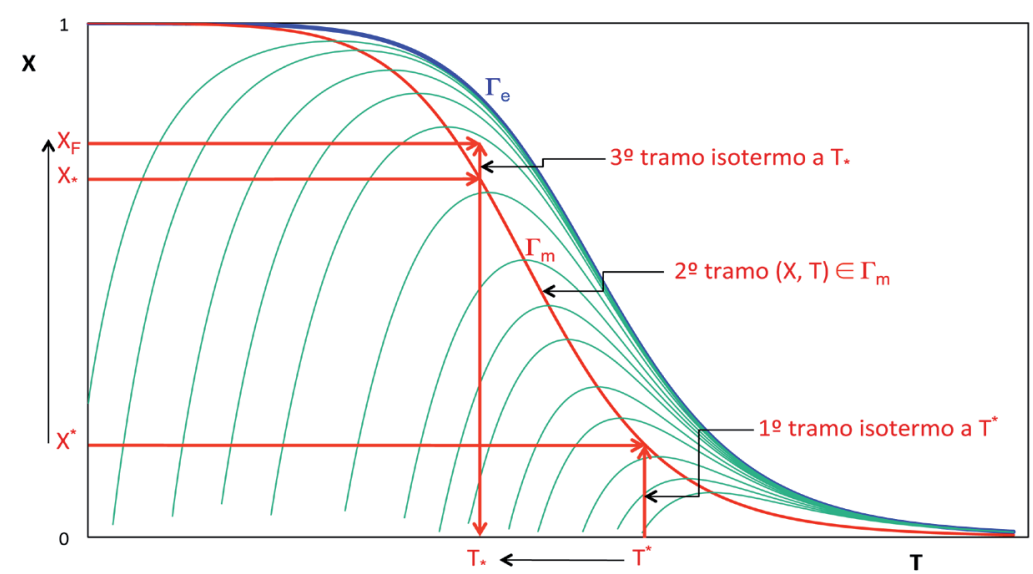

Figura 17. Ciclo óptimo de temperatura en reacciones exotérmicas reversibles

Tabla 2. Ciclo óptimo de temperatura en reacciones exotérmicas reversibles

\begin{tabular}{|c|c|c|c|}
\hline RFP & Intervalo de $\mathrm{X}$ & Intervalo de $\mathrm{T}$ & Velocidad de reacción \\
\hline Isotermo & $0 \leq \mathrm{X} \leq \mathrm{X}^{*}$ & $\mathrm{~T}=\mathrm{T}^{*}=$ constante & $\mathrm{r}=\mathrm{r}\left(\mathrm{X}, \mathrm{T}^{*}\right)$ \\
\hline Intermedio & $\mathrm{X}^{*} \leq \mathrm{X} \leq \mathrm{X}_{*}$ & $\mathrm{~T}^{*} \leq \mathrm{T} \leq \mathrm{T}_{*} /(\mathrm{X}, \mathrm{T}) \in \Gamma_{\mathrm{m}}$ & $\mathrm{r}=\mathrm{r}\left[\mathrm{X}, \mathrm{T}_{\mathrm{m}}(\mathrm{X})\right]$ \\
\hline Isotermo & $\mathrm{X}_{*} \leq \mathrm{X} \leq \mathrm{X}_{\mathrm{F}}$ & $\mathrm{T}=\mathrm{T}_{*}=$ constante & $\mathrm{r}=\mathrm{r}\left(\mathrm{X}, \mathrm{T}_{*}\right)$ \\
\hline
\end{tabular}

En las reacciones exotérmicas reversibles debe recordarse que el máximo grado de conversión que se puede alcanzar a cada temperatura fijada viene determinado por la curva de equilibrio.

\subsubsection{Sistemas con varias reacciones químicas}

En estos sistemas, el criterio que permite seleccionar el perfil de temperatura más adecuado consiste en optimizar la selectividad del producto principal, favoreciendo el transcurso de las reacciones por las que éste se forma y dificultando las reacciones por las que desaparece o aquellas que conducen a otros productos no deseados, procurando que el reactor opere a la temperatura más alta que convenga, para reducir a la vez (en lo posible) el volumen del reactor necesario.

A continuación se muestra de forma cualitativa un ejemplo correspondiente a un sistema de dos reacciones consecutivas irreversibles de primer orden:

$$
\begin{gathered}
\mathrm{A}_{1} \stackrel{\mathrm{i}=1}{\longrightarrow} \mathrm{A}_{2} \stackrel{\mathrm{i}=2}{\longrightarrow} \mathrm{A}_{3} \\
\mathrm{r}_{1}=\mathrm{k}_{1}(\mathrm{~T}) \cdot \mathrm{c}_{1}(\ell) \quad ; \quad \mathrm{r}_{2}=\mathrm{k}_{2}(\mathrm{~T}) \cdot \mathrm{c}_{2}(\ell)
\end{gathered}
$$

Hay que actuar sobre las velocidades de reacción dependiendo del producto que se desee obtener. Y en estas ecuaciones hay que recordar que intervienen dos parámetros: la temperatura, a través de la constante de velocidad, y la concentración 
de reactivo, que va variando con la longitud del reactor. Ambos factores hay que tenerlos en consideración en un razonamiento cualitativo.

Supóngase, por ejemplo, que se desea obtener $\mathrm{A}_{2}$, por lo que hay que favorecer la reacción $\mathrm{i}=1$ e intentar minimizar la reacción $\mathrm{i}=2$, y que la relación de las energías de activación es $\mathrm{E}_{1}<<<\mathrm{E}_{2}$.

En primer lugar conviene hacer una representación cualitativa de la variación de las constantes de velocidad con la temperatura (figura 18). Es decir, en general conviene operar a temperaturas bajas donde $\mathrm{k}_{1}>\mathrm{k}_{2}$

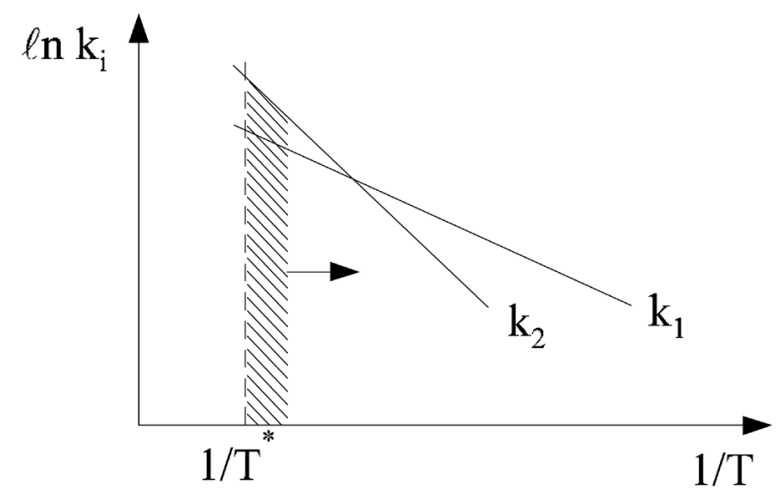

Figura 18. Variación de las constantes de velocidad con la temperatura

Ahora bien, a pesar de esta diferencia de las constantes de velocidad, es evidente que al principio de la reacción hay una elevada concentración de $\mathrm{A}_{1}$ pero muy pequeña de $\mathrm{A}_{2}$, por lo que se puede trabajar a temperaturas más altas (aunque sea $\mathrm{k}_{2}>\mathrm{k}_{1}$ ) porque probablemente se cumplirá que $\mathrm{r}_{1}>\mathrm{r}_{2}$. Obviamente, a medida que se avanza a lo largo del reactor aumenta la concentración de $\mathrm{A}_{2} \mathrm{y}$, para que se siga cumpliendo que $r_{1}>r_{2}$, el RFP ha de comenzar a operar a temperaturas cada vez menores donde $\mathrm{k}_{1}>\mathrm{k}_{2}$

En conclusión el principio del RFP comenzaría a operar a temperaturas altas y, a medida que la mezcla de reacción avanzara a lo largo del reactor, debería ir disminuyendo la temperatura (figura 18). 


\section{ANEXO I}

En la figura siguiente se muestra la geometría y los parámetros geométricos utilizados para el estudio de los reactores continuos tubulares de flujo de pistón.

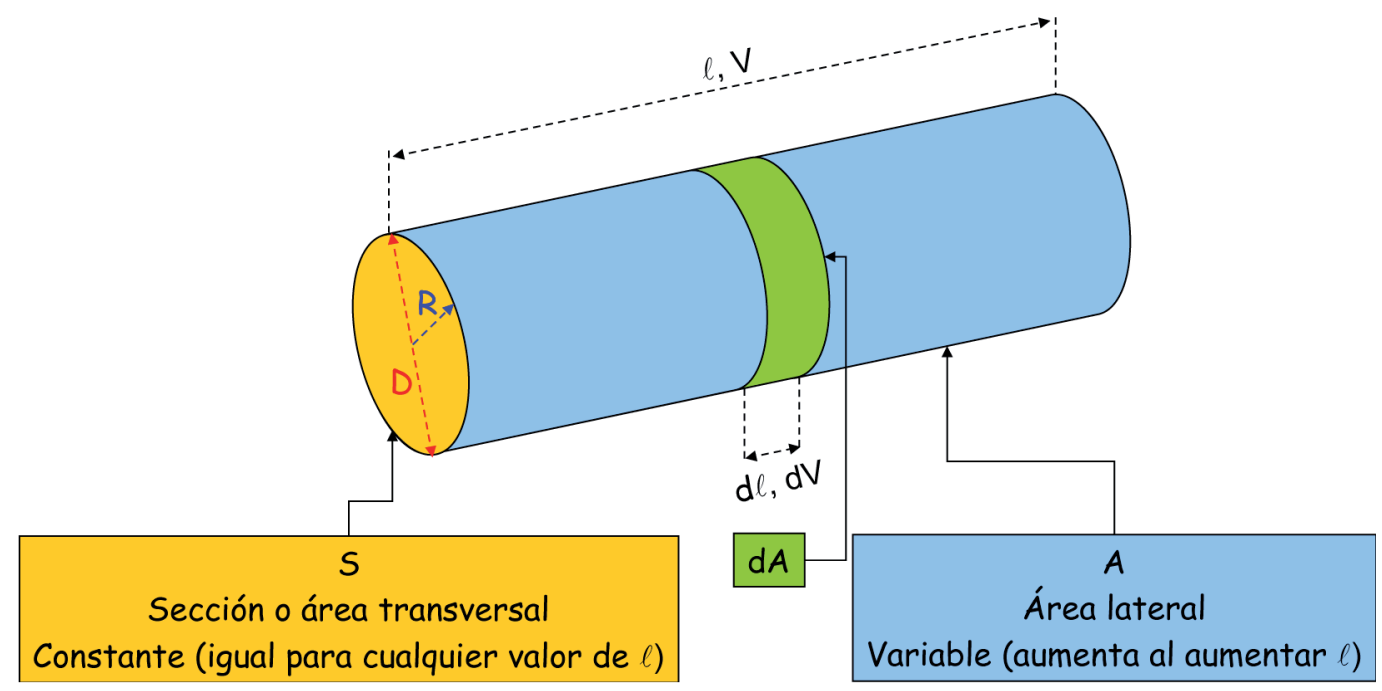

Así se definen las siguientes relaciones geométricas:

i) Área transversal o sección $\left(\mathrm{m}^{2}\right)$ :

$$
\mathrm{S}=\pi \cdot \mathrm{R}^{2}=\frac{\pi}{4} \cdot \mathrm{D}^{2} \quad \text { (constante) } \quad ; \quad \mathrm{d} S=0
$$

ii) Área lateral $\left(\mathrm{m}^{2}\right)$ :

$$
\mathrm{A}=2 \cdot \pi \cdot \mathrm{R} \cdot \ell=\pi \cdot \mathrm{D} \cdot \ell \quad ; \quad \mathrm{dA}=2 \cdot \pi \cdot \mathrm{R} \cdot \mathrm{d} \ell=\pi \cdot \mathrm{D} \cdot \mathrm{d} \ell
$$

iii) Área lateral por unidad de longitud $\left(\mathrm{m}^{2} / \mathrm{m}\right.$ o $\left.\mathrm{m}\right)$ :

$$
\begin{aligned}
& \mathrm{A}_{\mathrm{S}}=\frac{\mathrm{A}}{\ell}=\frac{2 \cdot \pi \cdot \mathrm{R} \cdot \ell}{\ell}=2 \cdot \pi \cdot \mathrm{R} \\
& \mathrm{A}_{\mathrm{S}}=\frac{\mathrm{A}}{\ell}=\frac{\pi \cdot \mathrm{D} \cdot \ell}{\ell}=\pi \cdot \mathrm{D} \\
& \mathrm{A}=\mathrm{A}_{\mathrm{S}} \cdot \ell ; \mathrm{dA}=\mathrm{A}_{\mathrm{S}} \cdot \mathrm{d} \ell
\end{aligned}
$$

iv) Radio hidráulico (m):

$$
\mathrm{R}_{\mathrm{h}}=\frac{\mathrm{S}}{\mathrm{A}_{\mathrm{S}}}=\frac{\mathrm{D}}{4}=\frac{\mathrm{R}}{2}
$$

v) Volumen $\left(\mathrm{m}^{3}\right)$ :

$$
\mathrm{V}=\pi \cdot \mathrm{R}^{2} \cdot \ell=\frac{\pi}{4} \cdot \mathrm{D}^{2} \cdot \ell \quad ; \quad \mathrm{dV}=\pi \cdot \mathrm{R}^{2} \cdot \mathrm{d} \ell=\frac{\pi}{4} \cdot \mathrm{D}^{2} \cdot \mathrm{d} \ell
$$


Otras variables útiles en el análisis de los RFP son:

i) Flujo molar $\left(\mathrm{kmol} \mathrm{A}_{\mathrm{j}} / \mathrm{s}\right)$ :

$$
\mathrm{F}_{\mathrm{k} 0}, \mathrm{~F}_{\mathrm{j} 0} \text { (constantes) ; } \mathrm{F}_{\mathrm{k}}, \mathrm{F}_{\mathrm{j}} \text { (variables con } \ell \text { ) }
$$

ii) Densidad de flujo molar $\left(\mathrm{kmol} \mathrm{A}_{\mathrm{j}} / \mathrm{s} \cdot \mathrm{m}^{2}\right)$ :

$$
\begin{aligned}
& G_{k 0}=\frac{F_{k 0}}{S}, G_{j 0}=\frac{F_{j 0}}{S}(\text { constantes }) ; \\
& G_{k}=\frac{F_{k}}{S}, G_{j}=\frac{F_{j}}{S}(\text { variables con } \ell)
\end{aligned}
$$

iii) Módulo de Stanton:

$$
\mathrm{St}=\frac{\mathrm{U}}{\mathrm{G}_{\mathrm{k} 0} \cdot \sum_{\mathrm{j}=1}^{\mathrm{S}} \theta_{\mathrm{j}} \cdot \overline{\mathrm{C}}_{\mathrm{pj}}}=\frac{\mathrm{U} \cdot \mathrm{S}}{\mathrm{F}_{\mathrm{k} 0} \cdot \sum_{\mathrm{j}=1}^{\mathrm{S}} \theta_{\mathrm{j}} \cdot \overline{\mathrm{C}}_{\mathrm{pj}}}=\frac{\mathrm{U} \cdot \mathrm{A}_{\mathrm{S}} \cdot \mathrm{R}_{\mathrm{h}}}{\mathrm{F}_{\mathrm{k} 0} \cdot \sum_{\mathrm{j}=1}^{\mathrm{S}} \theta_{\mathrm{j}} \cdot \overline{\mathrm{C}}_{\mathrm{pj}}}
$$

Finalmente, conviene también resaltar que al tratarse de un reactor continuo que consiste en la circulación de fluidos por una conducción cilíndrica, además de aplicar los balances de materia y de energía también se debe cumplir el balance de cantidad de movimiento (condición indispensable para que se produzca el flujo del fluido). A continuación se recogen los balances de cantidad de movimiento más habituales:

i) Ecuación de Hagen-Poiseuille (para valores del número de Reynolds $\operatorname{Re}<2100)$ :

$$
-\frac{\mathrm{dP}}{\mathrm{d} \ell}=\frac{32 \cdot \mu \cdot \mathrm{v}}{\mathrm{D}^{2}}
$$

ii) Ecuación de Fanning (para valores del número de Reynolds Re>4000):

$$
-\frac{\mathrm{dP}}{\mathrm{d} \ell}=2 \cdot \mathrm{f} \cdot \rho \cdot \frac{\mathrm{v}^{2}}{\mathrm{D}}
$$

iii) Ecuación de Ergun (para lechos porosos):

$$
-\frac{\mathrm{dP}}{\mathrm{d} \ell}=\left(\frac{1-\varepsilon}{\varepsilon}\right) \cdot\left[0.75+150 \cdot \frac{(1-\varepsilon)}{\mathrm{Re}_{\mathrm{g}}}\right] \cdot \frac{\rho \cdot \mathrm{v}^{2}}{\mathrm{~d}_{\mathrm{g}}}
$$

siendo $\varepsilon$ la porosidad del lecho, $\mathrm{d}_{\mathrm{g}}$ el diámetro de grano y calculándose el número de Reynolds como $\operatorname{Re}_{\mathrm{g}}=\frac{\mathrm{G} \cdot \mathrm{d}_{\mathrm{g}}}{\mu}$ 


\section{PROBLEMAS}

Problema 01. Un compuesto orgánico experimenta una pirolisis al hacerlo circular a través de un RFP consistente en un tubo cilíndrico que atraviesa el interior de un horno. Se desea desarrollar la reacción endotérmica a temperatura constante e igual a $675{ }^{\circ} \mathrm{C}$. Se sabe que su cinética es de primer orden y que la constante de velocidad, expresada en $\mathrm{s}^{-1}$, varía con la temperatura según la expresión:

$$
\ln \mathrm{k}=34.34-\frac{34000}{\mathrm{~T}}
$$

La temperatura del aire que rodea al tubo coincide prácticamente con la de las paredes del horno y es de $1000{ }^{\circ} \mathrm{C}$. El coeficiente global de transmisión de calor por convección y radiación desde el horno al interior del tubo vale $34 \mathrm{~J} / \mathrm{s} \cdot \mathrm{m}^{2} \cdot \mathrm{K}$. En estas condiciones la cantidad de calor que recibe la mezcla de reacción por unidad de tiempo es $2.11 \cdot 10^{6} \mathrm{~kJ} / \mathrm{h}$ para obtener un grado de conversión de 0.3 .

¿Cuáles serán las dimensiones del tubo de reacción si el caudal volumétrico del alimento es $4.245 \mathrm{~m}^{3} / \mathrm{s}$ ?

Problema 02. Se desea descomponer acetaldehído en metano y monóxido de carbono utilizando un RFP adiabático. La reacción en fase gas se desarrolla según el esquema $\mathrm{CH}_{3} \mathrm{CHO} \rightarrow \mathrm{CH}_{4}+\mathrm{CO}$. Si el alimento, acetaldehido puro, se introduce en el reactor a $780 \mathrm{~K}$, con un caudal molar de $0.1 \mathrm{~mol} / \mathrm{s}$ y la presión de operación es una atmósfera, calcular:

a) La longitud del reactor requerida para alcanzar un conversión de 0.95 , si la sección libre de paso es $0.15 \mathrm{~m}^{2}$.

b) La temperatura de salida de la mezcla de reacción.

Datos y notas

$$
\begin{aligned}
& \Delta \mathrm{H}_{\mathrm{k}}^{0}=-19050 \mathrm{~J} / \mathrm{mol} ; \mathrm{C}_{\mathrm{p}\left(\mathrm{CH}_{3} \mathrm{CHO}\right)}=134.3 \mathrm{~J} / \mathrm{mol} \cdot \mathrm{K} \\
& \mathrm{r}=3.3 \cdot 10^{-4} \mathrm{c}_{1}^{2} \exp \left(31.71-\frac{25080}{\mathrm{~T}}\right) \text { que viene en } \mathrm{mol} / \mathrm{s} \cdot \mathrm{m}^{3} \text { cuando la }
\end{aligned}
$$

concentración de acetaldehido se expresa en $\mathrm{mol} / \mathrm{m}^{3}$

Problema 03. La descomposición de la fosfamina tiene lugar a temperaturas elevadas según el esquema de reacción en fase gas $4 \mathrm{PH}_{3} \rightarrow \mathrm{P}_{4}+6 \mathrm{H}_{2}$. La reacción es endotérmica e irreversible, ajustándose bien a una cinética de primer orden según 
la ecuación $-\Re_{\mathrm{PH}_{3}}=\mathrm{k} \cdot \mathrm{c}_{\mathrm{PH}_{3}}$. Si se parte de fosfamina pura, a $945 \mathrm{~K}$, con caudal másico de $22.7 \mathrm{~kg}$ fosfamina $/ \mathrm{h}$ y la reacción se desarrolla en un RFP adiabático, calcular el tamaño del reactor requerido para alcanzar una conversión de 0.3 si el reactor opera a la presión atmosférica.

\section{Datos y notas}

Mediante un balance energético se ha determinado la variación de T con $\mathrm{X}$, que se detalla en la tabla siguiente, junto con los valores de $k$ a diferentes temperaturas.

\begin{tabular}{|c|c|c|c|c|c|}
\hline $\mathrm{X}$ & 0.00 & 0.10 & 0.20 & 0.25 & 0.30 \\
\hline $\mathrm{T}(\mathrm{K})$ & 945 & 885 & 826 & 797 & 768 \\
\hline $\mathrm{k}\left(\mathrm{s}^{-1}\right)$ & 37.2 & 1.42 & $3.65 \cdot 10^{-2}$ & $3.00 \cdot 10^{-3}$ & $5.83 \cdot 10^{-4}$ \\
\hline
\end{tabular}

Problema 04. La descomposición de la fosfamina tiene lugar a temperaturas elevadas según el esquema de reacción en fase gas $4 \mathrm{PH}_{3} \rightarrow \mathrm{P}_{4}+6 \mathrm{H}_{2}$. La reacción es endotérmica e irreversible, ajustándose bien a una cinética de primer orden según la ecuación $-\Re_{\mathrm{PH}_{3}}=\mathrm{k} \cdot \mathrm{c}_{\mathrm{PH}_{3}}$. La constante de velocidad, expresada en $\mathrm{s}^{-1}$, varía con la temperatura según la expresión:

$$
\log \mathrm{k}=-\frac{18963}{\mathrm{~T}}+2 \cdot \log \mathrm{T}+12.13
$$

En una instalación industrial se desea obtener fósforo por descomposición de la fosfamina en un RFP que operará a la presión atmosférica, al que se inyecta una corriente de fosfamina pura con un caudal másico de $45 \mathrm{~kg} / \mathrm{h}$ y a $680{ }^{\circ} \mathrm{C}$. Si el tubo de reacción está situado dentro de una cámara de calefacción en la que puede admitirse que la temperatura del fluido calefactor permanece constante a $700{ }^{\circ} \mathrm{C}$ :

a) Deducir las ecuaciones a partir de las que se podría calcular la variación de $\mathrm{X}$ y $\mathrm{T}$ a lo largo del reactor.

b) Calcular la longitud del reactor requerida para alcanzar una conversión de 0.6 .

\section{Datos y notas}

Diámetro del reactor: $0.10 \mathrm{~m}$

Peso molecular de la fosfamina: $34 \mathrm{~kg} / \mathrm{kmol}$

$\Delta \mathrm{H}_{\mathrm{k}}^{0}=23713 \mathrm{~kJ} / \mathrm{mol} ; \mathrm{C}_{\mathrm{p}\left(\mathrm{PH}_{3}\right)}=52.7 \mathrm{~kJ} / \mathrm{mol} \cdot \mathrm{K} ; \mathrm{C}_{\mathrm{p}\left(\mathrm{P}_{4}\right)}=62.4 \mathrm{~kJ} / \mathrm{kmol} \cdot \mathrm{K}$ 
Problema 05. La reacción elemental, reversible, en fase gaseosa catalizada por un sólido $A_{1}+A_{2} \leftrightarrow 2 A_{3}$ se lleva a cabo en el RFP autotérmico que se esquematiza a continuación:

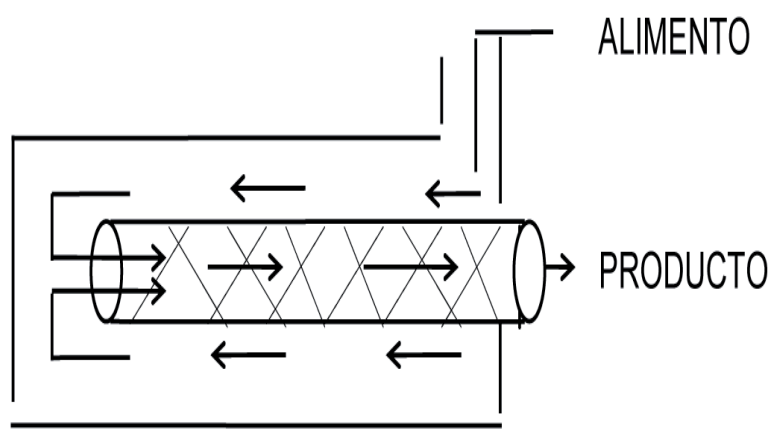

El alimento se introduce en el espacio anular existente entre el tubo exterior, aislado, y el tubo interior que contiene el catalizador. En el espacio anular se precalienta, pasando luego al tubo interior donde se desarrolla la reacción. En dicha zona anular no tiene lugar reacción química alguna. El intercambio de calor entre la mezcla gaseosa que circula por el tubo interior y el gas que circula por el espacio anular, en contracorriente, tiene lugar a lo largo de toda la longitud del primero.

El alimento consta exclusivamente de $\mathrm{A}_{1} \mathrm{y}_{\mathrm{A}}$ en la proporción estequiométrica de la reacción, siendo el caudal de $\mathrm{A}_{1}$ de $20 \mathrm{~mol} / \mathrm{s}$. El RFP trabaja a la presión de $5.8 \cdot$ $10^{5} \mathrm{~N} / \mathrm{m}^{2}$. La temperatura del alimento es $350 \mathrm{~K}$. Si se desea obtener una conversión final de 0.74 ,

a) Obtener la variación de la conversión y la temperatura de la mezcla reactante a lo largo del reactor y representar la curva correspondiente en un diagrama $\mathrm{X}-\mathrm{T}$.

b) Calcular el volumen de reactor necesario para alcanzar el grado de conversión indicado.

\section{Datos y notas}

Diámetro del reactor: $0.025 \mathrm{~m}$.

$\Delta \mathrm{H}_{\mathrm{k}}^{0}=-20000 \mathrm{~kJ} / \mathrm{mol} ; \mathrm{C}_{\mathrm{p}, 1}=25 \mathrm{~J} / \mathrm{mol} \cdot \mathrm{K} ; \mathrm{C}_{\mathrm{p}, 2}=15 \mathrm{~J} / \mathrm{kmol} \cdot \mathrm{K} ; \mathrm{C}_{\mathrm{p}, 3}=$ $20 \mathrm{~J} / \mathrm{mol} \cdot \mathrm{K}$

$\mathrm{k}=0.035 \mathrm{~m}^{3} / \mathrm{kmol} \cdot \min$ a $273 \mathrm{~K} ; \mathrm{E}=70000 \mathrm{~J} / \mathrm{mol} ; \mathrm{K}_{\mathrm{c}}=25000(\mathrm{a} 298 \mathrm{~K})$

Coeficiente global de transmisión de calor: $5 \mathrm{w} / \mathrm{m}^{2} \cdot \mathrm{K}$.

Problema 06. Se desea diseñar una planta piloto para la producción de $\mathrm{A}_{3}$, según la siguiente reacción irreversible en fase gas $A_{1}+A_{2} \rightarrow A_{3}+A_{4}$. El alimento al reactor consiste en una mezcla de 4 moles de $\mathrm{A}_{2}$ por cada mol de $\mathrm{A}_{1}$, a $200{ }^{\circ} \mathrm{C}$. El caudal molar total del alimento es $0.17 \cdot 10^{-3} \mathrm{kmol} / \mathrm{h}$. El reactor está constituido por un tubo de 5 pulgadas de diámetro interno y perfectamente aislado del exterior 
para que su comportamiento se pueda considerar adiabático. Teniendo en cuenta que el producto $\mathrm{A}_{3}$ se descompone a una temperatura superior a los $546 \mathrm{~K}$, calcular la máxima posible longitud del reactor para evitar la descomposición de $\mathrm{A}_{3}$

\section{Datos y notas}

La presión total es constante e igual a 2 atm

$\Delta \mathrm{H}_{\mathrm{k}}^{0}=-26800 \mathrm{kcal} / \mathrm{mol}$

$\mathrm{C}_{\mathrm{p}, 1}=8.6 \mathrm{kcal} / \mathrm{mol} \cdot \mathrm{K} ; \mathrm{C}_{\mathrm{p}, 2}=25.3 \mathrm{Kcal} / \mathrm{kmol} \cdot \mathrm{K} ; \mathrm{C}_{\mathrm{p}, 3}=28 \mathrm{kcal} / \mathrm{mol} \cdot \mathrm{K}$;

$\mathrm{C}_{\mathrm{p}, 4}=7.2 \mathrm{kcal} / \mathrm{kmol} \cdot \mathrm{K}$

$r=206 \cdot 10^{6} \cdot \exp \left[-\frac{14233}{T}\right] \cdot p_{1} \cdot p_{2}$, en la que si las presiones parciales se expresan en atmósferas la velocidad viene en $\mathrm{kmol} / \mathrm{m}^{3} \cdot \mathrm{h}$

Problema 07. La reacción en fase líquida $A_{1}+A_{2} \rightarrow 2 A_{3}$ se lleva a cabo en un RFP multitubular no isotermo. Los tubos del reactor (de $7 \mathrm{~m}$ de longitud y $2 \mathrm{~cm}$ de diámetro) están rodeados por un refrigerante que mantiene constante la temperatura de la pared. La reacción es de pseudoprimer orden con respecto a $\mathrm{A}_{1}$, siendo la constante de velocidad en $\mathrm{s}^{-1}$ :

$$
k=4.03 \cdot 10^{5} \cdot \exp \left[-\frac{5624}{T}\right]
$$

El caudal másico es constante y vale $0.06 \mathrm{~kg} / \mathrm{s}$; la densidad del sistema es constante y vale $1025 \mathrm{~kg} / \mathrm{m}^{3}$. La temperatura del alimento puede ser 350, 365, 400 y $425 \mathrm{~K}$.

Calcular y dibujar el perfil de T y X a lo largo de la longitud del RFP cuando la temperatura de la pared de los tubos es $350 \mathrm{~K}$.

\section{Datos y notas}

$\mathrm{C}_{10}=0.50 \mathrm{kmol} / \mathrm{m}^{3}$

$\Delta \mathrm{H}_{\mathrm{k}}^{0}=-210000 \mathrm{~kJ} / \mathrm{mol} ; \mathrm{C}_{\mathrm{p} \text {, fase liquida }}=4.2 \mathrm{~kJ} / \mathrm{kg} \cdot \mathrm{K}$

Coeficiente global de transmisión de calor: $1.59 \mathrm{~kJ} / \mathrm{s} \cdot \mathrm{m}^{2} \cdot \mathrm{K}$

Problema 08. La reacción irreversible $2 \mathrm{~A}_{1}+\mathrm{A}_{2} \rightarrow \mathrm{A}_{3}$ de segundo orden con respecto a $\mathrm{A}_{1} \mathrm{y}$ de orden cero con respecto a $\mathrm{A}_{2}$, tiene lugar en fase gas en el interior de un horno cilíndrico, cuyas paredes se encuentran a $1600 \mathrm{~K}$. Se alimenta una mezcla gaseosa, a $1500 \mathrm{~K}$, con un caudal molar total de $9072 \mathrm{~mol} / \mathrm{h}$; la composición de esta mezcla es $40 \%$ de $\mathrm{A}_{1}, 40 \%$ de $\mathrm{A}_{2}$ y $20 \%$ de un gas inerte (porcentajes volumétricos). La presión total del sistema se mantiene constante e igual a 5 atmósferas. 
Deducir el sistema de ecuaciones diferenciales y calcular la evolución del grado de conversión y de la temperatura de la mezcla reaccionante con el volumen del reactor (perfil V, T, X).

\section{Datos y notas}

$$
\begin{aligned}
& \Delta \mathrm{H}_{\mathrm{k}}^{0}=58110 \mathrm{~J} / \mathrm{mol} ; \mathrm{C}_{\mathrm{p}, 1}=\mathrm{C}_{\mathrm{p}, 2}=33.2 \mathrm{~J} / \mathrm{mol} \cdot \mathrm{K} ; \mathrm{C}_{\mathrm{p}, 3}=41.5 \mathrm{~J} / \mathrm{mol} \cdot \mathrm{K} ; \\
& \mathrm{C}_{\mathrm{p}, \text { inerte }}=28.8 \mathrm{~J} / \mathrm{mol} \cdot \mathrm{K} \\
& \mathrm{k}=9.442 \cdot 10^{12} \cdot \exp \left[-\frac{30000}{\mathrm{~T}}\right] \ell / \mathrm{mol} \cdot \mathrm{h}
\end{aligned}
$$

Coeficiente global de transmisión de calor: $670 \mathrm{~J} / \mathrm{h} \cdot 1 \cdot \mathrm{K}$ (obsérvese que este coeficiente viene referido por unidad de volumen (1) y no por unidad de área $\left(\mathrm{m}^{2}\right)$ como suele ser habitual).

Problema 09. Jeffreys estudió el diseño de una instalación para fabricar anhídrido acético y observó que una de las etapas clave era el craqueo en fase vapor de la acetona para producir cetena y metano, según el esquema de reacción $\mathrm{CH}_{3}-\mathrm{CO}-\mathrm{CH}_{3} \rightarrow \mathrm{H}_{2} \mathrm{C}=\mathrm{C}=\mathrm{O}+\mathrm{CH}_{4}$. Además llegó a la conclusión de que esta reacción era irreversible y de primer orden con respecto a la acetona, obteniendo que la constante de velocidad variaba con la temperatura según la expresión:

$$
\ln \mathrm{k}=34.34-\frac{34222}{\mathrm{~T}}
$$

donde la temperatura va en grados Kelvin y la constante de velocidad en $\mathrm{s}^{-1}$

Para llevar a cabo esta reacción se dispone de un RFP al que se alimenta $8000 \mathrm{~kg} / \mathrm{h}$ de acetona a $1035 \mathrm{~K}$ y $162 \mathrm{kPa}$. El interior del RFP está constituido por 1000 tubos que están en contacto con una corriente de gas (que se encuentra a una temperatura de $1150 \mathrm{~K}$ y se mantiene constante) que aporta la energía necesaria para que la reacción endotérmica tenga lugar.

Calcular el perfil del grado de conversión $(\mathrm{X})$ y temperatura $(\mathrm{T})$ para la mezcla reactante a lo largo del reactor.

\section{Datos y notas}

$$
\begin{aligned}
& \Delta \mathrm{H}_{\text {acetona }}^{0}=-216670 \mathrm{~kJ} / \mathrm{kmol} ; \Delta \mathrm{H}_{\text {cetena }}^{0}=-61090 \mathrm{~kJ} / \mathrm{kmol} ; \Delta \mathrm{H}_{\text {metano }}^{0}= \\
& -74810 \mathrm{~kJ} / \mathrm{mol} \\
& \text { Capacidades caloríficas } \\
& \mathrm{C}_{\text {p,acetona }}=26.63+0.183 \cdot \mathrm{T}-45.86 \cdot 10^{-6} \cdot \mathrm{T}^{2} \mathrm{~kJ} / \mathrm{kmol} \cdot \mathrm{K} \\
& \mathrm{C}_{\mathrm{p}, \text { cetena }}=20.04+0.0945 \cdot \mathrm{T}-30.95 \cdot 10^{-6} \cdot \mathrm{T}^{2} \mathrm{~kJ} / \mathrm{kmol} \cdot \mathrm{K} \\
& \mathrm{C}_{\mathrm{p}, \text { metano }}=13.39+0.077 \cdot \mathrm{T}-18.71 \cdot 10^{-6} \cdot \mathrm{T}^{2} \mathrm{~kJ} / \mathrm{kmol} \cdot \mathrm{K}
\end{aligned}
$$


Dimensiones de cada tubo: $\mathrm{D}_{\text {exterior }}=0.0334 \mathrm{~m} \mathrm{y} \mathrm{D}_{\text {interior }}=0.0266 \mathrm{~m}$ Coeficiente global de transmisión de calor referido al área interna de los tubos: $0.110 \mathrm{~kJ} / \mathrm{s} \cdot \mathrm{m}^{2} \cdot \mathrm{K}$

Problema 10. Se dispone de un RFP industrial que se calienta mediante una camisa de vapor de agua (que condensa a $114{ }^{\circ} \mathrm{C}$ ). El diámetro interno del reactor es de 10 ". Se pretende desarrollar una reacción irreversible de primer orden, en fase líquida, que responde al esquema de reacción $\mathrm{A}_{1} \rightarrow$ productos . Al reactor entra una corriente de $\mathrm{A}_{1}$ puro, a $75^{\circ} \mathrm{C}$, con un caudal másico de $4540 \mathrm{~kg} / \mathrm{h}$.

Calcular la longitud del reactor para alcanzar una conversión del 0.5.

\section{Datos y notas}

Densidad media de la mezcla reactiva: $1280 \mathrm{~kg} / \mathrm{m}^{3}$

$\mathrm{M}_{1}=200 \mathrm{~kg} / \mathrm{kmol}$

$\Delta \mathrm{H}_{\mathrm{k}}^{0}=93000 \mathrm{~kJ} / \mathrm{mol} ; \mathrm{C}_{\mathrm{p} \text {,medio }}=3138 \mathrm{~J} / \mathrm{kg} \cdot \mathrm{K}$

$\mathrm{k}=203 \cdot 10^{15} \cdot \exp \left[-\frac{13940}{\mathrm{~T}}\right] \mathrm{h}^{-1}$

Coeficiente global de transmisión de calor referido al diámetro interno del reactor: $2270 \mathrm{~J} / \mathrm{s} \cdot \mathrm{m}^{2} \cdot \mathrm{K}$ 


\section{Asociación de reactores continuos}

\subsection{Asociación de RCTA}

En los capítulos anteriores se ha considerado el diseño y comportamiento de un único reactor. Como ya se indicó en la asignatura previa EQ1021-Ingeniería de la Reacción Química, una secuencia o batería de RCTA conduce siempre, en el caso de tratarse de reacciones químicas con cinética normal, a un volumen total de reacción inferior al que se obtendría si la reacción se desarrollara en un único reactor, para alcanzar un determinado grado de conversión final. En el caso de tratarse de reacciones químicas con cinética autocatalítica la situación es generalmente, pero no necesariamente, igual a la descrita para reacciones con cinética normal. Por otra parte, si el sistema consta de varias reacciones químicas que se desarrollan simultáneamente la existencia de varios RCTA permite mejorar la selectividad. Existe, por tanto, una necesidad real para estudiar el diseño de baterías de tanques agitados conectados en serie para tratar de minimizar el volumen total de reacción o aumentar la selectividad por lo que el uso de series de RCTA es muy habitual en los procesos industriales.

En el Anexo I se recoge, a modo de recordatorio, los balances de materia que se aplican a una serie de RCTA y la nomenclatura correspondiente, según lo estudiado en la asignatura previa EQ1021-Ingeniería de la Reacción Química. Asimismo, en el Anexo II se muestran los balances de energía junto con la nomenclatura correspondiente aplicables a una serie de RCTA, según lo expuesto en el capítulo 1.

La temperatura de cada RCTA, $\mathrm{T}_{\mathrm{n}}$, se supone conocida puesto que todos deben operar a las condiciones de mayor velocidad de reacción química posible. Es decir, para reacciones endotérmicas (tanto reversibles como irreversibles) y exotérmicas irreversibles la temperatura de operación debe de ser la máxima permisible, $\mathrm{T}=\mathrm{T}^{*}$, mientras que para reacciones exotérmicas reversibles la temperatura debe ser la que hace máxima la velocidad de reacción, lo que se cumple cuando el estado estacionario se encuentra sobre la curva de velocidades de reacción máximas, $\left(\mathrm{X}_{\mathrm{n}}, \mathrm{T}_{\mathrm{n}}\right) \in \Gamma_{\mathrm{m}}$. 


\subsubsection{Cálculo del volumen de una serie de RCTA del mismo tamaño por el método de Aris}

Si los N RCTA conectados en serie tienen el mismo volumen, el balance de materia se puede escribir como:

$$
c_{k 0} \cdot \Delta X_{n}+v_{k} \cdot r_{n}\left(X_{n}, T_{n}\right) \cdot \tau=0 \quad n=1,2 \ldots N
$$

en la que se observa que el tiempo espacial es el mismo para cualquier reactor de la serie, no requiriéndose el subíndice $n$. Asimismo, en esta ecuación la temperatura de cada RCTA, $T_{n}$, es conocida puesto que todos deben operar a las condiciones de mayor velocidad de reacción química posible. Es decir, que la ecuación anterior se puede escribir como:

- para reacciones endotérmicas y exotérmicas irreversibles

$$
\mathrm{c}_{\mathrm{k} 0} \cdot \Delta \mathrm{X}_{\mathrm{n}}+v_{\mathrm{k}} \cdot \mathrm{r}_{\mathrm{n}}\left(\mathrm{X}_{\mathrm{n}}, T^{*}\right) \cdot \tau=0
$$

- para reacciones exotérmicas reversibles

$$
c_{k 0} \cdot \Delta X_{n}+v_{k} \cdot r_{n}\left(X_{n}, T_{n}\right) \cdot \tau=0 /\left(X_{n}, T_{n}\right) \in \Gamma_{m}
$$

En ambos casos, la ecuación se puede aplicar a cada RCTA n de la serie y calcular analíticamente:

- el grado de conversión a la salida $X_{n}$ si se conoce el de entrada y $\tau$

- $\tau$ si se conoce el grado de conversión a la entrada y a la salida

Para facilitar estos cálculos y tener una visión conjunta de las tres variables implicadas, $\bar{X}_{n}, X_{n} y \tau$, el profesor $R$. Aris propone un método gráfico que consiste en expresar el balance de materia en la forma:

$$
\bar{X}_{n}=X_{n}+\frac{v_{k} \cdot r_{n}\left[X_{n}, T_{n}\right] \cdot \tau}{c_{k 0}}=X_{n}+\frac{v_{k} \cdot r_{n}\left[X_{n}, T_{n}\right] \cdot V}{F_{k 0}}
$$

\section{Supuesto 1. Se conoce el valor de $\tau$ (o de V)}

En este caso se puede representar la función matemática $\bar{X}_{n}=f\left(X_{n}\right)$ simplemente dando valores a $X_{n}$. Dado que tanto $\bar{X}_{n}$, como $X_{n}$ se encuentran en el intervalo $[0,1]$, solo interesa la representación de la función matemática en ese intervalo. Así se obtienen curvas como la mostrada en la figura 1. Por otra parte la hipótesis de que para cualquier reactor $n$ de la serie se cumple que $\bar{X}_{n}=X_{n+1}(o$, lo que es lo mismo $X_{n}=\bar{X}_{n+1}$ ) representa matemáticamente la ecuación de una recta de pendiente unidad y ordenada en el origen cero $\left(\bar{X}_{n}=X_{n+1}\right)$ que es la diagonal del cuadrante positivo, siempre y cuando se emplee la misma escala tanto en el eje de abscisas como en el de ordenadas. Para cada uno de los N RCTA se cumple lo que se recoge en la siguiente tabla 1. 
Tabla 1. Valores de $\bar{X}_{n}$ y $X_{n}$

\begin{tabular}{|c|c|c|}
\hline RCTA $n$ & $\mathrm{X}_{n}$ & $\bar{X}_{n}$ \\
\hline $\mathrm{n}=1$ & $\mathrm{X}_{1}$ & $\bar{X}_{1}=0$ \\
\hline $\mathrm{n}=2$ & $\mathrm{X}_{2}$ & $\bar{X}_{2}=\mathrm{X}_{1}$ \\
\hline $\mathrm{n}=3$ & $\mathrm{X}_{3}$ & $\bar{X}_{3}=\mathrm{X}_{2}$ \\
\hline- & - & - \\
\hline $\mathrm{n}=\mathrm{n}$ & $\mathrm{X}_{\mathrm{n}}$ & $\bar{X}_{\mathrm{n}}=\mathrm{X}_{\mathrm{n}[1}$ \\
\hline- & - & - \\
\hline $\mathrm{n}=\mathrm{N}$ & $\mathrm{X}_{\mathrm{N}}=\mathrm{X}_{\mathrm{FINAL}}$ & $\bar{X}_{\mathrm{N}}=\mathrm{X}_{\mathrm{N} \square 1}$ \\
\hline
\end{tabular}

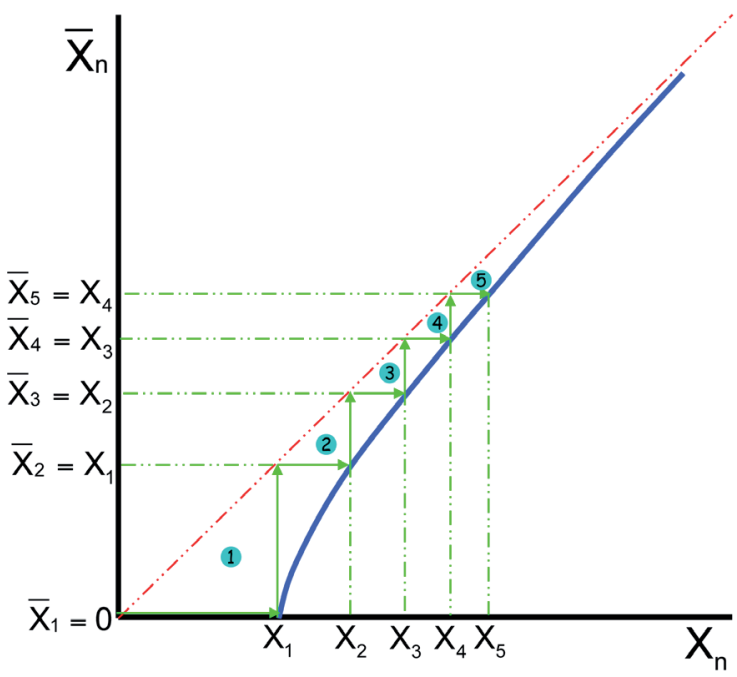

Figura 1. Relación entre $\bar{X}_{n}$ y $X_{n}$

Una vez que en la figura 1 se han representado simultáneamente el balance de materia (curva azul) y la condición de entrada (recta roja) se construye la triangulación mostrada en color verde trazando escalones entre la curva y la recta comenzando hasta alcanzar un valor de la conversión a la salida del último reactor igual o mayor que la conversión final deseada. El número de reactores necesario es igual al de triángulos rectángulos cuya hipotenusa coincide con la diagonal. Por supuesto, a cada valor de $t \mathrm{y} / \mathrm{o} \mathrm{T}$ le corresponde una curva diferente. La distancia existente entre la curva y la diagonal es proporcional a $t$. El punto de intersección entre la curva y la diagonal representa la conversión de equilibrio, a la T correspondiente, o $\mathrm{X}=1$ si se trata de una reacción irreversible.

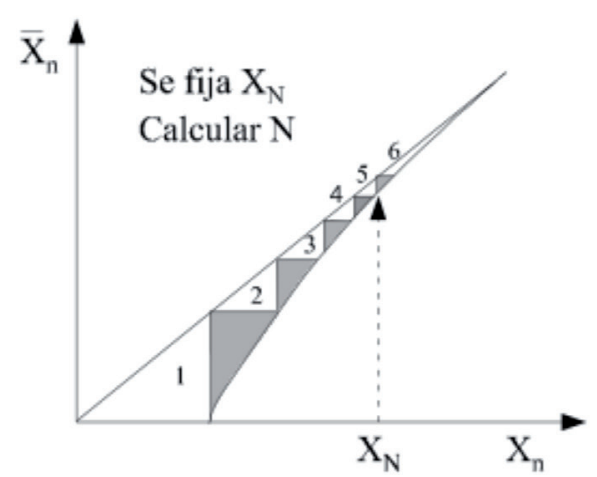

Figura 2. Se fija $X_{N}$ y se calcula $N$

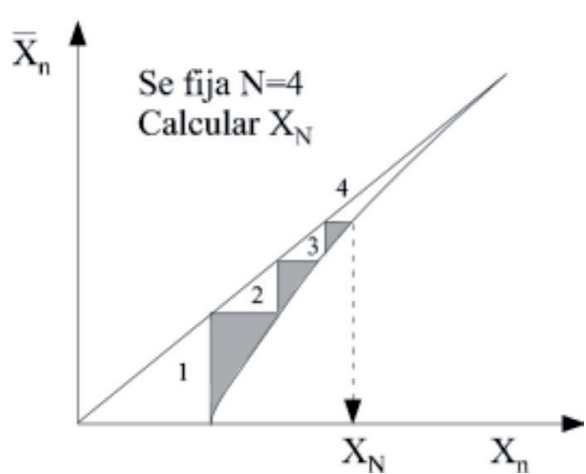

Figura 3. Se fija $\mathrm{N}$ y se calcula $X_{N}$

A partir de la figura 1 se puede fácilmente determinar bien cuántos RCTA se necesitan para alcanzar un determinado grado de conversión final o bien qué grado de conversión se puede alcanzar con un número determinado de RCTA, tal y como se muestra a continuación en las figuras 2 y 3 . 
Supuesto 2. Se conoce el valor de $N y X_{N}$

En este supuesto se procede de la misma forma que en el supuesto 1, mediante un proceso iterativo en el que se prueban diferentes valores de $\tau$ (o de V), hasta hallar el que cumple que se alcanza el grado de conversión final $\mathrm{X}_{\mathrm{N}}$ con el número $\mathrm{N}$ de RCTA de que se dispone.

\subsubsection{Cálculo del volumen de una serie de RCTA de diferente tamaño por el método de Jones}

Si los N RCTA conectados en serie tienen diferentes volúmenes, el balance de materia se puede escribir como:

$$
c_{k 0} \cdot \Delta X_{n}+v_{k} \cdot r_{n}\left(X_{n}, T_{n}\right) \cdot \tau_{n}=0 \quad n=1,2 \ldots N
$$

en la que se observa que el tiempo espacial es diferente para cada reactor. Asimismo, en esta ecuación la temperatura de cada RCTA, $T_{n}$, es conocida puesto que todos deben operar a las condiciones de mayor velocidad de reacción química posible. Es decir, que la ecuación anterior se puede escribir como:

- para reacciones endotérmicas y exotérmicas irreversibles

$$
c_{k 0} \cdot \Delta X_{n}+v_{k} \cdot r_{n}\left(X_{n}, T^{*}\right) \cdot \tau_{n}=0
$$

- para reacciones exotérmicas reversibles

$$
c_{k 0} \cdot \Delta X_{n}+v_{k} \cdot r_{n}\left(X_{n}, T_{n}\right) \cdot \tau_{n}=0 /\left(X_{n}, T_{n}\right) \in \Gamma_{m}
$$

En ambos casos, la ecuación se puede aplicar a cada RCTA $n$ de la serie. Los cálculos analíticos requieren el uso de métodos iterativos caso de que no se conozca el volumen de cada reactor de la serie. Para facilitar el proceso de cálculo el profesor Jones propone un método gráfico que consiste en expresar el balance de materia en la forma:

$$
\left(-v_{k}\right) \cdot r_{n}\left(X_{n}, T_{n}\right)=-\frac{c_{k 0}}{\tau_{n}} \cdot \bar{X}_{n}+\frac{C_{k 0}}{\tau_{n}} \cdot X_{n}
$$

A continuación se definen las funciones matemáticas:

- $\mathrm{R}\left[\mathrm{X}_{n}\right]=\left(-v_{k}\right) \cdot r_{n}\left(X_{n}, T_{n}\right)$ en la que la temperatura es conocida para cada valor discreto de $X_{n}$. Se puede obtener una función continua al representar $R$ frente a $X_{n}$, dando a esta última variable valores dentro del intervalo [0-1] (curva azul de la figura 4).

- $F_{\bar{X}_{n}}\left[X_{n}\right]=-\frac{C_{k 0}}{\tau} \cdot \bar{X}_{n}+\frac{C_{k 0}}{\tau} \cdot X_{n}$ para cada valor discreto de $X_{n}$. Se puede obtener una función continua al representar $F_{\bar{x}_{n}}$ frente a $X_{n}$, dando a esta última variable valores dentro del intervalo [0-1], para cada valor fijado de $\bar{X}_{n}$ (recuérdese que se cumple para cada RCTA que $\bar{X}_{n}=X_{n-1}$, rectas verdes). Se obtienen así 
líneas rectas de pendiente $c_{k 0} / \tau_{n}$ y ordenada en el origen $\left(-c_{k 0} \cdot \bar{x}_{n} / \tau_{n}\right)$ (rectas rojas de la figura 4).

- los puntos de corte entre las rectas y la curva de la figura 4 se recogen en la tabla 2 y satisfacen simultáneamente las $R\left[X_{n}\right]$ y $F_{\bar{x}_{n}}\left[x_{n}\right]$

Una vez construida la figura 4 se puede determinar el número de RCTA necesarios para alcanzar un grado de conversión final $\mathrm{X}_{\mathrm{N}} \mathrm{y}$ su volumen o bien qué grado de conversión se puede alcanzar con un número dado de RCTA de volumen conocido.

Obsérvese que las diferentes funciones $F_{\bar{x}_{n}}$, para cada valor de $\bar{X}_{n}$ fijado, son rectas paralelas si todos los RCTA tienen el mismo volumen. En consecuencia, este método también es aplicable al caso estudiado en el apartado 5.1.1. En este caso, a partir de la gráfica se puede fácilmente determinar bien cuántos RCTA se necesitan para alcanzar un determinado grado de conversión final o bien qué grado de conversión se puede alcanzar con un número determinado de RCTA, tal y como se muestra en las figuras 5 y 6 .

Tabla 2. Puntos de corte entre las rectas y la curva de la figura 4

\begin{tabular}{|c|c|c|c|}
\hline RCTA $\mathrm{n}$ & $\overline{\mathrm{X}}_{\mathrm{n}}$ & Pendiente & Punto de corte $\mathrm{R}\left[\mathrm{X}_{\mathrm{n}}\right]=\mathrm{F}_{\mathrm{X}_{\mathrm{n}}}\left[\mathrm{X}_{\mathrm{n}}\right]$ \\
\hline $\mathrm{n}=1$ & $\overline{\mathrm{X}}_{1}=0$ & $\mathrm{c}_{\mathrm{k} 0} / \tau_{1}$ & $\mathrm{X}_{1}, \mathrm{R}\left[\mathrm{X}_{1}\right]$ \\
\hline $\mathrm{n}=2$ & $\overline{\mathrm{X}}_{2}=\mathrm{X}_{1}$ & $\mathrm{c}_{\mathrm{k} 0} / \tau_{2}$ & $\mathrm{X}_{2}, \mathrm{R}\left[\mathrm{X}_{2}\right]$ \\
\hline $\mathrm{n}=3$ & $\overline{\mathrm{X}}_{3}=\mathrm{X}_{2}$ & $\mathrm{c}_{\mathrm{k} 0} / \tau_{3}$ & $\mathrm{X}_{3}, \mathrm{R}\left[\mathrm{X}_{3}\right]$ \\
\hline- & - & - & - \\
\hline $\mathrm{n}=\mathrm{n}$ & $\overline{\mathrm{X}}_{\mathrm{n}}=\mathrm{X}_{\mathrm{n}-1}$ & $\mathrm{c}_{\mathrm{k} 0} / \tau_{\mathrm{n}}$ & $\mathrm{X}_{\mathrm{n}}, \mathrm{R}\left[\mathrm{X}_{\mathrm{n}}\right]$ \\
\hline- & - & - & - \\
\hline $\mathrm{n}=\mathrm{N}$ & $\overline{\mathrm{X}}_{\mathrm{N}}=\mathrm{X}_{\mathrm{N}-1}$ & $\mathrm{c}_{\mathrm{k} 0} / \tau_{\mathrm{N}}$ & $\mathrm{X}_{\mathrm{N}}, \mathrm{R}\left[\mathrm{X}_{\mathrm{N}}\right]$ \\
\hline
\end{tabular}

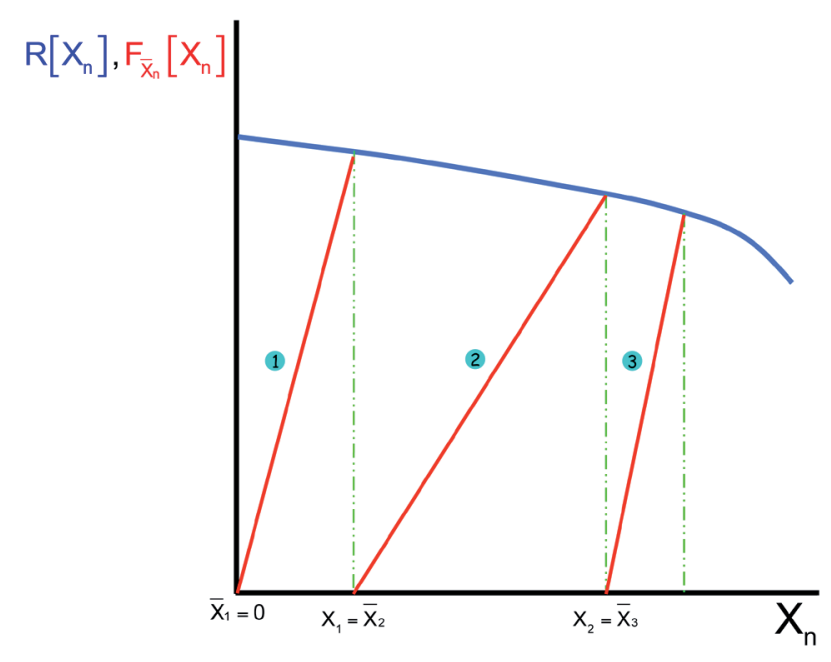

Figura 4. Forma de las funciones $R\left[x_{n}\right]$ y $F_{\bar{x}_{n}}\left[x_{n}\right]$ 


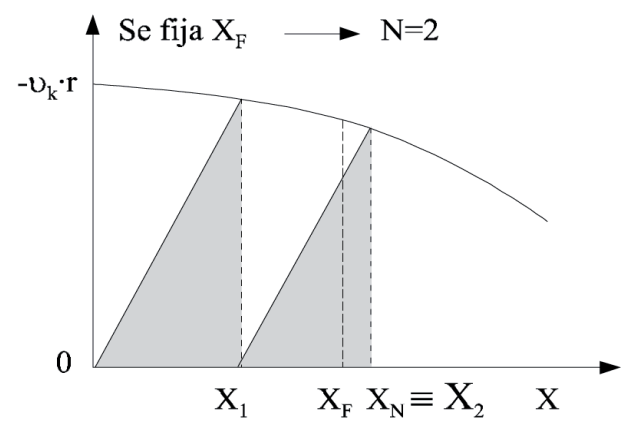

Figura 5. Se fija $\mathrm{X}_{\mathrm{N}}$ y se calcula $\mathrm{N}$

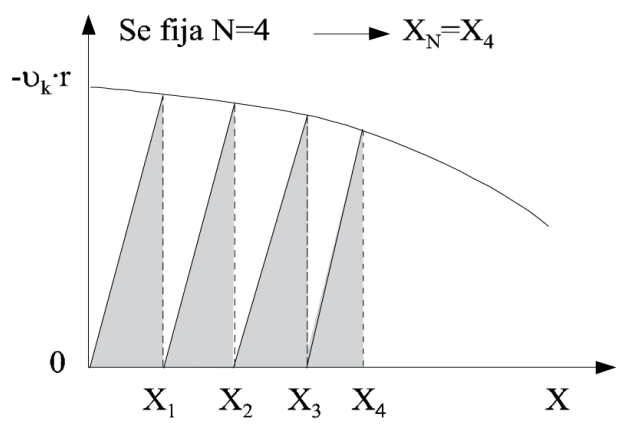

Figura 6. Se fija $\mathrm{N}$ y se calcula $\mathrm{X}_{\mathrm{N}}$

\subsubsection{Cálculo del volumen óptimo de cada RCTA de una serie}

En el caso de que sea preferible utilizar una secuencia de RCTA de distinto tamaño se plantea el problema de poder determinar el volumen óptimo de cada RCTA de la secuencia que minimiza el volumen total necesario para obtener una conversión dada a la salida del sistema. Uno de los posibles procedimientos es el método gráfico que se describe a continuación.

Supóngase un sistema de dos RCTA en serie en el que se va a llevar a cabo una reacción homogénea de cinética normal. La ecuación del balance de materia:

$$
\mathrm{c}_{\mathrm{k} 0} \cdot \Delta \mathrm{X}_{\mathrm{n}}+v_{\mathrm{k}} \cdot \mathrm{r}_{\mathrm{n}}\left(\mathrm{X}_{\mathrm{n}}, \mathrm{T}_{\mathrm{n}}\right) \cdot \tau_{\mathrm{n}}=0
$$

aplicada a cada reactor, puede escribirse en la forma:

$$
\begin{aligned}
& \text { RCTA } n=1 \quad c_{k 0} \cdot\left(x_{1}-\bar{X}_{1}\right)+v_{k} \cdot r_{1} \cdot \tau_{1}=c_{k 0} \cdot\left(x_{1}-0\right)+v_{k} \cdot r_{1} \cdot \tau_{1}=0 \rightarrow \frac{x_{1}}{\left(-v_{k}\right) \cdot r_{1}}=\frac{\tau_{1}}{c_{k 0}} \\
& \text { RCTA n }=2 \quad c_{k 0} \cdot\left(x_{2}-\bar{X}_{2}\right)+v_{k} \cdot r_{2} \cdot \tau_{2}=c_{k 0} \cdot\left(x_{2}-x_{1}\right)+v_{k} \cdot r_{2} \cdot \tau_{2}=0 \rightarrow \frac{\left(x_{2}-x_{1}\right)}{\left(-v_{k}\right) \cdot r_{2}}=\frac{\tau_{2}}{c_{k 0}}
\end{aligned}
$$

Dado que los dos reactores son de diferente tamaño solo son posibles las dos secuencias que se muestran, junto con el correspondiente diagrama de la inversa de la velocidad de reacción versus el grado de conversión, en la figura 7.

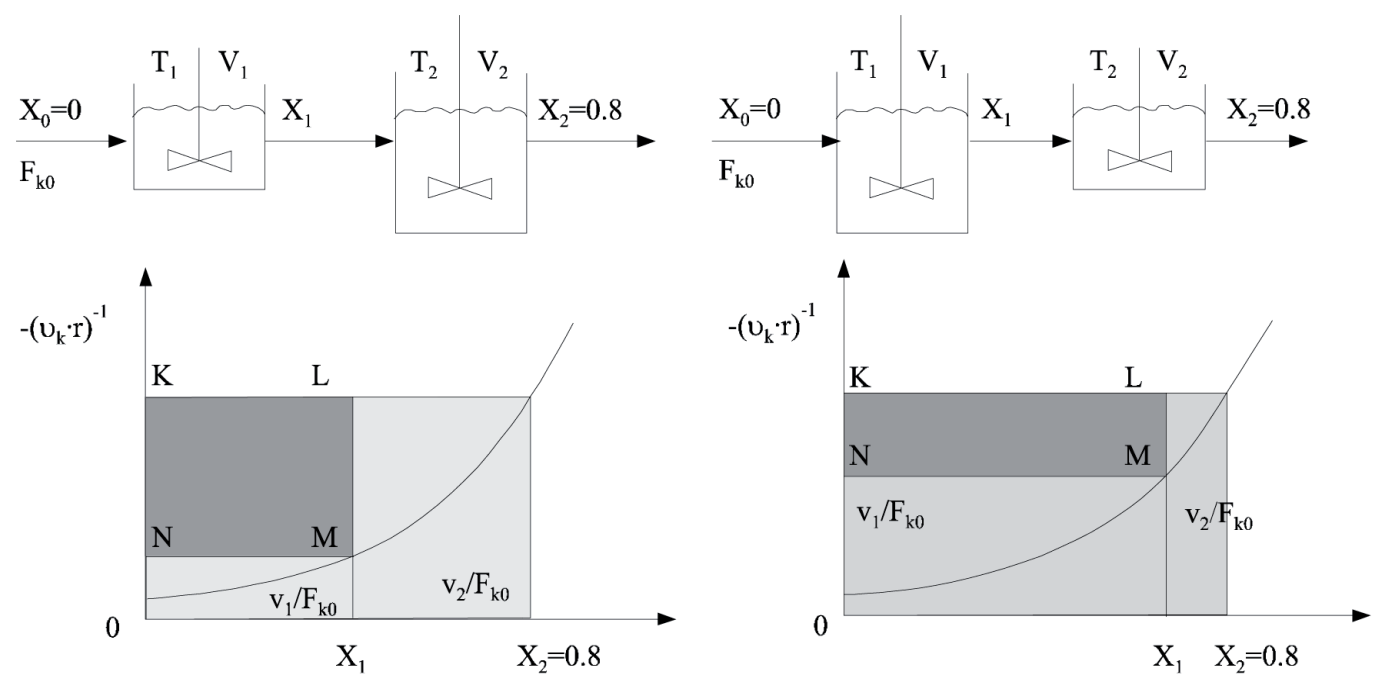

Figura 7. Secuencias posibles de dos reactores de diferente tamaño 
Independientemente de la secuencia elegida, minimizar el volumen total de reacción supone maximizar el área A del rectángulo KLMN. Si, para simplificar la nomenclatura, se define:

$$
y=\frac{1}{\left(-v_{k}\right) \cdot r}
$$

el área A se calcula como:

$$
A=\overline{L M} \cdot \overline{M N}=\left(y_{2}-y\right) \cdot(x-0)
$$

y aplicando la condición de máximo matemático:

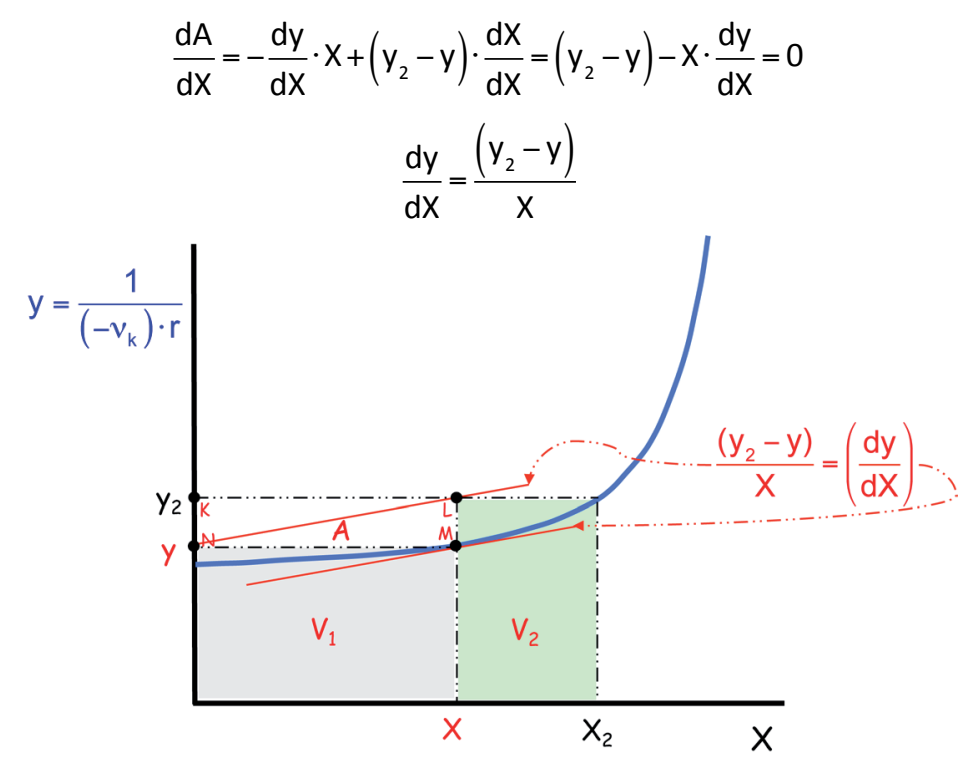

Figura 8. Condición de volumen mínimo en dos reactores

El segundo miembro de esta condición matemática representa la pendiente de la recta LN (diagonal del rectángulo KLMN) y el primero, la pendiente de la curva en el punto de coordenadas $(\mathrm{X}, \mathrm{y})$, según la figura 8 .

Como puede apreciarse, la condición de máximo para el rectángulo A es independiente de la conversión que se alcanzará en el segundo reactor $\left(\mathrm{X}_{2}\right)$.

Este procedimiento es también aplicable a sistemas de más de dos RCTA en serie, según se aprecia en la figura 9 que representa un sistema de tres reactores en serie. En consecuencia, tras maximizar el área A, hay que proceder a maximizar la A', como se detalla a continuación.

$$
\begin{gathered}
A^{\prime}=\overline{B C} \cdot \overline{C D}=\left(y_{3}-y\right) \cdot\left(x-x_{1}\right) \\
\frac{d A^{\prime}}{d X}=-\frac{d y}{d X} \cdot\left(x-x_{1}\right)+\left(y_{3}-y\right)=0 \\
\frac{d y}{d X}=\frac{\left(y_{3}-y\right)}{\left(x-x_{1}\right)}
\end{gathered}
$$




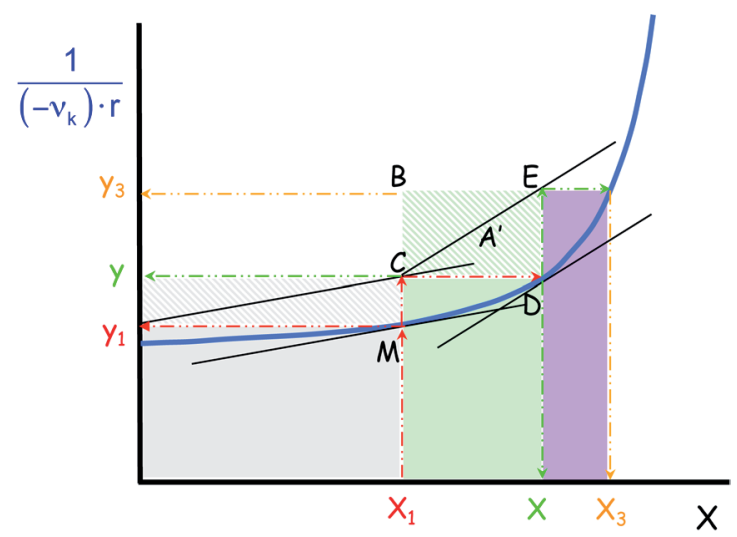

Figura 9. Condiciones de volumen mínimo en tres reactores

De nuevo, el segundo miembro de esta condición matemática representa la pendiente de la recta $\mathrm{CE}$ (diagonal del rectángulo $\mathrm{BCDE}$ ) y el primero, la pendiente de la curva en el punto de coordenadas (X, y) según la figura 9, siendo el resultado independiente de la conversión que se alcanzará en el tercer reactor $\left(\mathrm{X}_{3}\right)$.

El algoritmo general de resolución para el caso de una serie de N RCTA se detalla a continuación y se muestra en las figuras 10.1 a 10.7 :

1. Elegir un valor arbitrario de $X_{1}$ :

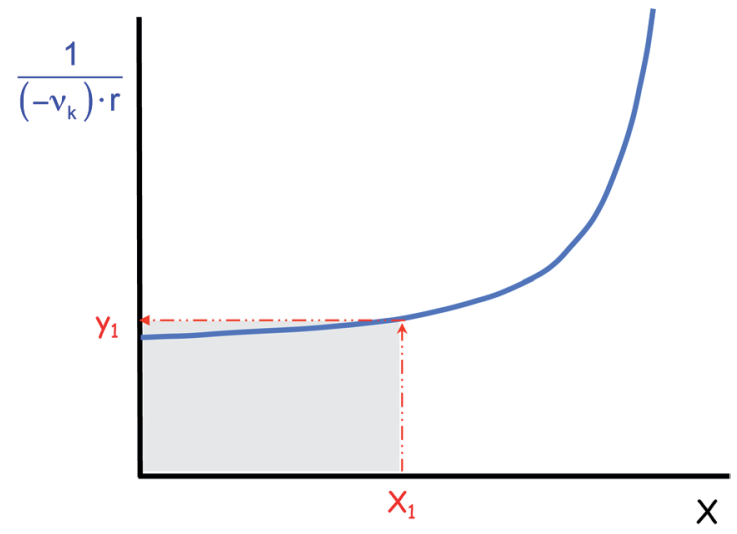

Figura 10.1

2. Trazar la tangente a la curva en el punto $\mathrm{M}$ de coordenadas $\left(\mathrm{X}_{1}, \mathrm{Y}_{1}\right)$ :

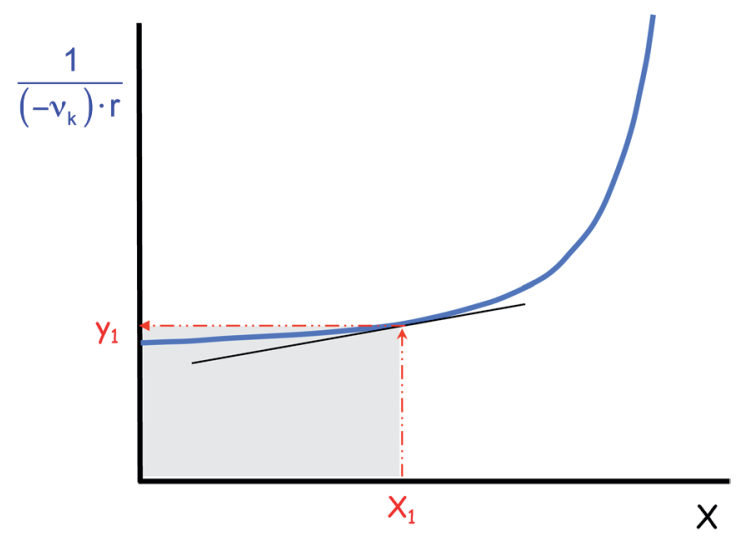

Figura 10.2 
3. Desde la proyección de este punto en el eje de ordenadas $\left(0, \mathrm{y}_{1}\right)$ se traza una recta paralela a la tangente anterior hasta determinar el valor de la ordenada correspondiente a $\mathrm{X}_{1}$ [punto $\mathrm{C}$, de coordenadas $\left(\mathrm{X}_{1}, \mathrm{y}_{2}\right)$ ]:

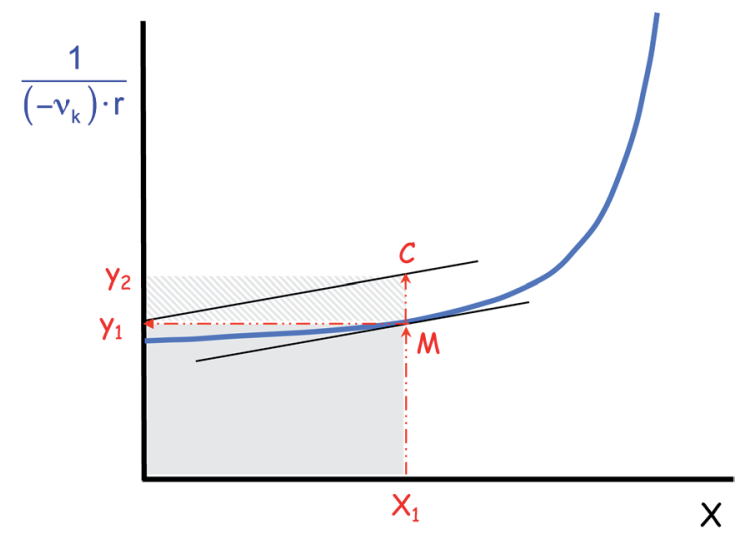

Figura 10.3

4. Desde el punto $\mathrm{C}$ se prolonga una paralela al eje de abscisas hasta cortar a la curva en el punto $\mathrm{D}$ de coordenadas $\left(\mathrm{X}_{2}, \mathrm{y}_{2}\right)$ :

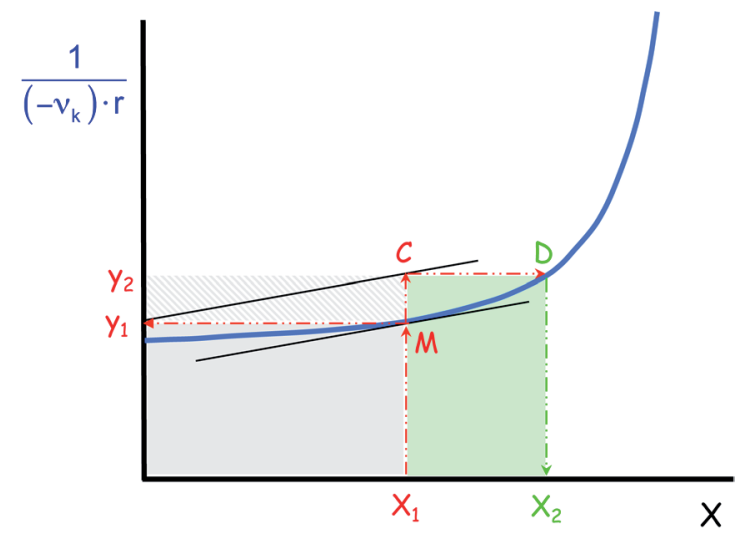

Figura 10.4

5. Se traza la tangente a la curva en el punto D:

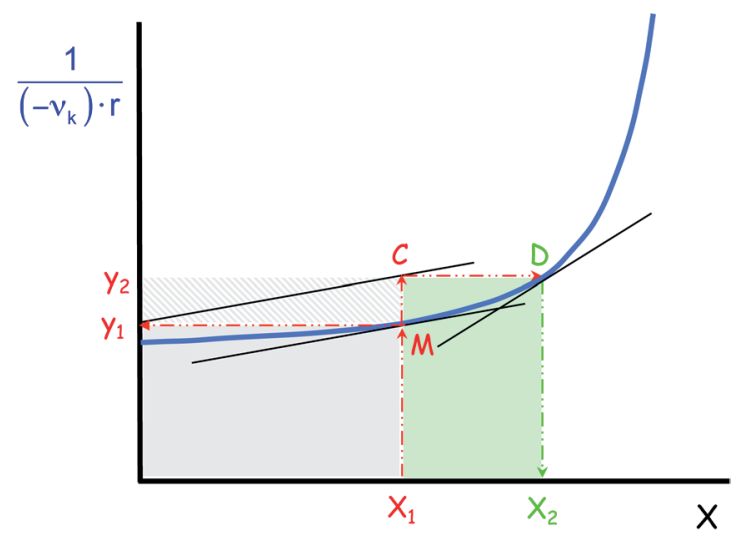

Figura 10.5 
6. Con origen en $\mathrm{C}$ se traza una recta paralela a dicha tangente para determinar el punto $\mathrm{E}$ de coordenadas $\left(\mathrm{X}_{2}, \mathrm{y}_{3}\right)$ :

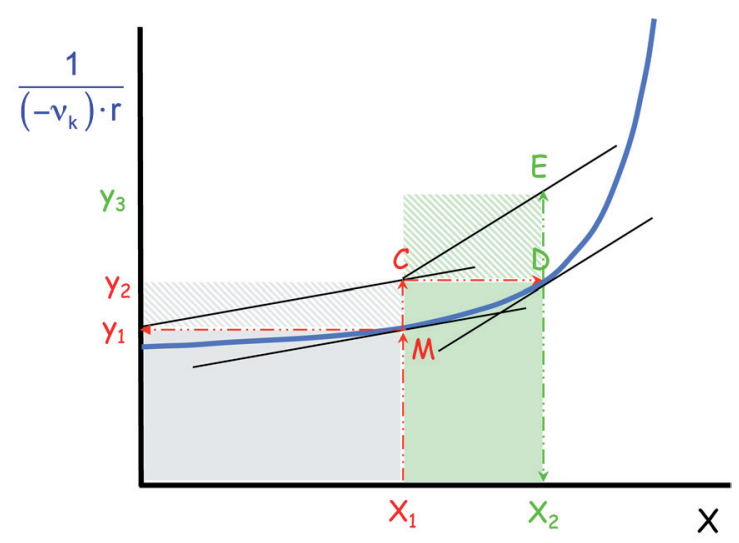

Figura 10.6

7. Repetir el proceso hasta llegar al $\mathrm{X}_{\mathrm{N}}$ establecido:

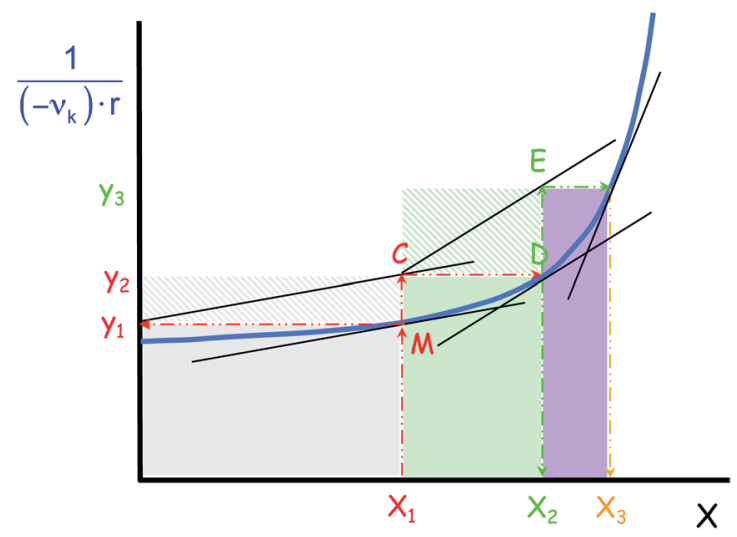

Figura 10.7

Si con el número de reactores de que se dispone no se alcanza el valor de $\mathrm{X}_{\mathrm{N}}$ deseado o se supera, se ha de repetir el tanteo hasta que la conversión final obtenida coincida con la deseada. Una vez determinadas las áreas de los rectángulos representativos de los reactores, se calculan los valores de $\mathrm{t}_{1} / \mathrm{c}_{\mathrm{k} 0}, \mathrm{t}_{2} / \mathrm{c}_{\mathrm{k} 0}, \ldots, \mathrm{t}_{\mathrm{N}} / \mathrm{c}_{\mathrm{k} 0}$ óptimos. Szepe y Levenspiel (1964) construyeron gráficas que permiten calcular los tamaños óptimos para distintas conversiones y diferentes valores del número de RCTA conectados en serie. Al advertir que era muy pequeña la diferencia entre el volumen total calculado cuando se utilizaba el montaje que determinaba el volumen total mínimo y el que se obtenía cuando todos los reactores eran iguales, llegaron a la conclusión de recomendar siempre el empleo de unidades de igual tamaño. En efecto, para reacciones de primer orden es óptimo utilizar reactores de igual tamaño, mientras que para reacciones de orden distinto de uno, la diferencia de volumen total entre montajes con reactores de igual tamaño o no, rara vez es superior al $10 \%$. 


\subsubsection{Sistemas con varias reacciones químicas}

En estos sistemas, el criterio que permite seleccionar la temperatura de trabajo más adecuada de cada reactor consiste en optimizar la selectividad del producto principal, favoreciendo el transcurso de las reacciones por las que éste se forma y dificultando las reacciones por las que desaparece o aquellas que conducen a otros productos no deseados, procurando que cada reactor opere a la temperatura más alta que convenga, para reducir, a la vez (en lo posible) el volumen del reactor necesario. Los cálculos necesarios implican una gran cantidad de trabajo algebraico, así que resulta conveniente comparar la respuesta final con algunas conclusiones de carácter cualitativo que pueden deducirse fácilmente del estudio de las reacciones que tienen lugar.

A continuación se mostrará de forma cualitativa, como ejemplo, un sistema de dos reacciones consecutivas irreversibles de primer orden que se lleva a cabo en una serie de dos RCTA:

$$
\begin{gathered}
\mathrm{A}_{1} \stackrel{\mathrm{i}=1}{\longrightarrow} \mathrm{A}_{2} \stackrel{\mathrm{i}=2}{\longrightarrow} \mathrm{A}_{3} \\
\mathrm{r}_{1}=\mathrm{k}_{1}(\mathrm{~T}) \cdot \mathrm{c}_{1} ; \quad \mathrm{r}_{2}=\mathrm{k}_{2}(\mathrm{~T}) \cdot \mathrm{c}_{2}
\end{gathered}
$$

Hay que actuar sobre las velocidades de reacción dependiendo del producto que se desee obtener. Y en estas ecuaciones hay que recordar que intervienen dos parámetros: la temperatura, a través de la constante de velocidad, y la concentración de reactivo, que va variando al pasar de un reactor al siguiente de la serie. Ambos factores hay que tenerlos en consideración en un razonamiento cualitativo.

Supóngase, por ejemplo, que se desea obtener $\mathrm{A}_{2}$, por lo que hay que favorecer la reacción $\mathrm{i}=1$ e intentar minimizar la reacción $\mathrm{i}=2$, y que la relación de las energías de activación es $\mathrm{E}_{1}<<<\mathrm{E}_{2}$

En primer lugar conviene hacer una representación cualitativa de la variación de las constantes de velocidad con la temperatura (figura 11). Es decir en general conviene operar a temperaturas bajas donde $\mathrm{k}_{1}>\mathrm{k}_{2}$.

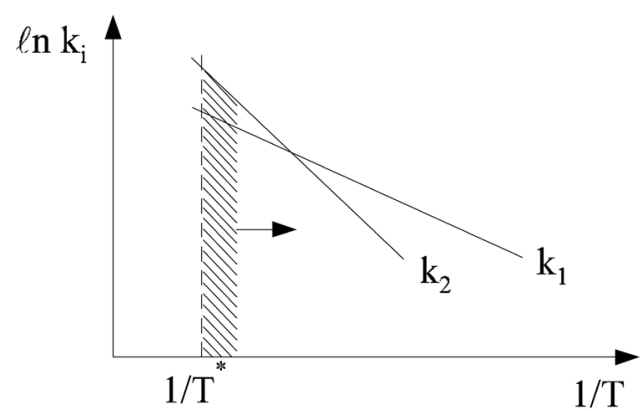

Figura 11. Variación de las constantes de velocidad con la temperatura 
Ahora bien, a pesar de esta diferencia de las constantes de velocidad, es evidente que en el primer reactor de la serie hay una elevada concentración de $\mathrm{A}_{1}$ pero muy pequeña de $\mathrm{A}_{2}$, por lo que se puede trabajar a temperaturas más altas (incluso aunque sea $\mathrm{k}_{2}>\mathrm{k}_{1}$ ) porque probablemente se cumplirá que $\mathrm{r}_{1}>\mathrm{r}_{2}$. Obviamente, en el segundo reactor de la serie aumenta la concentración de $\mathrm{A}_{2} \mathrm{y}$, para que se siga cumpliendo que $\mathrm{r}_{1}>\mathrm{r}_{2}$, este RCTA ha de operar a menor temperatura donde $\mathrm{k}_{1}>\mathrm{k}_{2}$

En conclusión el primer RCTA debe trabajar a temperaturas altas mientras que el segundo RCTA debe trabajar a una temperatura menor, como se muestra en la figura 11.

Si se deseara obtener $\mathrm{A}_{3}$ deberían potenciarse ambas reacciones, por lo que los dos reactores de la serie tendrían que operar a la máxima temperatura permisible.

Ejercicio 1. Hágase el mismo estudio cualitativo para el sistema de tres reacciones paralelas y consecutivas, irreversibles de primer orden, que se desarrollan en una serie de tres RCTA:

$$
\begin{array}{ll}
\mathrm{A}_{1} \stackrel{\mathrm{i}=1}{\longrightarrow} \mathrm{A}_{2} \stackrel{\mathrm{i}=3}{\longrightarrow} \mathrm{A}_{3} \\
\mathrm{~A}_{1} \stackrel{\mathrm{i}=2}{\longrightarrow} \mathrm{A}_{4} \\
\mathrm{r}_{1}=\mathrm{k}_{1}(\mathrm{~T}) \cdot \mathrm{c}_{1} ; \quad \mathrm{r}_{2}=\mathrm{k}_{2}(\mathrm{~T}) \cdot \mathrm{c}_{1} ; \quad \mathrm{r}_{3}=\mathrm{k}_{3}(\mathrm{~T}) \cdot \mathrm{c}_{2}
\end{array}
$$

en que $\mathrm{E}_{1}<<\mathrm{E}_{2}$ y el producto principal que se desea obtener sea $\mathrm{A}_{3}$.

\subsection{Asociación de RFP}

En los capítulos anteriores se ha considerado el diseño y comportamiento de un único reactor. Como ya se indicó en la asignatura previa EQ1021-Ingeniería de la Reacción Química, una secuencia o batería de RFP conduce al mismo volumen de reacción que si se utilizara un único RFP si se mantiene la temperatura de proceso constante. Sin embargo, el volumen total se puede minimizar si cada RFP de la serie trabaja a las condiciones de temperatura más adecuadas.

En el Anexo III se recoge, a modo de recordatorio, los balances de materia que se aplican a una serie de RFP y la nomenclatura correspondiente, según lo estudiado en la asignatura previa EQ1021-Ingeniería de la Reacción Química. Asimismo, en el Anexo IV se muestran los balances de energía junto con la nomenclatura correspondiente aplicables a una serie de RFP, según lo expuesto en el capítulo 1.

Cada RFP debe operar a las condiciones de mayor velocidad de reacción química posible.

Para este tipo de reactores basta con resolver simultáneamente los balances de materia y energía integrados entre las condiciones de entrada y salida de cada reactor de la serie: 


$$
\begin{aligned}
& d V_{n}=F_{k 0} \cdot \frac{d X}{\left(-v_{k}\right) \cdot r} \quad\left(m^{3}\right) \rightarrow V_{n}=F_{k 0} \cdot \int_{\bar{X}_{n}}^{X_{n}} \frac{d X}{\left(-v_{k}\right) \cdot r} \quad\left(m^{3}\right) \\
& d T-J \cdot d X=d Q_{n} \quad(K) \rightarrow \int_{\bar{T}_{n}}^{T_{n}} d T-J \cdot \int_{\bar{X}_{n}}^{X_{n}} d X=\int d Q_{n} \quad(K)
\end{aligned}
$$

Lógicamente, esta integración depende del sistema de intercambio de calor utilizado, puesto que determina la expresión de $\mathrm{Q}_{\mathrm{n}}$. Para cada RFP de la serie se procede como si se tratada de un único reactor, tal y como se ha detallado en el capítulo 4. 


\section{ANEXO I}

\section{BALANCE DE MATERIA EN ASOCIACIONES DE RCTA}

Sea la siguiente secuencia de N RCTA:

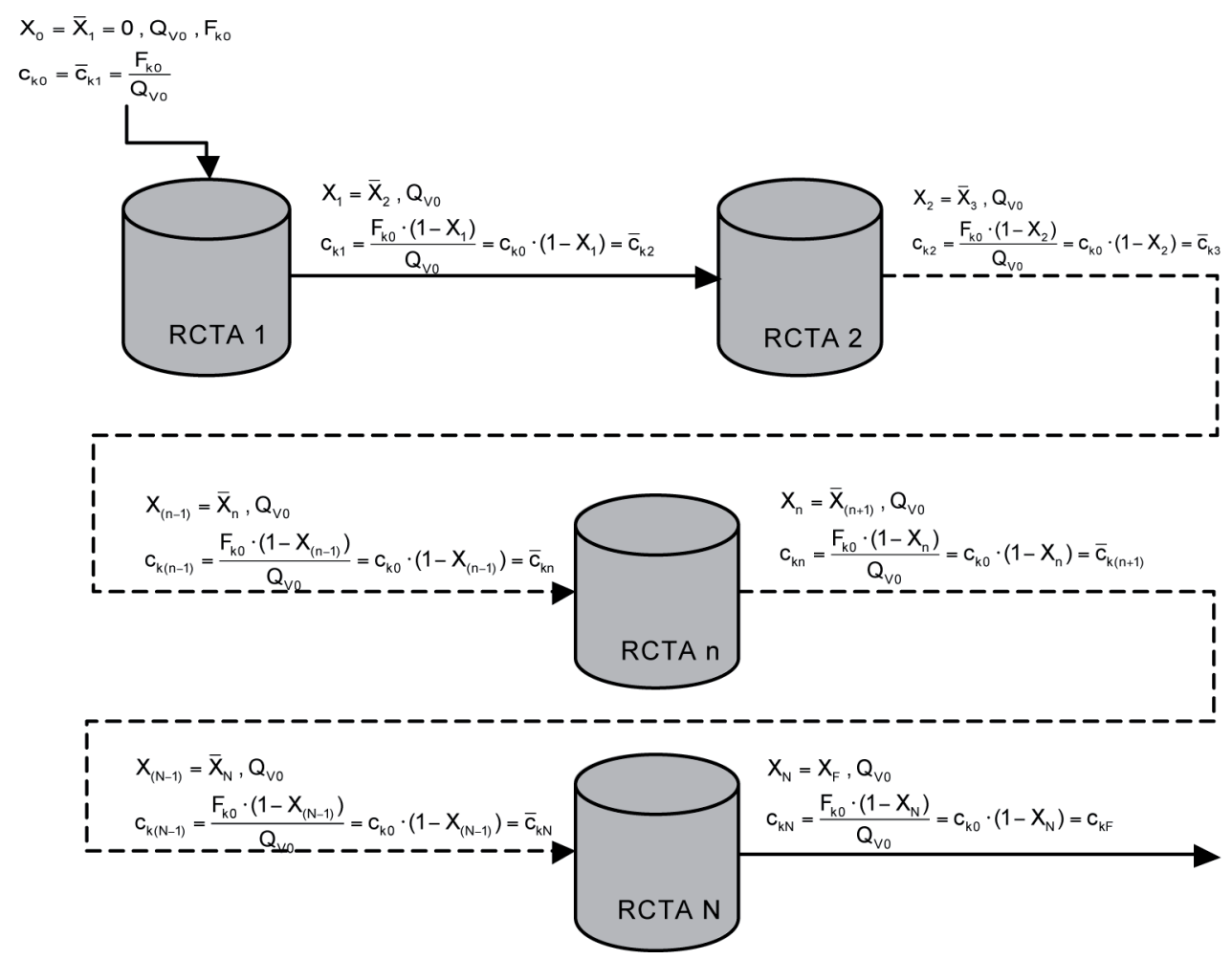

El balance general de materia un reactor $n$, cualquiera de una serie, será (siempre en $\left.\mathrm{kmol} \mathrm{A}_{\mathrm{j}} / \mathrm{s}\right)$ :

$$
F_{j n}-\bar{F}_{j n}+\frac{d}{d t} \int_{V} c_{j n} \cdot d V_{n}=\int_{V} \Re_{j n} \cdot d V_{n} \quad(j=1,2 \ldots S)
$$

para un reactor de tanque agitado (RTA)

$$
F_{\text {jn }}-\bar{F}_{\text {jn }}+\frac{d\left(c_{j n} \cdot V_{n}\right)}{d t}=\Re_{j n} \cdot V_{n}
$$

Si además de ser un RTA el reactor es continuo RCTA y funciona en régimen estacionario

$$
\bar{F}_{\mathrm{jn}}-\mathrm{F}_{\mathrm{jn}}+\Re_{\mathrm{jn}} \cdot \mathrm{V}_{\mathrm{n}}=0 \quad(\mathrm{j}=1,2 \ldots \mathrm{S})
$$

y para una reacción química se cumple que $\hat{\mathrm{A}}_{\mathrm{jn}}=\mathrm{v}_{\mathrm{j}} \cdot \mathrm{r}_{\mathrm{n}}$ :

$$
\bar{F}_{j n}-F_{j n}+v_{j} \cdot r_{n} \cdot v_{n}=0 \quad(j=1,2 \ldots S)
$$


Haciendo el siguiente cambio de variables, $\bar{c}_{\mathrm{jn}}=\bar{F}_{\mathrm{jn}} / \mathrm{Q}_{\mathrm{v} 0}, \mathrm{c}_{\mathrm{jn}}=\mathrm{F}_{\mathrm{jn}} / \mathrm{Q}_{\mathrm{vo}}$, y el tiempo espacial $\tau_{n}=V_{n} / Q_{v 0}$ :

$$
\bar{c}_{\mathrm{jn}}-\mathrm{c}_{\mathrm{jn}}+v_{\mathrm{j}} \cdot r_{\mathrm{n}} \cdot \tau_{\mathrm{n}}=0 \quad \mathrm{j}=1,2 \ldots \mathrm{S}
$$

Finalmente, relacionando concentración con grado de conversión para un sistema de densidad constante:

$$
\begin{aligned}
& \bar{c}_{j n}=c_{j 0}-c_{k 0} \cdot \frac{v_{j}}{v_{k}} \cdot \bar{x}_{n}=0 \quad(j=1,2 \ldots S) \\
& c_{j n}=c_{j 0}-c_{k 0} \cdot \frac{v_{j}}{v_{k}} \cdot x_{n}=0 \quad(j=1,2 \ldots S)
\end{aligned}
$$

se llega a la forma del balance de materia más útil para sistemas con una reacción química (el expresado en función del grado de conversión):

$$
c_{k 0} \cdot\left(X_{n}-\bar{X}_{n}\right)+v_{k} \cdot r_{n} \cdot \tau_{n}=c_{k 0} \cdot \Delta X_{n}+v_{k} \cdot r_{n} \cdot \tau_{n}=0
$$

En cualquiera de las dos formas anteriores del balance de materia se debe sustituir la velocidad de reacción por la correspondiente función de la temperatura y de la concentración o el grado de conversión $r_{n}=r_{n}\left(c_{j n}, T_{n}\right) \quad 0 \quad r_{n}=r_{n}\left(x_{n}, T_{n}\right)$ quedando:

$$
\bar{c}_{j n}-c_{j n}+v_{j} \cdot r_{n}\left(c_{j n}, T_{n}\right) \cdot \tau_{n}=0 \quad j=1,2 \ldots S
$$

o:

$$
c_{k 0} \cdot \Delta X_{n}+v_{k} \cdot r_{n}\left(X_{n}, T_{n}\right) \cdot \tau_{n}=0
$$

Estas dos ecuaciones indican que el volumen de reacción siempre es inversamente proporcional a la velocidad de reacción en cada RCTA. 


\section{ANEXO II \\ BALANCE DE ENERGÍA EN ASOCIACIONES DE RCTA}

Sea la siguiente secuencia de N RCTA:

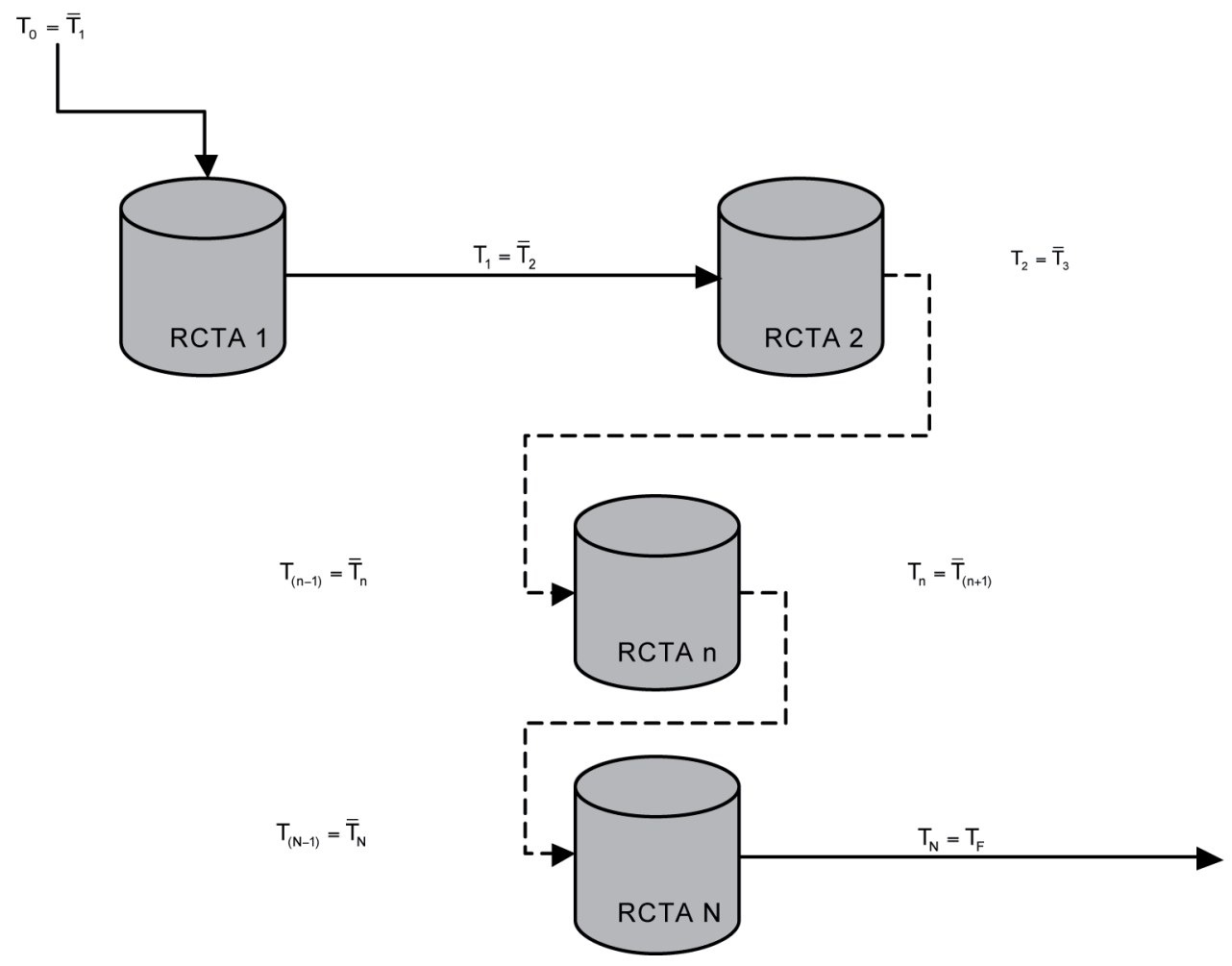

El balance general de energía para cualquier reactor $n$ de una serie será (siempre en $\mathrm{kJ} / \mathrm{s})$ :

$$
\sum_{j=1}^{S} F_{j n} \cdot h_{j n}-\sum_{j=1}^{S} \bar{F}_{j n} \cdot \bar{h}_{j n}+\frac{d}{d t} \int_{v}\left(e_{p}+e_{k}+u\right)_{n} \cdot c_{t n} \cdot d V_{n}=Q_{n}^{*}
$$

Para cada reactor continuo de tanque agitado (RCTA) de una batería que funciona en régimen estacionario:

$$
\sum_{j=1}^{S} F_{j n} \cdot h_{j n}-\sum_{j=1}^{S} \bar{F}_{j n} \cdot \bar{h}_{j n}=Q_{n}^{*}
$$

A continuación se introducen las variables más habituales $\left(\mathrm{X}_{\mathrm{n}} \mathrm{y}_{\mathrm{n}}\right)$ en lugar de $\mathrm{h}_{\mathrm{jn}}$, mediante una secuencia de cuatro pasos:

1) Se introduce $X_{n}$ a partir de la correspondiente relación estequiométrica:

$$
\begin{aligned}
& \bar{F}_{j n}=F_{j 0}-F_{k 0} \cdot \frac{v_{j}}{v_{k}} \cdot \bar{X}_{n} \\
& F_{j n}=F_{j 0}-F_{k 0} \cdot \frac{v_{j}}{v_{k}} \cdot X_{n}
\end{aligned}
$$




$$
\sum_{j=1}^{s} F_{j 0} \cdot\left(h_{j n}-\bar{h}_{j n}\right)+\frac{F_{k 0}}{\left(-v_{k}\right)} \cdot \sum_{j=1}^{s} v_{j} \cdot\left(h_{j n} \cdot X_{n}-\bar{h}_{j n} \cdot \bar{X}_{n}\right)=Q_{n}^{*}
$$

2) Se introduce la entalpía de reacción por kmol de componente clave, admitiendo que no varía con la temperatura y que vale lo mismo calculada a la temperatura de reacción y de la corriente de salida y calculada a la temperatura de la corriente de entrada:

$$
\begin{gathered}
\Delta H_{k}^{0}=\frac{\sum_{j=1}^{s} v_{j} \cdot \bar{h}_{j n}}{\left(-v_{k}\right)}=\frac{\sum_{j=1}^{s} v_{j} \cdot h_{j n}}{\left(-v_{k}\right)} \\
\sum_{j=1}^{s} F_{j 0} \cdot\left(h_{j n}-\bar{h}_{j n}\right)+F_{k 0} \cdot \Delta H_{k}^{0} \cdot\left(X_{n}-\bar{X}_{n}\right)=Q_{n}^{*}
\end{gathered}
$$

3) Se introduce la capacidad calorífica de cada especie química, que se considerará constante e independiente de la temperatura:

$$
\begin{gathered}
\overline{\mathrm{h}}_{\mathrm{jn}}=\left(\Delta \mathrm{H}_{\mathrm{f}, \mathrm{j}}^{0}\right)_{298}+\int_{298}^{\overline{\mathrm{T}}_{\mathrm{n}}} \mathrm{C}_{\mathrm{pj}} \cdot \mathrm{dT}=\left(\Delta \mathrm{H}_{\mathrm{f}, \mathrm{j}}^{0}\right)_{298}+\left(\overline{\mathrm{T}}_{\mathrm{n}}-298\right) \cdot \overline{\mathrm{C}}_{\mathrm{pj}} \\
\mathrm{h}_{\mathrm{jn}}=\left(\Delta \mathrm{H}_{\mathrm{f}, \mathrm{j}}^{0}\right)_{298}+\int_{298}^{\mathrm{T}_{\mathrm{n}}} \mathrm{C}_{\mathrm{pj}} \cdot \mathrm{dT}=\left(\Delta \mathrm{H}_{\mathrm{f}, \mathrm{j}}^{0}\right)_{298}+\left(\mathrm{T}_{\mathrm{n}}-298\right) \cdot \overline{\mathrm{C}}_{\mathrm{pj}} \\
\left(\mathrm{T}_{\mathrm{n}}-\overline{\mathrm{T}}_{\mathrm{n}}\right) \cdot \sum_{\mathrm{j}=1}^{\mathrm{S}} \mathrm{F}_{\mathrm{j} 0} \cdot \overline{\mathrm{C}}_{\mathrm{p} j}+\mathrm{F}_{\mathrm{k} 0} \cdot \Delta \mathrm{H}_{\mathrm{k}}^{0} \cdot\left(\mathrm{X}_{\mathrm{n}}-\overline{\mathrm{X}}_{\mathrm{n}}\right)=\mathrm{Q}_{\mathrm{n}}^{*}
\end{gathered}
$$

4) Se compacta la expresión definiendo nuevos módulos:

$$
\begin{gathered}
\theta_{j}=\frac{F_{j 0}}{F_{k 0}}\left(T_{n}-\bar{T}_{n}\right) \cdot F_{k 0} \cdot \sum_{j=1}^{S} \theta_{j} \cdot \bar{C}_{p j}+F_{k 0} \cdot \Delta H_{k}^{0} \cdot\left(X_{n}-\bar{X}_{n}\right)=Q_{n}^{*} \\
T_{n}-\bar{T}_{n}-\frac{\left(-\Delta H_{k}^{0}\right)}{\sum_{j=1}^{S} \theta_{j} \cdot \bar{C}_{p j}} \cdot\left(X_{n}-\bar{X}_{n}\right)=\frac{Q_{n}^{*}}{F_{k 0} \cdot \sum_{j=1}^{S} \theta_{j} \cdot \bar{C}_{p j}} \\
J=\frac{\left(-\Delta H_{k}^{0}\right)}{\sum_{j=1}^{S} \theta_{j} \cdot \bar{C}_{p j}} \\
Q_{n}=\frac{Q_{n}^{*}}{F_{k 0} \cdot \sum_{j=1}^{S} \theta_{j} \cdot \bar{C}_{p j}} \\
T_{n}-\bar{T}_{n}-J \cdot\left(X_{n}-\bar{X}_{n}\right)=Q_{n}
\end{gathered}
$$

Si X se sustituye por el balance de materia queda:

$$
T_{n}-\bar{T}_{n}+J \cdot \frac{v_{k} \cdot r_{n} \cdot \tau_{n}}{C_{k 0}}=Q_{n}
$$


En la primera ecuación, que es la forma habitual del balance de energía, se debe sustituir la velocidad de transmisión de calor por la correspondiente función del gradiente de temperaturas (entre el fluido intercambiador y la mezcla de reacción), del coeficiente global de transmisión de calor y del área de transmisión de calor:

$$
Q_{n}=\kappa_{n} \cdot\left(T_{f 0 n}-T_{n}\right)
$$

De acuerdo con los tres tipos de RCTA se puede generalizar a una única ecuación de diseño:

$$
T_{n}-\bar{T}_{n}-J \cdot\left(X_{n}-\bar{X}_{n}\right)=\kappa_{n} \cdot\left(T_{\text {fon }}-T_{n}\right)
$$

siendo:

\begin{tabular}{|c|l|}
\hline$\kappa_{\mathrm{n}}=\frac{\mathrm{U}_{\mathrm{n}} \cdot \mathrm{A}_{\mathrm{n}}}{\mathrm{F}_{\mathrm{k} 0} \cdot \sum_{\mathrm{j}=1}^{\mathrm{s}} \theta_{\mathrm{j}} \cdot \mathrm{C}_{\mathrm{pj}}}$ & $\begin{array}{l}\text { si el RCTA } n \text { posee un sistema de } \\
\text { intercambio de calor con } \mathrm{T}_{\mathrm{fn}} \text { cons- } \\
\text { tante }\end{array}$ \\
\hline$\kappa_{\mathrm{n}}=\frac{\mathrm{m}_{\mathrm{fn}} \cdot \mathrm{C}_{\mathrm{fn}}}{\mathrm{F}_{\mathrm{k} \mathrm{j}} \cdot \sum_{\mathrm{j}=1}^{\mathrm{s}} \theta_{\mathrm{j}} \cdot \mathrm{C}_{\mathrm{pj}}} \cdot\left\{1-\exp \left[\frac{-\mathrm{U}_{\mathrm{n}} \cdot \mathrm{A}_{\mathrm{n}}}{\left.\left.\mathrm{m}_{\mathrm{fn}} \cdot \mathrm{C}_{\mathrm{fn}}\right]\right\}}\right.\right.$ & $\begin{array}{l}\text { si el RCTA } n \text { posee un sistema de in- } \\
\text { tercambio de calor con } \mathrm{T}_{\mathrm{fn}} \text { variable }\end{array}$ \\
\hline$\kappa_{\mathrm{n}}=0$ & $\begin{array}{l}\text { si el RCTA } n \text { no intercambia calor } \\
\text { (tiene comportamiento adiabático) }\end{array}$ \\
\hline
\end{tabular}




\section{ANEXO III \\ BALANCE DE MATERIA EN ASOCIACIONES DE RFP}

Sea la siguiente secuencia de N RFP:

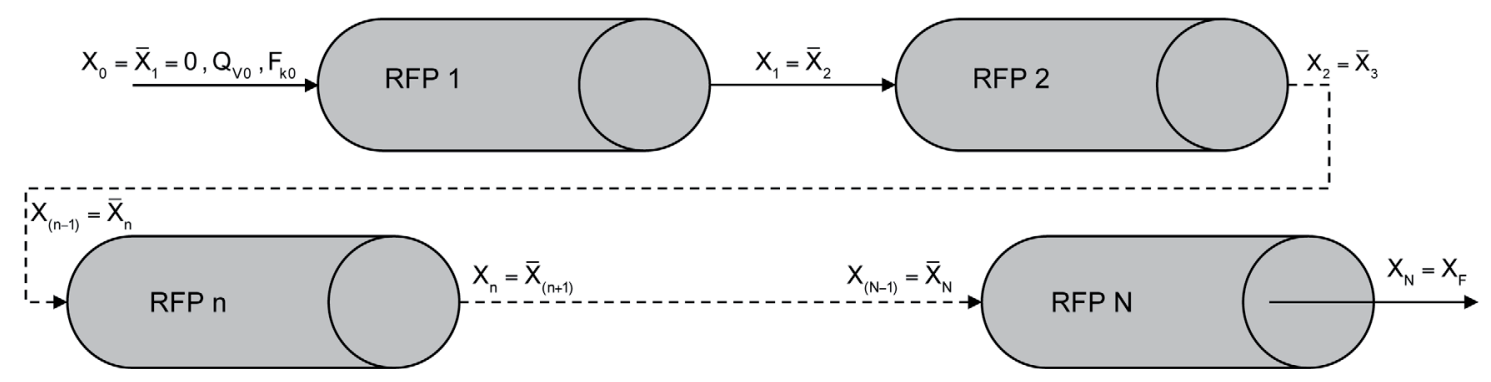

El balance general de materia a un reactor $n$, cualquiera de una serie, será (siempre en $\left.\mathrm{kmol} \mathrm{A}_{\mathrm{j}} / \mathrm{s}\right)$ :

$$
d F_{j}=\Re_{j} \cdot d V_{n}\left(\frac{k m o l A_{j}}{s}\right)
$$

para un reactor de flujo de pistón $n(\mathrm{RFP})$

$$
d V_{n}=F_{k 0} \cdot \frac{d X}{\left(-v_{k}\right) \cdot r}\left(m^{3}\right)
$$

e integrando:

$$
V_{n}=F_{k 0} \cdot \int_{\bar{x}_{n}}^{x_{n}} \frac{d X}{\left(-v_{k}\right) \cdot r}\left(m^{3}\right)
$$

donde $\mathrm{F}_{\mathrm{k} 0}$ es el caudal molar a la entrada del primer reactor de la serie. 


\section{ANEXO IV \\ BALANCE DE ENERGÍA EN ASOCIACIONES DE RFP}

Sea la siguiente secuencia de N RFP:
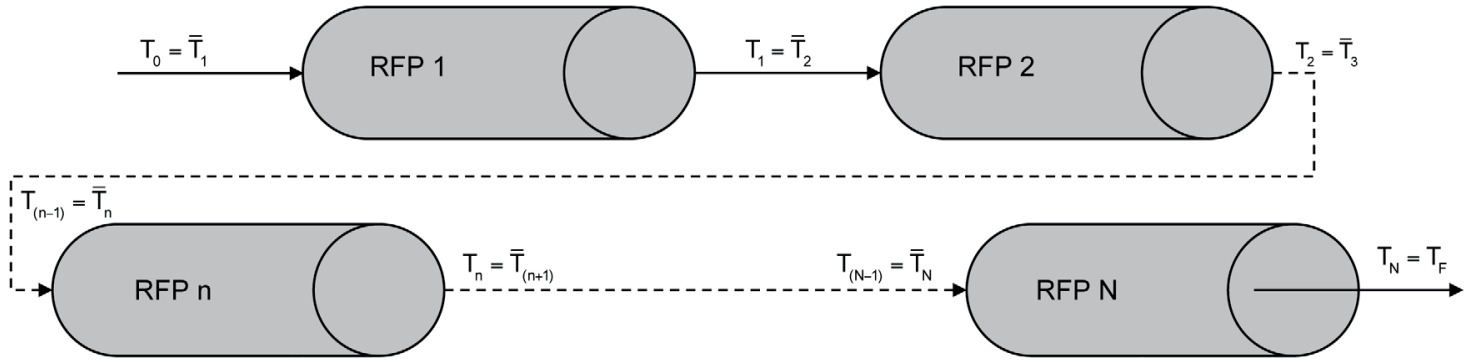

El balance general de energía para cualquier reactor $n$ de una serie será (siempre en $\mathrm{kJ} / \mathrm{s})$ :

$$
\sum_{j=1}^{S} F_{j} \cdot d h_{j}+\sum_{j=1}^{S} h_{j} \cdot d F_{j}=d Q_{n}^{*} \quad(k J / s)
$$

A continuación se introduce $\mathrm{X}$ a partir de la correspondiente relación estequiométrica:

$$
\begin{aligned}
& F_{j}=F_{j 0}-F_{k 0} \cdot \frac{v_{j}}{v_{k}} \cdot X \\
& d F_{j}=F_{k 0} \cdot \frac{v_{j}}{\left(-v_{k}\right)} \cdot d X
\end{aligned}
$$

resultando:

$$
\begin{aligned}
& \sum_{j=1}^{S}\left(F_{j 0}-F_{k 0} \cdot \frac{v_{j}}{v_{k}} \cdot X\right) \cdot d h_{j}+\sum_{j=1}^{S} h_{j} F_{k 0} \cdot \frac{v_{j}}{\left(-v_{k}\right)} \cdot d X=d Q_{n}^{*} \\
& \sum_{j=1}^{S} F_{j 0} \cdot d h_{j}+F_{k 0} \cdot X \cdot \frac{\sum_{j=1}^{S} v_{j} \cdot d h_{j}}{\left(-v_{k}\right)}+F_{k 0} \cdot d X \cdot \frac{\sum_{j=1}^{S} v_{j} \cdot h_{j}}{\left(-v_{k}\right)}=d Q_{n}^{*}(k J / s)
\end{aligned}
$$

Considerando la definición de la entalpía de una especie química, la entalpía de la reacción y sus correspondientes diferenciales, así como suponiendo que la entalpía de la reacción no cambia con la longitud del reactor (aunque cambien las condiciones de operación del reactor) se llega a:

$$
\left(\sum_{j=1}^{s} F_{j 0} \cdot \bar{C}_{p j}\right) \cdot d T+F_{k 0} \cdot \Delta H_{k}^{0} \cdot d X=d Q_{n}^{*} \quad(k J / s)
$$


Finalmente, considerando la definición del parámetro $\mathrm{J}$ de la reacción se obtiene:

$$
d T-J \cdot d X=\frac{d Q_{n}^{*}}{F_{k 0} \cdot \sum_{j=1}^{S} \theta_{j} \cdot \bar{C}_{p j}}(K)
$$

Si se define el parámetro $d Q_{n}=\frac{d Q_{n}^{*}}{s}$, el balance se puede reescribir como:

$$
\begin{aligned}
& F_{k 0} \cdot \sum_{j=1}^{S} \theta_{j} \cdot \bar{C}_{p j} \\
& d T-J \cdot d X=d Q_{n} \quad(K)
\end{aligned}
$$

o bien, a lo largo de la longitud del reactor:

$$
\frac{\mathrm{dT}}{\mathrm{d} \ell}-\mathrm{J} \cdot \frac{\mathrm{dX}}{\mathrm{d} \ell}=\frac{\mathrm{dQ}_{\mathrm{n}}}{\mathrm{d} \ell} \quad(\mathrm{K} / \mathrm{m})
$$

Integrando:

$$
\int_{\bar{T}_{n}}^{T_{n}} d T-J \cdot \int_{\bar{x}_{n}}^{X_{n}} d X=\int d Q_{n} \quad(K)
$$

Si en lugar de X se sustituye el correspondiente balance de materia, se obtiene otra expresión del balance de energía para un RFP de la serie en el que tiene lugar una reacción química:

$$
\frac{\mathrm{dT}}{\mathrm{d} \ell}+\frac{\mathrm{v}_{\mathrm{k}} \cdot \mathrm{r}}{\mathrm{G}_{\mathrm{k} 0}} \cdot \mathrm{J}=\frac{\mathrm{dQ}_{\mathrm{n}}}{\mathrm{d} \ell} \quad(\mathrm{K} / \mathrm{m})
$$




\section{PROBLEMAS}

Problema 01. Se desea llevar a cabo la reacción elemental reversible $2 \mathrm{~A}_{1} \leftrightarrow \mathrm{A}_{2}+\mathrm{A}_{3}$ en uno o más RCTA conectados en serie. El alimento se introduce con un caudal de $3.5 \mathrm{~m}^{3} / \mathrm{h}$, exento de $\mathrm{A}_{2} \mathrm{y} \mathrm{A}_{3}$, y con una concentración de $\mathrm{A}_{1}$ de $48 \mathrm{kmol} / \mathrm{m}^{3}$. Calcular:

a) el tamaño de reactor que se requiere si las concentraciones finales de $\mathrm{A}_{2} \mathrm{y} \mathrm{A}_{3}$ han de ser iguales al $85 \%$ de las de equilibrio;

b) el número de RCTA en serie requeridos para alcanzar el grado de conversión deseado si los recipientes de que se dispone tienen un volumen igual al $5 \%$ de la capacidad del reactor único;

c) una vez en funcionamento el conjunto de reactores determinado en el apartado anterior, hay que separar uno para proceder a su reparació. ¿En qué medida habrá que modificar el caudal volumétrico para seguir manteniendo la conversión deseada?

\section{Datos y notas}

La constante de velocidad de la reacción directa, a la temperatura de reacción (que es la misma para todos los reactores), vale $2 \cdot 10^{-4} \mathrm{~m}^{3} / \mathrm{kmol} \cdot \mathrm{s}$ y la constante de equilibrio es igual a 16.

Problema 02. En dos RCTA en serie de igual volumen tiene lugar una reacción endotérmica irreversible en fase líquida, entre los reactivos $A_{1} y_{2}$. La reacción es de segundo orden. Cada reactor va provisto de una camisa de vapor de calefacción. Calcular la relación entre las áreas de las dos camisas de calefacción que se requiere para que la temperatura de los tanques sea la misma, a igualdad de velocidad de agitación y demás variables que afecten al coeficiente de transmisión de calor.

\section{Datos y notas}

En unidades autoconsistentes el volumen de cada reactor es $10^{4}$, la constante de velocidad $10^{-2}$, el caudal molar de alimentación de cada reactivo 5 , el caudal volumétrico de la solución 12 y la temperatura de alimentación igual a la de reacción.

La temperatura de condensación del vapor de calefacción en ambos reactores es la misma.

Problema 03. Se desea diseñar una serie de dos RCTA para alcanzar una conversión final de 0.85 , cuando se desarrolle la reacción en fase líquida, irreversible, de segundo orden $A_{1} \rightarrow$ productos. Las opciones de diseño, teniendo en cuenta que los dos RCTA operarán a la misma temperatura, son: 
a) dos RCTA en serie, del mismo tamaño

b) dos RCTA en serie, de tamaño tal que el volumen total de reacción sea mínimo

El coste de cada RCTA es $290 € / \mathrm{m}^{3}$, pero se aplica un descuento del $10 \%$ en el caso de que los RCTA sean del mismo tamaño y geometría.

¿Cuál de las dos opciones de diseño supone una inversión menor?

\section{Datos y notas}

$\mathrm{k}=0.075 \mathrm{~m}^{3} / \mathrm{kmol} \cdot \min ($ a la temperatura de reacción)

$\mathrm{Q}_{\mathrm{V} 0}=0.025 \mathrm{~m}^{3} / \mathrm{min}$

$\mathrm{c}_{10}=0.040 \mathrm{kmol} / \mathrm{m}^{3}$

Problema 04. Un conjunto de 6 RCTA de igual tamaño, conectados en serie, se utiliza para desarrollar a escala industrial la reacción de descomposición de un reactivo $\mathrm{A}_{1}$ en disolución. La reacción es de primer orden e irreversible y todos los RCTA operan a la misma temperatura. Bajo determinadas condiciones de operación, la corriente que sale del último reactor tiene una concentración igual al $5 \%$ de la que se alimenta al primer reactor. Debido a un inadecuado funcionamiento del primer reactor de la serie, éste se inutiliza, introduciendo el alimento en el segundo reactor.

a) ¿Cuál será el valor de la concentración de reactivo $\mathrm{A}_{1}$ en la corriente de salida del último reactor, si se mantiene el mismo caudal de alimentación que en el primer caso?

b) ¿Qué caudal de alimentación conducirá a la misma composición de la corriente de salida que se tenía originalmente?

\section{Datos y notas}

A las condiciones de operación: $\mathrm{k}=10 \mathrm{~h}^{-1} ; \mathrm{c}_{10}=1 \mathrm{kmol} / \mathrm{m}^{3}$.

Problema 05. Wassermann y sus colaboradores estudiaron la reacción de adición del tipo Diels-Alder entre la benzoquinona y el ciclopentadieno para dar un aducto $\mathrm{A}_{1}+\mathrm{A}_{2} \rightarrow$ productos. El estudio lo llevaron a cabo a $25{ }^{\circ} \mathrm{C}$ y comprobaron que se trata de una reacción irreversible y elemental en la que se pueden despreciar los cambios de volumen y con una constante de velocidad de $9.92 \cdot 10^{-3} \mathrm{~m}^{3} / \mathrm{kmol} \cdot \mathrm{s}$. Se desea desarrollar esta reacción en un RCTA, a $25^{\circ} \mathrm{C}$, con una corriente alimento de caudal $2.78 \cdot 10^{-4} \mathrm{~m}^{3} / \mathrm{s}$ en la que la concentración de benzoquinona y ciclopentadieno son, respectivamente, 0.08 y $0.1 \mathrm{kmol} / \mathrm{m}^{3}$.

a) Calcular el volumen de reacción para conseguir un grado de conversión de 0.875 .

b) Para disminuir el volumen de reacción obtenido en el apartado anterior se piensa sustituir dicho RCTA por una batería de 3 RCTA (todos ellos del mismo volumen y operando a $25^{\circ} \mathrm{C}$ ). Calcular, utilizando el método de Jones, el volumen de cada uno de estos RCTA, el volumen total de reacción y los grados de conversión a la salida de cada RCTA. 


\section{Consideraciones sobre el funcionamiento de reactores continuos adiabáticos}

\subsection{Curvas de velocidad de reacción constante}

Como norma general, para determinar la temperatura de trabajo más adecuada para un reactor se utilizan los diagramas de velocidad de reacción constante (diagrama $\mathrm{X}$ versus $\mathrm{T}$ ) ya que permiten detectar las condiciones para que la velocidad de reacción en el reactor sea la mayor posible, lo que garantiza un volumen mínimo. Una vez conocidos los valores de X y T que hacen que se cumpla esta condición, las ecuaciones de diseño permiten determinar el volumen del reactor y el resto de variables de proceso (véanse los capítulos 2, 3 y 4). Las curvas de velocidad de reacción constante se obtienen fácilmente a partir de la ecuación de la velocidad de reacción química (véase la asignatura EQ1021-Ingeniería de la reacción química).

La aplicación concreta del principio general enunciado en el párrafo anterior requiere distinguir entre el tipo de intercambio de calor aplicado al reactor, siendo un caso particular, pero de gran importancia, el caso de que el reactor esté perfectamente aislado, de tal manera que no intercambie calor con los alrededores y su comportamiento sea adiabático.

Para determinar la temperatura de trabajo más adecuada en reactores continuos adiabáticos resulta muy conveniente utilizar los diagramas de velocidad de reacción constante junto con el balance de energía aplicando el mismo principio general:

1) El diagrama $X$ versus $T$ para velocidad de reacción constante permite aplicar la condición de que la velocidad de reacción en el reactor sea la mayor posible.

2) El balance de energía, representado también en un diagrama $X$ versus $T$ permite relacionar $\mathrm{X}$ con $\mathrm{T} \mathrm{y}_{0}$, escogiendo los valores que permitan que se cumpla la condición anterior.

Para los dos tipos de reactores continuos estudiados (RCTA y RFP) el balance de energía es formalmente idéntico:

$$
\mathrm{T}=\mathrm{T}_{0}+\mathrm{J} \cdot \mathrm{X}
$$


aunque para tratarlo conjuntamente con las curvas de velocidad de reacción constante conviene reordenarlo para también poderlo representar en la forma $\mathrm{X}$ versus $\mathrm{T}$ :

$$
X=-\frac{T_{0}}{J}+\frac{1}{J} \cdot T
$$

En el caso de que se trate de un reactor de una secuencia el balance de energía también es idénticos y se expresa como:

$$
\begin{gathered}
T_{n}=\bar{T}_{n}+J \cdot\left(X_{n}-\bar{X}_{n}\right) \\
X_{n}=-\frac{\left(\bar{T}_{n}-J \cdot \bar{X}_{n}\right)}{J}+\frac{1}{J} \cdot T_{n}
\end{gathered}
$$

En un diagrama $\mathrm{X}$ versus $\mathrm{T}$ esta ecuación representa una línea recta de pendiente $1 / \mathrm{J}$, positiva (caso de que la reacción química sea exotérmica) o negativa (caso de que la reacción química sea endotérmica), y de ordenada en el origen $-T_{0} / J$ o $-\left(\bar{T}_{n}-J \cdot \bar{X}_{n}\right) / J$.

Es muy importante destacar que según el tipo de reactor continuo la recta adiabática tiene un significado físico diferente. En efecto:

i) En el caso de los RCTA, solo representa la unión de dos puntos con existencia real: el correspondiente a las condiciones de entrada del alimento y el correspondiente a las condiciones de operación del RCTA. Este hecho pone de manifiesto que un RCTA siempre trabaja a temperatura constante, independientemente de las condiciones de intercambio de calor. Por lo tanto, de la recta matemática solo tienen existencia real dos puntos: el que representa la corriente de entrada y el que representa las condiciones de funcionamiento del reactor (que coincide con la corriente de salida).

ii) En el caso de los RFP, la recta adiabática representa todas y cada una de las posiciones de la mezcla de reacción en el interior del RFP, recogiendo la información en cada punto a lo largo de la longitud del reactor (1), desde las condiciones de entrada hasta las de salida. Por lo tanto, de la recta matemática solo tienen existencia real el conjunto de puntos (segmento) comprendidos entre el punto que representa la corriente de entrada y el que representa la corriente de salida.

\subsection{Sistemas de un RCTA}

Dado que los RCTA trabajan a temperatura constante, una vez alcanzado el régimen estacionario, las condiciones de trabajo más adecuadas dependen del tipo de reacción. Así, las condiciones óptimas de temperatura son las que permiten una mayor velocidad de reacción química, según la curva correspondiente a cada tipo de reacción química: 
- el RCTA en el que tenga lugar una reacción química endotérmica (reversible o irreversible) o una reacción exotérmica irreversible debe operar en un estado estacionario estable que corresponda a la temperatura máxima permisible $\left(\mathrm{T}_{\mathrm{RCTA}}=\mathrm{T}^{*}\right)$

- el RCTA en el que tenga lugar una reacción química exotérmica reversible debe operar en un estado estacionario estable que corresponda a una pareja $\mathrm{X}_{\mathrm{RCTA}}, \mathrm{T}_{\mathrm{RCTA}}$ perteneciente a la curva de máxima velocidad de reacción $\left[\left(\mathrm{X}_{\mathrm{RCTA}}, \mathrm{T}_{\mathrm{RCTA}}\right) \in \Gamma_{\mathbf{m}}\right]$

Reacciones endotérmicas (reversibles e irreversibles)

Condiciones de trabajo a fijar de forma consecutiva y en el orden que se indica:

1. Dado que $\mathrm{J}<0$, el alimento se enfría cuando entra al reactor, por lo que la condición fundamental es que la temperatura del alimento sea la más alta posible: $\mathrm{T}_{0}=\mathrm{T}^{*}$

2. El $\mathrm{X}_{\mathrm{RCTA}}$ debe fijarse atendiendo a la producción o al beneficio económico que se desee obtener

Condición de trabajo determinada por las anteriores:

3. Al haber fijado $\mathrm{T}_{0} \mathrm{y} \mathrm{X}_{\mathrm{RCTA}}$ la temperatura de operación queda determinada por la ecuación (11) según:

$$
\mathrm{T}_{\mathrm{RCTA}}=\mathrm{T}_{0}+\mathrm{J} \cdot \mathrm{X}_{\mathrm{RCTA}}
$$

Estas condiciones se observan fácilmente en la representación simultánea, en la forma $\mathrm{X}$ versus $\mathrm{T}$, de las curvas de velocidad de reacción constante y del balance de energía (figura 1):

- al fijar la temperatura de entrada, el punto $\mathrm{T}=\mathrm{T}_{0}=\mathrm{T}^{*} \mathrm{y} X=0$ representa el origen de la recta de pendiente $1 / \mathrm{J}$ correspondiente a la ecuación de la recta adiabática

- al fijar el grado de conversión de operación del RCTA, $\mathrm{X}_{\mathrm{RCTA}}$, queda definida la recta paralela al eje de abscisas de ecuación $\mathrm{X}=\mathrm{X}_{\mathrm{RCTA}}$

- el punto de corte de las dos rectas determina $\mathrm{T}_{\mathrm{RCTA}}$, la temperatura de operación del RCTA. 


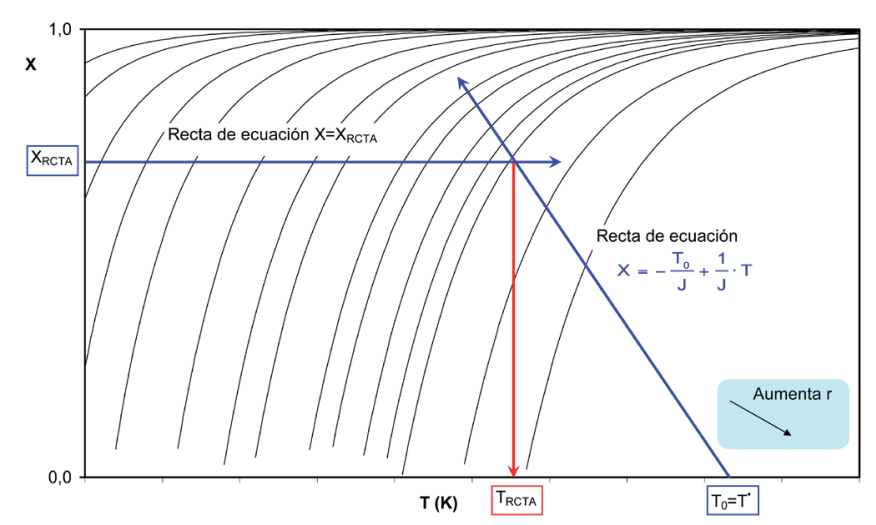

Figura 1. Condiciones de trabajo para reacciones endotérmicas

Obsérvese que para conseguir un $\mathrm{X}_{\mathrm{RCTA}}$ elevado a una velocidad de reacción aceptable (con la finalidad de que el volumen del RCTA no sea excesivamente grande) se requiere un valor de $1 / \mathrm{J}$ (pendiente de la recta adiabática) elevado, lo que es habitual en reacciones químicas con valores pequeños de entalpía. De no ser así se debería diseñar una secuencia de RCTA en lugar de un único RCTA, como se estudiará en el siguiente apartado.

Reacciones exotérmicas reversibles

Se distinguirá en esta ocasión dos casos posibles.

Caso 1

Condición de trabajo a fijar:

1. El $\mathrm{X}_{\mathrm{RCTA}}$ se fija atendiendo a la producción o al beneficio económico que se desee obtener

Condiciones de trabajo determinadas:

2. $\mathrm{La} \mathrm{T}_{\mathrm{RCTA}}$ queda automáticamente determinada puesto que el par $\left(\mathrm{X}_{\mathrm{RCTA}}, \mathrm{T}_{\mathrm{RCTA}}\right)$ debe pertenecer a la curva de velocidades de reacción máximas $\Gamma_{\mathrm{m}}$. Esta $\mathrm{T}_{\mathrm{RCTA}}$ se puede calcular fácilmente mediante la expresión matemática representativa de la curva $\Gamma_{\mathrm{m}}$ :

$$
T_{R C T A}=\frac{-\Delta H_{k}^{0}}{R \cdot \ln \left[\frac{E^{\prime}}{E} \cdot \frac{A^{\prime}}{A} \cdot \prod_{j=1}^{S} c_{j}^{v_{j}}\left(X_{R C T A}\right)\right]}
$$

3. Al haber fijado $\mathrm{X}_{\mathrm{RCTA}} \mathrm{y}$ calculado $\mathrm{T}_{\mathrm{RCTA}}, \mathrm{T}_{0}$ queda determinada por la ecuación (11) según:

$$
\mathrm{T}_{0}=\mathrm{T}_{\mathrm{RCTA}}-\mathrm{J} \cdot \mathrm{X}_{\mathrm{RCTA}}
$$


Estas condiciones se observan fácilmente en la representación simultánea, en la forma $\mathrm{X}$ versus $\mathrm{T}$, de las curvas de velocidad de reacción constante y del balance de energía (figura 2):

- al fijar el grado de conversión de operación del RCTA, $\mathrm{X}_{\mathrm{RCTA}}$, queda definida la recta paralela al eje de abscisas de ecuación $\mathrm{X}=\mathrm{X}_{\mathrm{RCTA}}$

- el punto de corte entre la recta $X=X_{\mathrm{RCTA}}$ y la curva $\Gamma_{\mathrm{m}}$ determina $\mathrm{T}_{\mathrm{RCTA}}$, la temperatura de operación del RCTA

- por el punto de corte indicado en el párrafo anterior pasa la recta de pendiente $1 / \mathrm{J}$ correspondiente a la ecuación de la recta adiabática. El punto de corte entre esta recta y el eje de abscisas representa la temperatura de entrada del alimento (punto $\mathrm{T}=\mathrm{T}_{0}, \mathrm{X}=0$ )

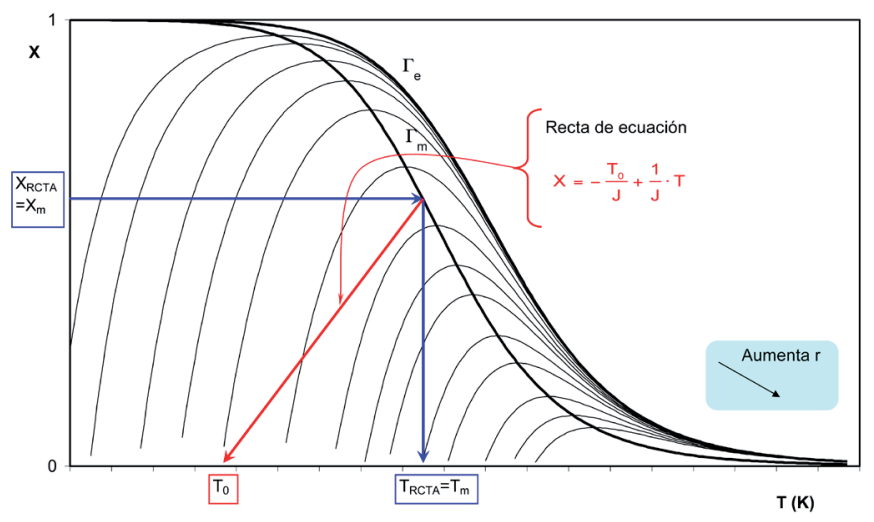

Figura 2. Condiciones de trabajo para reacciones exotérmicas reversibles (se fija X)

\section{Caso 2}

Condición de trabajo a fijar:

1. Se fija la temperatura de entrada del alimento, $\mathrm{T}_{0}$, al RCTA.

Condiciones de trabajo determinadas:

2. En este caso, teniendo en cuenta la forma de las curvas de velocidad de reacción constante y que la recta adiabática tiene una pendiente positiva, de valor $1 / \mathrm{J}$, se observa que puede existir un punto de tangencia entre una curva de velocidad de reacción constante y una recta adiabática. Dicho punto de tangencia representa la máxima velocidad de reacción a la que puede operar el RCTA, para un valor previamente fijado de $\mathrm{T}_{0}$.

Para una reacción química, con un valor determinado de $\mathrm{J}$, al modificar $\mathrm{T}_{0}$ se obtiene un punto de tangencia diferente con una curva de velocidad de reacción constante distinta. El conjunto de puntos de tangencia que se obtienen para diferentes $T_{0}$ define una curva denominada $\Gamma_{\mathrm{Jm}}$. 
La ecuación de la curva $\Gamma_{\mathrm{Jm}}$ se puede obtener fácilmente de su propia definición:

- Ecuación de la recta adiabática: $X=-\frac{T_{0}}{\mathrm{~J}}+\frac{1}{\mathrm{~J}} \cdot T$

- Pendiente de la recta adiabática: $\mathrm{dX} / \mathrm{dT}=1 / \mathrm{J}$

- Ecuación de cualquier curva de velocidad de reacción química constante: $r=r(X, T)$

Tangente a cualquier curva de velocidad de reacción química constante:

$$
\begin{aligned}
& d r=\left|\frac{\partial r}{\partial X}\right|_{T} \cdot d X+\left|\frac{\partial r}{\partial T}\right|_{X} \cdot d T \\
& r=\text { constante } \rightarrow d r=0 \\
& \frac{d X}{d T}=-\frac{\left|\frac{\partial r}{\partial T}\right|_{X}}{\left|\frac{\partial r}{\partial X}\right|_{T}}
\end{aligned}
$$

Por lo tanto:

$$
\frac{1}{J}=-\frac{\left|\frac{\partial r}{\partial T}\right|_{X}}{\left|\frac{\partial r}{\partial X}\right|_{T}}
$$

Esta ecuación representa el lugar geométrico de los puntos $\left(\mathrm{X}_{\mathrm{Jm}}, \mathrm{T}_{\mathrm{Jm}}\right)$ que pertenecen a la curva $\Gamma_{\mathrm{Jm}}$. Cada punto corresponde a una $\mathrm{T}_{0}$ diferente (para una reacción química representada por $\mathrm{J}$ ). Dicha $\mathrm{T}_{0}$ se puede calcular fácilmente porque el par $\left(\mathrm{X}_{\mathrm{Jm}}, \mathrm{T}_{\mathrm{Jm}}\right)$ también pertenece a la ecuación del balance de energía o recta adiabática $\mathrm{y}$, en consecuencia, se cumple:

$$
T_{0}=T_{J m}-J \cdot X_{J m}
$$

Estas condiciones se observan fácilmente en la representación simultánea, en la forma $\mathrm{X}$ versus $\mathrm{T}$, de las curvas de velocidad de reacción constante y del balance de energía (figura 3):

- por el valor fijado de $\mathrm{T}_{0}$ pasa la recta de pendiente $1 / \mathrm{J}$ correspondiente a la recta adiabática

- el punto de corte entre la recta adiabática y la curva $\Gamma_{\mathrm{Jm}}$ determina simultáneamente $\mathrm{T}_{\text {RCTA }}$ y $\mathrm{X}_{\mathrm{RCTA}}$ a los que debe operar el RCTA 


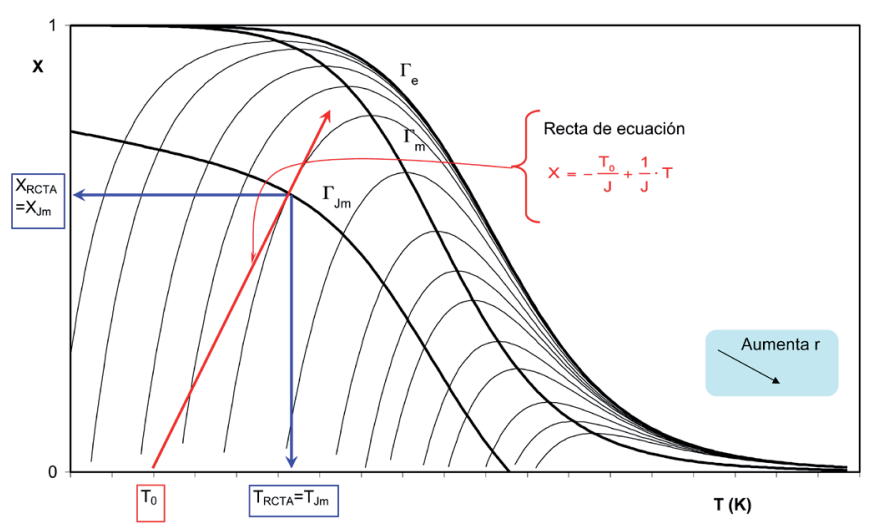

Figura 3. Condiciones de trabajo para reacciones exotérmicas reversibles (se fija $\mathrm{T}_{0}$ )

Es decir, para cada valor de $\mathrm{T}_{0}$ existe el correspondiente par $\left(\mathrm{X}_{\mathrm{Jm}}, \mathrm{T}_{\mathrm{Jm}}\right)$ en el que el RCTA está operando a la máxima velocidad de reacción posible. Para el valor de $\mathrm{T}_{0}$ fijado previamente el par $\left(\mathrm{X}_{\mathrm{Jm}}, \mathrm{T}_{\mathrm{Jm}}\right)$ representa el par $\left(\mathrm{X}_{\mathrm{RCTA}}, \mathrm{T}_{\mathrm{RCTA}}\right)$ al que debe operar el reactor.

Reacciones exotérmicas irreversibles

Se distinguirá en esta ocasión dos casos posibles.

Caso 1

Condiciones de trabajo a fijar de forma consecutiva y en el orden que se indica:

1. Dado que $\mathbf{J}>0$, el alimento se calienta cuando entra al reactor, por lo que la condición fundamental es que la temperatura de trabajo del reactor sea la más alta posible: $\mathrm{T}_{\mathrm{RCTA}}=\mathrm{T}^{*}$

2. El $\mathrm{X}_{\mathrm{RCTA}}$ debe fijarse atendiendo a la producción o al beneficio económico que se desee obtener

Condición de trabajo determinada por las anteriores:

3. Al haber fijado $\mathrm{X}_{\mathrm{RCTA}} \mathrm{y} \mathrm{T}_{\mathrm{RCTA}}, \mathrm{T}_{0}$ queda determinada por el balance de energía según:

$$
T_{0}=T_{\text {RCTA }}-J \cdot X_{\text {RCTA }}
$$

Estas condiciones se observan fácilmente en la representación simultánea, en la forma $\mathrm{X}$ versus $\mathrm{T}$, de las curvas de velocidad de reacción constante y del balance de energía (figura 4):

- al fijar la temperatura y el grado de conversión del reactor, el punto de intersección de las rectas $\mathrm{T}=\mathrm{T}_{\mathrm{RCTA}}=\mathrm{T}^{*} \mathrm{y} \mathrm{X}=\mathrm{X}_{\mathrm{RCTA}}$ representa un punto de la recta de pendiente $1 / \mathrm{J}$ correspondiente a la recta adiabática 
- el punto de corte entre la recta que pasa por el punto $\left(\mathrm{T}_{\mathrm{RCTA}}, \mathrm{X}_{\mathrm{RCTA}}\right)$ y de pendiente $1 / \mathrm{J}$ y la recta $X=0$ (eje de abscisas) indica la temperatura de entrada del alimento $\mathrm{T}_{0}$

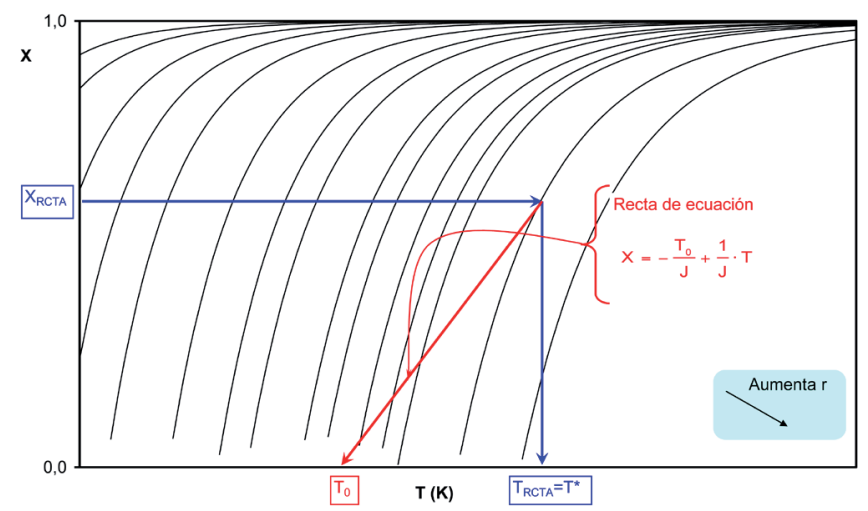

Figura 4. Condiciones de trabajo para reacciones exotérmicas irreversibles (se fija X)

\section{Caso 2}

Condición de trabajo a fijar:

1. Se fija la temperatura de entrada del alimento, $\mathrm{T}_{0}$, al RCTA.

Condiciones de trabajo determinadas:

2. En este caso, teniendo en cuenta la forma de las curvas de velocidad de reacción constante y que la recta adiabática tiene una pendiente positiva, de valor $1 / \mathrm{J}$, se observa que puede existir un punto de tangencia entre una curva de velocidad de reacción constante y una recta adiabática. Dicho punto de tangencia representa la máxima velocidad de reacción a la que puede operar el RCTA, para un valor previamente fijado de $\mathrm{T}_{0}$

Para una reacción química, con un valor determinado de $\mathrm{J}$, al modificar $\mathrm{T}_{0}$ se obtiene un punto de tangencia diferente con una curva de velocidad de reacción constante distinta. El conjunto de puntos de tangencia que se obtienen para diferentes $\mathrm{T}_{0}$ define una curva denominada $\Gamma_{\mathrm{Jm}}$.

El procedimiento para obtener la ecuación de la curva $\Gamma_{\mathrm{Jm}}$ es totalmente análogo al expuesto para las reacciones exotérmicas reversibles. Cada punto de la citada curva corresponde a una $\mathrm{T}_{0}$ diferente (para una reacción química representada por $\mathrm{J}$ ). Dicha $T_{0}$ se puede calcular fácilmente porque el par $\left(\mathrm{X}_{\mathrm{Jm}}, \mathrm{T}_{\mathrm{Jm}}\right)$ también pertenece al balance de energía o recta adiabática y, en consecuencia, se cumple:

$$
\mathrm{T}_{0}=\mathrm{T}_{\mathrm{Jm}}-\mathrm{J} \cdot \mathrm{X}_{\mathrm{Jm}}
$$


Estas condiciones se observan fácilmente en la representación simultánea, en la forma $\mathrm{X}$ versus $\mathrm{T}$, de las curvas de velocidad de reacción constante y del balance de energía (figura 5):

- por el valor fijado de $\mathrm{T}_{0}$ pasa la recta de pendiente $1 / \mathrm{J}$ correspondiente al balance de energía

- el punto de corte entre la recta adiabática y la curva $\Gamma_{\mathrm{Jm}}$ determina simultáneamente $\mathrm{T}_{\mathrm{RCTA}}$ y $\mathrm{X}_{\mathrm{RCTA}}$ a los que debe operar el RCTA

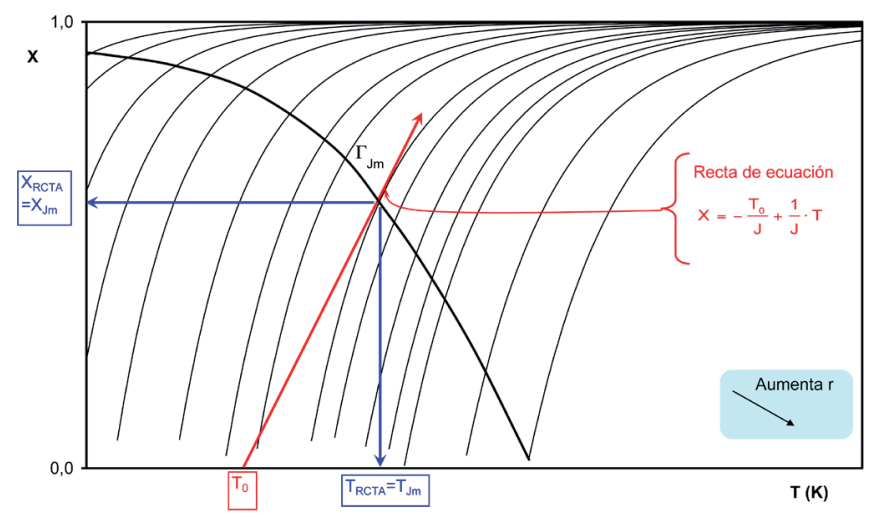

Figura 5. Condiciones de trabajo para reacciones exotérmicas irreversibles (se fija $\mathrm{T}_{0}$ )

Es decir, para cada valor de $\mathrm{T}_{0}$ existe el correspondiente par $\left(\mathrm{X}_{\mathrm{Jm}}, \mathrm{T}_{\mathrm{Jm}}\right)$ en el que el RCTA está operando a la máxima velocidad de reacción posible. Para el valor de $\mathrm{T}_{0}$ fijado previamente el par $\left(\mathrm{X}_{\mathrm{Jm}}, \mathrm{T}_{\mathrm{Jm}}\right)$ representa el par $\left(\mathrm{X}_{\mathrm{RCTA}}, \mathrm{T}_{\mathrm{RCTA}}\right)$ al que debe operar el reactor.

A continuación se resume en la tabla 1 la influencia de las características termodinámicas de una reacción química sobre su cinética y sobre las condiciones de operación de un RCTA adiabático.

Tabla 1. Condiciones de operación de un RCTA adiabático

\begin{tabular}{|c|c|c|c|c|c|}
\hline $\begin{array}{l}\text { ENTAL- } \\
\text { PÍA }\end{array}$ & $\begin{array}{l}\text { EQUILI- } \\
\text { BRIO }\end{array}$ & $\begin{array}{l}\text { Temperatura del } \\
\text { alimento }\end{array}$ & $\begin{array}{l}\text { Conver- } \\
\text { sión }\end{array}$ & Temperatura del RCTA & Caso \\
\hline \multirow{2}{*}{ ENDO } & REV & \multirow{2}{*}{$\mathrm{T}_{0}=\mathrm{T}^{*}$} & \multirow{2}{*}{$\mathrm{X}_{\mathrm{RCTA}}$} & \multirow{2}{*}{$\mathrm{T}_{\mathrm{RCTA}}=\mathrm{T}_{0}+\mathrm{J} \cdot \mathrm{X}_{\mathrm{RCTA}}$} & - \\
\hline & IRREV & & & & - \\
\hline \multirow{4}{*}{ EXO } & \multirow[t]{2}{*}{ REV } & $\begin{array}{c}\mathrm{T}_{0}=\mathrm{T}_{\mathrm{RCTA}}- \\
\mathrm{J} \cdot \mathrm{X}_{\mathrm{RCTA}}\end{array}$ & $\mathrm{X}_{\mathrm{RCTA}}$ & $\left(\mathrm{X}_{\mathrm{RCTA}}, \mathrm{T}_{\mathrm{RCTA}}\right) \in \Gamma_{\mathrm{m}}$ & 1 \\
\hline & & $\mathrm{T}_{0}$ & \multicolumn{2}{|c|}{$\left(\mathrm{X}_{\mathrm{RCTA}}, \mathrm{T}_{\mathrm{RCTA}}\right) \in \Gamma_{\mathrm{Jm}}$} & 2 \\
\hline & \multirow[t]{2}{*}{ IRREV } & $\begin{array}{c}\mathrm{T}_{0}=\mathrm{T}_{\mathrm{RCTA}}- \\
\mathrm{J} \cdot \mathrm{X}_{\mathrm{RCTA}}\end{array}$ & $\mathrm{X}_{\mathrm{RCTA}}$ & $\mathrm{T}_{\mathrm{RCTA}}=\mathrm{T}^{*}$ & 1 \\
\hline & & $\mathrm{T}_{0}$ & & $\left.{ }_{\mathrm{TA}}, \mathrm{T}_{\mathrm{RCTA}}\right) \in \Gamma_{\mathrm{Jm}}$ & 2 \\
\hline \multicolumn{3}{|c|}{ Valores fijados } & \multicolumn{3}{|c|}{ Valores calculados } \\
\hline
\end{tabular}




\subsection{Secuencia de RCTA}

La ventaja de utilizar una secuencia de RCTA estriba en que el volumen total de reacción necesario es menor que si se utiliza un solo reactor. Si los reactores son adiabáticos hay que intercalar sistemas de calefacción o enfriamiento entre cada dos RCTA consecutivos, a fin de que la temperatura de operación de cada uno esté lo más próxima posible a su valor óptimo. Para cada reactor de la serie las condiciones de trabajo a fijar son exactamente las mismas que las indicadas en el apartado 6.2 , en función de las características termodinámicas de la reacción que tenga lugar. A continuación se muestra un ejemplo para cada tipo de reacción suponiendo una secuencia de tres RCTA.

\section{Reacciones endotérmicas (reversibles e irreversibles)}

Como se ha indicado reiteradamente, para reacciones endotérmicas el criterio de optimización consiste en hacer operar los reactores a la temperatura más alta posible, por lo que conviene que el alimento se introduzca, en cada uno de ellos, a la máxima temperatura permisible (figura 6).

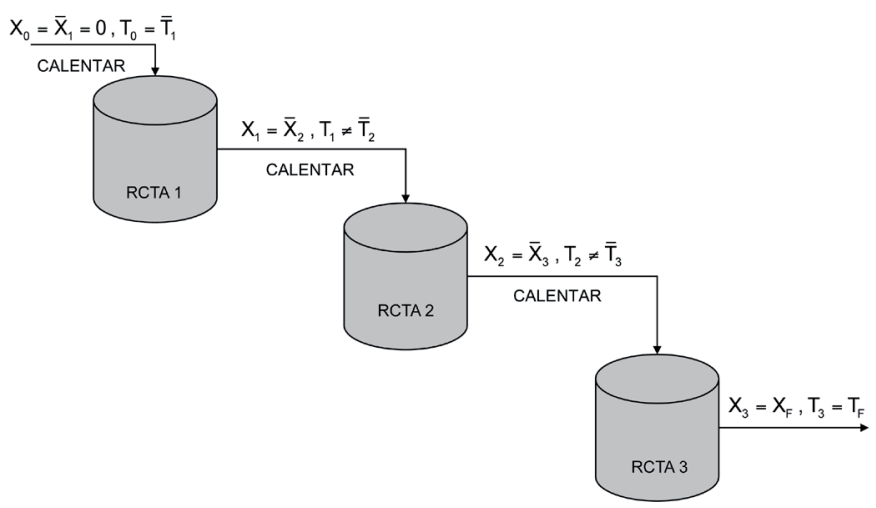

Figura 6. Secuencia de tres RCTA

En cualquier caso, si la pendiente de la adiabática es muy pequeña (elevado valor de J), ha de desecharse la utilización de RCTA adiabáticos para este tipo de reacciones, por la dificultad de alcanzar una conversión final elevada (figura 7).

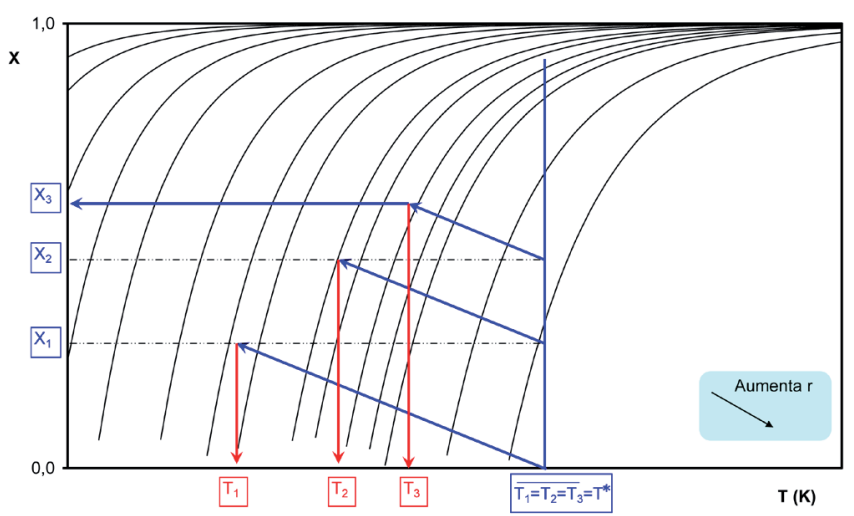

Figura 7. Condiciones de trabajo para reacciones endotérmicas 
En cada uno de los RCTA hay que tomar dos decisiones que se detallan secuencialmente en la primera columna de la tabla 2.

Tabla 2. Condiciones de trabajo para reacciones endotérmicas

\begin{tabular}{|c|c|c|c|}
\hline RCTA $\mathrm{n}$ & Temperatura del alimento & Conversión & Temperatura del reactor \\
\hline 1 & $\overline{\mathrm{T}}_{1}=\mathrm{T}^{*}$ & $\mathrm{X}_{1}$ & $\mathrm{~T}_{1}=\overline{\mathrm{T}}_{1}+\mathrm{J} \cdot\left(\mathrm{X}_{1}-\overline{\mathrm{X}}_{1}\right)=\mathrm{T}^{*}+\mathrm{J} \cdot\left(\mathrm{X}_{1}-0\right)$ \\
\hline 2 & $\overline{\mathrm{T}}_{2}=\mathrm{T}^{*}$ & $\mathrm{X}_{2}$ & $\mathrm{~T}_{2}=\overline{\mathrm{T}}_{2}+\mathrm{J} \cdot\left(\mathrm{X}_{2}-\overline{\mathrm{X}}_{2}\right)=\mathrm{T}^{*}+\mathrm{J} \cdot\left(\mathrm{X}_{2}-\mathrm{X}_{1}\right)$ \\
\hline 3 & $\overline{\mathrm{T}}_{3}=\mathrm{T}^{*}$ & $\mathrm{X}_{3}$ & $\mathrm{~T}_{3}=\overline{\mathrm{T}}_{3}+\mathrm{J} \cdot\left(\mathrm{X}_{3}-\overline{\mathrm{X}}_{3}\right)=\mathrm{T}^{*}+\mathrm{J} \cdot\left(\mathrm{X}_{3}-\mathrm{X}_{2}\right)$ \\
\hline \multicolumn{2}{|c|}{ Valores fijados } & Valores calculados \\
\multicolumn{2}{|c|}{} & \multicolumn{2}{r}{}
\end{tabular}

En consecuencia, en una secuencia de 3 RCTA hay que fijar 6 variables, de las que $\mathrm{X}_{1} \mathrm{y} \mathrm{X}_{2}$ son arbitrarias, mientras que las tres temperaturas de entrada se fijan por el tipo de reacción química y $\mathrm{X}_{3}$ según la producción deseada. El sistema se optimiza por tanteos sucesivos, resolviendo para distintos valores de $\mathrm{X}_{1} \mathrm{y} \mathrm{X}_{2}$ hasta obtener el volumen total mínimo. (También se podrían fijar arbitrariamente $\mathrm{T}_{1} \mathrm{y}$ $\mathrm{T}_{2}$, calculándose en ese caso $\mathrm{X}_{1}$ y $\mathrm{X}_{2}$ mediante la ecuación de la recta adiabática.) En general, para una secuencia de $\mathrm{N}$ RCTA hay que fijar $2 \mathrm{~N}$ variables.

También se han de diseñar los intercambiadores de calor necesarios, calculando las áreas de intercambio respectivas $\left(\mathrm{A}_{1}, \mathrm{~A}_{2}, \mathrm{y}_{3}\right)$, una vez conocida la temperatura de la mezcla reactante a la entrada y a la salida de cada uno de ellos.

\section{Reacciones exotérmicas irreversibles}

En este caso conviene que todos los reactores operen a la máxima temperatura posible, si se fija el grado de conversión, o sobre la curva $\Gamma_{\mathrm{Jm}}$, si se fija la temperatura de entrada. En este apartado se tratará solo el primer caso. Para ello se ha de precalentar el alimento a la entrada del primer reactor y luego se ha de refrigerar la mezcla reactante entre dos RCTA consecutivos (figura 8).

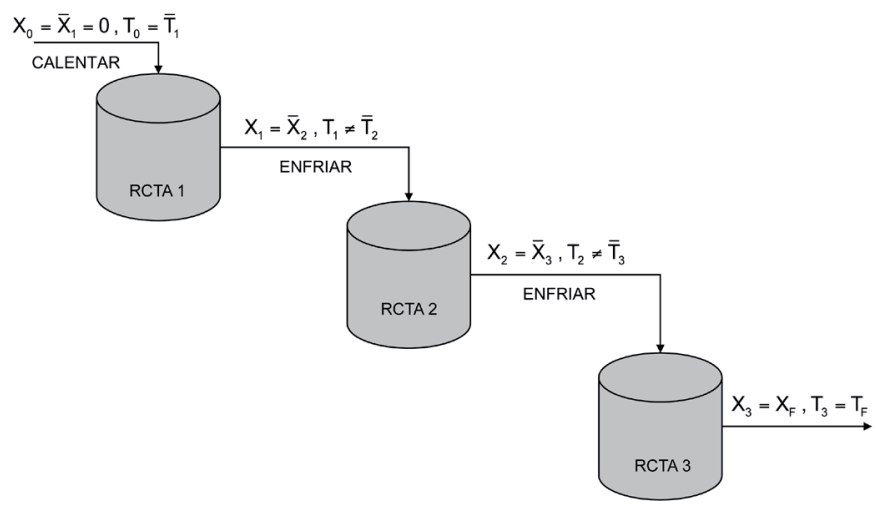

Figura 8. Secuencia de tres RCTA 
En la figura 9 se muestra las condiciones de trabajo para cada RCTA, una vez se ha fijado X.

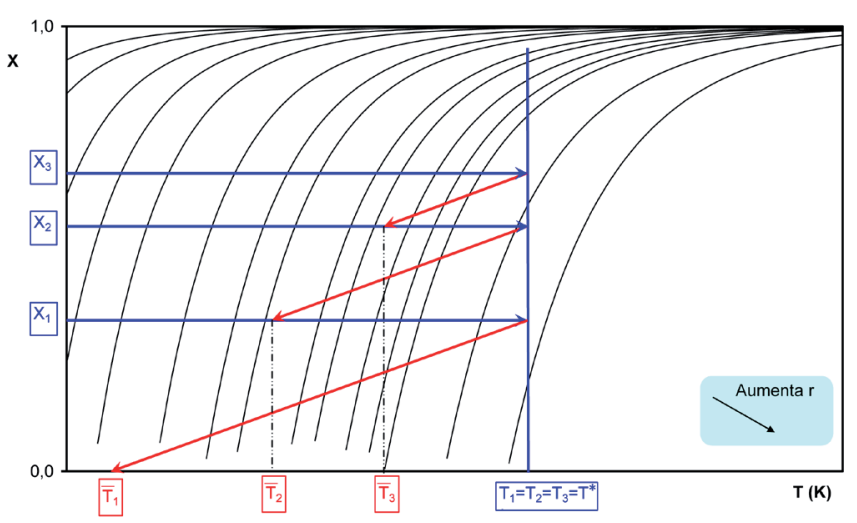

Figura 9. Condiciones de trabajo para reacciones exotérmicas irreversibles (se fija X)

En cada uno de los RCTA hay que tomar dos decisiones que se detallan secuencialmente en la primera columna de la tabla 3.

Tabla 3. Condiciones de trabajo para reacciones exotérmicas irreversibles (se fija X)

\begin{tabular}{|c|c|c|c|}
\hline RCTA $\mathrm{n}$ & Temperatura del reactor & Conversión & Temperatura del alimento \\
\hline 1 & $\mathrm{~T}_{1}=\mathrm{T}^{*}$ & $\mathrm{X}_{1}$ & $\overline{\mathrm{T}}_{1}=\mathrm{T}_{1}-\mathrm{J} \cdot\left(\mathrm{X}_{1}-\overline{\mathrm{X}}_{1}\right)=\mathrm{T}^{*}-\mathrm{J} \cdot\left(\mathrm{X}_{1}-0\right)$ \\
\hline 2 & $\mathrm{~T}_{2}=\mathrm{T}^{*}$ & $\mathrm{X}_{2}$ & $\overline{\mathrm{T}}_{2}=\mathrm{T}_{2}-\mathrm{J} \cdot\left(\mathrm{X}_{2}-\overline{\mathrm{X}}_{2}\right)=\mathrm{T}^{*}-\mathrm{J} \cdot\left(\mathrm{X}_{2}-\mathrm{X}_{1}\right)$ \\
\hline 3 & $\mathrm{~T}_{3}=\mathrm{T}^{*}$ & $\mathrm{X}_{3}$ & $\overline{\mathrm{T}}_{3}=\mathrm{T}_{3}-\mathrm{J} \cdot\left(\mathrm{X}_{3}-\overline{\mathrm{X}}_{3}\right)=\mathrm{T}^{*}-\mathrm{J} \cdot\left(\mathrm{X}_{3}-\mathrm{X}_{2}\right)$ \\
\hline \multicolumn{2}{|r|}{ Valores fijados } & Valores calculados \\
\hline \multicolumn{2}{|r|}{}
\end{tabular}

En consecuencia, en una secuencia de 3 RCTA hay que fijar 6 variables, de las que $\mathrm{X}_{1} \mathrm{y} \mathrm{X}_{2}$ son arbitrarias, mientras que las tres temperaturas de reacción se fijan por el tipo de reacción química y $\mathrm{X}_{3}$ según la producción deseada. El sistema se optimiza por tanteos sucesivos, resolviendo para distintos valores de $\mathrm{X}_{1} \mathrm{y}_{2}$ hasta obtener el volumen total mínimo. (También se podrían fijar arbitrariamente las temperaturas de alimento a los reactores 1 y 2 , calculándose en ese caso $\mathrm{X}_{1}$ y $\mathrm{X}_{2}$ mediante la ecuación de la recta adiabática.) En general, para una secuencia de $\mathrm{N}$ RCTA hay que fijar $2 \mathrm{~N}$ variables.

También se han de diseñar los intercambiadores de calor necesarios, calculando las áreas de intercambio respectivas $\left(\mathrm{A}_{1}, \mathrm{~A}_{2}, \mathrm{y} \mathrm{A}_{3}\right)$, una vez conocida la temperatura de la mezcla reactante a la entrada y a la salida de cada uno de ellos. 


\section{Reacciones exotérmicas reversibles}

En este caso, los puntos representativos de las parejas de valores $\left(\mathrm{X}_{\mathrm{n}}, \mathrm{T}_{\mathrm{n}}\right)$ correspondientes a las condiciones de estado estacionario de cada reactor de la serie deben estar situados en la curvas $\Gamma_{\mathrm{m}}$ (si se fija el grado de conversión) o $\Gamma_{\mathrm{Jm}}$ (si se fija la temperatura de entrada). En este apartado se tratará solo el primer caso. Por tratarse de una reacción exotérmica entre reactores hay que enfriar la mezcla de reacción. Es habitual utilizar dos tipos de intercambio de calor: refrigeración con intercambiadores de calor (figura 10) o refrigeración por inyección de alimento frío (figura 12).

Refrigeración entre RCTA con intercambiadores de calor

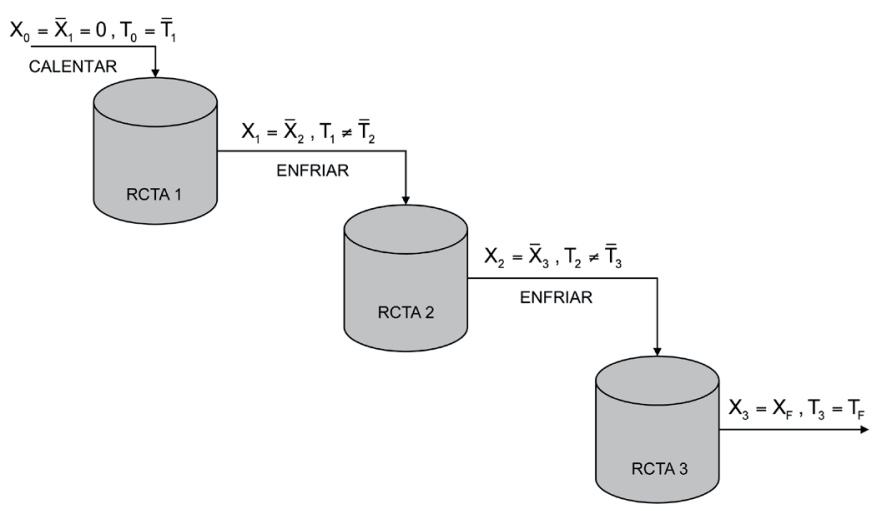

Figura 10. Secuencia de tres RCTA

En la figura 11 se muestra las condiciones de trabajo para cada RCTA, una vez se ha fijado X.

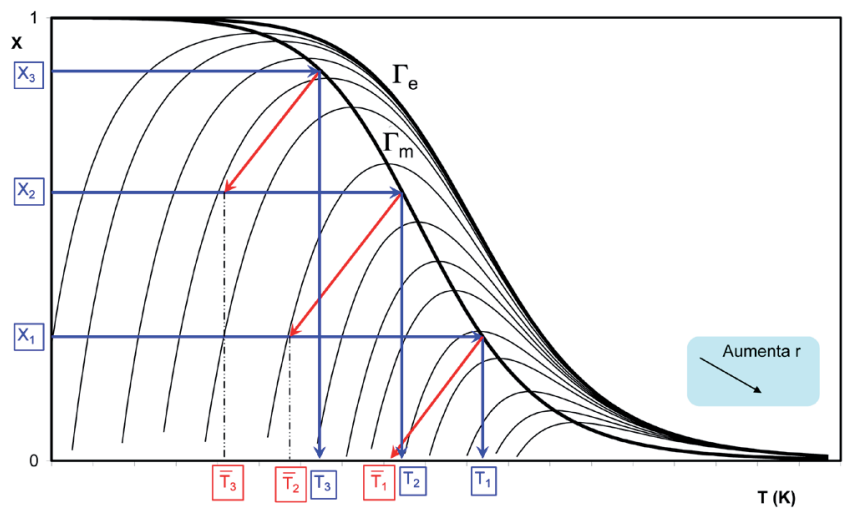

Figura 11. Condiciones de trabajo para reacciones exotérmicas reversibles (se fija X)

En cada uno de los RCTA hay que tomar dos decisiones que se detallan secuencialmente en la primera columna de la tabla 4. 
Tabla 4. Condiciones de trabajo para reacciones exotérmicas reversibles (se fija X)

\begin{tabular}{|c|c|c|c|}
\hline RCTA $n$ & Temperatura del reactor & Conversión & Temperatura del alimento \\
\hline 1 & $T_{1}=T_{m}\left(X_{1}\right)$ & $\mathrm{x}_{1}$ & $\bar{T}_{1}=T_{1}-J \cdot\left(X_{1}-\bar{X}_{1}\right)=T_{m}\left(X_{1}\right)-J \cdot\left(X_{1}-0\right)$ \\
\hline 2 & $T_{2}=T_{m}\left(X_{2}\right)$ & $x_{2}$ & $\bar{T}_{2}=T_{2}-J \cdot\left(X_{2}-\bar{X}_{2}\right)=T_{m}\left(X_{2}\right)-J \cdot\left(X_{2}-X_{1}\right)$ \\
\hline 3 & $T_{3}=T_{m}\left(X_{3}\right)$ & $x_{3}$ & $\overline{\mathrm{T}}_{3}=\mathrm{T}_{3}-\mathrm{J} \cdot\left(\mathrm{X}_{3}-\overline{\mathrm{X}}_{3}\right)=\mathrm{T}_{\mathrm{m}}\left(\mathrm{X}_{3}\right)-\mathrm{J} \cdot\left(\mathrm{X}_{3}-\mathrm{X}_{2}\right)$ \\
\hline & \multicolumn{2}{|c|}{ Valores fijados } & Valores calculados \\
\hline
\end{tabular}

En consecuencia, en una secuencia de 3 RCTA hay que fijar 6 variables, de las que $\mathrm{X}_{1} \mathrm{y}_{2}$ son arbitrarias, mientras que las tres temperaturas de reacción se fijan por el tipo de reacción química y $\mathrm{X}_{3}$ según la producción deseada. El sistema se optimiza por tanteos sucesivos, resolviendo para distintos valores de $\mathrm{X}_{1} \mathrm{y}_{2}$ hasta obtener el volumen total mínimo. (También se podrían fijar arbitrariamente las temperaturas de alimento a los reactores 1 y 2, calculándose en ese caso $\mathrm{X}_{1} \mathrm{y} \mathrm{X}_{2}$ de las temperaturas de velocidad de reacción máxima.) En general, para una secuencia de $\mathrm{N}$ RCTA hay que fijar $2 \mathrm{~N}$ variables.

También se han de diseñar los intercambiadores de calor necesarios, calculando las áreas de intercambio respectivas $\left(\mathrm{A}_{1}, \mathrm{~A}_{2}, \mathrm{y}_{3}\right)$, una vez conocida la temperatura de la mezcla reactante a la entrada y a la salida de cada uno de ellos.

\section{Refrigeración mediante inyección de alimento frío}

Si los reactores se refrigeran mediante este sistema, la mezcla reactante no se alimenta en su totalidad en el primer reactor, sino que parte de ella se introduce en los reactores siguientes. Con ello se consigue no solo refrigerar el reactor, sino también aumentar la velocidad de reacción, al haber incrementado la concentración de los reactantes en el interior del mismo. Suele necesitarse un intercambiador de calor que precaliente la mezcla reactante y dos inyecciones de alimento frío (figura 12).

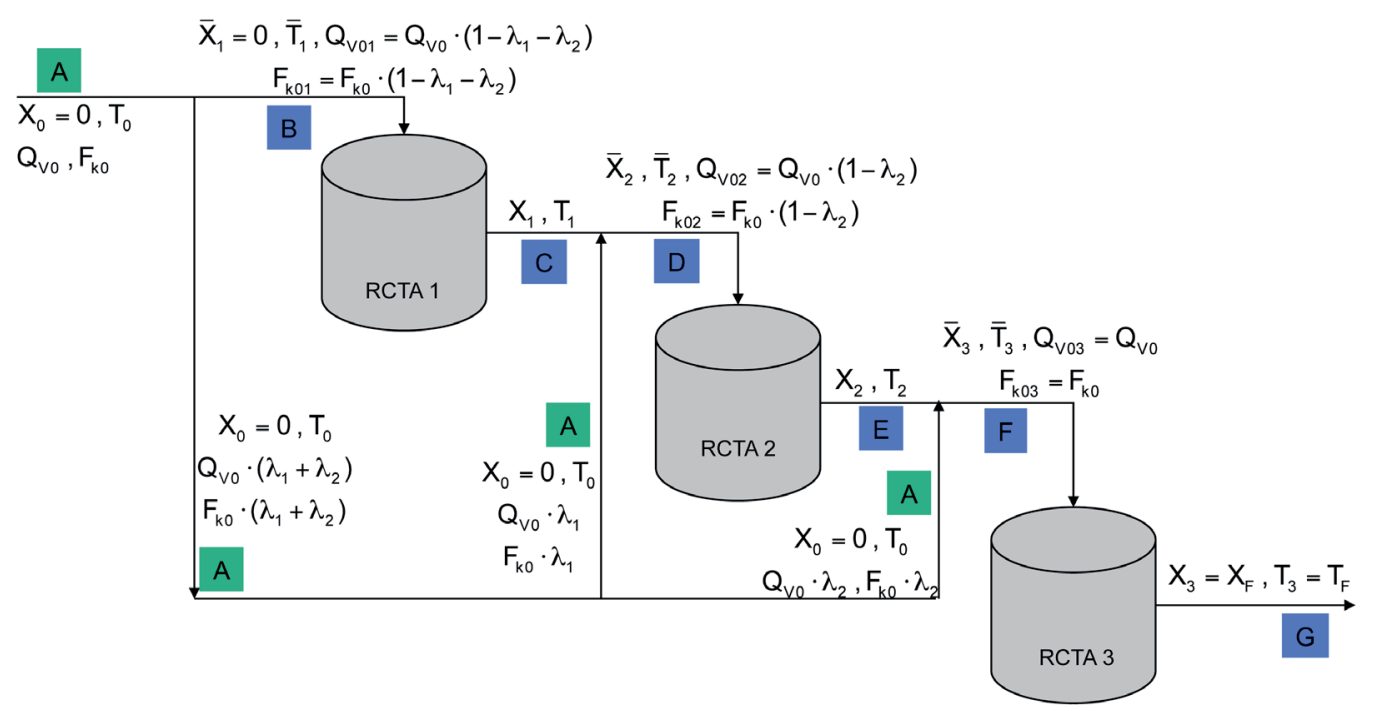

Figura 12. Secuencia de tres RCTA 
Si se representan los puntos que definen las distintas corrientes del sistema en un diagrama conversión-temperatura se obtiene la figura 13.

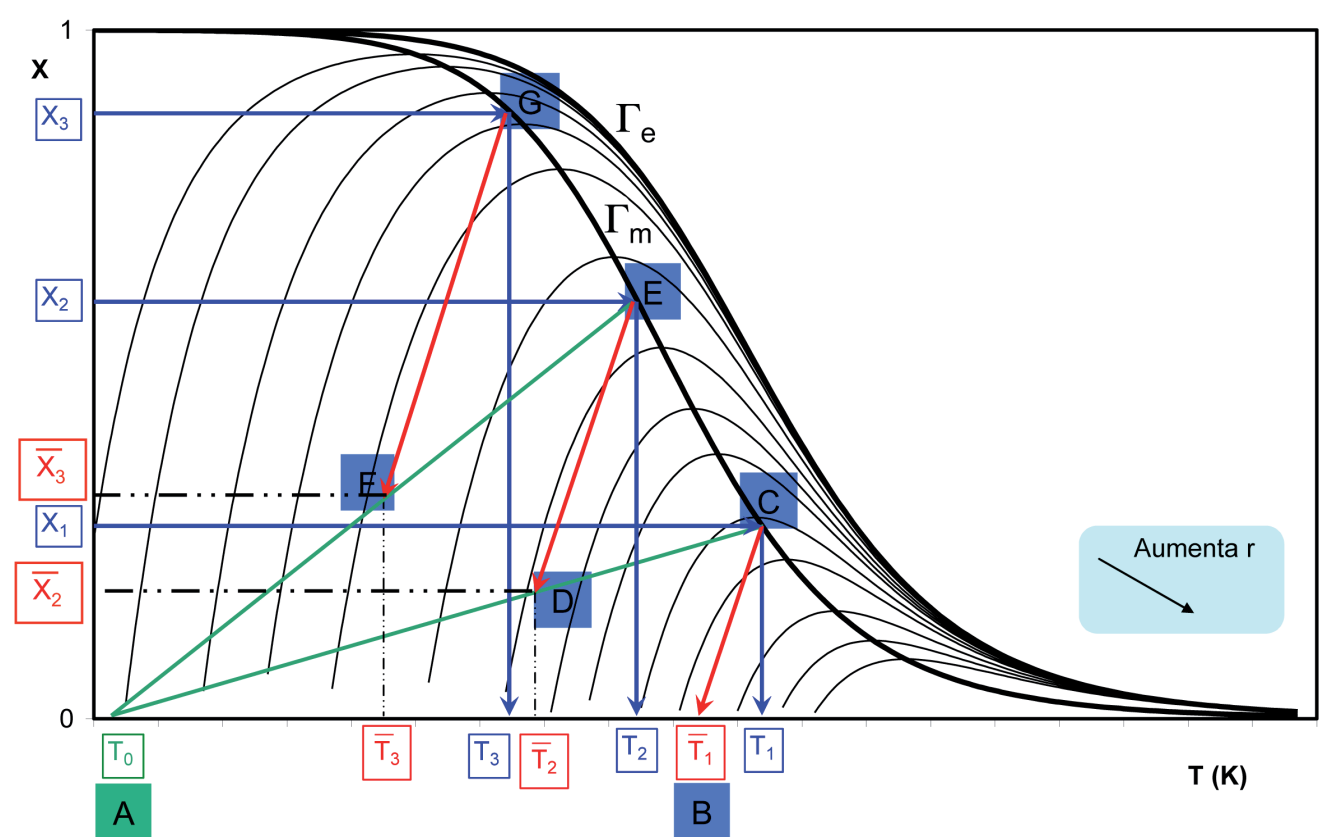

Figura 13. Condiciones de trabajo para reacciones exotérmicas reversibles (se fija X)

Algunas consideraciones sobre la figura 13 son:

1. Conviene que el punto $B$, correspondiente $a\left(\bar{T}_{1} ; \bar{X}_{1}=0\right)$, se encuentre suficientemente alejado del punto $\mathrm{A}\left(\mathrm{T}_{0} ; \mathrm{X}_{0}=0\right)$ para estar en una zona de velocidades de reacción razonablemente elevadas.

2. Los puntos $\mathrm{C}, \mathrm{E}$ y $\mathrm{G}$ deben estar situados sobre la curva de velocidad máxima $\Gamma_{\mathrm{m}}$, si se fijan $\mathrm{X}_{1}, \mathrm{X}_{2} \mathrm{y} \mathrm{X}_{3}$

3. Las corrientes representadas por los puntos $\mathrm{C}$ y A se unen para formar la corriente representada por el punto $\mathrm{D}$ que se sitúa en el gráfico donde más convenga (sobre la recta $\overline{\mathrm{AC}}$ ) para que al trazar la adiabática, ésta corte a la curva $\Gamma_{\mathrm{m}}$ en el punto $E$ de ordenada $\mathrm{X}_{2}$ suficientemente próxima a la $\mathrm{X}_{3}$ final deseada.

4. Las corrientes representadas por los puntos E y A se unen para formar la corriente representada por el punto $\mathrm{F}$ que se sitúa en el gráfico donde más convenga (sobre la recta $\overline{\mathrm{AE}}$ ) para que al trazar la adiabática, ésta corte a la curva $\Gamma_{\mathrm{m}}$ en el punto $\mathrm{G}$ de ordenada la $\mathrm{X}_{3}$ final deseada.

5. Los caudales de las corrientes respectivas son proporcionales a las longitudes de las líneas adyacentes del diagrama X-T, de modo que se cumple siempre la ley de la palanca, según la cual: 
En el punto D: $\quad \frac{\mathrm{F}_{\mathrm{k} 0} \cdot\left(1-\lambda_{1}-\lambda_{2}\right)}{\mathrm{F}_{\mathrm{k} 0} \cdot \lambda_{1}}=\frac{\mathrm{Q}_{\mathrm{v} 0} \cdot\left(1-\lambda_{1}-\lambda_{2}\right)}{\mathrm{Q}_{\mathrm{v} 0} \cdot \lambda_{1}}=\frac{\overline{\mathrm{AD}}}{\overline{\mathrm{CD}}}$

En el punto $F: \quad \frac{F_{\mathrm{k} 0} \cdot\left(1-\lambda_{2}\right)}{\mathrm{F}_{\mathrm{k} 0} \cdot \lambda_{2}}=\frac{\mathrm{Q}_{\mathrm{vo}} \cdot\left(1-\lambda_{2}\right)}{\mathrm{Q}_{\mathrm{v} 0} \cdot \lambda_{2}}=\frac{\overline{\mathrm{AF}}}{\overline{\mathrm{EF}}}$

Así pues, si se mide la longitud de los tramos $\overline{\mathrm{AD}}, \overline{\mathrm{CD}}, \overline{\mathrm{AF}}$ y $\overline{\mathrm{EF}}$ sobre la figura solo quedan dos incógnitas, $\lambda_{1} \mathrm{y} \lambda_{2}$, que se pueden calcular. De ese modo se pueden calcular las fracciones de la corriente alimento que se han de introducir entre el primer y segundo reactor y entre el segundo y el tercero.

La refrigeración por inyección de alimento frío tiene algunas limitaciones:

a) No puede utilizarse si $\mathrm{J}^{-1}$ es muy pequeña. Esto se debe a que la intersección de la adiabática trazada desde $\mathrm{T}_{0}$ con la curva de equilibrio determina la conversión máxima que se puede alcanzar con un conjunto de RCTA adiabáticos $\left(\mathrm{X}_{\max }\right)$. Si la pendiente de la adiabática es muy baja, la conversión máxima también lo es (figura 14).

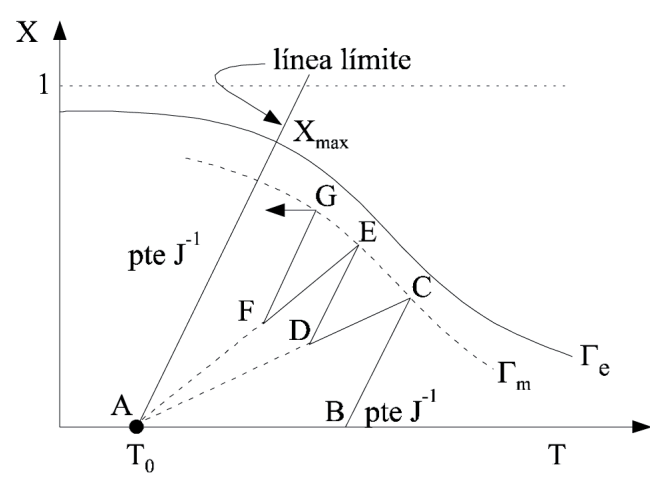

Figura 14. Influencia del valor de $\mathrm{J}^{-1}$

b) Si $\mathrm{J}^{-1}$ es suficientemente grande, es conveniente utilizar este montaje solo si la temperatura del alimento es bastante más baja que la temperatura a la que tiene lugar la reacción. Además interesa que $\mathrm{T}_{0}$ sea lo suficientemente pequeña para que el corte de la adiabática con la curva de equilibrio dé como resultado un valor de $X_{\max }$ suficientemente grande (figura 15).
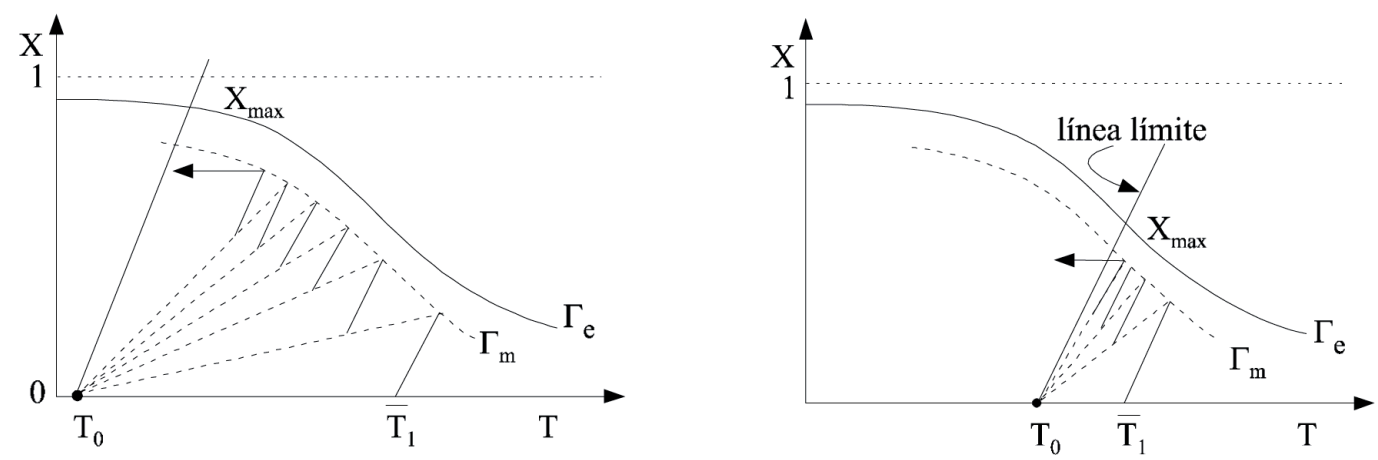

Figura 15. Influencia de $\mathrm{T}_{0}$ 
En cada uno de los RCTA hay que tomar dos decisiones que se detallan secuencialmente en la primera columna de la tabla 5.

Tabla 5. Condiciones de trabajo para reacciones exotérmicas reversibles (se fija X)

\begin{tabular}{|c|c|c|c|}
\hline RCTA $n$ & Temperatura del reactor & Conversión & Temperatura del alimento \\
\hline 1 & $\mathrm{~T}_{1}=\mathrm{T}_{\mathrm{m}}\left(\mathrm{X}_{1}\right)$ & $\mathrm{X}_{1}$ & $\bar{T}_{1}=T_{1}-J \cdot\left(X_{1}-\bar{X}_{1}\right)=T_{m}\left(X_{1}\right)-J \cdot\left(X_{1}-\bar{X}_{1}\right)$ \\
\hline 2 & $T_{2}=T_{m}\left(x_{2}\right)$ & $\mathrm{X}_{2}$ & $\overline{\mathrm{T}}_{2}=\mathrm{T}_{2}-\mathrm{J} \cdot\left(\mathrm{X}_{2}-\overline{\mathrm{X}}_{2}\right)=\mathrm{T}_{\mathrm{m}}\left(\mathrm{X}_{2}\right)-\mathrm{J} \cdot\left(\mathrm{X}_{2}-\overline{\mathrm{X}}_{2}\right)$ \\
\hline 3 & $T_{3}=T_{m}\left(x_{3}\right)$ & $x_{3}$ & $\overline{\mathrm{T}}_{3}=\mathrm{T}_{3}-\mathrm{J} \cdot\left(\mathrm{X}_{3}-\overline{\mathrm{X}}_{3}\right)=\mathrm{T}_{\mathrm{m}}\left(\mathrm{X}_{3}\right)-\mathrm{J} \cdot\left(\mathrm{X}_{3}-\overline{\mathrm{X}}_{3}\right)$ \\
\hline & \multicolumn{2}{|c|}{ Valores fijados } & Valores calculados \\
\hline
\end{tabular}

Por lo tanto, en una secuencia de 3 RCTA hay que fijar 6 variables, de las que $X_{1}$ $\mathrm{y}_{2}$ son arbitrarias, mientras que las tres temperaturas de reacción se fijan por el tipo de reacción química y $\mathrm{X}_{3}$ según la producción deseada. El sistema se optimiza por tanteos sucesivos, resolviendo para distintos valores de $\mathrm{X}_{1} \mathrm{y} \mathrm{X}_{2}$ hasta obtener el volumen total mínimo. (También se podrían fijar arbitrariamente las temperaturas de alimento a los reactores 1 y 2, calculándose en ese caso $\mathrm{X}_{1}$ y $\mathrm{X}_{2}$ de las temperaturas de velocidad de reacción máxima.) En general, para una secuencia de $\mathrm{N}$ RCTA hay que fijar $2 \mathrm{~N}$ variables.

A diferencia del resto de casos estudiados, el cálculo del volumen de cada reactor debe hacerse mediante la ecuación del balance de materia, pero teniendo en cuenta que el caudal molar $\mathrm{F}_{\mathrm{k} 0 \mathrm{n}}$ referido al alimento no es el mismo para los tres reactores considerados, y hay que calcularlo según se muestra en la tabla 6.

Tabla 6. Cálculo del volumen molar que se alimenta a cada RCTA

\begin{tabular}{|c|c|c|c|}
\hline $\mathrm{F}_{\mathrm{k} 0}$ & RCTA $\mathrm{n}$ & $\mathrm{F}_{\mathrm{k} 0 \mathrm{n}}$ & Balance de materia para calcular $\mathrm{V}_{\mathrm{n}}$ \\
\hline \multirow{3}{*}{$\mathrm{F}_{\mathrm{k} 0}$} & 1 & $\mathrm{~F}_{\mathrm{k} 01}=\mathrm{F}_{\mathrm{k} 0} \cdot\left(1-\lambda_{1}-\lambda_{2}\right)$ & $\begin{aligned} & F_{k 0}\left(1-\lambda_{1}-\lambda_{2}\right) \cdot\left(x_{1}-\bar{X}_{1}\right)+v_{k} \cdot r\left[X_{1}, T_{1}\right] \cdot v_{1}= \\
= & F_{k 0}\left(1-\lambda_{1}-\lambda_{2}\right) \cdot x_{1}+v_{k} \cdot r\left[x_{1}, T_{m}\left(x_{1}\right)\right] \cdot v_{1}=0\end{aligned}$ \\
\hline & 2 & $\mathrm{~F}_{\mathrm{k} 02}=\mathrm{F}_{\mathrm{k} 0} \cdot\left(1-\lambda_{2}\right)$ & $\begin{array}{c}F_{k 0} \cdot\left(1-\lambda_{2}\right) \cdot\left(X_{2}-\bar{X}_{2}\right)+v_{k} \cdot r\left[X_{2}, T_{2}\right] \cdot v_{2}= \\
=F_{k 0} \cdot\left(1-\lambda_{2}\right) \cdot\left(X_{2}-\bar{X}_{2}\right)+v_{k} \cdot r\left[X_{2}, T_{m}\left(X_{2}\right)\right] \cdot v_{2}=0\end{array}$ \\
\hline & 3 & $\mathrm{~F}_{\mathrm{k} 03}=\mathrm{F}_{\mathrm{k} 0}$ & $\begin{array}{c}F_{k 0} \cdot\left(X_{3}-\bar{X}_{3}\right)+v_{k} \cdot r\left[X_{3}, T_{3}\right] \cdot v_{3}= \\
=F_{k 0} \cdot\left(X_{3}-\bar{X}_{3}\right)+v_{k} \cdot r\left[X_{3}, T_{m}\left(X_{3}\right)\right] \cdot v_{3}=0\end{array}$ \\
\hline
\end{tabular}




\subsection{Sistemas de un RFP}

Ejercicio 1. Para el caso de un RFP adiabático en el que tiene lugar una reacción química con velocidad de reacción normal (no autocatalítica):

a) Hágase el mismo estudio que el mostrado en el apartado 6.2 para un RCTA. Analícese los tres tipos de reacciones que pueden tener lugar, endotérmicas (reversibles e irreversibles), exotérmicas irreversibles y exotérmicas reversibles, indicando cuáles son las mejores condiciones de funcionamiento en el supuesto de que se fije el grado de conversión final a la salida del reactor (no la temperatura de entrada).

b) ¿Qué sucede al tratar de fijar las condiciones de operación de un RFP adiabático en el que tiene lugar una reacción exotérmica reversible? ¿Cómo cree que se debería operar en este caso?

\subsection{Secuencia de RFP}

En el caso de los RFP, dada la forma diferencial de los balances de materia y energía, si se conecta en serie un conjunto de reactores adiabáticos o isotermos no se obtiene una disminución de volumen respecto a si se utiliza uno solo con el mismo volumen y diámetro e igual forma de operar. Ahora bien, si se refrigera o calienta la mezcla entre cada dos reactores consecutivos, sí que se obtiene una disminución del volumen total de reacción.

Este tipo de montaje se suele utilizar en la práctica con reactores catalíticos en los que se desarrollan reacciones químicas muy exotérmicas y reversibles.

El montaje que se propone es utilizar N reactores en serie. Para cada reactor de la serie las condiciones de trabajo a fijar dependen de las características termodinámicas de la reacción que tenga lugar. A continuación se muestra un ejemplo para cada tipo de reacción suponiendo una secuencia de tres RFP.

\section{Reacciones endotérmicas (reversibles e irreversibles)}

En el caso de reacciones endotérmicas, tanto reversibles como irreversibles, hay que calentar la mezcla entre cada dos etapas consecutivas y la temperatura de alimentación a la primera etapa conviene que sea $\mathrm{T}^{*}$ (la máxima permisible). 
Ejercicio 2. Para el caso de una serie de tres RFP adiabáticos en el que tiene lugar una reacción química endotérmica (reversible o irreversible), en el caso de que se fijen los grados de conversión a la salida de cada reactor:

a) Dibújese el esquema de los tres RFP.

b) Constrúyase de forma cualitativa el diagrama $\mathrm{X}$ versus $\mathrm{T}$ en el que se indiquen las condiciones de operación de cada RFP.

c) Indíquese en una tabla las variables que se fijan y las que se calculan, indicando cómo hacerlo, en cada RFP.

\section{Reacciones exotérmicas irreversibles}

Para reacciones exotérmicas irreversibles conviene también que todos los reactores operen a la máxima temperatura posible utilizándose la refrigeración mediante intercambiadores de calor intercalados entre los reactores.

Ejercicio 3. Para el caso de una serie de tres RFP adiabáticos en el que tiene lugar una reacción química exotérmica irreversible, en el caso de que se fijen los grados de conversión a la salida de cada reactor:

a) Dibújese el esquema de los tres RFP.

b) Constrúyase de forma cualitativa el diagrama $\mathrm{X}$ versus $\mathrm{T}$ en el que se indiquen las condiciones de operación de cada RFP.

c) Indíquese en una tabla las variables que se fijan y las que se calculan, indicando cómo hacerlo, en cada RFP.

\section{Reacciones exotérmicas reversibles}

Si la reacción que se desarrolla en el RFP es exotérmica y reversible, el volumen mínimo de reacción referido se obtendrá en el caso de que la variación de $\mathrm{X}-\mathrm{T}$, en cada uno de los diferentes RFP, esté próxima a la curva $\Gamma_{\mathrm{m}}$, ante la imposibilidad de conseguir que la variación de $\mathrm{X}$ a lo largo del reactor coincida con dicha curva $\Gamma_{\mathrm{m}}$. Esto se consigue mediante una secuencia de RFP adiabáticos, enfriando la mezcla reactante entre cada dos reactores consecutivos. Es habitual utilizar dos tipos de intercambio de calor: refrigeración con intercambiadores de calor (figura 16) o refrigeración por inyección de alimento frío (figura 18). 


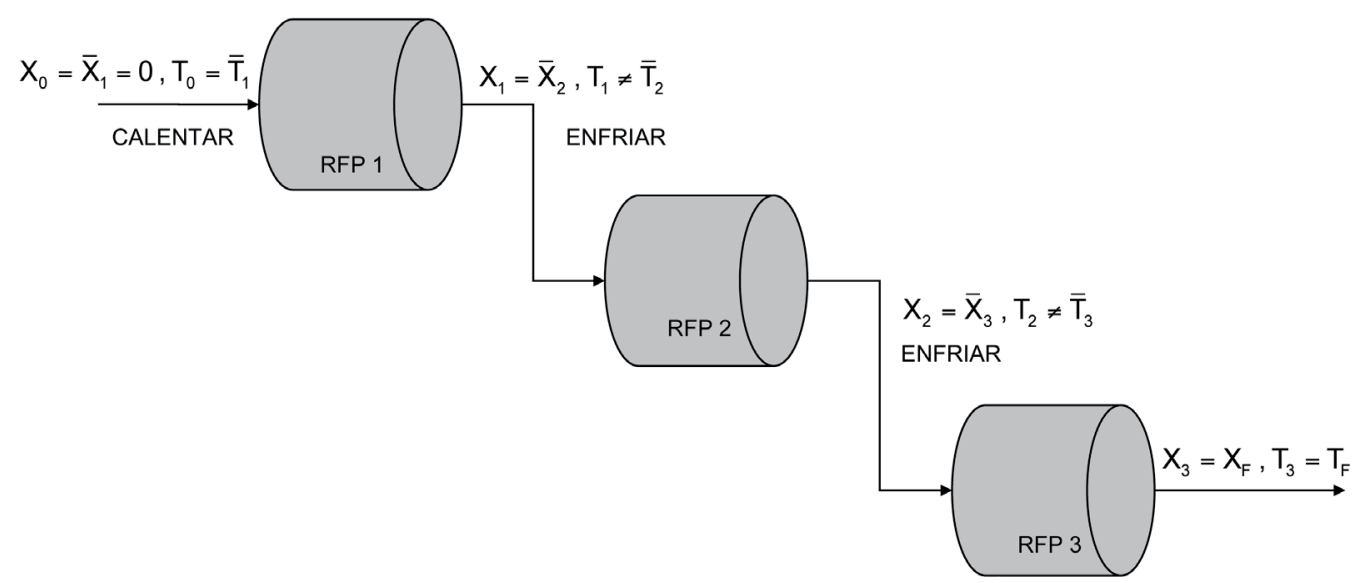

Figura 16. Secuencia de tres RFP

Conviene que los puntos representativos de las condiciones de salida de los distintos reactores se encuentren un poco por encima de la curva $\Gamma_{m}$. Las mejores condiciones se obtienen cuando la corriente de salida de un reactor y la de entrada al siguiente se sitúan en la misma curva de velocidad de reacción constante (figura 17).

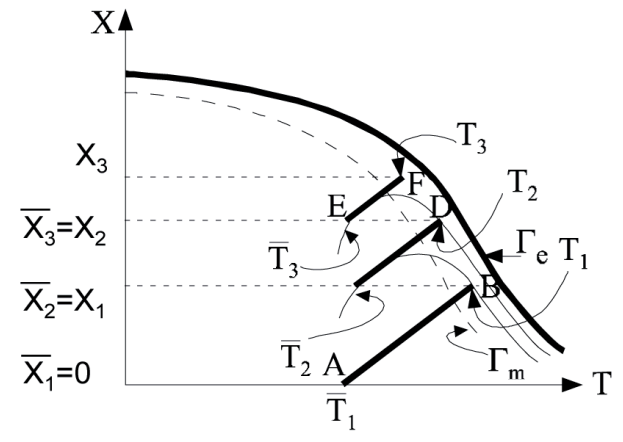

Figura 17. Condiciones de trabajo para reacciones exotérmicas reversibles

\section{Refrigeración por inyección de alimento frio}

En este caso, el alimento que se introduce en el sistema se divide en varias corrientes. Una de ellas se introduce directamente en el primer RFP tras, generalmente, ser precalentada; otra se introduce en el segundo RFP y otra en el tercero. 


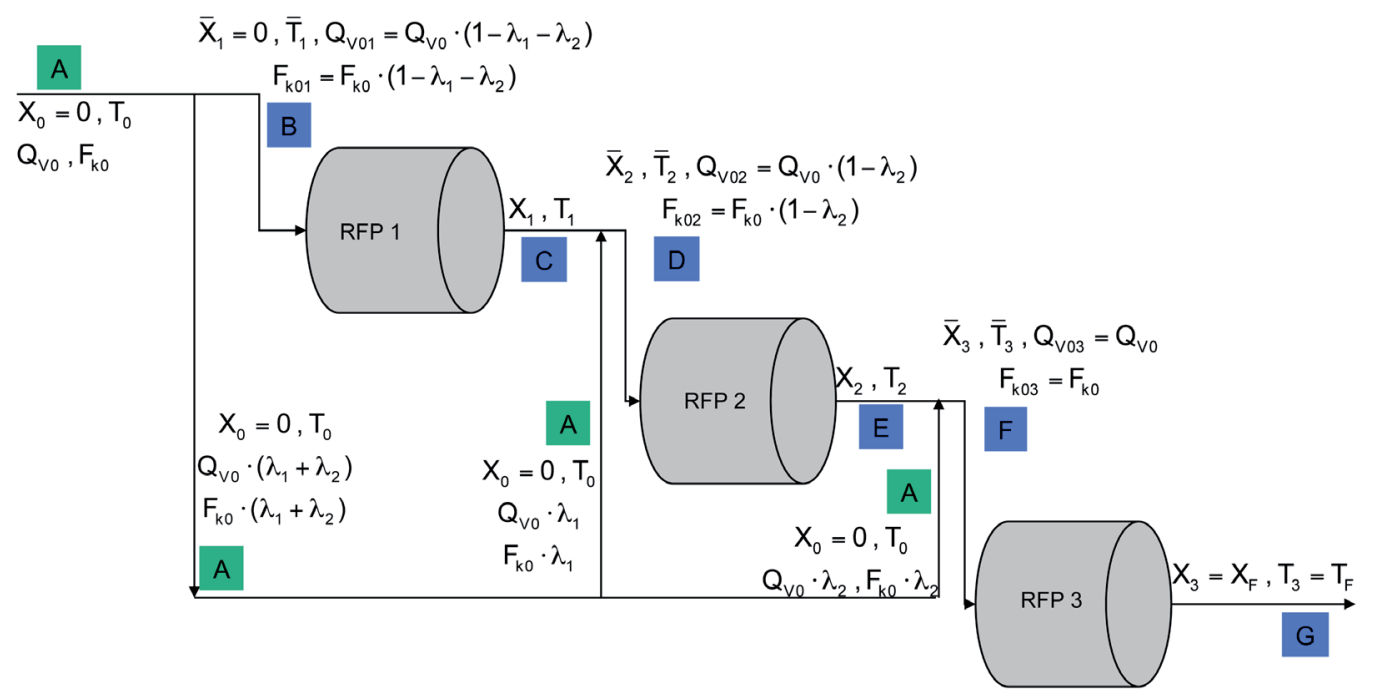

Figura 18. Secuencia de tres RFP

Ejercicio 4. Para el caso de una serie de tres RFP adiabáticos en el que tiene lugar una reacción química exotérmica reversible, en al caso de que se fijen los grados de conversión a la salida de cada reactor:

a) Constrúyase de forma cualitativa el diagrama $\mathrm{X}$ versus $\mathrm{T}$ en el que se indiquen las condiciones de operación de cada RFP, recordando que para minimizar el volumen resulta conveniente que la corriente de salida de un reactor y la de entrada al siguiente se sitúan en la misma curva de velocidad de reacción constante: $r\left(X_{n}, T_{n}\right)=r\left(\bar{X}_{n+1}, \bar{T}_{n+1}\right)$

b) A la vista de ese diagrama extrapólense las consideraciones que considere oportunas, para el mismo caso estudiado con tres RCTA en serie.

c) Indíquese en un tabla las variables que se fijan y las que se calculan, indicando cómo hacerlo, en cada RFP.

Recuérdese que, a diferencia del resto de casos estudiados, el cálculo del volumen de cada reactor debe hacerse mediante la ecuación del balance de materia, pero teniendo en cuenta que el caudal molar $\mathrm{F}_{\mathrm{k} 0 \mathrm{n}}$ referido al alimento no es el mismo para los tres reactores considerados, y hay que calcularlo según se muestra en la tabla 7.

Tabla 7. Cálculo del volumen molar que se alimenta a cada RFP

\begin{tabular}{|c|c|c|c|}
\hline $\mathrm{F}_{\mathrm{k} 0}$ & RFP $\mathrm{n}$ & $\mathrm{F}_{\mathrm{k} 0 \mathrm{n}}$ & Balance de materia para calcular $\mathrm{V}_{\mathrm{n}}$ \\
\hline & 1 & $\mathrm{~F}_{\mathrm{k} 01}=\mathrm{F}_{\mathrm{k} 0} \cdot\left(1-\lambda_{1}-\lambda_{2}\right)$ & $\mathrm{dV}_{1}=\mathrm{F}_{\mathrm{k} 0}\left(1-\lambda_{1}-\lambda_{2}\right) \cdot \frac{\mathrm{dX}}{\left(-v_{\mathrm{k}}\right) \cdot \mathrm{r}(\mathrm{X}, \mathrm{T})}$ \\
\cline { 2 - 4 } $\mathrm{F}_{\mathrm{k} 0}$ & 2 & $\mathrm{~F}_{\mathrm{k} 02}=\mathrm{F}_{\mathrm{k} 0} \cdot\left(1-\lambda_{2}\right)$ & $\mathrm{dV}_{2}=\mathrm{F}_{\mathrm{k} 0} \cdot\left(1-\lambda_{2}\right) \cdot \frac{\mathrm{dX}}{\left(-v_{\mathrm{k}}\right) \cdot \mathrm{r}(\mathrm{X}, \mathrm{T})}$ \\
\cline { 2 - 4 } & 3 & $\mathrm{~F}_{\mathrm{k} 03}=\mathrm{F}_{\mathrm{k} 0}$ & $\mathrm{dV}_{3}=\mathrm{F}_{\mathrm{k} 0} \cdot \frac{\mathrm{dX}}{\left(-v_{\mathrm{k}}\right) \cdot \mathrm{r}(\mathrm{X}, \mathrm{T})}$ \\
\hline
\end{tabular}




\section{PROBLEMAS}

Problema 01. En un sistema de dos reactores de flujo de pistón adiabáticos, en serie, con refrigeración entre ambos mediante un intercambiador de calor adecuado, según el esquema:

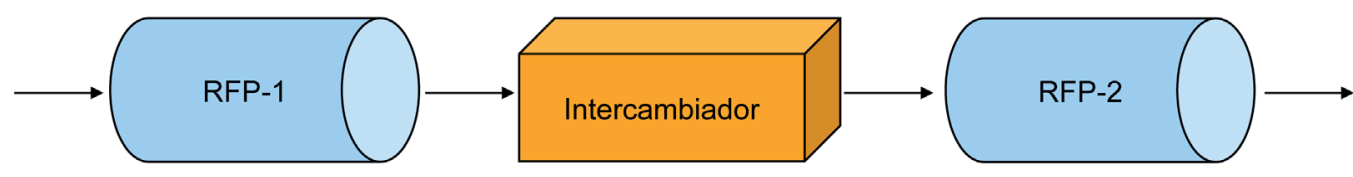

se quiere desarrollar la reacción reversible $\mathrm{SO}_{2}(\mathrm{~g})+1 / 2 \mathrm{O}_{2}(\mathrm{~g}) \leftrightarrow \mathrm{SO}_{3}(\mathrm{~g})$ en presencia de un catalizador de $\mathrm{V}_{2} \mathrm{O}_{5}$ sobre un soporte de piedra pómez. Ambos reactores operan a la presión atmosférica y el alimento se introduce en el primer reactor a una temperatura de $760 \mathrm{~K}$, con un caudal molar de $45 \mathrm{kmol} / \mathrm{h}$ y con la composición molar siguiente: $\mathrm{SO}_{2}\left(\mathrm{~A}_{1}\right)(11 \%), \mathrm{O}_{2}\left(\mathrm{~A}_{2}\right)(10 \%), \mathrm{SO}_{3}\left(\mathrm{~A}_{3}\right)(0 \%)$ y $\mathrm{N}_{2}\left(\mathrm{~A}_{4}\right)(79 \%)$. La temperatura de salida del primer reactor es de $900 \mathrm{~K}$. Calcular:

a) El volumen o masa de catalizador del primer reactor.

b) La temperatura a que debería enfriarse la mezcla que sale del primer reactor para que la conversión a la salida del segundo reactor sea máxima (admítase que ambos reactores contienen el mismo peso de catalizador).

c) El volumen o masa de catalizador que debería tener el segundo reactor si la temperatura de los gases se enfriara en el intercambiador de calor hasta $750 \mathrm{~K}$ y se deseara alcanzar un grado de conversión final de 0.71 .

\section{Datos y notas}

La ecuación de velocidad referida al $\mathrm{SO}_{2}$ es:

$\mathrm{r}=\mathrm{k} \cdot\left[\frac{\mathrm{p}_{1}}{\mathrm{p}_{3}}\right]^{1 / 2} \cdot\left[\mathrm{p}_{2}-\left(\frac{\mathrm{p}_{3}}{\mathrm{~K}_{\mathrm{p}} \cdot \mathrm{p}_{1}}\right)^{2}\right](\mathrm{kmol} / \mathrm{s} \cdot \mathrm{kg}$ de catalizador $)$, que es aplicable para valores de grado de conversión superiores a 0.05 . Para valores inferiores del grado de conversión la velocidad de reacción permanece constante e igual al valor que se obtiene al sustituir las presiones parciales por su valor a $\mathrm{X}=0.05$ y la temperatura respectiva.

Para el intervalo de temperaturas comprendido entre 750 y $950 \mathrm{~K}$ la constante de velocidad y la de equilibrio pueden calcularse mediante las expresiones:

$$
\begin{aligned}
& \ln \mathrm{k}=\frac{-97782}{\mathrm{~T}}-110.1 \cdot \ln \mathrm{T}+848.1 \mathrm{y} \mathrm{k}=\exp \left[\frac{-97782}{\mathrm{~T}}-110.1 \cdot \ln \mathrm{T}+848.1\right](\mathrm{kmol} / \mathrm{s} \cdot \mathrm{kg} \text { de catalizador } \cdot \mathrm{atm}) \\
& \mathrm{K}_{\mathrm{p}}=1.314 \cdot 10^{-5} \cdot \exp \left[\frac{11818}{\mathrm{~T}}\right] \quad \mathrm{atm}^{-1 / 2}
\end{aligned}
$$


La masa de catalizador y el volumen del reactor pueden relacionarse mediante $\mathrm{m}_{\text {cat }}=\rho_{\text {lecho }} \cdot \mathrm{V}$ en la que la densidad global del lecho catalítico vale $500 \mathrm{~kg} / \mathrm{m}^{3}$

$\Delta \mathrm{H}_{\mathrm{k}}^{0}=-98370 \quad \mathrm{~kJ} / \mathrm{kmol}$

$\overline{\mathrm{C}}_{\mathrm{p} 1}=54.4 \mathrm{~kJ} / \mathrm{kmol} \cdot \mathrm{K} \quad \overline{\mathrm{C}}_{\mathrm{p} 2}=35.2 \quad \mathrm{~kJ} / \mathrm{kmol} \cdot \mathrm{K} \quad \overline{\mathrm{C}}_{\mathrm{p} 3}=83.7 \quad \mathrm{~kJ} / \mathrm{kmol} \cdot \mathrm{K} \quad \overline{\mathrm{C}}_{\mathrm{p} 4}=33.1 \quad \mathrm{~kJ} / \mathrm{kmol} \cdot \mathrm{K}$

Problema 02. Se pretende desarrollar la reacción reversible de primer orden $\mathrm{A}_{1}(\ell) \leftrightarrow \mathrm{A}_{2}(\ell)$ en uno o varios RCTA adiabáticos, partiendo de una corriente exenta de $\mathrm{A}_{2} \mathrm{y}$ con una concentración de $\mathrm{A}_{1}$ igual $\mathrm{a}_{10}$. Se dispone de los siguientes datos de la reacción:

$$
\begin{aligned}
& \mathrm{K}=1.8747 \cdot 10^{-11} \cdot \exp \left[\frac{9058}{\mathrm{~T}}\right] \quad \Delta \mathrm{H}_{\mathrm{k}}^{\mathrm{o}}=-75312 \mathrm{~kJ} / \mathrm{kmol} \text { a } 298 \mathrm{~K} \\
& \mathrm{k}_{1}=2.9503 \cdot 10^{7} \cdot \exp \left[-\frac{5837.4}{\mathrm{~T}}\right] \quad\left(\min ^{-1}\right) \quad \mathrm{k}_{2}=1.5738 \cdot 10^{18} \cdot \exp \left[-\frac{14895.4}{\mathrm{~T}}\right] \quad\left(\mathrm{min}^{-1}\right)
\end{aligned}
$$

a) Construir la gráfica con $\mathrm{X}$ versus $\mathrm{T}$ de las curvas de velocidad de reacción constante, de la curva de velocidad máxima de reacción y de la curva de equilibrio comprobando que tiene la siguiente forma:

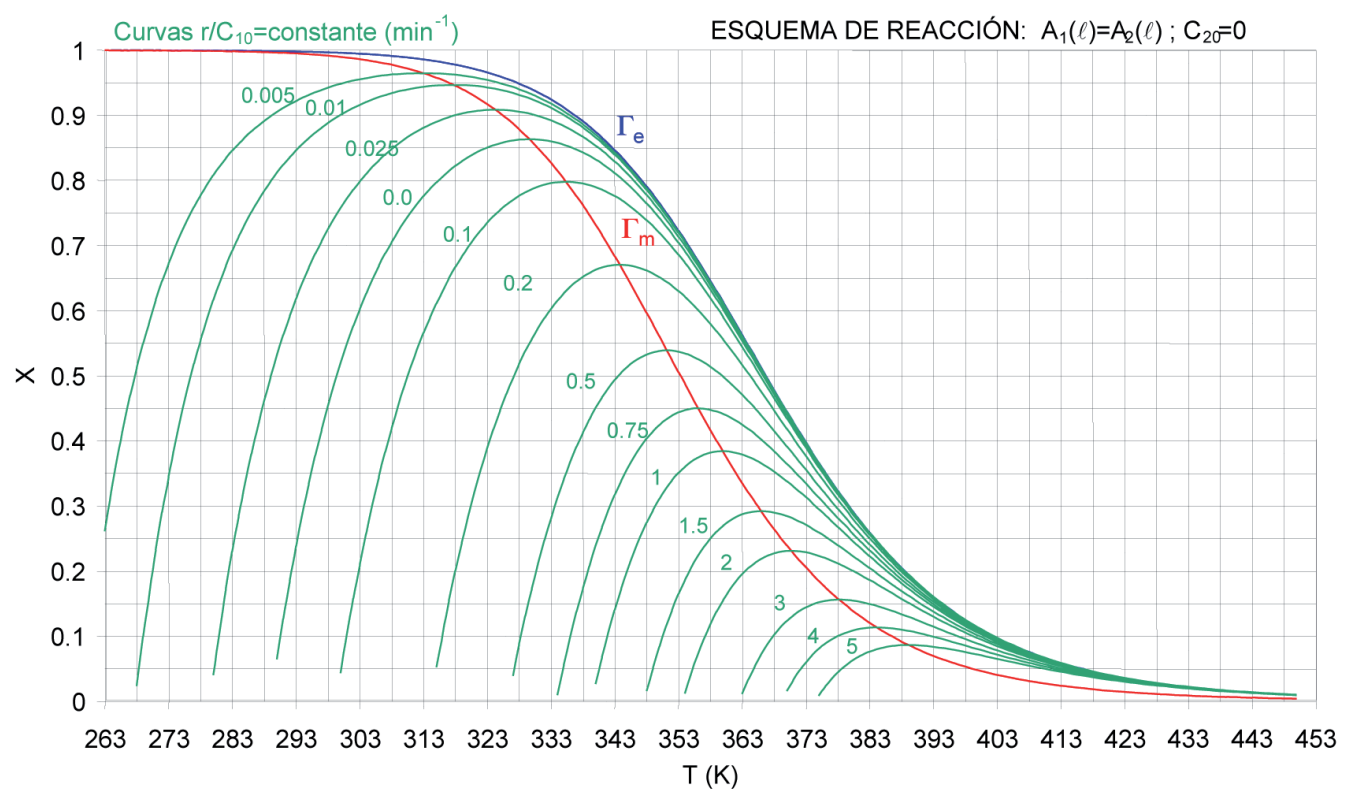

b) Si la corriente de alimento a un RCTA adiabático consiste en una disolución acuosa de la especie $\mathrm{A}_{1}$ con $\mathrm{c}_{10}=5 \mathrm{kmol} / \mathrm{m}^{3}, \mathrm{~F}_{10}=1 \mathrm{kmol} / \mathrm{min}$ y $\mathrm{T}_{0}=5{ }^{\circ} \mathrm{C}$, pudiéndose admitir que el calor específico medio de la disolución coincide con el del agua (recuérdese que para el agua $\rho=1000 \mathrm{~kg} / \mathrm{m}^{3}, \mathrm{M}=18 \mathrm{~kg}$ / $\left.\mathrm{kmol} \mathrm{y} \mathrm{C}_{\mathrm{p}}=4.184 \mathrm{~kJ} / \mathrm{kg} \cdot \mathrm{K}\right)$ : 
i) Calcular el valor de $\mathrm{J}$ y escribir la ecuación de la recta adiabática

ii) Calcular el volumen del RCTA si la velocidad de reacción fuera la máxima posible para el grado de conversión que se alcance. Determinar la pareja de valores X,T a la que trabajará el RCTA. Dibujar en la gráfica de velocidad de reacción constante la recta adiabática y el estado estacionario.

iii) Calcular el volumen del RCTA si la velocidad de reacción fuera la máxima posible para la temperatura de alimentación. Determinar la pareja de valores X,T a la que trabajará el RCTA. Dibujar en la gráfica de velocidad de reacción constante la recta adiabática y el estado estacionario.

c) Si las condiciones de entrada del alimento fueran las citadas en el apartado anterior, excepto la temperatura del alimento que es ahora de $25^{\circ} \mathrm{C}$, y se dispone de tres RCTA adiabáticos, completar la siguiente tabla para obtener el menor volumen de reacción posible. Dibujar en la gráfica de las curvas de velocidad de reacción constante las correspondientes rectas adiabáticas y, según proceda, de enfriamiento o calentamiento.

\begin{tabular}{|c|c|c|c|c|c|}
\hline RCTA & $\mathrm{T}_{0}(\mathrm{~K})$ & $\mathrm{X}$ & $\mathrm{T}(\mathrm{K})$ & $\mathrm{r}\left(\mathrm{kmol} / \mathrm{m}^{3} \cdot \mathrm{min}\right)^{(1)}$ & $\mathrm{V}\left(\mathrm{m}^{3}\right)^{(2)}$ \\
\hline 1 & & & & & \\
\hline 2 & & 0.75 & & & \\
\hline 3 & & 0.9 & & & \\
\hline
\end{tabular}

(1) Indicar cómo se ha procedido para el cálculo de r.

${ }^{(2)}$ Indicar cómo se ha procedido para calcular $\mathrm{V}$ y determinar el volumen mínimo.

Problema 03. Se pretende desarrollar la reacción exotérmica reversible de primer orden $\mathrm{A}_{1}(\ell) \leftrightarrow \mathrm{A}_{2}(\ell)$ en tres RCTA adiabáticos, partiendo de una corriente exenta de $\mathrm{A}_{2}$. El reactivo $\mathrm{A}_{1}$ se encuentra a la temperatura de $283 \mathrm{~K}$ y parte de él se va a utilizar como refrigerante, inyectándolo a la salida de los reactores $\mathrm{n}=1$ y $\mathrm{n}=2$. Se ha encontrado que, para minimizar el volumen total de reacción, es conveniente que en el primer reactor se alcance un grado de conversión de 0.3 y en el segundo de 0.5 , de forma que se pueda alcanzar un grado de conversión final de 0.8. El esquema de funcionamiento de los tres RCTA es:

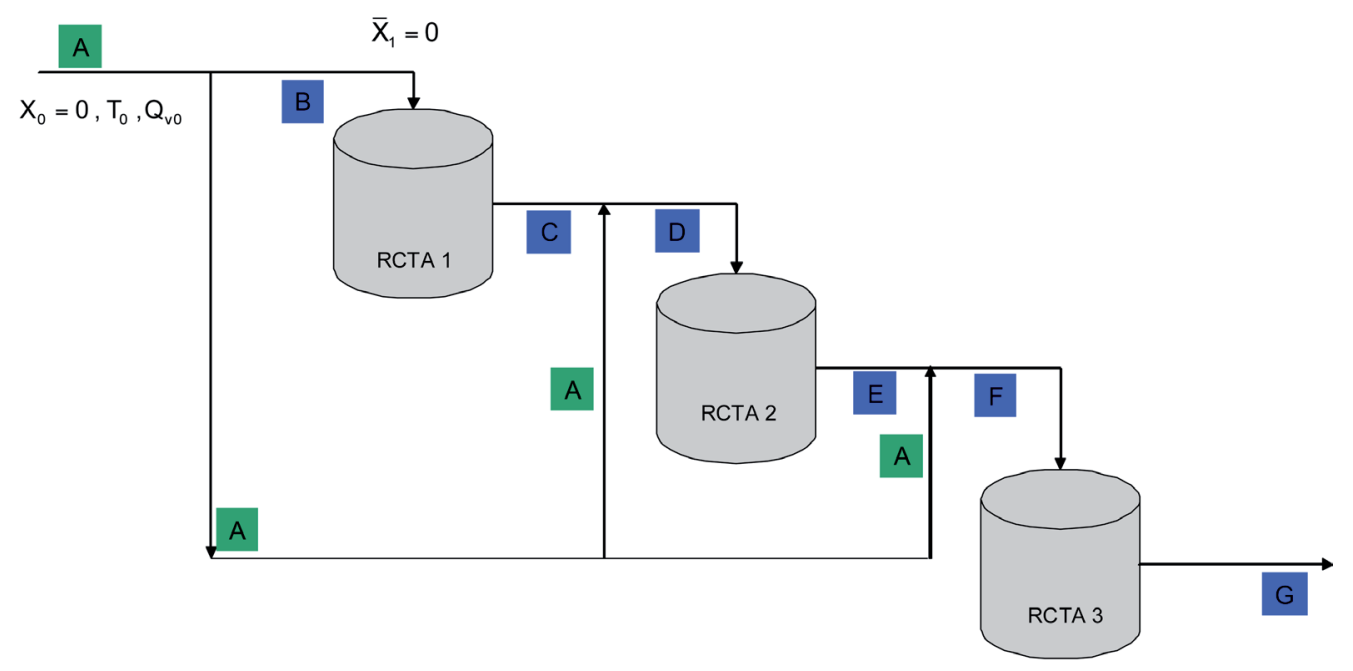


En la siguiente figura se muestran las correspondientes curvas de velocidad de reacción constante, junto con una recta de pendiente $1 / \mathrm{J}$.

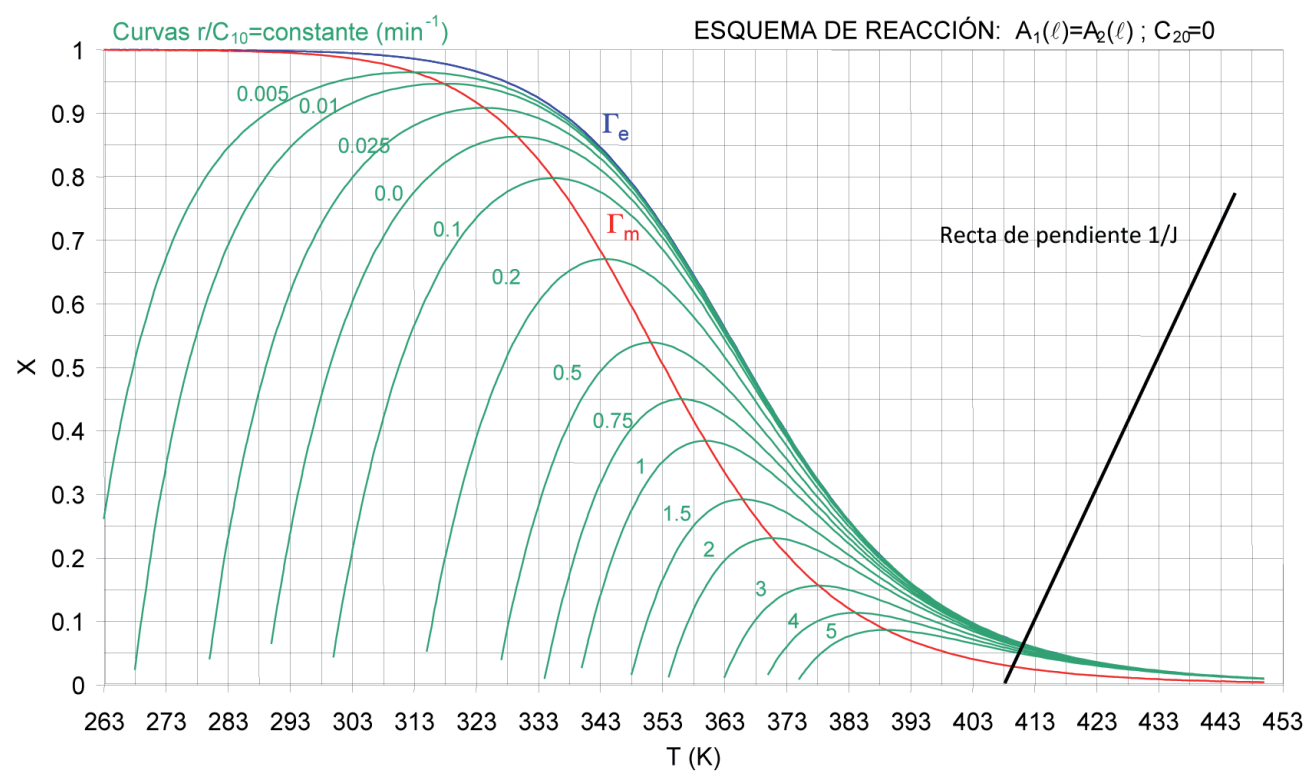

a) Situar cuidadosamente en la figura los puntos A, B, C, D, E, F, G marcados en el esquema de funcionamiento

b) Calcular, realizando la construcción gráfica necesaria, los grados de conversión a la entrada de los reactores $n=2$ y $n=3$ y las temperaturas de las corrientes a la entrada a los reactores $\mathrm{n}=1, \mathrm{n}=2$ y $\mathrm{n}=3$

c) Determinar las temperaturas de reacción en los reactores $\mathrm{n}=1, \mathrm{n}=2$ y n $=3$

d) Si se dispone de un caudal volumétrico de $\mathrm{Q}_{\mathrm{v} 0}=1000 \mathrm{l} / \mathrm{h}$, calcular los caudales volumétricos $\left(\mathrm{Q}_{\mathrm{v} 1}, \mathrm{Q}_{\mathrm{v} 2}, \mathrm{Q}_{\mathrm{v} 3}\right)$ con que se alimenta cada uno de los tres reactores. Justificar los cálculos. 


\section{CAPÍTULO 7}

\section{Reactores bioquímicos. Fermentadores}

\subsection{Procesos celulares}

El uso de células vivas para producir productos químicos es cada vez más importante. En el año 2000 los productos químicos, agrícolas y alimenticios obtenidos por biosíntesis había alcanzado el valor de 17.000 millones (en 1990 solo era de 275 millones de dólares). Tanto microorganismos como células animales se están utilizando para producir una amplia variedad de productos como insulina, antibióticos y polímeros. Se espera que en el futuro un gran número de productos químicos orgánicos, actualmente derivados del petróleo, se producirán a partir de células vivas. Las ventajas de las bioconversiones son: condiciones de reacción suaves, elevados rendimientos (por ejemplo, $100 \%$ de conversión de glucosa a ácido glucónico con la Aspergillus niger), que los organismos contienen varios enzimas que pueden catalizar sucesivos pasos de una reacción, y, más importante, que los organismos actúan como catalizadores estereoespecíficos (por ejemplo, en la conversión del ácido cis-propenilfónico al antibiótico ácido (-)cis-1,2epoxipropil-fosfónico, al utilizar un proceso químico se obtiene una mezcla de isómeros mientras que una bioconversión permite producir el único isómero deseado).

El término fermentación en su sentido estricto hace referencia a la producción de alcohol a partir de azúcar. Etimológicamente significa acción burbujeante o de ebullición, y se empleó por primera vez en la producción de vino. En la actualidad el significado del término es mucho más amplio, haciendo referencia a cualquier transformación molecular en la que una materia prima orgánica se convierte en producto por la acción directa de microorganismos o por la acción de los enzimas segregados por los mismos.

El objetivo de la fermentación puede ser la producción de microorganismos, la desaparición del sustrato, la producción de un producto o una combinación de ellos según el esquema general:

$$
\text { sustrato } \stackrel{\text { células }}{\longrightarrow} \text { más células }+ \text { producto }
$$

En biosíntesis, las células, también referidas como biomasa, consumen nutrientes para crecer y producir más células e importantes productos. Internamente una célula utiliza sus nutrientes para producir energía y más células. Esta transformación de nutrientes en energía y bioproductos se realiza mediante el uso de las células 
de un número de diferentes enzimas (catalizadores) en una serie de reacciones que dan productos metabólicos. Estos productos pueden permanecer en la célula (intracelulares) o ser segregados por las células (extracelulares). En el primer caso, las células se someten a una celulisis (ruptura) y el producto purificado del cultivo (mezcla de reacción).

En general, el crecimiento de un organismo anaeróbico sigue la ecuación:

$$
\begin{aligned}
& {[\text { células }]+\left[\begin{array}{c}
\text { fuente de } \\
\text { carbono }
\end{array}\right]+\left[\begin{array}{c}
\text { fuente de } \\
\text { nitrógeno }
\end{array}\right]+\left[\begin{array}{c}
\text { fuente de } \\
\text { oxígeno } \\
\text { (orgánica o inorgánica) }
\end{array}\right]+\left[\begin{array}{c}
\text { fuente de } \\
\text { fosfato }
\end{array}\right]+\ldots \stackrel{\text { condiciones del medio de cultivo }}{\longrightarrow} } \\
& \stackrel{(\mathrm{pH}, \text { temperatura, etc.) }}{\longrightarrow}\left[\begin{array}{c}
\text { más } \\
\text { células }
\end{array}\right]+[\text { productos }]+\mathrm{H}_{2} \mathrm{O}+\mathrm{CO}_{2}
\end{aligned}
$$

y el aeróbico:

$$
\begin{gathered}
{[\text { células }]+\left[\begin{array}{c}
\text { fuente de } \\
\text { carbono }
\end{array}\right]+\left[\begin{array}{l}
\text { fuente de } \\
\text { nitrógeno }
\end{array}\right]+\left[\begin{array}{l}
\mathrm{O}_{2}
\end{array}\right]+\left[\begin{array}{c}
\text { fuente de } \\
\text { fosfato }
\end{array}\right]+\ldots \stackrel{\text { condiciones del medio de cultivo }}{\longrightarrow}} \\
\stackrel{(\mathrm{pH} \text {, temperatura, etc. })}{\longrightarrow}\left[\begin{array}{c}
\text { más } \\
\text { células }
\end{array}\right]+[\text { productos }]+\mathrm{H}_{2} \mathrm{O}+\mathrm{CO}_{2}
\end{gathered}
$$

Como se acaba de indicar la forma abreviada generalmente utilizada es:

$$
\text { sustrato } \stackrel{\text { células }}{\longrightarrow} \text { más células }+ \text { producto }
$$

y los productos incluyen $\mathrm{CO}_{2}, \mathrm{H}_{2} \mathrm{O}$, proteínas y otras especies específicas de una reacción particular.

El medio de cultivo o sustrato contiene todos los nutrientes (carbono, nitrógeno, etc.) junto con otros productos químicos necesarios para el crecimiento. La reacción es autocatalítica ya que la velocidad de reacción es proporcional a la concentración celular.

\subsection{Crecimiento celular}

Las etapas de crecimiento celular en un RDTA se muestran en la figura 1, en la forma del logaritmo de la concentración de células vivas $\left(\mathrm{kg} / \mathrm{m}^{3}\right)$ versus tiempo. Inicialmente se inocula (se carga al RDTA) un pequeño número de células junto con los nutrientes y el proceso de crecimiento comienza.

Fase I o de latencia. Hay un pequeño aumento de la concentración celular. Las células se adaptan a su nuevo entorno, sintetizan enzimas y se preparan para comenzar a reproducirse. Durante este tiempo las células realizan funciones como sintetizar proteínas de transporte para mover el sustrato al interior de la célula, sintetizar enzimas para utilizar el nuevo sustrato y comenzar el trabajo para replicar el material genético celular. La duración de la fase depende del medio de crecimiento 
del que se tomó el inóculo en relación al medio de reacción en que es colocado. Si el inóculo es similar al medio del RDTA esta fase será prácticamente inexistente. Sin embargo, si el inóculo se sitúa en un medio con un nutriente diferente o si el inóculo se encontraba en una fase estacionaria o de muerte, las células deberán readaptar su camino metabólico para que les sea posible consumir los nutrientes de su nuevo entorno.

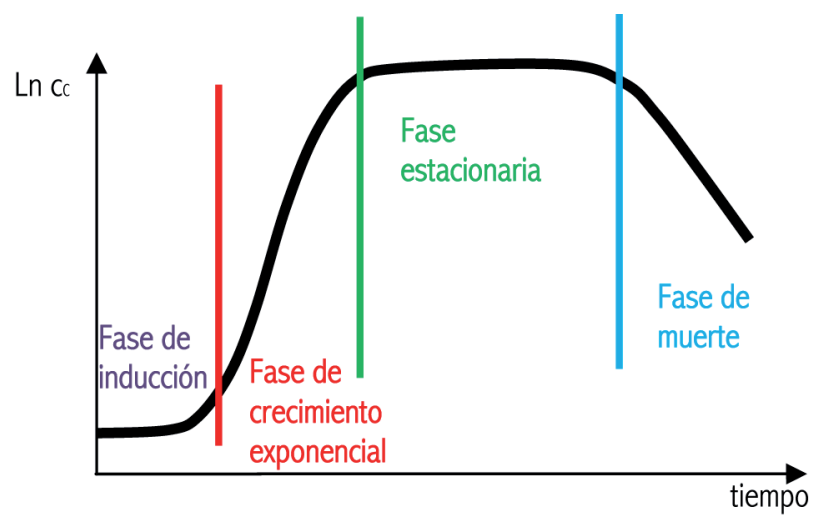

Figura 1. Etapas de crecimiento celular

Fase II o de crecimiento exponencial. La velocidad de crecimiento celular es proporcional a la concentración de células. En esta fase las células se dividen a la máxima velocidad posible porque todos los caminos de reacción enzimáticos para metabolizar el medio están activos (como resultado de la fase de latencia) y las células son capaces de utilizar los nutrientes con mucha eficiencia.

Fase III o estacionaria. Las células alcanzan un mínimo espacio biológico en el que la falta de uno o más nutrientes limita el crecimiento celular. Durante esta fase la velocidad de crecimiento es cero como resultado de la disminución o agotamiento de nutrientes y metabolitos esenciales. Muchos importantes productos de fermentación, incluyendo muchos de los antibióticos, se producen en la fase estacionaria (por ejemplo, la penicilina que se produce industrialmente a partir del hongo Penicillium chrysogenum se forma solo después de que el crecimiento celular ha cesado). El crecimiento celular también puede disminuir por la acumulación de ácidos orgánicos y materiales tóxicos generados durante la fase de crecimiento.

Fase IV final o de muerte. Se produce una disminución de la concentración de células vivas como consecuencia de los subproductos tóxicos y/o la disminución del suministro de nutrientes.

\subsection{Estequiometría: coeficientes de rendimiento estequiométrico}

La estequiometría del crecimiento celular es muy compleja y varía con el sistema microorganismo/nutriente y con las condiciones ambientales como $\mathrm{pH}$, temperatura, potencial redox, etc. Esta complejidad es especialmente grave cuando más de 
un nutriente contribuye al crecimiento celular, como suele ser habitual. Este capítulo, introductorio a los reactores bioquímicos, se centrará en una versión simplificada del crecimiento celular, limitado, a su vez, por un único nutriente del medio. En general el crecimiento celular se puede expresar mediante la reacción:

$$
\begin{gathered}
\text { células }+ \text { sustrato } \rightarrow \text { más células }+ \text { producto } \\
\qquad \mathrm{S} \stackrel{\text { células }}{\longrightarrow} \mathrm{Y}_{\mathrm{c} / \mathrm{s}} \mathrm{C}+\mathrm{Y}_{\mathrm{p} / \mathrm{s}} \mathrm{P}
\end{gathered}
$$

donde los coeficientes de rendimiento estequiométrico son:

$$
\begin{gathered}
Y_{c / s}=\frac{\text { masa de nuevas células formadas }}{\text { masa de sustrato consumida }} \text { con } Y_{c / s}=\frac{1}{Y_{s / c}} \\
Y_{p / s}=\frac{\text { masa de producto formada }}{\text { masa de sustrato consumida }} \text { con } Y_{p / s}=\frac{1}{Y_{s / p}}
\end{gathered}
$$

Además se puede definir el cociente entre ambos coeficientes:

$$
\mathrm{Y}_{\mathrm{p} / \mathrm{c}}=\frac{\text { masa de producto formada }}{\text { masa de nuevas células formadas }} \text { con } \mathrm{Y}_{\mathrm{p} / \mathrm{c}}=\frac{1}{\mathrm{Y}_{\mathrm{c} / \mathrm{p}}}
$$

Para los tres casos, en sistemas de densidad constante (que es lo más habitual), se cumplen las relaciones:

$$
\begin{aligned}
& c_{c}-c_{c 0}=Y_{c / s} \cdot\left(c_{s 0}-c_{s}\right) \\
& c_{p}-c_{p 0}=Y_{p / s} \cdot\left(c_{s 0}-c_{s}\right) \\
& c_{p}-c_{p 0}=Y_{p / c} \cdot\left(c_{c}-c_{c 0}\right)
\end{aligned}
$$

\subsection{Cinética microbiana}

\subsubsection{Ecuación de velocidad para el crecimiento celular \\ i) Ecuación de Monod}

Aunque existen muchas leyes sobre la velocidad de crecimiento celular de nuevas células:

$$
\text { células }+ \text { sustrato } \rightarrow \text { más células }+ \text { producto }
$$

la expresión más utilizada es la ecuación de Monod para la fase II de crecimiento exponencial: 


$$
\mathrm{r}_{\mathrm{c}}=\mu \cdot \mathrm{c}_{\mathrm{c}}
$$

siendo:

$\mathrm{r}_{\mathrm{c}}$ : velocidad de crecimiento celular $\left(\mathrm{kg} / \mathrm{m}^{3} \times \mathrm{s}\right)$

$\mathrm{c}_{\mathrm{c}}$ : concentración de células $\left(\mathrm{kg} / \mathrm{m}^{3}\right)$

$\mu$ : velocidad específica de crecimiento $\left(\mathrm{s}^{-1}\right)$

Obsérvese que la velocidad específica de crecimiento es una variable intensiva $\left(\mu=r_{c} / c_{c}\right.$ en s$\left.^{-1}\right)$ independiente de la concentración celular.

A su vez, la velocidad específica de crecimiento específico se puede expresar como:

$$
\mu=\mu_{\max } \cdot \frac{\mathrm{c}_{\mathrm{s}}}{\mathrm{K}_{\mathrm{s}}+\mathrm{c}_{\mathrm{s}}}
$$

donde:

$\mu_{\max }:$ velocidad específica de crecimiento máxima $\left(\mathrm{s}^{-1}\right)$

$\mathrm{c}_{\mathrm{s}}$ : concentración de sustrato $\left(\mathrm{kg} / \mathrm{m}^{3}\right)$

$\mathrm{K}_{\mathrm{s}}$ : parámetro análogo a la constante de Michaelis-Menten $\left(\mathrm{kg} / \mathrm{m}^{3}\right)$

Combinando ambas ecuaciones:

$$
\mathrm{r}_{\mathrm{c}}=\mu \cdot \mathrm{c}_{\mathrm{c}}=\mu_{\max } \cdot \frac{\mathrm{c}_{\mathrm{s}}}{\mathrm{K}_{\mathrm{s}}+\mathrm{c}_{\mathrm{s}}} \cdot \mathrm{c}_{\mathrm{c}}
$$

Puede observarse que formalmente esta ecuación matemática es muy parecida a la ecuación de Michaelis-Menten para la cinética enzimática (véase Anexo I). Esto parece razonable ya que los enzimas intracelulares toman parte en las mayoría de las reacciones que tienen lugar en el interior de la célula.

Para muchas bacterias la constante $\mathrm{K}_{\mathrm{s}}$ es pequeña reduciéndose la ley de velocidad a:

$$
\mathrm{r}_{\mathrm{c}}=\mu \cdot \mathrm{c}_{\mathrm{c}} \cong \mu_{\max } \cdot \mathrm{c}_{\mathrm{c}}
$$

La velocidad de crecimiento depende frecuentemente de la concentración de más de un nutriente, sin embargo la concentración del nutriente limitante es la que habitualmente se suele utilizar en las expresiones anteriores.

En muchos sistemas el producto inhibe la velocidad de crecimiento. Un ejemplo clásico de esta inhibición se da en la fabricación de vino, donde la fermentación de la glucosa para producir etanol es inhibida por el producto etanol. Hay diferentes ecuaciones para tener en cuenta la inhibición; una de estas leyes de velocidad toma la forma: 


$$
\mathrm{r}_{\mathrm{c}}=\mathrm{k}_{\mathrm{obs}} \cdot \mu_{\max } \cdot \frac{\mathrm{c}_{\mathrm{s}}}{\mathrm{K}_{\mathrm{s}}+\mathrm{c}_{\mathrm{s}}} \cdot \mathrm{c}_{\mathrm{c}}=\left(1-\frac{\mathrm{c}_{\mathrm{p}}}{\mathrm{c}_{\mathrm{p}}^{*}}\right)^{\mathrm{n}} \cdot \mu_{\max } \cdot \frac{\mathrm{c}_{\mathrm{s}}}{\mathrm{K}_{\mathrm{s}}+\mathrm{c}_{\mathrm{s}}} \cdot \mathrm{c}_{\mathrm{c}}
$$

siendo:

$\mathrm{c}_{\mathrm{p}}$ : concentración de producto $\left(\mathrm{kg} / \mathrm{m}^{3}\right)$

$\mathrm{c}_{\mathrm{p}}$ : concentración de producto a la que todo metabolismo cesa $\left(\mathrm{kg} / \mathrm{m}^{3}\right)$

n: constante empírica

Para el ejemplo de la fermentación de glucosa a etanol los valores típicos de los parámetros de inhibición son: $\mathrm{n}=0.5 \mathrm{y} \mathrm{c}_{\mathrm{p}}{ }^{*}=93 \mathrm{~kg} / \mathrm{m}^{3}$.

ii) Otras ecuaciones de velocidad para el crecimiento celular

Además de la ecuación de Monod, otras dos ecuaciones se utilizan habitualmente para describir la velocidad de crecimiento celular:

Ecuación de Tessier

$$
\mathrm{r}_{\mathrm{c}}=\mu_{\max } \cdot\left[1-\exp \left(\frac{\mathrm{c}_{\mathrm{s}}}{\mathrm{k}}\right)\right] \cdot \mathrm{c}_{\mathrm{c}}
$$

Ecuación de Moser

$$
\mathrm{r}_{\mathrm{c}}=\frac{\mu_{\max }}{\left[1+\mathrm{k} \cdot \mathrm{c}_{\mathrm{s}} \cdot \exp (-\lambda)\right]} \cdot \mathrm{c}_{\mathrm{c}}
$$

siendo tanto $\mathrm{k}$ como $\lambda$ constantes empíricas determinadas por el ajuste de los datos experimentales.

Las leyes de crecimiento de Tessier y de Moser se utilizan frecuentemente porque suelen ajustar mejor los resultados experimentales al inicio o al final de la fermentación.

\subsubsection{Ecuación de velocidad para el mantenimiento celular}

Además de consumirse sustrato para producir nuevas células, parte del sustrato debe utilizarse exclusivamente para el mantenimiento de la actividad diaria de las células, fundamentalmente como suministro de energía ya que se trata de seres vivos. La ecuación de velocidad, independientemente de si las células crecen o no, es de la forma: 


$$
\mathrm{r}_{\mathrm{sm}}=\mathrm{k}_{\mathrm{sm}} \cdot \mathrm{c}_{\mathrm{c}}
$$

donde:

$$
\mathrm{k}_{\mathrm{sm}}=\frac{\text { masa de sustrato consumida para mantenimiento }}{\text { masa de células } \cdot \text { tiempo }}
$$

Un valor habitual de $\mathrm{k}_{\mathrm{sm}}$ es $0.05 \mathrm{~kg}$ de sustrato $/ \mathrm{kg}$ de células seco $\cdot \mathrm{h}=0.05 \mathrm{~h}^{-1}$, aunque depende en gran medida de las condiciones del medio de cultivo siendo esta dependencia poco conocida.

\subsubsection{Ecuación de velocidad para la muerte celular}

La velocidad de muerte celular viene dada por la ecuación:

$$
\mathrm{r}_{\mathrm{m}}=\left(\mathrm{k}_{\mathrm{m}}+\mathrm{k}_{\mathrm{t}} \cdot \mathrm{c}_{\mathrm{t}}\right) \cdot \mathrm{c}_{\mathrm{c}}
$$

siendo $\mathrm{c}_{\mathrm{t}}$ la concentración de una sustancia tóxica para la célula. Las constantes $\mathrm{k}_{\mathrm{m}}$ $\mathrm{y} \mathrm{k}_{\mathrm{t}}$ hacen referencia a la muerte natural y a la muerte debida a una sustancia tóxica, respectivamente. Valores representativos de $\mathrm{k}_{\mathrm{m}}$ oscilan entre $0.1 \mathrm{~h}^{-1}$ y menos incluso de $0.0005 \mathrm{~h}^{-1}$. El valor de $\mathrm{k}_{\mathrm{t}}$ depende de la naturaleza de la toxina.

\subsubsection{Ecuación de velocidad para la formación de producto}

El producto se obtiene durante la fase II de crecimiento.

La formación de producto puede tener lugar durante diferentes fases del crecimiento celular. Cuando el producto se forma solo durante la fase II de crecimiento se cumple:

$$
r_{p}=Y_{p / c} \cdot r_{c}
$$

El producto se obtiene durante la fase III estacionaria.

Sin embargo, cuando el producto se forma durante la fase III estacionaria, se puede relacionar la formación de producto con el consumo de sustrato mediante una ley de velocidad similar a la ecuación de Monod:

$$
\mathrm{r}_{\mathrm{p}}=\mathrm{k}_{\mathrm{p}} \cdot \frac{\mathrm{c}_{\mathrm{s}}}{\mathrm{K}_{\mathrm{s}}+\mathrm{c}_{\mathrm{s}}} \cdot \mathrm{c}_{\mathrm{c}}
$$


donde:

$\mathrm{k}_{\mathrm{p}}$ : constante de velocidad $\left(\mathrm{s}^{-1}\right)$

$\mathrm{c}_{\mathrm{s}}$ : concentración de sustrato secundario $\left(\mathrm{kg} / \mathrm{m}^{3}\right)$

$\mathrm{c}_{\mathrm{c}}$ : concentración de células $\left(\mathrm{kg} / \mathrm{m}^{3}\right)$

$\mathrm{K}_{\mathrm{s}}$ : constante $\left(\mathrm{kg} / \mathrm{m}^{3}\right)$

Caso de que no exista una relación clara entre formación de producto y de células suele expresarse la velocidad de formación de producto mediante una relación de primer orden:

$$
\mathrm{r}_{\mathrm{p}}=\mathrm{k}_{\mathrm{nc}} \cdot \mathrm{c}_{\mathrm{c}}
$$

siendo $\mathrm{k}_{\mathrm{nc}}$ la constante de velocidad de formación de producto no asociada al crecimiento $\left(\mathrm{h}^{-1}\right)$.

\section{El producto se obtiene en ambas fases}

Aunque se acaban de mostrar dos casos límite (formación de producto en la fase II o en la fase III), a veces ninguna de estas dos situaciones es aplicable. Por ejemplo, en la fermentación que utiliza lactobacillus se produce ácido láctico en ambas fases. En estos casos en que el producto se forma tanto en la fase II como en la fase III, la ecuación de velocidad de formación del producto tiene la forma:

$$
\mathrm{r}_{\mathrm{p}}=\mathrm{Y}_{\mathrm{p} / \mathrm{c}} \cdot \mathrm{r}_{\mathrm{c}}+\mathrm{k}_{\mathrm{nc}} \cdot \mathrm{c}_{\mathrm{c}}
$$

\subsubsection{Ecuación de velocidad para la desaparición de sustrato}

Para calcular la la velocidad de consumo de nutriente o sustrato $\left(r_{s}\right)$ hay que relacionarla con las velocidades de crecimiento celular $\left(\mathrm{r}_{\mathrm{c}}\right)$, de generación de producto $\left(\mathrm{r}_{\mathrm{p}}\right)$ y de mantenimiento $\left(\mathrm{r}_{\mathrm{sm}}\right)$.

En general se puede escribir el siguiente balance de materia aplicado al sustrato:

$$
\begin{aligned}
{\left[\begin{array}{c}
\text { velocidad de } \\
\text { consumo de } \\
\text { sustrato }
\end{array}\right] } & =\left[\begin{array}{c}
\text { velocidad de } \\
\text { crecimiento celular }
\end{array}\right]+\left[\begin{array}{c}
\text { velocidad de } \\
\text { formación de producto }
\end{array}\right]+\left[\begin{array}{c}
\text { velocidad para } \\
\text { mantenimiento celular }
\end{array}\right] \\
r_{\mathrm{s}} & =\mathrm{Y}_{\mathrm{s} / \mathrm{c}} \cdot \mathrm{r}_{\mathrm{c}}+\mathrm{Y}_{\mathrm{s} / \mathrm{p}} \cdot \mathrm{r}_{\mathrm{p}}+\mathrm{k}_{\mathrm{sm}} \cdot \mathrm{c}_{\mathrm{c}}
\end{aligned}
$$

y se pueden distinguir dos casos según el producto se forme en la fase II de crecimiento o en la fase III estacionaria. 
En este caso puede no ser posible distinguir entre la cantidad de sustrato consumida para el crecimiento celular de la consumida para formar producto. Bajo estas circunstancias todo el sustrato consumido se agrupa en el coeficiente de rendimiento estequiométrico $\mathrm{Y}_{\mathrm{s} / \mathrm{c}}$ y la velocidad de desaparición del sustrato es:

$$
\begin{aligned}
{\left[\begin{array}{c}
\text { velocidad de } \\
\text { consumo de } \\
\text { sustrato }
\end{array}\right] } & =\left[\begin{array}{c}
\text { velocidad de } \\
\text { crecimiento celular } \\
\mathrm{y} \\
\text { formación de producto }
\end{array}\right]+\left[\begin{array}{c}
\text { velocidad para } \\
\text { mantenimiento celular }
\end{array}\right] \\
\mathrm{r}_{\mathrm{s}} & =\quad \mathrm{Y}_{\mathrm{s} / \mathrm{c}} \cdot \mathrm{r}_{\mathrm{c}}+\mathrm{k}_{\mathrm{sm}} \cdot \mathrm{c}_{\mathrm{c}}
\end{aligned}
$$

El producto se obtiene durante la fase III estacionaria.

En esta fase $r_{c}=0$ y la velocidad de consumo de sustrato es:

$$
\mathrm{r}_{\mathrm{s}}=\mathrm{Y}_{\mathrm{s} / \mathrm{p}} \cdot \mathrm{r}_{\mathrm{p}}+\mathrm{k}_{\mathrm{sm}} \cdot \mathrm{c}_{\mathrm{c}}
$$

o bien:

$$
\mathrm{r}_{\mathrm{s}}=\mathrm{Y}_{\mathrm{s} / \mathrm{p}} \cdot\left(\mathrm{k}_{\mathrm{p}} \cdot \frac{\mathrm{c}_{\mathrm{s}}}{\mathrm{K}_{\mathrm{s}}+\mathrm{c}_{\mathrm{s}}} \cdot \mathrm{c}_{\mathrm{c}}\right)+\mathrm{k}_{\mathrm{sm}} \cdot \mathrm{c}_{\mathrm{c}}
$$

\subsection{Reactores bioquímicos: características y tipos de fermentadores}

El diseño de un biorreactor depende de las demandas propias de todo reactor químico más las específicas del proceso biológico. Por lo tanto, la selección de un determinado biorreactor dependerá, fundamentalmente, del factor que limite en mayor medida el proceso de conversión. Así, por ejemplo,

1. Los reactores de mezcla completa (RTA) permiten mantener la concentración de substrato por debajo del nivel en que se presentan problemas de inhibición y, al mismo tiempo, lograr una operación eficaz. Además permiten aplicar sistemas de alimentación adecuados con cada uno de los sistemas que propongan.

2. Los reactores de mezcla completa (RTA) permiten minimizar los efectos derivados de los fenómenos de inhibición por producto, mediante la eliminación del producto obtenido a medida que se va formando. 
3. Los reactores de flujo de pistón (RFP) permiten minimizar los efectos derivados de los fenómenos de inhibición por producto, debido al propio modelo de flujo próximo al flujo en pistón.

4. La recirculación celular es un mecanismo muy útil para aumentar la concentración celular en el sistema.

5. En los casos en que la trasferencia de materia es una etapa limitante de la velocidad global del proceso, como por ejemplo la difusión del oxígeno molecular, se puede mejorar el sistema de dispersión de gas mediante boquillas, difusores, etc., que permiten aumentar el área interfacial, o se puede modificar el sistema de agitación.

Los biorreactores o fermentadores se clasifican de acuerdo con la terminología utilizada en el diseño de reactores químicos en:

1. Fermentadores de Tanque Agitado (FTA)

2. Fermentadores Continuos Tubulares de Flujo de Pistón (FFP)

\subsubsection{Fermentadores de Tanque Agitado (FTA)}

i) Fermentador Discontinuo de Tanque Agitado (FDTA)

Es el fermentador más ampliamente utilizado a escala industrial. El tiempo de operación necesario puede ir desde horas a varias semanas, dependiendo de la conversión y de las condiciones de operación. Durante la operación ha de evitarse la contaminación, mantener la agitación, y controlar $\mathrm{pH}$ y temperatura. Operan con baja densidad celular, sobre todo en el periodo inicial, y debe evitarse la alimentación de concentraciones elevadas de substrato para evitar inhibiciones por éste o por el producto. Se emplea mayoritariamente en la industria alimentaria, farmacéutica y biotecnológica, en general, ya que en ellos es fácil alcanzar y mantener condiciones asépticas durante la operación.

En este tipo de fermentadores se produce, por ejemplo, ácido acético, vitamina $\mathrm{C}$ y B12, levadura de panificación, penicilina y también se utilizan para la digestión anaerobia de fangos.

ii) Fermentador Discontinuo de Tanque Agitado (FDTA) con alimentación por lotes

En este caso se trata de un FDTA en el que la alimentación se hace mediante cargas sucesivas sin retirar producto alguno; en consecuencia el volumen de reacción va aumentando progresivamente. Esta forma de operar se aplica, por ejemplo, en la producción de levadura de pan y de antibióticos. 
El Fermentador Continuo de Tanque Agitado (FCTA) también se denomina quimiostato. Suele operar en régimen estacionario.

Si el caudal volumétrico es demasiado elevado puede suceder que la producción de microorganismos sea inferior al número de éstos que son arrastrados por la corriente de salida y, en consecuencia, se llegaría a la situación de que no quedara ningún microorganismo en el interior del reactor: se indica este caso diciendo que los microorganismos han sido lavados del reactor. Se puede evitar esta situación mediante un proceso de recirculación celular en el que la corriente de salida del FCTA se trata en un sedimentador o en una centrifugadora, separándose una solución concentrada de microorganismos que se retroalimenta la FCTA.

En la industria también suele ser frecuente el uso de una serie de FCTA para aumentar la productividad o cambiar las condiciones de operación en cada FCTA.

\subsubsection{Fermentadores Continuos Tubulares de Flujo de Pistón (FFP)}

En principio, en los Fermentadores Continuos Tubulares de Flujo de Pistón (FFP) no se puede alimentar un medio estéril, ya que se trata de un proceso autocatalítico. Este inconveniente se soluciona o bien con una corriente de recirculación o bien con un diseño ad hoc que permite que los microorganismos estén inmovilizados en el interior del reactor.

En este último caso los FFP se pueden dividir; según la disposición de los microorganismo se dividen en dos tipos.

i) Fermentador Continuo Tubular de Flujo de Pistón (FFP) con flóculos en suspensión

Un flóculo es un agregado de microorganismos cuyo tamaño es un orden de magnitud superior al tamaño del microorganismo. El tamaño del flóculo depende, entre otras variables, de la agitación y de los agentes floculantes utilizados.

ii) Fermentador Continuo Tubular de Flujo de Pistón (FFP) de película

Los microorganismos se disponen en forma de película que crece sobre la superficie de un relleno inerte. Este relleno actúa como un lecho de inerte a través del que circula el líquido. 


\subsection{Balance de materia de fermentadores de tanque agitado (FTA)}

Hay dos formas de abordar el crecimiento de microorganismos: contar el número de células vivas o pesar la masa de células vivas. En este capítulo se considerará solo la segunda forma. El balance de materia en un Fermentador de Tanque Agitado (FTA) de volumen constante se puede aplicar

a las células:

$\left[\begin{array}{c}\text { caudal de células } \\ \text { que salen del reactor }\end{array}\right]-\left[\begin{array}{c}\text { caudal de células } \\ \text { que entran al reactor }\end{array}\right]+\left[\begin{array}{c}\text { acumulación } \\ \text { de células }\end{array}\right]=\left[\begin{array}{c}\text { generación de } \\ \text { células vivas }\end{array}\right]$

$$
\mathrm{Q}_{\mathrm{v}} \cdot \mathrm{c}_{\mathrm{c}} \quad-\quad \mathrm{Q}_{\mathrm{v} 0} \cdot \mathrm{c}_{\mathrm{c} 0} \quad+\mathrm{V} \cdot \frac{\mathrm{dc}_{\mathrm{c}}}{\mathrm{dt}}=\left(\mathrm{r}_{\mathrm{c}}-\mathrm{r}_{\mathrm{m}}\right) \cdot \mathrm{V}
$$

al sustrato:

$\left[\begin{array}{c}\text { caudal de sustrato } \\ \text { que sale del reactor }\end{array}\right]-\left[\begin{array}{c}\text { caudal de sustrato } \\ \text { que entra al reactor }\end{array}\right]+\left[\begin{array}{c}\text { acumulación } \\ \text { de sustrato }\end{array}\right]=\left[\begin{array}{c}\text { generación } \\ \text { de sustrato }\end{array}\right]$

$$
\mathrm{Q}_{\mathrm{v}} \cdot \mathrm{c}_{\mathrm{s}} \quad-\quad \mathrm{Q}_{\mathrm{v} 0} \cdot \mathrm{c}_{\mathrm{s} 0} \quad+\mathrm{V} \cdot \frac{\mathrm{dc}_{\mathrm{s}}}{\mathrm{dt}}=-\mathrm{r}_{\mathrm{s}} \cdot \mathrm{V}
$$

al producto:

$$
\begin{gathered}
{\left[\begin{array}{l}
\text { caudal de producto } \\
\text { que sale del reactor }
\end{array}\right]-\left[\begin{array}{l}
\text { caudal de producto } \\
\text { que entra al reactor }
\end{array}\right]+\left[\begin{array}{c}
\text { acumulación } \\
\text { de producto }
\end{array}\right]=\left[\begin{array}{c}
\text { generación } \\
\text { de producto }
\end{array}\right]} \\
\mathrm{Q}_{\mathrm{v}} \cdot \mathrm{c}_{\mathrm{p}}-\mathrm{Q}_{\mathrm{v} 0} \cdot \mathrm{c}_{\mathrm{p} 0}+\mathrm{V} \cdot \frac{\mathrm{dc}}{\mathrm{dt}}=\mathrm{r}_{\mathrm{p}} \cdot \mathrm{V}
\end{gathered}
$$

\subsubsection{Fermentador discontinuo de tanque agitado (FDTA)}

Los balances de materia que quedan se pueden aplicar tanto a las células como al sustrato y al producto.

Balance de materia a las células:

Aplicando las condiciones de un sistema discontinuo se llega a :

$$
\mathrm{V} \cdot \frac{\mathrm{dc}_{\mathrm{c}}}{\mathrm{dt}}=\left(\mathrm{r}_{\mathrm{c}}-\mathrm{r}_{\mathrm{m}}\right) \cdot \mathrm{V}
$$




$$
\frac{\mathrm{dc}_{\mathrm{c}}}{\mathrm{dt}}=\left(\mathrm{r}_{\mathrm{c}}-\mathrm{r}_{\mathrm{m}}\right)
$$

Balance de materia al sustrato:

Análogamente, aplicando las condiciones de un sistema discontinuo se obtiene:

$$
\mathrm{V} \cdot \frac{\mathrm{dc}_{\mathrm{s}}}{\mathrm{dt}}=-\mathrm{r}_{\mathrm{s}} \cdot \mathrm{V}
$$

donde:

i) si el sistema se encuentra en la fase II de crecimiento $\mathrm{r}_{\mathrm{s}}=\mathrm{Y}_{\mathrm{s} / \mathrm{c}} \cdot \mathrm{r}_{\mathrm{c}}+\mathrm{m} \cdot \mathrm{c}_{\mathrm{c}}$ y se obtiene:

$$
\frac{\mathrm{dc}_{\mathrm{s}}}{\mathrm{dt}}=-\left(\mathrm{Y}_{\mathrm{s} / \mathrm{c}} \cdot \mathrm{r}_{\mathrm{c}}+\mathrm{m} \cdot \mathrm{c}_{\mathrm{c}}\right)
$$

ii) si el sistema se encuentra en la fase III estacionaria, donde no hay crecimiento y el mantenimiento celular y la formación de producto son las únicas reacciones que consumen sustrato $\mathrm{r}_{\mathrm{ss}}=\mathrm{m} \cdot \mathrm{c}_{\mathrm{c}}+\mathrm{Y}_{\mathrm{ss} / \mathrm{p}} \cdot \mathrm{r}_{\mathrm{p}} \mathrm{o}$ bien para este caso $\mathrm{r}_{\mathrm{s}}=\mathrm{m} \cdot \mathrm{c}_{\mathrm{c}}+\mathrm{Y}_{\mathrm{s} / \mathrm{p}} \cdot \mathrm{r}_{\mathrm{p}}$ y se obtiene:

$$
\frac{d c_{s}}{d t}=-\left(m \cdot c_{c}+Y_{s / p} \cdot r_{p}\right)
$$

Balance de materia al producto:

Aplicando de nuevo las condiciones de un sistema discontinuo se obtiene:

$$
\mathrm{V} \cdot \frac{\mathrm{dc}_{\mathrm{p}}}{\mathrm{dt}}=\mathrm{r}_{\mathrm{p}} \cdot \mathrm{V}
$$

donde:

i) si el sistema se encuentra en la fase II de crecimiento $r_{p}=Y_{p / c} \cdot r_{c}$ y se obtiene:

$$
\frac{d c_{p}}{d t}=Y_{p / c} \cdot r_{c}
$$

ii) si el sistema se encuentra en la fase III estacionaria, donde no hay crecimiento y el mantenimiento celular y la formación de producto son las únicas reacciones que consumen sustrato $r_{p}=Y_{p / s} \cdot r_{s}$ y se obtiene:

$$
\frac{d c_{p}}{d t}=Y_{p / s} \cdot r_{s}
$$


Las ecuaciones anteriores constituyen un sistema de ecuaciones diferenciales lineales de primer orden que se pueden resolver simultáneamente utilizando cualquiera de los métodos numéricos habituales.

\subsubsection{Fermentador continuo de tanque agitado (FCTA), o quimiostato, en régimen estacionario y estéril}

Los denominados quimiostatos son básicamente reactores continuos de tanque agitado que contienen microorganismos. Una de sus características más importante es que permite controlar la velocidad de crecimiento celular mediante el ajuste del caudal volumétrico de alimentación (aquí expresado habitualmente velocidad de dilución).

Se parte de los balances de materia a reactores de tanque agitado, considerando que los caudales volumétricos de entrada y de salida son iguales, que funciona en régimen estacionario y que la corriente de entrada no contiene células vivas (estéril). Asimismo, conviene definir el parámetro D conocido como velocidad de dilución:

$\mathrm{D}=\frac{\mathrm{Q}_{\mathrm{v} 0}}{\mathrm{~V}} \quad\left(=\mathrm{s}=\frac{1}{\tau} \quad\right.$; véase los conceptos de velocidad espacial y tiempo espacial definidos para un RCTA $)$

y que resulta ser la conocida velocidad espacial (inversa del tiempo espacial). Aplicando estas condiciones a los balances de materia:

a las células:

$$
\begin{gathered}
\mathrm{Q}_{\mathrm{v}} \cdot \mathrm{c}_{\mathrm{c}}-\mathrm{Q}_{\mathrm{v} 0} \cdot \mathrm{c}_{\mathrm{c} 0}=\left(\mathrm{r}_{\mathrm{c}}-\mathrm{r}_{\mathrm{m}}\right) \cdot \mathrm{V} \\
\mathrm{Q}_{\mathrm{v} 0} \cdot \mathrm{c}_{\mathrm{c}}-0=\left(\mathrm{r}_{\mathrm{c}}-\mathrm{r}_{\mathrm{m}}\right) \cdot \mathrm{V} \\
\mathrm{D} \cdot \mathrm{c}_{\mathrm{c}}=\left(\mathrm{r}_{\mathrm{c}}-\mathrm{r}_{\mathrm{m}}\right)
\end{gathered}
$$

al sustrato:

$$
\begin{gathered}
\mathrm{Q}_{\mathrm{v}} \cdot \mathrm{c}_{\mathrm{s}}-\mathrm{Q}_{\mathrm{v} 0} \cdot \mathrm{c}_{\mathrm{s} 0}=-\mathrm{r}_{\mathrm{s}} \cdot \mathrm{V} \\
\mathrm{Q}_{\mathrm{v} 0} \cdot \mathrm{c}_{\mathrm{s}}-\mathrm{Q}_{\mathrm{v} 0} \cdot \mathrm{c}_{\mathrm{s} 0}=-\mathrm{r}_{\mathrm{s}} \cdot \mathrm{V} \\
\mathrm{D} \cdot \mathrm{c}_{\mathrm{s}}-\mathrm{D} \cdot \mathrm{c}_{\mathrm{s} 0}=-\mathrm{r}_{\mathrm{s}} \\
\mathrm{D} \cdot\left(\mathrm{c}_{\mathrm{s} 0}-\mathrm{c}_{\mathrm{s}}\right)=\mathrm{r}_{\mathrm{s}}
\end{gathered}
$$

al producto:

$$
\begin{array}{r}
\mathrm{Q}_{\mathrm{v}} \cdot \mathrm{c}_{\mathrm{p}}-\mathrm{Q}_{\mathrm{v} 0} \cdot \mathrm{c}_{\mathrm{p} 0}=\mathrm{r}_{\mathrm{p}} \cdot \mathrm{V} \\
\mathrm{Q}_{\mathrm{v} 0} \cdot \mathrm{c}_{\mathrm{p}}-0=\mathrm{r}_{\mathrm{p}} \cdot \mathrm{V}
\end{array}
$$

$$
\mathrm{D} \cdot \mathrm{c}_{\mathrm{p}}=\mathrm{r}_{\mathrm{p}}
$$


i) Ecuación de diseño: relación entre la velocidad de dilución y el estado estacionario. Condición de lavado.

Si en el balance de materia a las células se desprecia $\mathrm{r}_{\mathrm{m}}$ porque se procurará estar en la fase II de crecimiento:

$$
\mathrm{D} \cdot \mathrm{c}_{\mathrm{c}}=\mathrm{r}_{\mathrm{c}}
$$

y para una cinética de crecimiento de Monod

$$
\begin{gathered}
D \cdot c_{c}=\mu \cdot c_{c} \\
D=\mu
\end{gathered}
$$

lo que pone de manifiesto que la velocidad específica de crecimiento celular se puede controlar mediante la velocidad de dilución.

\begin{tabular}{|c|c|c|}
\hline Concentración del sustrato & Concentración celular & $\begin{array}{l}\text { Concentración } \\
\text { de producto }\end{array}$ \\
\hline $\begin{array}{l}\text { Dado que: } \\
\mu=\mu_{\max } \cdot \frac{\mathrm{c}_{\mathrm{s}}}{\mathrm{K}_{\mathrm{s}}+\mathrm{c}_{\mathrm{s}}} \\
\mathrm{c}_{\mathrm{s}}\end{array}$ & $\begin{array}{l}\text { Aplicando la correspon- } \\
\text { diente relación estequio- } \\
\text { métrica: } \\
\mathrm{c}_{\mathrm{c}}-\mathrm{c}_{\mathrm{c} 0}=\mathrm{Y}_{\mathrm{c} / \mathrm{s}} \cdot\left(\mathrm{c}_{\mathrm{s} 0}-\mathrm{c}_{\mathrm{s}}\right) \\
\text { se obtiene: }\end{array}$ & $\begin{array}{l}\text { Aplicando la correspon- } \\
\text { diente relación estequio- } \\
\text { métrica } \\
\mathrm{c}_{\mathrm{p}}-\mathrm{c}_{\mathrm{p} 0}=\mathrm{Y}_{\mathrm{p} / \mathrm{s}} \cdot\left(\mathrm{c}_{\mathrm{s} 0}-\mathrm{c}_{\mathrm{s}}\right) \\
\text { se obtiene: }\end{array}$ \\
\hline $\begin{array}{l}\mathrm{D}=\mu_{\max } \cdot \frac{\mathrm{s}}{\mathrm{K}_{\mathrm{s}}+\mathrm{c}_{\mathrm{s}}} \\
\text { de donde: }\end{array}$ & $c_{c}=Y_{c / s} \cdot\left(c_{s 0}-\frac{D \cdot K_{s}}{\mu_{\max }-D}\right)$ & $c_{p}=Y_{p / s} \cdot\left(c_{s 0}-\frac{D \cdot K_{s}}{\mu_{\max }-D}\right)$ \\
\hline $\mathrm{c}_{\mathrm{s}}=\frac{\mathrm{D} \cdot \mathrm{K}_{\mathrm{s}}}{\mu_{\max }-\mathrm{D}}$ & & \\
\hline
\end{tabular}

La concentración de cada especie se puede obtener a partir de esta expresión. En efecto:

En la figura 2 se muestra la concentración celular (la de producto tendría una forma análoga a la celular) y la de sustrato para cada estado estacionario según el valor de D al que se esté operando. Fácilmente se pueden extraer las siguientes conclusiones:

a) Curva $\mathrm{c}_{\mathrm{s}}$. Al operar con caudales volumétricos pequeños, el valor de $\mathrm{D}$ se aproxima a 0 y así lo hace $\mathrm{c}_{\mathrm{s}}$. La interpretación es que el sustrato se consume inmediatamente al entrar en el reactor. Contrariamente, al operar con caudales volumétricos grandes, D también es grande, con lo que $\mathrm{c}_{\mathrm{s}}$ aumenta en el interior del reactor: primero linealmente y luego bruscamente a medida que $\mathrm{D}$ se aproxima al valor de $\mu_{\max }$. Cuando esto se produce $\mathrm{c}_{\mathrm{s}}$ tendería a infinito, lo cual no tiene sentido ya que su valor máximo es $\mathrm{c}_{\mathrm{s} 0}$. 
b) Curva $\mathrm{c}_{\mathrm{c}}$. Al operar con caudales volumétricos pequeños, el valor de $\mathrm{D}$ se aproxima a $0 \mathrm{y} \mathrm{c}_{\mathrm{c}}$ lo hace hacia $\mathrm{Y}_{\mathrm{c} / \mathrm{s}} \cdot \mathrm{c}_{\mathrm{s} 0}$. Contrariamente, al operar con caudales volumétricos grandes $\mathrm{D}$ también es grande, con lo que $\mathrm{c}_{\mathrm{c}}$ disminuye en el interior del reactor: primero linealmente y luego bruscamente a medida que $\mathrm{D}$ se aproxima al valor de $\mu_{\max }$. Cuando esto se produce $\mathrm{c}_{\mathrm{c}}=0 \mathrm{y} \mathrm{c}_{\mathrm{s}}=\mathrm{c}_{\mathrm{s} 0}$. Esta situación se conoce como lavado del reactor y pone de manifiesto que ya no hay células en el quimiostato.

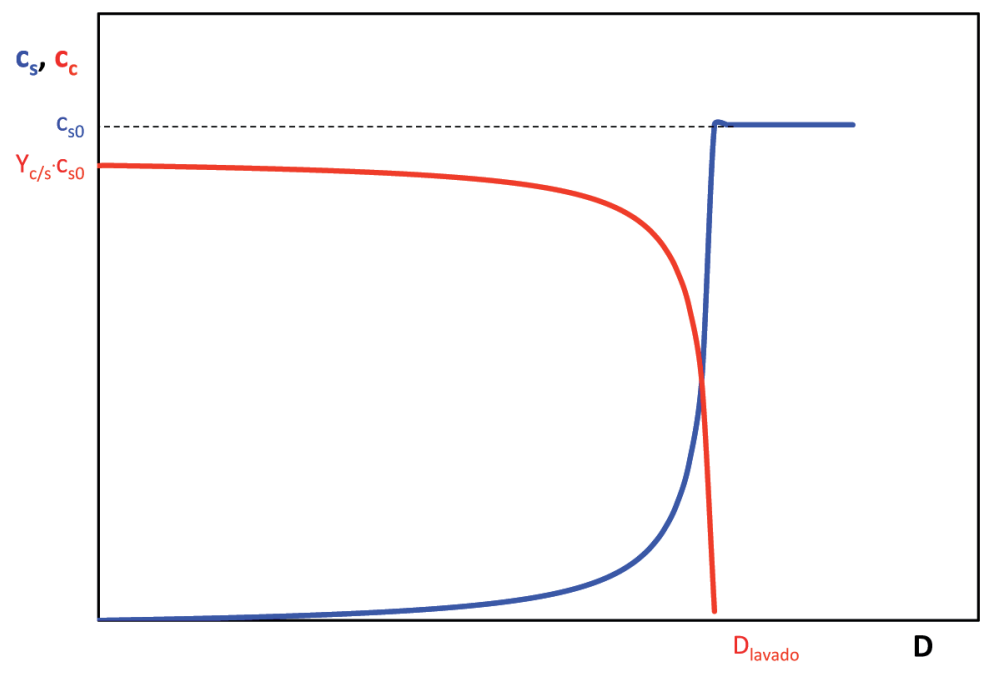

Figura 2. Concentración celular y de sustrato para cada estado estacionario según el valor de D al que se esté operando

Ejercicio 1. Proponer el procedimiento para determinar experimentalmente los parámetros cinéticos que se basen en la relación $\mathrm{c}_{\mathrm{s}}=\frac{\mathrm{D} \cdot \mathrm{K}_{\mathrm{s}}}{\mu_{\text {max }}-\mathrm{D}}$. Representar en una gráfica la expresión $\frac{1}{\mathrm{c}_{\mathrm{s}}}=-\frac{1}{\mathrm{~K}_{\mathrm{s}}}+\frac{\mu_{\max }}{\mathrm{K}_{\mathrm{s}}} \cdot \frac{1}{\mathrm{D}}$ indicando la forma de proceder para obtener los parámetros clave.

El valor de $\mathrm{D}$ para el que se produce el lavado $\left(\mathrm{D}_{\mathrm{w}}\right)$ del quimiostato, es decir, para el que $\mathrm{c}_{\mathrm{c}}=0 \mathrm{y} \mathrm{c}_{\mathrm{s}}=\mathrm{c}_{\mathrm{s} 0}$, se denomina velocidad de dilución de lavado y se puede calcular a partir de la expresión de la concentración de sustrato aplicando la condición de que su valor sea $\mathrm{c}_{\mathrm{s} 0}$. En efecto:

$\mathrm{c}_{\mathrm{s}}=\frac{\mathrm{D} \cdot \mathrm{K}_{\mathrm{s}}}{\mu_{\max }-\mathrm{D}} \quad$ si c $\mathrm{s}_{\mathrm{s}}=\mathrm{c}_{\mathrm{s} 0} \mathrm{y} \mathrm{D}=\mathrm{D}_{\mathrm{w}} \rightarrow \mathrm{c}_{\mathrm{s} 0}=\frac{\mathrm{D}_{\mathrm{w}} \cdot \mathrm{K}_{\mathrm{s}}}{\mu_{\text {max }}-\mathrm{D}_{\mathrm{w}}}$

y despejando:

$$
\mathrm{D}_{\mathrm{w}}=\frac{\mu_{\max } \cdot \mathrm{c}_{\mathrm{s} 0}}{\mathrm{~K}_{\mathrm{s}}+\mathrm{c}_{\mathrm{s} 0}}
$$


ii) Velocidad de dilución óptima y volumen óptimo del FCTA

Para determinar el valor de la velocidad de dilución óptima a la que debe operar el reactor $\left(\mathrm{D}_{\mathrm{opt}}\right)$ hay que definir la producción celular por unidad de volumen como:

$$
\mathrm{D} \cdot \mathrm{c}_{\mathrm{c}} \quad\left(\mathrm{kg} \text { de células } / \mathrm{m}^{3} \cdot \mathrm{s}\right)
$$

Sustituyendo el valor de la concentración por la expresión correspondiente se obtiene:

$$
\mathrm{D} \cdot \mathrm{c}_{\mathrm{c}}=\mathrm{D} \cdot \mathrm{Y}_{\mathrm{c} / \mathrm{s}} \cdot\left(\mathrm{c}_{\mathrm{s} 0}-\frac{\mathrm{D} \cdot \mathrm{K}_{\mathrm{s}}}{\mu_{\text {max }}-\mathrm{D}}\right)
$$

cuya forma se muestra en la figura 3.

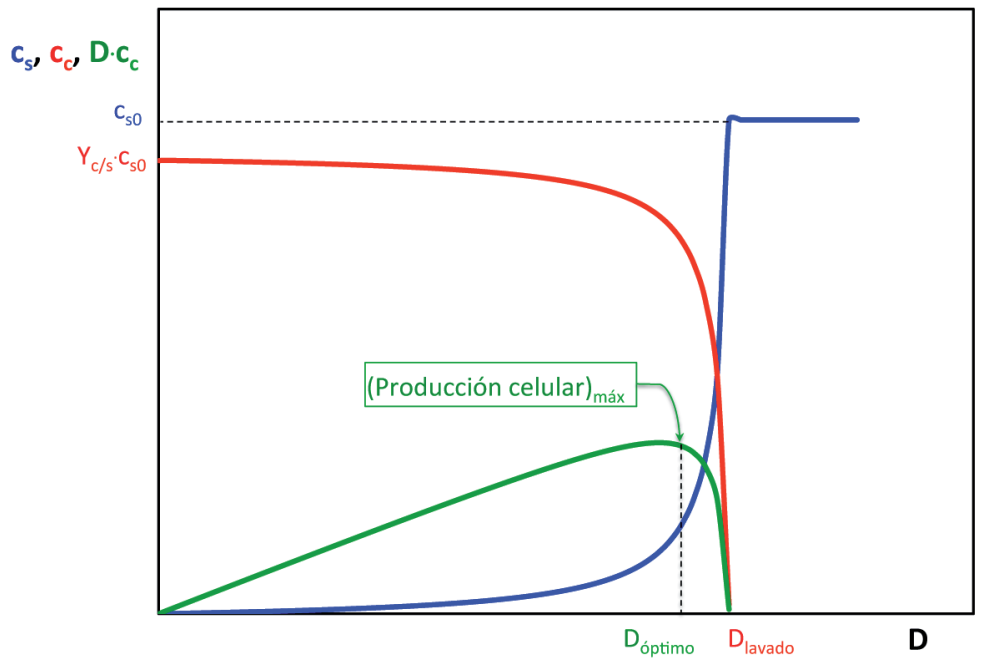

Figura 3. Velocidad óptima de dilución

Derivando con respecto a $\mathrm{D}$ e igualando a cero se puede obtener el valor de $\mathrm{D}$ $\left(\mathrm{D}_{\mathrm{opt}}\right)$ que hace que la producción celular por unidad de volumen sea máxima:

$$
\frac{\mathrm{d}}{\mathrm{dD}}\left(\mathrm{D} \cdot \mathrm{c}_{\mathrm{c}}\right)=\frac{\mathrm{d}}{\mathrm{dD}}\left[\mathrm{D} \cdot \mathrm{Y}_{\mathrm{c} / \mathrm{s}} \cdot\left(\mathrm{c}_{\mathrm{s} 0}-\frac{\mathrm{D} \cdot \mathrm{K}_{\mathrm{s}}}{\mu_{\max }-\mathrm{D}}\right)\right]=0
$$

resultando:

$$
\mathrm{D}_{\mathrm{opt}}=\mu_{\max } \cdot\left(1-\sqrt{\frac{\mathrm{K}_{\mathrm{s}}}{\mathrm{K}_{\mathrm{s}}+\mathrm{c}_{\mathrm{s} 0}}}\right)
$$

Aunque $c_{s}, c_{c} y_{p}$ se pueden obtener sustituyendo $D$ por el valor de $D_{\text {opt }}$ en las correspondientes ecuaciones, resulta todavía más sencillo obtener estos valores de la gráfica anterior. 
Lógicamente se puede obtener el volumen del reactor correspondiente:

$$
\mathrm{D}_{\mathrm{opt}}=\frac{\mathrm{Q}_{\mathrm{v} 0}}{\mathrm{~V}_{\mathrm{opt}}}
$$

iii) Velocidad de crecimiento celular máxima y volumen mínimo del FCTA

Adicionalmente a lo visto en el apartado anterior, se puede calcular la concentración de sustrato en el reactor para que la velocidad de crecimiento celular sea máxima. En efecto, la velocidad de crecimiento celular era:

$$
\mathrm{r}_{\mathrm{c}}=\mu \cdot \mathrm{c}_{\mathrm{c}}=\mu_{\max } \cdot \frac{\mathrm{c}_{\mathrm{s}}}{\mathrm{K}_{\mathrm{s}}+\mathrm{c}_{\mathrm{s}}} \cdot \mathrm{c}_{\mathrm{c}}
$$

y considerando la relación estequiométrica entre $\mathrm{c}_{\mathrm{c}} \mathrm{y} \mathrm{c}_{\mathrm{s}}$ :

$$
\mathrm{c}_{\mathrm{c}}-\mathrm{c}_{\mathrm{c} 0}=\mathrm{Y}_{\mathrm{c} / \mathrm{s}} \cdot\left(\mathrm{c}_{\mathrm{s} 0}-\mathrm{c}_{\mathrm{s}}\right)
$$

se obtiene la expresión:

$$
\mathrm{r}_{\mathrm{c}}=\mu_{\max } \cdot \frac{\mathrm{c}_{\mathrm{s}}}{\mathrm{K}_{\mathrm{s}}+\mathrm{c}_{\mathrm{s}}} \cdot\left[\mathrm{c}_{\mathrm{c} 0}+\mathrm{Y}_{\mathrm{c} / \mathrm{s}} \cdot\left(\mathrm{c}_{\mathrm{s} 0}-\mathrm{c}_{\mathrm{s}}\right)\right]
$$

Esta expresión se puede hacer máxima para una determinada concentración de sustrato, que se puede calcular aplicando la condición de máximo:

$$
\frac{\mathrm{dr}_{\mathrm{c}}}{\mathrm{dc}_{\mathrm{s}}}=\frac{\mathrm{d}}{\mathrm{dc}_{\mathrm{s}}}\left\{\mu_{\max } \cdot \frac{\mathrm{c}_{\mathrm{s}}}{\mathrm{K}_{\mathrm{s}}+\mathrm{c}_{\mathrm{s}}} \cdot\left[\mathrm{c}_{\mathrm{c} 0}+\mathrm{Y}_{\mathrm{c} / \mathrm{s}} \cdot\left(\mathrm{c}_{\mathrm{s} 0}-\mathrm{c}_{\mathrm{s}}\right)\right]\right\}=0
$$

obteniéndose:

$$
c_{s}\left(r_{c, \max }\right)=-K_{s}+\sqrt{K_{s}^{2}+K_{s} \cdot\left(c_{s 0}+Y_{s / c} \cdot c_{c 0}\right)}
$$

A esta velocidad de reacción máxima le debe corresponder un volumen mínimo de FCTA, que se puede calcular a partir del balance de materia:

$$
\begin{gathered}
\mathrm{D} \cdot\left(\mathrm{c}_{\mathrm{s} 0}-\mathrm{c}_{\mathrm{s}}\right)=\mathrm{r}_{\mathrm{s}} \\
\mathrm{D}=\frac{\mathrm{r}_{\mathrm{s}}}{\left(\mathrm{c}_{\mathrm{s} 0}-\mathrm{c}_{\mathrm{s}}\right)} \\
\frac{1}{\mathrm{D}_{\mathrm{V} \min }}=\left(\frac{1}{\mathrm{r}_{\mathrm{s}}}\right) \cdot\left(\mathrm{c}_{\mathrm{s} 0}-\mathrm{c}_{\mathrm{s}}\right)=\frac{\mathrm{V}_{\min }}{\mathrm{Q}_{\mathrm{v} 0}}
\end{gathered}
$$


Por lo que al representar la inversa de $\mathrm{r}_{\mathrm{s}}$ frente a $\mathrm{c}_{\mathrm{s}}$ se obtiene la figura 4, en la que el volumen mínimo es proporcional al área del rectángulo de base $\left(\mathrm{c}_{\mathrm{s} 0}-\mathrm{c}_{\mathrm{s}}\right) \mathrm{y}$ de altura $1 / \mathrm{r}_{\mathrm{s}}\left(\mathrm{c}_{\mathrm{s}}\right)$.

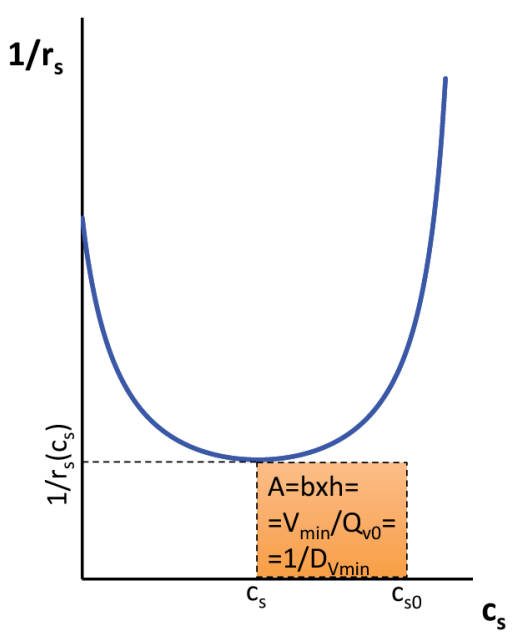

Figura 4. Volumen mínimo de un FCTA

\subsubsection{Fermentador continuo de tanque agitado (FCTA), o quimiostato, en régimen estacionario y estéril, con envenenamiento por producto}

Los balances de materia al quimiostato son, obviamente, los utilizados en el apartado 8.6.2, ya que solo cambia la expresión de la ley de velocidad de reacción. Por lo tanto,

a las células:

$$
\mathrm{D} \cdot \mathrm{c}_{\mathrm{c}}=\left(\mathrm{r}_{\mathrm{c}}-\mathrm{r}_{\mathrm{m}}\right)
$$

al sustrato:

$$
\mathrm{D} \cdot\left(\mathrm{c}_{\mathrm{s} 0}-\mathrm{c}_{\mathrm{s}}\right)=\mathrm{r}_{\mathrm{s}}
$$

al producto:

$$
D \cdot c_{p}=r_{p}
$$


i) Ecuación de diseño: relación entre la velocidad de dilución y el estado estacionario. Condición de lavado.

Al tratarse de un proceso con envenenamiento por producto la ecuación de velocidad tipo Monod a aplicar es (apartado 8.5.1):

$$
\mathrm{r}_{\mathrm{c}}=\mu_{\max } \cdot \frac{\mathrm{c}_{\mathrm{s}}}{\mathrm{K}_{\mathrm{s}}+\mathrm{c}_{\mathrm{s}}} \cdot \mathrm{c}_{\mathrm{c}} \cdot\left(1-\frac{\mathrm{c}_{\mathrm{p}}}{\mathrm{c}_{\mathrm{p}}^{*}}\right)^{\mathrm{n}}
$$

Se tratará a continuación el caso más sencillo en que $\mathrm{n}=1 \mathrm{y} \mathrm{c}_{\mathrm{s}} \gg>\mathrm{K}_{\mathrm{s}}$ quedando la ecuación de velocidad como:

$$
\mathrm{r}_{\mathrm{c}} \approx \mu_{\max } \cdot\left(1-\frac{\mathrm{c}_{\mathrm{p}}}{\mathrm{c}_{\mathrm{p}}^{*}}\right) \cdot \mathrm{c}_{\mathrm{c}}
$$

Si la corriente de entrada al reactor es estéril $\left(\mathrm{c}_{\mathrm{c} 0}=0\right)$ y no contiene producto $\left(\mathrm{c}_{\mathrm{p} 0}=\right.$ $0)$ a partir del balance de materia al producto:

$$
\mathrm{D} \cdot \mathrm{c}_{\mathrm{p}}=\mathrm{r}_{\mathrm{p}}
$$

y considerando que $r_{p}=Y_{p / c} \cdot r_{c}$ :

$$
\mathrm{D} \cdot \mathrm{c}_{\mathrm{p}}=\mathrm{r}_{\mathrm{p}}=\mathrm{Y}_{\mathrm{p} / \mathrm{c}} \cdot \mathrm{r}_{\mathrm{c}}=\mathrm{Y}_{\mathrm{p} / \mathrm{c}} \cdot \mu_{\max } \cdot\left(1-\frac{\mathrm{c}_{\mathrm{p}}}{\mathrm{c}_{\mathrm{p}}^{*}}\right) \cdot \mathrm{c}_{\mathrm{c}}
$$

Mediante la correspondiente relación estequiométrica:

$$
c_{p}-c_{p 0}=Y_{p / c} \cdot\left(c_{c}-c_{c 0}\right) \rightarrow c_{c}-c_{c 0}=Y_{c / p} \cdot\left(c_{p}-c_{p 0}\right) \rightarrow c_{c}=Y_{c / p} \cdot c_{p}
$$

se obtiene:

$$
\begin{gathered}
D \cdot c_{p}=r_{p}=Y_{p / c} \cdot r_{c}=Y_{p / c} \cdot \mu_{\max } \cdot\left(1-\frac{c_{p}}{c_{p}^{*}}\right) \cdot Y_{c / p} \cdot c_{p} \\
D=\mu_{\max } \cdot\left(1-\frac{c_{p}}{c_{p}^{*}}\right)
\end{gathered}
$$

o bien la conocida como ecuación de diseño:

$$
\frac{\mu_{\max }}{D}=\frac{1}{1-\frac{c_{p}}{c_{p}^{*}}}=\frac{c_{p}^{*}}{c_{p}^{*}-c_{p}}
$$

en la que se cumple que si $\mathrm{c}_{\mathrm{p}} / \mathrm{c}_{\mathrm{p}}^{*}<1$ entonces $\mu_{\max } / \mathrm{D}>1$. 
En la figura 5 se representa la ecuación de diseño. Puede observarse que la condición de lavado se alcanza cuando $c_{p}=0$ y por tanto $\mu_{\max } / D_{w}=1$, siendo independiente de las condiciones de alimentación.

La velocidad de dilución a la que la velocidad de reacción es máxima y el volumen mínimo del FCTA se determinarán en el apartado siguiente.

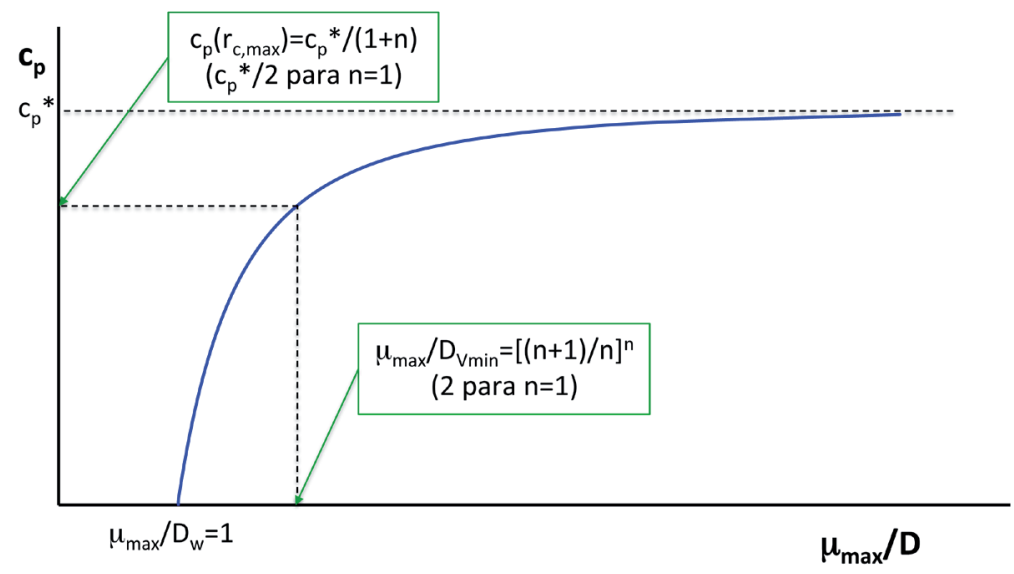

Figura 5. Representación de la ecuación de diseño

Ejercicio 2. Proponer el procedimiento para determinar experimentalmente los parámetros cinéticos que se base en la relación $\frac{\mu_{\max }}{D}=\frac{c_{p}^{*}}{c_{p}^{*}-c_{p}}$. Representar en una gráfica la expresión $c_{p}=c_{p}^{*}-\frac{c_{p}^{*}}{\mu_{\max }} \cdot D$ indicando la forma de proceder para obtener los parámetros clave.

Ejercicio 3. Compruébese que bajo las mismas hipótesis, pero con un valor de $n$ distinto de 1, la ecuación de diseño tiene la forma:

$$
\frac{\mu_{\max }}{D}=\frac{1}{\left(1-\frac{c_{p}}{c_{p}^{*}}\right)^{n}}
$$

Ejercicio 4. Si el valor de $\mathrm{n}$ es distinto de 1, es necesario determinar en primer lugar $\mathrm{c}_{\mathrm{p}}{ }^{*}$ en un FDTA utilizando un exceso de sustrato y tiempo tendiendo a infinito o bien utilizando un FCTA con valores de D muy pequeños. Demuéstrese que, una vez conocido $\mathrm{c}_{\mathrm{p}}^{*}$, los otros parámetros cinéticos se pueden estimar a partir de la regresión lineal:

$$
\ln \mathrm{D}=\ln \mu_{\max }+\mathrm{n} \cdot \ln \left(1-\frac{\mathrm{c}_{\mathrm{p}}}{\mathrm{c}_{\mathrm{p}}^{*}}\right)
$$


ii) Velocidad de crecimiento celular máxima y volumen mínimo del FCTA

A partir de la velocidad de crecimiento celular:

$$
r_{c} \approx \mu_{\max } \cdot\left(1-\frac{c_{p}}{c_{p}^{*}}\right) \cdot c_{c}
$$

y considerando que $r_{p}=Y_{p / c} \cdot r_{c}$ :

$$
\mathrm{r}_{\mathrm{c}}=\mu_{\max } \cdot\left(1-\frac{\mathrm{c}_{\mathrm{p}}}{\mathrm{c}_{\mathrm{p}}^{*}}\right) \cdot \mathrm{c}_{\mathrm{c}}=\mu_{\max } \cdot\left(1-\frac{\mathrm{c}_{\mathrm{p}}}{\mathrm{c}_{\mathrm{p}}^{*}}\right) \cdot \mathrm{Y}_{\mathrm{c} / \mathrm{p}} \cdot \mathrm{c}_{\mathrm{p}}
$$

se puede calcular la velocidad de reacción máxima:

$$
\frac{d r_{c}}{d c_{p}}=\mu_{\max } \cdot Y_{c / p} \cdot\left[\left(1-\frac{c_{p}}{c_{p}^{*}}\right)+c_{p} \cdot\left(\frac{-1}{c_{p}^{*}}\right)\right]=0
$$

de donde:

$$
\mathrm{c}_{\mathrm{p}}\left(\mathrm{r}_{\mathrm{c}, \max }\right)=\frac{1}{2} \cdot \mathrm{c}_{\mathrm{p}}^{*}
$$

Introduciendo este valor en la ecuación de diseño se puede determinar el volumen mínimo del FCTA:

$$
\begin{gathered}
\frac{\mu_{\max }}{D}=\frac{c_{p}^{*}}{c_{p}^{*}-c_{p}}=\frac{c_{p}^{*}}{c_{p}^{*}-\frac{1}{2} \cdot c_{p}^{*}}=2 \\
D_{V \min }=\frac{Q_{V 0}}{V_{\min }}=\frac{1}{2} \cdot \mu_{\max }
\end{gathered}
$$

así como calcular la producción máxima:

$$
\mathrm{Q}_{\mathrm{V} 0} \cdot \mathrm{c}_{\mathrm{p}}\left(\mathrm{r}_{\mathrm{c}, \text { max }}\right)=\frac{\mathrm{Q}_{\mathrm{V} 0}}{\mathrm{~V}_{\text {min }}} \cdot \mathrm{c}_{\mathrm{p}}\left(\mathrm{r}_{\mathrm{c}, \text { max }}\right) \cdot \mathrm{V}_{\text {min }}=\mathrm{D}_{\mathrm{V} \text { min }} \cdot \mathrm{c}_{\mathrm{p}}\left(\mathrm{r}_{\mathrm{c}, \text { max }}\right) \cdot \mathrm{V}_{\text {min }}=\frac{1}{2} \cdot \mu_{\text {max }} \cdot \frac{1}{2} \cdot \mathrm{c}_{\mathrm{p}}^{*} \cdot \mathrm{V}_{\text {min }}=\frac{1}{4} \cdot \mu_{\text {max }} \cdot \mathrm{c}_{\mathrm{p}}^{*} \cdot \mathrm{V}_{\text {min }}
$$




\subsubsection{Fermentador continuo de tanque agitado (FCTA), o quimiostato, en régimen estacionario y con recirculación (no estéril)}

En este caso necesariamente el alimento al reactor es no estéril como consecuencia de la corriente de recirculación que aporta células (figura 6). Esta recirculación se produce tras la adecuada separación y concentración de microorganismos, tal y como se muestra en la figura.

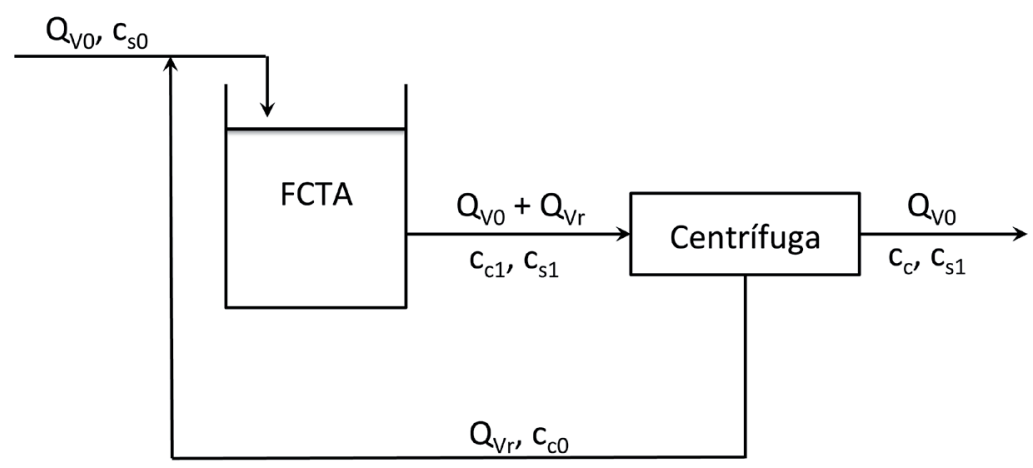

Figura 6. FCTA en régimen estacionario con recirculación no estéril

Aplicando un balance de materia a los microorganismos una vez se alcanza el régimen estacionario, despreciando $\mathrm{r}_{\mathrm{m}}$ :

$$
\begin{gathered}
\left(\mathrm{Q}_{\mathrm{v} 0}+\mathrm{Q}_{\mathrm{Vr}}\right) \cdot \mathrm{c}_{\mathrm{cl}}-\mathrm{Q}_{\mathrm{vr}} \cdot \mathrm{c}_{\mathrm{c} 0}=\mathrm{r}_{\mathrm{c}} \cdot \mathrm{V} \\
\left(\mathrm{Q}_{\mathrm{v} 0}+\mathrm{Q}_{\mathrm{Vr}}\right) \cdot \mathrm{c}_{\mathrm{c} 1}-\mathrm{Q}_{\mathrm{vr}} \cdot \mathrm{c}_{\mathrm{c} 0}=\mu \cdot \mathrm{c}_{\mathrm{c} 1} \cdot \mathrm{V} \\
\mathrm{Q}_{\mathrm{vr}} \cdot \mathrm{c}_{\mathrm{c} 0}-\left(\mathrm{Q}_{\mathrm{v} 0}+\mathrm{Q}_{\mathrm{vr}}\right) \cdot \mathrm{c}_{\mathrm{c} 1}+\mu \cdot \mathrm{c}_{\mathrm{c} 1} \cdot \mathrm{V}=0
\end{gathered}
$$

Si se define:

$$
\alpha=\frac{\mathrm{Q}_{\mathrm{Vr}}}{\mathrm{Q}_{\mathrm{v} 0}} \quad \mathrm{y} \quad \beta=\frac{\mathrm{c}_{\mathrm{c} 0}}{\mathrm{c}_{\mathrm{c} 1}}
$$

(donde, si se considera que los microorganismos en la corriente de recirculación están más concentrados que en la corriente de salida del biorreactor, entonces $\beta>1$ ) fácilmente se llega a la ecuación de diseño:

$$
\mathrm{D}=\frac{\mu}{1-\alpha \cdot(\beta-1)}
$$

$\mathrm{Si}$, como se ha indicado, $\beta>1$ entonces $\mathrm{D}>\mu$. Es decir, con la misma $\mu$ la recirculación permite tratar más alimento por unidad de tiempo y de volumen que sin recirculación. Así si:

$$
\mathrm{D}_{\text {sin recirculación }}=\frac{\mathrm{V}}{\mathrm{Q}_{\mathrm{V} 0}}=\mu
$$


entonces:

$$
\mathrm{D}_{\text {con recirculación }}=\frac{\mathrm{D}_{\text {sin recirculación }}}{1-\alpha \cdot(\beta-1)}
$$

Ejercicio 5. Demuéstrese que, si se cumple la cinética de Monod, la recirculación de células aumenta el caudal de lavado en la cantidad $[1-\alpha \cdot(\beta-1)]$.

La fracción de células recirculadas se puede calcular mediante la expresión:

$$
\frac{\mathrm{Q}_{\mathrm{vr}} \cdot \mathrm{c}_{\mathrm{c} 0}}{\left(\mathrm{Q}_{\mathrm{v} 0}+\mathrm{Q}_{\mathrm{vr}}\right) \cdot \mathrm{c}_{\mathrm{c} 1}}=\frac{\mathrm{Q}_{\mathrm{vr}} / \mathrm{Q}_{\mathrm{v} 0}}{\left(1+\mathrm{Q}_{\mathrm{vr}} / \mathrm{Q}_{\mathrm{v} 0}\right)} \cdot \frac{\mathrm{c}_{\mathrm{c} 0}}{\mathrm{c}_{\mathrm{c} 1}}=\frac{\alpha}{1+\alpha} \cdot \beta
$$

Ejercicio 6. A partir del balance de células a la centrífuga:

$$
\left(\mathrm{Q}_{\mathrm{v} 0}+\mathrm{Q}_{\mathrm{Vr}}\right) \cdot \mathrm{c}_{\mathrm{c} 1}=\mathrm{Q}_{\mathrm{v} 0} \cdot \mathrm{c}_{\mathrm{c}}+\mathrm{Q}_{\mathrm{vr}} \cdot \mathrm{c}_{\mathrm{c} 0}
$$

demuéstrese que $\mathrm{c}_{\mathrm{c}}=\mathrm{c}_{\mathrm{cl}} \cdot[1-\alpha \cdot(\beta-1)]$.

Ejercicio 7. A partir del balance de células al FCTA:

$$
\mathrm{Q}_{\mathrm{vr}} \cdot \mathrm{c}_{\mathrm{c} 0}-\left(\mathrm{Q}_{\mathrm{v} 0}+\mathrm{Q}_{\mathrm{Vr}}\right) \cdot \mathrm{c}_{\mathrm{c} 1}+\mu \cdot \mathrm{c}_{\mathrm{cl}} \cdot \mathrm{V}=0
$$

y teniendo en cuenta que $\mu=\mu_{\max } \cdot \frac{\mathrm{c}_{\mathrm{s} 1}}{\mathrm{~K}_{\mathrm{s}}+\mathrm{c}_{\mathrm{s} 1}}$ demuéstrese que:

$$
\mathrm{c}_{\mathrm{s} 1}=\frac{\mathrm{K}_{\mathrm{s}} \cdot \mathrm{D} \cdot[1-\alpha \cdot(\beta-1)]}{\mu_{\max }-\mathrm{D} \cdot[1-\alpha \cdot(\beta-1)]}
$$

\subsection{Balance de materia de fermentadores continuos tubulares de flujo de pistón (FFP) con flóculos en suspensión}

Los fermentadores continuos tubulares de flujo de pistón suelen funcionar en régimen estacionario, de manera que los caudales volumétricos de entrada y de salida son iguales, y la corriente de entrada siempre tiene que contener células vivas (no estéril). 
El parámetro D, utilizado en los fermentadores continuos de tanque agitado, también se aplica a los fermentadores continuos tubulares de flujo de pistón manteniendo la misma definición:

$\mathrm{D}=\frac{\mathrm{Q}_{\mathrm{v} 0}}{\mathrm{~V}} \quad\left(=\mathrm{S}=\frac{1}{\tau} \quad\right.$; véase los conceptos de velocidad espacial y tiempo espacial definidos para un RCTA $)$

El balance de materia en un Fermentador Continuo Tubular de Flujo de Pistón (FFP) con flóculos en suspensión se puede aplicar

a las células: $\mathrm{Q}_{\mathrm{V} 0} \cdot \mathrm{dc}_{\mathrm{c}}=\mathrm{r}_{\mathrm{c}} \cdot \mathrm{dV}$

al sustrato: $\quad \mathrm{Q}_{\mathrm{V} 0} \cdot \mathrm{dc}_{\mathrm{s}}=\mathrm{r}_{\mathrm{s}} \cdot \mathrm{dV}$

al producto: $\mathrm{Q}_{\mathrm{V} 0} \cdot \mathrm{dc}_{\mathrm{p}}=\mathrm{r}_{\mathrm{p}} \cdot \mathrm{dV}$

\subsubsection{Fermentador continuo tubular de flujo de pistón (FFP) con flóculos en suspensión, en régimen estacionario}

Al tratarse de un proceso con envenenamiento por producto la ecuación de velocidad tipo Monod a aplicar es (apartado 8.5.1):

$$
\mathrm{r}_{\mathrm{c}}=\mu \cdot \mathrm{c}_{\mathrm{c}}=\mu_{\max } \cdot \frac{\mathrm{c}_{\mathrm{s}}}{\mathrm{K}_{\mathrm{s}}+\mathrm{c}_{\mathrm{s}}} \cdot \mathrm{c}_{\mathrm{c}}
$$

i) Balance de materia a las células

Para la fase de crecimiento y admitiendo una cinética de Monod, el balance de materia a las células queda como sigue:

$$
\begin{gathered}
\int_{c_{c 0}}^{c_{c}} \frac{d_{c}}{r_{c}}=\int_{0}^{v} \frac{d V}{Q_{V}}=\frac{V}{Q_{V 0}}=\frac{1}{D}=\theta=\tau \\
\tau \cdot \mu_{\max }=\frac{\mu_{\max }}{D}=\int_{c_{c 0}}^{c_{c}} \frac{\left(K_{s}+c_{s}\right)}{c_{s}} \cdot \frac{d_{c}}{c_{c}}
\end{gathered}
$$

$\mathrm{y}$ considerando la relación estequiométrica entre $\mathrm{c}_{\mathrm{c}} \mathrm{y} \mathrm{c}_{\mathrm{s}}$ :

$$
\begin{gathered}
\mathrm{c}_{\mathrm{c}}-\mathrm{c}_{\mathrm{c} 0}=\mathrm{Y}_{\mathrm{c} / \mathrm{s}} \cdot\left(\mathrm{c}_{\mathrm{s} 0}-\mathrm{c}_{\mathrm{s}}\right) \rightarrow \mathrm{c}_{\mathrm{s}}=\mathrm{c}_{\mathrm{s} 0}+\mathrm{Y}_{\mathrm{s} / \mathrm{c}} \cdot \mathrm{c}_{\mathrm{c} 0}-\mathrm{Y}_{\mathrm{s} / \mathrm{c}} \cdot \mathrm{c}_{\mathrm{c}} \\
\tau \cdot \mu_{\max }=\frac{\mu_{\max }}{\mathrm{D}}=\int_{\mathrm{c}_{\mathrm{c} 0}}^{\mathrm{c}_{\mathrm{c}}} \frac{\left(\mathrm{K}_{\mathrm{s}}+\mathrm{c}_{\mathrm{s}}\right)}{\mathrm{c}_{\mathrm{s}}} \cdot \frac{\mathrm{dc} \mathrm{c}_{\mathrm{c}}}{\mathrm{c}_{\mathrm{c}}}=\int_{\mathrm{c}_{\mathrm{c} 0}}^{\mathrm{c}_{\mathrm{c}}} \frac{\mathrm{K}_{\mathrm{s}}}{\mathrm{c}_{\mathrm{s}} \cdot \mathrm{c}_{\mathrm{c}}} \cdot \mathrm{dc}_{\mathrm{c}}+\int_{\mathrm{c}_{\mathrm{c} 0}}^{\mathrm{c}_{\mathrm{c}}} \frac{\mathrm{dc}_{\mathrm{c}}}{\mathrm{c}_{\mathrm{c}}} \\
\tau \cdot \mu_{\max }=\frac{\mu_{\max }}{\mathrm{D}}=\int_{\mathrm{c}_{\mathrm{c} 0}}^{\mathrm{c}_{\mathrm{c}}} \frac{\mathrm{K}_{\mathrm{s}}}{\left(\mathrm{c}_{\mathrm{s} 0}+\mathrm{Y}_{\mathrm{s} / \mathrm{c}} \cdot \mathrm{c}_{\mathrm{c} 0}-\mathrm{Y}_{\mathrm{s} / \mathrm{c}} \cdot \mathrm{c}_{\mathrm{c}}\right) \cdot \mathrm{c}_{\mathrm{c}}} \cdot \mathrm{dc} \mathrm{c}_{\mathrm{c}}+\ln \frac{\mathrm{c}_{\mathrm{c}}}{\mathrm{c}_{\mathrm{c} 0}}
\end{gathered}
$$


cuya solución es la ecuación de diseño:

$$
\begin{aligned}
& \tau \cdot \mu_{\max }=\frac{\mu_{\max }}{\mathrm{D}}=\left(1+\frac{\mathrm{K}_{\mathrm{s}}}{\mathrm{c}_{\mathrm{s} 0}+\mathrm{Y}_{\mathrm{s} / \mathrm{c}} \cdot \mathrm{c}_{\mathrm{c} 0}}\right) \cdot \ln \frac{\mathrm{c}_{\mathrm{c}}}{\mathrm{c}_{\mathrm{c} 0}}-\frac{\mathrm{K}_{\mathrm{s}}}{\mathrm{c}_{\mathrm{s} 0}+\mathrm{Y}_{\mathrm{s} / \mathrm{c}} \cdot \mathrm{c}_{\mathrm{c} 0}} \cdot \ln \left(\frac{\mathrm{c}_{\mathrm{s} 0}+\mathrm{Y}_{\mathrm{s} / \mathrm{c}} \cdot \mathrm{c}_{\mathrm{c} 0}-\mathrm{Y}_{\mathrm{s} / \mathrm{c}} \cdot \mathrm{c}_{\mathrm{c}}}{\mathrm{c}_{\mathrm{s} 0}+\mathrm{Y}_{\mathrm{s} / \mathrm{c}} \cdot \mathrm{c}_{\mathrm{c} 0}-\mathrm{Y}_{\mathrm{s} / \mathrm{c}} \cdot \mathrm{c}_{\mathrm{c} 0}}\right) \\
& \tau \cdot \mu_{\max }=\frac{\mu_{\max }}{\mathrm{D}}=\left(1+\frac{\mathrm{K}_{\mathrm{s}}}{\mathrm{c}_{\mathrm{s} 0}+\mathrm{Y}_{\mathrm{s} / \mathrm{c}} \cdot \mathrm{c}_{\mathrm{c} 0}}\right) \cdot \ln \frac{\mathrm{c}_{\mathrm{c}}}{\mathrm{c}_{\mathrm{c} 0}}-\frac{\mathrm{K}_{\mathrm{s}}}{\mathrm{c}_{\mathrm{s} 0}+\mathrm{Y}_{\mathrm{s} / \mathrm{c}} \cdot \mathrm{c}_{\mathrm{c} 0}} \cdot \ln \frac{\mathrm{c}_{\mathrm{s}}}{\mathrm{c}_{\mathrm{s} 0}}
\end{aligned}
$$

Ejercicio 8. Resuélvase la integral racional anterior comprobando que se llega a la solución indicada.

Esta expresión sería análoga para un FDTA isotermo simplemente sustituyendo el tiempo espacial $(\mathrm{t})$ por el tiempo real $(\mathrm{t})$.

Se pueden extrapolar dos casos particulares:

1. Caso en que $c_{s}>>>K_{s}$

La ecuación de diseño es:

$$
\tau \cdot \mu_{\max }=\frac{\mu_{\max }}{D}=\ln \frac{\mathrm{c}_{\mathrm{c}}}{\mathrm{c}_{\mathrm{c} 0}}
$$

\section{Caso en que $c_{s}<<<K_{s}$}

La ecuación de diseño es:

$$
\tau \cdot \mu_{\max }=\frac{\mu_{\max }}{\mathrm{D}}=\frac{\mathrm{K}_{\mathrm{s}}}{\mathrm{c}_{\mathrm{s} 0}+\mathrm{Y}_{\mathrm{s} / \mathrm{c}} \cdot \mathrm{c}_{\mathrm{c} 0}} \cdot \ln \left(\frac{\mathrm{c}_{\mathrm{c}}}{\mathrm{c}_{\mathrm{c} 0}} \cdot \frac{\mathrm{c}_{\mathrm{s} 0}}{\mathrm{c}_{\mathrm{s}}}\right)
$$

Ejercicio 9. Obténgase esta ecuación a partir de la ecuación de velocidad de Monod, considerando la aproximación: $\mathrm{r}_{\mathrm{c}}=\mu \cdot \mathrm{c}_{\mathrm{c}}=\mu_{\max } \cdot \frac{\mathrm{c}_{\mathrm{s}}}{\mathrm{K}_{\mathrm{s}}+\mathrm{c}_{\mathrm{s}}} \cdot \mathrm{c}_{\mathrm{c}} \approx \mu_{\max } \cdot \frac{\mathrm{c}_{\mathrm{s}} \cdot \mathrm{c}_{\mathrm{c}}}{\mathrm{K}_{\mathrm{s}}}$.

ii) Balance de materia al sustrato

Si se aplica el balance de materia al sustrato:

$$
\int_{\mathrm{c}_{\mathrm{s} 0}}^{\mathrm{c}_{\mathrm{s}}} \frac{\mathrm{dc}_{\mathrm{s}}}{\mathrm{r}_{\mathrm{s}}}=\int_{0}^{\mathrm{V}} \frac{\mathrm{dV}}{\mathrm{Q}_{\mathrm{V} 0}}=\frac{\mathrm{V}}{\mathrm{Q}_{\mathrm{V} 0}}=\frac{1}{\mathrm{D}}=\theta=\tau
$$


y considerando que la velocidad de desaparición del sustrato en la fase de crecimiento responde a la cinética:

$$
\begin{gathered}
\mathrm{r}_{\mathrm{s}}=\mathrm{Y}_{\mathrm{s} / \mathrm{c}} \cdot \mathrm{r}_{\mathrm{c}}+\mathrm{k}_{\mathrm{sm}} \cdot \mathrm{c}_{\mathrm{c}} \approx \mathrm{Y}_{\mathrm{s} / \mathrm{c}} \cdot \mathrm{r}_{\mathrm{c}} \\
\tau=\frac{1}{\mathrm{D}}=\int_{\mathrm{c}_{\mathrm{s} 0}}^{\mathrm{c}_{\mathrm{s}}} \frac{\mathrm{dc}_{\mathrm{s}}}{\mathrm{Y}_{\mathrm{s} / \mathrm{c}} \cdot \mathrm{r}_{\mathrm{c}}}
\end{gathered}
$$

\subsubsection{Fermentador continuo tubular de flujo de pistón (FFP) con flóculos en suspensión, en régimen estacionario, con envenenamiento por producto}

Al tratarse de un proceso con envenenamiento por producto la ecuación de velocidad tipo Monod a aplicar es (apartado 8.5.1):

$$
\mathrm{r}_{\mathrm{c}}=\mu_{\max } \cdot \frac{\mathrm{c}_{\mathrm{s}}}{\mathrm{K}_{\mathrm{s}}+\mathrm{c}_{\mathrm{s}}} \cdot \mathrm{c}_{\mathrm{c}} \cdot\left(1-\frac{\mathrm{c}_{\mathrm{p}}}{\mathrm{c}_{\mathrm{p}}^{*}}\right)^{\mathrm{n}}
$$

Se tratará a continuación el caso más sencillo en que $n=1$ y $c_{s}>>K_{s}$ quedando la ecuación de velocidad como:

$$
\mathrm{r}_{\mathrm{c}} \approx \mu_{\max } \cdot\left(1-\frac{\mathrm{c}_{\mathrm{p}}}{\mathrm{c}_{\mathrm{p}}^{*}}\right) \cdot \mathrm{c}_{\mathrm{c}}
$$

i) Balance de materia a las células

El balance de materia a las células queda como:

$$
\begin{gathered}
\int_{c_{c 0}}^{c_{c}} \frac{d_{c}}{r_{c}}=\int_{0}^{v} \frac{d V}{Q_{V 0}}=\frac{V}{Q_{V 0}}=\frac{1}{D}=\theta=\tau \\
\tau \cdot \mu_{\max }=\frac{\mu_{\max }}{D}=\int_{c_{c 0}}^{c_{c}} \frac{d_{c}}{\left(1-\frac{c_{p}}{c_{p}^{*}}\right) \cdot c_{c}}
\end{gathered}
$$

ii) Balance de materia al producto

Si se aplica el balance de materia al producto:

$$
\int_{c_{p} 0}^{c_{p}} \frac{d_{p}}{r_{p}}=\int_{0}^{V} \frac{d V}{Q_{V 0}}=\frac{V}{Q_{V 0}}=\frac{1}{D}=\theta=\tau
$$


y considerando que la velocidad de formación del producto en la fase de crecimiento responde a la cinética:

$$
\begin{gathered}
r_{p}=Y_{p / c} \cdot r_{c} \\
\tau=\frac{1}{D}=\int_{c_{p 0}}^{c_{p}} \frac{d_{p}}{Y_{p / c} \cdot r_{c}}
\end{gathered}
$$

donde teniendo en consideración la expresión anteriormente indicada para $\mathrm{r}_{\mathrm{c}} \mathrm{y}$ la relación estequiométrica:

$$
\begin{gathered}
c_{p}-c_{p 0}=Y_{p / c} \cdot\left(c_{c}-c_{c 0}\right) \rightarrow Y_{p / c} \cdot c_{c}=c_{p}-c_{p 0}+Y_{p / c} \cdot c_{c 0} \\
\tau \cdot \mu_{\max }=\frac{\mu_{\max }}{D}=\int_{c_{p 0}}^{c_{p}} \frac{d c_{p}}{Y_{p / c} \cdot\left(1-\frac{c_{p}}{c_{p}^{*}}\right) \cdot c_{c}} \\
\tau \cdot \mu_{\max }=\frac{\mu_{\max }}{D}=\int_{c_{p 0}}^{c_{p}} \frac{d c_{p}}{\left.1-\frac{c_{p}}{c_{p}^{*}}\right) \cdot\left(c_{p}-c_{p 0}+Y_{p / c} \cdot c_{c 0}\right)}
\end{gathered}
$$

cuya solución es la ecuación de diseño:

$$
\begin{aligned}
& \tau \cdot \mu_{\max }=\frac{\mu_{\max }}{D}=\left(\frac{c_{p}^{*}}{c_{p}^{*}-c_{p 0}+Y_{p / c} \cdot c_{c 0}}\right) \cdot \ln \frac{c_{p}^{*}-c_{p 0}}{c_{p}^{*}-c_{p}}+\left(\frac{c_{p}^{*}}{c_{p}^{*}-c_{p 0}+Y_{p / c} \cdot c_{c 0}}\right) \cdot \ln \left(\frac{c_{p}-c_{p 0}+Y_{p / c} \cdot c_{c 0}}{c_{p 0}-c_{p 0}+Y_{p / c} \cdot c_{c 0}}\right) \\
& \tau \cdot \mu_{\max }=\frac{\mu_{\max }}{D}=\left(\frac{c_{p}^{*}}{c_{p}^{*}-c_{p 0}+Y_{p / c} \cdot c_{c 0}}\right) \cdot \ln \frac{\left(c_{p}^{*}-c_{p 0}\right) \cdot c_{c}}{\left(c_{p}^{*}-c_{p}\right) c_{c 0}}
\end{aligned}
$$

Ejercicio 10. Resuélvase la integral racional anterior comprobando que se llega a la solución indicada. 


\section{ANEXO I \\ CINÉTICA ENZIMÁTICA}

Una reacción catalizada por un enzima responde a un esquema de reacción global del tipo:

$$
\mathrm{S} \stackrel{\mathrm{E}}{\longrightarrow} \mathrm{P}
$$

donde el sustrato $\mathrm{S}$ se transforma en el producto $\mathrm{P}$ gracias a la actividad catalítica del enzima $\mathrm{E}$.

Se supondrá que la reacción global considerada responde a un mecanismo de reacción en el que, en primer lugar, el enzima forma un complejo intermedio (SE)* con el sustrato $\mathrm{S}$ según la reacción reversible:

$$
\mathrm{S}+\mathrm{E} \leftrightarrow(\mathrm{SE})^{*} \quad \mathrm{i}=1
$$

y que, posteriormente, este complejo se descompone irreversiblemente en el producto P y el enzima original:

$$
(\mathrm{SE})^{*} \rightarrow \mathrm{P}+\mathrm{E} \quad \mathrm{i}=2
$$

Las velocidades a las que transcurren ambas reacciones elementales son de la forma:

$$
\begin{aligned}
& \mathrm{r}_{1}=\mathrm{k}_{1} \cdot \mathrm{c}_{\mathrm{s}} \cdot \mathrm{c}_{\mathrm{E}}-\mathrm{k}_{1}^{\prime} \cdot \mathrm{c}_{\mathrm{sE}} \\
& \mathrm{r}_{2}=\mathrm{k}_{2} \cdot \mathrm{c}_{\mathrm{sE}}
\end{aligned}
$$

En el supuesto de que las reacciones se desarrollen en un RDTA isotermo, puede escribirse (aplicando la ecuación de conservación de materia):

$$
\begin{gathered}
\frac{\mathrm{dc}_{\mathrm{s}}}{\mathrm{dt}}=-\mathrm{k}_{1} \cdot \mathrm{c}_{\mathrm{s}} \cdot \mathrm{c}_{\mathrm{E}}+\mathrm{k}_{1}^{\prime} \cdot \mathrm{c}_{\mathrm{sE}} \\
\frac{\mathrm{dc}_{\mathrm{sE}}}{\mathrm{dt}}=\mathrm{k}_{1} \cdot \mathrm{c}_{\mathrm{s}} \cdot \mathrm{c}_{\mathrm{E}}-\left(\mathrm{k}_{1}^{\prime}+\mathrm{k}_{2}\right) \cdot \mathrm{c}_{\mathrm{sE}} \\
\frac{\mathrm{dc}_{\mathrm{E}}}{\mathrm{dt}}=-\mathrm{k}_{1} \cdot \mathrm{c}_{\mathrm{s}} \cdot \mathrm{c}_{\mathrm{E}}+\left(\mathrm{k}_{1}^{\prime}+\mathrm{k}_{2}\right) \cdot \mathrm{c}_{\mathrm{sE}}
\end{gathered}
$$

obsérvese que $\mathrm{d}\left(\mathrm{c}_{\mathrm{E}}+\mathrm{c}_{\mathrm{sE}}\right) / \mathrm{dt}=0$, lo que significa que la suma de las concentraciones $\left(\mathrm{c}_{\mathrm{E}}+\mathrm{c}_{\mathrm{AE}}\right)$ es constante para cualquier valor de $\mathrm{t} y$, por tanto, igual a su valor inicial $\mathrm{c}_{\mathrm{E} 0}$ si se admite que, al comienzo de la reacción, $\mathrm{c}_{\mathrm{s}}=\mathrm{c}_{\mathrm{s} 0}, \mathrm{c}_{\mathrm{p}}=\mathrm{c}_{\mathrm{sE}}=0, \mathrm{y}_{\mathrm{E}}=$ $\mathrm{c}_{\mathrm{E} 0}$. 
Sustituyendo $\mathrm{c}_{\mathrm{E}}=\mathrm{c}_{\mathrm{E} 0}-\mathrm{c}_{\mathrm{sE}}$ en la primera y la segunda de las tres ecuaciones diferenciales anteriores, resulta:

$$
\begin{gathered}
\frac{\mathrm{dc}_{\mathrm{s}}}{\mathrm{dt}}=-\mathrm{k}_{1} \cdot \mathrm{c}_{\mathrm{s}} \cdot \mathrm{c}_{\mathrm{E} 0}+\left(\mathrm{k}_{1}^{\prime}+\mathrm{k}_{1} \cdot \mathrm{c}_{\mathrm{s}}\right) \cdot \mathrm{c}_{\mathrm{sE}} \\
\frac{\mathrm{dc}_{\mathrm{sE}}}{\mathrm{dt}}=-\mathrm{k}_{1} \cdot \mathrm{c}_{\mathrm{s}} \cdot \mathrm{c}_{\mathrm{E} 0}+\left(\mathrm{k}_{1}^{\prime}+\mathrm{k}_{1} \cdot \mathrm{c}_{\mathrm{s}}+\mathrm{k}_{2}\right) \cdot \mathrm{c}_{\mathrm{sE}}
\end{gathered}
$$

Se trata de dos ecuaciones diferenciales no lineales, para las que no puede darse una solución analítica sencilla. No obstante, ambas ecuaciones pueden reducirse a una sola si se admite la hipótesis del estado pseudoestacionario. Dicha hipótesis consiste en admitir que, cuando $\mathrm{c}_{\mathrm{E} 0}$ es mucho más pequeña que $\mathrm{c}_{\mathrm{s} 0}, \mathrm{c}_{\mathrm{sE}}$ nunca es muy grande y varía muy lentamente, de modo que puede aceptarse que $\mathrm{dc}_{\mathrm{sE}} / \mathrm{dt}=0$. Haciendo $\mathrm{dc}_{\mathrm{sE}} / \mathrm{dt}=0$ se puede obtener de la segunda ecuación diferencial:

$$
\mathrm{c}_{\mathrm{sE}}=\frac{\mathrm{k}_{1} \cdot \mathrm{c}_{\mathrm{s}} \cdot \mathrm{c}_{\mathrm{E} 0}}{\mathrm{k}_{1}^{\prime}+\mathrm{k}_{1} \cdot \mathrm{c}_{\mathrm{s}}+\mathrm{k}_{2}}
$$

valor que, sustituido en la primera de las dos últimas ecuaciones diferenciales, permite su resolución como sigue:

$$
\frac{\mathrm{dc}_{\mathrm{s}}}{\mathrm{dt}}=-\mathrm{k}_{1} \cdot \mathrm{c}_{\mathrm{s}} \cdot \mathrm{c}_{\mathrm{E} 0}+\left(\mathrm{k}_{1}^{\prime}+\mathrm{k}_{1} \cdot \mathrm{c}_{\mathrm{s}}\right) \cdot \mathrm{c}_{\mathrm{sE}}=-\mathrm{k}_{1} \cdot \mathrm{c}_{\mathrm{s}} \cdot \mathrm{c}_{\mathrm{E} 0}+\left(\mathrm{k}_{1}^{\prime}+\mathrm{k}_{1} \cdot \mathrm{c}_{\mathrm{s}}\right) \cdot \frac{\mathrm{k}_{1} \cdot \mathrm{c}_{\mathrm{s}} \cdot \mathrm{c}_{\mathrm{E} 0}}{\mathrm{k}_{1}^{\prime}+\mathrm{k}_{1} \cdot \mathrm{c}_{\mathrm{s}}+\mathrm{k}_{2}}
$$

y, reordenando términos se llega a:

$$
\frac{\mathrm{dc}_{\mathrm{s}}}{\mathrm{dt}}=-\frac{\mathrm{k}_{2} \cdot \mathrm{c}_{\mathrm{E} 0} \cdot \mathrm{c}_{\mathrm{s}}}{\mathrm{c}_{\mathrm{s}}+\frac{\mathrm{k}_{1}^{\prime}+\mathrm{k}_{2}}{\mathrm{k}_{1}}}=-\frac{\mathrm{k} \cdot \mathrm{c}_{\mathrm{s}}}{\mathrm{c}_{\mathrm{s}}+\mathrm{K}_{\mathrm{m}}}
$$

y:

$$
\mathrm{r}=\frac{\mathrm{k} \cdot \mathrm{c}_{\mathrm{s}}}{\mathrm{c}_{\mathrm{s}}+\mathrm{K}_{\mathrm{m}}}
$$

siendo $\mathrm{K}_{\mathrm{m}}$ la constante de Michaelis. Por consiguiente puede afirmarse que se cumple la estequiometría de la reacción global $\mathrm{S} \rightarrow \mathrm{P}$, pero la ecuación de velocidad es una función racional de la concentración de $\mathrm{S}\left(\mathrm{c}_{\mathrm{s}}\right)$. 


\section{PROBLEMAS}

Problema 01. Bender and Marshall han estudiado la hidrólisis del p-nitrofenol por la acción de la enzima elastasa. Estos autores han propuesto el siguiente mecanismo para la citada reacción:

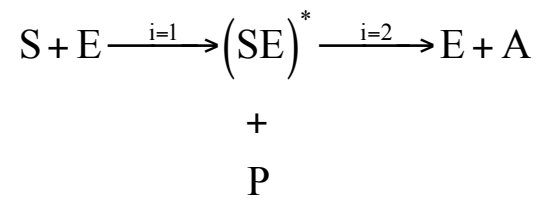

siendo $\mathrm{P}$ el producto y $\mathrm{A}$ el ácido trimetilacético.

Deducir una ecuación de velocidad para la producción de $\mathrm{P}$ en función de $\mathrm{k}_{1}, \mathrm{k}_{2}, \mathrm{c}_{\mathrm{E} 0}$ (concentración total de elastasa en las dos formas, $\mathrm{E}$ y $(\mathrm{SE})^{*}$ ) y $\mathrm{c}_{\mathrm{S}}$, concentración instantánea de sustrato.

Badger Chemical Company está investigando la producción del p-nitrofenol mediante este proceso en un tanque agitado continuo con un volumen de $0.3 \mathrm{~m}^{3}$. Si se ha de convertir el $90 \%$ del sustrato en producto, determinar el caudal de alimentación que puede tratarse cuando las concentraciones de elastasa y sustrato sean de 10 y 100 moles $/ \mathrm{m}^{3}$ respectivamente.

\section{Datos y notas}

$\mathrm{k}_{1}=150 \mathrm{~m}^{3} / \mathrm{mol} \cdot \mathrm{ks}$

$\mathrm{k}_{2}=2.60 \mathrm{ks}^{-1}$

Problema 02. En los últimos años se ha incrementado la utilización de enzimas como catalizadores. El proceso implica la fijación de éstas en un soporte sólido y la disposición del mismo en un tubo por el que circula un líquido. Se ha pensado el tratamiento de una solución acuosa de lactosa para convertirla en glucosa y galactosa, lo que permitiría la transformación de residuos de la industria de quesos en productos útiles. La velocidad de reacción para este tipo de procesos, por unidad de volumen de lecho, puede escribirse como:

$$
\mathrm{r}_{\mathrm{s}}=\frac{\mathrm{k} \cdot \mathrm{c}_{\mathrm{s}}}{\mathrm{c}_{\mathrm{s}}+\mathrm{K}_{\mathrm{M}} \cdot\left(1+\frac{\mathrm{c}_{\mathrm{p} 1}}{\mathrm{~K}}\right)}
$$

para una reacción del tipo $\mathrm{S} \rightarrow \mathrm{P}_{1}+\mathrm{P}_{2}$.

A $40{ }^{\circ} \mathrm{C}, \mathrm{K}_{\mathrm{M}}=0.0528 \mathrm{~mol} / \mathrm{l}, \mathrm{K}=0.0054 \mathrm{~mol} / 1 \mathrm{y} \mathrm{k}=5.53 \mathrm{~mol} / \mathrm{l} \cdot \mathrm{min}$. Si la concentración inicial de lactosa es de $0.149 \mathrm{~mol} / \mathrm{l}$, calcular el tiempo espacial de un reactor tubular necesario para alcanzar una conversión de lactosa del $80 \%$. 
Problema 03. El crecimiento de un cultivo de la bacteria Escherichia coli en un substrato de manitol puede representarse por la ecuación de velocidad:

$$
\mathrm{r}_{\mathrm{c}}=\frac{1.2 \cdot \mathrm{c}_{\mathrm{s}} \cdot \mathrm{c}_{\mathrm{c}}}{2+\mathrm{c}_{\mathrm{s}}} \quad\left(\frac{\mathrm{g} \text { de células }}{\mathrm{m}^{3} \cdot \mathrm{h}}\right)
$$

siendo $\mathrm{c}_{\mathrm{s}}$ los gramos de manitol por $\mathrm{m}^{3}$. El coeficiente estequiométrico $\mathrm{Y}_{\mathrm{c} / \mathrm{s}}$ vale 0.1 $\mathrm{g}$ de células por g manitol.

1. Determinar la concentración de salida de las células producidas al alimentar un FCTA de $1 \mathrm{~m}^{3}$ de volumen con una corriente de $1 \mathrm{~m}^{3} / \mathrm{h}$ de solución de manitol $\left(\mathrm{c}_{\mathrm{s} 0}=6 \mathrm{~g} / \mathrm{m}^{3}\right)$.

2. Calcular la velocidad de dilución de lavado.

3. Determinar la concentración de salida de las células si el FCTA fuera de $5 \mathrm{~m}^{3}$.

Problema 04. El crecimiento del microbio Escherichia coli en glucosa sigue la cinética de Monod de acuerdo con la expresión:

$$
\mathrm{r}_{\mathrm{c}}=\frac{1.333 \cdot \mathrm{c}_{\mathrm{s}} \cdot \mathrm{c}_{\mathrm{c}}}{4+\mathrm{c}_{\mathrm{s}}}\left(\frac{\mathrm{g} \text { de células }}{\mathrm{m}^{3} \cdot \mathrm{h}}\right)
$$

siendo $\mathrm{c}_{\mathrm{s}}$ los gramos de glucosa por $\mathrm{m}^{3}$. El coeficiente estequiométrico $\mathrm{Y}_{\mathrm{c} / \mathrm{s}}$ vale 0.1 $\mathrm{g}$ de células por g glucosa.

Determinar el caudal de alimentación de una solución de glucosa $\left(\mathrm{c}_{\mathrm{s} 0}=60 \mathrm{~g} / \mathrm{m}^{3}\right)$ a un FCTA de $1 \mathrm{~m}^{3}$ que daría una velocidad máxima de consumo de glucosa y una velocidad máxima de producción de células. Determinar también esta velocidad máxima de producción de células.

Problema 05. Un substrato A se convierte por fermentación en un alcohol (P) según la siguiente cinética, en la que se ha supuesto que el producto es el único factor limitante:

$$
\mathrm{r}_{\mathrm{p}}=\mu \cdot\left(1-\frac{\mathrm{c}_{\mathrm{p}}}{\mathrm{c}_{\mathrm{p}}^{*}}\right)^{\mathrm{n}} \cdot \mathrm{c}_{\mathrm{c}}
$$

Donde $\mu=1.73 \mathrm{~h}^{-1}, \mathrm{n}=0$ en verano, $\mathrm{n}=1$ en primavera, $\mathrm{n}=2$ en invierno, $\mathrm{c}_{\mathrm{p}}{ }^{*}=$ $0.12 \mathrm{~g}$ de alcohol por $\mathrm{g}$ de solución y $\rho=1000 \mathrm{~kg} / \mathrm{m}^{3}$. Calcular la cantidad máxima de alcohol que se podrá producir en cada una de les estaciones ( $\mathrm{kg} /$ día $)$ en un FCTA comercial de $30 \mathrm{~m}^{3}$ para un caudal de alimentación arbitraria. 
Problema 06. Producir glucosa en un FCTA a escala piloto de 501 se utiliza papel triturado que se ataca con la ayuda de un microbio. En presencia de un exceso de papel triturado (materia incompresible), la glucosa producida resulta ser el factor limitante de la conversión. Los resultados obtenidos son los siguientes:

\begin{tabular}{|c|c|}
\hline $\mathrm{Q}_{\mathrm{V} 0}(\mathrm{l} / \mathrm{h})$ & $\mathrm{c}_{\mathrm{p}}(\mathrm{mmol} / \mathrm{l})$ \\
\hline 16 & 54 \\
\hline 4 & 75 \\
\hline 0 & 90 \\
\hline
\end{tabular}

Determinar el caudal necesario para maximizar la producción de glucosa y encontrar su velocidad de producción.

Problema 07. El crecimiento de una bacteria sigue el modelo cinético siguiente:

$$
\mathrm{r}_{\mathrm{c}}=\mu_{\max } \cdot\left(1-\frac{\mathrm{c}_{\mathrm{c}}}{\mathrm{c}_{\mathrm{c}}^{*}}\right) \cdot \mathrm{c}_{\mathrm{c}}
$$

siendo $\mu_{\max }=0.5 \mathrm{~h}^{-1} \mathrm{y} \mathrm{c}_{\mathrm{c}}{ }^{*}=20 \mathrm{~g} / 1$ y encontrándose es sustrato en exceso.

1. Deducir la velocidad de dilución de lavado de un quimiostato con alimentación estéril.

2. ¿Cuál sería la concentración de biomasa si la velocidad de dilución fuese 0.4 $\mathrm{h}^{-1}$ ?

3. En caso de hacer funcionar el reactor anterior de manera discontinua, determinar el tiempo necesario para alcanzar una concentración celular de $10 \mathrm{~g} / 1$ si la concentración de inoculo fuera de $0.4 \mathrm{~g}$ de células (despreciar la fase de adaptación o iniciación de las células).

Problema 08. La fermentación de glucosa a etanol se lleva a cabo en un RDTA utilizando el organismo Saccharomyces cerevisiae. Represéntese las concentraciones de células, sustrato y producto así como la velocidad de crecimiento celular en función del tiempo. La concentración celular inicial es $1 \mathrm{~kg} / \mathrm{m}^{3}$ y la concentración de sustrato (glucosa) de $250 \mathrm{~kg} / \mathrm{m}^{3}$.

\section{Datos y notas}

$\mathrm{Y}_{\mathrm{c} / \mathrm{s}}=0.08 \mathrm{~kg} / \mathrm{kg} ; \mathrm{Y}_{\mathrm{p} / \mathrm{s}}=0.45 \mathrm{~kg} / \mathrm{kg} ; \mathrm{Y}_{\mathrm{p} / \mathrm{c}}=5.60 \mathrm{~kg} / \mathrm{kg}$

$\mathrm{c}_{\mathrm{p}}{ }^{*}=93 \mathrm{~kg} / \mathrm{m}^{3}$

$\mathrm{n}=0.52 ; \mathrm{m}=0.03 \mathrm{~kg}$ de sustrato $/ \mathrm{kg}$ de células $\cdot \mathrm{h}$

$\mu_{\max }=0.33 \mathrm{~h}^{-1} ; \mathrm{K}_{\mathrm{s}}=1.7 \mathrm{~kg} / \mathrm{m}^{3} ; \mathrm{k}_{\mathrm{m}}=0.01 \mathrm{~h}^{-1}$ 
Problema 09. A partir del problema anterior se pueden extraer un gran número de hechos interesantes cuando se modifica el valor de parámetros como $c_{p}^{*}, \mathrm{Y}_{\mathrm{p} / \mathrm{s}}, \mathrm{Y}_{\mathrm{c} / \mathrm{s}}$, $\mathrm{c}_{\mathrm{s} 0} \mathrm{y} \mathrm{c}_{\mathrm{c} 0}$. Describa los resultados que se obtienen.

Problema 10. El crecimiento de la bacteria Stepinpoopi se adapta bien a la ley de crecimiento:

$$
\mathrm{r}_{\mathrm{c}}=\mu_{\mathrm{m}} \cdot\left(1-\frac{\mathrm{c}_{\mathrm{c}}}{\mathrm{c}_{\mathrm{m}}}\right) \cdot \mathrm{c}_{\mathrm{c}}
$$

siendo $\mu_{\mathrm{m}}=0.5 \mathrm{~h}^{-1} \mathrm{y} \mathrm{c}_{\mathrm{m}}=20 \mathrm{~kg} / \mathrm{m}^{3}$.

El sustrato se encuentra en exceso.

a) El crecimiento celular se lleva a cabo en un RDTA de 2 litros. Represéntese la velocidad de crecimiento y la concentración de células $\left(\mathrm{k} / \mathrm{m}^{3}\right)$ como funciones del tiempo tras inocular 0.4 gramos de células en el reactor (ignórese la fase de latencia).

b) El reactor del apartado anterior se convierte en un RCTA. Obténgase una ecuación para la velocidad de lavado. Escójanse valores del caudal volumétrico del sustrato entrante y represéntese la concentración celular como una función del tiempo tras la inoculación.

Problema 11. Los datos siguientes se han obtenido para el Pyrodictium occultum a $98^{\circ} \mathrm{C}$. El ciclo 1 se ha realizado en ausencia de extracto de levadura y el ciclo 2 con extracto de levadura. Ambos ciclos contenían inicialmente $\mathrm{Na}_{2} \mathrm{~S}$. El porcentaje volumétrico de $\mathrm{H}_{2} \mathrm{~S}$ recogido (producto del crecimiento) se muestra en la tabla.

\begin{tabular}{|c|c|c|c|c|c|c|c|c|c|}
\hline \multicolumn{10}{|c|}{ Ciclo 1} \\
\hline $\mathrm{t}(\mathrm{h})$ & 0 & 10 & 15 & 20 & 30 & 40 & 50 & 60 & 70 \\
\hline $\begin{array}{c}\text { Densidad celular } \\
(\text { células·10-4/ml) }\end{array}$ & 2.7 & 2.8 & 15 & 70 & 400 & 600 & 775 & 600 & 525 \\
\hline $\begin{array}{c}(\% \text { volumétrico } \\
\left.\mathrm{H}_{2} \mathrm{~S}\right)\end{array}$ & 0.5 & 0.8 & 1.0 & 1.2 & 6.8 & 4.7 & 7.5 & 8.0 & 8.2 \\
\hline
\end{tabular}

\begin{tabular}{|c|c|c|c|c|c|c|c|c|c|}
\hline \multicolumn{10}{|c|}{ Ciclo 2} \\
\hline $\mathrm{t}(\mathrm{h})$ & 0 & 5 & 10 & 15 & 20 & 30 & 40 & 50 & 60 \\
\hline $\begin{array}{c}\text { Densidad celular } \\
(\text { células·10-4/ml) }\end{array}$ & 2.7 & 7 & 11 & 80 & 250 & 350 & 350 & 250 & - \\
\hline $\begin{array}{c}(\% \text { volumétrico } \\
\left.\mathrm{H}_{2} \mathrm{~S}\right)\end{array}$ & 0.1 & 0.7 & 0.7 & 0.8 & 1.2 & 4.3 & 7.5 & 11.0 & 12.3 \\
\hline
\end{tabular}

a) ¿Cuál es el tiempo de latencia con y sin extracto de levadura?

b) ¿Cuál es la diferencia en las velocidades de crecimiento específico, $\mu_{\max }$, de la bacteria con y sin extracto de levadura? 
c) ¿Cuánto dura la fase estacionaria?

d) ¿En qué fase se produce la mayor cantidad de $\mathrm{H}_{2} \mathrm{~S}$ ?

e) El volumen de líquido en el RDTA que se desarrolla la reacción es 0.2 litros. Si el reactor se transformara en un RCTA, ¿cuál sería la velocidad de lavado?

Problema 12. El crecimiento celular con inhibición no competitiva por sustrato tiene lugar en un RCTA. La ley de crecimiento celular es:

$$
\mathrm{r}_{\mathrm{c}}=\frac{\mu_{\max } \cdot \mathrm{c}_{\mathrm{s}} \cdot \mathrm{c}_{\mathrm{c}}}{\mathrm{K}_{\mathrm{s}}+\mathrm{c}_{\mathrm{s}} \cdot\left(1-\frac{\mathrm{c}_{\mathrm{s}}}{\mathrm{K}_{1}}\right)}
$$

$\mu_{\max }=1.5 \mathrm{~h}^{-1} ; \mathrm{K}_{\mathrm{s}}=1 \mathrm{~kg} / \mathrm{m}^{3} ; \mathrm{K}_{1}=50 \mathrm{~kg} / \mathrm{m}^{3}$

$\mathrm{c}_{\mathrm{s} 0}=30 \mathrm{~kg} / \mathrm{m}^{3} ; \mathrm{c}_{\mathrm{c} 0}=0.5 \mathrm{~kg} / \mathrm{m}^{3}$

$\mathrm{Y}_{\mathrm{c} / \mathrm{s}}=0.08 \mathrm{~kg} / \mathrm{kg}$

$\mathrm{D}=0.75 \mathrm{~h}^{-1}$

a) Represéntese en una misma gráfica la concentración celular en estado estacionario, $\mathrm{c}_{\mathrm{c}}$, $\mathrm{y}$ la concentración de sustrato, $\mathrm{c}_{\mathrm{s}}$, como una función de $\mathrm{D}$.

b) Inicialmente se había introducido $0.5 \mathrm{~kg} / \mathrm{m}^{3}$ de bacteria en el tanque de reacción que ya contenía el sustrato y en el que ya se había establecido el flujo de entrada y de salida. Represéntese las concentraciones de bacteria y de sustratos como funciones del tiempo.

Problema 13. Una solución que contiene una bacteria con una concentración de $0.001 \mathrm{~kg} / \mathrm{m}^{3}$ se alimenta a un RSCTA. El nutriente se encuentra en exceso y la ley de crecimiento celular es de primer orden respecto a la concentración celular. El reactor estaba vacío al iniciarse el proceso. Si la concentración de la bacteria en el reactor al cabo de dos horas es $0.025 \mathrm{~kg} / \mathrm{m}^{3}$, ¿cuál es la velocidad de crecimiento específica $\mathrm{k}$ en $\mathrm{min}^{-1}$ ?

Problema 14. El crecimiento de una bacteria B se lleva a cabo en un RCTA. La ley de velocidad del crecimiento de la bacteria es:

$$
\mathrm{r}_{\mathrm{Bc}}=\frac{\mu_{\text {max }} \cdot \mathrm{c}_{\mathrm{s}} \cdot \mathrm{c}_{\mathrm{B}}}{\mathrm{K}_{\mathrm{s}}+\mathrm{c}_{\mathrm{s}}} \cong \mu_{\max } \cdot \mathrm{c}_{\mathrm{B}}
$$

ya que el sustrato se encuentra en gran exceso. Desafortunadamente, de forma inadvertida, una ameba se introduce en el reactor y depreda la bacteria. La ley de crecimiento de la ameba A es:

$$
\mathrm{r}_{\mathrm{Ac}}=\mathrm{k}_{\mathrm{A}} \cdot \mathrm{c}_{\mathrm{A}} \cdot \mathrm{c}_{\mathrm{B}}
$$

y su velocidad de muerte:

$$
\mathrm{r}_{\mathrm{Am}}=\mathrm{k}_{\mathrm{m}} \cdot \mathrm{c}_{\mathrm{A}}
$$


a) Escribir los balances de materia de la ameba y de la bacteria. A continuación asúmase las condiciones de estado estacionario para llegar a obtener una ecuación para la velocidad de dilución. Suponiendo que la velocidad de dilución es la única variable de control, descríbase los pasos a realizar para rectificar la situación y deshacerse de la ameba (supóngase que las condiciones de estado estacionario se alcanzan inmediatamente tras cualquier cambio realizado).

b) Representar la concentración de ameba y de bacteria en función del tiempo.

c) Varíese los valores de los parámetros $\mathrm{k}_{\mathrm{A}} \mathrm{y} \mu_{\max }$ y represéntese la concentración de ameba y de bacteria en función del tiempo. Búsquese cualquier oscilación inusual y/o comportamiento caótico.

\section{Datos y notas}

En el momento en el que la ameba entra en el tanque:

$$
\begin{aligned}
& \mathrm{c}_{\mathrm{B} 0}=1 \mathrm{~kg} / \mathrm{m}^{3} ; \mathrm{c}_{\mathrm{A} 0}=0.1 \mathrm{~kg} / \mathrm{m}^{3} \\
& \mu_{\max }=0.1 \mathrm{~h}^{-1} ; \mathrm{K}_{\mathrm{s}}=0.01 \mathrm{~kg} / \mathrm{m}^{3} ; \mathrm{k}_{\mathrm{A}}=0.5 \mathrm{~m}^{3} / \mathrm{kg} \cdot \mathrm{h}^{-1} ; \mathrm{k}_{\mathrm{m}}=0.01 \mathrm{~h}^{-1} \\
& \mathrm{Y}_{\mathrm{A} / \mathrm{B}}=0.5 \mathrm{~kg} / \mathrm{kg} \\
& \text { (pruébese también con } \mu_{\max }=0.5 \mathrm{~h}^{-1} \mathrm{y} \mathrm{c}_{\mathrm{A} 0}=0.5 \mathrm{~kg} / \mathrm{m}^{3} \text { ) }
\end{aligned}
$$

No hay bacteria ni ameba en la corriente de entrada.

Problema 15. Una corriente de sustrato $\mathrm{S}\left(\mathrm{c}_{\mathrm{s} 0}=3 \mathrm{~kg} / \mathrm{m}^{3}, \mathrm{c}_{\mathrm{c} 0}=0 \mathrm{y} \mathrm{c}_{\mathrm{p} 0}=0\right)$ se descompone de acuerdo con la fermentación microbiana $\mathrm{S}+\mathrm{C} \rightarrow \mathrm{P}+\mathrm{C}$ y según la ley de velocidad:

$$
\mathrm{r}_{\mathrm{c}}=\frac{2 \cdot \mathrm{c}_{\mathrm{s}} \cdot \mathrm{c}_{\mathrm{c}}}{1+\mathrm{c}_{\mathrm{s}}} \quad\left(\frac{\mathrm{kg} \text { de células }}{\mathrm{m}^{3} \cdot \mathrm{h}}\right)
$$

El coeficiente estequiométrico $\mathrm{Y}_{\mathrm{c} / \mathrm{s}}$ es $0.5 \mathrm{~kg}$ células por $\mathrm{kg}$ de sustrato. Determinar el valor de $\mathrm{c}_{\mathrm{s}}$ que se podría obtener en un FCTA de $1 \mathrm{~m}^{3}$ con recirculación con $\alpha=$ 2 y $\beta=1.3$, siendo el caudal de alimentación fresca de $1 \mathrm{~m}^{3} / \mathrm{h}$. Comparar con el correspondiente valor sin recirculación.

Problema 16. Se desea diseñar un proceso para reducir, en condiciones óptimas, la concentración de un sustrato en una corriente estéril desde $\mathrm{c}_{\mathrm{s} 0}=60 \mathrm{a} \mathrm{c}_{\mathrm{s} 0}=3 \mathrm{~g} /$ $\mathrm{m}^{3}$, consistente en una combinación en serie de dos biorreactores, un quimiostato seguido de un reactor en flujo en pistón. El modelo cinético es:

$$
\mathrm{r}_{\mathrm{c}}=\frac{1.333 \cdot \mathrm{c}_{\mathrm{s}} \cdot \mathrm{c}_{\mathrm{c}}}{4+\mathrm{c}_{\mathrm{s}}}\left(\frac{\mathrm{g} \text { de células }}{\mathrm{m}^{3} \cdot \mathrm{h}}\right)
$$

El factor estequiométrico $\mathrm{Y}_{\mathrm{s} / \mathrm{c}}$ vale $10 \mathrm{y}$ el caudal de alimento $2.5 \mathrm{~m}^{3} / \mathrm{h}$. 
1. Determinar el volumen total mínimo y comparar con el resultado obtenido utilizando un solo FCTA.

2. Calcular la razón de recirculación óptima en el caso de utilizar un solo reactor tubular con recirculación. Comparar el volumen de reactor necesario con los valores obtenidos en el apartado anterior.

Problema 17. Se quiere un sistema de fermentación continuo para tratar una alimentación estéril de caudal $250 \mathrm{l} / \mathrm{h}$ con una concentración de sustrato $\mathrm{c}_{\mathrm{s} 0}=25 \mathrm{~g} / \mathrm{l}$, para producir $2100 \mathrm{~kg} /$ año de producto $\mathrm{P}$, considerando 300 días/año y $24 \mathrm{~h} /$ día.

1. ¿Recomendaría un sistema de fermentación con un único biorreactor o con dos biorreactores conectados en serie?

2. Diseñe un sistema de fermentación que, manteniendo la producción señalada, minimice el volumen total de fermentación. Calcular los volúmenes, concentraciones de biomasa, sustrato y producto de todo el sistema.

\section{Datos y notas}

Para obtener el modelo cinético y los coeficientes estequiométricos se han realizado dos experimentos continuos a escala de laboratorio con un quimiostato de 1 litro utilizando una alimentación estéril con una concentración de substrato $\mathrm{c}_{\mathrm{s} 0}=30 \mathrm{~g} / \mathrm{l}$. Los resultados se indican en la tabla siguiente:

\begin{tabular}{|c|c|c|c|}
\hline $\mathrm{Q}_{\mathrm{V} 0}(\mathrm{ml} / \mathrm{h})$ & $\mathrm{c}_{\mathrm{s}}(\mathrm{g} / \mathrm{l})$ & $\mathrm{c}_{\mathrm{c}}(\mathrm{g} / \mathrm{l})$ & $\mathrm{c}_{\mathrm{p}}(\mathrm{g} / \mathrm{l})$ \\
\hline 27.5 & 10.00 & 12.0 & 1.04 \\
\hline 18.8 & 2.32 & 16.7 & 1.44 \\
\hline
\end{tabular}

Problema 18. Para reducir el impacto ambiental y al mismo tiempo producir ácido láctico, el efluente de una fábrica de quesos, con un caudal de $1 \mathrm{~m}^{3} / \mathrm{h}$ y una concentración de $60 \mathrm{~kg}$ de lactosa $/ \mathrm{m}^{3}$, se trata en una planta de fermentación que consiste en un FCTA de $3 \mathrm{~m}^{3}$. El modelo cinético encontrado en la bibliografía señala que la producción del ácido láctico sigue el modelo:

$$
\mathrm{r}_{\mathrm{c}}=\mu_{\max } \cdot\left(1-\frac{\mathrm{c}_{\mathrm{p}}}{\mathrm{c}_{\mathrm{p}}^{*}}\right) \cdot \frac{\mathrm{c}_{\mathrm{s}} \cdot \mathrm{c}_{\mathrm{c}}}{\mathrm{K}_{\mathrm{c}}+\mathrm{c}_{\mathrm{s}}}
$$

donde $\mu_{\max }=0.5 \mathrm{~h}^{-1}, \mathrm{~K}_{\mathrm{c}}=0.3 \mathrm{~kg} / \mathrm{m}^{3}, \mathrm{c}_{\mathrm{p}}{ }^{*}=50 \mathrm{~kg} / \mathrm{m}^{3}$ y los coeficientes estequiométricos valen $\mathrm{Y}_{\mathrm{c} / \mathrm{s}}=0.1 \mathrm{~kg}$ biomasa $/ \mathrm{kg}$ lactosa $\mathrm{e}_{\mathrm{p} / \mathrm{c}}=8 \mathrm{~kg}$ ácido láctico $/ \mathrm{kg}$ biomasa.

1. Determinar si la corriente tratada se puede enviar a la red de aguas residuales considerando que la concentración máxima permitida de lactosa es $12 \mathrm{~kg} / \mathrm{m}^{3}$. 
2. En caso de incumplimiento de la normativa se ha pensado instalar a la salida del FCTA un segundo fermentador de tipo tubular para rebajar la concentración de lactosa hasta los niveles permitidos. ¿Cuál ha de ser el volumen de este segundo reactor?

3. Otra alternativa para cumplir la normativa es la de substituir el FCTA por uno igual pero más grande. ¿Qué volumen debería tener? 


\section{Bibliografía básica}

AtKInson, Bernard, Reactores bioquímicos [versión castellana de Juan Mata Álvarez y José Costa López], Barcelona, Reverte, SA, 1986.

ARIs, Rutherford, Elementary chemical reactor analysis, Boston [etc.], Butterworths cop., 1989.

- Análisis de reactores [versión castellana de José Costa López], Madrid [etc.], Alhambra, 1973.

EsCARDINo, Agustín, Introducció a l'enginyeria dels reactors químics, València, Universitat de València, 2003.

Fogler, H. Scott, Elementos de ingeniería de las reacciones químicas [versión castellana de Roberto Luis Escalona García], México [etc.], Pearson Educación, 2001.

Levenspiel, Octave, El omnilibro de los reactores químicos [versión castellana de J. Costa López y L. Puigjaner Corbella], Barcelona, Reverté cop., 1986.

- Ingeniería de las reacciones químicas [versión castellana de Gabriel Tojo Barreiro], Barcelona [etc.], Reverté cop., 1990.

\section{Bibliografía complementaria}

Belfiore, Lawrence A., Transport phenomena for chemical reactor design, Hoboken, NJ, Wiley \& Sons cop., 2003.

ButT, John B., Reaction kinetics and reactor design, Englewood Cliffs, NJ, Prentice-Hall cop., 1980.

Carberry, J. J. y A. Varma, Chemical reaction and reactor engineering, Nueva York, Marcel Dekker, 1986.

Coker, A. Kayode, Modeling of chemical kinetics and reactor design, Boston [etc.], Gulf Professional Publishing cop., 2001.

Davis, M. E. y R. J. Davis, Fundamentals of chemical reaction engineering, Boston, McGraw-Hill, 2003.

Denbigh, Kenneth George, Chemical reactor theory: an introduction, Cambridge [Cambridgeshire], Nueva York, Cambridge University Press, 1984.

DoraisWAMY, L. K. y M. M. SHARMA, Heterogeneous reactions: analysis, examples and reactor design, Nueva York [etc.], John Wiley and Sons, 1984.

Fogler, H. Scott, Elements of chemical reaction engineering, Upper Saddle River, NJ, Prentice Hall, 2001.

Froment, Gilbert F., Chemical reactor analysis and design, Nueva York, John Wiley \& Sons cop., 1990.

GonzÁlez-Velasco, J. R., et al. Cinética química aplicada, Madrid, Editorial Síntesis, SA, 1999.

HiLl, Charles G., An introduction to chemical engineering kinetics \& reactor design, Nueva York, John Wiley \& Sons cop., 1977.

Holland, Charles D., Fundamentals of chemical reaction engineering, Englewood Cliffs, NJ, Prentice-Hall cop., 1989. 
Joshi, J. B. y L. K. Doraiswamy, «Chemical reaction engineering», Chapter 11, en Chemical Engineer's Handbook, CRC Press, Boca Raton, FL, Ed. L. F. Albright, 2009.

LeE, H. H., Heterogeneous reactor design, Boston, Butteworth, 1984.

LeVenspiel, Octave, The chemical reactor minibook, Corvallis, Oregon [s.n.], osu Bookstores cop., 1979.

Missen, Ronald W., Introduction to chemical reaction engineering and kinetics, Nueva York, 1999.

Nauman, E. B., Chemical reactor design, Nueva York, Wiley cop., 1987.

Petersen, E. E., Chemical reaction analysis, Englewood Cliffs, NJ, Prentice Hall, 1965.

RodríGuez-Renuncio, J. A., et al. Termodinámica química, Madrid, Editorial Síntesis, SA, 1999.

SAntamaría, Jesús, et al. Ingeniería de reactores, Madrid, Editorial Síntesis, SA, 1999.

Schmidt, Lanny D., The engineering of chemical reactions, Nueva York [etc.], Oxford University Press, 1998.

Sмiтн, J. M., Introduction to chemical engineering thermodynamics, Nueva York [etc.], McGraw-Hill, 2001.

Thoenes, Dirk, Chemical reactor development: from laboratory synthesis to industrial production, Dordrecht [etc.], Kluwer Academic Publishers cop., 1994.

WaLAs, Stanley M., Chemical reaction engineering handbook of solved problems, Australia [etc.], Gordon and Breach cop., 1995.

- Reaction kinetics for chemical engineers, Boston, Butterworths cop., 1989.

Westerterp, K. R., Chemical reaction and reactor design, Chischester [etc.], John Wiley, 1997. 